\title{
EFFECTS OF ADDED DRAG ON CETACEANS: FISHING GEAR ENTANGLEMENT AND EXTERNAL TAG ATTACHMENT
}

\author{
by \\ Julie M. van der Hoop \\ B.Sc., Dalhousie University (2010)
}

Submitted in partial fulfillment of the requirements for the degree of

Doctor of Philosophy

at the

MASSACHUSETTS INSTITUTE OF TECHNOLOGY

and the

WOODS HOLE OCEANOGRAPHIC INSTITUTION

February 2017

(C)2017 Julie M. van der Hoop. All rights reserved.

The author hereby grants to MIT and WHOI permission to reproduce and to distribute publicly paper and electronic copies of this thesis document in whole or in part in any medium now known or hereafter created.

Author

Joint Program in Applied Ocean Science \& Engineering Massachusetts Institute of Technology \& Woods Hole Oceanographic Institution October 4, 2016

Certified by

Dr. Michael J. Moore Senior Research Specialist Biology Department Woods Hole Oceanographic Institution

Thesis Supervisor

Accepted by

Dr. Ann Tarrant

Chair, Joint Committee for Biological Oceanography Massachusetts Institute of Technology Woods Hole Oceanographic Institution 


\title{
EFFECTS OF ADDED DRAG ON CETACEANS: FISHING GEAR ENTANGLEMENT AND EXTERNAL TAG ATTACHMENT
}

by

Julie M. van der Hoop

\begin{abstract}
Submitted to the MIT-WHOI Joint Program in Oceanography and Applied Ocean Science and Engineering on October 4, 2016, in partial fulfillment of the requirements for the degree of Doctor of Philosophy in Biological Oceanography
\end{abstract}

\begin{abstract}
Animal movement is motivated in part by energetic constraints, where fitness is maximized by minimizing energy consumption. The energetic cost of movement depends on the resistive forces acting on an animal; changes in this force balance can occur naturally or unnaturally. Fishing gear that entangles large whales adds drag, often altering energy balance to the point of terminal emaciation. An analog to this is drag from tags attached to cetaceans for research and monitoring. This thesis quantifies the effects of drag loading from these two scenarios on fine-scale movements, behaviors and energy consumption.

I measured drag forces on fishing gear that entangled endangered North Atlantic right whales and combined these measurements with theoretical estimates of drag on whales' bodies. Entanglement in fishing gear increased drag forces by up to 3 fold. Bio-logging tags deployed on two entangled right whales recorded changes in the diving and fine-scale movement patterns of these whales in response to relative changes in drag and buoyancy from fishing gear and through disentanglement: some swimming patterns were consistently modulated in response. Disentanglement significantly altered dive behavior, and can affect thrust production. Changes in the force balance and swimming behaviors have implications for the survival of chronically entangled whales. I developed two bioenergetics approaches to estimate that chronic, lethal entanglements cost approximately the same amount as the cost of pregnancy and supporting a calf to near-weaning. I then developed a method to estimate drag, energy burden and survival of an entangled whale at detection. This application is essential for disentanglement response and protected species management.

Experiments with tagged bottlenose dolphins suggest similar responses to added drag: I determined that instrumented animals slow down to avoid additional energetic costs associated with drag from small bio-logging tags, and incrementally decrease swim speed as drag increases. Metabolic impacts are measurable when speed is constrained. I measured the drag forces on these tags and developed guidelines depending on the relative size of instruments to study-species.

Together, these studies quantify the magnitude of added drag in complementary systems, and demonstrate how animals alter their movement to navigate changes in their energy landscape associated with increased drag.

Thesis Supervisor: Dr. Michael J. Moore

Title: Senior Research Specialist

Biology Department

Woods Hole Oceanographic Institution
\end{abstract}




\section{ACKNOWLEDGMENTS}

Throughout graduate school, my research has been funded by the M. S. Worthington Foundation, the North Pond Foundation, Sloan and Hardwick Simmons, the Herrington-Fitch Family Foundation, the National Oceanographic Partnership Program [National Science Foundation via the Office of Naval Research N00014-11-1-0113], the Cooperative Institute for the North Atlantic Region [CINAR; NA14OAR4320158], and NOAA Cooperative Agreements POEA133F09SE4792 and NA09OAR4320129. A Postgraduate Scholarship from the Natural Sciences and Engineering Research Council of Canada, and the MIT Martin Family for Sustainability and WHOI-Duke Fellowships gave me flexibility in pursuing my academic interests.

Collaboration has made this work possible. I gratefully acknowledge Florida FWC, EcoHealth Alliance, Georgia DNR, the Center for Coastal Studies, Georgia Aquarium, St. Johns County's Beach Services and Environmental Division, Hubbs-Sea World, University of Florida, the Atlantic Large Whale Disentanglement Network, Doug Nowacek, and many others who assisted with right whale tagging, disentanglement and necropsy. Permits include NOAA Permit 932-1905-00/MA-009526 issued to T. Rowles and NMFS Permit 14791 issued to D. Nowacek. Four chapters of this thesis rely on data from a single day on the RV Tioga - thank you to the crew for assistance and to members of the ALWDN for collecting and cataloging of entangling fishing gear, the North Atlantic Right Whale Consortium for data access, and all who contribute to the database. I give special thanks to Dolphin Quest Oahu whose dolphin interactive facility served as a critically important controlled research environment. Thank you to Julie Rocho-Levine and the crew at DQ, who along with Alex Shorter, showed me the importance of patience and positivity in field work. Thank you to Scott Kraus for the use of the RC boat, which made my thesis "so much cooler" according to some. Thank you to all my co-authors, listed at the beginning of each chapter. And of course, to the animals, who contributed to this research and to a greater understanding of issues facing many marine mammal populations.

Beyond this, I have to thank those who have contributed to my thesis, my research, my intellectual development and my personal growth.

To my committee members - Peter Corkeron, Andreas Fahlman, Aran Mooney and Michael Triantafyllou - your diverse backgrounds and knowledge helped me view this thesis as a body of work with complementary pieces. I am thankful to Becky Gast for her support as Education Coordinator, as previous chair of JCBO and as the chair of my defense. Thank you to the Biology department, JCBO, and WHOI's academic programs for supporting my participation in diverse initiatives and conferences throughout my career so far. Thank you to my undergraduate advisor, Christopher Taggart, for being rigorous and critical yet so encouraging. 
To my advisor - Michael Moore. I cannot believe that a two-line email exchange in 2010 and your phone call from a necropsy site in Argentina could have led me to this point. Thank you for pushing me to answer the toughest questions, for your patience as I explored how to answer them rigorously, and for including me from the beginning. You have shown tremendous example of tenacity and dedication in science, balanced with appreciation for life and family.

Michael has been extremely supportive of my undertaking personal research involving oxygen consumption, power output and drag. Cycling has offered me another environment in which to challenge myself while helping balance the rigors of academic life. I am grateful to my teammates and friends in Boston, on the MIT Cycling team, and in the NECX community for their support on and off the bike.

In 2011, I embarked on the SV Corwith Cramer with a bunch of strangers as we began our PhDs. Since then I have been so fortunate to have been surrounded by the most lively and loving individuals as we have grown in this small community. Thank you for world travels and local adventures, P-Parties and P-set parties, and both side-splitting laughter and times of support. Thank you to \#w1lson, the supreme egg coop, and the fish bowl. Finally, thank you to my family for always reminding me of my roots but never questioning my desire to pursue opportunities far from home.

and Michael, I feel pretty triumphant right now.

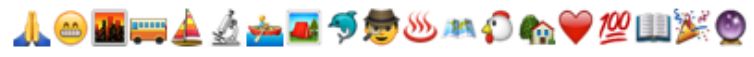




\section{Contents}

1 Introduction $\quad 21$

1.1 Hydrostatic equilibrium . . . . . . . . . . . . . . . . . . . . 22

1.2 Cetacean swimming . . . . . . . . . . . . . . . . . . 23

1.3 Changes in force balance . . . . . . . . . . . . . . . . . . 23

1.4 Changes in swimming movements . . . . . . . . . . . . . . . 25

1.5 Thesis overview . . . . . . . . . . . . . . . . . 26

1.5.1 North Atlantic right whale entanglement . . . . . . . . . . . . 26

1.5.2 Tag drag . . . . . . . . . . . . . . . . . . . . . . 27

2 Behavioral impacts of disentanglement of a right whale under sedation $\begin{array}{ll}\text { and the energetic cost of entanglement } & 29\end{array}$

2.1 Abstract . . . . . . . . . . . . . . . . . . . . . . 30

2.2 Introduction . . . . . . . . . . . . . . . . . . 30

2.3 Methods . . . . . . . . . . . . . . . . . . . . 31

2.3.1 Sedation . . . . . . . . . . . . . . . . . 32

2.3 .2 Tagging and behavior . . . . . . . . . . . . . . . 33

2.3.3 Dive parameters . . . . . . . . . . . . . . . . . . . . 34

2.3 .4 Dive shape . . . . . . . . . . . . . . . . . . . 35

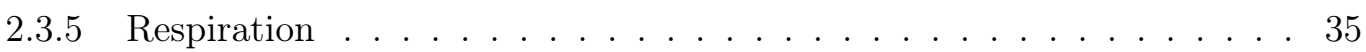

2.3.6 Proxies for thrust . . . . . . . . . . . . . . . . . . . 35

2.3.7 Overall Dynamic Body Acceleration (ODBA) . . . . . . . . . . . 36

2.3.8 Phase definitions and statistical analyses . . . . . . . . . . . 36

2.3 .9 Gear towing . . . . . . . . . . . . . . . . . . 37

2.3.10 Energy requirements . . . . . . . . . . . . . . . . . . . . 39

2.4 Results . . . . . . . . . . . . . . . . . . . . . . . . 42

2.4.1 Tagging and behavior . . . . . . . . . . . . . . 42

2.4 .2 Dive shape . . . . . . . . . . . . . . . . . . . . . 43

2.4 .3 Respiration . . . . . . . . . . . . . . . . 44

2.4.4 Proxies for thrust . . . . . . . . . . . . . . . . . . 44

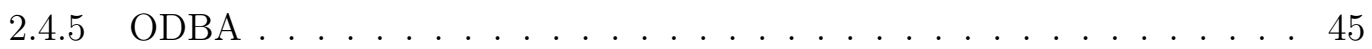

2.4.6 Gear towing . . . . . . . . . . . . . . . . . . . 47

2.4 .7 Energy requirements . . . . . . . . . . . . . . . . . . 47 


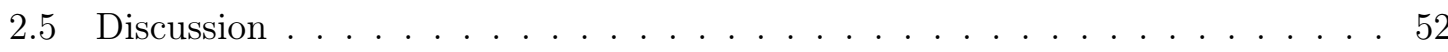

3 Drag from fishing gear entangling North Atlantic right whales $\quad 57$

3.1 Abstract . . . . . . . . . . . . . . . . . . . . 58

3.2 Introduction . . . . . . . . . . . . . . . . . . . 58

3.3 Materials and Methods . . . . . . . . . . . . . . . . . . . . . . 59

3.3.1 Experimental gear description . . . . . . . . . . . . . . . . . 59

3.3 .2 Tow experiment . . . . . . . . . . . . . . . . . . 60

3.3 .3 Analysis . . . . . . . . . . . . . . . . . . . . . . . 62

3.3.4 Entangling gear description . . . . . . . . . . . . . . . . 62

3.3.5 Effect of partial gear removal . . . . . . . . . . . . . . . . . . 64

3.3.6 Entangled whale drag . . . . . . . . . . . . . . . . . . . . . . . . 64

3.4 Results . . . . . . . . . . . . . . . . . . . 68

3.4.1 Entangling gear description . . . . . . . . . . . . . . 68

3.4 .2 Effect of partial gear removal . . . . . . . . . . . . . . . 71

3.4 .3 Entangled whale drag . . . . . . . . . . . . . . . . . . . . 71

3.5 Discussion . . . . . . . . . . . . . . . . . . . 73

3.6 Conclusions . . . . . . . . . . . . . . . . . . . . 78

4 Swimming kinematics and efficiency of entangled North Atlantic right $\begin{array}{ll}\text { whales } & 81\end{array}$

4.1 Abstract . . . . . . . . . . . . . . . . . . . . . 82

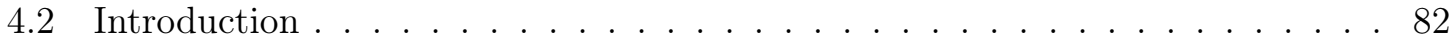

4.3 Methods . . . . . . . . . . . . . . . . . . . . . 84

4.3 .1 Case histories . . . . . . . . . . . . . . . . . . . 84

4.3.2 Tag data analysis . . . . . . . . . . . . . . . . . . . 85

4.3.3 Thrust, efficiency and power . . . . . . . . . . . . . . 87

4.3 .4 Statistical analysis . . . . . . . . . . . . . . . . . . . . 89

4.4 Results . . . . . . . . . . . . . . . . . . . . . . . 90

4.4 .1 Case histories . . . . . . . . . . . . . . . . . . . . 90

4.4 .2 Dive parameters . . . . . . . . . . . . . . . . . . . . 91

4.4 .3 Kinematic parameters . . . . . . . . . . . . . . . . . 91

4.4.4 Thrust, efficiency and power . . . . . . . . . . . . . . . 98

4.5 Discussion . . . . . . . . . . . . . . . . . . . . . . . . . . . . . 99

4.5.1 Diving behavior . . . . . . . . . . . . . . . . . . 101

4.5.2 Kinematic responses . . . . . . . . . . . . . . . . . . 103

4.5.3 Changes in efficiency . . . . . . . . . . . . . . . . . . . . . . . . 104

4.5.4 Efficiency considerations . . . . . . . . . . . . . . . . . 105

4.5.5 Conclusions . . . . . . . . . . . . . . . . . . . 107 
5 Entanglement is a costly life history stage in large whales 109

5.1 Abstract . . . . . . . . . . . . . . . . . . . 110

5.2 Introduction . . . . . . . . . . . . . . . . . . . 110

5.3 Methods . . . . . . . . . . . . . . . . . . . . . . . 112

5.3.1 Changes in blubber thickness and volume . . . . . . . . . . . 112

5.3 .2 Increased power requirements . . . . . . . . . . . . . . . . . 114

5.3 .3 Life history context . . . . . . . . . . . . . . . . 115

5.4 Results . . . . . . . . . . . . . . . . . . . . . 118

$5.4 .1 \quad$ Blubber thickness . . . . . . . . . . . . . . . . . . . . 118

5.4 .2 Increased power requirements . . . . . . . . . . . . . . . . 118

5.4 .3 Life history context . . . . . . . . . . . . . . . . . 124

5.5 Discussion . . . . . . . . . . . . . . . . . . . . 125

5.5.1 Entanglement cost and survival . . . . . . . . . . . . . . 126

5.5 .2 Entanglement in the context of life history . . . . . . . . . . . . . . 127

5.5 .3 Energetic costs and reproduction . . . . . . . . . . . . 127

5.5.4 Comparing the bioenergetic methods . . . . . . . . . . . . . . 129

5.6 Conclusions . . . . . . . . . . . . . . . . . . . . . . . . 131

6 Predicting lethal entanglements as a consequence of drag from fishing gear 133

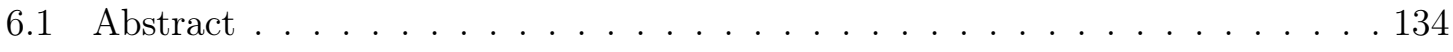

6.2 Highlights . . . . . . . . . . . . . . . . . . . . . 134

6.3 Graphical abstract . . . . . . . . . . . . . . . . . . . 134

6.4 Introduction . . . . . . . . . . . . . . . . . . . . 135

6.5 Methods . . . . . . . . . . . . . . . . . 136

6.5.1 Measured gear sets . . . . . . . . . . . . . . 137

6.5.2 Non-Measured gear sets . . . . . . . . . . . . . . . . . . 138

6.5.3 Disentanglement response . . . . . . . . . . . . . . . . . . . . . . . 139

6.5.4 Sensitivity to gear parameters . . . . . . . . . . . . . . . . 140

6.6 Results . . . . . . . . . . . . . . . . . . . . 140

6.6 .1 Non-measured gear sets . . . . . . . . . . . . . . . . . 140

6.6 .2 Disentanglement response . . . . . . . . . . . . . . . . . . 145

6.6 .3 Sensitivity to gear parameters . . . . . . . . . . . . . . . 145

6.7 Discussion . . . . . . . . . . . . . . . . . . . . . 146

6.7.1 Assessing documented entanglements . . . . . . . . . . . . . . 147

6.7.2 Application to entanglement assessment: Real-time . . . . . . . . . . . 149

6.7.3 Application to entanglement assessment: Federal injury assessment . . 150

6.7.4 Methodological considerations . . . . . . . . . . . . . . . . . . . 152

6.7 .5 Acknowledging variability . . . . . . . . . . . . . . . . 154 
7 Bottlenose dolphins modify behavior to reduce metabolic effect of tag attachment $\quad 157$

7.1 Abstract . . . . . . . . . . . . . . . . . . . . . 158

7.2 Introduction . . . . . . . . . . . . . . . . . . . . 158

7.3 Results . . . . . . . . . . . . . . . . . . . . 160

7.4 Discussion . . . . . . . . . . . . . . . . . . . . . . . . . . 162

7.5 Conclusions . . . . . . . . . . . . . . . . . . . . . 168

7.6 Materials and Methods . . . . . . . . . . . . . . . . . 168

7.6.1 CFD and conceptual model . . . . . . . . . . . . . . . . . 168

7.6 .2 Experiment . . . . . . . . . . . . . . . . . . . 170

7.6 .3 Tags . . . . . . . . . . . . . . . . . . . . . . 171

7.6 .4 Respirometry . . . . . . . . . . . . . . . 171

7.6 .5 Statistical analysis . . . . . . . . . . . . . . . . 173

8 Hydrodynamic drag of bio-logging tags on bottlenose dolphins $\quad 175$

8.1 Abstract . . . . . . . . . . . . . . . . . . . . 176

8.2 Introduction . . . . . . . . . . . . . . . . . 176

8.3 Methods . . . . . . . . . . . . . . . . . . . . 178

8.3.1 Computational Fluid Dynamics (CFD) . . . . . . . . . . 178

8.3 .2 Experimental setup . . . . . . . . . . . . . . . . . 180

8.3 .3 Video analysis . . . . . . . . . . . . . . . . . . . . . . . . 180

8.3.4 Video $C_{d}$ : Time-varying . . . . . . . . . . . . . . . . . . . 181

8.3.5 Video $C_{d}$ : Velocity fit . . . . . . . . . . . . . . . . . 182

8.3 .6 Drag comparison . . . . . . . . . . . . . . . . . . . 183

8.3 .7 Statistical analysis . . . . . . . . . . . . . . . . . . 184

8.4 Results . . . . . . . . . . . . . . . . . . . . . . . . . 184

8.4.1 Drag Coefficients - Computational Fluid Dynamics . . . . . . . . . . . 184

8.4.2 Drag coefficients - Video - Time-varying . . . . . . . . . . . . . . . . . 184

8.4.3 Drag coefficients - Video - Velocity fit . . . . . . . . . . . . . . 185

8.4 .4 Comparison of methods . . . . . . . . . . . . . . . . 185

8.4 .5 Tag effect . . . . . . . . . . . . . . . . 187

8.5 Discussion . . . . . . . . . . . . . . . . . . . . . . . . . 188

8.5.1 Comparison of methods . . . . . . . . . . . . . . . . . . 189

8.5.2 Use of tag data to estimate drag . . . . . . . . . . . . . . 190

8.5.3 Simulated, measured and natural speeds . . . . . . . . . . . . . . 191

8.5.4 Tag effect . . . . . . . . . . . . . . . . . . . . . . . 192

8.5.5 Tag size recommendations . . . . . . . . . . . . . 193 
9 Energy economy in bottlenose dolphins under different levels of instru$\begin{array}{lr}\text { mentation drag loading } & 197\end{array}$

9.1 Abstract . . . . . . . . . . . . . . . . . . . . . . . . 198

9.2 Introduction . . . . . . . . . . . . . . . . . . . . . 198

9.3 Methods . . . . . . . . . . . . . . . . . . . . . . . . . . . . 199

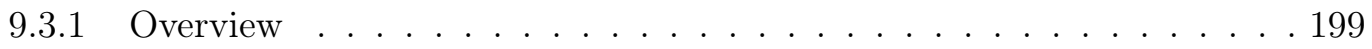

9.3.2 Drag conditions . . . . . . . . . . . . . . . . 200

9.3.3 Swimming task 1: Metabolic trials . . . . . . . . . . . . . . 201

9.3.4 Swimming task 2: Incremental loading trials . . . . . . . . . . . . 202

9.3.5 Respirometry . . . . . . . . . . . . . . . . . . . . . 202

9.3 .6 Lap timing . . . . . . . . . . . . . . . . . . . . . . . . . . . . . . 204

9.3.7 Aerial video . . . . . . . . . . . . . . . . . . . . . . . . 204

9.3.8 Expected drag and speeds . . . . . . . . . . . . . . 204

9.3 .9 Tag analysis . . . . . . . . . . . . . . . . 206

9.3 .10 Statistical analysis . . . . . . . . . . . . . . . 206

9.4 Results . . . . . . . . . . . . . . . . . . . . . 206

9.4.1 Metabolic trials . . . . . . . . . . . . . 206

9.4 .2 Incremental loading trials . . . . . . . . . . . . . . . . 207

9.4 .3 Tag analysis . . . . . . . . . . . . . . . . . . . . . 210

9.5 Discussion . . . . . . . . . . . . . . . . . . . . . . . 210

9.6 Conclusions . . . . . . . . . . . . . . . . . . . 215

10 Consequences of tag-related drag loading in dolphins when circumstances $\begin{array}{lr}\text { preclude slowing down } & 217\end{array}$

10.1 Abstract . . . . . . . . . . . . . . . . . . . 218

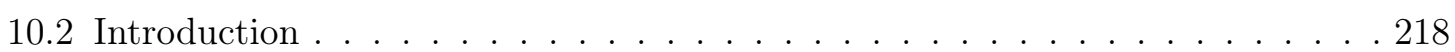

10.3 Methods . . . . . . . . . . . . . . . . . . . . . . . 219

10.3.1 Overview . . . . . . . . . . . . . . . . . . . . . . 219

10.3.2 Drag conditions . . . . . . . . . . . . . . . . . 220

10.3.3 Swimming task . . . . . . . . . . . . . . . . . . . . . 221

10.3.4 Respirometry . . . . . . . . . . . . . . . . . . . . . 221

10.3.5 Aerial video . . . . . . . . . . . . . . . . . . . . . . . . . 222

10.4 Results . . . . . . . . . . . . . . . . . . . . . . 222

10.5 Discussion . . . . . . . . . . . . . . . . . . . . . . 223

11 Conclusion and Outlook 227

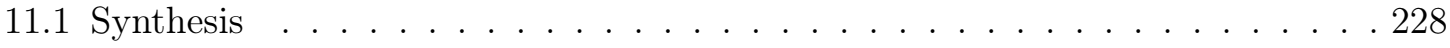


11.1.1 Energy economy . . . . . . . . . . . . . . . . . . . . 228

11.1.2 Swimming efficiency . . . . . . . . . . . . . . . . . . . 229

11.1.3 Positioning of added drag elements . . . . . . . . . . . . . . . 229

11.1.4 Duration of impact . . . . . . . . . . . . . . . . . . 230

11.1.5 Relative impact of tags vs. entanglement in dolphins vs. whales . . . . 231

11.2 Next steps . . . . . . . . . . . . . . . . . . . . . . . . . 231

11.2 .1 Tag drag . . . . . . . . . . . . . . . . . . . . . 231

11.2 .2 Entanglement drag . . . . . . . . . . . . . . . . . . 232

A Chapter 2 Supplemental Information $\quad 239$

A.1 Supplemental Material . . . . . . . . . . . . . . . . . . . . . . . 240

B Chapter 3 Supplemental Information $\quad 241$

B.1 Estimating wetted surface area and Froude number of gear configurations. . . 242

B.1.1 Wetted surface area estimates . . . . . . . . . . . . . . . . . . . 242

B.1.2 Froude number estimates . . . . . . . . . . . . . . . . 244

B.2 Sets of fishing gear removed from entangled North Atlantic right whales used in this experiment . . . . . . . . . . . . . . . . . . . 245

B.3 Supplemental Figures . . . . . . . . . . . . . . . . . . . . . . 261

C Chapter 4 Supplemental Information $\quad 263$

C.1 Supplemental Figures . . . . . . . . . . . . . . . . . . . . . . 264

D Chapter 5 Supplemental Information $\quad 267$

D.1 Supplemental Figures . . . . . . . . . . . . . . . . . . . . . . 268

E Chapter 6 Supplemental Information $\quad 269$

E.1 Wetted areas of floats or traps on unmeasured gear sets. . . . . . . . . . 270

E.2 Guide for calculating minimum entanglement duration for Serious Injury (SI) determinations . . . . . . . . . . . . . . . . . . 271

E.2.1 Motivation . . . . . . . . . . . . . . . . 271

E.2.2 Procedure . . . . . . . . . . . . . . . . . . . . . 271

E.3 Changes in whale drag with body dimensions . . . . . . . . . . . . . 272

E.3.1 Motivation . . . . . . . . . . . . . . . . 272

E.3.2 Methods . . . . . . . . . . . . . . . . . . . 273

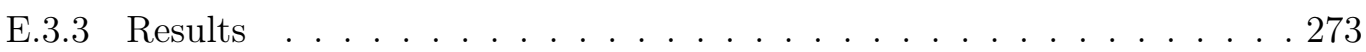

$\begin{array}{lll}\text { F Chapter } 8 \text { Supplemental Information } & 277\end{array}$

F.1 Supplemental Figures . . . . . . . . . . . . . . . . . . . . . 278 
G Chapter 9 Supplemental Information $\quad 279$

G.1 Supplemental Figures . . . . . . . . . . . . . . . . . . . . 280

H Chapter 10 Supplemental Information 281

H.1 Supplemental Figures . . . . . . . . . . . . . . . . . . . . . . . . . . . . . 282 


\section{List OF FiguRES}

1-1 Forces on a swimming cetacean . . . . . . . . . . . . . . . 23

1-2 Trade-offs an animal faces under drag loading . . . . . . . . . . . . . 26

2-1 Aerial photograph of right whale Eg 3911 on 30 Dec 2010 . . . . . . . . . . . 32

2-2 Satellite telemetry track of right whale Eg 3911 swimming entangled from 25 December 2010 to 15 January 2011 . . . . . . . . . . . . . . . . 33

2-3 Location of attachment of a suction-cup attached Dtag on right whale Eg 391134

2-4 Fishing gear removed from right whale Eg 3911 on 15 January 2011 . . . . . . 38

2-5 Dive profile of right whale Eg 3911 . . . . . . . . . . . . . . . 43

2-6 Dive parameters of right whale Eg 3911 separated into phases. . . . . . . . . . 44

2-7 Dive profiles, maximum dive areas, and the calculated Dive Area Ratio (DAR) of Eg 3911 during phases of disentanglement . . . . . . . . . . . . . . . . 45

2-8 Kinematic parameters of right whale Eg 3911 in different phases of disentanglement . . . . . . . . . . . . . . . . . 46

2-9 Drag coefficient of right whale Eg 3911 when nonentangled or entangled in tested gear configurations . . . . . . . . . . . . . . . . . 51

2-10 Power input of right whale Eg 3911 when nonentangled and entangled in tested gear configurations . . . . . . . . . . . . . . 51

3-1 The experimental setup . . . . . . . . . . . . . . . . 60 60

3-2 Entangling gear configurations on North Atlantic right whales . . . . . . . . 61

3-3 Drag on sets of entangling fishing gear is highly variable . . . . . . . . . . 69

3-4 Hierarchical clustering of entangling fishing gear sets . . . . . . . . . . . 70

3-5 Predictions of mean drag from gear weight and length . . . . . . . . . . . 72

3-6 Drag force decreases with towed line length . . . . . . . . . . . . 73

3-7 Estimated total body drag on entangled and nonentangled whales and entangling gears . . . . . . . . . . . . . . . . . . . . . . 74

3-8 Contributions of whale body drag, estimated interference drag, and measured drag on entangling gear for 15 entangled North Atlantic right whales . . . . . 74

4-1 Depth distributions and dive profiles of right whales Eg 3911 and Eg 4057 . . 85

4-2 Right whales show different fluke stroke patterns between individuals, dive phases and drag conditions. . . . . . . . . . . . . . . . . . . . . . 94 
4-3 Right whales alter fluke stroke frequency and amplitude, and glide behavior, under low- and high-drag and buoyancy conditions . . . . . . . . . . . . 95

4-4 Depth distributions and dive profiles of right whales Eg 3911 and Eg 4057 . . 96

4-5 Disentanglement significantly reduces thrust coefficients and increases ideal efficiency for an entangled right whale Eg 3911 . . . . . . . . . . . . . . . . . 99

4-6 Strouhal number and propulsive efficiency are significantly different between dive descents and ascents but do not differ between low drag and high drag . 100

4-7 Thrust power is significantly lower on dive descent than ascent and in low drag compared to high drag conditions for right whale Eg 3911 . . . . . . . 100

5-1 Blubber volume estimation for a North Atlantic right whale . . . . . . . . . 120

5-2 Energetic costs from drag associated with entanglement in fishing gear compared to costs of other life history events . . . . . . . . . . . . . . . . . . . 121

5-3 Dorsal axillary blubber thickness measured at necropsy in juvenile and adult North Atlantic right whales whose cause of death was not related to entanglement or due to entanglement. . . . . . . . . . . . . . . . . . 122

5-4 Power and work output required for entangled right whales . . . . . . . . . . 124

5-5 Relative impacts of entanglement on female energy budgets . . . . . . . . 125

6-1 Expected theoretical forces vs. measured drag forces on five lengths rope . . . 141

6-2 Expected theoretical forces vs. measured drag forces on fishing gear removed from or similar to that which entangles North Atlantic right whales . . . . . . 142

6-3 Drag forces, total work, additional work, estimated critical durations and observed entanglement durations for ten North Atlantic right whales . . . . . 143

6-4 Partial disentanglement reduces drag and work, and increases critical entanglement duration . . . . . . . . . . . . . . . . . . . . . . 145

6-5 Uncertainty in gear configuration affects critical duration estimate by $\sim 3$ months 146

6-6 Minimum and maximum entanglement durations of North Atlantic right whales149

6-7 Sighting and entanglement histories of three North Atlantic right whales . . . 151

7-1 Bio-logging tags increase the drag forces experienced by bottlenose dolphins . 161

7-2 Bio-logging tags do not significantly affect the metabolic rate of bottlenose dolphins, but tagged dolphins swim at significantly lower speeds . . . . . . . . 162

7-3 The experimental setup . . . . . . . . . . . . . . . . . . . 164

7-4 Lift and drag forces on a DTAG2 increase with speed . . . . . . . . . . 165

8-1 The experimental setup _ . . . . . . . . . . . . . . . . . . . 179

8-2 Methods to estimate drag coefficients from underwater video data . . . . . . . 182

8-3 Computational Fluid Dynamics simulation results from the dolphin and combined dolphin-tag models . . . . . . . . . . . . . . . . . . 185 
8-4 Computational Fluid Dynamics simulation results from the dolphin and combined dolphin-tag models . . . . . . . . . . . . . . . . . . . . . 187

8-5 Drag coefficients of dolphins and porpoises estimated in historical data compared to this study . . . . . . . . . . . . . . . . . . . . 188

8-6 Drag coefficients and forces on tagged dolphins with three methods . . . . . 188

8-7 Success rates of glide performance of two male bottlenose dolphins . . . . . . 189

8-8 Recommended tag dimensions for cetacean species based on their body length 194

9-1 The experimental setup _. . . . . . . . . . . . . . . . . . . 201

9-2 Drag forces on a bottlenose dolphin and a dolphin instrumented with tags of increasing size . . . . . . . . . . . . . . . . . . . . . 205

9-3 Physical Activity Ratio of swimming trials . . . . . . . . . . . . . . . 207

9-4 Oxygen consumption rates of dolphins after swimming trials . . . . . . . 208

9-5 Mean lap duration increases with drag loading . . . . . . . . . . . . . . . 208

9-6 Dolphins progressively changed swimming paths with drag unloading and loading . . . . . . . . . . . . . . . . . . . . . . . . . 209

9-7 Dolphins changed speed with incremental changes in drag loading, in line with what is expected from simulation models . . . . . . . . . . . . . . . 210

9-8 Dolphins swam in surface waters in both drag conditions . . . . . . . . . . . 211

9-9 Observed water flow patterns over the tag were similar to those simulated with CFD . . . . . . . . . . . . . . . . . . . . . 211

10-1 The experimental setup _ . . . . . . . . . . . . . . . . 220

10-2 Energy expenditure of bottlenose dolphins increased under drag loading . . . 223

10-3 Dolphins maintained their lap duration when following a remote-controlled boat . . . . . . . . . . . . . . . . . . . . . . . 224

10-4 Energy expenditure of bottlenose dolphins increased under drag loading . . . 225

B.1 The satellite telemetry buoy used for tracking entangled whales . . . . . . . 261

B.2 Groups of gear have similar drag coefficients and responses to depth and speed. . . . . . . . . . . . . . . . . . . . 261

B.3 Froude number with speed fishing gear removed from entangled right whales and the satellite telemetry buoy . . . . . . . . . . . . . . . . . 262

B.4 Changes in drag coefficient with towed line length, Reynolds number and speed 262

C.1 Drag forces on two right whales when not entangled and when entangled in fishing gear . . . . . . . . . . . . . . . . . . . . . . . 264

C.2 Description of the estimation of propulsive efficiency from tag data and studies on flexible foils. . . . . . . . . . . . . . . . . . . . . . 265

C.3 Thrust coefficients on dive descent and ascent of right whale Eg 3911 . . . . 266 
D.1 Time course of disentanglement for two North Atlantic right whales . . . . . 268

E.1 Critical Duration and Serious Injury evaluation flow chart . . . . . . . . 272

E.2 Drag forces and coefficients of right whales of different body dimensions . . 274

E.3 Total body drag force and coefficient as a right whale ages . . . . . . . . . . 275

E.4 Total body drag force and coefficient as a right whale loses body condition . . 275

F.1 The dolphin-tag model in the computational domain . . . . . . . . . . 278

F.2 Percentage increase in drag coefficient with increasing tag wetted and frontal area contribution to the tag wearer . . . . . . . . . . . . 278

G.1 Lap duration for trials paced with a remote controlled boat and with no prescribed swimming pace . . . . . . . . . . . . . . . . . . 280

H.1 End-expiratory carbon dioxide concentrations and the relationship between instantaneous oxygen consumption and carbon dioxide production for two bottlenose dolphins . . . . . . . . . . . . . . . . . . . . . . . 282 


\section{LIST OF TABLES}

2-1 Timeline of events on 15 January 2011 in Sedation/Entangled, Disentangled, and Recovery phases of Eg $3911 \ldots$. . . . . . . . . . . . . . . . 37

2-2 List of symbols and abbreviations . . . . . . . . . . . . . . . . . . 48

2-3 Respiration rate and dive parameters for Eg 3911 before and following sedation injection, but prior to gear and buoy removal . . . . . . . . . . . . . . . 49

2-4 Drag forces exerted by fishing gear and buoy configurations . . . . . . . . . . 49

2-5 Drag forces and consequences on Eg 3911 swimming entangled in various gear configurations . . . . . . . . . . . . . . . . . 5 50

3-1 List of symbols and abbreviations . . . . . . . . . . . . . . . . 63

3-2 Identification numbers, age, length and weight of North Atlantic right whales at the onset of their entanglement, and their entanglement duration, minimum distance traveled, and fold increase in drag from the entangling gear . . . . . 66

4-1 List of symbols and abbreviations . . . . . . . . . . . . . . . . . 86

4-2 Added drag and buoyant forces on right whales Eg 3911 and Eg 4057 before and after disentanglement response efforts . . . . . . . . . . . . . . . . 90

4-3 Observed and expected descent and ascent rates for right whales Eg 3911 and 4057 under low and high drag conditions . . . . . . . . . . . . . . . . . . . 92

4-4 Fluke stroke cycle duration and fluke stroke rates for two right whales Eg 3911 and $\operatorname{Eg} 4057 \ldots \ldots$. . . . . . . . . . . . . . . . . . . . . 97

4-5 Propulsive efficiency and thrust power of right whale Eg 3911 in low and high drag and buoyancy conditions, on descents and ascents of dives to $>5 \mathrm{~m}$. . . . 101

5-1 List of symbols and abbreviations . . . . . . . . . . . . . . . . . . 113

5-2 Identification number, age, sex, length, weight, date of necropsy and measured dorsal blubber thickness of right whales that died from entanglement or other causes . . . . . . . . . . . . . . . . . . . . . . . 119

5-3 Details of fishing gear entanglements whose drag forces were measured . . . . 123

5-4 Additional energy expenditure and power output of entangled North Atlantic right whales . . . . . . . . . . . . . . . . . . . . . . . 124

6-1 List of symbols and abbreviations . . . . . . . . . . . . . . 136

6-2 Details of fishing gear entanglements whose drag forces were estimated . . . . 144 
7-1 Body sizes, resting metabolic rates and order of experimental trials . . . . . . 161

7-2 Oxygen consumption rates, physical activity ratios, respiratory exchange ratios and costs of transport . . . . . . . . . . . . . . . . . . . . 163

7-3 List of symbols and abbreviations . . . . . . . . . . . . . . . . . . 169

8-1 Measurements of the dolphins in this study and the number of trials they performed . . . . . . . . . . . . . . . . . . . . 181

$8-2 \quad$ List of symbols and abbreviations . . . . . . . . . . . . . . 183

8-3 Dimensions and drag coefficients of the tag and tag + configurations from three methods . . . . . . . . . . . . . . . . . . . . . 186

9-1 Measurements and number and order of experimental metabolic trials . . . . 200

9-2 List of symbols and abbreviations . . . . . . . . . . . . . . . . . 203

9-3 Relationships between drag and speed for a dolphin without a tag and with tags of increasing size . . . . . . . . . . . . . . . . . . 209

10-1 Measurements and number and order of experimental trials performed under different drag-loading conditions. . . . . . . . . . . . . . . . . . . . . . . 219

10-2 List of symbols and abbreviations. . . . . . . . . . . . . . . . . 220

11-1 A comparison of changes in drag and speed as reported in this thesis . . . . . 229

A-1 Timeline of events on 15 January 2011 in Sedation/Entangled, Disentangled, and Recovery phases of Eg 3911. . . . . . . . . . . . . . . . . . . . . 240

E-1 Individual ID, age, measured length, weight and diameter, and notes on the body condition or cause of death of four North Atlantic right whales . . . . . 274 
Chapter 1

\section{INTRODUCTION}


Animal movement has evolved in part by energetic constraints, where fitness is maximized by minimizing energy consumption. The economical approach is to perform a task while expending the least amount of energy as possible (Alexander, 1989; Sparrow and Newell, 1998): for example, humans adjust their step length or stride frequency when carrying loads to minimize energy expenditure, and horses change from trot to canter to gallop because these gaits require less energy than their intermediates. By moving at speeds where transport costs are minimized (Williams, 1999; Wickler et al., 2000; Rosen and Trites, 2002a; Weber et al., 2009; Halsey, 2016), or by taking advantage of environmental factors such as winds (Hedenstrom, 1993; Weimerskirch et al., 2000), tides (Weihs, 1978; Metcalfe et al., 1990; Forward and Tankersley, 2001), or buoyancy (Webb et al., 1998; Fahlman, 2008; Aoki et al., 2011; Miller et al., 2012b; Adachi et al., 2014), animals can improve their energy economy.

Swimming animals adjust their fine-scale movements to optimize their performance, addressing trade-offs between thrust generation and efficiency (Chopra and Kambe, 1977; Bose and Lien, 1989; Daniel, 1991; Fish and Rohr, 1999). Whales and other marine mammals make repetitive or transient gait changes; for example, employing intermittent swimming strategies (Williams, 2001) or reducing thrust production to take advantage of net buoyancy on dive descents or ascents (Webb et al., 1998; Fahlman, 2008; Aoki et al., 2011; Miller et al., $2012 b)$. Economy includes but is not the same as efficiency. Efficiency is the ratio of work done relative to the metabolic energy required at multiple levels in the system (e.g. muscular, hydraulic, propulsive; Zamparo et al., 2002; Blake, 2005). Together with elements such as frequency and speed, efficiency contributes to economy, the energetic cost of a task.

\subsection{Hydrostatic equilibrium}

The energetic cost of movement depends on the magnitude of resistive forces acting on the animal and how they are balanced; in water, this hydrostatic equilibrium consists of weight, buoyancy, and lift in the vertical direction, and drag and thrust in the horizontal (Figure 1). The weight of an animal in water is balanced by a combination of buoyancy and lift forces generated as water flows along the animal's horizontal surfaces (e.g., pectoral fins). Water flows more quickly across one side of these hydrofoils than the other, creating a pressure differential across them; the resultant is a lift force generated perpendicular to the foil (Figure 1). Drag forces resist horizontal movement. An immersed body alters fluid flow around it, removing momentum from that fluid. This rate of removal of momentum is the drag force, which has separate pressure and frictional components (for reviews see e.g., Webb, 1975a; Vogel, 1994; Fish, 1998). Pressure drag results from a pressure differential across different parts of the body, distorting the flow. Gravitational effects at fluid interfaces (e.g., at the water's surface) also arise due to a pressure differential as fluid is unable to flow 
around the body, dissipating energy. Induced drag occurs as lift is generated by other body surfaces (e.g., pectoral fins) which also contribute interference drag. Finally, form drag arises from the shape of the body, where streamlining reduces pressure effects and flow separation. Frictional or viscous drag forces arise from shear stresses on the body, and are a function of size and boundary layer flow conditions. These components interact and form the passive drag on a body; active movement alters the various components of drag by influencing boundary layer thickness and separation, entraining additional fluid mass, increasing surface area due to body oscillation, and increasing induced drag from vortices generated in swimming.

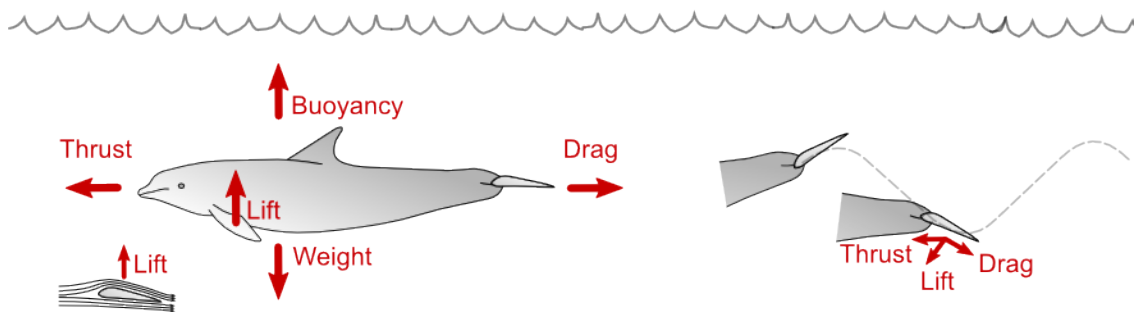

Figure 1-1: Forces on a swimming cetacean.

\subsection{Cetacean swimming}

Resistive drag forces must be balanced by thrust generated by the animal. Whales and dolphins (cetaceans) generate thrust by vertically oscillating their caudal fin as a propulsor (Figure 1). Bending is mostly restricted to the caudal portion of the body (Lang and Daybell, 1963; Videler and Kammermans, 1985) though the body is not rigid; the rostrum and forebody tend to move in phase (Fish, 2003). The flukes themselves are pitched throughout the stroke cycle altering the angle of incident flow (angle of attack; Figure 1; Fish and Rohr, 1999). These coordinated rhythmic body and tail motions result in thrust from the horizontal component of lift generated over the caudal fin, as well as the generation and interaction with vortices (Triantafyllou et al., 1993, 2000). Swimming movements strike a balance between thrust generation and propulsive efficiency - the ratio of power produced by vs. supplied to the system (Fish and Rohr, 1999).

\subsection{Changes in force balance}

The force balance on a swimming animal can change periodically over seconds (e.g., with swimming movements), weeks (e.g., with changes in body condition), months (e.g., with pregnancy), and years (e.g., with body growth or maturation). Dynamic movement causes short-term changes in force balance. For example, right (Eubalaena glacialis) and bowhead (Balaenoptera mysticetus) whales both employ ram filter feeding strategy, where individuals 
swim at slow speeds with their mouths agape to expose their large baleen plates to filter small copepod prey species. Doing so can double drag forces in right whales (McGregor, 2010) or increase drag approximately $6 \times$ in ram feeding bowhead whales (Simon et al., 2009). The more energetic lunge-feeding strategy characteristic of balaenopterid (e.g., humpback (Megaptera novaeangliae) and blue (B. musculus)) whales can lead to order of magnitude increases in drag over those experienced in steady swimming (Goldbogen et al., 2007).

While foraging and other movements lead to short-term changes in force balance that often feed back into locomotory strategies, forces can also change over the long-term. These changes in an animal's force balance can occur naturally. Body shape changes with age and growth affect the ratio of body length to width, affecting the body's overall streamlining (see e.g. Appendix E3, Chapter 6; Woodward, 2006; Fish et al., 2008).

Body shape is also considerably altered by pregnancy, affecting pressure drag. North Atlantic and Southern right whales experience significant changes in body width with pregnancy (Miller et al., 2012a); however, the increase in surface area is estimated to increase drag by only 3-4\% (McGregor, 2010). A more considerable effect is in smaller cetaceans. Pregnant bottlenose dolphins (Tursiops truncatus) show marked increases in girth with abdominal distention, doubling the drag coefficient (Noren et al., 2011). These changes in girth are most pronounced mid-body, affecting the position of maximal thickness and therefore the streamlined body shape (Blake, 1983; Fish et al., 2008). Significantly thicker blubber (Dunkin et al., 2005) and decreased body density (Dunkin et al., 2010) have been measured during pregnancy, though effect relative to the weight of the fetus is uncertain.

Epibiosis - where one organism grows on another - is an example of drag loading, which is defined in this thesis as where an additional or external structure contributes surplus drag forces to a body. Drag and lift forces on a host organism will change as its shape and surface properties are altered by the settlement, attachment, and growth of different epibionts (Wahl, 1989, 1996). Biofouling from e.g., barnacles is known to occur in turtles, seals (e.g., Setsaas and Bester, 2006) and cetaceans (Fertl and Newman, 2009). In dolphins, barnacle settlement and attachment is often limited to trailing edges of dorsal and caudal fins due to water flow patterns (e.g. as reviewed in Carrillo et al., 2015); the extent to which barnacles alter flow around these fins or increase drag has yet to be quantified.

There have been many approaches to experimentally alter the force balance of animals to determine how or whether individuals perceive and respond. Drag harnesses (Cornick et al., 2006), collars (Lang and Daybell, 1963), pipes (Aoki et al., 2011; Suzuki et al., 2014), wooden blocks (Boyd et al., 1997) have been attached to marine mammals while measuring their long-term energy consumption with radio-isotope labeled water (Costa and Gentry, 1986), heat flux (Willis et al., 2005) their diving and swimming behaviors (Boyd et al., 1997; Aoki et al., 2011), or their colony return rates (Costa and Gentry, 1986; Boyd et al., 1997). 
There are also unnatural, unanticipated changes in force balance: animals may become entangled in marine debris (Fowler, 1987; Wells et al., 2008), or more commonly in actively fished gear (Read et al., 2006; van der Hoop et al., 2013b). Larger whales are often able to break free of anchor points. In doing so, some whales are able to continue to swim for days to years while carrying a portion of gear with them. Entangling gear contributes considerable drag and weight to the animal (Feldkamp, 1985; Feldkamp et al., 1988). An analogue is when bio-logging or -tracking tags are attached to animals for research and monitoring. These devices have revolutionized the fields of animal movement, ecology and physiology (Rutz and Hays, 2009; Goldbogen et al., 2014; Hussey et al., 2015; Wilson et al., 2014b), but add drag and weight to tagged animals (Wilson et al., 2006; McMahon et al., 2008, 2011; Jones et al., 2013).

How animals respond to these changes in drag depends on physical and biological constraints. For example, two strategies tend to be adopted in animals when they experience natural, experimental or inadvertent drag loading: (1) speed is decreased to minimize costs of transport (Lang and Daybell, 1963; Wilson et al., 1986; Skrovan et al., 1999; van der Hoop et al., 2014b) and overall energy expenditure is limited by shifting behavioral budgets (Figure 2 closed square; Feldkamp et al., 1988; Blomqvist and Amundin, 2004; Elliott et al., 2014); or (2) speeds are maintained, incurring additional energy demands due to drag from the tags (Figure 2 open square; Costa and Gentry, 1986; Culik et al., 1993; Methling et al., 2011; Tudorache et al., 2014; Jepsen et al., 2015; Maresh et al., 2015). Individuals can also adopt a strategy somewhere between these two end points (Culik and Wilson, 1991; Boyd et al., 1997; Cornick et al., 2006) depending on social, environmental, or time constraints. At the upper end of performance, critical speeds (the speed at which individuals fatigue; Brett, 1964) are often reduced, limiting the maximum swimming capacity of animals with additional drag loading (reviewed in e.g., Tudorache et al., 2014; Jepsen et al., 2015). Sea otters are an excellent example of changes in behavior in response to drag tolerance: during surface swimming, individuals experience higher drag forces, and there appears to be a certain limit where individuals switch from surface to submerged swimming behaviors to reduce transport costs (Williams, 1989).

\subsection{Changes in swimming movements}

A major component of swimming behavior that animals can alter are their kinematics the form, pattern, or sequence of movement with respect to time. Swimming animals often alter their kinematics in response to changes in force balance, increasing swimming effort by increasing fluke stroke rate and/or amplitude (Williams, 1989; Cornick et al., 2006; Aoki et al., 2011) or reducing the use of stroke-and-glide gaits (Cornick et al., 2006; Simon et al., 2009). Swimming animals therefore show plasticity in their ability to adjust their fine-scale 


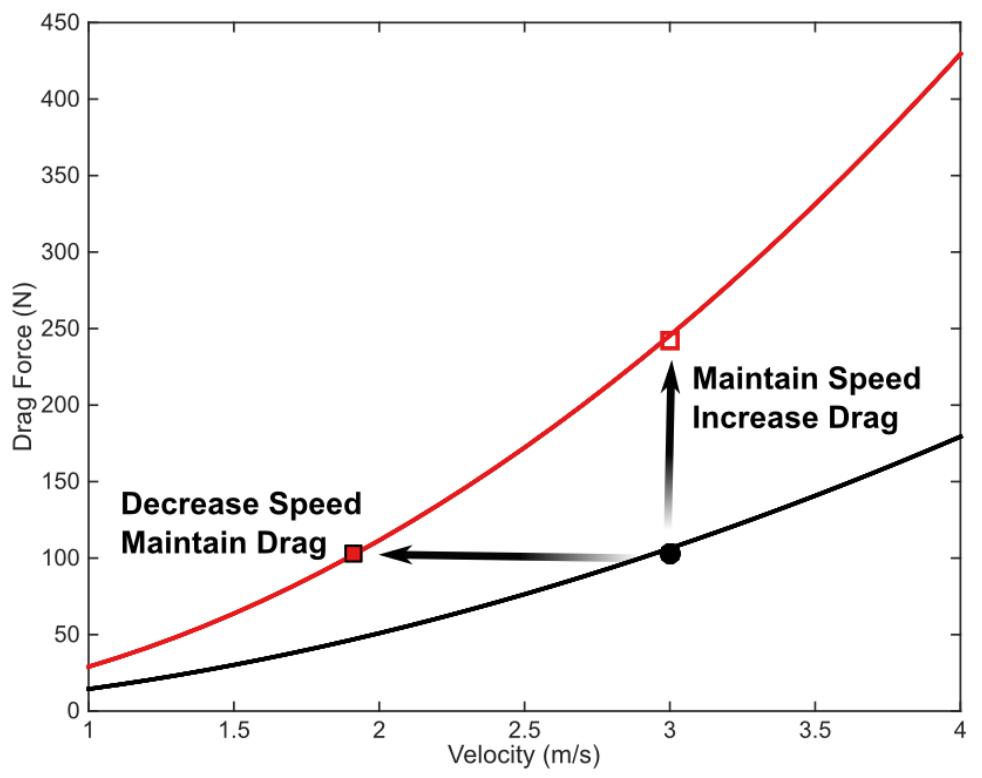

Figure 1-2: Illustration of the trade-offs an animal faces under drag loading. The black line illustrates the drag force experienced by the animal in its standard state. Under drag loading (red line), the animal may maintain speed, which would increase the drag forces it experiences (open square); alternatively, it could slow down to maintain the same amount of drag force it experienced in the non-loaded state (closed square).

movement patterns in response to changes in drag and buoyant forces and moments to effectively navigate their energy landscape (Fish and Rohr, 1999).

\subsection{Thesis overview}

This thesis quantifies and addresses the consequences of drag loading in two scenarios: entanglement in fishing gear of North Atlantic right whales and tag attachment in a small cetacean, the bottlenose dolphin. I use overlapping and complimentary approaches to quantify drag in these scenarios, to consider the constraints on the systems, and to evaluate animal responses in light of these constraints. This thesis provides insight into how drag loading affects individual behavior, energetics, movement from the order of seconds and minutes to multi-year calving cycles of large whales. The right whale model provided an extensive case series of anthropogenic drag loading in a wild cetacean. The tag drag model allowed a more controlled experimental approach with dolphins under managed care.

\subsubsection{North Atlantic right whale entanglement}

Entanglement in fishing gear is the leading cause of death to large whales in the western North Atlantic (van der Hoop et al., 2013b), and within this region, sublethal entanglement 
affects $83 \%$ of the North Atlantic right whale population (hereafter right whale; Knowlton et al., 2012a). Most recorded right whale entanglement cases involve whales that swim while towing gear that is no longer anchored (NMFS, 2003). These whales are subject to additional drag forces, likely increasing the cost of locomotion and in some cases leading to negative energy balance (Feldkamp et al., 1988; van der Hoop et al., 2013a). The onset of entanglement is unpredictable, and the duration hours to years; chronically entangled whales often consume much of their body lipid stores and ultimately die from emaciation (Cassoff et al., 2011; Moore et al., 2012; Barratclough et al., 2014). Entanglement in, and disentanglement from, fishing gear therefore presents a unique context in which to examine individual responses to changes in force balance.

This thesis addresses four main questions on the magnitude and consequences of drag from right whale entanglement in fishing gear:

1. How much drag does entanglement add to these whales? Chapters 2,3

2. How does this drag loading affect their swimming and diving behaviors? Chapters 2, 4

3. How does this drag loading affect their rates of energy consumption? Chapters 2,5

4. And finally, can we use this information estimate the drag on entangled whales at the time of their detection or to determine the potential time frame of their survival? Chapter 6

\subsubsection{Tag drag}

Tagging studies strive to collect novel data in an environment where observation is difficult - where tags are needed to measure animal movement as well as environmental and physiological variables to help interpret animal behavior or performance (Johnson et al., 2009; Crossin et al., 2014; Hussey et al., 2015). Tags do, however, contribute additional weight and bulk, and more relevant in the marine realm, buoyancy, lift and drag. Few studies directly assess the consequences of adding tags (Wilson et al., 2006; McMahon et al., 2011; McIntyre, 2014), which may impact not only on the tag subject's vital rates (e.g., Barron et al., 2010; Best et al., 2014; van der Hoop et al., 2014b), but also their behavior which is often assumed to be representative of the untagged population (Ropert-Coudert and Wilson, 2004; Vandenabeele et al., 2011; Broell et al., 2016). Quantifying drag forces added by tags is also useful to refine designs and improve the longevity of attachment systems (e.g., Shorter et al., 2013).

This thesis addresses three main questions related to tag-related drag in bottlenose dolphins. 
1. How much drag do current and leading suction-cup tag designs (DTAG2, DTAG3) add to a small cetacean? Chapters 7,8

2. Are there measurable metabolic effects of this additional drag? Chapters 7, 910

3. Are there significant effects of drag loading on instrumented animals' behavior? Chapters $7,9,10$ 
Chapter 2

\section{BEHAVIORAL IMPACTS OF DISENTANGLEMENT OF A RIGHT WHALE UNDER SEDATION AND THE ENERGETIC COST OF ENTANGLEMENT}

This chapter was originally published as van der Hoop, J. M., Moore, M. J., Fahlman, A., Bocconcelli, A., George, C., Jackson, K., Miller, C., Morin, D., Pitchford, T., Rowles, T., Smith, J., and Zoodsma, B. (2013). Behavioral impacts of disentanglement of a right whale under sedation and the energetic cost of entanglement. Marine Mammal Science 30, 282-307.

JvdH, MJM, CG, KJ, DM, TP performed fieldwork; TP, TR, BZ provided support and permits; JvdH and MJM developed concepts; JvdH processed and analyzed the data; MJ, AF, CM guided analysis; JvdH wrote the manuscript. MJM, AF, AB, CG. KJ, CM, DM, TP, TR, BZ contributed to the manuscript.

The supplemental material for this chapter can be found in Appendix A. 


\section{$2.1 \quad$ Abstract}

Protracted entanglement in fishing gear often leads to emaciation through reduced mobility and foraging ability, and energy budget depletion from the added drag of towing gear for months or years. We examined changes in kinematics of a tagged entangled North Atlantic right whale (Eg 3911), before, during, and after disentanglement on 15 January 2011. To calculate the additional drag forces and energetic demand associated with various gear configurations, we towed three sets of gear attached to a load-cell tensiometer at multiple speeds. Tag analyses revealed significant increases in dive depth and duration; ascent, descent and fluke stroke rates; and decreases in root mean square fluke amplitude (a proxy for thrust) following disentanglement. Conservative drag coefficients while entangled in all gear configurations (mean $\pm \mathrm{SD} C_{d, e, g o}=3.4 \times 10^{-3} \pm 0.0003$, $\left.C_{d, e, g b}=3.7 \times 10^{-3} \pm 0.0003, C_{d, e, s l}=3.8 \times 10^{-3} \pm 0.0004\right)$ were significantly greater than in the nonentangled case $\left(C_{d, n}=3.2 \times 10^{-3} \pm 0.0003 ; P=0.0156,0.0312,0.0078\right.$, respectively). Increases in total power input (including standard metabolism) over the nonentangled condition ranged from $1.6 \%$ to $120.9 \%$ for all gear configurations tested; locomotory power requirements increased $60.0 \%-164.6 \%$. These results highlight significant alteration to swimming patterns, and the magnitude of energy depletion in a chronically entangled whale.

\subsection{Introduction}

Entanglement in fishing gear is the leading cause of detected mortalities of large whales in the Northwest Atlantic (van der Hoop et al., 2013a). Upon initial entanglement, a number of outcomes are possible: individuals may die anchored in gear, or may break free, either cleanly or carrying all or a portion of the entangling gear (Clapham and Mead, 1999). Chronic effects of entanglement in free-swimming individuals include systemic infection and debilitation from extensive tissue damage (Cassoff et al., 2011). More common in protracted cases is severe emaciation due to the inability to cope with a negative energy budget, driven by the combined effects of reduced mobility and foraging ability, and increased energetic demand imposed by towing accessory gear for months to years (Moore et al., 2006; Moore and van der Hoop, 2012).

Whereas disentanglement efforts were first developed to release large whales entangled and anchored in fixed fishing gear (Ledwell et al., 2010), techniques have been adapted to address the issue in free-swimming individuals (Moore et al., 2010). Disentanglement response efforts are coordinated by multiple agencies with the primary goal of removing all entangling gear. During a disentanglement procedure, buoys or floats are often added to trailing gear to increase a whale's drag through the water and slow its movement (Moore 
et al., 2010). To further reduce boat aversion and allow for close approaches necessary for successful disentanglement, methods have been developed to lightly sedate large whales at sea (Moore et al., 2010).

No data exist for large whales on the behavioral impacts of sedation and disentanglement or on the energetic cost of entanglement in fishing gear due to drag. Through detailed spatial and behavioral monitoring by means of a biologging tag (Dtag) (Johnson and Tyack, 2003), we examined changes in dive behavior and kinematics of a tagged entangled North Atlantic right whale (North Atlantic Right Whale Catalog No. 3911, hereafter Eg 3911; Hamilton et al., 2007), before, during, and after disentanglement procedures on 15 January 2011. Further, we estimate drag forces experienced by the whale based on its body proportions, and the additional drag forces and energetic demand experienced while entangled in various gear configurations.

\subsection{Methods}

Eg 3911, a female born in 2009 (NARWC, 2011), was first sighted entangled and displaying consequent emaciation on 25 December 2010 by an aerial survey team offshore Ponte Vedra Beach near Jacksonville, Florida. The entanglement involved attachment at a minimum of six sites around the mouth, wraps around both pectoral fins, and approximately $30 \mathrm{~m}$ of line trailing aft of the flukes (Fig. 1; Moore et al., 2012). We conducted disentanglement attempts on 29 and 30 December 2010, though the whale remained entangled and was tracked by a satellite telemetry buoy. A third and final multiagency disentanglement effort took place 15 January 2011 near Melbourne, Florida, during which we tagged Eg 3911 with a biologging device (Dtag). Subsequently, we sedated, partially disentangled to the extent possible, administered antibiotics, and tracked the whale for six days via satellite with a Low Impact Minimally-Percutaneous External-electronics Transmitter (Fig. 2; LIMPET; Andrews et al., 2008). We observed Eg 3911 dead at sea by aerial survey on 1 February 2011, and towed it ashore for necropsy performed on 3 February 2011. The ultimate cause of death was premortem shark predation, though the proximate cause was chronic constrictive deep rope lacerations and severe emaciation (Moore et al., 2012, McLellan and Costidis ${ }^{1}$ ). Upon necropsy, we systematically removed, photographed, and described the remaining entangling gear. In total, the entanglement involved approximately $132 \mathrm{~m}$ of $1.12 \mathrm{~cm}$ diameter floating synthetic line, including six gangions and two fragments of vinyl coated trap mesh. This gear was consistent with that used in fixed trap/pot fisheries, though the target species could not be identified (Morin and Kenney, 2011). We used a portion of the entangling gear in the experiments, below.

\footnotetext{
${ }^{1}$ Unpublished necropsy report from William McLellan, Biology and Marine Biology Department, UNC Wilmington, 601 South College Road Wilmington, NC 28403.
} 


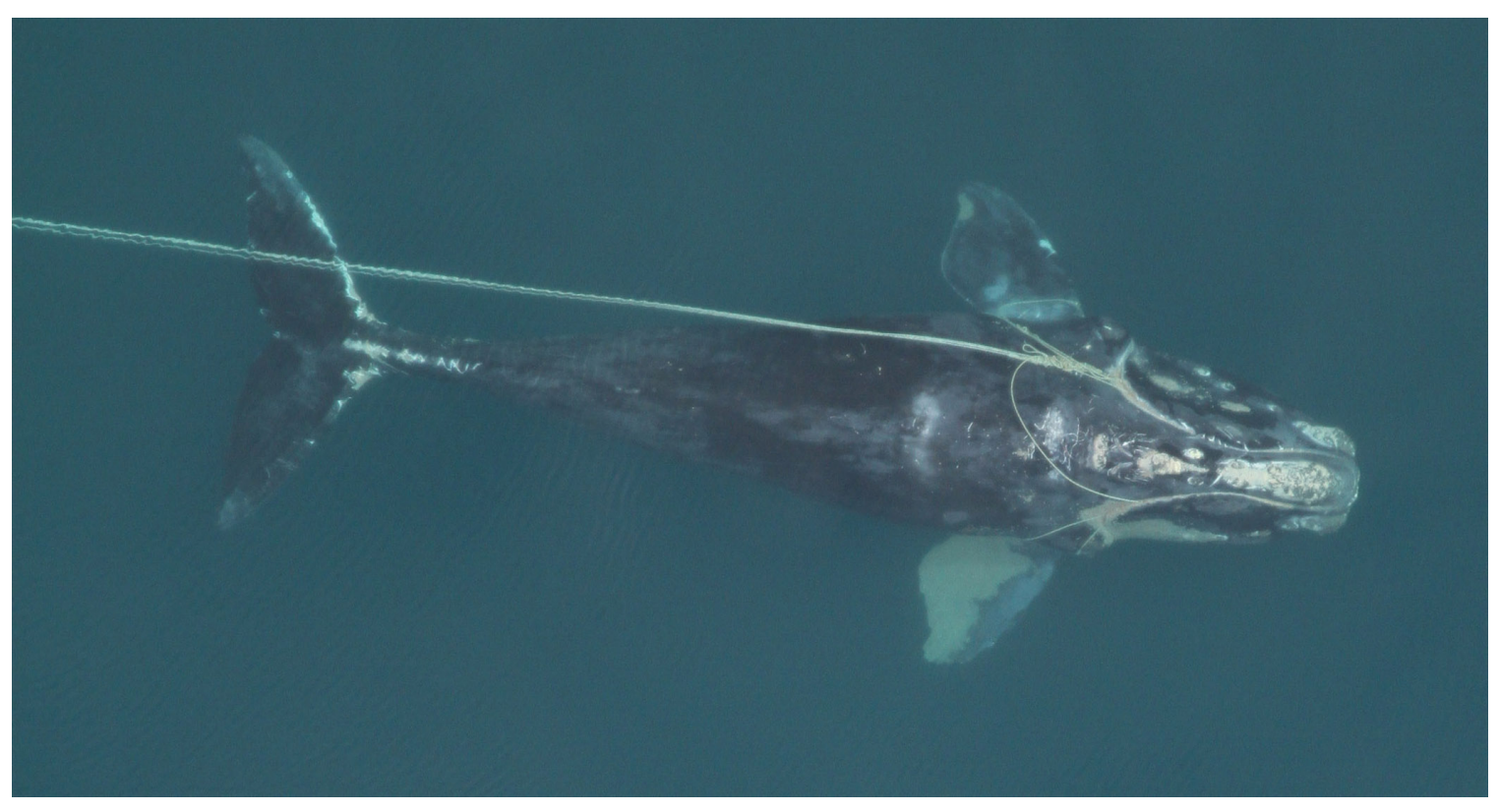

Figure 2-1: Aerial photograph of right whale Eg 3911 on 30 Dec 2010, showing complex entanglement in the head and pectoral fins. Photo under NOAA Fisheries Permit No. 594-1759.

\subsubsection{Sedation}

To determine appropriate sedative dosages, we calculated a range of weight estimates based on a body length estimate $(945 \mathrm{~cm})$ obtained from aerial photographs of Eg 3911 next to a vessel of known dimensions and four length-to-weight methodologies (Appendix A). We found Eg 3911 to be $20 \%$ thinner than adult female right whales (see Appendix A for details Miller et al., 2012b). To consider this emaciation, we reduced weight estimates by $20 \%$, to $\sim 7,000 \mathrm{~kg}$.

We administered sedative via injection (Moore et al., 2010) of $14 \mathrm{~mL}(0.1 \mathrm{mg} / \mathrm{kg}$ body weight) each of $50 \mathrm{mg} / \mathrm{mL}$ Butorphanol and Midazolam (ZooPharm Inc., Windsor, CO), and sedative reversal via $7 \mathrm{~mL}(0.05 \mathrm{mg} / \mathrm{kg})$ of $50 \mathrm{mg} / \mathrm{mL}$ Naloxone and $49 \mathrm{~mL}$ of 0.1 $\mathrm{mg} / \mathrm{mL}$ Flumazenil. The reversal needle inserted fully, but on recovery it was discovered that the syringe had malfunctioned and the dose remained in the syringe barrel and was not administered. We also administered two doses of antibiotics (56 mL each; total $17.6 \mathrm{~g}$ of 220 $\mathrm{mg} / \mathrm{mL}$ Ceftiofur; Pfizer Inc, Madison, NJ). Injections occurred via a ballistic syringe system (Fig. 3; Paxarms, Timaru, New Zealand; Moore et al., 2010), with the syringe attached to a stainless steel leader tied to $20 \mathrm{~m}$ of $80 \mathrm{~kg}$ test line spooled at the projector barrel tip, and then tied to a custom float. The float is designed to extract the needle and provide a visual marker for retrieval (Moore et al., 2010). 


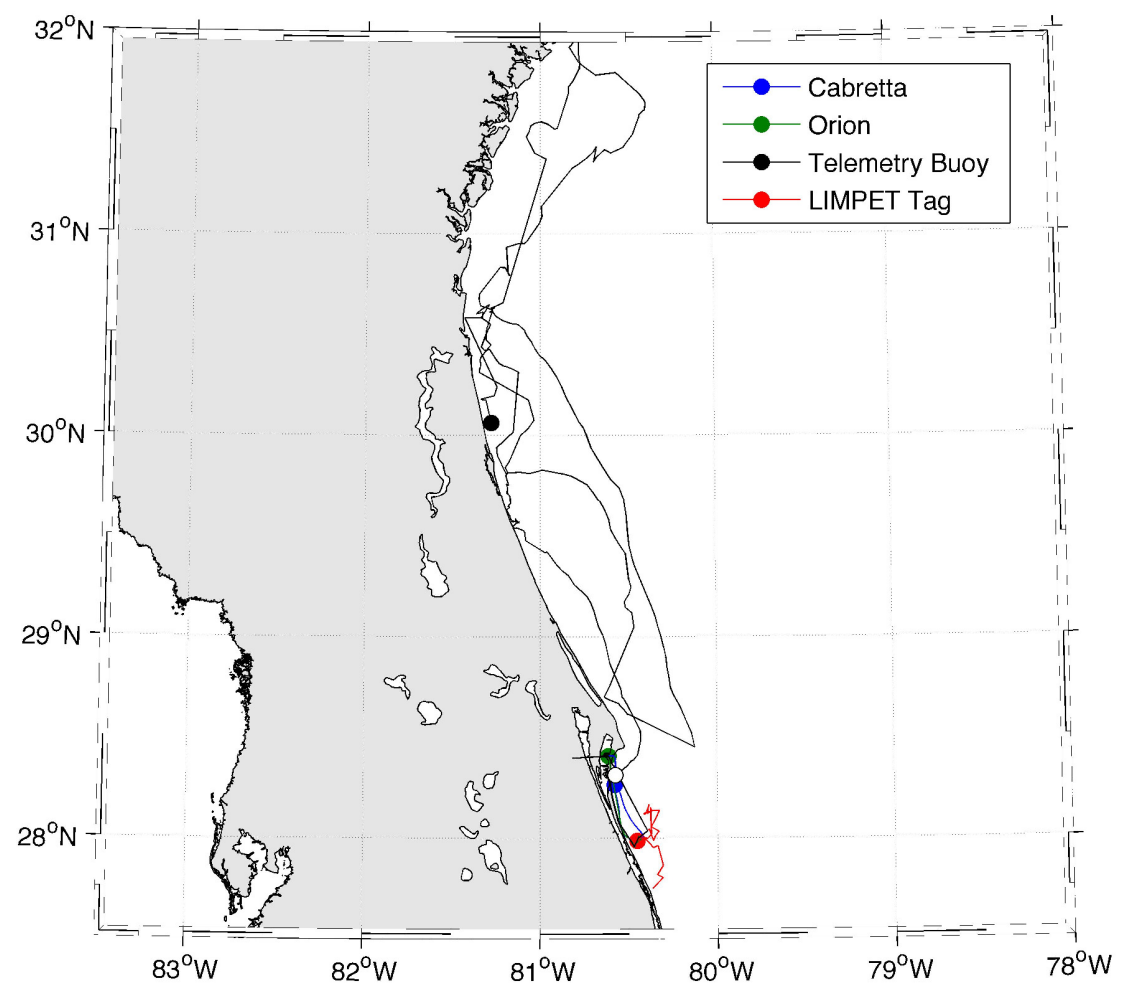

Figure 2-2: Satellite telemetry track of right whale Eg 3911 (black) swimming entangled from 25 December 2010 to 15 January 2011, and following disentanglement (red; 15 January 2011 to 21 January 2011) performed from vessels Cabretta (blue) and Orion (green). Colored circles represent track starting points. The white circle represents Eg 3911's track at the beginning of the disentanglement effort on 15 January.

\subsubsection{Tagging and behavior}

Prior to the disentanglement, we attached a Dtag at 1004 EDT on 15 January 2011 via suction cup just above the right lateral midline, midway between the blowhole and tail (Fig. 3). Deployment lasted 6:11 (h:min).

The Dtag is equipped with depth and temperature sensors, 3-axis accelerometers and magnetometers sampling at $50 \mathrm{~Hz}$, and a hydrophone sampling at $96 \mathrm{kHz}$ (Johnson and Tyack, 2003). We down-sampled sensor data to $5 \mathrm{~Hz}$, and calibrated accelerometer and magnetometer measurements to account for the orientation of the tag on the whale (Johnson and Tyack, 2003). We derived pitch and roll from the accelerometer and heading from the magnetometer measurements. 


\subsubsection{Dive parameters}

We defined dives as depths $>5 \mathrm{~m}$, representing the top $29 \%-38 \%$ of the water column where Eg 3911 was tagged. We estimated bottom depth from bathymetric charts with coordinates of pursuit and disentanglement operations. Tidal range for 15 January 2011 was only 30-70 $\mathrm{cm}$ above chart datum for Cape Canaveral, Florida. We calculated proportional depth as the amount of the water column explored relative to available (depth of dive/approximate depth of dive location). We manually detected descent and ascent periods of each dive, reflecting periods of sustained motion to depth and to the surface, respectively. Dive profiles appeared in randomized order for the manual determination of descent and ascent periods to reduce potential bias. We calculated descent and ascent rates as the distance traveled from the surface to the depth at which the descent period ends (or from depth to surface for ascents), over the duration of that period.

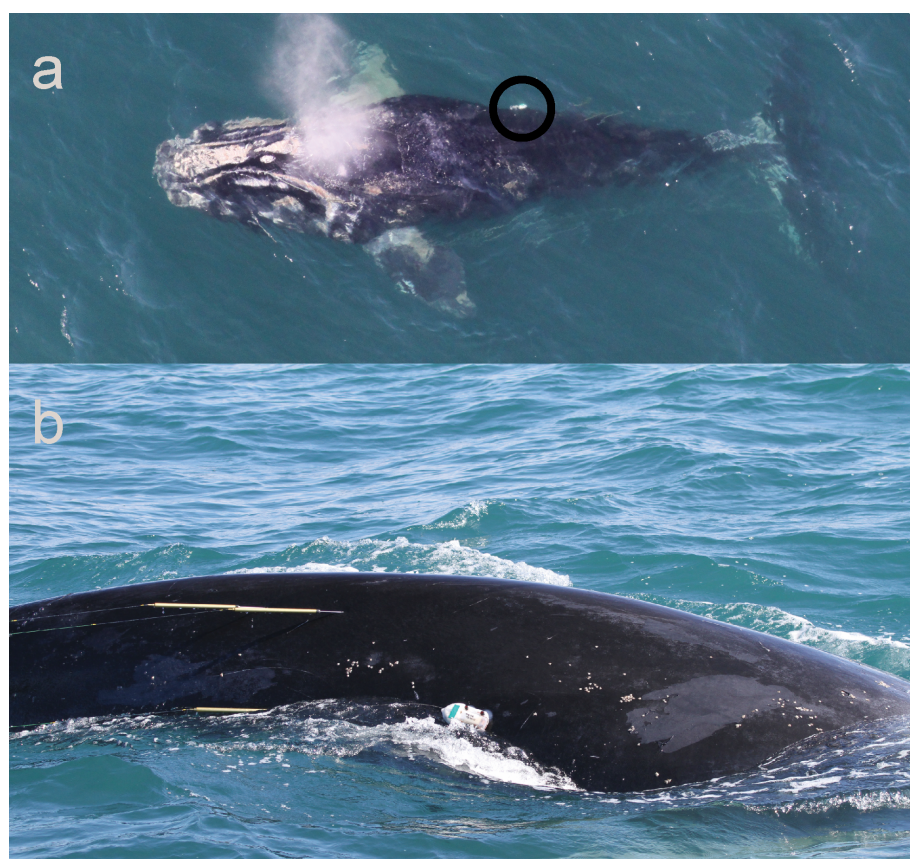

Figure 2-3: Location of attachment of a suction-cup attached Dtag on right whale Eg 3911. (a) Aerial view, with the Dtag visible on right flank, circled in black. (b) Lateral view of right flank with the Dtag just above waterline. Three partially extruded darts are shown caudal to the tag. The darts have all folded at the skin surface through water drag. Photos under NOAA Permit No. 932-1905-00/MA-009526.

Wave drag is greatest when the ratio between the submergence depth $h$ of a body of diameter $d$ is $h / d=0.5$, and becomes negligible at $h / d=3$ (Hertel, 1969). To determine the relative amount of time spent swimming in more costly conditions, we compared the ratio of time spent above vs. below this wave drag limit $(h / d=0.5)$ between phases. We calculated dive duration (s) from when the animal left the surface (to a depth $>5 \mathrm{~m}$ ) until returning to $<1 \mathrm{~m}$ depth. 


\subsubsection{Dive shape}

We created a dimensionless, depth- and duration-independent index to compare dive shapes under entangled and nonentangled conditions. The Dive Area Ratio (DAR), similar to the Time Allocation at Depth (TAD) Index (Fedak et al., 2001), is based on the concept of a time-depth area, being the area enclosed by a dive profile or the integral of dive depth over the dive duration. We therefore calculate the DAR as the ratio of the total dive area (the integral of the dive profile) and the maximum dive area,

$$
D A R=\frac{A_{a}}{D T}=\frac{\sum_{i=2}^{n} \frac{\left(d_{d, j}+d_{d, j-1}\right) \times \frac{1}{f s}}{2}}{D T},
$$

where $A_{a}=$ integrated actual dive area, $d_{d}=\operatorname{tag}$-derived depth $(\mathrm{m})$ at $n$ intervals during dive, $D=$ maximum depth of dive $(\mathrm{m}), f s=\operatorname{tag}$ sampling rate $(\mathrm{Hz})$, and $T=$ total dive duration (s).

The DAR differs from the TAD Index in that it does not remove the "necessary travel area" (the area required to descend and ascend to and from maximum depth) from each dive. The time to descend and ascend is of particular interest in this analysis, as changes in drag and buoyancy due to the presence of entangling gear will have the greatest effect in these portions of the dive cycle. The DAR thus provides greater information on the difference in dive shapes over the entire duration of the dive, not only the bottom period between descent and ascent.

\subsubsection{Respiration}

We determined respiration rate from aerial observer counts of the number of visual respiration cues per 5 min interval, from 40 min prior to and 3:45 h:min following tag attachment.

\subsubsection{Proxies for thrust}

The Dtag captures individual fluke strokes as cyclic oscillations in the deviation of the pitch angle (degrees) from mean orientation. We considered three tag-obtained measures of thrust production: (1) fluke stroke rate, the inverse of the time between peaks in pitch angle averaged over $30 \mathrm{~s}$ bins (fluke strokes per second, Hz; Johnson and Tyack, 2003), which is a relative indicator of thrusting intensity; (2) the root mean square (RMS) energy of fluke amplitude, a measure of signal average and variability, proportional to power (Semmlow, 2012), measured only within dives to discount large changes in pitch associated with surfacing events; and (3) glides, characterized by periods where no fluke oscillation occurs in the pitch rate signal. We identified glides as segments where the absolute value of the Hilbert transform of the pitch rate signal was $<0.05$ (Woodward et al., 2006b), and visually checked 
these sequences. Based on previously described gliding behaviors in right whales (Nowacek et al., 2001; Woodward et al., 2006b), we defined the minimum glide duration as $5 \mathrm{~s}$.

\subsubsection{Overall Dynamic Body Acceleration (ODBA)}

Following Wilson and McMahon (2006) and Fahlman (2008), we calculated Overall Dynamic Body Acceleration (ODBA, g) by smoothing accelerometer measurements in three separate axes, with a window size of $3 \mathrm{~s}$. We then subtracted these smoothed data (static acceleration) from the unsmoothed data to estimate the dynamic acceleration in each axis. Finally, we then calculated ODBA as the sum of the absolute value of dynamic acceleration in each axis. We observed peaks and identified outliers in ODBA at each surfacing event, and therefore calculated mean ODBA values within dives, between dives, and during descent and ascent periods of each dive.

\subsubsection{Phase definitions and statistical analyses}

We defined three phases of the sedation and disentanglement of Eg 3911 (Table 1) hereafter referred to as (1) Sedation/Entangled: animal towing gear and attached buoys, and sedative injection; (2) Disentangled: following removal of most of trailing gear and buoys, administration of antibiotics, and attachment of the satellite LIMPET tag (Andrews et al., 2008); and (3) Recovery: retrieval of injection darts, dart tethers and floats (Moore et al., 2010), and the end of active boat approaches.

To determine the behavioral effects of sedation on an entangled whale, we used Wilcoxon rank sum tests to compare dive parameters and respiration rates within the Sedation/Entangled phase, between the 21 min prior to and the 50 min following sedative injection, but prior to removal of the gear and buoys. We used Three-sample Kruskal-Wallis single factor analysis of variance tests with tied ranks and post hoc Bonferroni-corrected $(\alpha=0.05 / 3=0.0167)$ Wilcoxon rank sum tests to compare the distributions of various dive parameters between Sedation/Entangled, Disentangled and Recovery phases. To compare the observed vs. expected ratio of time spent above and below the wave drag limit between phases, we used Chi-square contingency tables.

We compared fluke stroke rate, RMS, and the frequency and duration of glides across phases within the single tag deployment to infer changes in thrust intensity and power requirements. As propulsive (thrusting) forces should equal resistive forces (net buoyancy and drag), we expect thrusting intensity (stroke rate and RMS) to be greater and for fewer and shorter glides to occur in entangled vs. nonentangled conditions. We present all dive parameters as median (IQR) unless otherwise stated. 
Table 2-1: Timeline of events on 15 January 2011 in Sedation/Entangled, Disentangled, and Recovery phases of Eg 3911.

\begin{tabular}{|c|c|c|c|}
\hline Phase & $\begin{array}{l}\text { Dtag elapsed } \\
\text { time }(\mathrm{s})\end{array}$ & $\begin{array}{l}\text { GPS time } \\
(\mathrm{EST})\end{array}$ & Event \\
\hline \multirow[t]{8}{*}{ Sedation/Entangled } & 0 & 10:04:18 & Dtag attachment \\
\hline & 1,217 & 10:24:00 & Sedation Induction \\
\hline & 5,048 & 11:28:00 & Possible cut with spring knife \\
\hline & 5,348 & 11:33:00 & Cut \\
\hline & 5,648 & 11:38:00 & Cut \\
\hline & 6,008 & 11:44:00 & Cut \\
\hline & 6,188 & 11:47:00 & Cut \\
\hline & 6,428 & 11:51:00 & Cut \\
\hline \multirow[t]{6}{*}{ Disentangled } & 6,667 & 11:55:00 & Buoys slack and removed \\
\hline & 9,223 & $12: 36: 00$ & Attachment of LIMPET Tag \\
\hline & 9,548 & 12:43:00 & $\begin{array}{l}\text { Sedation reversal dart: did not } \\
\text { deploy }\end{array}$ \\
\hline & 9,548 & 12:43:00 & Antibiotic dart \\
\hline & 12,248 & $13: 28: 00$ & $\begin{array}{l}\text { Antibiotic dart unsuccessful at- } \\
\text { tempt }\end{array}$ \\
\hline & 13,808 & 13:54:00 & Antibiotic dart \\
\hline \multirow[t]{2}{*}{ Recovery } & 15,248 & $14: 18: 00$ & $\begin{array}{l}\text { Dart tethers, floats, and } 2 / 4 \\
\text { darts recovered. Vessell Cabretta } \\
\text { left scene; Vessel Orion following } \\
\text { at } 50-300 \mathrm{~m} \text { distance }\end{array}$ \\
\hline & 22,268 & $16: 15: 00$ & Tag off \\
\hline
\end{tabular}

\subsubsection{Gear towing}

We conducted a series of tests in Marion Harbor, Massachusetts, on 13 May 2011 towing three sets of gear off the side of a $7.3 \mathrm{~m}$ (24 ft), 25 HP motor-propelled Carolina Skiff: (1) $24.93 \mathrm{~m}$ of $1.12 \mathrm{~cm}$ diameter floating line removed from Eg 3911 in the disentanglement procedure on 15 January 2011, "gear-only"; (2) this same line with two buoys as attached during disentanglement, "gear-and-buoys"; and (3) $160 \mathrm{~m}$ of $0.89 \mathrm{~cm}$ sinking line for comparison, "sinkline", all detailed below.

To measure drag force, we used an MLP-100 load cell tensiometer (Transducer Techniques, Temecula, CA) between two eyebolts threaded into opposite sides of the cell. One eyebolt suspended the load cell parallel to a vertical spar on the side of the Skiff. The second eyebolt attached to a leader running through the pulley at the base of the spar, then immediately attached to the gear (i.e., the leader produced drag that was negligible compared to the gear). We held the base of the spar at the surface and at $2 \mathrm{~m}$ depth, consistent with the animal's body depth of $2.20 \mathrm{~m}$. 
We modified the drag force signal from the load cell as in Cavatorta et al. (2005) and recorded it through the serial port on a laptop, sampled at $250 \mathrm{~ms}$. We calculated mean $( \pm \mathrm{SD})$ drag forces from the data record for a given gear configuration (gear-only, gear-andbuoys, or sinkline), anchor point (surface or $2 \mathrm{~m}$ depth), and boat speed $(0.772-2.98 \mathrm{~m} / \mathrm{s})$. We measured boat speed via a handheld GPS unit and used this speed as a relative indicator of the effect of whale swimming speed. These speeds are biologically relevant, as right whales are known to swim in the range of 0.52 (Mayo and Marx 1990) to $2.05 \mathrm{~m} / \mathrm{s}$ (Baumgartner et al., 2003) and maximum speeds for balaenids have been recorded between 4 and $4.5 \mathrm{~m} / \mathrm{s}$ (Hamner et al., 1988). Tide was $<0.5$ knot.

The entangling gear removed 15 Jan 2011 (Configuration 1: gear-only) measured 24.93 $\mathrm{m}$ in length, and consisted of parallel arrangements of six line segments for the first $0.7 \mathrm{~m}$, three segments for the next $1.50 \mathrm{~m}$ and two segments for the next $2.20 \mathrm{~m}$; the remaining $20.53 \mathrm{~m}$ was a single piece of line with one gangion (a large knot connecting a second line) and three figure-eight knots (Fig. 4). The combined length of all line segments was 33.63 m.

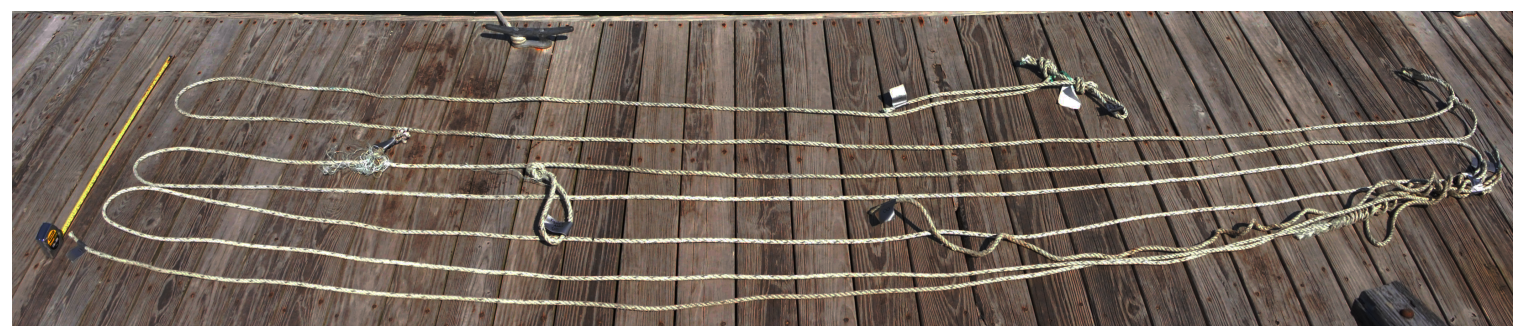

Figure 2-4: Fishing gear removed from right whale Eg 3911 on 15 January 2011. The total length of the configuration is approximately $24.93 \mathrm{~m}$, with a combined line length of $33.63 \mathrm{~m}$. A tape measure (left) is drawn to $1 \mathrm{~m}$ for spatial reference.

To mimic the configuration on the animal, we attached the buoys added during disentanglement (Configuration 2: gear-and-buoys), an A3 Polyform buoy (42.5 $\mathrm{cm}$ diameter) and an NB60 Scanmarin buoy (45.4 cm diameter) to the aft-most figure-eight knots on the removed gear (i.e., Configuration 1). We connected each buoy to its respective figure-eight knot by an $11.4 \mathrm{~cm}$ karabiner and an approximately $1 \mathrm{~m}$ long lanyard of $0.95 \mathrm{~cm}$ diameter polysteel. The buoys and karabiners used in the tow deployments were identical to those used in the disentanglement procedure; however, during the disentanglement, we attached buoys to the fore-most and aft-most knots. We assume this difference in the gear configuration does not change the results materially.

As a control, we towed $160 \mathrm{~m}$ of $0.89 \mathrm{~cm}$ diameter sinkline (Configuration 3: sinkline) in a single-line configuration with no knots, gangions, or buoys. 


\subsubsection{Energy requirements}

We applied the following calculations to determine the forces acting on Eg 3911. Symbols are listed in Table 2. The Reynolds number, Re, describes the relative importance of viscous and inertial forces acting on a body, calculated as

$$
R e=\frac{l U}{v}
$$

where $l$ is the length of the body $(\mathrm{m}), U$ is the velocity or swimming speed $(\mathrm{m} / \mathrm{s})$ and $v$ is the kinematic viscosity of the surrounding medium $\left(1 \times 10^{-6} \mathrm{~m}^{2} / \mathrm{s}\right.$ for seawater $)$. Reynolds numbers $>5 \times 10^{6}$, as calculated here and is the case for other large whales, indicate a turbulent boundary layer. Total drag on a body is composed of frictional, pressure, interference, and surface components. Frictional drag, $D_{f}(\mathrm{~N})$, is given by

$$
D_{f}=\frac{1}{2} \rho U^{2} A_{w} C_{f}
$$

where $\rho$ is the density of the surrounding medium (here seawater, $1,025 \mathrm{~kg} / \mathrm{m}^{3}$ ), $A_{w}$ is the total wetted surface area $\left(\mathrm{m}^{2}\right.$; Alexander, 1990)) calculated from body weight $W(\mathrm{~kg})$ as $A_{w}=0.08 W^{0.65}$ (Fish, 1993a). $C_{f}$ is a frictional drag coefficient, which depends on boundary layer flow characteristics (e.g., Blake, 1983). For a turbulent boundary condition, as calculated above,

$$
C_{f}=0.072\left(R e^{-1 / 5}\right)
$$

The pressure drag coefficient, $C_{p}$, is relatively constant for $R e>10^{6}$. By convention, we calculated $C_{p}$ as a fraction of $C_{f}$ by calculating $C D_{0}$, the profile drag coefficient,

$$
C_{d}=C_{f}\left[1+1.5\left(\frac{d_{\max }}{l}\right)^{3 / 2}+7\left(\frac{d_{\max }}{l}\right)^{3}\right]
$$

where $d$ is the maximum width of the body (or diameter; $\mathrm{m}$ ) estimated from photographs using width-to-length ratios of the widest point of the body.

We added three drag augmentation factors. (1) Appendages increase interference, frictional, and pressure drag over the theoretical condition due to protrusion from a streamlined body. We used $g=1.3$ to account for $\sim 30 \%$ increases in drag due to flukes and fins (Fish, 1999). (2) $k$ accounts for the oscillation of the flukes and body during active swimming, which alters body shape and increases frontal area and $C_{p}$ (Fish, 1999). Further, boundary 
layer thinning is expected when the amplitude of the propulsive movement is much greater than the maximum body diameter (Lighthill, 1971). Thinning of the boundary layer increases skin friction, $C_{f}$, over a greater proportion of the body than if the body were rigid, increasing drag by up to a factor of five (Lighthill, 1971). Due to uncertainties on the degree to which whale swimming affects anterior oscillation, we employed values of $k=1$ and $k=$ $3^{2}$.

The effect of surface, or wave drag on an object varies with submergence depth $(h$, measured from the surface to the center line of the object; $\mathrm{m}$ ) relative to body diameter, $d$. Critical relative submergence depth $(h / d)$ values have been established experimentally (Hertel and Technica, 1966; Hertel, 1969) and theoretically (Hoerner, 1965) describing the relative contribution of wave drag with depth. Wave drag is highest at the surface $(h / d=0.5)$ and decreases with submergence, becoming negligible at $h / d=3$ (Hertel, 1969). To account for surface drag (Hertel and Technica, 1966; Fish, 1993a), we determined the augmentation factor $\gamma$ for entangled $(\gamma=1.6)$ and nonentangled $(\gamma=1.0)$ conditions from tag-derived relative submergence depths $(1.81 \mathrm{~m}$ and $4.25 \mathrm{~m}$, respectively). We then calculated the drag on the body, $D_{w}(\mathrm{~N})$, as

$$
D_{w}=\frac{1}{2} \rho U^{2} A_{w} C_{D 0} \gamma k g
$$

Line lying flush with the body surface produces a surface protuberance that may disrupt fluid flow over the body, affecting body drag. The total drag of the system is not simply the sum of the drag on the body and on the element, but also the interference between the elements (interference drag; Blake, 1983). The magnitude of interference drag varies nonlinearly with the position ( $\%$ of $l$ ) and height of the protuberance $(p, \mathrm{~m})$ compared to the length of the body $(l, \mathrm{~m})$ (Jacobs, 1934; Blake, 1983). As protuberance height is increased from $p=0$ to $p=0.001 l$ (e.g., from 0 to $1.25 \mathrm{~cm}$ diameter line) interference drag is comparatively small, on the order of $10 \%$ of the drag of the element. Increases in drag over this height scale are slow due to the protuberance being in the body's boundary layer $(\delta)$; however, they should not be considered negligible (Jacobs, 1934). For this height scale, the interference drag coefficient of a protuberance $j\left(C_{D I, j}\right)$ is

$$
C_{d I_{n ; j}}=\left(\frac{p_{j}}{\delta_{j}}\right)^{1 / 3}
$$

where we calculated boundary layer thickness $(\delta, \mathrm{m})$ at the location of protuberance $j$ (distance from leading edge, $l_{x, j} ; \mathrm{m}$ ) based on the ratio between the maximum diameter

\footnotetext{
${ }^{2}$ Personal communication from Dr. Frank Fish, Professor of Biology, West Chester University, West Chester, PA 19383-2112, January 2013.
} 
$\left(d_{\max }\right)$ and the diameter at the location of protuberance $j\left(d_{x, j}\right)$ as

$$
\delta_{j}=\left(\frac{d_{\max }}{d_{j}}\right) 0.02 l_{x, j}
$$

We then calculated the total interference drag, $D_{I}(\mathrm{~N})$, as the sum of the interference drag associated with all $n$ protuberances on the frontal projection of the body (Hoerner, 1965):

$$
D_{I}=\sum_{j=1}^{n} D I_{j}=\sum_{j=1}^{n} \frac{1}{2} \rho U^{2} A_{p, j} C_{D I, j}
$$

Bodies in water have a shielding effect that reduces drag on objects floating in their wake (Hoerner, 1965). In the wake of the first body, the dynamic pressure is reduced and drag is decreased over the distance of $x / d=2$, where $x$ is the distance between the two bodies $(\mathrm{m})$. Organisms take advantage of reduced drag in a wake by forming queues (e.g., Fish, 1995; Bill and Herrnkind, 1976), and the same theory holds for an animal towing accessory gear in its wake. Any object at a distance $x / d<2$ should experience a reduction in drag by a factor of approximately 0.75 (Hoerner, 1965).

We calculated the total drag, $D_{T}(\mathrm{~N})$, on an entangled whale:

$$
D_{T}=D_{w}+a\left(D_{b}+D_{l}\right)+D_{I}
$$

where $D_{b}$ is the drag on tethered buoys or other accessory gear, $D_{l}$ is the drag on the attached line, $D_{I}$ is the interference drag, and a is the shielding factor, based on the spacing distance, $x$, between the body and the towed gear where if $x / d<2, a=0.75$, and if $x / d>1$, $a=1$. In this study, we measured $\left(D_{b}+D_{l}\right)$ empirically.

We derived the total power input $\left(P_{I, T} ; \mathrm{W}\right)$ required for propulsion at a certain speed under any calculated drag condition (generic $D$ ) as

$$
P_{I, T}=P_{L}+P_{I, B}=\frac{D U}{\eta}+P_{I}, B
$$

where $P_{L}$ is locomotory power, and $P_{I, B}$ is power input for standard metabolism, both in $\mathrm{W}$, and $\eta$ is an efficiency coefficient of 0.15 (Fish, 1993a; Hind and Gurney, 1997). Given the uncertainties in appropriate metabolic rate estimation for cetaceans (Gallivan, 1992), we estimated minimum and maximum standard metabolism (W) using Kleiber $\left(3.4 W^{0.75}\right.$; where $W$ is body weight in $\mathrm{kg}$ ), and $3 \times$ Kleiber. Facing an increase in drag, an individual 
can: (1) maintain a characteristic velocity and exponentially increase energy expenditure to overcome added drag; or (2) swim at a reduced speed in order to maintain the same power output as if under normal conditions (Jones et al., 2011). For the latter case, the decrease in velocity $\left(U_{\text {red }}, \mathrm{m} / \mathrm{s}\right)$ to maintain the same power output in an entangled drag scenario $\left(D_{T}\right)$, is

$$
U_{\text {red }}=\left[\frac{P_{L} \eta U^{2}}{D_{T}}\right]^{1 / 3} .
$$

To determine the additional power demands experienced by Eg 3911 while entangled, we compared $P_{I, T}$ for the drag conditions of a nonentangled whale, with surface drag factor $\gamma$ following disentanglement (i.e., $\gamma=1.0$ ), to the conditions of an entangled whale, towing three gear configurations tested in this experiment, with surface drag factor $\gamma$ calculated for the mean $\pm \mathrm{SD}$ dive depth prior to disentanglement (i.e., $\gamma=1.6$ ).

\subsection{Results}

\subsubsection{Tagging and behavior}

Eg 3911 completed $n=152$ dives over the $6 \mathrm{~h}$ deployment period, to a median (IQR) depth of 11.50 (10.97) $\mathrm{m}$ and duration of 98.7 (82.1) s (Fig. 5).

Within the Sedation/Entangled phase, there was no significant difference between the depth or duration of dives completed in the $21 \mathrm{~min}$ prior to $(n=7)$ and the $50 \mathrm{~min}$ following $(n=45)$ sedative injection $(Z=0.402$ and $0.188 ; P=0.6876$ and 0.8511 , respectively; Table $3)$.

Dive depth increased significantly with every phase $\left(\chi^{2}=26.66, P<0.0001\right.$; Fig. 6). Median dive depth was significantly $(138 \%)$ shallower in Sedation/Entangled compared to Disentangled $(Z=-6.121, P<0.0001)$. Significant increases in dive depth occurred between Disentangled and Recovery $(Z=4.607, P<0.0001)$, though only by $19 \%$. Even when considering increases in approximate regional water column depth with time, proportional dive depth was significantly shallower in Sedation/ Entangled (by 95\%) compared to following the removal of gear and buoys (i.e., in Disentangled; $Z=-5.216, P<0.0001$; Fig. 6). Further, we observed no significant difference in proportional dive depth between Disentangled and Recovery phases $(Z=-0.679, P=0.497)$.

Descent rates $(\mathrm{m} / \mathrm{s})$ during dives differed significantly between phases $\left(\chi^{2}=49.87, P<\right.$ 0.0001; Fig. 6), where descents during Sedation/Entanglement were $57 \%$ slower than in Disentangled $(Z=-6.287, P<0.0001)$. There was no significant difference between the descent rates in Disentangled and Recovery $(Z=0.535, P=0.5927)$.

Ascent rates $(\mathrm{m} / \mathrm{s})$ during dives also differed significantly between phases $\left(\chi^{2}=46.22, P<\right.$ 


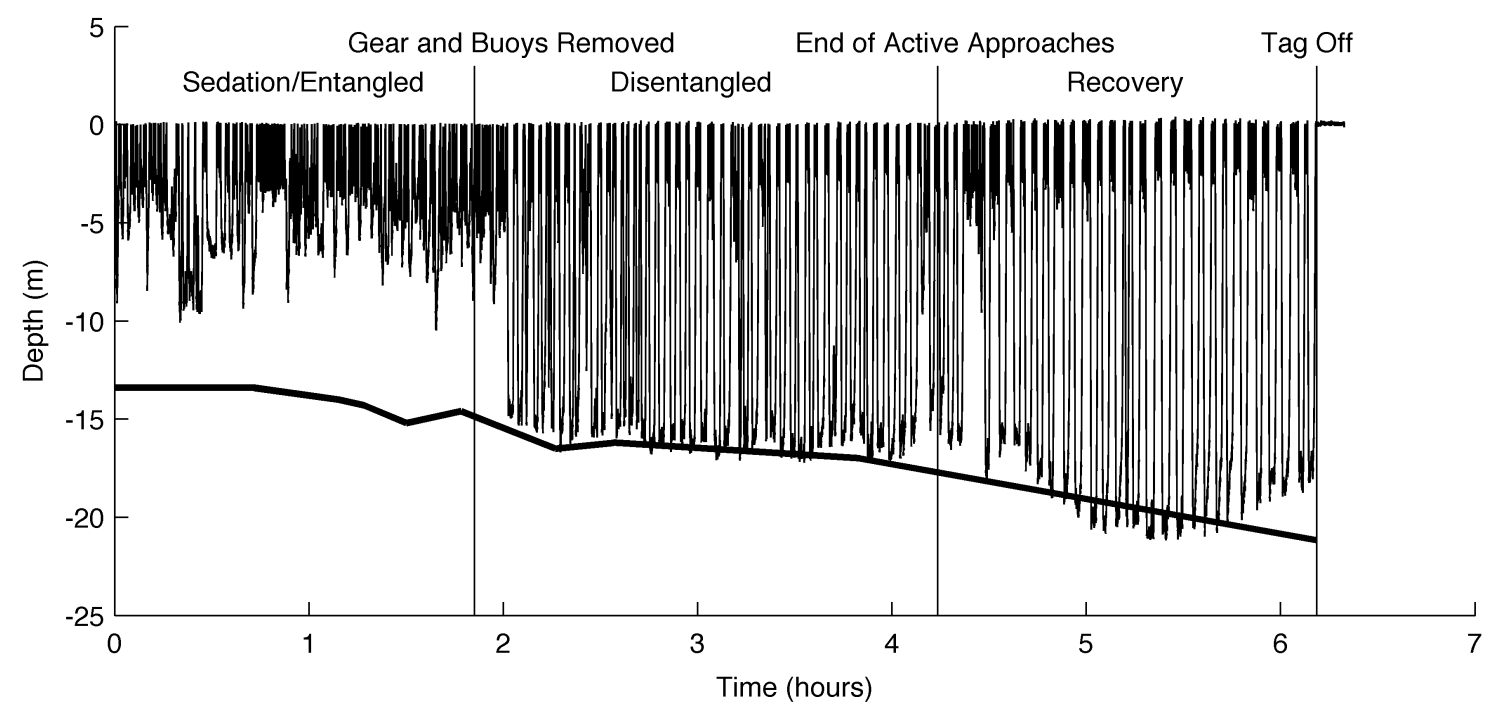

Figure 2-5: Dive profile of right whale Eg 3911 over the course of a 6:11 (h:min) Dtag attachment. Estimated bottom depth (meters; horizontal black line) and event markers are plotted for reference.

0.0001; Fig. 6), with significantly slower ascents (31\%) during Sedation/ Entanglement compared to in Disentanglement $(Z=-5.948, P<0.0001)$. Similar to descent rate, ascent rate did not differ between Disentanglement and Recovery $(Z=0.090, P=0.9285)$.

For Eg $3911(h=1 \mathrm{~m}, d=2.20 \mathrm{~m})$, wave drag is maximal within $0.1 \mathrm{~m}$ of the surface, and becomes negligible below $5.58 \mathrm{~m}$ depth $(h=6.58 \mathrm{~m})$. The ratio of time spent above vs. below the wave drag limit $(5.58 \mathrm{~m})$ over the entire deployment was 1.06, meaning Eg 3911 spent almost equal amounts of time above and below the threshold. However, significantly more time was spent in surface waters where energy requirements are higher before $(7.02: 1)$ vs. following sedative injection $\left(2.47: 1 ; \chi^{2}=141, P<0.0001\right.$; Table 3$)$, and while entangled (i.e., during Sedation/Entangled; 2.87:1) vs. during Disentangled (0.6656:1) and Recovery phases $\left(0.4405: 1 ; \chi^{2}=3,220, P<0.0001\right)$.

Dive duration (s) differed significantly between phases $\left(\chi^{2}=26.67, P<0.0001\right.$; Fig. $6)$, where dives during Sedation/Entangled were $56 \%$ shorter than in Disentanglement $(Z=-3.151 ; P<0.0016)$. Dive duration also increased significantly, by $30 \%$, from Disentanglement to Recovery $(Z=3.4218, P=0.0006)$.

\subsubsection{Dive shape}

Dive shape, as measured by the DAR, differed significantly between phases $\left(\chi^{2}=19.1083, P=\right.$ 0.0001; Fig. 7), with significantly lower DAR during Sedation/Entangled than in Disentangled or Recovery phases $(Z=-3.1615,4.3410, P=0.0016,<0.0001$, respectively). There was no significant difference in the DAR between Disentangled and Recovery phases $(Z=0.9443, P=0.3450)$. 

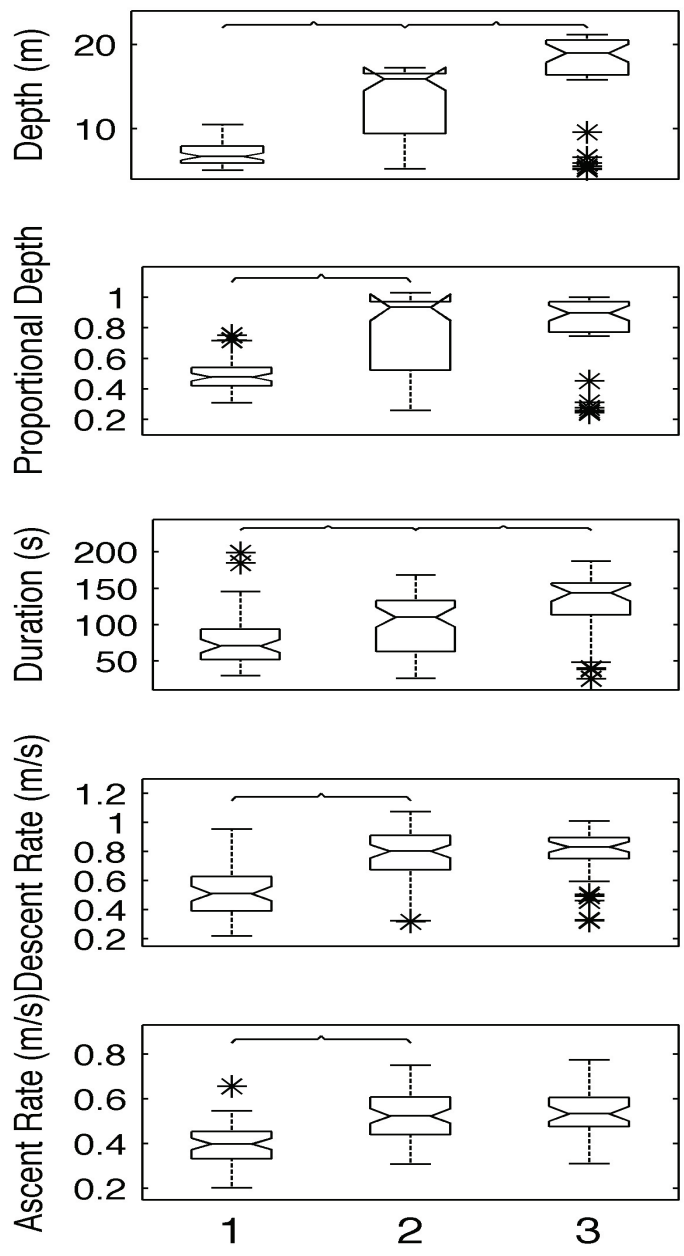

Figure 2-6: Boxplots of dive parameters of right whale Eg 3911 separated into phases (1) Sedation/Entangled, (2) Disentangled, and (3) Recovery. Brackets denote significant differences between two phases. Asterisks indicate outliers.

\subsubsection{Respiration}

Respiration rate per $5 \mathrm{~min}$ interval did not change following sedative delivery $(P=0.4312$; Table 3). We detected no significant difference between respiration rate before (5.00 (2.00) $/ 5 \mathrm{~min})$ and after $(5.00(1.75) / 5 \mathrm{~min})$ buoy and gear removal $(P=0.1679)$.

\subsubsection{Proxies for thrust}

Fluke stroke rate increased significantly following sedative injection $(Z=-8.417, P<$ 0.0001; Table 3). Fluke stroke rate within dives differed significantly between phases $\left(\chi^{2}=\right.$ 

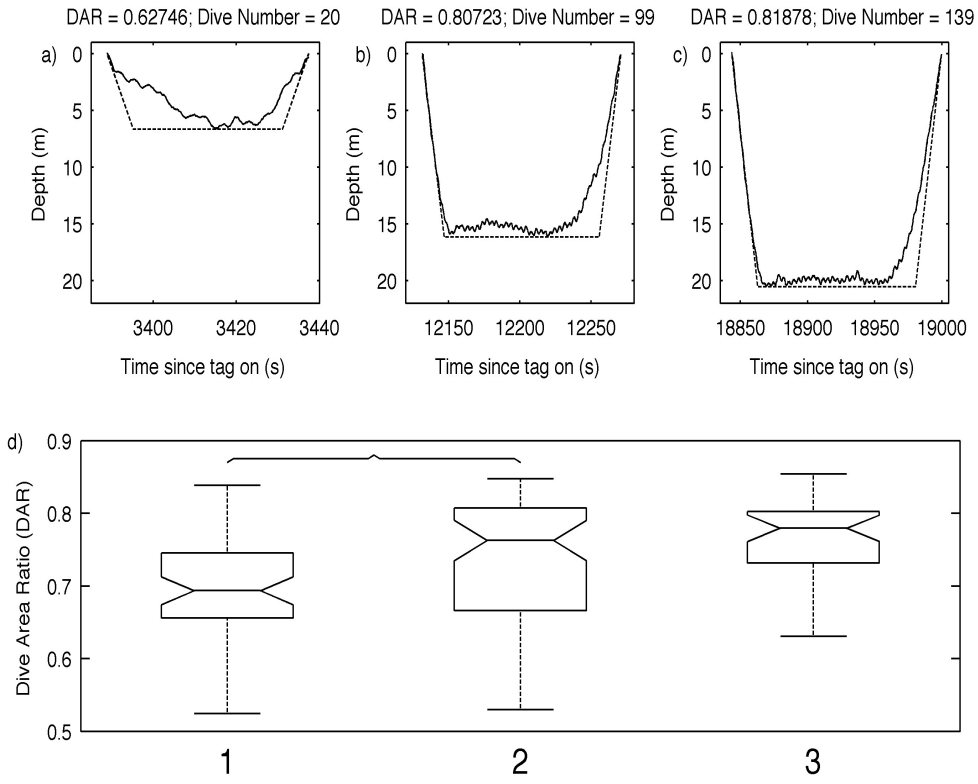

Figure 2-7: Representative dive profiles (black solid line), maximum dive areas (black dashed line), and the calculated Dive Area Ratio (DAR), for phases of (a) Sedation/Entanglement, (b) Disentangled, and (c) Recovery in the Dtag record of right whale Eg 3911. The distribution of the DAR for each phase is shown in (d), with brackets to denote significant differences between two phases. See text for phase definition and details.

18.7179, $P=0.0001$; Fig. 8), being significantly lower during Sedation/Entangled compared to the Disentangled phase $(Z=-3.928, P<0.0001)$. Fluke stroke rate did not differ in Disentangled and Recovery phases $(Z=-0.0323, P=0.9742)$.

Following sedative injection, RMS energy within dives increased significantly, by $28 \%$ $(Z=-3.0832, P=0.0020$; Table 3$)$. RMS energy was $12 \%$ lower after gear and buoy removal $(Z=3.1943, P=0.0014)$. From Disentangled to Recovery phases, RMS energy within dives significantly decreased $(Z=-2.5960, P=0.0094)$. Glide duration did not differ significantly before and after sedative injection $(P=0.1993)$, or before and after the removal of the gear and buoys $(Z=0.334, P=0.9734)$. While glides occurred in all phases, the portion of the dive cycle in which gliding occurred differed between phases. When entangled ( $n=18$ ), $50 \%$ of glides occurred during the bottom period, $33 \%$ during descent, and $17 \%$ on ascent. However, following disentanglement $(n=41), 85 \%$ of glides were performed during the bottom period and $15 \%$ during ascent. No glides were performed during descent following disentanglement.

\subsubsection{ODBA}

Within dives, ODBA did not differ significantly between phases $\left(\chi^{2}=5.4288, P=0.0662\right)$. During dive descents, ODBA differed significantly between phases $\left(\chi^{2}=8.2055, P=\right.$ 

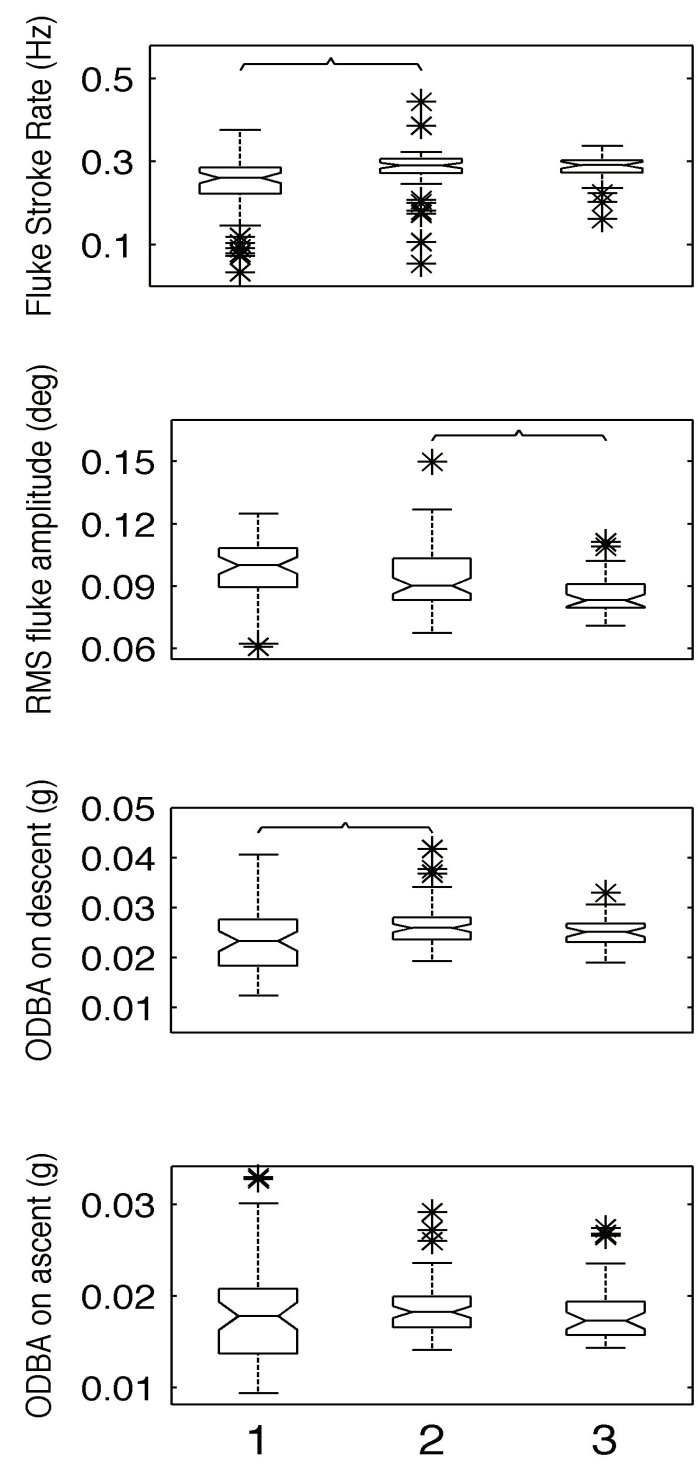

Figure 2-8: Boxplots of fluke stroke rate, Root Mean Square (RMS) fluke amplitude, and Overall Dynamic Body Acceleration (ODBA) on dive descent and ascent, separated into phases (1) Sedation/Entangled, (2) Disentangled, and (3) Recovery in the DTAG record of right whale Eg 3911. Brackets denote significant differences between two phases. Asterisks indicate outliers.

0.0165), being significantly (10\%) lower during Sedation/Entangled than in the Disentangled phase $(Z=-2.7230, P=0.0065$; Fig. 8). There was no significant difference between ODBA in dive descents between Disentangled and Recovery phases $(Z=$ $-1.2603, P=0.2076)$. During ascents, ODBA did not differ significantly between phases 
$\left(\chi^{2}=2.8613, P=0.2392 ;\right.$ Fig. 8$)$.

\subsubsection{Gear towing}

Mean drag forces (N) of gear removed from Eg 3911 were consistently though not significantly greater at all speeds with buoys attached (Table 4). Sinkline drag forces were intermediate between gear-only and gear-and-buoy configurations (Table 4). Mean drag forces showed no significant difference between surface and $2 \mathrm{~m}$ anchor points for gear-only $(P=0.4595)$, gear-and-buoys $(P=0.4888)$ or sinkline $(P=0.4965)$ configurations (Devore, 2008).

\subsubsection{Energy requirements}

The mean theoretical drag coefficient of a nonentangled right whale $\left(C_{d, n}\right)$ of Eg 3911's dimensions, swimming at $0.75-2.9 \mathrm{~m} / \mathrm{s}$ ranged from $3.7 \times 10^{-3}$ to $2.9 \times 10^{-3}$, respectively (mean $\pm \mathrm{SD} ; C_{d, n}=3.2 \times 10^{-3} \pm 0.0003$; Fig. 9). The drag coefficient for each entangled gear scenario was calculated by applying Equation $6\left(C_{d}=D_{T} /(1 / 2) \rho U^{2} A_{w} \gamma k g\right)$. Though drag coefficients for $\mathrm{Eg} 3911$ entangled in all gear configurations differed based on the value of $k$ (Fig. 10), the most conservative estimates with $k=3\left(C_{d, e, g o}=3.4 \times 10^{-3} \pm 0.0003, C_{d, e, g b}=\right.$ $\left.3.7 \times 10^{-3} \pm 0.0003, C_{d, e, s l}=3.8 \times 10^{-3} \pm 0.0004\right)$ were significantly greater than in the nonentangled case (Wilcoxon signed rank, $P=0.0156,0.0312,0.0078$, respectively).

Having made low (Kleiber) and high $(3 \times$ Kleiber $)$ estimates of BMR, and using two values of $k$ (1 and 3), we present drag and power requirements as the lower $(k=1, \mathrm{BMR}=$ Kleiber $)$ and upper $(k=3, \mathrm{BMR}=3 \times$ Kleiber $)$ bounds of the model results. Drag forces on Eg 3911 while not entangled ranged from $37.2 \mathrm{~N}$ to $1,263 \mathrm{~N}$ at $0.75-2.9 \mathrm{~m} / \mathrm{s}$. The associated total power requirements in the nonentangled condition (Eq. 11) ranged from 2,791 W to $16,140 \mathrm{~W}$ (Fig 10). Locomotory power requirements ranged from $191 \mathrm{~W}$ to $25,021 \mathrm{~W}$.

Drag forces on Eg 3911 entangled in various gear configurations are summarized in Table 5. Across all gear configurations, mean entangled drag values ranged from $62.1 \mathrm{~N}$ to $2,421 \mathrm{~N}$. Increases in total power input over the normal (nonentangled) condition ranged from $4.1 \%$ to $58.8 \%$ for the gear-only configuration, $4.9 \%$ to $82.5 \%$ for the sinkline configuration, and $4.8 \%$ to $120.9 \%$ for the gear-and-buoy configuration (Fig. 9). Locomotory power requirements increased on average $70.5 \%$ (SD 9.5) for the gear-only configuration, $91.0 \%$ (22.5) for the sinkline configuration, and $101.9 \%$ (31.9) for the gear-and-buoy configuration (total range $60.0 \%-164.6 \%$ ). Alternatively, to maintain the same power output over the range of swimming speeds, an individual entangled in gear-only, sinkline, and gear-and-buoy configurations would need to decrease swimming speed by $16.2 \%$ (SD 1.5), $19.2 \%$ (3.0), or $20.5 \%$ (3.9), respectively (total range $14.5 \%-27.7 \%$ ). 
Table 2-2: List of symbols and abbreviations.

\begin{tabular}{|c|c|c|}
\hline Symbol & Definition & Unit \\
\hline$a$ & Shielding factor & \\
\hline$A$ & Wetted surface area & $\mathrm{m}^{2}$ \\
\hline$\overline{A_{a}}$ & Integrated dive area & $\mathrm{m}^{2}$ \\
\hline$\overline{A_{p}}$ & Protuberance frontal area & $\mathrm{m}^{2}$ \\
\hline$C_{d}$ & Drag coefficient & \\
\hline$C_{D} I$ & Interference drag coefficient & \\
\hline$C_{D} 0$ & Profile drag coefficient & \\
\hline$C_{f}$ & Frictional drag coefficient & \\
\hline$C_{p}$ & Pressure drag coefficient & \\
\hline$d_{\max }$ & Maximum body diameter & $\mathrm{m}$ \\
\hline$d_{d}$ & Tag-derived depth & $\mathrm{m}$ \\
\hline$d_{x}$ & Body diameter at distance $l_{x}$ from leading edge & $\mathrm{m}$ \\
\hline$D$ & Maximum dive depth & $\mathrm{m}$ \\
\hline$D_{b}$ & Buoy drag & $\mathrm{N}$ \\
\hline$D_{f}$ & Frictional drag & $\mathrm{N}$ \\
\hline$D_{I}$ & Interference drag & $\mathrm{N}$ \\
\hline$D_{l}$ & Line drag & $\mathrm{N}$ \\
\hline$D_{T}$ & Total drag & $\mathrm{N}$ \\
\hline$D_{W}$ & Total whale drag & $\mathrm{N}$ \\
\hline fs & Tag sampling rate & $\mathrm{N}$ \\
\hline$g$ & Appendage drag augmentation factor & \\
\hline$h$ & Submergence depth & $\mathrm{m}$ \\
\hline$k$ & Profile drag augmentation factor & $\mathrm{N}$ \\
\hline$l$ & Length & $\mathrm{m}$ \\
\hline$l_{x}$ & Distance from leading edge & $\mathrm{m}$ \\
\hline$p$ & Protuberance height & $\mathrm{m}$ \\
\hline$P$ & Power & $\mathrm{W}$ \\
\hline$R e$ & Reynolds number & \\
\hline$T$ & Total dive duration & $\mathrm{m}^{2} / \mathrm{s}$ \\
\hline$U$ & Speed & $\mathrm{m} / \mathrm{s}$ \\
\hline$U_{\text {red }}$ & Reduced speed & $\mathrm{m} / \mathrm{s}$ \\
\hline$v$ & Kinematic viscosity & $\mathrm{m}^{2} / \mathrm{s}$ \\
\hline$W$ & Weight & $\mathrm{kg}$ \\
\hline$x$ & Distance between whale and first towed body & $\mathrm{m}$ \\
\hline$\delta$ & Boundary layer thickness & $\mathrm{m}$ \\
\hline$\gamma$ & Surface wave drag factor & \\
\hline$\eta$ & Efficiency coefficient & \\
\hline$\rho$ & Fluid density & $\mathrm{kg} / \mathrm{m}^{3}$ \\
\hline
\end{tabular}


Table 2-3: Median (IQR) respiration rate (/5 min), dive depth $(\mathrm{m})$, proportional dive depth, dive duration (s) and surface interval (s), time spent above:below the significant wave drag depth, fluke stroke rate $(\mathrm{Hz})$ and fluke stroke root-mean-square (RMS) energy (degrees) before and following sedation injection, but prior to gear and buoy removal. Significance values $(P)$ from Wilcoxon rank sum tests are presented.

\begin{tabular}{|l|l|l|l|}
\hline & Preinjection & Postinjection & $P$ \\
\hline Respiration rate $(/ 5 \mathrm{~min})$ & $5.00(4.50)$ & $5.00(1.75)$ & 0.4312 \\
\hline Dive depth $(\mathrm{m})$ & $6.70(3.07)$ & $6.67(1.86)$ & 0.6876 \\
\hline Proportional dive depth & $0.500(0.229)$ & $0.477(0.122)$ & 0.2835 \\
\hline Dive duration (s) & $70.40(15.55)$ & $71.00(45.80)$ & 0.8511 \\
\hline Time above:below significant depth & $7.02: 1$ & $2.87: 1$ & $<0.0001$ \\
\hline Fluke stroke rate $(\mathrm{Hz} ;$ flukes/s) & $0.277(0.049)$ & $0.288(0.058)$ & $<0.0001$ \\
\hline Fluke stroke RMS energy (degrees) & $0.0798(0.0124)$ & $0.1023(0.0163)$ & 0.002 \\
\hline
\end{tabular}

Table 2-4: Mean (SD) drag forces (N) exerted by (1) $33.63 \mathrm{~m}$ of fishing gear and (2) gear and buoy configurations removed from Eg 3911, and (3) $160 \mathrm{~m}$ of sinkline at surface and bottom (2 m) towpoints at various boat speeds $(\mathrm{m} / \mathrm{s})$.

\begin{tabular}{|c|c|c|c|}
\hline Tow Point & Configuration & Vessel speed $(\mathrm{m} / \mathrm{s})$ & Drag Force $(\mathrm{N})$ \\
\hline \multirow{11}{*}{ Surface } & \multirow{3}{*}{ Gear only } & 0.772 & $2.9(2.0)$ \\
\hline & & 1.49 & $21.6(3.9)$ \\
\hline & & 2.83 & $59.8(4.9)$ \\
\hline & \multirow{3}{*}{ Gear and buoys } & 0.772 & $16.7(2.9)$ \\
\hline & & 1.49 & $55.9(12.7)$ \\
\hline & & 2.73 & $377.6(36.3)$ \\
\hline & \multirow{5}{*}{ Sinkline $160 \mathrm{~m}$} & 0.772 & $11.8(2.9)$ \\
\hline & & 0.772 & $8.8(3.9)$ \\
\hline & & 0.772 & $11.8(3.9)$ \\
\hline & & 1.49 & $80.4(2.9)$ \\
\hline & & 2.73 & $202.0(23.5)$ \\
\hline \multirow{10}{*}{ Bottom } & \multirow{4}{*}{ Gear only } & 0.772 & $2.9(2.0)$ \\
\hline & & 1.49 & $36.3(3.9)$ \\
\hline & & 2.52 & $77.5(9.8)$ \\
\hline & & 2.73 & $80.4(13.7)$ \\
\hline & \multirow{3}{*}{ Gear and buoys } & 0.772 & $12.7(2.9)$ \\
\hline & & 1.49 & $76.5(6.9)$ \\
\hline & & 2.98 & $415.8(28.4)$ \\
\hline & \multirow{3}{*}{ Sinkline $160 \mathrm{~m}$} & 0.772 & $29.4(3.9)$ \\
\hline & & 1.49 & $70.6(6.7)$ \\
\hline & & 2.83 & $194.2(24.8)$ \\
\hline
\end{tabular}


Table 2-5: Total drag forces (N) on, and power output (W) required by Eg 3911 swimming entangled in various configurations (Gear Only, Gear and Buoys, and Sinkline) of fishing gear, and the percentage increase in power, or percent decrease in swimming velocity due to increased drag over the normal nonentangled) condition. Ranges represent the lower and upper bounds of values of $k$ (profile drag augmentation factor) and metabolic rate (see text).

\begin{tabular}{|c|c|c|c|c|c|c|c|}
\hline & $\begin{array}{l}\text { Velocity } \\
(\mathrm{m} / \mathrm{s})\end{array}$ & $\begin{array}{l}\text { Total drag } \\
(\mathrm{N})\end{array}$ & $\begin{array}{l}\text { Total power } \\
(\mathrm{W})\end{array}$ & $\begin{array}{l}\text { Locomotor } \\
\text { power }(\mathrm{W})\end{array}$ & $\begin{array}{l}\text { Percent total } \\
\text { power increase }\end{array}$ & $\begin{array}{l}\text { Percent locomo- } \\
\text { tor power in- } \\
\text { crease }\end{array}$ & $\begin{array}{l}\text { Percent velocity } \\
\text { decrease }\end{array}$ \\
\hline \multirow{5}{*}{ Gear only } & 0.77 & $62.1-178.4$ & $2,920-8,718$ & $320-918$ & $4.1-4.6$ & $60.0-67.2$ & $14.5-15.8$ \\
\hline & 1.49 & $223.0-603.9$ & $4,818-13,806$ & $2,218-6,006$ & $20.8-26.4$ & $65.3-83.2$ & $15.4-18.3$ \\
\hline & 2.52 & $577.4-1556.5$ & 12304-33957 & 9704-26157 & $44.0-56.5$ & $65.8-84.5$ & $15.5-18.5$ \\
\hline & 2.73 & $656.8-1784.3$ & 14538-40234 & 11938-32434 & $46.5-58.8$ & $65.0-82.2$ & $15.4-18.1$ \\
\hline & 2.83 & $676.5-1881.8$ & 15361-43297 & 12671-35497 & $46.3-55.7$ & $62.8-75.6$ & $15.0-17.1$ \\
\hline \multirow{4}{*}{ Gear and buoys } & 0.77 & 73.9-190.2 & $2980-8778$ & $380-978$ & $4.8-6.8$ & $70.6-98.9$ & $20.5-16.3$ \\
\hline & 1.49 & $260.3-641.1$ & $5189-14176$ & $2589-6376$ & $24.0-36.2$ & $75.5-113.8$ & $17.1-22.4$ \\
\hline & 2.73 & 953.9-2081.5 & 19939-45635 & 17339-37835 & $66.2-117.9$ & $92.5-164.6$ & $19.6-27.7$ \\
\hline & 2.98 & $1094.7-2420.9$ & $24376-55957$ & $21776-48157$ & 69.1-120.9 & $90.3-158.2$ & $19.3-27.1$ \\
\hline \multirow{4}{*}{ Sinkline } & 0.77 & $74.4-190.7$ & $2983-8780$ & $383-981$ & 4.39-6.9 & $71.0-100.2$ & $16.7-21.4$ \\
\hline & 1.49 & $268.7-649.5$ & $5272-14260$ & $2672-6460$ & $24.7-38.3$ & $77.8-120.7$ & $19.5-27.4$ \\
\hline & 2.73 & $775.9-1903.5$ & $16704-42400$ & 14103-34600 & $54.4-82.5$ & $76.0-115.3$ & $17.2-22.6$ \\
\hline & 2.83 & $808.2-2013.5$ & $17844-45780$ & $15244-37980$ & $54.6-80.1$ & $74.2-109.7$ & $16.9-21.9$ \\
\hline
\end{tabular}




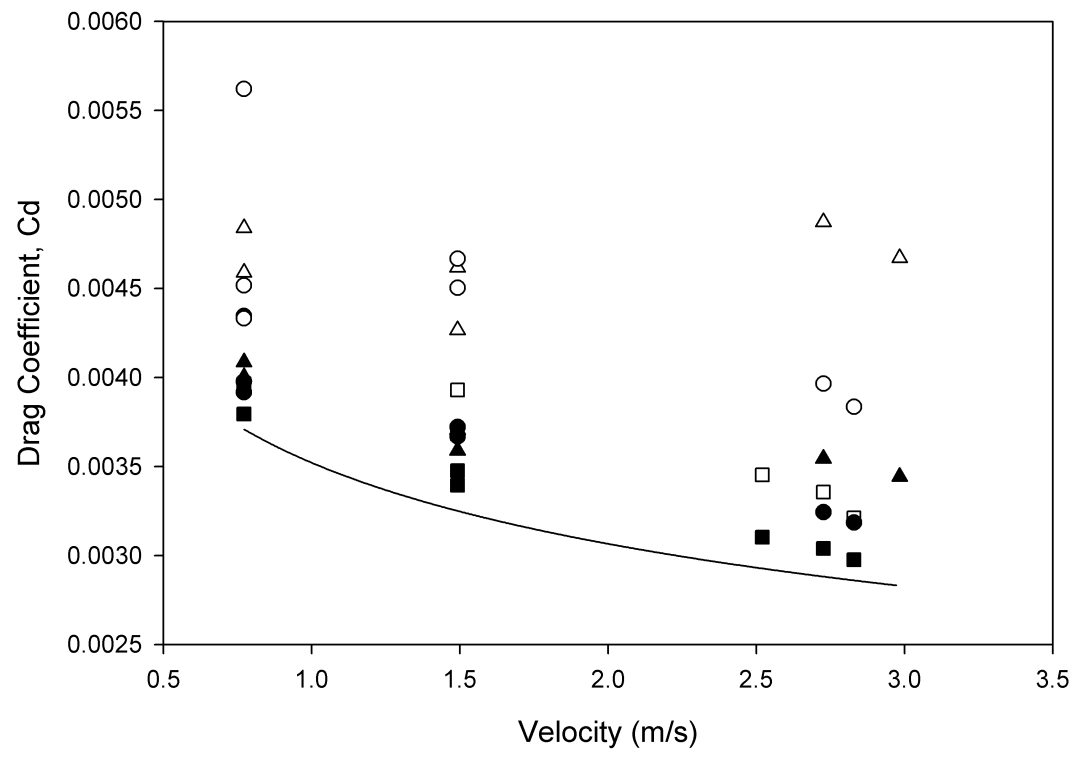

Figure 2-9: Drag coefficient of right whale Eg 3911 at various swimming velocities in the nonentangled condition (line), and while entangled in gear-only (squares), gear-and-buoys (triangles) and sinkline (circles) configurations using minimum (closed symbol) and maximum (open symbol) parameter estimates.

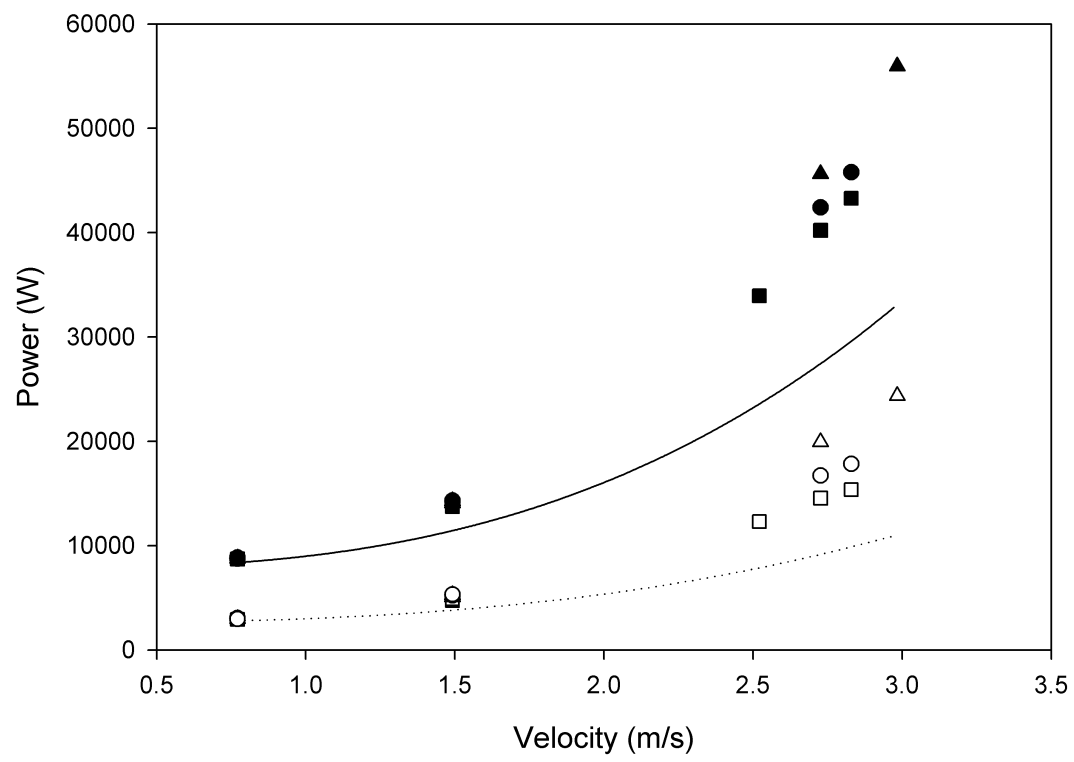

Figure 2-10: Minimum (open symbol, dotted line) and maximum (closed symbol, solid line) estimates of total power input (W) of right whale Eg 3911 while nonentangled (lines) and entangled in gear-only (squares), gear-and-buoys (triangles) and sinkline (circles) configurations. 


\subsection{Discussion}

We describe the effect of sedation and near-complete disentanglement of a free-swimming entangled right whale, Eg 3911. Tag data show major changes in locomotion before and after disentanglement. Modeling the drag forces of the removed gear, we show that entangled whales can have significantly increased energetic demand.

Sedative injection had little to no effect on dive parameters or respiration rate. It is likely that in this condition, behavior is dominated by the effect of entangling gear rather than of a light sedative. At the dosage level $(0.1 \mathrm{mg} / \mathrm{kg})$, Midazolam has not been found to cause cardiovascular, respiratory, or airway reflex changes in humans (Reves et al., 1985), though a previous study reports increased respiration rates following sedation in right whales (Moore et al., 2010).

After sedation, Eg 3911 spent a greater proportion of time below the wave-drag threshold $(5.58 \mathrm{~m})$, though showed no difference in maximum dive depth. This increased submergence time may be linked to the lethargy associated with sedation. Moore et al. (2010) describe less forceful surfacing events in sedated right whales. However, increased fluke rate and RMS energy postsedation may suggest the drugs had an analgesic effect in reducing entanglementassociated pain, and therefore freeing the animal to locomote more strongly.

The near-complete disentanglement of Eg 3911 resulted in significant increases in dive duration and depth. Similarly, (Williams and Kooyman, 1985) found that increased drag loading in harbor seals led to shortened dive times. As dive duration is considered limited by the total amount and rate of consumption of body oxygen stores, the elevated energetic cost associated with additional entanglement drag likely quickly depletes available oxygen, leading to premature dive termination.

Changes in kinematics and dive parameters indicate the whale altered its behavior immediately following disentanglement. Previous studies suggest that propulsive forces are increased in response to changes in resistive forces, where elephant seals adjust stroke intensity when buoyancy is experimentally altered (Aoki et al., 2011). Animals may also actively alter swimming dynamics or posture to compensate for an added load. As suggested by Watson and Granger (1998), animals facing an increase in drag may either (1) maintain characteristic velocity, exponentially increasing energy expenditure; or (2) reduce swimming

speed in an attempt to reduce the cost of locomotion. Fluke stroke rate, which has been shown to correlate with speed in dolphins (Fish, 1993a) and other cetaceans (Fish, 1998), increased significantly following disentanglement. Further, Eg 3911 showed descent and ascent speeds $57 \%$ and $31 \%$ faster (respectively) after disentanglement, greater than the expected $14.5 \%-27.7 \%$ as calculated above. While changes in swimming speed were likely due to a combination of factors rather than energy conservation alone (e.g., sedation, pursuit by a vessel), this case suggests that entanglement significantly alters swimming modes. 
The greater increase in descent speed (57\%) vs. ascent speed (31\%) following disentanglement likely highlights the effects of both drag and buoyancy related to the entangling gear and buoys. In order to dive to depth, an individual must overcome resistive buoyant forces. More active swimming is thus required on descent, while ascents can be passive (Nowacek et al., 2001). Such buoyant effects are also evident in dive shape. The overall depth- and duration-normalized dive area (DAR) was significantly lower while entangled. Dive descents to, and ascents from maximum depth were more gradual, and less time was spent in the bottom phase of the dive while the animal was entangled as compared with the behavior following disentanglement.

Given that the added buoys were further from the whale than the water column was deep, the buoys should have never been submerged to provide an upwards buoyant force that Eg 3911 could take advantage of to conserve energy in diving (Nowacek et al., 2001). Glides occurred in all phases of the dive cycle, indicating that passive swimming was not timed to take advantage of changes in buoyancy by gliding on ascent while entangled. The emaciated condition of Eg 3911 may have led to negative buoyancy, as has been found in emaciated bottlenose dolphins (Dunkin et al., 2010), and dive depths were much shallower than the predicted depth of lung collapse in cetaceans (30-235 m; Fahlman, 2008). It is thus likely that glides were employed to conserve energy (Videler and Weihs, 1982; Williams, 2001) rather than to optimize the benefits of buoyancy.

ODBA has shown to be a reliable estimator for activity and metabolic rate in freeswimming animals (Fahlman, 2008). It was thus expected that ODBA be greater under the entangled condition; however, ODBA was often lower while entangled, compared to after disentanglement. We suggest that restraint by the drag and buoyancy of the gear may have reduced Eg 3911's ability to make large dynamic movements. Accelerometer measurements determine only the movement of the animal (i.e., net movement) and those forces associated, but not the forces required to move against any materials that may be restraining movement (i.e., total exertion). Consider a running parachute: the runner expends considerably more energy with the parachute, though their motion is more limited and is slower than without the apparatus. The application of ODBA to free-swimming and restrained cases likely requires separate metabolic calibrations for each condition, which are not available for entangled large whales at this time.

Together, the effects of added buoyancy, added drag, and reduced swimming speed due to towing accessory gear pose many threats to entangled whales. If buoyancy overwhelms an animal's ability to descend to the depth of its preferred prey, its foraging ability may be significantly compromised, accelerating the transition to a negative energy balance. Increased time spent in surface waters results in greater overall drag, due to surface effects (Hoerner, 1965; Hertel, 1969), and places individuals at greater ship strike risk (Nowacek et al., 2001; Parks et al., 2012). Reduced swimming speed will lead to increases in travel 
time, potentially delaying an entangled individual's arrival to feeding or breeding grounds in the case of migratory species (Watson and Granger, 1998; Jones et al., 2011).

Most significant, however, is the energy drain associated with added drag. The drag experienced by an animal is significantly affected by the size of the animal relative to the entangling gear, and its configuration, position of attachment, placement in the animal's wake, and surface area (Feldkamp, 1985). The addition of buoys to entangling gear during disentanglement procedures to increase surface area, buoyancy, and turbulence does significantly increase drag forces; however, this method has been used successfully to disentangle whales that have survived to breed (Robbins, 2012; Robbins and Knowlton, 2012). Therefore, we suggest that current practice be continued in adding buoys only for shortterm operations, such as a single disentanglement attempt. The benefits of partial or full gear-removal likely outweigh the short-term energetic impact buoy-addition may incur.

Since not all entanglements can be resolved during a single attempt, a $36 \mathrm{~cm}$ diameter satellite/VHF telemetry buoy is the current method of tracking entangled individuals for later re-sighting and disentanglement. In eight cases, these buoys have also provided sufficient drag to allow whales to remove some or all remaining gear. ${ }^{3}$ Since the current telemetry buoy does create drag force (ca. $76 \mathrm{~N}$ at $1.3 \mathrm{~m} / \mathrm{s}$; Woodward et al., 2006a) entanglement responders should continue to make every effort to: use telemetry on a case-by-case basis, strategically place the telemetry buoy to minimize impacts, remove as much of the original trailing gear to minimize additional drag force and reduce the duration of buoy placement. Longer-duration, lower drag telemetry buoy designs should continue to be developed for tracking entangled individuals for later disentanglement.

To reduce locomotory costs, marine mammals have adapted low drag coefficients. Drag has been estimated from Dtag records (Miller et al., 2004; Simon et al., 2009; McGregor, 2010), though this method requires a measure of speed, which cannot be obtained from this tagging event due to boat noise and low pitch angles. Still, the theoretical coefficient we estimated for Eg $3911\left(3.7 \times 10^{-3}\right.$ to $2.8 \times 10^{-3}$ over a range of speeds) falls well within the range of previously estimated drag coefficients for large whales $\left(5.2 \times 10^{-3}-1.4 \times 10^{-2}\right.$; Miller et al., 2004; McGregor, 2010). Significant increases (2.3\%-69.2\%) in the drag coefficient occur in the entangled scenario, leading to $60.0 \%-164.6 \%$ increases in locomotory power output.

These energy requirements are only related to propulsion in an entanglement scenario and do not consider increased thermoregulation to compensate for loss of body fat, or stressrelated changes in metabolic rate, which have increased up to $16.25 \%$ in entangled northern fur seals despite increased resting time (Feldkamp et al., 1988). Though fecal glucocorticoid studies have shown markedly elevated stress hormone levels in a severely entangled right whale (Hunt et al., 2006), the relationships between entanglement stress and metabolic rate

\footnotetext{
${ }^{3}$ Personal communication from Scott Landry, Provincetown Center for Coastal Studies Marine Animal Entanglement Response, 5 Holway Ave, Provincetown, MA 02657, February 2013.
} 
are too complex to be considered here.

High energy requirements and negative energy balance are not uncommon in large whales. Right whales routinely enter a phase of energy deficit during the fasting cycle associated with annual migrations between high-latitude foraging habitats and low latitude calving areas. Sufficient endurance to survive the fasting phase and subsequently recoup losses in the following foraging season are likely adaptations, though prolonged periods of an imbalance of greater magnitude may impact an individual's energy reserve to a point beyond which recovery is not possible (Millar and Hickling, 1990). The magnitude of power output due to drag of entangling gear almost certainly would make such long distance $(\sim 2,900 \mathrm{~km}$, from the Gulf of Maine to Florida; Kraus et al., 1986) fasting migrations much more energetically costly for an entangled whale.

A simple calculation can illustrate both the effects of increased drag, and of reduced swimming speed (Watson and Granger, 1998; Jones et al., 2011). Using our most conservative estimate, a nonentangled right whale swimming $2,900 \mathrm{~km}$, at an average speed of 1.5 $\mathrm{m} / \mathrm{s}$ could complete a one-way migration in $22 \mathrm{~d}$, expending $7.3 \times 10^{9} \mathrm{~J}$ of energy. Entangled in the gear-only configuration, an individual could migrate at the same speed, arriving on time and expending $9.3 \times 10^{9} \mathrm{~J}$ of energy (a $27 \%$ increase) or could swim at a reduced speed to arrive $5 \mathrm{~d}$ late, expending $9.6 \times 10^{9} \mathrm{~J}$ (a $31 \%$ increase). If this same calculation is made with a more energetically costly entanglement scenario (e.g., gear-and-buoys), the entangled individual could arrive on-time, expending $1.0 \times 10^{10} \mathrm{~J}$ (a $37 \%$ increase), or 5 $\mathrm{d}$ late expending essentially the same $1.0 \times 10^{10} \mathrm{~J}$. Under both entanglement and speed maintenance or reduction scenarios, the energy store budgeted for a nonentangled one-way migration $\left(7.3 \times 10^{9} \mathrm{~J}\right)$ would be exhausted between $71 \%$ and $78 \%$ of the distance to the destination.

These results provide the first visualization of significant alteration to swimming patterns associated with entanglement. Understanding the major behavioral and energetic implications of towing accessory gear is crucial in considering the sub-lethal effects of persistent entanglement in a critically endangered population. 


\section{Chapter 3}

\section{DRAG FROM FISHING GEAR ENTANGLING NoRTH AtLANTIC RIGHT WHALES}

This chapter was originally published as van der Hoop, J.M., P. Corkeron, J. Kenney, S. Landry, D. Morin, J. Smith, M. J. Moore (2015). Drag from fishing gear entangling North Atlantic right whales. Marine Mammal Science 32, 619-642.

JvdH and MJM developed concepts; JK, SL, JS provided historical data and gear; JvdH, DM, MJM performed fieldwork; JvdH processed and analyzed the data; PC provided statistical guidance; JvdH, PC, JK, SL, DM, JS, MJM contributed to the manuscript.

The supplemental material for this chapter can be found in Appendix B. 


\subsection{Abstract}

Lethal and sublethal fishing gear entanglement is pervasive in North Atlantic right whales (Eubalaena glacialis). Entanglement can lead to direct injury and is likely to incur substantial energetic costs. This study (1) evaluates drag characteristics of entangled right whales, (2) contextualizes gear drag measurements for individual whales, and (3) quantifies the benefits of partial disentanglement. A load cell measured drag forces on 15 sets of fishing gear removed from entangled right whales, a towed satellite telemetry buoy, and 200 $\mathrm{m}$ of polypropylene line as it was shortened to $25 \mathrm{~m}$, as they were towed behind a vessel at $\sim 0.77,1.3$, and $2.1 \mathrm{~m} / \mathrm{s}(\sim 1.5,2.5$, and 4 knots $)$ and $\sim 0,3$, and $6 \mathrm{~m}$ depth. Mean drag ranges from $8.5 \mathrm{~N}$ to $315 \mathrm{~N}$, and can be predicted from the dry weight or length of the gear. Combining gear drag measurements with theoretical estimates of drag on whales' bodies suggests that on average, entanglement increases drag and propulsive power by 1.47 fold. Reducing trailing line length by $75 \%$ can reduce parasitic gear drag by $85 \%$, reinforcing current disentanglement response practices. These drag measurements can be incorporated into disentanglement response, serious injury determination, and evaluation of sublethal effects on population dynamics.

\subsection{Introduction}

Lethal and sublethal trauma to North Atlantic right whales (hereafter right whales; Eubalaena glacialis) from entanglement in fishing gear is pervasive in this endangered species (Knowlton et al., 2012a; van der Hoop et al., 2013a). The majority of recorded right whale entanglement cases involve free-swimming whales that are no longer anchored by gear (NMFS, 2003). The impacts of entanglement can then become protracted as portions of the entangling gear are carried for months to years (Moore et al., 2006; Moore and van der Hoop, 2012). Entangling gear may wrap and abrade body parts, while towing large portions of trailing gear likely affects an individual's energy balance: emaciation was documented in $56 \%$ of a case series of entanglement mortalities (Cassoff et al., 2011).

That emaciation is so commonly reported in chronic entanglement cases is not surprising. The theoretical basis is that towed bodies add drag to the system (Fridman and Dvernik, 1973; Batchelor, 2000), which then requires more thrust for forward movement, involving additional energy output by the animal (Webb, 1975b). The literature on scientific instrumentation ethics provides direct evidence for changes in body condition associated with drag and weight of tags (e.g., reviewed in Barron et al., 2010). While the energetic consequences imposed by entangling fishing gear have been described (Fowler, 1987; Wells et al., 2008; Barco and Moore, 2010; Cassoff et al., 2011; Wegner and Cartamil, 2012; Barratclough et al., 2014), only recently have they been quantified (Feldkamp et al., 1988; van der Hoop et al., 
2013b). van der Hoop et al. (2013b) showed that towing gear increased power requirements of one entangled right whale by $70 \%$ to $102 \%$ when maintaining preferred swimming speeds. Quantifying the parasitic drag imposed by entangling gear is the first step in evaluating these energetic impacts, and is critical in assessing the potential for survival and recovery of an entangled whale.

Disentanglement groups (e.g., the Center for Coastal Studies, CCS; Provincetown, MA) and networks (e.g., the Atlantic Large Whale Disentanglement Network, ALWDN [USA]; International Whaling Commission Global Whale Entanglement Response Network) have been established to train responders to address the growing number of whale entanglements reported worldwide. Ultimately, disentanglement response seeks to reduce the potentially lethal portions of gear (not necessarily all gear) while maintaining high human and whale safety (IWC, 2011). While new tools for at-sea tranquilization (Moore et al., 2010) and gear removal (Moore et al., 2012) have been developed to enhance disentanglement effectiveness, success continues to vary, often requiring multiple disentanglement attempts, especially in right whales. Satellite telemetry buoys (Fig. B1) are sometimes attached to trailing gear to track whales until conditions (e.g., equipment, personnel, weather or time of day) favor further attempts at gear removal. In some attempts, trailing gear is cut to minimize the risk of further entanglement and to reduce parasitic drag and energetic impacts, especially in chronic entanglement cases.

The fishing gear removed through disentanglement operations can and should be archived and studied to better understand the causes and effects of entanglement (Johnson et al., 2005; Knowlton et al., 2015). To quantify the amount of drag imposed by entangling gear, and the energetic impacts potentially incurred, a load cell tensiometer measured drag forces (Fig. 1) from 15 sets of fishing gear removed from entangled whales (Fig. 2, Appendix B2), and an additional six sets similar to those found on entangling whales (21 total configurations). This study (1) describes and compares the drag characteristics of sets of fishing gear entangling right whales, (2) puts the drag measurements in the context of individual whales entangled by the gear, and (3) quantifies the benefits of partial gear removal.

\subsection{Materials and Methods}

\subsubsection{Experimental gear description}

Fifteen sets of fishing gear removed from past entanglements of right whales were selected from those available through the NOAA (National Oceanic and Atmospheric Administration) Gear Research Team; and where whale ID, specific entanglement dates, and individual fate were known. Chain-of-custody protocols were followed during all transfers. Some gear sets had been altered to return components to their rightful owner, or for use in other previous experiments. Gear sets were reassembled as needed, and towed in configurations that 


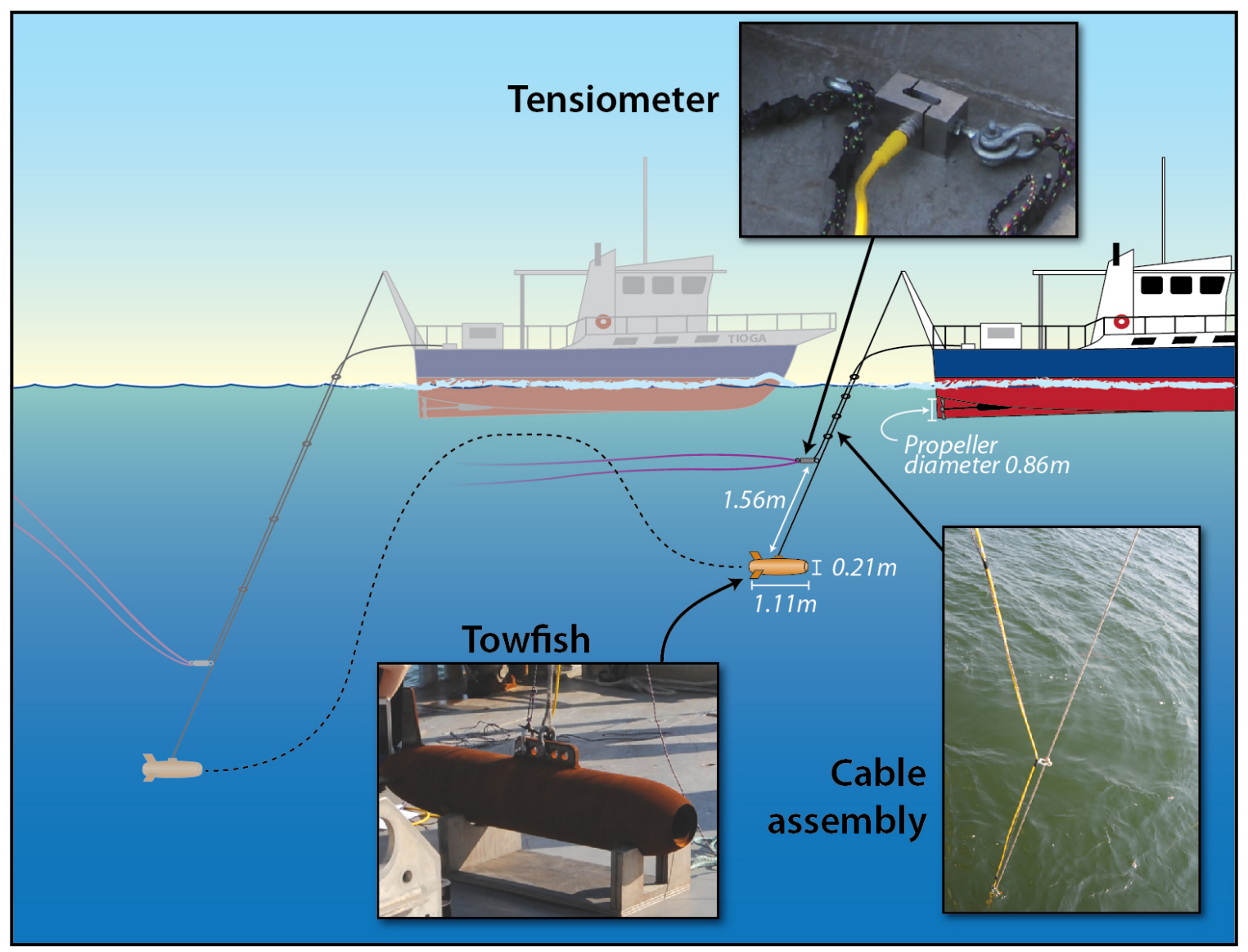

Figure 3-1: The experimental setup. The towfish is towed from the R/V Tioga, below the tensiometer's attachment to fishing gear (orange and purple lines). The winch line controls the depth of the towfish, from 0 to $6 \mathrm{~m}$ measured at the depth of the tensiometer. Changing the depth of the towfish has an effect on the gear's orientation in the water column (orange vs. purple gear).

best replicated the entanglement scenario of each case as documented by the disentanglement team (see, e.g., Fig. 2) as practical.

Two additional set-ups were measured: (1) $200 \mathrm{~m}$ of $8 \mathrm{~mm}$ diameter polypropylene line as it was successively shortened to $25 \mathrm{~m}$, and (2) a satellite telemetry buoy (Fig. B1) used in relocating entangled whales by members of the ALWDN (). The satellite telemetry buoy is a $0.36 \mathrm{~m}$ diameter deep-water trawl buoy rated to $600 \mathrm{~m}$, fitted with a stainless steel counterbalanced harness for satellite and VHF radio telemetry transmitters. The buoy is appropriately weighted and designed to retain an upward orientation, to allow for satellite and radio communication, when towed at speeds of up to $3.6 \mathrm{~m} / \mathrm{s}$, or when floating carrying up to $6.8 \mathrm{~kg}$ of entangling gear and attachment hardware, should it come free from a whale.

\subsubsection{Tow experiment}

On 12 September 2012, the 15 sets of fishing gear removed from right whales, $8 \mathrm{~mm}$ diameter line, and a satellite telemetry buoy (Fig. B1) were towed behind the R/V Tioga in Buzzards 

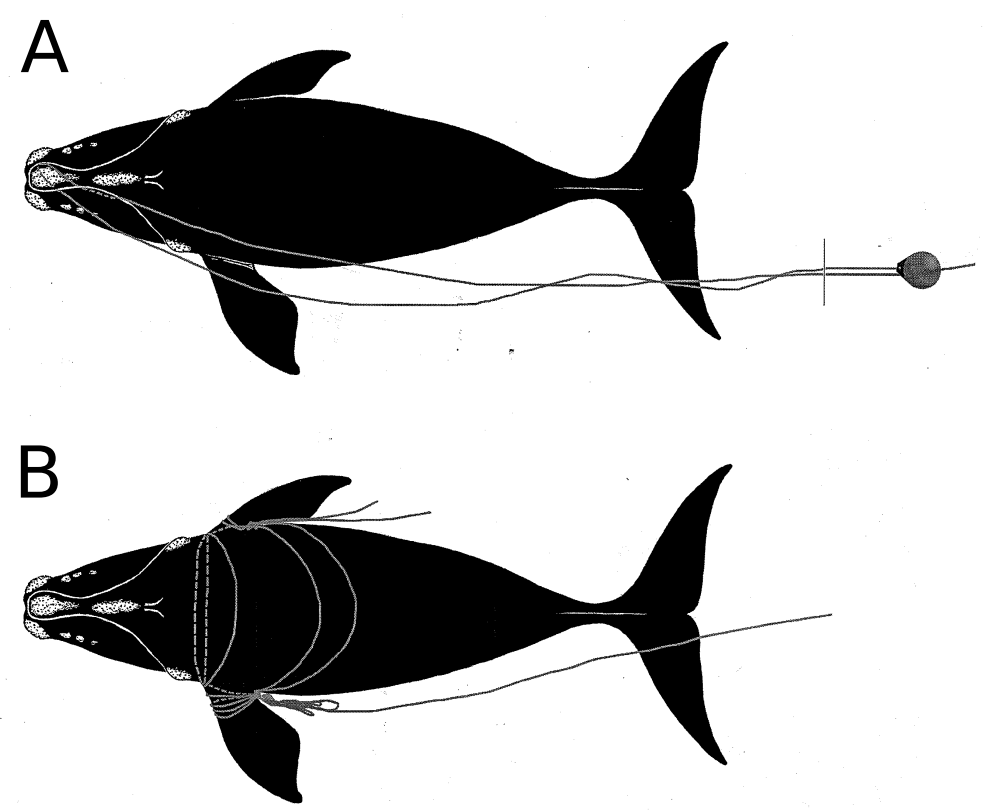

Figure 3-2: Entangling gear can have very different configurations on North Atlantic right whales. A. Eg 1427, showing a rostrum wrap with $82 \mathrm{~m}(269 \mathrm{ft})$ of trailing line. B. Eg 2030, showing extensive body and flipper wraps, with little line trailing. Dashed lines are used to illustrate rope on the underside of the animal.

Bay, Massachusetts, to measure drag forces with a tensiometer (Fig. 1; van der Hoop et al.,

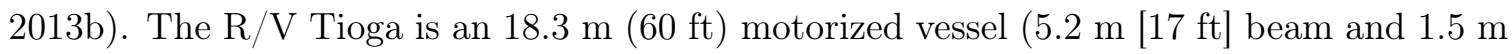
[5 ft] draft) with Twin Series 60 Detroit Diesel 750 horsepower engines. At the transom, the Tioga's stern hull is $0.84 \mathrm{~m}$ below the mean waterline, and the hubs of the $0.86 \mathrm{~m}$ diameter propellers sit $0.68 \mathrm{~m}$ below the mean waterline (Fig. 1). Average wind speeds were $6 \mathrm{~km} / \mathrm{h}$. Slack water occurred at 08:14, and maximum current speeds of $1.0 \mathrm{knot}(0.5 \mathrm{~m} / \mathrm{s})$ were reached at 10:40 before the next slack water at 14:21. All gear sets were towed between the hours of 09:14 and 15:00.

Each set of fishing gear was attached to an HRS-1K load cell tensiometer (Load Cell Central, Monroeton, PA; Fig. 1), connected to a towline $1.56 \mathrm{~m}$ above a $1.1 \mathrm{~m}$ long 9 $0.21 \mathrm{~m}$ diameter tow fish (Fig. 1). The load cell was therefore located outside of (above) the hydrodynamic influence of the tow fish, but within the draft of the vessel. The cable to the load cell was tied to a $4 \mathrm{~mm}$ line attached to 12 shackles closed around the winch cable (Fig. 1). This thin line could then be hauled in, similar to a shower curtain, to allow the load cell to be retrieved without cable strain. The load cell was connected to a laptop computer through a WeightSense OM-232-D Digital Signal Conditioner and read through custom graphical user interface software (ScaleWatch LITE, Load Cell Central, Monroeton, PA), sampling at $60 \mathrm{~Hz}$, and accurate to $0.14 \mathrm{~kg}$.

Drag on each gear set was measured at three speeds: approximately $1.5,2.5$, and 4.0 knots $(0.77,1.3$, and $2.1 \mathrm{~m} / \mathrm{s})$. Speed over ground was measured by a shipboard GPS 
(NavNet VX2; Furuno USA, Camas, WA) at $1 \mathrm{~Hz}$, and handheld GPS (Triton 1500; Magellan Navigation, San Dimas, CA) at approximately $3.05 \mathrm{~Hz}$ (range 0.5-60 Hz). Speed through the water was calculated by subtracting current speeds measured by a shipboard ADCP (300 $\mathrm{KHz}$ WorkHorse Mariner, Teledyne RDI Instruments, Poway, CA). At each speed, gear sets were towed at depths of $0 \mathrm{~m}, 3 \mathrm{~m}$, and $6 \mathrm{~m}$, measured at $1 \mathrm{~Hz}$ by a ReefNet Sensus Pro depth recorder (Mississauga, ON, Canada). Depth was limited by the cable length and pressure sensitivity of the load cell. It was expected that drag would increase with depth, as an increasing angle between rope and flow direction would lead to a greater projected frontal area as gear is buoyed to the surface (e.g., Fig 1).

\subsubsection{Analysis}

Mean $( \pm \mathrm{SD})$ drag forces were calculated from the tensiometer measurements over the 30 $\mathrm{s}$ period with the lowest variance in drag for each depth and speed combination $(n=9$ measurement points per gear set). Doing so isolated the measurement period to one where drag measurements were most stable, to reduce variability introduced by current flow and/or turbulence, and where vessel speed and tow depth were not intentionally being changed. The relationship between drag and speed at each depth was fit with a power model for each tow based on theory.

Gear sets removed from whales $(n=15)$ were weighed and measured when dry, prior to towing. The relationship between average drag measured across all depths and speeds and the dry weight $(\mathrm{kg})$ was fit with a linear model; the presence of floats or buoys was added as a complex covariate for the relationship between drag and length $(\mathrm{m})$ of a gear set $(n=$ 21). ANOVA was used to test for a difference in slopes between gear sets with and without floats. Outliers were detected and removed when Cook's $D>4 / n$ (Mendenhall and Sincich, 2011).

A list of symbols, abbreviations, and units are provided in Table 1.

\subsubsection{Entangling gear description}

From measured drag values, the drag coefficient $\left(C_{d}\right.$; dimensionless) of each gear set at each measurement point was estimated by

$$
C_{d}=\frac{2 D_{G}}{\rho U^{2} A_{w}}
$$

where $D_{G}$ is the drag force $(\mathrm{N})$ on the gear measured from the tensiometer, $\rho$ is seawater density $\left(1,025 \mathrm{~kg} / \mathrm{m}^{3}\right), U$ is the tow speed $(\mathrm{m} / \mathrm{s})$, and $A_{w}$ is the wetted surface area $\left(\mathrm{m}^{2}\right)$ of the gear set. Wetted surface area was estimated based on the area of a cylinder with a specified length, $l$, and radius, $r$ : 
Table 3-1: List of symbols and abbreviations.

\begin{tabular}{|l|l|l|}
\hline Symbol & Definition & Unit \\
\hline$a_{g}$ & Gravitational acceleration & $\mathrm{m} / \mathrm{s}^{2}$ \\
\hline$A_{w}$ & Wetted surface area & $\mathrm{m}^{2}$ \\
\hline$C_{d}$ & Drag coefficient & \\
\hline$C_{d} I$ & Interference drag coefficient & \\
\hline$C_{f}$ & Friction coefficient & \\
\hline$d$ & Body diameter & $\mathrm{m}$ \\
\hline$d_{m a x}$ & Maximum body diameter & $\mathrm{m}$ \\
\hline$D_{G}$ & Tensiometer-measured gear drag & $\mathrm{N}$ \\
\hline$D_{I}$ & Interference drag & $\mathrm{N}$ \\
\hline$D_{W}$ & Total whale drag & $\mathrm{N}$ \\
\hline$F$ & Theoretical drag & $\mathrm{N}$ \\
\hline$F n$ & Froude number & \\
\hline$g$ & Appendage drag augmentation factor & \\
\hline$h$ & Height of gear off body & $\mathrm{m}$ \\
\hline$l$ & Length & $\mathrm{m}$ \\
\hline$P_{p}$ & Propulsive power & $\mathrm{W}$ \\
\hline$r$ & Radius & $\mathrm{m}$ \\
\hline$R e$ & Reynolds number & \\
\hline$U$ & Speed & $\mathrm{m} / \mathrm{s}$ \\
\hline$v$ & Kinematic viscosity & $\mathrm{m}{ }^{2} / \mathrm{s}$ \\
\hline$W$ & Weight & $\mathrm{kg}$ \\
\hline$\delta$ & Boundary layer thickness & $\mathrm{m}$ \\
\hline$\gamma$ & Surface wave drag factor & \\
\hline$\eta$ & Efficiency coefficient & \\
\hline$\rho$ & Fluid density & \\
\hline & & \\
\hline & & \\
\hline & & \\
\hline & & \\
\hline & & \\
\hline & & \\
\hline & & \\
\hline
\end{tabular}

$$
A_{w}=2 \pi r l
$$

For most gear sets, this calculation was straightforward and based on the single line diameter and dimensions; however, six gear sets consisted of multiple line types, floats, or buoys, and total wetted surface area was estimated as the sum of surface areas of the components of each gear set. For all attached floats and buoys, half of the wetted surface area was calculated, assuming that half of each item was submerged (see Appendix B1).

The Reynolds number of each gear set was calculated as 


$$
R e=\frac{l U}{v},
$$

where $v$ is the kinematic viscosity for $16^{\circ} \mathrm{C}$ seawater $\left(1.17 \times 10^{-6} \mathrm{~m}^{2} / \mathrm{s}\right)$.

To compare the relative influence of surface wave drag, the Froude number ( $F n$; dimensionless) was calculated for floats on certain gear sets, including the telemetry buoy (see Appendix B1), across the range of measured tow speeds $(U ; \mathrm{m} / \mathrm{s})$ as

$$
F n=\frac{U}{\sqrt{a_{g} l}}
$$

where $a_{g}$ is the acceleration due to gravity $\left(9.8 \mathrm{~m} / \mathrm{s}^{2}\right)$ and $l$ is the length of the float $(\mathrm{m})$ at the water line level, assuming that each float is half submerged.

To determine whether certain gear configurations have similar drag coefficients or responses to depth and speed, hierarchically clustering using Manhattan distance dissimilarity metric and Ward's minimal variance clustering algorithm were performed in $\mathrm{R}$ (Warnes et al., 2014). Within identified clusters, the effects of depth and speed are described, including their relative influences, calculated as the percent change in drag coefficient for each gear set between different levels of depth and speed.

\subsubsection{Effect of partial gear removal}

To determine the incremental effect of line length on drag, a piece of $8 \mathrm{~mm}$ diameter polypropylene line was repeatedly measured on the tow cell as it was shortened in stages from $200 \mathrm{~m}$ to $25 \mathrm{~m}$ in length $(200,150,100,50,25 \mathrm{~m})$. Power functions were fit to mean drag measurements across measured speeds for each length of line. Due to significant effects of depth (see Results), only surface drag measurements were used. From these curves, the percent reduction in drag was calculated between decreasing lengths of line, across speeds of 0.2 to $2.15 \mathrm{~m} / \mathrm{s}$.

\subsubsection{Entangled whale drag}

The drag on each whale entangled by the 15 sets of fishing gear towed was estimated to illustrate the relative contribution of drag from gear to the entangled whale system (Rayleigh, 1876; Batchelor, 2000).

The theoretical rigid-body drag force $(F ; \mathrm{N})$ was calculated based on a turbulent spindle model (Webb, 1975b), 


$$
F=\frac{1}{2} \rho U^{2} A_{w} C_{d}
$$

where $\rho$ is seawater density $\left(1,025 \mathrm{~kg} / \mathrm{m}^{3}\right), U$ is swimming speed $(\mathrm{m} / \mathrm{s})$, and $A$ is the total wetted surface area $\left(\mathrm{m}^{2}\right)$ calculated from body weight $W(\mathrm{~kg})$ as $A=0.08 W^{0.65}$ (Fish, 1993b). $C_{d}$ is the drag coefficient, calculated as

$$
C_{d}=C_{f}\left[1+1.5\left(\frac{d_{\max }}{l}\right)^{3 / 2}+7\left(\frac{d_{\max }}{l}\right)^{3}\right]
$$

where $C_{f}$ is the frictional drag component computed from the Reynolds number $(R e)$,

$$
C_{f}=0.072\left(R e^{-1 / 5}\right)
$$

and $d_{\max }$ and $l$ are the maximum body diameter $(\mathrm{m})$ and total body length $(\mathrm{m})$.

Body dimensions were obtained for each individual whale (Table 2). Body length (m) and weight $(\mathrm{kg})$ were estimated from age at the first sighting of entanglement based on Moore et al. (2004). For four individuals, birth year was not known. Length and weight were estimated from minimum age in two cases (Egs 1102 and 3294), and expected ages based on size-at-age approximations by staff at the New England Aquarium in the two other cases. The minimum age of Eg 3610, estimated to be a juvenile, was increased from 1 to 3 yr. Eg 2030 was identified as an adult; her estimated age was therefore increased from 9 to $12 \mathrm{yr}$, the age at which right whales attain $95 \%$ of their total body length (Fortune et al., 2012). Maximum body diameter was estimated from body length as presented in Fortune et al. $(2012)^{1}$ :

\footnotetext{
${ }^{1}$ Personal communication from Sarah Fortune, Marine Mammal Research Unit, Institute for Oceans and Fisheries, University of British Columbia, Vancouver, British Columbia V6T 1Z4, Canada, 29 September 2015. There is an error in the equation printed in Fortune et al. (2012), where the reported values of slope and intercept are reversed. Equation 7 is the correct equation.
} 
Table 3-2: Catalog identification number, entangling gear identification number, age, and estimated length (m) and weight (kg; from Moore et al. 2004) of North Atlantic right whales at the onset of their entanglement, and their minimum and maximum entanglement duration (d), minimum swimming distance while entangled $(\mathrm{km})$, and average fold increase in drag from their entangling gear.

\begin{tabular}{|l|l|l|l|l|l|l|l|}
\hline $\begin{array}{l}\text { Catalog } \\
\text { number }\end{array}$ & Gear number & Age & Length $(\mathrm{m})$ & Weight $(\mathrm{kg})$ & $\begin{array}{l}\text { Entanglement } \\
\text { duration (min - } \\
\text { max; days) }\end{array}$ & $\begin{array}{l}\text { Minimum swim } \\
\text { distance } \\
\text { entangled (km) }\end{array}$ & $\begin{array}{l}\text { Mean (SD) } \\
\text { fold increase } \\
\text { in drag }\end{array}$ \\
\hline 1102 & J060801 & 21 & 14.35 & 40,416 & $100-3,328$ & 5,504 & $1.05(0.01)$ \\
\hline 1427 & J071202 & 18 & 14.13 & 35,095 & $5-487$ & 962 & $1.22(0.04)$ \\
\hline 2030 & J051099 & 12 & 13.57 & 24,453 & $163-769$ & 1,839 & $1.61(0.12)$ \\
\hline 2212 & J091298 & 5 & 12.35 & 12,037 & $1-23$ & 10 & $3.07(0.42)$ \\
\hline 2212 & J072498 & 6 & 12.60 & 13,811 & $332-346$ & 492 & $1.19(0.08)$ \\
\hline 2223 & J081800 & 8 & 13.00 & 17,359 & $263-300$ & 2,524 & $1.20(0.04)$ \\
\hline 2710 & J072199 & 3 & 11.64 & 8,490 & $68-397$ & 119 & $1.39(0.08)$ \\
\hline 3107 & J070602 & 1 & 10.11 & 4,943 & $57-297$ & 128 & $1.38(0.11)$ \\
\hline 3294 & J120808 & 6 & 12.60 & 13,811 & $11-293$ & 53 & $1.47(0.11)$ \\
\hline 3311 & J011409 & 7 & 12.82 & 15,585 & $51-2,510$ & 5,232 & $1.71(0.20)$ \\
\hline 3314 & J120604 & 2 & 11.08 & 6,717 & $25-98$ & 659 & $1.92(0.23)$ \\
\hline 3420 & J013109 & 5 & 12.35 & 12,038 & $12-352$ & 1,506 & $1.11(0.01)$ \\
\hline 3445 & J120305 & 2 & 11.08 & 6,717 & $9-2,459$ & 1,213 & $1.69(0.19)$ \\
\hline 3610 & J092706 & 3 & 11.64 & 8,490 & $119-435$ & 2,619 & $1.06(0.02)$ \\
\hline 3714 & J020709 & 2 & 11.08 & 6,717 & $5-64$ & 169 & $1.07(0.02)$ \\
\hline
\end{tabular}




$$
d_{\max }=0.21 l+38.63
$$

and diameter $\left(d_{j} ; \mathrm{cm}\right)$ at multiple $(j)$ stations along the body from width-to-length ratio of mesomorphic right whales (van der Hoop et al., 2013b). Although length, weight, and width data are available for some of these cases at the time of death, postmortem body dimensions would reflect body shape following the impact of the course of an entanglement, rather than at its onset.

The total drag on each whale $\left(D_{W}\right)$ is calculated as

$$
D_{W}=F g k,
$$

where $g$ and $k$ are drag augmentation factors. The appendage drag factor $g=1.3$ accounts for increases in interference, frictional and pressure drag by $\sim 30 \%$ from fins and flukes (Fish and Rohr, 1999). The body oscillation drag factor $k$ accounts for increases in frontal area and pressure drag due to the oscillation of the flukes and body during active swimming (Fish and Rohr, 1999). Due to uncertainties on the magnitude of anterior oscillation, $k$ $=1.5$ with a range of $1.35-1.65( \pm 10 \%)$. Compared to van der Hoop et al. $(2013 \mathrm{~b})$, the effect of surface wave drag $(\gamma)$ is not included, as recent data suggest that whales show variable and opposite responses in submergence due to drag and buoyant forces associated with entanglement (van der Hoop et al., unpublished data; Chapter 4).

Case-specific drag measurements were added to each individual whale's body drag, as in van der Hoop et al. (2013b). Briefly, for all n cases, the interference drag coefficient was calculated for all $j$ gear attachment points $\left(C_{d I_{n ; j}}\right)$

$$
C_{d I_{n ; j}}=\left(\frac{h_{j}}{\delta_{j}}\right)^{1 / 3},
$$

where $h$ is the height $(\mathrm{m})$ of the gear and $\mathrm{d}$ is the boundary layer thickness $(\mathrm{m})$ at all $j$ gear attachment locations (proportion of body length; $l_{j}$ ), calculated as

$$
\delta_{j}=\left(\frac{d_{\max }}{d_{j}}\right) 0.02 l_{j}
$$

Total interference drag for each $n$ case is then the sum of interference drag at all $j$ gear attachment points: 


$$
D_{I ; n}=\sum_{j} D_{I ; n, j}=\sum_{j} \frac{1}{2} \rho U^{2} A_{w ; n, j} C_{d I_{n ; j}} .
$$

Compared to van der Hoop et al. (2013b), the shielding effects of the whale's body $(\sim 12$ m) are ignored here, as similar shielding likely occurred behind the tow fish and in the wake of the vessel $(18 \mathrm{~m})$. The total drag for each entangled whale $\left(D_{T}: \mathrm{N}\right)$ is then

$$
D_{T}=D_{W}+D_{G}+D_{I}
$$

where $D_{G}(\mathrm{~N})$ is the drag on the entangling gear measured from the tensiometer at all depths and speeds, fitted with power functions by nonlinear least squares. Lower and upper estimates of $D_{T}$ are obtained by incorporating the $95 \%$ prediction intervals of $D_{G}$ and calculating $D_{I}$ with $C_{d I} \pm 10 \%$ (Fig. 3).

Propulsive power $\left(P_{p}, \mathrm{~W}\right)$ is computed as

$$
P_{p}=\frac{D_{T} U}{\eta}
$$

where $\eta$ is an efficiency coefficient of 0.15 (Fish and Rohr, 1999; van der Hoop et al., 2013b).

Because individual whales vary in their dimensions, gear varies in the amount of measured drag and estimated interference drag, and measured drag and interference drag do not necessarily correlate, the contributions of each drag component $\left(D_{W}, D_{G}, D_{I}\right)$ to $D_{T}$ were calculated.

\subsection{Results}

\subsubsection{Entangling gear description}

Fifteen sets of fishing gear removed from entangled right whales were used in this study (Appendix B2). Ten sets were made up of line only, while five sets included floats or buoys; one of these five included a two-brick lobster trap. Identified gear types were trap/pot $(n=6)$, longline $(n=1)$, gill net $(n=1)$, and unidentified fixed gear $(n=1)$; six sets were unable to be identified. Gear sets with floating rope only $(n=11)$, sinking rope only $(n=1)$, and both floating and sinking rope $(n=3)$ were recovered. The majority of lines were twisted three-strand polypropylene, 0.8-1.6 cm (5/16"-5/8") in diameter.

There is considerable variation in the magnitude of drag forces measured from entangling 
fishing gear (Fig. 3). At the lowest tested speeds $(\sim 0.5 \mathrm{~m} / \mathrm{s})$ the drag measured on all 21 gear configurations ranged 0 to $83 \mathrm{~N}$; at the highest tested speeds $(\sim 2 \mathrm{~m} / \mathrm{s})$, drag forces ranged 18 to $630 \mathrm{~N}$. Coefficients of variation ranged 0.0132 to 10.08 across all measured depths and speeds for all gear sets. The median CV was 0.089; high CVs occurred at the lowest speeds, because $\mathrm{CV}$ approaches infinity as mean measured drag values approach zero. Drag coefficients $\left(C_{d}\right)$ range from $9.2 \times 10^{-3} \pm 0.0029$ to $0.45 \pm 0.13$ across gear configurations (Fig. 4, Fig. B2). The mean \pm SD power relating drag and speed across all gear sets $(n=21)$ was $1.43 \pm 0.52$, less than the expected theoretical $\mathrm{drag} \approx$ speed $^{2}$ relationship.

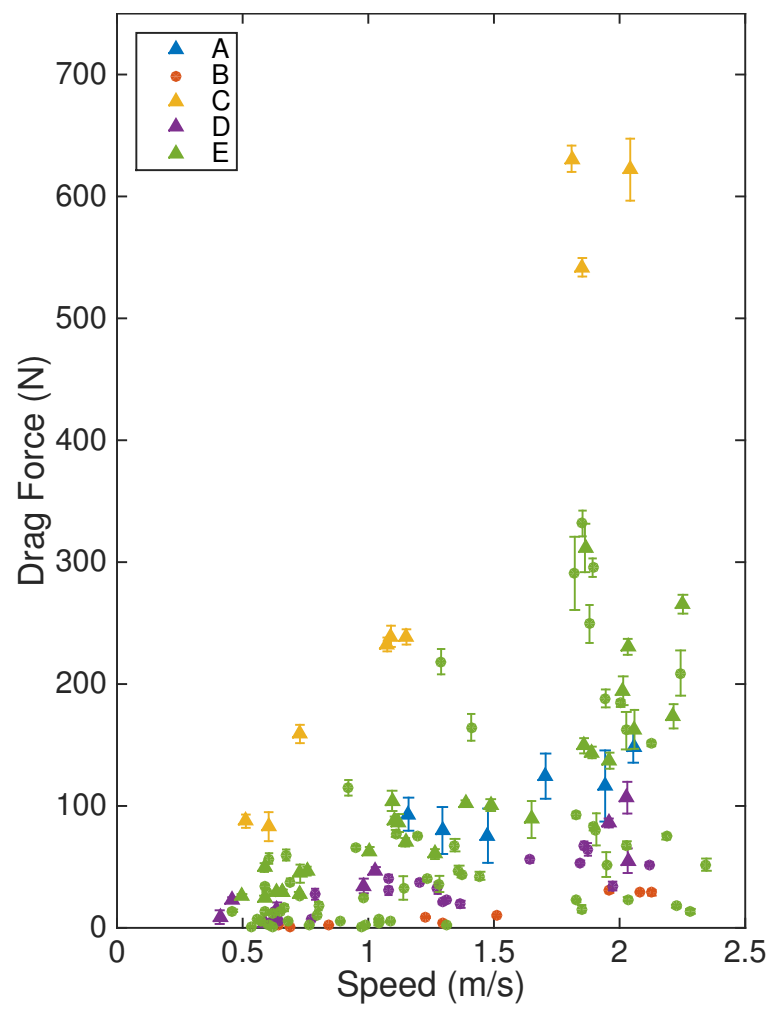

Figure 3-3: Drag on sets of entangling fishing gear is highly variable. Measured drag force $(\mathrm{N})$ of 15 sets of fishing gear (colored by cluster; see text, Fig. 4) removed from entangled right whales and a satellite telemetry buoy used to track entangled whales (blue, cluster A) towed across a range of speeds, averaged over depths of 0,3 , and $6 \mathrm{~m}$. Gear sets made up of line only are represented as circles, and gear sets with floats or buoys as triangles.

Hierarchical cluster analysis separated gear configurations into five groups based on drag coefficient and its response to changes in depth and speed (Fig. 4). The telemetry buoy, a two-brick lobster trap (J091298) and an extremely short gear configuration (J092706) cluster independently from the rest of the gear sets towed. A small cluster of four gear configurations is also apparent. The presence of buoys, traps, or floats on the gear sets is not directly related to clustering, as gear sets with these features occur in many clusters (Fig. 4). Further, the drag coefficients, lengths, and weights of gear sets with buoys, traps, or floats do not fall at the extremes of those within each cluster. Across speeds of 0.5-2.5 
$\mathrm{m} / \mathrm{s}$, the Froude number $(F n)$ of floats on three gear sets (Appendix B1, Fig. B3) ranged 0.16-0.95 and 0.27-1.34 for the telemetry buoy.

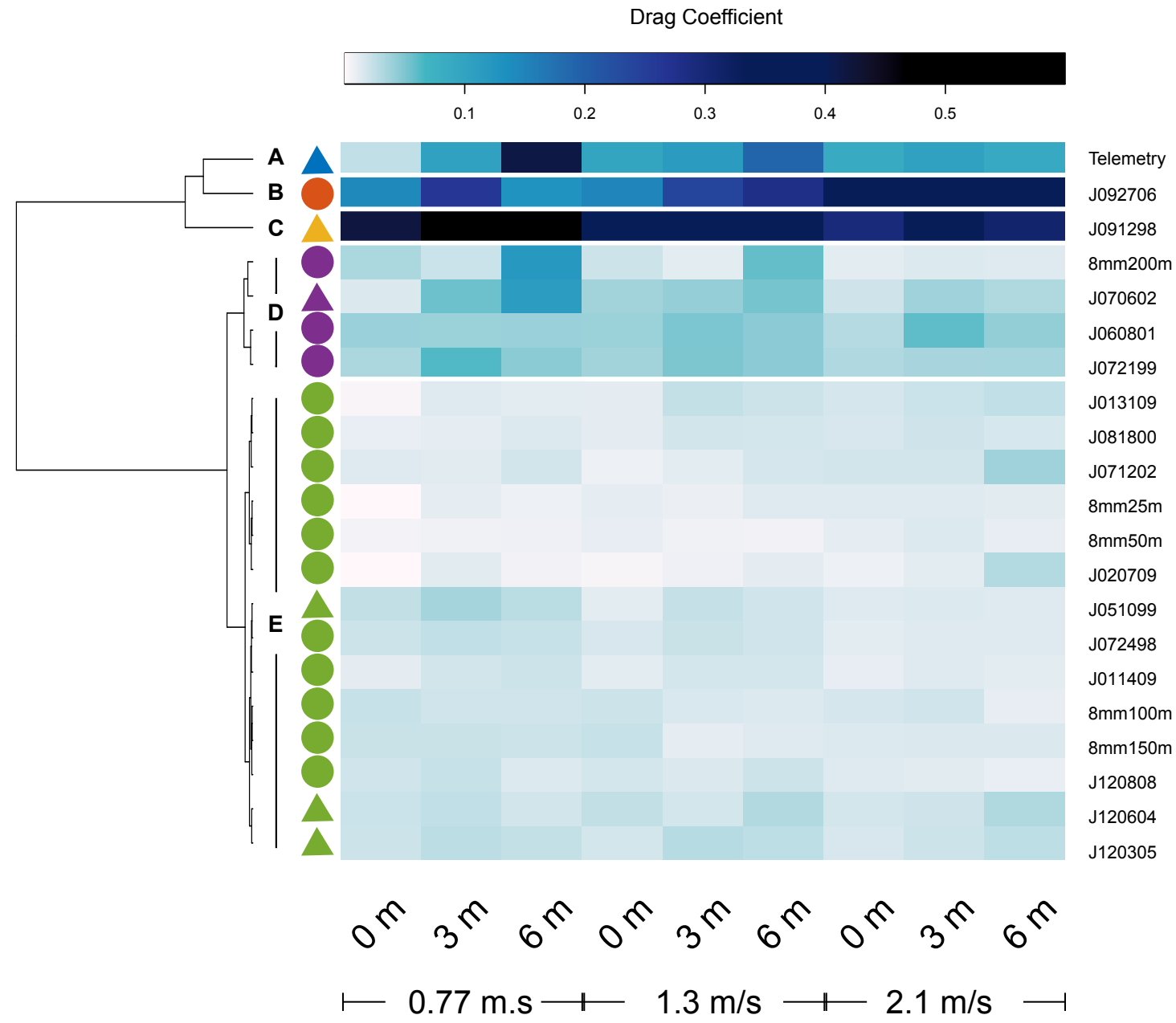

Figure 3-4: Hierarchical clustering separates entangling fishing gear sets into five groups. Drag coefficients of 21 fishing gear configurations measured at different depths and speeds, separated by row-wise hierarchical minimal variance Ward's clustering. Five clusters (A through E) are identified by separate colors. Gear sets made up of line only are represented as circles, and gear sets with floats or buoys as triangles.

Depth and speed differentially affect the drag coefficients of gear sets in these five clusters (Fig. B2). Drag coefficients in clusters A, B, D, and E decrease with increasing speed, whereas the drag coefficient of cluster C (J092706) increases with speed. Drag coefficients of gear in clusters $\mathrm{A}$ and $\mathrm{D}$ increase with depth. In clusters $\mathrm{C}$ and $\mathrm{E}$, drag coefficients are lower at greater depth at the lowest speeds, but become greatest at the greatest depth and speed. The gear in cluster B shows multiple interacting effects of drag and speed on drag coefficient.

Percent differences in drag coefficient range 22\%-81\% (median 37\%) with depth, and 18\%-74\% (median 48\%) with speed. For $14 / 21$ cases, occurring in all gear clusters, drag 
coefficients vary more with speed than with depth. There is no pattern for whether gear sets with floats are more affected by depth or by speed.

Linear models suggest that mean drag forces $(\mathrm{N})$ measured over all combinations of depths and speeds can be predicted from dry weight $(\mathrm{kg})$, as Drag $=5.9+9.1 \times$ Weight $\left(R^{2}=0.983, \mathrm{RMSE}=8.63 ; F_{1,12}=680, P<0.0001 ;\right.$ Fig. 5A $)$. More practically, drag can also be predicted from the length of the gear $(\mathrm{m})$, as:

$$
\text { Drag }=8.67+0.47 \times \text { Length }+39.26 \times \text { Float }+0.01 \times \text { Length } \times \text { Float },
$$

where Float is a binary variable depending on whether the gear set includes floats or buoys $\left(R^{2}=0.812\right.$, RMSE $=21.2 ; F_{3,16}=23.1, P<0.0001 ;$ Fig. $\left.5 \mathrm{~B}\right)$. There is no detectable difference in the slopes of float vs. nonfloat gear $\left(F_{1,16}=0.0011, P=0.9729\right)$, but note the much higher intercept for gear with floats. The lobster trap (J091298) was deemed an outlier and was not used in weight or length regression analyses based on Cook's $D=1.2$ and 1.64 (» $4 / n$ in both cases), respectively.

\subsubsection{Effect of partial gear removal}

To understand the incremental gain in removing entangled gear to reduce parasitic gear drag and the energetic impact on an individual, a piece of $8 \mathrm{~mm}$ diameter polypropylene line was shortened from $200 \mathrm{~m}$ to $25 \mathrm{~m}$. By comparing drag measurements at the surface across a range of speeds for each length of line, the incremental effect of line length on parasitic drag was determined. As expected, the magnitude of the drag force at a given speed decreases with shorter lengths of line (Fig. 6A).

Similarly, drag coefficients systematically decrease with towed line length, from $0.041 \pm 0.039$ at $200 \mathrm{~m}$ to $0.011 \pm 0.0043$ at $25 \mathrm{~m}$ (Fig. B4). Comparing drag estimates from fitted curves indicates that considerable reductions in parasitic drag can be achieved by reducing the length of a set of entangling gear (Fig. 6B, C). For example, a $200 \mathrm{~m}$ long line cut to 50 $\mathrm{m}$ results in an $83.4 \% \pm 6.0 \%$ reduction in drag across speeds (Fig. $6 \mathrm{~B}$ ); removing $75 \%$ of a line's original length reduces parasitic gear drag by $85.0 \% \pm 7.4 \%$ across speeds (Fig. 6C). The greatest gains in parasitic drag reduction are achieved at the lowest swimming speeds, where $75 \%$ removal yields a $93.8 \% \pm 3.3 \%$ parasitic drag reduction at $0.5 \mathrm{~m} / \mathrm{s}$ compared to $78.8 \% \pm 1.0 \%$ at $2.00 \mathrm{~m} / \mathrm{s}$ (Fig. $6 \mathrm{C}$ ).

\subsubsection{Entangled whale drag}

Average drag on nonentangled whales ranged between $40 \pm 16$ and $721 \pm 297 \mathrm{~N}$ across speeds of $0.5-2.5 \mathrm{~m} / \mathrm{s}$ (Fig. 7). Modeled drag coefficients for nonentangled whales ranged 0.00290.0040, similar to as modeled in (0.0028-0.0037 van der Hoop et al., 2013b) and measured 

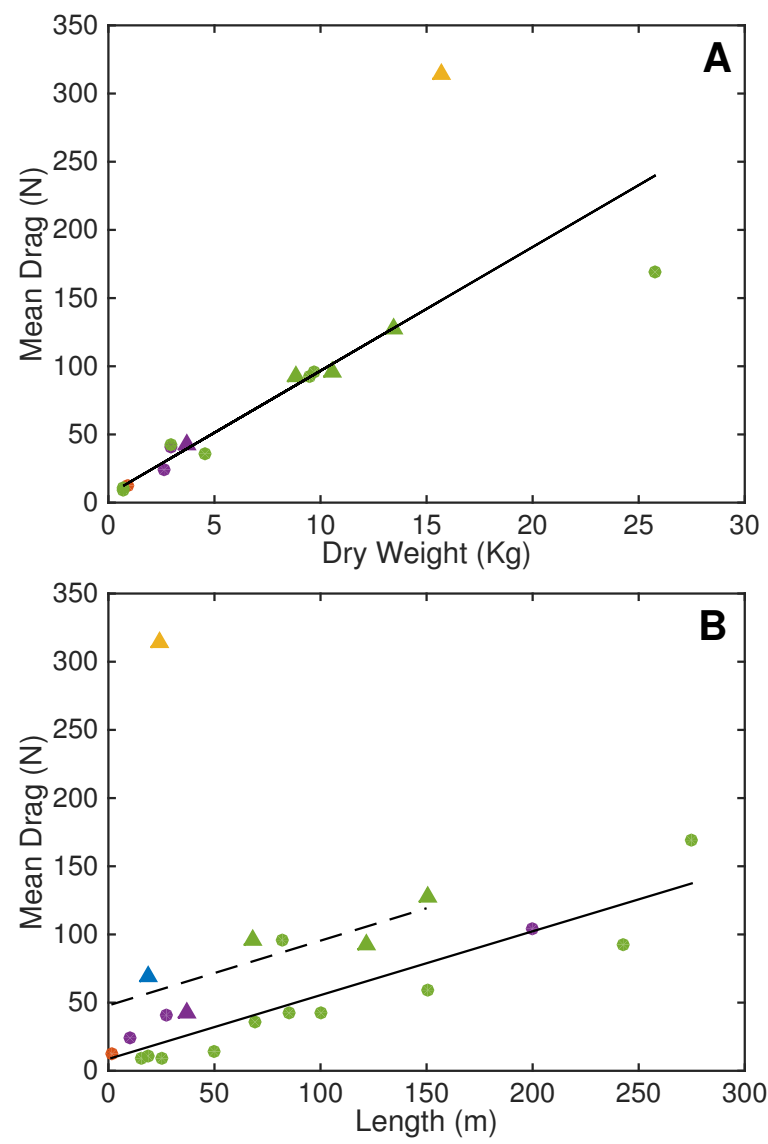

Figure 3-5: Mean drag (N) measured across speeds and depths can be predicted by the dry weight (kg; A) or length $(\mathrm{m} ; \mathrm{B})$ of a gear set. Colors represent different groupings of gear identified by hierarchical clustering (see text). Gear sets made up of line only are represented as circles, and gear sets with floats or buoys as triangles. Black lines illustrate linear model fits of mean drag and (A) weight; and (B) length, with the presence of floats (dashed line) as a categorical covariate. See text for equations.

during traveling (0.0036-0.0052) and foraging (0.0091-0.024; McGregor, 2010). At the upper $95 \%$ CI swimming speed of nonentangled right whales of $1.23 \mathrm{~m} / \mathrm{s}$ (Baumgartner and Mate, 2005), the average drag for these 15 individuals is $193 \pm 79 \mathrm{~N}$. When entangled in their specific gear configurations at $1.23 \mathrm{~m} / \mathrm{s}$, drag is significantly increased to $268 \pm 115 \mathrm{~N}$ (paired $t$-test; $t_{14},=3.35, P=0.0031$ ), by $1.42 \pm 0.46$ fold (Fig. 8 ). The mean increase in drag from entanglement across speeds, is $1.47 \pm 0.52$ fold, with a maximum of $3.07 \pm 0.42$ fold, i.e., three times over the nonentangled condition (Fig. 7).

Drag from entangling fishing gear is $0.35 \pm 0.38$ of the drag on the whale's body across speeds (Fig. 8). Drag from entangling gear approaches or exceeds the magnitude of drag from the whale's body alone in four cases, primarily at the lowest speeds $(0.5-0.7 \mathrm{~m} / \mathrm{s})$. Additionally, gear drag is 1.78-3.45 fold greater than whale body drag across speeds for case J091298. Estimated interference drag $\left(D_{I}\right)$ is variable among all gear sets, ranging from 1.3 $\mathrm{N}$ to $147.1 \mathrm{~N}$, contributing on average $39 \%$ to the total drag from entangling gear $\left(D_{G}+D_{I}\right.$; 

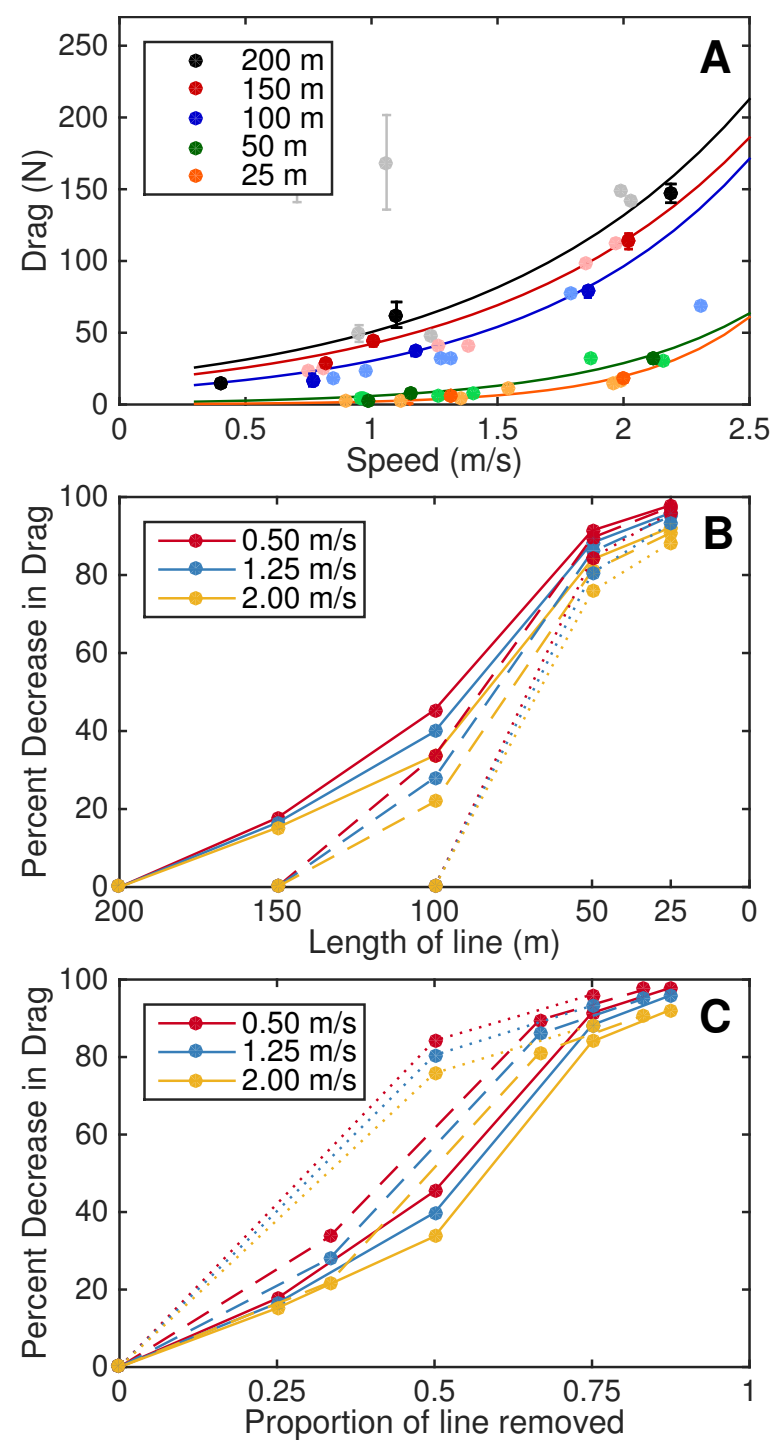

Figure 3-6: Drag force decreases with towed line length. (A) Measured mean \pm SD drag forces (N) with speed $(\mathrm{m} / \mathrm{s})$ on different lengths of $8 \mathrm{~mm}$ diameter polypropylene fishing rope towed at the surface (darker colors) and at 3 and $6 \mathrm{~m}$ depth (lighter colors). Lines represent power functions fit to surface drag values. (B) Percent decrease in drag as a line is shortened from $200 \mathrm{~m}$ (solid line), $150 \mathrm{~m}$ (dashed line), $100 \mathrm{~m}$ (dotted line), and $50 \mathrm{~m}$ (no line) to its new length (x-axis) at speeds of $0.50,1.25$ and $2.00 \mathrm{~m} / \mathrm{s}$. (C) Percent decrease in drag with the proportion of line removed from 200 $\mathrm{m}$ (solid line), $150 \mathrm{~m}$ (dashed line), $100 \mathrm{~m}$ (dotted line), and $50 \mathrm{~m}$ (no line) to its new length (x-axis) at speeds of $0.50,1.25$, and $2.00 \mathrm{~m} / \mathrm{s}$. For example: trimming a $200 \mathrm{~m}$ line to $150 \mathrm{~m}$, equivalent to having removed 0.25 of the line, results in a $14 \%$ decrease in drag at $2.00 \mathrm{~m} / \mathrm{s}$.

Fig. 8).

\subsection{Discussion}

Entanglement in fishing gear contributes significant mortality to many large whale species (van der Hoop et al., 2013a). The fatality of an entanglement, its time course, candidacy 


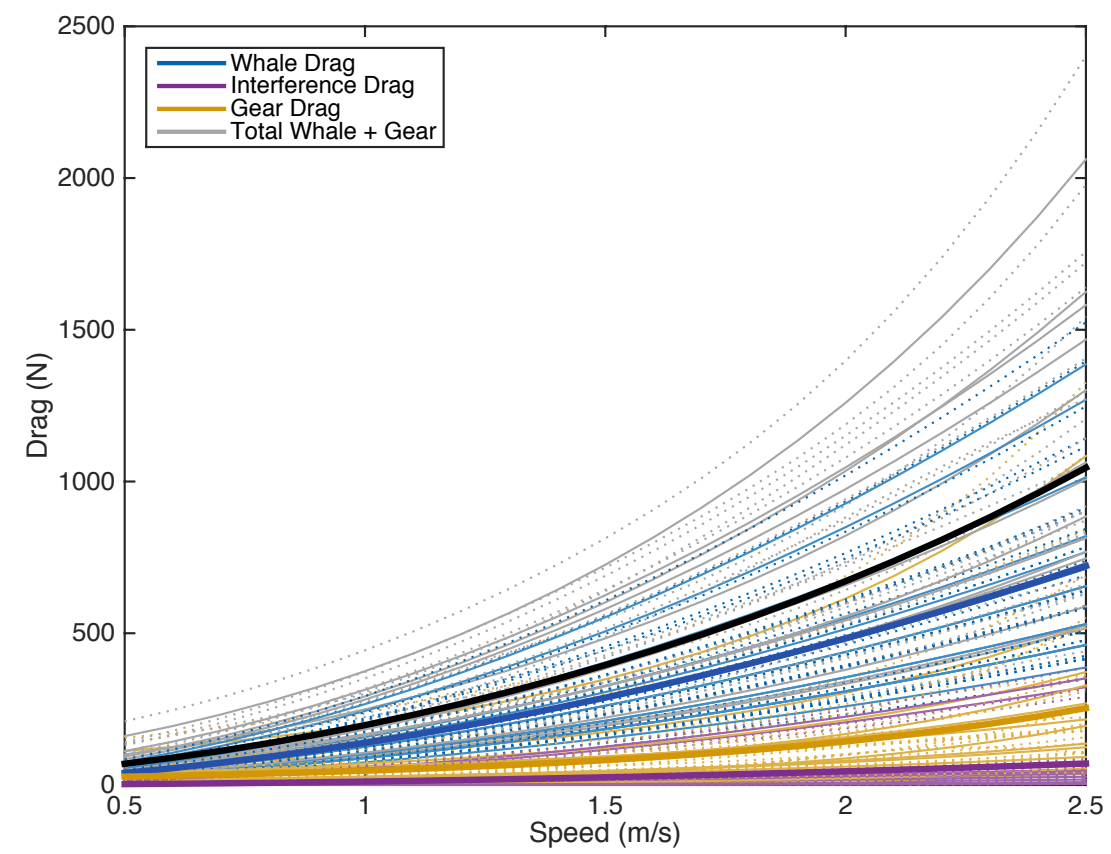

Figure 3-7: Estimated total body drag on entangled and nonentangled whales and entangling gears. Drag force $(\mathrm{N})$ of 15 individual right whales when not entangled (blue), their entangling fishing gear (yellow), interference drag from the entangling gear (purple), and when entangled in these fishing gear (black). Thicker lines represent means.

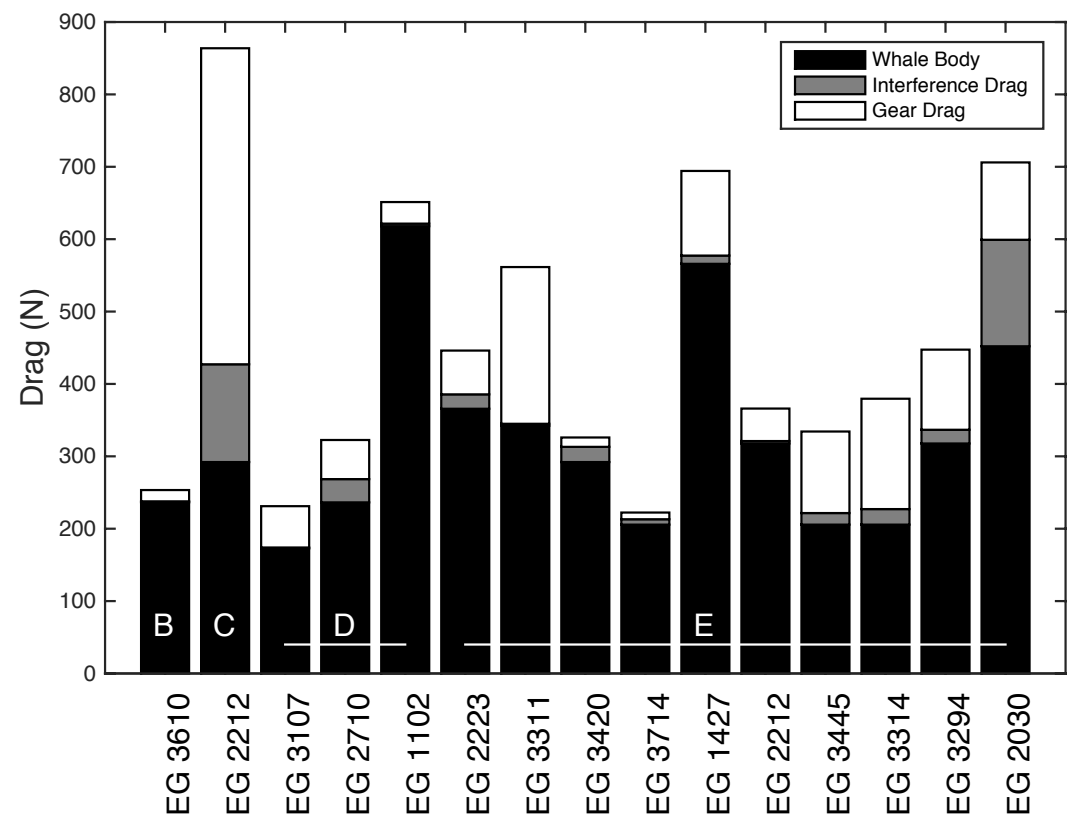

Figure 3-8: Contributions of whale body drag, estimated interference drag, and measured drag on entangling gear for 15 entangled North Atlantic right whales (identified by EG code). Letters identify gear type clusters (see text; note cluster A is not attributed to a specific right whale case) 
for disentanglement, and sublethal effects are all complex aspects of the same incident, largely related to the amount of gear involved and its configuration on the animal. This study sought to measure drag on sets of fishing gear that have entangled or are similar to those entangling North Atlantic right whales, describe differences in their responses to drag and speed, and put these differences in the context of the whales that the gear entangled. The average drag forces and coefficients of measured gear sets range up to two and four orders of magnitude. Such variation is expected, as the cases selected for this experiment represent the great diversity of sizes, shapes and types of fishing gear entangling whales (Johnson et al., 2005). Minimum values are therefore especially notable: seemingly small entanglements (short pieces of line, small floats) can still impart significant drag.

Estimated drag coefficients can be compared with those reported elsewhere. The highest drag coefficient of the gear set with a lobster trap measured in this study was 0.69 (6.4 $\mathrm{m}, 0.51 \mathrm{~m} / \mathrm{s}, R e=3 \times 10^{7}$ ), considerably lower than the 2.3 reported by Budiman et al. (2004) for a similar size crab trap at low flow speeds $(0.1-0.5 \mathrm{~m} / \mathrm{s})$ and Reynolds number $\left(R e=1.0 \times 10^{3}-6.7 \times 10^{3}\right)$. This disparity is likely due to different experimental setups, where a large amount of low drag-coefficient rope was attached to the pot in this study. Rope drag coefficients range from 0.12 to 1.30, depending on material and degree of wear, but primarily increase with the angle between rope and flow direction (Fridman, 1986). In this study, drag coefficients increased with depth on average (Fig. 4, B2), likely due to the increase in frontal area when lines are buoyed to the water surface (Fig. 1, orange line); those that did not follow this trend may have been neutrally buoyant, trailing at the same depth instead of being taut to the surface. Buoyancy will greatly affect the shape of gear underwater (Baldwin and Pickett, 2009), as will the tension on the line, which increases with ship speed. These considerations constrain the application of standard formulae to estimate the effect of depth on drag of unmeasured gear sets.

Measured drag forces are also comparable to those in other studies. van der Hoop et al. (2013b) present a similar range of drag measurements for a $25 \mathrm{~m}$ line-only configuration (3-70 $\mathrm{N}$ across $0.77-2.8 \mathrm{~m} / \mathrm{s}$ ), which increased by $\sim 72 \%$ with the addition of two $42-45 \mathrm{~cm}$ spherical buoys. Bullet-style lobster buoys on short $(0.5 \mathrm{~m})$ tethers can have measured drag as low as $22 \mathrm{~N}$ at $2 \mathrm{~m} / \mathrm{s}$, while a standard, single double-brick lobster pot may add $222 \mathrm{~N}$ (Woodward et al., 2006a). Gear configurations with multiple floats and buoys, as observed in five cases in this study, can have especially high drag: a single 40" circumference Scanmarin float with two 6" x 4" buoys on a $183 \mathrm{~m}$ line can be as high as 1,245 $\mathrm{N}$ at the same speeds (Salvador and Kenney, 2002). These measured differences in drag with the addition of accessory items support the use of separate equations (with and without floats) when estimating drag from gear set length (Fig. 5B).

Drag measurements were performed in the wake of a vessel, which may lead to differences in the absolute values of drag as experienced behind a whale. Although speed through 
water was calculated from current speeds, the ship's wake may have reduced flow speeds experienced by the gear, leading to underestimates of $C_{d}$. Measurements of drag from the gear sets therefore ignore the shielding effects of the vessel. When combining with a theoretical whale, no shielding effects are added, as it is assumed that the vessel $(\mathrm{R} / \mathrm{V}$ Tioga $=18 \mathrm{~m}$ ) likely imparts similar shielding effects as a right whale's body (10.1-14.3 $\mathrm{m}$ in this study). Turbulence from vortices from the ship's propellers, and conversely, the whale's locomotory movements, could additionally generate transverse forces which would interact with gear and its features in an unpredictable manner based on buoyancy, depth, etc. Mean gear drag measurements in this study were taken from the $30 \mathrm{~s}$ period with the lowest variance in drag to account for potential variability from small changes in hydrodynamics. The drag forces measured on the gear in this study were all performed in the same regime, which at least allows for within-study comparisons to be made.

Mean drag on a set of entangling gear can be predicted from the dry weight or length of the gear (Fig. 5, Eq. 14). This allows for the average drag forces experienced by an entangled animal to be estimated at the time of its detection. Floats, including the telemetry buoy, add $39 \mathrm{~N}$ of drag. Although floats do not drive the separation of clusters in drag coefficient, it has a significant effect on the drag-length relationship. Floats on measured fishing gear have a Froude number in the range of 0.16-0.95, while the telemetry buoy has a higher $F n$, $0.27-1.34$, over the $0.5-2.5 \mathrm{~m} / \mathrm{s}$ speed range (Fig. B3). Especially at routine right whale swimming speeds $(1-1.5 \mathrm{~m} / \mathrm{s}) F n$ is on average $0.43 \pm 0.06$ for fishing gear or $0.64 \pm 0.08$ for the telemetry buoy. Wave drag is greatest at $F n=0.5$ (Lighthill, 1978). The wave drag from these gear sets is a component of the total resistance measured by the tensiometer $\left(D_{G}\right)$. Calculating and comparing $F n$ shows that gear sets with floats have high wave drag, especially at average swimming speeds of right whales.

This study estimates the increase in drag and propulsive power experienced by the right whales entangled by each gear set (Fig. 7, 8), based on gear-specific measurements and individual-specific morphometrics. Inter- and intraspecific body shape changes affect drag and buoyant forces on a whale's body (e.g., Woodward et al., 2006b; Nousek-McGregor et al., 2013). While individual variation in body shape at the onset of entanglement is included, the change in body condition that typically occurs through the course of the entanglement as energy is depleted is ignored (van der Hoop, unpublished data; Ahlborn et al., 2009; Barratclough et al., 2014). Almost all (49/50; Robbins et al., 2015) photoidentified entangled right whales are in good body condition at their last sighting prior to entanglement detection. The rate of deterioration in body condition is a function of a number of known factors, e.g., increased drag and power, but is highly dependent on a number of unknowns, including the location and feeding status of each individual at the time of onset.

Factors such as interference drag and the point(s) of gear attachment have been esti- 
mated, as they alter boundary layer flow. Entangling gear that is raised off the body more than $0.001 \times$ body length (e.g., a float in the mouth, a gill net across the back) can produce serious disturbance to fluid flow (Jacobs, 1934). The relative contributions of interference drag to total drag (Fig. 8) are highly variable across gear sets, and are also influenced by the point of attachment: forward of the maximum-thickness position, flow-disturbance is greatest, having similar effects to air brakes (Jacobs, 1934).

There are at least two broad factors contributing to the lethality of an entanglement configuration: the presence or absence of wraps of body parts and drag (at those body parts and to the whale as a whole). This study addresses some of the issues surrounding one of those factors: drag. By lessening the energy cost of swimming as well as the tension on entangled body parts, reducing drag certainly could benefit a whale with $300 \mathrm{~m}$ of line trapped in its baleen (Fig. 2A); however, drag reduction is unlikely to save a whale with a rostrum or body wrap with only $10 \mathrm{~m}$ of line trailing (Fig. 2B), as trained responders need at least some trailing line to address body wraps in follow-up disentanglement attempts. Reducing drag, as seen in these results, can help in some whale entanglement cases, but will not resolve those that involve wraps of body parts (e.g., Eg 3346 in Moore et al., 2013).

This study reinforces the current practice of reducing trailing gear to roughly a whale's body length prior to adding the telemetry buoy for subsequent disentanglement efforts, which ultimately enhance survival (Robbins et al., 2015). While this practice originated with the desire to reduce the chance that trailing line would reentangle the whale or other gear, these results highlight the practice of minimizing trailing line from an entirely different perspective. Responders can be urged to reduce drag to a "reasonable" minimum while allowing them enough access to the other elements of the entanglement (e.g., wraps of body parts). Simply cutting off all trailing gear is not going to solve an entanglement, especially if doing so reduces access to the remaining entanglement configuration. Drag can also be useful for whales to disentangle themselves, e.g., when drag on the trailing end of gear is sufficient to pull rope from baleen (Cavatorta et al., 2005). As scarring rates in many different whale populations (Neilson et al., 2009; Knowlton et al., 2012a; Robbins and Knowlton, 2012) greatly exceed entanglement mortalities, reported entanglements, and sightings of animals with gear, many whales are able to shed gear on their own.

Gear drag contributes to the immediate sublethal effects of entanglement: direct injury and physiological disturbances, such as stress and metabolic responses (Wilson et al., 2014a). Greater drag loading leads to more severe entanglement-related injuries, increasing both the depth and length of furrows (Woodward et al., 2006a). Epidermal penetration occurs when tensions exceed regional tissue compliance (Winn et al., 2008): nine and six (60\% and 40\%) of the 15 right whale cases presented here exceed tensions that create 0.27 and $0.40 \mathrm{~cm}$ deep furrows, respectively, in right whale peduncles over the equivalent of five days of swimming at $2 \mathrm{~m} / \mathrm{s}$ (Woodward et al., 2006a). These injuries were modeled over a much shorter duration 
than the 15 cases presented here (Table 2). Consistent drag on gear cutting into the tissues has led to near (e.g., cases VAQS2005-1008Eg (Eg 2301) and MH02-736-Eg (Eg 3107) in Moore et al., 2013) and complete (Urban et al., 2004; IWC, 2011) pectoral and caudal fin amputations.

Physiological responses to stress, injury, wound repair, and metabolic disturbance due to drag loading and altered swimming behaviors all interact (Hunt et al., 2006; Archie, 2013); health impairment (Pettis et al., 2004; Robbins et al., 2015) and energetic costs can begin to be estimated in terms of cost to an individual. The 15 entanglements studied here lead to significant increases in drag and propulsive power output in right whales (Fig. 7). When swimming at $2.0 \mathrm{~m} / \mathrm{s}$, nonentangled whales expend on average $2.3 \% \pm 0.1 \%$ and $9.4 \% \pm 0.2 \%$ of their estimated maximal and submaximal force outputs, respectively (Arthur et al., 2015). When entangled, these force outputs increase to $3.3 \% \pm 1.0 \%$ and $13.2 \% \pm 4.5 \%$. Sustained over long periods (mean \pm SD minimum $81 \pm 100$ and maximum $810 \pm 1,044$ d in these 15 cases; Table 2), such increases have the potential to lead to significant alteration to time and energy budgets and reductions in body condition (Feldkamp, 1985). Disentanglement has shown to increase survival in life-threatening entanglement cases, although health impacts are most predictive of subsequent survival (Robbins et al., 2015). Despite concerted and dedicated efforts to remove or reduce the impacts of entangling gear, postdisentanglement mortality occasionally occurs, likely due to significant reductions in heath and body condition prior to entanglement detection or response (Moore et al., 2010, 2012). Additionally, body condition is a major contributor to reproductive success in many mammals, including right whales (Cassoff et al., 2011; Moore et al., 2013; van der Hoop et al., 2013b). It is conceivable that energy reserves may be sufficiently affected by chronic gear drag so as to limit future individual reproductive success. Further analysis of the impact of fishing gear entanglement drag on energy balance is therefore warranted. These hidden, sublethal costs of entanglement are not currently considered in the annual North Atlantic right whale stock assessment reports, which form the basis of the U.S. Government management of this endangered species, but are likely a significant contributor to the variability in annual right whale recruitment.

\subsection{Conclusions}

Entanglement in fishing gear remains a significant issue for marine animal species worldwide (Hofmeyr et al., 2006; Moore et al., 2009; Moore and Barco, 2013; van der Hoop et al., 2013a).While mortality is the simplest indicator of a negative entanglement outcome, the subtler sublethal effects in the form of stress response, metabolic disturbance, and behavioral impairment can and should be considered (Wilson et al., 2014a), especially in cetaceans, where mortality detection probabilities are remarkably low (Williams et al., 2011; Wells et al., 2014). The amount of drag imposed from entangling gear is a factor in defining entanglement 
cases as "Serious Injury" for the purposes of the U.S. Marine Mammal Protection Act and/or U.S. Endangered Species Act (NOAA, 2008). The ability to determine gear drag from length (which can be estimated from photos) and the drag reductions of gear removal enables the prediction of a drag scenario of free-swimming entangled individual when detected, to be applied when planning disentanglement response and in case-by-case evaluations of serious injuries and stock assessment reports for U.S. Federally mandated endangered species conservation. 


\section{Chapter 4}

\section{SWIMMING KINEMATICS AND EFFICIENCY OF ENTANGLED NoRTH ATLANTIC RIGHT WHALES}

This chapter has been submitted for publication: van der Hoop, J.M., Nowacek, D.P., Moore, M.J. and Triantafyllou, M.S.Swimming kinematics and efficiency of entangled North Atlantic right whales Endangered Species Research Submission ID 201604008

JvdH proposed the project; DPN and MJM performed fieldwork; DPN, MT, MJM guided the analysis; JvdH processed and analyzed data; JvdH wrote the paper; JvdH, DPN, MJM, MT contributed to the manuscript.

The supplemental materials for this chapter can be found in Appendix C. 


\subsection{Abstract}

Marine mammals are streamlined for efficient movement in their relatively viscous fluid environment, and are able to alter their kinematics (e.g., fluke stroke frequency, amplitude, or both) in response to changes in force balance. Entanglement in fishing gear adds significant drag and buoyant forces that can impact swimming behaviors across a range of timescales. We deployed bio-logging tags during the disentanglement of two North Atlantic right whales (Eubalaena glacialis; Egs 3911 and 4057) to (1) examine how their kinematics changed in response to drag and buoyancy from entanglement in fishing gear, and (2) calculate resultant changes in swimming efficiency for one individual (Eg 3911). We observed variable responses in dive behavior, but neither whale appeared to exploit added buoyancy to reduce energy expenditure. While some of the observed changes in behavior were individually specific, some swimming kinematics were consistently modulated in response to high drag and buoyancy associated with entangling gear, affecting thrust production. In high drag and buoyancy conditions, fluke strokes were significantly shorter and more variable in shape, and gliding was less frequent. Thrust and efficiency significantly differed among dive phases. Disentanglement reduced thrust coefficients by $\sim 4$ fold, leading to 1.2-1.8 fold lower power (W). Ideal propulsive efficiency was significantly lower when entangled, though we detected no difference in observed propulsive efficiency between the conditions. Similar to carrying heavy objects or changing shoes, we present another condition where animals perceive unique movement constraints over seconds to minutes and develop compensatory strategies, altering their movement accordingly.

\subsection{Introduction}

Marine mammals are streamlined to optimize efficient movement in their relatively viscous fluid environment. Changes in the vertical and horizontal force balances on marine animals have been shown to affect energy consumption (Ponganis et al., 1990; Kooyman and Ponganis, 1994) while also influencing swimming behavior and kinematics - the form, pattern, or sequence of movement with respect to time. To overcome additional drag forces, individuals increase thrust by altering fluke stroke frequency, amplitude, or a combination of both (Skrovan et al., 1999; Williams, 1999; Cornick et al., 2006; Aoki et al., 2011); alternatively, individuals can minimize the increase in drag by swimming at slower speeds (Lang and Daybell, 1963; Boyd et al., 1997; Cornick et al., 2006; van der Hoop et al., 2014a). Marine mammals tend to respond to natural (e.g. lipid loss or gain, lung compression; Biuw et al., 2003; Beck et al., 2000; Nousek-McGregor et al., 2013) or experimental (Webb et al., 1998; Skrovan et al., 1999; Aoki et al., 2011) changes in buoyancy by reducing thrust output or by gliding in the buoyancy-aided direction to maximize swimming efficiency when possible. 
However, despite behavioral changes there appear to be compensatory mechanisms that help prevent changes in energy requirements at least during experimentally induced changes in buoyancy (Fahlman, 2008). Swimming animals therefore show plasticity in their ability to adjust their fine-scale movement patterns in response to changes in drag and buoyant forces and moments to optimize their movement - maximizing swimming efficiency and minimizing energetic cost given conflicting forces, factors, and constraints (Fish, 1999).

Entanglement in fishing gear is the leading cause of death to large whales in the western North Atlantic (van der Hoop et al., 2013a), and within this region, sublethal entanglement affects $83 \%$ of the North Atlantic right whale population (Eubalaena glacialis, hereafter right whale; Knowlton et al., 2012). Most recorded right whale entanglement cases involve whales that swim while towing gear that is no longer anchored (NMFS, 2003). These whales are subject to 1.47 fold increases in drag on average, with some gear configurations increasing drag up to 3.07 fold (van der Hoop et al., 2015). An additional consideration in buoyancy. Right whale buoyancy likely ranges $\pm 1000 \mathrm{~N}$ depending on nutritional condition and life stage; most right whales are positively buoyant (Miller et al., 2004; Nowacek et al., 2001; Nousek-McGregor et al., 2013). Some entangling sets of fishing gear include floats and buoys that can add 26 - $665 \mathrm{~N}$ of buoyancy. Disentanglement response teams are trained to add buoys to increase drag and buoyancy to slow entangled whales and facilitate their disentanglement, a process referred to as 'kegging' (NMFS, 2003). Further, many disentanglement operations require the addition of a satellite telemetry buoy to track entangled whales until conditions (e.g., equipment, personnel, weather or time of day) favor further attempts at gear removal; this contributes more drag and buoyancy. Entanglement in, and disentanglement from, fishing gear therefore presents a unique context in which to examine individual responses to changes in force balance.

If swimming animals optimize their performance, especially during sub-optimal conditions such as entanglement, changes in their swimming kinematics (i.e., their fluke stroke frequency or amplitude) or gaits in response to drag and buoyancy loading should be detectable. The effects of entanglement-related drag and buoyancy on the swimming behavior of one right whale were quantified by deploying a bio-logging tag (DTAG; Johnson and Tyack, 2003) during a disentanglement operation in 2011 (van der Hoop et al., 2013b). We deployed a second tag prior to a disentanglement attempt on a second whale; a portion of entangling gear was removed and a satellite telemetry buoy added to track the whale for another disentanglement attempt the following day. Here, we examine tag data from these two entangled whales with differences in natural and added drag and buoyant forces, manipulated during disentanglement procedures, with the expectations that: (1) fluke strokes and swimming speed would be altered to maintain normal power output under high drag loading conditions (Skrovan et al., 1999; Simon et al., 2012; van der Hoop et al., 2014a); (2) individuals should take advantage of added buoyancy, reducing thrust and increasing speed 
in the buoyancy-aided direction (Aoki et al., 2011; Miller et al., 2012b; Nousek-McGregor et al., 2013); and (3) swimming efficiency will be reduced when entangled. Together, these hypotheses address whether animals maintain optimal swimming under drag loading (maximizing efficiency and economy), or whether physiological and anatomical constraints limit the plasticity of these parameters and the maintenance of efficient swimming movements under high loading conditions (Methling et al., 2011; Nudds et al., 2014; Shimojo et al., 2014).

\subsection{Methods}

We used DTAG data from two entangled right whales (North Atlantic Right Whale Consortium Catalog Numbers Eg 3911 and Eg 4057) to resolve changes in dive behavior, kinematics, swimming efficiency and power output. We tagged the two entangled whales prior to their partial disentanglement, described below. Due to the differences in their disentanglement procedures and equipment deployed and recovered, we hypothesized that Eg 3911 and Eg 4057 experienced periods of relative low and high drag and buoyancy loading from the gear (Fig. 1); we therefore compared diving behavior and kinematics between these periods. We provide a list of symbols and abbreviations in Table 1.

\subsubsection{Case histories}

We compiled individual entanglement histories from data from the North Atlantic Right Whale Consortium Database (NARWC, 2015) and from members of the Atlantic Large Whale Disentanglement Network involved in the separate disentanglement attempts. For each whale, we estimated the drag forces $(\mathrm{N})$ on the entangling fishing gear from gear length $(\mathrm{m})$ using the length-drag relationship established in van der Hoop et al. (2015). We estimated these drag forces throughout the course of disentanglement procedures as portions of entangling gear were removed. We estimated buoyant forces from available manufacturer information (Scanmarin, 2008; Polyform, 2015). We estimated the total drag on both whales based on a turbulent spindle model as in van der Hoop et al. (2013b) with drag augmentation factors for appendages $(g=1.3)$ body oscillation $(k=1.5 \pm 10 \%$; Fig. C1 $)$.

Eg 3911 was a severely emaciated (approximately 20\% thinner than normal right whales; van der Hoop et al., 2013b), two-year-old, $10 \mathrm{~m}$ female at the time of the third and final multi-agency disentanglement effort on 15 January 2011 near Melbourne, FL. To facilitate this disentanglement, we injected a mixture of analgesic and anti-anxiety drugs via ballistic syringe 20 minutes after we attached the DTAG; the effect of this drug mixture on right whales has been briefly described but remains only partially understood (Moore et al., 2010; van der Hoop et al., 2013b). We placed the tag just above the right lateral midline, midway between the blowhole and tail (Fig. 3 in van der Hoop et al., 2013b). 
Eg 4057 was a three-year-old male when first sighted entangled 16 February 2014 off Jacksonville, FL. His most recent pre-entanglement sighting was 11 months prior on 18 March 2013 in Cape Cod Bay, MA; his body condition was observed and evaluated as fair (i.e., not emaciated) in both sightings. Prior to the disentanglement effort, we attached a DTAG on the left flank (Fig. 1D).

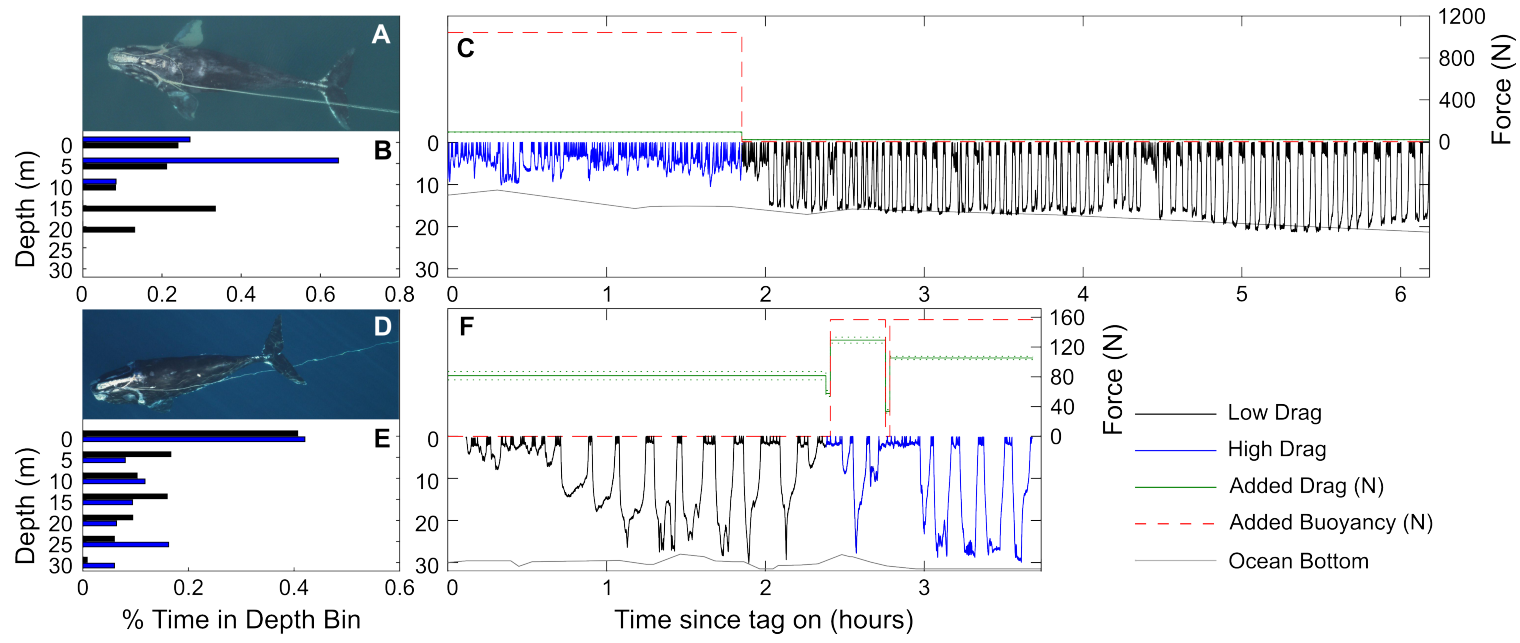

Figure 4-1: Percentage of time spent at different depths (B, E) and dive profiles from complete DTAG records (C, F) of right whales Eg 3911 (A) and Eg 4057 (D) in low (black) and high (blue) drag and buoyancy conditions. Drag (green solid; dotted $=95 \%$ CI of estimate) and buoyant (red dashed) forces are estimated from gear dimensions from van der Hoop et al. (2015) and from manufacturer information (see text for details).

\subsubsection{Tag data analysis}

The DTAG includes a pressure sensor and three-axis accelerometers and magnetometers sampling at $50 \mathrm{~Hz}$, though we down-sampled sensor data to $5 \mathrm{~Hz}$ for analysis. We derived pitch, roll and heading from the accelerometer and magnetometer signals after correcting for the orientation of the tags on the whales (Johnson and Tyack, 2003; Johnson, 2015), and for tag movement during the deployment on Eg 4057.

\section{Dive parameters}

We defined dives as departures from the surface to $>5 \mathrm{~m}$. We obtained water column depth from nautical charts (GPS Geoplaner, www.geoplaner.com) for GPS waypoints from follow vessels and aircraft during the disentanglement procedures. We visually determined descent and ascent portions of dives from the DTAG depth record. We calculated descent and ascent rates of each dive as the change in depth/time. We used these rates as observed swimming speeds $\left(U_{o b s} ; \mathrm{m} / \mathrm{s}\right)$ in further analyses, and note they are a lower bound of speed as they represent change in only one dimension. We estimated the expected descent and ascent rates $\left(U_{\text {exp }} ; \mathrm{m} / \mathrm{s}\right)$ as those at which the whales would have to swim to maintain non-entangled 
Table 4-1: List of symbols and abbreviations

\begin{tabular}{|l|l|l|}
\hline Symbol & Definition & Unit \\
\hline$\alpha$ & Angle of attack & $\circ$ \\
\hline$A$ & Peak-to-peak Amplitude & $\mathrm{m}$ \\
\hline$C_{T}$ & Thrust coefficient & \\
\hline$D_{h}$ & High-drag drag force & $\mathrm{N}$ \\
\hline$D_{l}$ & Low-drag drag force & $\mathrm{N}$ \\
\hline$D_{n}$ & Non-entangled drag force & $\mathrm{N}$ \\
\hline$\eta_{a}$ & Overall efficiency & \\
\hline$\eta_{i}$ & Ideal efficiency & \\
\hline$\eta_{m}$ & Metabolic efficiency & \\
\hline$\eta_{p}$ & Propulsive efficiency & \\
\hline$f$ & Frequency & $\mathrm{Hz}$ \\
\hline$L$ & Body length & $\mathrm{m}$ \\
\hline$P_{O}$ & Overall power & $\mathrm{W}$ \\
\hline$P_{T}$ & Thrust power & $\mathrm{W}$ \\
\hline$\rho$ & Density & $\mathrm{kg} / \mathrm{m}^{3}$ \\
\hline$S$ & Fluke span & $\mathrm{m}$ \\
\hline$S t$ & Strouhal Number & \\
\hline$U$ & Speed & $\mathrm{m} / \mathrm{s}$ \\
\hline$U_{e x p}$ & Expected speed & $\mathrm{m} / \mathrm{s}$ \\
\hline & & \\
\hline
\end{tabular}

drag forces under the entangled condition (Fig. C1). To do so, we calculated the drag force for each dive descent and ascent with their observed descent or ascent rates in either the high drag or low drag conditions. We fit polynomial functions to the relationship between speed and drag for each condition, and then determined the speed $\left(U_{\text {exp }}\right)$ that would result in the same low-drag force in the high-drag condition, for each descent and ascent. We calculated the observed change in swimming speed as:

$$
\Delta U_{o b s}=\frac{\overline{U_{l o w}}-\overline{U_{h i g h}}}{\overline{U_{l o w}}}
$$

and the expected decrease in swimming speed as:

$$
\Delta U_{\text {exp }}=\frac{\overline{U_{l o w}}-\overline{U_{e x p}}}{\overline{U_{l o w}}}
$$

\section{Kinematic parameters}

We calculated pitch deviation as the difference between the mean and instantaneous body posture accelerometer and magnetometer matrices as in Simon et al. (2012). We used peak 
detection algorithms to detect peaks in the pitch deviation signal which represent individual fluke strokes (Johnson and Tyack, 2003; Johnson, 2015). We calculated the duration of each fluke stroke (sec; i.e., the time between peaks) for the descent, ascent, and bottom phases of each dive, and at the surface between dives. We calculated fluke stroke rate $(\mathrm{Hz})$ as the number of fluke strokes divided by the duration of each phase (sec). Following convention (Woodward, 2006; Nousek-McGregor et al., 2013; van der Hoop et al., 2013b; Johnson, 2015), we transformed the pitch deviation signal with the Hilbert transform to detect glides as periods of oscillations $<0.4$ radians for $>5 \mathrm{~s}$.

\subsubsection{Thrust, efficiency and power}

We were only able to calculate peak-to-peak amplitude, thrust, efficiency and power estimates for Eg 3911; fluke stroke amplitudes could not be estimated from the tag on Eg 4057 because it moved during the deployment. The tag sensors measure movements at the point of tag attachment and not at the position of the flukes. We therefore estimated fluke stroke amplitude by calculating maxima and minima of the pitch deviation oscillations as measured on the tag, and then scaled the average amplitude to body length. We assumed that for normal swimming, peak-to-peak amplitude is a constant proportion of body length (i.e., insensitive to speed), $\sim 0.2 \times L$ (Webb, 1975b; Fish, 2003). When accelerating, peak-to-peak amplitudes in dolphins can be as high as $0.5 \times L$ (Skrovan et al., 1999). We scaled the mean measured amplitudes in the low drag condition, representing 'normal' swimming behavior, to body length (10 $\mathrm{m}$ for $\mathrm{Eg} 3911$ so mean amplitude $=2 \mathrm{~m}$ ) and then applied this scaling factor to the recorded pitch amplitudes in the high drag condition.

We calculated the total drag on Eg 3911 based on a turbulent spindle model as in van der Hoop et al. (2013b) with drag augmentation factors for appendages $(g=1.3)$ body oscillation $(k=1.5 \pm 10 \%)$. We calculated the surface wave drag augmentation factor $(\gamma)$ from Eg 3911's median submergence depth when entangled (3.68 m, $\gamma=1.75)$ and after disentanglement $(10.68 \mathrm{~m}, \gamma=1)$. We calculated drag on the whale when it was entangled and after disentanglement, when some gear remained, by adding the estimated average drag on the gear (with floats) established in van der Hoop et al. (see Case Histories; 2015). We used this relationship instead of the values measured in van der Hoop et al. (2013b) because only a portion of the gear present during the tag deployment was measured in that study.

We were interested in determining if the kinematic changes observed in Eg 3911 affected thrust production and overall swimming efficiency. Thrust forces are better reported as a non-dimensionalized thrust coefficient, $C_{T}$, which allows for better comparison across e.g., speed or size. For reference, $C_{T}$ ranges from 0.3-1.6 in odontocetes (Fish and Lauder, 2006).

Efficiency can be estimated from $C_{T}$ : we calculated the average thrust coefficient for each dive descent and ascent from tag data when the whale was entangled (high drag) and after 
disentanglement (low drag), and for the whale when non-entangled as

$$
C_{T}=\frac{2 D}{\rho U^{2} A S}
$$

where $D$ is the drag force estimated for the whale when entangled, disentangled and non-entangled ( $\mathrm{N} ; D_{e}, D_{d}$, and $D_{n}$ respectively); $\rho$ is seawater density $\left(1025 \mathrm{~kg} / \mathrm{m}^{3}\right) ; U$ is swimming speed; $A$ is the peak-to-peak fluke stroke amplitude (m; hereafter amplitude); and $S$ is the span of the tail fluke $(\mathrm{m})$ calculated from the morphometric relationship based on body length in Moore et al. (2004). For reference, we also estimated $C_{T}$ for the nonentangled condition across the full range of speeds using the drag of the non-entangled whale $\left(D_{n}\right)$ and the mean \pm SD fluke stroke amplitude of the whale when disentangled as these provide the best-informed approximation.

We calculated the ideal efficiency, $\eta_{i}$, from $C_{T}$ based on actuator disc theory (Prandtl, 1952; Anderson et al., 1998) as

$$
\eta_{i}=\frac{2}{1+\sqrt{1+C_{T}}}
$$

Ideal efficiency represents the upper limit of achievable propulsive efficiency, always greater than propulsive efficiency, $\eta_{p}$, the actual efficiency achieved by the whale. Ideal efficiency does not account for viscous, rotational losses and inefficiencies from non-uniform loading (Rayner, 1979; Blake, 1983; Muijres et al., 2011). Ideal efficiency therefore illustrates the general trends and the degree to which the optimal performance of the system is affected by different entanglement conditions.

Propulsive efficiency can be estimated from a combination of swimming movements, animal morphology and an understanding of wake structure. Highly efficient swimming or flying animals use their propulsors (tails or wings) to generate vortices and then coordinate their body movements to take further advantage of those vortices in their wakes (Triantafyllou et al., 1993). The Strouhal number $(S t)$ is a dimensionless number that describes the spacing and size of shed vortices based on the frequency $(f, \mathrm{~Hz})$ and amplitude $(A, \mathrm{~m})$ of the propulsor, and the speed of the flow $(U, \mathrm{~m} / \mathrm{s})$ :

$$
S t=\frac{f A}{U}
$$

Optimal swimming efficiency is achieved within the range of $0.2<S t<0.4$, when staggered vortices form in unstable wakes where disturbances are amplified (Triantafyllou et al., 1993; Eloy, 2011). We calculated $S t$ on the descent and ascent portion of each dive in low and high drag conditions to describe the patterns in the wake of the swimming whale, and to determine if $S t$ was within the optimal range in either condition.

We used the values of $C_{T}$ and $S t$ for each dive descent and ascent to estimate the 
angle of attack $(\alpha)$ and propulsive efficiency $\left(\eta_{p}\right)$ from flexible foil experiments and theory (Anderson et al., 1998; Hover et al., 2004, Fig. C2). The angle of attack is the angle between the oncoming fluid velocity and the chord of the foil; lift is perpendicular to the velocity and drag along it. In flexible foils an estimated angle of attack is obtained using an average chord direction. We assumed the tail to follow a symmetric sawtooth wave profile in Hover et al. (2004, Fig. C2) except for where $S t<0.4$ where we assumed harmonic waves. Harmonic motion in heave and pitch is not optimal when $S t$ is high $(>0.4)$ because of the arc-tangent in the heave velocity term (Hover et al., 2004). At these values of St, multi-harmonic or sawtooth motions provide far better efficiency and thrust. After estimating the angle of attack for each dive portion, we estimated propulsive efficiency from $S t$ based on sawtooth and harmonic contours of $\alpha$ from Figure 6 in Hover et al. (2004, Fig. C2). Points where $S t>$ 1.6 and $C_{T} / S t^{2}>10$ were not included in the angle of attack, efficiency, or power estimates.

We used our derived estimates of propulsive efficiency to estimate two forms of power (W), separately for the descent and ascent of each dive in high and low drag conditions. Thrust power, $P_{T}$, was estimated as

$$
P_{T}=\frac{D U}{\eta_{p}}
$$

based on propulsive efficiency, $\eta_{p}$, alone, while overall power, $P_{O}$, included metabolic efficiency $\left(\eta_{m}\right)$ of 0.25 (Webb, 1975b):

$$
P_{T}=\frac{D U}{\eta_{p} \times \eta_{m}}
$$

\subsubsection{Statistical analysis}

We compared average descent and ascent rates within individuals between conditions with a Bonferroni corrected two-sample $t$-test $(\alpha=0.025)$. We expected that $(1)$ both descent and ascent rates would be slower in the high drag condition, but that (2) ascent speeds in the high drag/buoyancy condition may not be reduced as much as descent speed, due to buoyant forces acting in the direction of travel. To determine whether fluke stroke timing differs with drag, we compared the duration of each fluke stroke and mean dive fluke stroke frequencies with three-way ANOVA with effects of individual, loading condition, and dive phase (descent, bottom, ascent, and surface) and Tukey's post-hoc HSD (R Core Team, 2015). We expected that higher drag conditions would result in shorter fluke stroke durations, or that fluke stroke rate would be greater in response to increased drag, especially on descent (the buoyancy-hindered direction). We compared theoretical drag loading conditions with a two-sample $t$-test to determine whether disentanglement significantly reduced drag. We compared thrust coefficients, ideal efficiency, Strouhal number, propulsive efficiency, and thrust and overall power estimated for Eg 3911 with two-way ANOVA with effects loading 
condition and dive phase (descent, bottom, ascent, and surface) and Tukey's post-hoc HSD (R Core Team, 2015). To determine whether the thrust coefficient $\left(C_{T}\right)$ was affected by drug treatment, we fit the relationship between $C_{T}$ and time with a linear regression model (MATLAB, 2014).

\subsection{Results}

\subsubsection{Case histories}

Eg 3911 was entangled in a total of $72 \mathrm{~m}$ of line, with two gangions (short lengths of moderate-weight line leading from traps to the groundline which connects the traps; McCarron and Tetreault, 2012) and two Scanmarin buoys of $42 \mathrm{~cm}$ and $45 \mathrm{~cm}$ diameter. At the time of the DTAG attachment on 15 January 2011, the entangling gear added an estimated $93 \mathrm{~N}$ of drag to the whale (Fig. 1A; Table 2). During this deployment, the disentanglement response team removed $50 \mathrm{~m}$ of gear, including one gangion and both buoys. The tag recorded through 4:20 h:m after the gear was removed. Eg 3911's tag deployment lasted 6:11 $\mathrm{h}: \mathrm{m}$. Drug injection of a mixture of Midazolam and Butorphanol occurred 20 min after the tag was attached. The first 1:51 h:m of the recording were under high drag and buoyancy conditions, and the last 4:20 h:m were low drag and buoyancy.

Table 4-2: Added drag and buoyant forces (N) on right whales Eg 3911 and Eg 4057 before and after disentanglement response efforts.

\begin{tabular}{|l|l|l|l|l|l|}
\hline & Drag before & Drag after & $\begin{array}{l}\text { Change in } \\
\text { drag }\end{array}$ & $\begin{array}{l}\text { Buoyancy } \\
\text { before }\end{array}$ & $\begin{array}{l}\text { Buoyancy af- } \\
\text { ter }\end{array}$ \\
\hline Eg 3911 & $93 \mathrm{~N}$ & $19 \mathrm{~N}$ & $-79.5 \%$ & $1058 \mathrm{~N}$ & - \\
\hline Eg 4057 & $81.5 \mathrm{~N}$ & $124 \mathrm{~N}$ & $+51.2 \%$ & - & $157 \mathrm{~N}$ \\
\hline
\end{tabular}

While the measured drag on a $34 \mathrm{~m}$ portion of the removed gear with buoys at $1.5 \mathrm{~m} / \mathrm{s}$ was $66 \mathrm{~N}$ (van der Hoop et al., 2013b), an additional segment of the entangling gear was lost during disentanglement, expected to have added $8 \mathrm{~N}$ at $1.5 \mathrm{~m} / \mathrm{s}$ (van der Hoop et al., 2015); the total drag of the removed gear was therefore $74 \mathrm{~N}$. Based on their dimensions, the buoys added $1058 \mathrm{~N}$ of positive buoyancy. The short amount of gear that remained on the whale added $19 \mathrm{~N}$ of drag, and the effect of buoyancy was likely negligible (Fig. 1C). This gear remained on Eg 3911 until she was discovered dead 1 February 2011. The disentanglement effort reduced drag by $79.5 \%$.

Eg 4057 was entangled in $155 \mathrm{~m}$ of three-strand synthetic rope entering and exiting the left side of the mouth, with one end just above the eye and the other end trailing more than $30 \mathrm{~m}$ aft of the flukes before sinking out of view. The entangling gear was estimated to have added an average of $82 \mathrm{~N}$ of drag across speeds of 0.5 to $3.0 \mathrm{~m} / \mathrm{s}$ (van der Hoop et al., 2015). The disentanglement team removed $104 \mathrm{~m}$ of line $(-24 \mathrm{~N})$ before attaching a satellite 
telemetry buoy $(+72 \mathrm{~N}$ drag, $+157 \mathrm{~N}$ buoyancy). The team then removed another $12.3 \mathrm{~m}$ of line $(-6 \mathrm{~N})$ before reattaching the telemetry buoy. The tag continued recording 1:18 h:m following the addition of the telemetry buoy. Overall, $116 \mathrm{~m}$ of rope was removed, reducing drag by $30 \mathrm{~N}$; however, the attachment of the telemetry buoy resulted in a $51 \%$ increase in drag over the original entangled condition, totalling 124 N (Fig. 1F; Table 2). Eg 4057's tag deployment was 3:34 h:m long; the first 2:16 h with low drag and buoyancy and the latter 1:18 h:m with high drag and buoyancy (Fig. 1C, F). The telemetry buoy and almost all of the entangling gear were successfully removed the following day. The whale was sighted and confirmed gear-free 20 April 2015 in Cape Cod Bay, MA.

\subsubsection{Dive parameters}

Eg 3911 completed 154 dives over the 6:11 h:m deployment period, with 101 and 53 in low and high drag conditions, respectively. Significant changes in diving behavior of Eg 3911 associated with disentanglement have been previously described (van der Hoop et al., 2013b). Eg 4057 made a total of 20 dives over the 3:34 h:m deployment, with 12 and 8 dives under low and high drag conditions, respectively, to 5-30 m (Fig. 1F). In contrast to Eg 3911, the depth distribution of Eg 4057 is very similar between low- and high-drag conditions (Fig. $1 \mathrm{E})$. Tag data therefore indicate variable responses in diving behavior between these two individuals.

The observed changes in ascent and descent rates of Egs 3911 and 4057 in response to drag and buoyancy were different (Table 3). Eg 3911 swam significantly slower in high drag, with descents on average $0.32 \mathrm{~m} / \mathrm{s}(46 \%)$ and ascents $0.14 \mathrm{~m} / \mathrm{s}(32 \%)$ slower than ascents. Observed speeds were greater than the expected reduction necessary to maintain low-drag drag forces under the high-drag condition on descents (67\%) and ascents (49\%; Table 3). Eg 4057 descended significantly faster in high drag (by $0.07 \mathrm{~m} / \mathrm{s} ; 24 \%$ ). There was no detectable difference in ascent rate (Table 3). Observed speeds were considerably faster than those expected if Eg 4057 were attempting to maintain the same drag forces as would be experienced in the low-drag condition.

\subsubsection{Kinematic parameters}

Kinematic parameters were estimated from all 154 dives of Eg 3911. Due to movement of the tag on Eg 4057, fluke stroke kinematics were only estimated for 17 dives total, 11 in low drag and 6 in high drag. The number of individual fluke strokes in various conditions and dive phases are shown in Fig. 2.

We derived kinematic parameters from all 154 dives of Eg 3911. Due to movement of the tag on Eg 4057, we could only derive fluke stroke kinematics for 17 dives total, 11 in low drag and 6 in high drag. The number of individual fluke strokes in various conditions and dive phases are shown in Fig. 2. 
Table 4-3: $M e a n( \pm \mathrm{SD})$ descent and ascent rates for right whales Eg 3911 and Eg 4057 in low and high drag conditions, along with the percent change in observed speeds between low and high drag conditions and the expected reduction in speed (in $\mathrm{m} / \mathrm{s}$ and percent) to maintain the same power output as in low drag. Values for $t$ statistic and $P$ from two-sample $t$-tests are included, with Bonferonni-corrected $\alpha=0.025$.

\begin{tabular}{|l|l|l|l|l|}
\hline & \multicolumn{3}{|c|}{ Eg 3911 $\mathrm{Eg} 4057$} \\
\hline & $\begin{array}{l}\text { Vertical descent } \\
\text { rate }\end{array}$ & $\begin{array}{l}\text { Vertical ascent } \\
\text { rate }\end{array}$ & $\begin{array}{l}\text { Vertical descent } \\
\text { rate }\end{array}$ & $\begin{array}{l}\text { Vertical ascent } \\
\text { rate }\end{array}$ \\
\hline Low Drag & $0.80( \pm 0.27) \mathrm{m} / \mathrm{s}$ & $0.44( \pm 0.13) \mathrm{m} / \mathrm{s}$ & $0.29( \pm 0.14) \mathrm{m} / \mathrm{s}$ & $0.13( \pm 0.11) \mathrm{m} / \mathrm{s}$ \\
\hline High Drag & $0.39( \pm 0.10) \mathrm{m} / \mathrm{s}$ & $0.31( \pm 0.10) \mathrm{m} / \mathrm{s}$ & $0.36( \pm 0.12) \mathrm{m} / \mathrm{s}$ & $0.21( \pm 0.10) \mathrm{m} / \mathrm{s}$ \\
\hline $\begin{array}{l}\text { Percent change ob- } \\
\text { served }\end{array}$ & $-51 \%$ & $-30 \%$ & $+24 \%$ & $+50 \%$ \\
\hline$t, p$ & $\begin{array}{l}t=-9.77 p< \\
0.0001\end{array}$ & $\begin{array}{l}t=-7.02 p< \\
0.0001\end{array}$ & $\begin{array}{l}t=3.02 p= \\
0.0073\end{array}$ & $\begin{array}{l}t=1.42 p \\
0.1733\end{array}$ \\
\hline Expected high drag & $0.38( \pm 0.13) \mathrm{m} / \mathrm{s}$ & $0.24( \pm 0.07) \mathrm{m} / \mathrm{s}$ & $0.14( \pm 0.06) \mathrm{m} / \mathrm{s}$ & $0.13( \pm 0.07) \mathrm{m} / \mathrm{s}$ \\
\hline $\begin{array}{l}\text { Percent change ex- } \\
\text { pected }\end{array}$ & $-53 \%$ & $-45 \%$ & $-52 \%$ & $-7 \%$ \\
\hline
\end{tabular}

There were significant, interactive effects on dive phase and drag condition on individual fluke strokes in these two whales (Fig. 2). First, the duration of the fluke stroke cycle was significantly shorter in $\operatorname{Eg} 3911$ compared to $\operatorname{Eg} 4057$ ( $F_{1,50155}=9866.8, p<0.0001$; Fig. $2 \mathrm{~A}-\mathrm{H}$ vs. Fig. 2I-P). Their response to drag condition was significantly different (Whalex Condition, $\left.F_{1,50155}=3113.4, p<0.0001\right)$ where in Eg 3911, individual fluke strokes were significantly longer in the high drag condition compared to low (Tukey's HSD $p<0.0001$; Fig. 2A-D vs. Fig. 2E-H). Although this result is statistically significant, it is likely not biologically significant, with a difference of 0.09 (95\% CI 0.05-0.13) sec. In contrast, Eg 4057 swam with significantly shorter fluke strokes in high drag $(3.95 \pm 0.71 \mathrm{sec})$ compared to low drag (5.42ś0.16 sec; Tukey's HSD $p<0.0001$; Fig. 2M-P vs. Fig. 2I-L). Dive phase had a significant effect on fluke stroke duration, especially in high drag. Fluke strokes were significantly shorter during the bottom phase of dives in both whales (Fig. 2B, F, J, N).

The longer fluke stroke durations observed for Eg 4057 in low drag, and especially on dive descents (e.g. Fig. 2I), corresponded with glides. Both individuals performed fewer glides in high drag (4057: $n=5,2.6 \times 10^{-3} / \mathrm{min}$; 3911: $\left.n=1,1.5 \times 10^{-4} / \mathrm{min}\right)$ compared to in low drag (4057: $n=40,4.9 \times 10^{-3} / \mathrm{min} ; 3911: n=7,4.5 \times 10^{-4} / \mathrm{min}$ ) conditions (Fig. $3)$.

Related to fluke stroke duration but averaged over each dive phase for all dives, fluke stroke rates were significantly different between the two whales (three-way ANOVA, $F_{1,642}=$ 186.9, $p<0.0001$; Fig. 4); across all dive phases, the fluke stroke rate of Eg 3911 (0.296 \pm 0.072 $\mathrm{Hz})$ was $40 \%$ greater than $\mathrm{Eg} 4057(0.178 \pm 0.058 \mathrm{~Hz})$. Fluke stroke rates also differed between dive phases (three-way ANOVA, $F_{3,642}=26.55, p<0.0001$ ), being significantly greater at 
the surface $(0.326 \pm 0.128 \mathrm{~Hz})$ compared to all other dive phases (Tukey's HSD $p<0.0001$ for each; Table 4). Average fluke stroke rate did not, however, appear to differ between low and high drag conditions $\left(F_{1,642}=0.3505, p=0.554\right.$; Fig. 4$)$. 

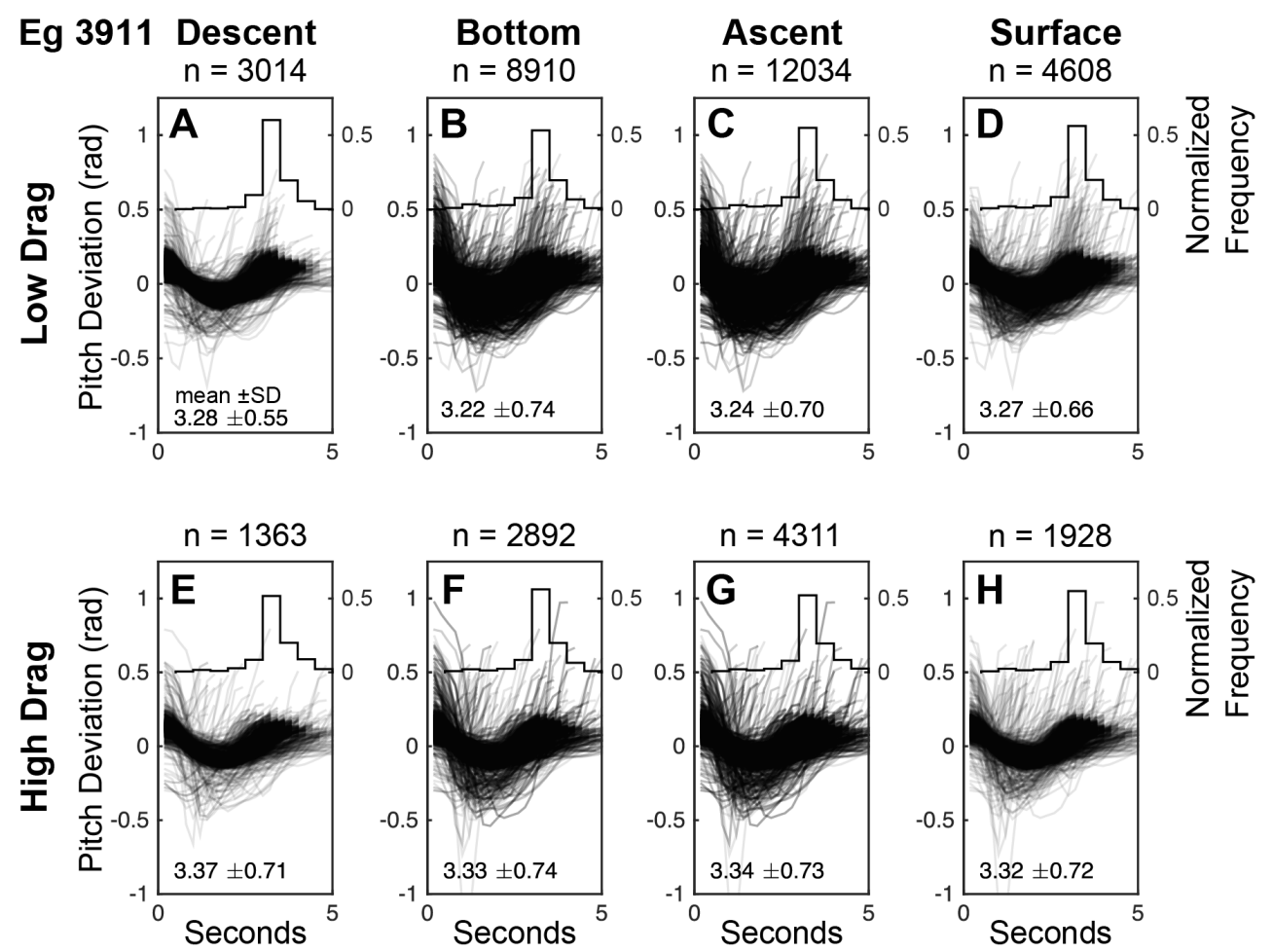

Eg 4057 Descent
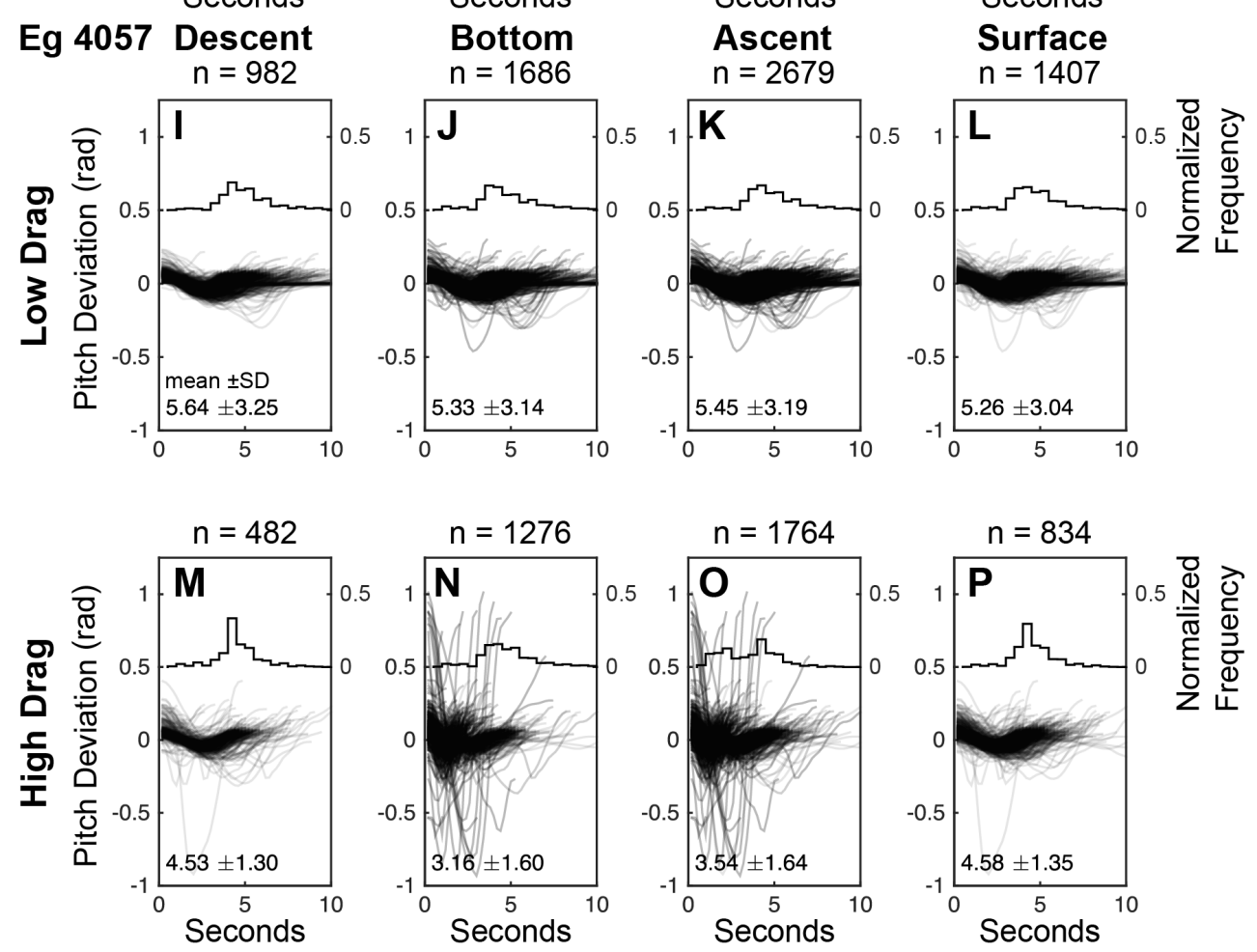

Figure 4-2: Right whales show different fluke stroke patterns between individuals, dive phases and drag conditions. Individual fluke strokes measured from pitch deviation (degrees) in low (A-D, I-L) and high (E-H, M-P) drag conditions on the dive descents (A, E, I, M), bottom (B, F, J, N), ascents $(\mathrm{C}, \mathrm{G}, \mathrm{K}, \mathrm{O})$ and surface periods (D, H, L, P) for two right whales (Eg $3911 \mathrm{~A}-\mathrm{H}$; Eg $4057 \mathrm{I}-\mathrm{P}$ ). Histograms show the frequency of observations of each duration, normalized by the total number of observations. Numbers represent mean \pm SD fluke stroke duration; sample sizes $(n)$ of the number of fluke strokes are denoted above each panel. 

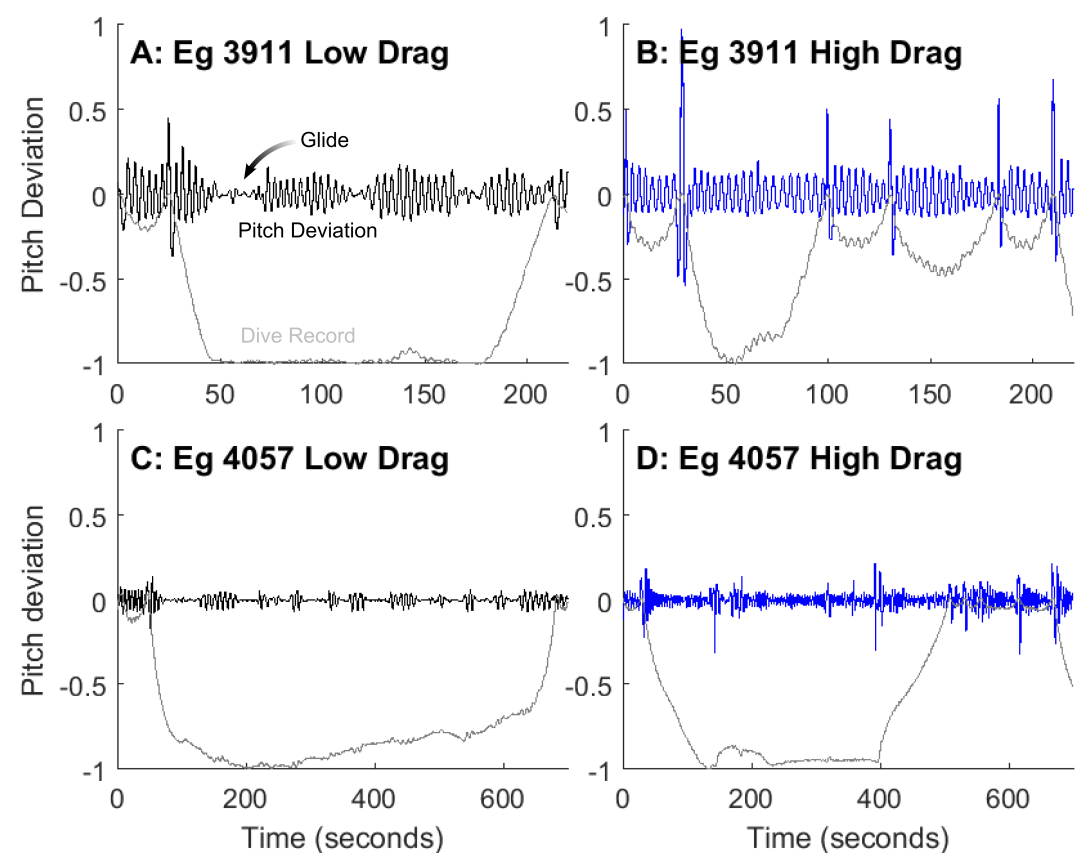

Figure 4-3: Right whales alter fluke stroke frequency and amplitude, and glide behavior, under low- and high-drag and buoyancy conditions. Pitch Deviation, showing individual fluke strokes and periods of gliding, in low (black; A, C) and high (blue; B, D) drag conditions during the course of dives (grey lines) in two right whales (Eg 3911 A, B; Eg 4057 C, D). 


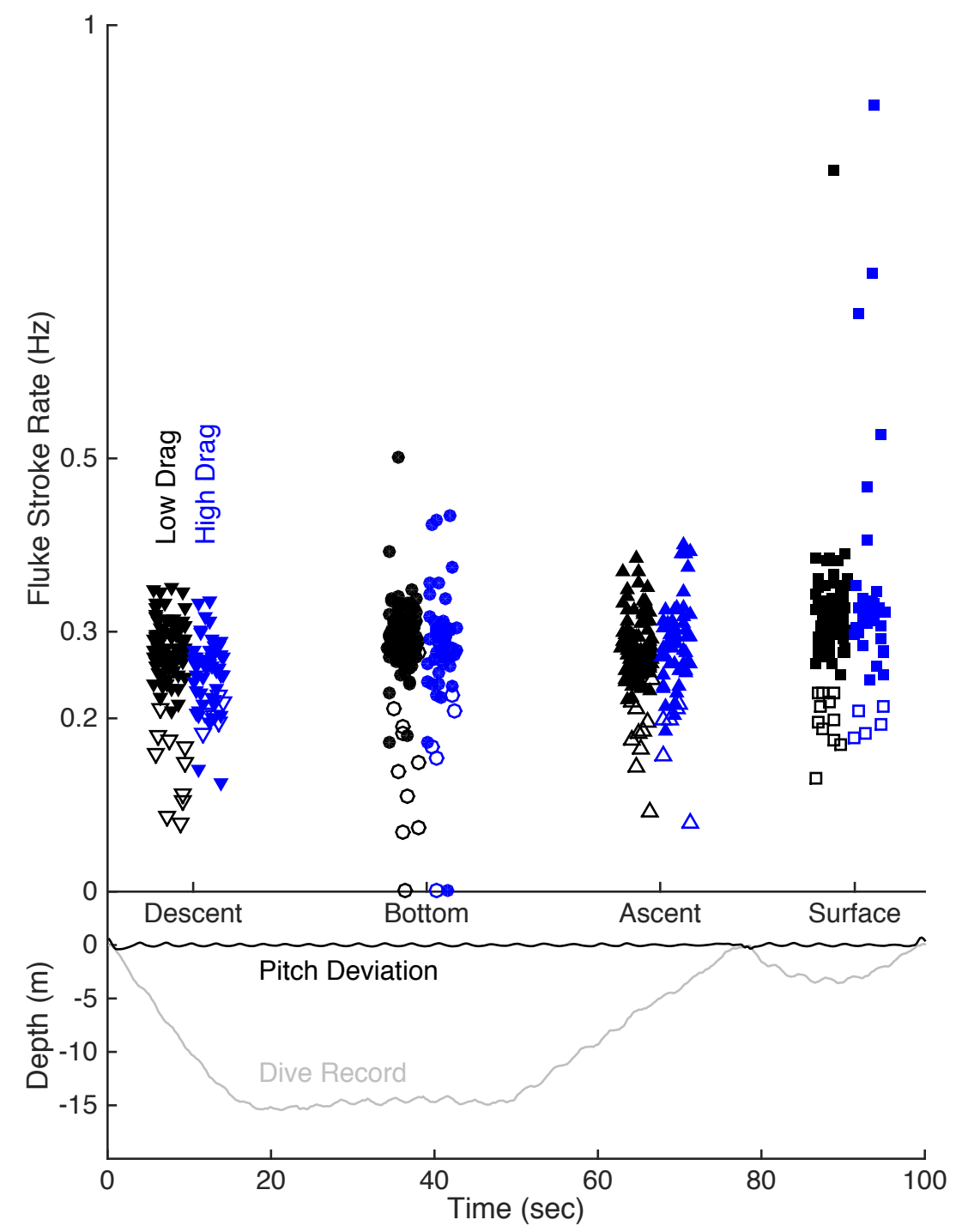

Figure 4-4: Average fluke stroke rates are different between whales (Eg 3911 closed symbols, Eg 4057 open symbols) and dive phases, but not between low (black) and high (blue) drag conditions (bottom). Representative dive profile (grey; top) and pitch record (black; top) illustrates the periods over which fluke stroke rates are calculated: descent (downward triangles), bottom (circles), ascent (upward triangles) and surface (squares). 
Table 4-4: $\operatorname{Mean}( \pm \mathrm{SD})$ fluke stroke cycle duration and dive-phase averaged fluke stroke rates for descent, bottom, ascent, and surface portions of the dive records of two right whales Eg 3911 and Eg 4057.

\begin{tabular}{|l|l|l|l|l|l|l|l|l|l|l|}
\hline Dive Phase & Drag Condition & \multicolumn{2}{|c|}{ Descent } & \multicolumn{3}{c|}{ Bottom } & \multicolumn{3}{c|}{ Ascent } & \multicolumn{2}{c|}{ Surface } \\
\hline Whale & & 3911 & 4057 & 3911 & 4057 & 3911 & 4057 & 3911 & 4057 \\
\hline Fluke Stroke Cycle & \multirow{2}{*}{ Low } & 3.28 & 5.64 & 3.22 & 5.33 & 3.24 & 5.45 & 3.27 & 5.26 \\
Duration (sec) & & $(0.55)$ & $(3.25)$ & $(0.74)$ & $(3.14)$ & $(0.70)$ & $(3.19)$ & $(0.66)$ & $(3.04)$ \\
& \multirow{2}{*}{ High } & 3.37 & 4.53 & 3.33 & 3.16 & 3.34 & 3.54 & 3.32 & 4.58 \\
& & $(0.71)$ & $(1.30)$ & $(0.74)$ & $(1.60)$ & $(0.73)$ & $(1.64)$ & $(0.72)$ & $(1.35)$ \\
\hline Mean Fluke Stroke & \multirow{2}{*}{ Low } & 0.282 & 0.153 & 0.295 & 0.152 & 0.285 & 0.188 & 0.335 & 0.198 \\
Rate (Hz) & \multirow{4}{*}{ High } & $(0.029)$ & $(0.057)$ & $(0.037)$ & $(0.087)$ & $(0.033)$ & $(0.047)$ & $(0.047)$ & $(0.031)$ \\
& & 0.253 & 0.206 & 0.289 & 0.171 & 0.288 & 0.176 & 0.362 & 0.195 \\
& & $(0.041)$ & $(0.015)$ & $(0.063)$ & $(0.094)$ & $(0.047)$ & $(0.052)$ & $(0.139)$ & $(0.017)$ \\
\hline
\end{tabular}


Mean body pitch amplitudes were variable across dive phases and drag conditions (Figs. $2,3)$. We could not make quantitative comparisons of amplitude could not be made for $\mathrm{Eg}$ 4057 due to tag movement during the deployment, or between the tags due to differences in tag placement. In both animals, fluke strokes appeared to be more variable in duration and shape in high drag/buoyancy (Fig. 3B, D vs. A, C), and during bottom and ascent phases (Fig. 2B, C, F, G, N, O).

\subsubsection{Thrust, efficiency and power}

We estimated drag on non-entangled Eg 3911 to be 10 - $463 \mathrm{~N}$ across speeds of $0.3-2.5$ m/s (Fig. C1). When entangled, drag on Eg 3911 was significantly (on average 230 $\pm 228 \%$ ) greater, ranging 118 to $909 \mathrm{~N}$ (two-sample $t$-test; $t=10.8881, p<0.0001$ ). Following disentanglement, drag was significantly reduced by on average $53 \pm 7 \%$, to $37-489 \mathrm{~N}$ across speeds (Fig. C1; $t=-9.6970, p<0.0001$ ).

Both drag condition and dive phase had a significant effect on average thrust coefficients. When entangled, average thrust coefficients $\left(C_{T}\right)$ during dive descents were $0.164( \pm 0.082)$ and ascents were significantly greater than following disentanglement $(0.311 \pm 0.135$; twoway ANOVA, $F_{1,304}=83.10, p<0.0001$; Fig. 5A). Eg 3911's disentanglement procedure significantly reduced thrust coefficients on descent and ascent by 3.91 and 3.99 fold, to $0.042( \pm 0.012)$ and $0.078( \pm 0.018)$, respectively (two-way ANOVA, $F_{1,305}=425.8, p<$ 0.0001; Fig. 5A). Because a portion of gear remained, thrust coefficients when disentangled were still on average 1.31 and 1.53 fold greater than estimated for the non-entangled condition on descent $(0.047 \pm 0.002)$ and ascent $(0.051 \pm 0.004$; Fig. $5 \mathrm{~A}) . C_{T}$ was almost always greater on each dive's ascent than on descent, and is confined in a very narrow range in low drag (Fig. C3A). In high drag, $C_{T}$ was much more variable (Fig. C3A) and showed a significant decrease with time, prior to disentanglement and following drug injection (Fig. C3B). Specifically, the thrust coefficient decreased on descent $\left(C_{T, \text { desc }}\right)$ and ascent $\left(C_{T, a s c}\right)$ as:

$$
\begin{gathered}
C_{T, \text { desc }}=0.28-2.97 \times 10^{-5} \times \text { time }(\mathrm{s})\left[\mathrm{R}^{2}=0.34, p<0.0001\right] . \\
C_{T, \text { asc }}=0.41-2.53 \times 10^{-5} \times \operatorname{time}(\mathrm{s})\left[\mathrm{R}^{2}=0.12, p=0.0231\right] .
\end{gathered}
$$

When entangled, ideal efficiency $\left(\eta_{i}\right)$ was $96.3( \pm 1.7) \%$ and $93.4( \pm 2.4) \%$ on descent and ascent, respectively (Fig. 5B). After disentanglement, ideal efficiency for descent and ascent increased to $99.0( \pm 0.3) \%$ and $98.1( \pm 0.4) \%$, respectively (two-way ANOVA, $F_{1,305}=$ $526.8, p<0.0001$; Fig. 5B). For the non-entangled whale, ideal efficiency was $99.2( \pm 0.1) \%$ on descent and $98.8( \pm 0.1) \%$ on ascent (Fig. 5B).

The Strouhal number $(S t)$ was significantly lower on descent compared to ascent (Fig. 

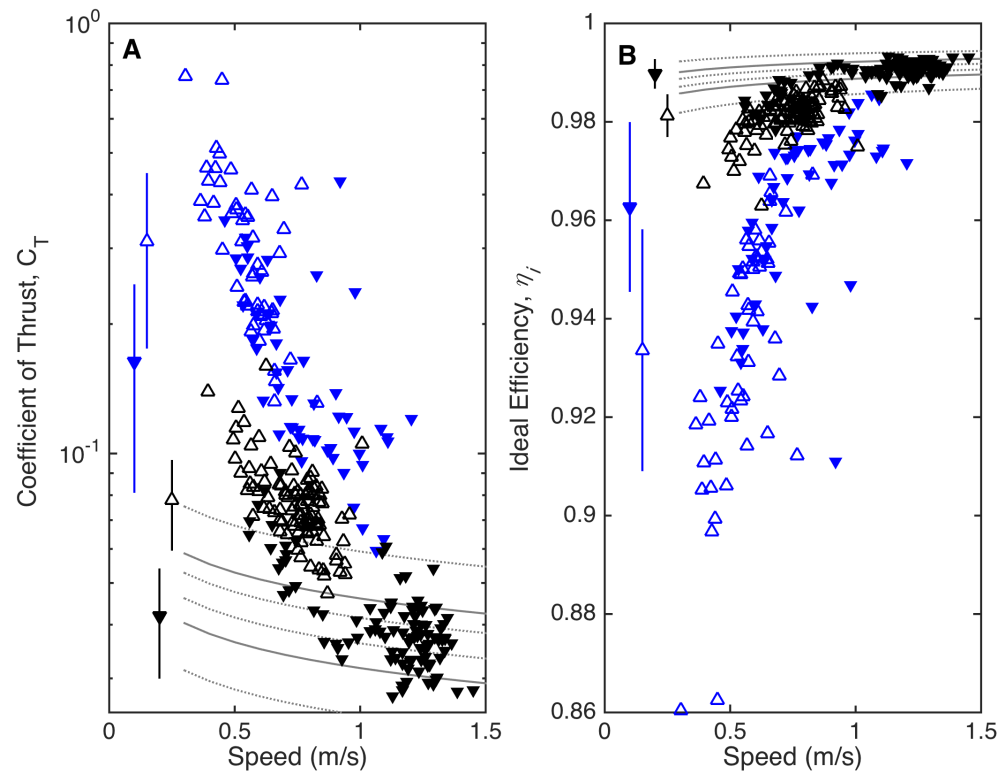

Figure 4-5: Disentanglement significantly reduces thrust coefficients and increases ideal efficiency for an entangled right whale Eg 3911. Coefficient of Thrust $\left(C_{T} ; \mathrm{A}\right)$ and ideal efficiency $\left(\eta_{i} ; \mathrm{B}\right)$ calculated for each descent (inverted filled triangles) and ascent (open triangles) of entangled (blue) and disentangled (black) dives, and estimates of $C_{T}$ and $\eta_{i}$ for the non-entangled condition (grey lines). Mean values (error bars $=\mathrm{SD}$ ) are presented for each condition and dive portion. Note the $\log \mathrm{y}$ axis in A.

6 A ; two-way ANOVA, $\left.F_{1,305}=11.13, p<0.0010\right)$ but we detected no significant difference between low and high drag (Fig. 6A; $\left.F_{1,305}=2.636, p=0.1056\right)$. St on descents were $0.726( \pm 0.257)$ compared to $0.833( \pm 0.292)$ on ascent. Similarly, propulsive efficiency $\left(\eta_{p}\right)$ was significantly higher on descent $(0.19 \pm 0.09)$ compared to ascent $(0.14 \pm 0.09$; Fig. 6B; Table 5; two-way ANOVA, $\left.F_{1,294}=15.93, p<0.0001\right)$, but we detected no difference as drag was decreased by disentanglement $\left(F_{1,294}=0.1413, p=0.7073\right.$; Table 5$)$. Thrust power $\left(P_{T}\right)$ was 1.25 fold greater on dive ascents compared to descents (Fig. 7; Table 5; two-way ANOVA, $\left.F_{1,294}=6.0186, p=0.0147\right)$. Drag from entangling gear significantly increased thrust power, by 1.8 and 1.2 fold on descent and ascent, respectively (Fig 7; Table $\left.5 ; F_{1,294}=13.33, p=0.0003\right)$.

\subsection{Discussion}

Animals, including humans, constantly navigate an energy landscape where kinematics or gait parameters are tuned to minimize metabolic cost in response to changing constraints (Bertram, 2005, 2015; Selinger et al., 2015). Swimmers tune their fluke strokes to address trade-offs between thrust generation and efficiency to optimize performance, especially in response to changes in their natural force balance. Laboratory studies on humans and 

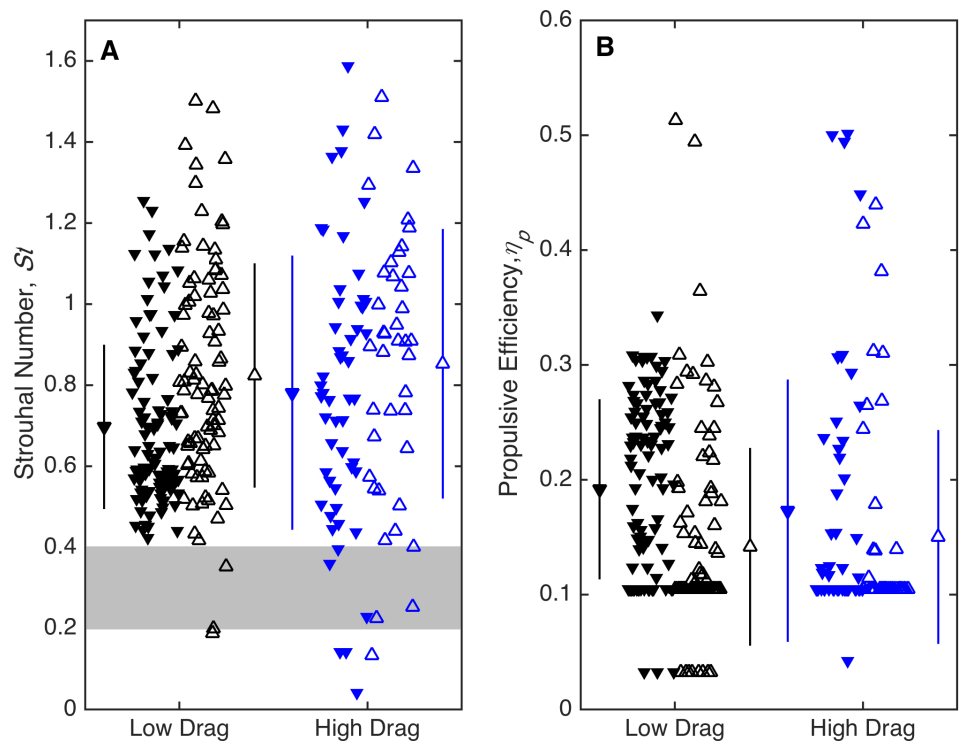

Figure 4-6: Strouhal number $(S t ; \mathrm{A})$ and propulsive efficiency $\left(\eta_{p}\right.$ : B) are significantly different between dive descents (filled inverted triangles) and ascents (open triangles) but do not differ between low drag (black, disentangled) and high drag (blue, entangled) conditions for right whale Eg 3911. Mean values (error bars $=\mathrm{SD}$ ) are presented for each condition and dive portion. The shaded region represents the optimal range of $0.2<S t<0.4$.
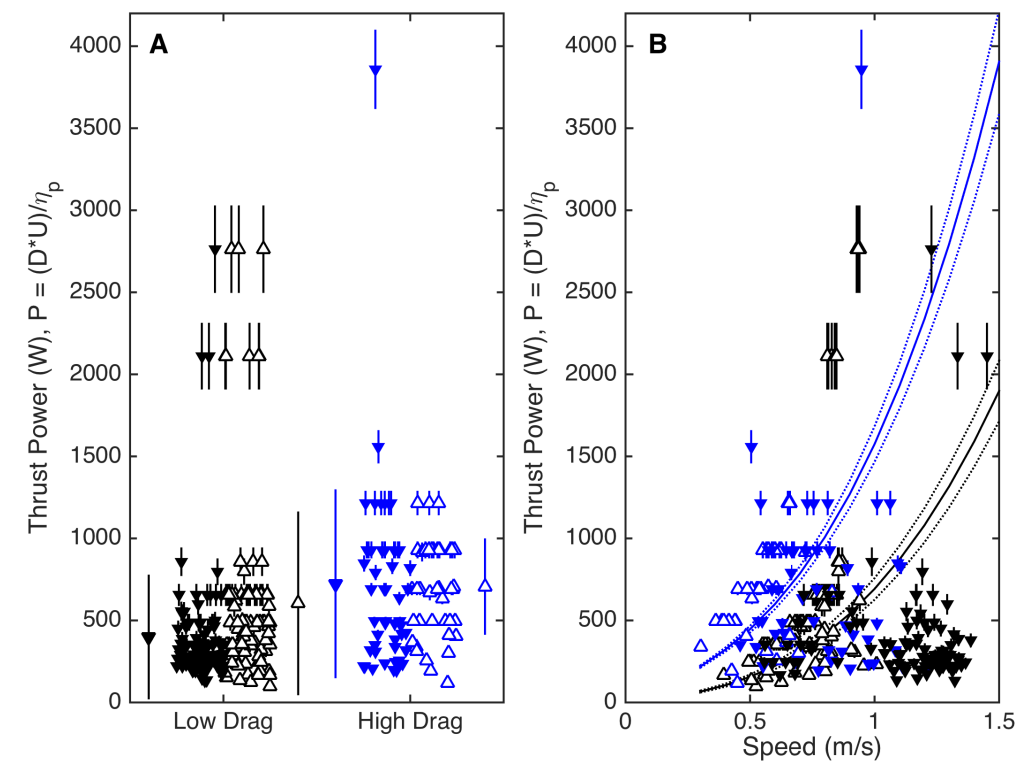

Figure 4-7: A. Thrust power $\left(P_{T}\right)$ is significantly lower on dive descent (filled inverted triangles) than ascent (open triangles) and in low drag (black, disentangled) compared to high drag (blue, entangled) conditions for right whale Eg 3911. B. Thrust power estimates derived from swimming kinematics (symbols) approximate theoretical calculations related to speed (lines). 
Table 4-5: Propulsive efficiency and thrust power of right whale Eg 3911 in low and high drag and buoyancy conditions, on descents and ascents of dives to $>5 \mathrm{~m}$.

\begin{tabular}{|l|l|l|l|l|l|}
\hline & & \multicolumn{2}{|c|}{ Low Drag } & \multicolumn{2}{c|}{ High Drag } \\
\hline & & Descent & Ascent & Descent & Ascent \\
\hline $\begin{array}{l}\text { Propulsive Effi- } \\
\text { ciency }\left(\eta_{p}\right)\end{array}$ & Mean(SD) & $0.025(0.09)$ & $0.26(0.13)$ & $0.22(0.13)$ & $0.16(0.09)$ \\
\hline $\begin{array}{l}\text { Overall Efficiency } \\
\left(\eta_{a}\right)\end{array}$ & Max & 0.53 & 0.58 & 0.57 & 0.48 \\
\hline $\begin{array}{l}\text { Thrust Poan(SD) } \\
\left(P_{T} ; \mathrm{W}\right)\end{array}$ & $0.06(0.02)$ & $0.07(0.03)$ & $0.06(0.03)$ & $0.04(0.02)$ \\
\hline $\begin{array}{l}\text { Overall Power } \\
\left(P_{O} ; \mathrm{W}\right)\end{array}$ & Mean(SD) & $273.6(133.1)$ & $263.6(110.9)$ & $537.6(326.1)$ & $628.0(258.5)$ \\
\hline
\end{tabular}

other animals are able to apply external forces in order to understand motor reorganization strategies and motor control (Bonnard and Pailhous, 1991). Similarly, in this study we attached fine-scale movement tags to two right whales that were entangled in fishing gear; recording continued while the forces on the whales were decreased (Eg 3911, via removal of the fishing gear) and increased ( $\mathrm{Eg} \mathrm{4057,} \mathrm{via} \mathrm{attachment} \mathrm{of} \mathrm{a} \mathrm{satellite} \mathrm{telemetry} \mathrm{tracking}$ buoy). Many of the changes in behavior we observed were individually specific; swimming kinematics were modulated in response to high drag and buoyant forces associated with entanglement in fishing gear, but not necessarily consistently between animals. Observed changes in gait parameters and swimming speed can significantly affect thrust production and efficiency, but may not be applicable to all entangled whales.

\subsubsection{Diving behavior}

While the diving behavior of Eg 3911 was significantly affected by drag and buoyancy (Fig. 1C; van der Hoop et al., 2013b), such a striking difference was not observed in Eg 4057. As both whales were tagged on the breeding grounds in Florida, similar diving behaviors were expected in comparable water depths, times of year and point in the annual migration pattern (e.g. see McGregor, 2010). The variable responses in dive patterns may be attributed to the difference in forces experienced by the individuals: while drag forces were comparable (93 N and $105 \mathrm{~N}$ ), the buoyant forces on $\mathrm{Eg} 3911$ are an order of magnitude greater than for Eg 4057 (1058 N vs. 157 N; Fig. 1C, F; Table 2). Eg 3911's more abrupt response may be due to relative change in force balance if feedback to loading is intensity dependent, as it is in humans and other vertebrates (Duysens et al., 2000). For both tagged right whales, the length of the tether to the buoys was greater than the maximum dive depth $(\operatorname{Eg} 3911=\sim 15$ $\mathrm{m}$ tether; Eg $4057=\sim 33 \mathrm{~m}$ tether). However, visual observations of Eg 4057 describe the telemetry buoy being pulled underwater during dives (Katie Jackson, Pers Comm ${ }^{1}$ ). The

\footnotetext{
${ }^{1}$ Florida Fish and Wildlife Conservation Commission, Jacksonville, FL, U.S.A.
} 
short tag deployment durations, especially for Eg 4057, make it difficult to assess changes in diving behavior associated drag and buoyancy. Future disentanglement operations should continue attaching tags to collect similar data to enable additional comparisons of individual responses to entanglement drag.

Differences in body condition between the whales are also relevant to differences in dive responses. Because the total buoyancy of these individuals is unknown, it is difficult to know just how the ratio of body to added buoyancy may affect their diving behavior. Being $20 \%$ thinner than mesomorphic right whales, Eg 3911 was negatively buoyant, though the entangling gear added a considerable amount of positive buoyancy. If Eg 3911 were to take advantage of added buoyant force, we would expect little change in dive ascent rate, and significantly greater fluke stroke kinematics and thrust on descent in high drag. Contrary to this expectation, $C_{T}$ and thrust power were significantly greater on ascent than descent in high drag (Fig. 5A, Fig. 7, Fig. C3A). The only observation suggesting buoyancy-aided ascents was that gear removal led to increases in descent speeds by $46 \%$ and ascent speeds by $32 \%$; gear had reduced speeds in both directions, but more so in the direction opposite the net force. Eg 3911 did not slow as much as was expected to maintain drag forces (Table 3). The additional buoyancy should have been more inhibitive for Eg 4057 on descent, as the whale was positively buoyant (Nowacek et al., 2001). However, Eg 4057 was able to reach comparable depths in both low- and high-drag and buoyancy conditions and swam at greater speeds in high vs. low drag (Table 3); Eg 4057 did not slow down to reduce power output in either direction. The tags here measured vertical displacement rates, rather than speed relative to water flow; tags with impellers may better elucidate more fine-scale changes in speed including how or whether individuals were taking advantage of current flow (e.g., Bose and Lien, 1990; Pike, 2008).

It is important to acknowledge that Eg 3911 was treated with a mixture of Midazolam and Butorphanol $20 \mathrm{~min}(0.34 \mathrm{~h})$ after the DTAG was attached in order to facilitate the disentanglement procedure (Moore et al., 2010; van der Hoop et al., 2013b). Midazolam produces reliable hypnosis, amnesia, and antianxiety effects (Reves et al., 1985), while Butorphanol is an analgesic (Pircio et al., 1976). Thus a mix of these drugs was appropriate for an entangled whale that had undergone months of pain from tissue constriction and laceration, with granulation and scarring, and increased work from the drag of the entanglement. The short-term effect of this drug mixture as it is absorbed from an intra muscular injection over a period of approximately $30 \mathrm{~min}$ would be to increasingly lessen pain, forget past stress, and reduce anxiety. Thus it would be likely to resume a more normal gait for the duration of effective blood levels of the drug mixture, which might last for an hour or more. During this time, fluke stroke amplitude and vertical speed indeed increased, leading to a slight reduction in thrust coefficient and an increase in swimming efficiency (Fig. C3B). Further, van der Hoop et al. (2013b) found significantly higher fluke stroke rate and RMS fluke 
stroke energy after injection, but still prior to disentanglement. In a different right whale (Eg 3311), a similar drug mixture led to a significant increase in respiratory frequency an hour after injection, along with increases in swimming speed and a marked reduction of boat evasion (Moore et al., 2010). Together, these data suggest that antianxiety and analgesic drugs likely reduce pain to enable standard locomotion at higher speeds.

\subsubsection{Kinematic responses}

There are a number of trade-offs that must be balanced when altering kinematics. For example, thrust increases, but, beyond a certain threshold, efficiency decreases with flukestroke frequency (Daniel, 1991) and, again, beyond a certain threshold, with the angle of attack (Chopra and Kambe, 1977). Increasing frequency is commonly observed in swimming animals in response to changes in force balance (Skrovan et al., 1999; Williams, 1999; Cornick et al., 2006; Aoki et al., 2011). Adjusting frequency instead of amplitude may be a strategy to reduce body distortion required for large-amplitude movements, that would further increase drag (Fish, 2003); there may also be limitations on the amount of work per stroke (Lovvorn et al., 2004). Changes in amplitude are less common in the literature compared to frequency, but may be especially useful in generating high thrust when especially needed, such as rapid accelerations (Fish et al., 2014) or at the initiation of dives (Fig. 3B).

It was expected that, similar to bowhead whales (Simon et al., 2009), right whales would reduce swimming speeds and adopt continuous swimming gaits (i.e., increase fluke stroke frequency) in higher drag scenarios; fluke stroke duration would therefore reflect the relative strength of opposing forces for the two whales. Both whales are subject to higher drag when swimming at the surface (Hertel, 1969; Webb, 1975b), resulting in shorter fluke strokes and higher fluke stroke rates (Fig. 2, 4). For Eg 3911, it was expected that fluke strokes would be longer on descent, as the animal was likely negatively buoyant, and shorter on ascents. The opposite was expected for Eg 4057, a healthy and likely positively buoyant individual: fluke stroke durations would be shorter on descent compared to ascent. Overall, drag loading should shorten fluke strokes, especially in the direction opposing movement. While the overall expectation of shorter fluke stroke durations with drag loading was observed for Eg 4057 (Fig 2. I-L vs. M-P), the expected pattern of fluke stroke duration with respect to dive phase was not observed. If whales were taking advantage of added buoyancy, the inverted pattern of what is observed in entangled 4057 would be expected: shorter on descent and higher on ascent (Fig. 2I vs. K). Again, it does not appear that these whales were taking advantage of the buoyancy added by the entangling gear. This may be because the buoyancy was not acting at the center of mass, but instead is trailing 15-30 m behind the whales.

The significantly longer fluke strokes in Eg 4057, especially in low drag, correspond to periods of gliding. Both individuals show fewer instances of gliding in high drag, consistent with more propulsion to counter greater drag forces as has been observed in other balaenids 
during high-drag filter feeding (Simon et al., 2012). Alternative gaits may not always be an option (Maresh et al., 2014) and these data suggest that despite considerable drag loading, whales still appear to use stroke and glide swimming when entangled (Eg 4057; Fig 3C, D), albeit less frequently, and are able to exploit some cost-effective swimming techniques.

\subsubsection{Changes in efficiency}

Whales swim by oscillating their tails and body, the rate, amplitude and angle of which can be altered to optimize propulsive efficiency. The relationship between these kinematic parameters and swimming speed is represented by the Strouhal number (St; Eq. 5), where the greatest swimming efficiencies occur in the range $0.2<S t<0.4$ (Triantafyllou et al., 1993; Eloy, 2011). We expected that frequency, amplitude and speed would be adjusted to maintain efficiency or a given $S t$ in low and high drag conditions. Some swimmers are able to compensate and maintain St (Noren et al., 2011; Nudds et al., 2014) whereas others have significantly greater and suboptimal St with drag loading (Lang and Daybell, 1963; Methling et al., 2011). In this study, St of right whale Eg 3911 was significantly lower on descent than ascent, but did not change significantly following removal of entangling fishing gear (Fig. 6A). Propulsive efficiencies were significantly greater on descents vs. ascents, but were maintained between drag conditions. Eg 3911 appears to be able to alter kinematics to compensate for the effects of drag loading to maintain similar, although sub-optimal, propulsive efficiency.

Individual and context-specific compensatory strategies occur on a range of temporal scales. On the short term, humans respond on the order of seconds to constraints in step frequency, stride length, or walking speed (Bertram, 2005) and converge on new energetic optima within minutes to realize seemingly small savings in energetic cost $(<5 \%$; Selinger et al., 2015). Ants and other load-carrying insects show plasticity in their step patterns and leg positions (Zollikofer, 1994) and high heel wearers adjust ankle, knee and hip touchdown angles and flexion systematically with heel height to maintain heart rate and oxygen consumption to a height threshold (Ebbeling et al., 1994). Prior exposure to specific limitations, e.g. experienced vs. inexperienced high heel wearers (Opila-Correia, 1990) or previous study subjects (Selinger et al., 2015) enhances the speed and plasticity of individual responses. Whales and other marine mammals make small gait changes in response to repetitive and transient actions, such as reducing thrust production in the buoyancy-aided direction on dives (Nowacek et al., 2001; Aoki et al., 2011; Miller et al., 2012b; Nousek-McGregor et al., 2013; Adachi et al., 2014). These case studies show yet another condition in which animals perceive changes in movement constraints over the course of seconds to minutes, here resulting from disentanglement response, and alter their movement accordingly (Duysens et al., 2000).

Compensation can also occur over the long term as whales are entangled for months 
to years. Certain postural diseases (e.g. flatback syndrome; Sarwahi et al., 2002) lead to specific gait modifications to reduce the cost of transport but can cause abnormal joint loading and eventual degeneration. Similarly, as amputees alter their biomechanics while establishing a prosthetic gait, loading imbalances lead to osteoarthritis of the intact limb and osteopenia due to insufficient loading of the residual limb (Gailey et al., 2008). Sustained compensation may be a mechanism for musculoskeletal deformations observed in entangled animals.

Trauma and consistent compensation for the disproportionate forces and moments associated with chronic entanglement is assumed to have deformed the developing vertebrae in a juvenile right whale, CALO0901, to the point of severe scoliosis that led to its debilitation and live stranding (Moore et al., 2013). Newly remodeled bony structures along the observed spinal deformation suggests that healing had occurred under different local conditions in mechanical strain (Lagier, 1977). Spinal scoliosis has also been observed in another free-swimming right whale with entanglement injuries (Eg 2110 in 2008; Henry et al., 2012) and in a chronically entangled shortfin mako shark (Isurus oxyrinchus; Wegner and Cartamil, 2012). The development and permanence of these musculoskeletal deformations associated with entanglement drag forces, and their impacts on swimming behaviors and efficiency, are entirely unknown.

\subsubsection{Efficiency considerations}

We present the first estimates of ideal and propulsive efficiencies for right whales. Being a relatively rotund cetacean with a large aspect-ratio tail, the right whale has evolved for slow cruising (Woodward et al., 2006b). Average propulsive efficiencies for a right whale following disentanglement were $0.25( \pm 0.09)$ and $0.26( \pm 0.12)$ on descent and ascent, respectively; maximum propulsive efficiencies were 0.50 and 0.51 . These estimates are considerably lower than values for other cetaceans. Fish (1998) estimated maximum propulsive efficiencies of 0.75-0.98 for various toothed whale species, which swim at much higher speeds (6.01-7.91 $\mathrm{m} / \mathrm{s}$ ) and therefore face greater selective pressures to develop locomotor efficiency to counter especially high drag. Bose and Lien (1989) estimated propulsive efficiencies of 0.84-0.87 for a fin whale, a continuous swimming species that cruises at $4-12 \mathrm{~m} / \mathrm{s}$. While propulsive efficiency can be expected to be greater than 0.7 at routine swimming speeds, it reaches minima at speeds $<0.5 \mathrm{~L} / \mathrm{s}$ (Webb, 1975b; Fish, 1993a, 1998, 1999). The right whale is a much slower swimmer than the odontocetes and cruising balaenopterids that have largely been the focus of marine mammal swimming efficiency studies, with routine speeds of 0.36 $1.6 \mathrm{~m} / \mathrm{s}$ (Hain et al., 2013), or 0.03-0.16 L/s for Eg 3911's dimensions. Both whales in this study show extremely slow vertical speeds of 0.14-0.68 m/s in low drag $(0.01-0.07 \mathrm{~L} / \mathrm{s}$; Table $3)$. Other slow-moving marine mammals such as manatees and belugas have low propulsive efficiencies of 0.67-0.81 and 0.82-0.84, respectively (Fish, 1998; Kojeszewski and Fish, 2007). 
It is therefore not surprising that a right whale may have lower propulsive efficiency due to its body dimensions, lifestyle and swimming characteristics (Woodward et al., 2006b), even in the non-entangled case.

Anderson et al. (1998) showed that the conditions to achieve high efficiency of a flapping foil are: (a) an amplitude of heave motion comparable to chord length, (b) an angle of attack at about 20 degrees and (c) a Strouhal number between 0.25-0.35. The average chord length for right whales (distance from the caudal peduncle to the fluke notch) is $0.083 \times \mathrm{L}$ (Woodward et al., 2006b), so $0.83 \mathrm{~m}$ for Eg 3911 - smaller than the estimated fluke stroke amplitudes of $2.0 \mathrm{~m}$ and $1.6 \mathrm{~m}$ in low and high drag, respectively. Estimated angles of attack generally fell within the 10-15 degree range, especially in the low-drag condition (Fig. C2), lower than the 10-30 degrees observed in highly-efficient bottlenose dolphins (Fish, 1993a). $S t$ was mostly $>0.5$. The dimensions and kinematics of right whales therefore approach but do not reach the conditions optimal for propulsion, and additional constraints reduce realized propulsive efficiency.

Overall swimming efficiency, often termed aerobic efficiency, $\eta_{a}$, is a combination of propulsive (also propeller) efficiency, $\eta_{p}$, and muscular (also metabolic) efficiency, $\eta_{m}$ (Webb, 1975a). Muscular efficiency is often assumed to be 0.25 for mammals (Kleiber, 1961). To consider losses from both propulsion and from muscular energy conversion, overall swimming efficiency must be multiplicative, i.e., $\eta_{a}=\eta_{p} \times \eta_{m}$. Some previous studies (Goldbogen et al., 2011; Potvin et al., 2012) sum the total losses of 0.75 and 0.10 to obtain to obtain $\eta_{a}=$ 0.15 , instead of multiplying $(0.25 \times 0.90=0.225)$, though 0.15 represents a conservative estimate.

Combining the average propulsive efficiencies $\left(\eta_{p}\right)$ for this right whale with losses due to muscle activity $\left(\eta_{m}=0.25\right)$ leads to overall efficiency factors $\left(\eta_{a}\right)$ of 0.05 and 0.04 and maximum efficiencies of 0.13 and 0.13 for descent and ascent respectively (Table 5), supporting the conservative 0.15 efficiency factor used in previous studies (Goldbogen et al., 2011; Potvin et al., 2012; van der Hoop et al., 2013b). Ideal efficiency was significantly lower when entangled; although it does not represent the realized case, it illustrates a simpler estimate (based on $C_{T}$ alone) and that the optimal performance of the system is affected by these different entanglement conditions. However, we did not detect a significant increase in observed propulsive efficiency following disentanglement (Table 5). The observed changes in kinematics may have served to maintain swimming efficiency across the two conditions. It is critical to consider that these efficiency estimates reflect those of a slow-moving, chronically entangled, emaciated and therefore negatively buoyant right whale that was within 18 days of death.

The most efficient methods of load carrying place the load at the center of mass, as is observed in animals (e.g. crabs and ants, Duysens et al. (2000); aboriginal tribespeople, Heglund et al. (1995)). The distribution of these loads can be more important than mass, 
affecting metabolic rates in humans (Laursen et al., 2000) and kinematics of load-carrying ants (Zollikofer, 1994). There is considerable variation in drag forces over the 30-second measurement periods of drag forces on entangling fishing gear in van der Hoop et al. (2015). Due to changing flow conditions, whales towing gear will experience variable forces and moments, not necessarily at the center of mass. The mouth is the most common point of attachment of entangling gear for right whales (77.4\%) and $51.6 \%$ of entanglements involve only the mouth (Johnson et al., 2005). Peduncle entanglements, well behind the center of mass, are less common in right whales, though gear often attaches at many points along the body (Johnson et al., 2005). The dynamics of variation in forces and moments at attachment locations other than the center of mass likely have different effects on whale propulsion, efficiency, and gait, though they are unknown at this time.

\subsubsection{Conclusions}

Chronic entanglement significantly affects locomotion, the specifics of which depend on the individual. Though the sample size is small, opportunities to tag entangled whales are few and far between, emphasizing the importance of continuing efforts to tag entangled whales with short-duration tags. Even with these limited data, it is apparent that drag significantly affects swimming behaviors, and their variability, which contribute to propulsive efficiency. 


\section{Chapter 5}

\section{ENTANGLEMENT IS A COSTLY LIFE HISTORY STAGE IN LARGE WHALES}

This chapter has been submitted for publication: van der Hoop, J.M., Corkeron, P., and Moore, M.J. Entanglement is a costly life history stage in large whales Ecology and Evolution ECE-2016-07-00675

JvdH, PC and MJM developed concepts; JvdH processed and analyzed the data; PC and MJM guided the analysis; JvdH wrote the manuscript; PC and MJM contributed to the manuscript.

The supplemental materials for this chapter can be found in Appendix D. 


\subsection{Abstract}

1. Individuals store energy to balance deficits in natural cycles; however, unnatural events can also lead to unbalanced energy budgets. Entanglement in fishing gear is one example of an unnatural but relatively common circumstance that imposes energetic demands of a similar order of magnitude and duration of life history events such as migration and pregnancy in large whales.

2. We present two complementary bioenergetic approaches to estimate the energy associated with entanglement in North Atlantic right whales, and compare these estimates to the natural energetic life history of each individual whale.

3. Differences in measured blubber thicknesses and estimated blubber volumes between normal and entangled, emaciated whales indicate between $7.4 \times 10^{10} \mathrm{~J}$ and $1.2 \times 10^{11}$ $\mathrm{J}$ of energy are consumed during the course-to-death of a lethal entanglement. Increased thrust power requirements to overcome drag forces suggest that when entangled, whales require $3.95 \times 10^{9} \mathrm{~J}$ to $4.08 \times 10^{10} \mathrm{~J}$ more energy to swim. Individuals who died from their entanglements performed significantly more work (energy expenditure $\times$ time) than those that survived; entanglement duration is therefore critical in determining whales' survival.

4. Significant sublethal energetic impacts also occur, especially in reproductive females. Drag from fishing gear contributes up to $8 \%$ of the four-year female reproductive energy budget, delaying time of energetic equilibrium (to restore energy lost by a particular entanglement) for reproduction by months to years.

5. In certain populations, chronic entanglement in fishing gear can be viewed as a costly unnatural life history stage, rather than a rare or short-term incident.

\subsection{Introduction}

Periods of fasting and feeding are natural for wild animals, with consequent adaptation to withstand food limitations imposed by their environment or their life histories. Energy is stored for times of deficit, and the energy budget is balanced over the long term. Migratory baleen whales go through periods of fattening and thinning, storing energy on the foraging grounds in recovery from and preparation to their depletion during migration, breeding, and lactation where individuals may be fasting and energetic costs can be high (Miller et al., 2012b; Christiansen et al., 2013).

Major changes in nutritive condition and reproduction can occur in response to good or bad prey years or environmental conditions, being natural, unforeseen circumstances 
(Williams et al., 2013; Rolland et al., 2016). Human-induced factors may also contribute. Entanglement in fishing gear is now almost ubiquitous in some large whale populations. Scarring of individually-identified humpback whales (Megaptera novaeangliae) indicates that more than a quarter of the North Pacific (29-60\%; NFWF, 2007), over half the Alaskan (52\%; Neilson et al., 2009), and over three-quarters of the North Atlantic (78\%; Robbins, 2012) populations have been entangled at least once. Further, 8-25\% of the individuals in these populations acquire new entanglement scars every year. Many individuals in some populations have been observed carrying gear (11\% of North Atlantic right whales; Eubalaena glacialis; Robbins et al., 2015), and do so for months to years (van der Hoop et al., 2015). Entanglement in fishing gear is the leading cause of death for large whales in the western North Atlantic (van der Hoop et al., 2013a) and contributes mortality to marine mammal species worldwide (Fowler, 1987; Clapham and Mead, 1999; Read et al., 2006), but the issue also affects individual whales that survive the incident.

Entangled whales are subject to additional drag forces, likely increasing the cost of locomotion and in some cases leading to negative energy balance (Feldkamp et al., 1988; van der Hoop et al., 2013b). The drag imposed by gear varies considerably based on its dimensions and configuration: based on 15 sets of gear measured by van der Hoop et al. (2015), entanglement increases drag forces by 1.5 fold on average, and up to 3.1 fold in the case of a weighted lobster trap. The onset of entanglement is unpredictable, and the duration hours to years (e.g., van der Hoop et al., 2015).

Blubber and lipid stores can be considered an energy currency with which to evaluate the energetic consequences and sublethal impacts of various stressors in cetaceans and many other mammal species (e.g., Miller et al., 2011; Williams et al., 2013). Excess energy is deposited in lipid stores which become the primary energy source during fasting (Lockyer, 1986; Worthy and Lavigne, 1987), illness (Koopman et al., 2002; Dunkin et al., 2010), or increased nutritional demand (e.g. pregnancy; Miller et al., 2011). Lipid catabolism can be directly observed and measured as significant reductions in body girths and blubber thicknesses; differences in blubber thicknesses, volumes, or masses between individuals with time or under different conditions represent the amount of energy derived from stores, and can be attributed to changes in environmental conditions or particular life history events (Lockyer, 1981b, 1986; Worthy and Lavigne, 1987). Separately, changes in the force balance on an animal can elicit behavioral or postural responses (Feldkamp et al., 1988; van der Hoop et al., 2013b, 2014a). Increased drag requires increased thrust or propulsive power and therefore energy expenditure (Webb, 1975b; Feldkamp, 1985). As a result, chronically entangled marine animals are often emaciated (Cassoff et al., 2011; Barratclough et al., 2014). Estimates of additional drag forces and thrust power over time can indicate how much additional work is required by entangled whales compared to their non-entangled conspecifics. However, these estimates evaluate only the additional cost for locomotion, not 
the associated costs of stress, injury, or healing.

How much additional energy do entangled whales expend, and what are the relative costs of entanglement compared to other energetically costly life history events? We present two separate but complementary bioenergetic approaches to estimate the amount of additional work and energy associated with entanglement in fishing gear in a large whale species: (1) changes in blubber thicknesses and volumes between normal and entangled, emaciated whales; and (2) increased thrust power requirements to overcome measured drag forces.

The North Atlantic right whale (hereafter right whale) is a species with exceptionally high entanglement rates (83\% of the population; Knowlton et al., 2012a), and where population health and reproductive rates are variable and in recent decline (Fujiwara and Caswell, 2001; Pettis et al., 2004; Robbins et al., 2015; Rolland et al., 2016). Long-term individual sightings, life history and health data are available, as is information on entanglements, including the gear involved. We use observations, measurements, theory, and available literature to outline the energetic life history of right whales in particular, to contextualize the demands, time course and extent of entanglement in fishing gear.

\subsection{Methods}

\subsubsection{Changes in blubber thickness and volume}

We obtained dorsal axillary blubber thicknesses and body lengths of dead right whales measured at necropsy from the North Atlantic Right Whale Consortium (NARWC) Necropsy database (NARWC, 2015). We assumed that individuals that were not described as emaciated and whose cause of death was not related to entanglement were in normal body condition. We have provided a list of symbols and abbreviations for reference (Table 1). We estimated total body volumes $\left(V ; \mathrm{m}^{3}\right)$ for each individual by approximating whales as rotationally symmetric ellipsoids (Figure 1 ), with major axis $a=$ half the body length $(l$; $\mathrm{cm}$ ), and minor axes $b_{n}=c_{n}=$ half the body width $(w ; \mathrm{cm})$ as in Klansjcek et al. (2007). We calculated body widths for whales in normal body condition as in Fortune and Trites (2012) and reduced body widths of entangled animals $\left(b_{e}=c_{e}\right)$ by the difference in measured blubber thicknesses between normal $\left(t_{n}\right)$ and entangled $\left(t_{e}\right)$ whales:

$$
\begin{gathered}
b_{e}=b_{n}-\left(t_{n}-t_{e}\right) \\
c_{e}=c_{n}-\left(t_{n}-t_{e}\right),
\end{gathered}
$$

We calculated total body volumes of each whale based on normal body proportions $\left(V_{n}\right)$ 
and with thinner blubber layers $\left(V_{e}\right.$, using $b_{e}$ and $\left.c_{e}\right)$, as:

$$
V=\frac{4}{3} \pi a b c
$$

We assumed the difference between the normal and entangled body volumes of each individual $\left(V_{n}-V_{e}\right)$ represents the volume of blubber catabolized between the two conditions (Figure 1).

Table 5-1: List of symbols and abbreviations.

\begin{tabular}{|l|l|l|}
\hline Symbol & Definition & Unit \\
\hline$a$ & Ellipsoid major axis & $\mathrm{m}$ \\
\hline$b$ & Ellipsoid minor axis & $\mathrm{m}$ \\
\hline$B a$ & Basal cost & \\
\hline$B r$ & Breeding cost & \\
\hline$c$ & Ellipsoid minor axis & $\mathrm{m}$ \\
\hline$d$ & Entanglement duration & $\mathrm{sec}$ \\
\hline$D$ & Drag force & $\mathrm{N}$ \\
\hline$F r$ & Foraging cost & \\
\hline$F d$ & Fat deposition cost & \\
\hline$\eta$ & Overall efficiency & \\
\hline$\eta_{m}$ & Muscular efficiency & \\
\hline$\eta_{p}$ & Propulsive efficiency & \\
\hline$l$ & Body length & $\mathrm{m}$ \\
\hline$L$ & Lactation cost & \\
\hline$M$ & Migration cost & \\
\hline$P$ & Pregnancy cost & \\
\hline$P_{T}$ & Thrust power & $\mathrm{W}$ \\
\hline$\rho$ & Density & $\mathrm{kg} / \mathrm{m}^{3}$ \\
\hline$T$ & Measured blubber thickness & $\mathrm{cm}$ \\
\hline$U$ & Speed & $\mathrm{m} / \mathrm{s}$ \\
\hline$V$ & Total body volume & $\mathrm{m}^{3}$ \\
\hline$W_{a}$ & Additional work & $\mathrm{J}$ \\
\hline
\end{tabular}

We converted blubber volumes to energy $(\mathrm{J})$ based on published values of lipid content (61.8\%; Montie et al., 2010), blubber density (900 kg/m³; Parry, 1949), caloric content of lipid (9450 kcal/kg) and energy (4184 J/kcal; Lockyer, 1978; Schmidt-Nielsen, 1997). Because lipid content measurements can vary with sampling method (Woodley et al., 1991; Krahn et al., 2004), blubber depth, and body location (Koopman et al., 2002; Struntz et al., 2004), we established high and low estimates of $61.8 \pm 25 \%$ and assumed constant lipid content and density. 
Because adults for which blubber thicknesses were available had significantly greater body lengths $(1418 \pm 387 \mathrm{~cm})$ than juveniles $\left(1097 \pm 129 \mathrm{~cm}\right.$; one-way ANOVA $F_{1,15}=32.788, p<$ 0.0001), they also had significantly larger estimated body widths. We therefore separated individuals into two life stages for statistical analysis. We used two-way ANOVA to determine whether blubber thicknesses were significantly different between life stages (juvenile, adult) or condition (normal, entangled).

\subsubsection{Increased power requirements}

To overcome the increased drag forces associated with fishing gear, entangled whales increase power for propulsion (thrust power, $P_{T}$; Watts, W). Over time, this requires additional work $\left(W_{a} ; \mathrm{J}\right)$ and therefore energy consumed $(\mathrm{J})$ by the animal. We calculated thrust power from measured drag forces $(D ; \mathrm{N})$ for 15 right whales whose entangling gear was measured with a tensiometer (van der Hoop et al., 2015) and estimates of swimming efficiency $(\eta)$ specific to the entangled (subscript $e ; P_{T, e}, D_{e}, \eta_{e}$ ) and non-entangled (subscript $n ; P_{T, n}, D_{n}, \eta_{n}$ ) conditions:

$$
\begin{gathered}
P_{T, e}=\frac{D_{e} U_{e}}{\eta_{e}} \\
P_{T, n}=\frac{D_{n} U_{n}}{\eta_{n}}
\end{gathered}
$$

Swimming efficiency is a combination of propulsive efficiency $\left(\eta_{p}\right)$ and muscular efficiency $\left(\eta_{m}=0.25\right)$. We based values of swimming efficiency on the maximum propulsive efficiencies calculated for right whale Eg 3911 averaged over dive descents and ascents when entangled and following disentanglement (Chapter 4):

$$
\begin{gathered}
\eta_{e}=\eta_{p} \times \eta_{m}=0.50 \times 0.25=0.13 \\
\eta_{n}=\eta_{p} \times \eta_{m}=0.51 \times 0.25=0.13 .
\end{gathered}
$$

We applied maximum propulsive efficiencies as this whale's health and energy remained compromised following disentanglement, which provide a conservative estimate. We assumed a constant-velocity scenario $\left(U_{e}=U_{n}\right)$, i.e., that animals do not slow down once entangled.

We calculated the additional thrust power $\left(P_{T, a} ; \mathrm{W}\right)$ as the difference in propulsive power between the entangled and non-entangled conditions, and the additional work $\left(W_{a}\right.$; $\mathrm{J})$ required for propulsion by an entangled whale as this additional power sustained over the minimum and maximum durations (seconds; $d_{\min }$ and $d_{\max }$, respectively) of each entangle- 
ment:

$$
\begin{gathered}
W_{a, \min }=d_{\min }\left(P_{T, e}-P_{T, n}\right), \\
W_{a, \max }=d_{\max }\left(P_{T, e}-P_{T, n}\right),
\end{gathered}
$$

We built entanglement timelines and associated increases in drag, power, and work from sightings records and disentanglement histories for all whales (Supplemental Figure D1). We calculated maximum entanglement durations $\left(d_{\max }\right)$ from the last gear-free sighting before entanglement and either the first gear-free sighting following disentanglement, confirmed death (carcass detection and identification), or presumed death (once an individual has not been sighted in 6 years; Knowlton et al., 1994). We calculated minimum entanglement durations $\left(d_{\min }\right)$ from the first entangled sighting and either the date (i) of disentanglement (including partial disentanglement), (ii) last seen entangled, or (iii) that the telemetry buoy ceased transmissions. At the onset of entanglement, we estimated increased power from entangled drag measurements $\left(D_{e}\right)$ from (van der Hoop et al., 2015). We incorporated information from each whale's history to reflect changes in drag from disentanglement response. We included drag from adding the satellite telemetry buoy based on measured values from van der Hoop et al. (2015). We reduced the total drag of entangled animals $\left(D_{e}\right)$ in the event of disentanglement attempts that were successful in reducing the length of trailing line or removing floats; we calculated the change in expected mean drag from the linear relationship in van der Hoop et al. (2015) with dimensions of the original and altered entangling gear. We ignored drag added for single-day disentanglement events (e.g., to slow the animal through a process known as kegging) as (i) these details were not consistently recorded, (ii) the drag of these buoys used were never measured, and (iii) these events occur over short $(<12 \mathrm{hr})$ durations.

We sought to determine whether the fate of each whale was related to the additional power output $\left(P_{T, a}\right)$, or minimum or maximum additional work $\left(W_{a, \min }, W_{a, \max }\right)$ associated with its entanglement. To do so, we compared these variables for individuals who died vs. survived their entanglements with paired $t$-tests. We defined a critical level of minimum additional work associated with entanglement-related mortality as the 0.75 quantile of the minimum additional work performed by whales that did not survive (i.e., of $W_{a, \min }$ ( fate $==$ dead)).

\subsubsection{Life history context}

To put the energetic demands of entanglement in context, we compared the estimated cost per day and duration of other right whale life history events from the literature (Figure 2). For example, van der Hoop et al. (2013b) estimate a one-way, 22-day migration costs $7.3 \times 10^{9}$ 
$\mathrm{J}$, or $3.3 \times 10^{8} \mathrm{~J} /$ day. Foraging requires energy for diving and searching, and to counter the increased drag associated with filter feeding - approximately $5 \times 10^{8} \mathrm{~J} /$ day (Simon et al., 2009; McGregor, 2010). While Klansjcek et al. (2007) estimated combined two-year reproductive costs of pregnancy and lactation at $7.9 \times 10^{8} \mathrm{~J} /$ day, Fortune et al. (2013) estimated the costs of pregnancy and lactation separately: the difference between daily energetic requirements of resting females $\left(1.9 \times 10^{9} \mathrm{~J} /\right.$ day $)$ and of pregnant $\left(2.1 \times 10^{9} \mathrm{~J} /\right.$ day $)$ and lactating females $\left(4.1 \times 10^{9} \mathrm{~J} /\right.$ day $)$, suggests daily costs of pregnancy and lactation are $1.8 \times 10^{8} \mathrm{~J} /$ day and $2.2 \times 10^{9} \mathrm{~J} /$ day, respectively. We projected daily energy costs of life history events over their duration to provide a comparison of daily additional energetic costs to entangled whales over the minimum and maximum durations of their entanglements.

We also represented seasonal variability in these costs and compared their relative contributions to individual energy budgets for non-entangled adult male and female right whales. Above basal $\left(B_{a}\right)$ costs, annual migration $(M)$, foraging $(F r)$, and breeding $(B r)$ contribute to varying energetic demands including periods of nutritional excess (hyperphagia) and deficit (hypophagia; McNamara and Houston, 2008). Pregnancy $(P)$ and lactation $(L)$ add especially costly demands in females. We represented the annual cost of living for a right whale following e.g., Gessaman (1973) and West (1960), by estimating monthly relative costs of life history events through the year separately for males (Eq. 7) and females (Eq. 8):

$$
\begin{gathered}
M E B=\sum_{m=1}^{1} 2 B a_{m}+M_{m}+F r_{m}+F d_{m}+B r_{m} . \\
\frac{M E B}{12}=1 \\
F E B=\sum_{m=1}^{4} 8 B a_{m}+M_{m}+F r_{m}+F d_{m}+B r_{m}+P_{m}+L_{m} . \\
\frac{F E B}{48}=1
\end{gathered}
$$

For a male, energy equilibrium is reached after a full annual cycle. For females, equilibrium is reached after 4 years: pregnancy, lactation and a 2-year resting period, reflecting the mean calving interval in the right whale population in the recent past (Knowlton et al., 1994, 2012a). We then calculated relative additional costs due to entanglement, and added these costs to the budget.

We assumed basal existence energy costs $\left(B_{a}\right.$; for thermoregulation, digestion and excretion) as $50 \%$ of the budget, remaining fairly continuous throughout the year; there is little evidence for seasonal resting metabolic rate fluctuations in cetaceans (Rechsteiner, 2013). We constrained the schematic to have a maximum sustained energy of $2.4 \times$ and $4.5 \times$ basal 
costs for males and females, respectively, on the conservative end of the maximum sustained energy of $4-7 \times$ basal metabolic rates across species (Weiner, 1992; Speakman and Krol, 2010).

We estimated migration costs $(M)$ from the relative movement of right whales between habitats (Vanderlaan and Taggart, 2010; Schick et al., 2013; Brillant et al., 2015), where movement is greatest in January-February and April-May. Males and females have similar overall movement patterns, though destinations and transition probabilities between habitats can differ (Schick et al., 2013). To reflect residence times on the calving grounds, we reduced movement costs in February-March (Fortune et al., 2013; Schick et al., 2013) and in feeding areas in June-August (Vanderlaan and Taggart, 2010). Although monthly residence probabilities can be high for some habitats (e.g. $66 \%$ in the Bay of Fundy; Vanderlaan 2010), individual whales can be highly transitory between north-temperate habitats on shorter timescales (Mate et al., 1997; Brillant et al., 2015).

Right whales forage seasonally on Calanoid copepods in surface waters (e.g. in Cape Cod Bay; Mayo and Marx, 1990; Parks et al., 2012) and at depth (e.g. in the Bay of Fundy, Roseway Basin); foraging costs (Fr) increase to a maximum in July-September, when foraging rates are greatest (Baumgartner and Mate, 2003).

On the feeding grounds, large amounts of energy are stored in lipid reserves (Miller et al., 2011; Christiansen et al., 2013). Fat deposition is efficient, roughly 5-15\% per kilocalorie of metabolizable energy; protein deposition costs can be much higher $(2.25-2.38 \mathrm{~kJ} / \mathrm{kJ}$ deposited; Pullar and Webster, 1977; van Es, 1977; Roberts and Young, 1988). We assume fat deposition $(F d)$ to be $2 \%$ of foraging costs. Costs of breeding $(B r)$ are incurred for mate searching and social displays (Kraus et al., 2001).

We included breeding costs only prior to pregnancy for females. Pregnancy costs $1.09 \times$ as much as the non-reproductive female (Fortune et al., 2013); fetal development is minimally costly and abdominal distention increases body drag only 3-4\% (McGregor, 2010). Lactation is the most energetically expensive life history event, costing $2.17 \times$ the non-reproductive female budget (Fortune et al., 2013). We assume weaning lasts 12 months (Hamilton and Cooper, 2010). Pregnant and lactating females have reduced fat deposition $(F d=1 \%)$ and slightly reduced foraging effort as has been suggested for humpback (Szabo and Duffus, 2008) and southern right (Taber and Thomas, 1982; Thomas and Taber, 1984) whales.

We converted the additional energy from entanglement over the course of one day $\left(W_{a, d a y}\right.$, e.g., Eq 5$)$ for each case to relative additional energetic costs $\left(W_{a, r e l}\right)$ by comparing them to the daily (subscript day) and relative (subscript rel) costs of foraging $(F)$ and migration $(M)$ :

$$
W_{a, r e l}=\frac{\left.\left(W_{a, d a y} / F_{d a y}\right) \times F_{r e l}+\left(W_{a, d a y} / M_{d a y}\right) \times M_{r e l}\right)}{2} .
$$

We added these relative, monthly costs to the budget over the minimum and maxi- 
mum durations of each entanglement case to obtain an entangled energy budget $\left(M E B_{E}=\right.$ $\left.M E B+W_{a, r e l} ; F E B_{E}=F E B+W_{a, r e l}\right)$. We calculated the contribution of pregnancy, lactation and maintenance to the 4-year breeding cycle budget, to which we compared entanglement costs over minimum and maximum durations; we then calculated the additional energetic demand of entanglement on top of the total 4-year budget. Assuming finite entanglements, we calculated the time for return to energetic equilibrium (for entanglement costs to be recouped and for recovery in preparation for the next pregnancy) as the surplus energy required for entanglement divided by the monthly energy available in the absence of reproduction.

\subsection{Results}

\subsubsection{Blubber thickness}

Entangled juveniles $(n=3,8.3 \pm 2.5 \mathrm{~cm})$ and adults $(n=3,11.3 \pm 3.3 \mathrm{~cm})$ had significantly thinner dorsal blubber layers $\left(t_{e}\right)$ at necropsy compared to non-entangled individuals in the same life stages $\left(t_{n}\right.$; juveniles $n=7,13.8 \pm 2.7 \mathrm{~cm}$; adults $n=4,13.4 \pm 1.8 \mathrm{~cm}$; Two-way ANOVA, $F_{1,14}=7.16, p=0.018$; Figure 3A; Table 2). Whales that died from entanglement had thinner blubber layers, even considering seasonality in blubber stores (Figure 3B).

Based on average body lengths of adults $(1418 \pm 87 \mathrm{~cm})$ and juveniles $(1097 \pm 129 \mathrm{~cm})$, the difference in blubber volumes between the entangled and non-entangled conditions was 3.4 and $5.4 \mathrm{~m}^{3}$ in these two age classes, respectively. These volume losses suggest $7.4(4.4-$ $10.3) \times 10^{10} \mathrm{~J}$ and $1.2(0.7-1.7) \times 10^{11} \mathrm{~J}$ of energy were consumed during the course-to-death of lethal entanglements in adults and juveniles, respectively.

\subsubsection{Increased power requirements}

Based on the histories of 15 right whale entanglements for which gear drag was measured, entanglements increased propulsive power requirements $1.48 \pm 0.52$ fold (range $1.04-4.45$ fold; Figure 4A) if swimming speed was maintained; these entanglements were sustained $92 \pm 101$ (range 1 - 332) to $810 \pm 1044$ (range 23 - 3328) days (Table 3). Mean( \pm SD) daily energetic costs were $2.13( \pm 0.92) \times 10^{8} \mathrm{~J} /$ day, and ranged $1.03 \times 10^{8}-3.96 \times 10^{8} \mathrm{~J} /$ day $($ Figure $2 \mathrm{~A})$. Over the duration of their entanglements, individuals required $3.95( \pm 4.84) \times 10^{9}$ to $4.08( \pm 7.19) \times 10^{10} \mathrm{~J}$ more energy than non-entangled whales to complete the additional propulsive work to overcome entanglement drag forces (Figure 4B). Individuals who died from entanglements had significantly higher minimum and maximum additional energy expenditures over their entanglement durations compared to those that survived (Table 4). The 0.75 quantile of minimum additional work performed by whales that did not survive their entanglements was $8.57 \times 10^{9} \mathrm{~J}$. There was no detectable difference in the increases 
Table 5-2: New England Aquarium Catalog and Field identification number, age, sex, length $(\mathrm{cm})$, weight $(\mathrm{kg})$, date of necropsy and measured dorsal blubber thickness of juvenile and adult North Atlantic right whales that died as a result of entanglement or due to other causes.

\begin{tabular}{|c|c|c|c|c|c|c|c|}
\hline & & $\begin{array}{l}\text { Catalog } \\
\text { Number }\end{array}$ & $\begin{array}{c}\text { Field } \\
\text { Number }\end{array}$ & Age & Sex & $\begin{array}{c}\text { Length } \\
(\mathrm{cm})\end{array}$ & $\begin{array}{c}\text { Date of } \\
\text { Necropsy }\end{array}$ \\
\hline \multirow{12}{*}{ 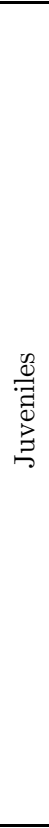 } & \multirow{9}{*}{ 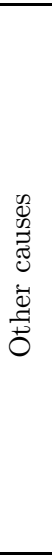 } & & $\begin{array}{c}\text { NY-2680- } \\
2001 \\
\end{array}$ & & $\mathrm{~F}$ & 910 & 19-Jun-01 \\
\hline & & 3710 & $\begin{array}{c}\text { CALO } \\
0901 \\
\end{array}$ & & M & 975 & 26-Jan-09 \\
\hline & & 1504 & \begin{tabular}{|c|} 
MH86-142- \\
Eg \\
\end{tabular} & & $\mathrm{F}$ & 1090 & 7-Aug-86 \\
\hline & & & JGM 415 & \multirow{3}{*}{3} & \multirow{3}{*}{ M } & \multirow{3}{*}{1100} & \multirow{3}{*}{ 5-Mar-79 } \\
\hline & & & 504886 & & & & \\
\hline & & & $\begin{array}{c}\text { MH-76-056- } \\
\text { Eg } \\
\end{array}$ & & & & \\
\hline & & 2450 & & $\min 2$ & $\mathrm{~F}$ & 1259 & 21-Aug-97 \\
\hline & & 3508 & $\begin{array}{c}\text { GA2006025 } \\
\text { Eg }\end{array}$ & 2 & M & 1260 & 30-Dec-06 \\
\hline & & 2250 & & $\min 3$ & $\mathrm{M}$ & 1266 & 20-Oct-95 \\
\hline & \multirow{3}{*}{ 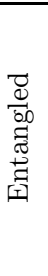 } & 3911 & $\begin{array}{c}\text { EgNEFL11 } \\
03\end{array}$ & 2 & $\mathrm{~F}$ & 1000 & 2-Feb-11 \\
\hline & & 1907 & $\begin{array}{c}\text { RKB-1420 } \\
\text { MH91-762- } \\
\text { Eg } \\
\end{array}$ & 2 & $\mathrm{~F}$ & 1005 & 13-Mar-91 \\
\hline & & 3107 & $\begin{array}{c}\text { MH02-726- } \\
\text { Eg }\end{array}$ & 1 & $\mathrm{~F}$ & 1100 & 13-Oct-02 \\
\hline \multirow{7}{*}{$\frac{\stackrel{n}{\rightleftarrows}}{\frac{3}{4}}$} & \multirow{4}{*}{ 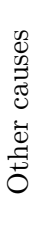 } & 1223 & & $\min 12$ & $\mathrm{~F}$ & 1360 & 13-Sep-92 \\
\hline & & 1014 & \begin{tabular}{|} 
MH99-601- \\
Eg \\
\end{tabular} & $\min 28$ & $\mathrm{~F}$ & 1370 & 21-Apr-99 \\
\hline & & 1623 & RKB-1429 & $\min 12$ & $\mathrm{M}$ & 1415 & 1-Feb-96 \\
\hline & & 1004 & $\begin{array}{c}\text { VMSM } \\
2004-1004\end{array}$ & 30 & & 1600 & $11-F e b-04$ \\
\hline & \multirow{3}{*}{ 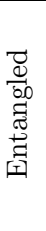 } & 2030 & $\begin{array}{c}\text { CCSN99- } \\
143 \\
\end{array}$ & $\min 10$ & $\mathrm{~F}$ & 1350 & 21-Oct-99 \\
\hline & & 2301 & $\begin{array}{c}\text { VAQS- } \\
2005- \\
1008 \mathrm{Eg}\end{array}$ & 12 & $\mathrm{~F}$ & 1380 & 4-Mar-03 \\
\hline & & 1238 & & $\min 19$ & $\mathrm{M}$ & 1455 & 4-Nov-01 \\
\hline
\end{tabular}

in propulsive power associated with the entanglement configurations of cases that died vs. those that survived their entanglements (Figure 4A; Table 4). 


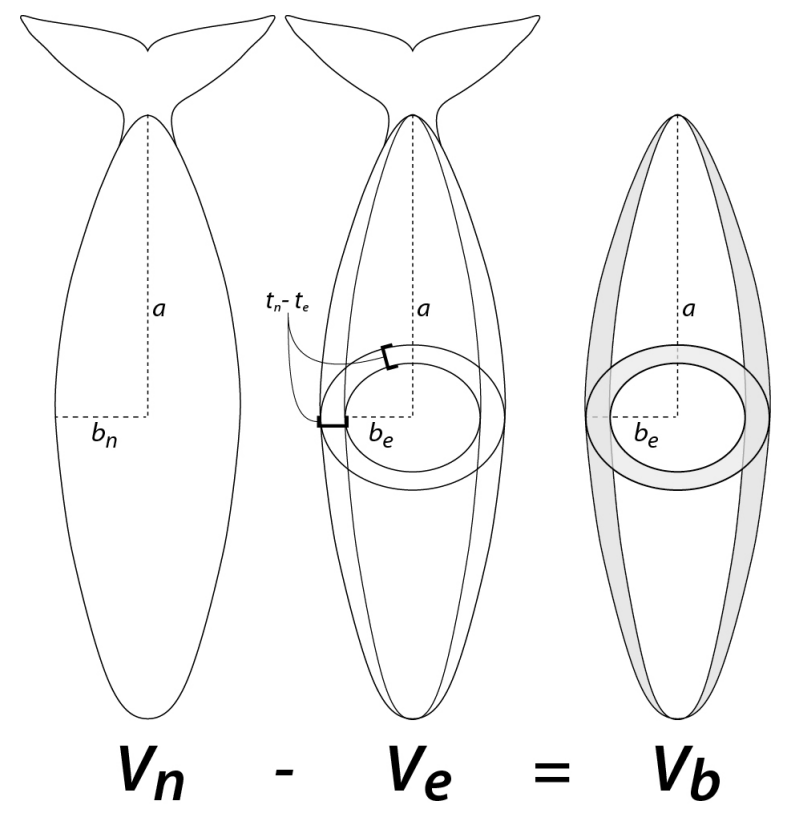

Figure 5-1: Schematic representing blubber volume estimation $\left(V_{b}\right)$ for a North Atlantic right whale of body length $a \times 2$. Total body volume of normal whales $\left(V_{n}\right)$ was estimated from length and body radius $b_{n}=c_{n}$. Body volumes of entangled whales $\left(V_{e}\right)$ were based on length and body radius $b_{e}$, based on the difference in blubber thicknesses between normal $\left(t_{n}\right)$ and entangled $\left(t_{e}\right)$ individuals measured at necropsy. See text for detail. 

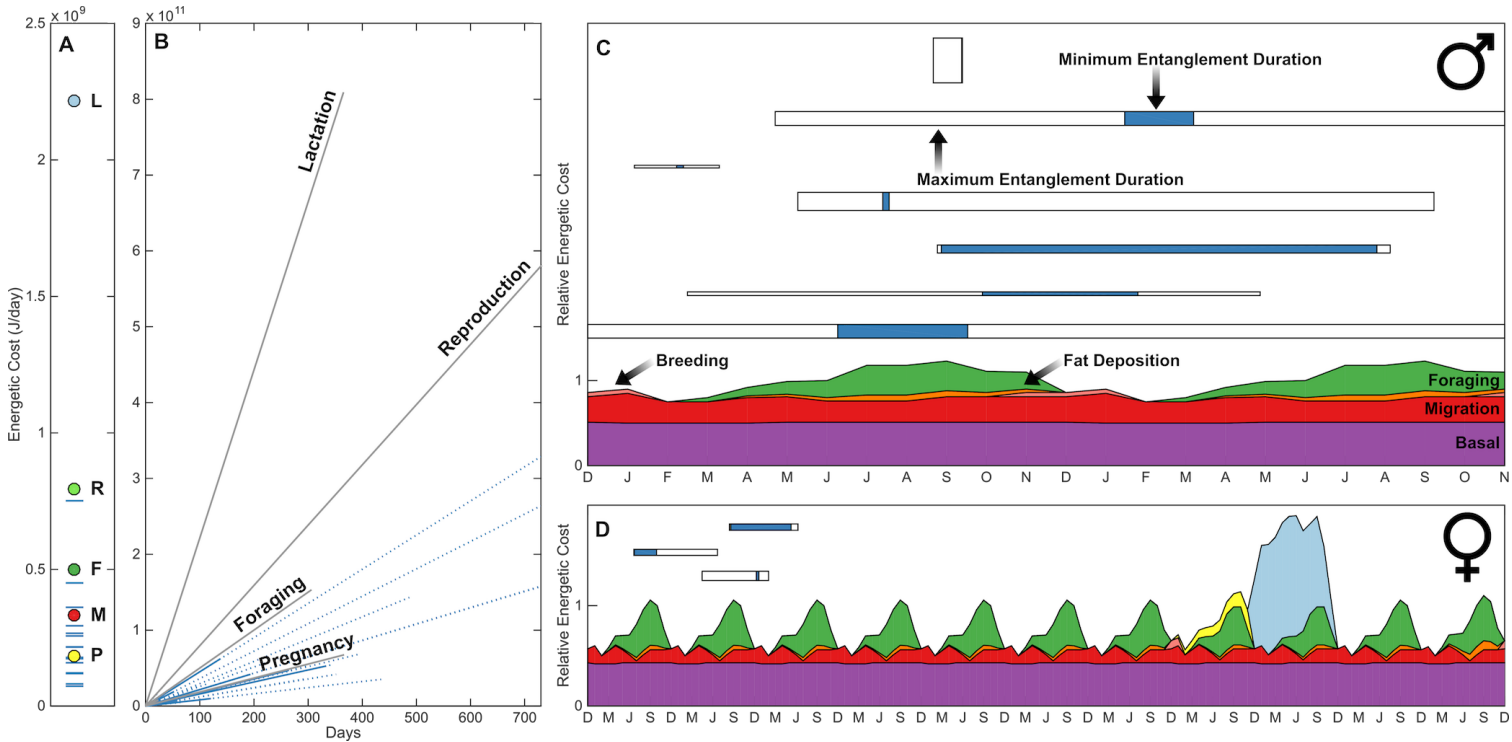

Figure 5-2: Daily energetic costs $(\mathrm{J} /$ day $)$ from drag associated with entanglement in fishing gear (blue lines), compared to costs of other life history events: pregnancy (yellow, P; Fortune et al., 2013), migration (red, M; van der Hoop et al., 2013b), foraging (green, F; McGregor, 2010), reproduction (green, R; pregnancy + lactation; Klansjcek et al., 2007) and lactation (light blue, L; Fortune et al., 2013) (A) and those daily energetic costs projected over the duration of each event (B). For entanglements, solid lines show the minimum entanglement duration and dotted lines the maximum entanglement duration. Relative energetic timelines of males (C) and females (D) show cyclic energy demands in migratory whales. Horizontal bars show the temporal onset and extent of the minimum (blue) and maximum (white) entanglement durations; the height of each bar indicates the cost of each entanglement case relative to other life history events ( $n=7$ males, $n=3$ females). The three female entanglements are shown relative to their known calving histories; five females in the study but not in this figure have unknown calving histories. 

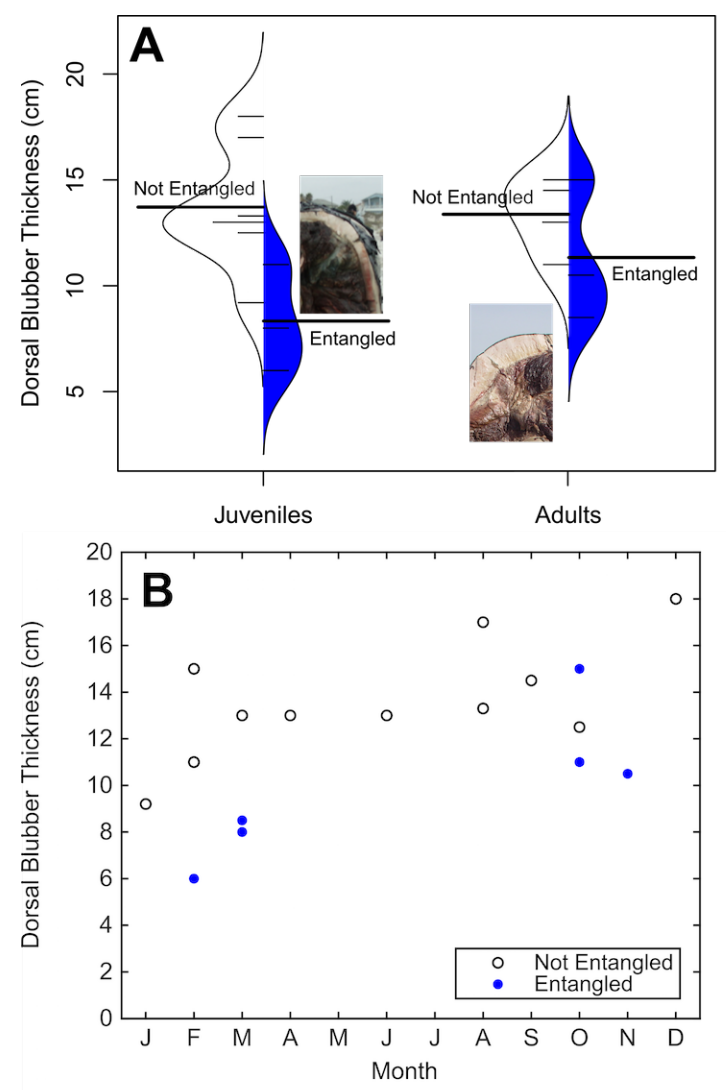

Figure 5-3: Dorsal axillary blubber thickness $(\mathrm{cm})$ measured at necropsy in juvenile and adult North Atlantic right whales whose cause of death was not related to entanglement (white) or due to entanglement (blue). A. Curved vertical lines show the distributions of each sub-group; short horizontal lines are individual samples, thick horizontal lines are population means (Kampstra 2008). Inset photos are equally scaled and show blubber of an entangled juvenile (Eg 3911) and a nonentangled adult (Eg 1004). B. Dorsal blubber thicknesses by month of death. 
Table 5-3: Details of fishing gear entanglements whose drag forces were measured ( $\mathrm{n}=15)$. Sightings data provided the minimum and maximum entanglement periods (days), individual fate $(\mathrm{S}=$ survived; $\mathrm{D}=$ died) and age at entanglement (years) for all whales, from which we estimated length and mass from Moore et al. (2004).

\begin{tabular}{|c|c|c|c|c|c|c|c|c|c|c|c|c|c|}
\hline \multirow{2}{*}{$\begin{array}{l}\text { Catalog } \\
\text { Number }\end{array}$} & \multirow{2}{*}{$\begin{array}{c}\text { Age at } \\
\text { Entanglement }\end{array}$} & \multirow{2}{*}{$\begin{array}{l}\text { Length } \\
(\mathrm{cm})\end{array}$} & \multirow[t]{2}{*}{$\begin{array}{c}\text { Mass } \\
(\mathrm{kg})\end{array}$} & \multicolumn{2}{|c|}{$\begin{array}{l}\text { Entanglement } \\
\text { duration (days) }\end{array}$} & \multirow{2}{*}{$\begin{array}{c}\text { Fold } \\
\text { increase } \\
\text { in Drag }\end{array}$} & \multirow[t]{2}{*}{ Floats } & \multirow{2}{*}{\begin{tabular}{|c|}
$\begin{array}{c}\text { Non- } \\
\text { entangled } \\
\text { Power }\end{array}$ \\
$\left(P_{T, n}, \mathrm{~W}\right)$ \\
\end{tabular}} & \multirow{2}{*}{$\begin{array}{c}\begin{array}{c}\text { Entangled } \\
\text { Power }\end{array} \\
\left(P_{T, e}, \mathrm{~W}\right)\end{array}$} & \multirow{2}{*}{$\begin{array}{c}\begin{array}{c}\text { Additional } \\
\text { Power }\end{array} \\
\left(P_{T, a}, \mathrm{~W}\right)\end{array}$} & \multicolumn{2}{|c|}{$\begin{array}{l}\text { Additional Work }\left(W_{a} \text {; }\right. \\
\text { J) }\end{array}$} & \multirow[t]{2}{*}{ Fate } \\
\hline & & & & Min & $\operatorname{Max}$ & & & & & & Min & Max & \\
\hline EG 1427 & 18 & 1413.35 & 35095 & 5 & 487 & 1.27 & 0 & 3126 & 3713 & 586 & $3.88 \times 10^{8}$ & $1.04 \times 10^{10}$ & $\mathrm{~S}$ \\
\hline EG 2030 & 12 & 1356.91 & 24453 & 163 & 769 & 1.68 & 1 & 2497 & 3926 & 1429 & $1.80 \times 10^{10}$ & $9.61 \times 10^{10}$ & $\mathrm{D}$ \\
\hline EG 2212 & 5 & 1235.05 & 12038 & 1 & 23 & 3.05 & 1 & 1614 & 4587 & 2973 & $1.28 \times 10^{8}$ & $5.91 \times 10^{9}$ & $\mathrm{~S}$ \\
\hline EG 2710 & 3 & 1163.95 & 8490 & 68 & 397 & 1.46 & 0 & 1306 & 1776 & 470 & $3.16 \times 10^{9}$ & $4.46 \times 10^{9}$ & S \\
\hline EG 3107 & 1 & 1011.03 & 4943 & 57 & 297 & 1.43 & 1 & 954 & 1273 & 319 & $1.68 \times 10^{9}$ & $7.47 \times 10^{9}$ & $\mathrm{D}$ \\
\hline EG 3294 & 6 & 1260.43 & 13811 & 11 & 293 & 1.52 & 0 & 1755 & 2493 & 737 & $7.50 \times 10^{8}$ & $1.65 \times 10^{10}$ & $\mathrm{~S}$ \\
\hline EG 3311 & 7 & 1281.89 & 15585 & 51 & 2510 & 1.74 & 0 & 1890 & 3063 & 1173 & $5.17 \times 10^{9}$ & $2.46 \times 10^{11}$ & $\mathrm{D}$ \\
\hline EG 3314 & 2 & 1107.51 & 6717 & 25 & 98 & 1.94 & 1 & 1136 & 2059 & 923 & $2.36 \times 10^{9}$ & $8.35 \times 10^{9}$ & $\mathrm{~S}$ \\
\hline EG 3714 & 2 & 1107.51 & 6717 & 5 & 64 & 1.13 & 0 & 1136 & 1196 & 60 & $1.76 \times 10^{8}$ & $3.86 \times 10^{8}$ & $\mathrm{~S}$ \\
\hline
\end{tabular}



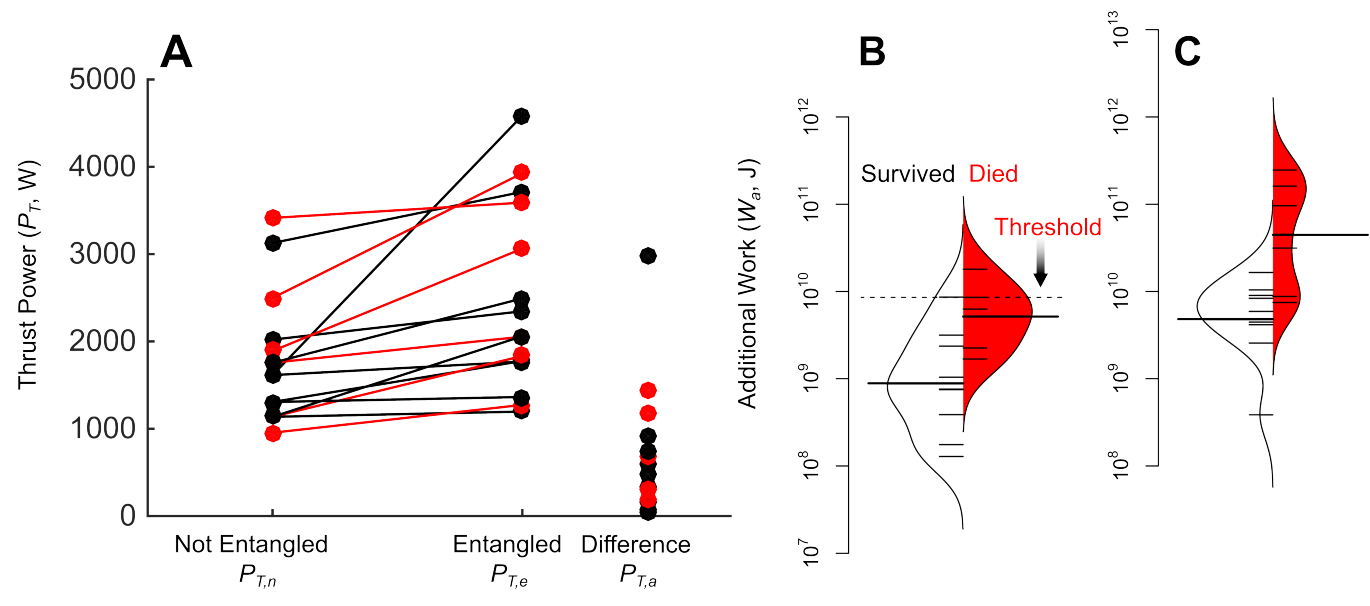

Figure 5-4: A: Thrust power $(\mathrm{W})$ of right whales swimming at $1.23 \mathrm{~m} / \mathrm{s}$ when not entangled $\left(P_{T, n}\right)$ and when entangled in fishing gear $\left(P_{T, e}\right)$, as well as the additional thrust power $\left(P_{T, a}=P_{T, e}-P_{T, n}\right)$ required when entangled. Red dots represent whales that died from their entanglement; black represents cases that survived. $\mathrm{B}$ and $\mathrm{C}$ : Additional work $\left(W_{a}, \mathrm{~J}\right)$ required for entangled right whales over their minimum (B) and maximum (C) entanglement durations, separated for whales who survived their entanglements (white) and those who died (red). The dashed line in panel B indicates the 0.75 quantile 'critical additional energy' threshold used to estimate survival.

Table 5-4: Minimum and maximum additional energy expenditures $\left(W_{a, \min }, W_{a, \max } ; \mathrm{J}\right)$ and thrust power output $\left(P_{T}, \mathrm{~W}\right)$ of entangled North Atlantic right whales that died or survived their entanglements. $t$ and $p$ statistics are presented for two-sample $t$-tests.

\begin{tabular}{|l|l|l|l|l|}
\hline & Died $(n=6)$ & Survived $(n=9)$ & $t_{13}$ & $p$ \\
\hline $\begin{array}{l}\text { Minimum additional Energy } \\
\left(W_{a, \text { min }} ; \mathrm{J}\right)\end{array}$ & $6.99 \pm 5.96 \times 10^{9}$ & $1.93 \pm 2.71 \times 10^{9}$ & -2.25 & 0.0424 \\
\hline $\begin{array}{l}\text { Maximum Additional Energy } \\
\left(W_{a, \max } ; \mathrm{J}\right)\end{array}$ & $9.18 \pm 9.62 \times 10^{10}$ & $6.86 \pm 4.83 \times 10^{9}$ & -2.7 & 0.0183 \\
\hline $\begin{array}{l}\text { Additional Power } \\
\left(P_{T} ; \mathrm{W}\right)\end{array}$ & $682 \pm 516$ & $699 \pm 904$ & 0.04 & 0.9687 \\
\hline
\end{tabular}

\subsubsection{Life history context}

The additional energetic costs of drag from fishing gear entanglement ranged $7.24 \times 10^{7}-$ $7.52 \times 10^{8} \mathrm{~J} /$ day (Figure $2 \mathrm{~A}$ ). These costs exceeded daily costs of pregnancy, are comparable to those of migration and foraging, and are 3-34\% as much as daily costs of lactation (Figure 2A, B). The time of onset of entanglements are unpredictable and occur throughout seasonal and reproductive cycles; as such, their costs can be incurred during times of high or low energy demand or availability (Figure 2C, D).

For females, entanglement costs were dwarfed by the high energetic demands of lactation (Figure 2D). We estimated that female right whales spend $2 \%$ of their energy budget during a 4 -year reproductive cycle on pregnancy and $23 \%$ on lactation; $75 \%$ is allocated 
to maintenance and recovery of energetic stores (Figure 5A). The drag from entanglements we measured required $0.3-6.2 \%$ to $1.0-7.8 \%$ of the 4 -year female energy budget based on minimum and maximum entanglement durations, respectively. The median time to energetic equilibrium, to restore energy lost by a particular entanglement, is 1.0-3.3 months (minimum-maximum entanglement durations), with one case requiring 12.5-15.8 months (Figure 5C). Assuming no change in energy availability or individual behavior to compensate for energetic allocation, these added costs likely would not affect calving intervals for 5 females ( $63 \%$ of cases), requiring a minimum of $<2$ months to reach equilibrium, but may increase calving intervals by 1 year for 3 females (37\% of cases) who require between 2-16 months (Figure 5C).
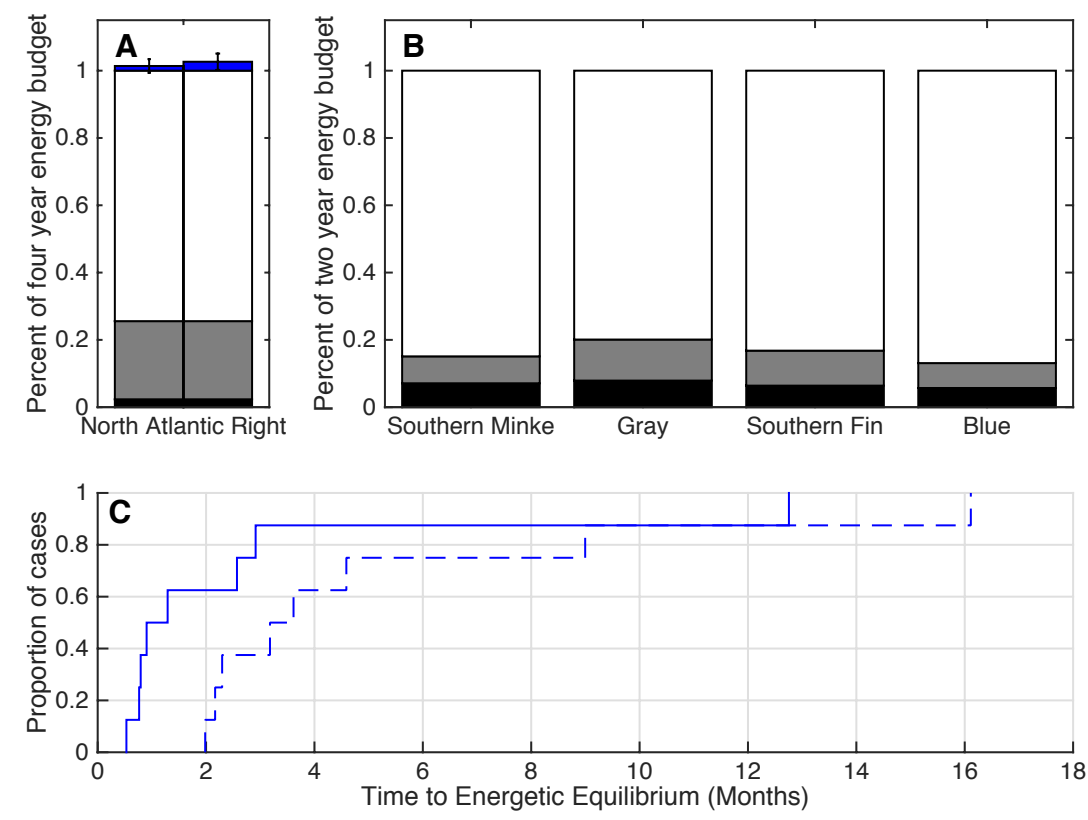

Figure 5-5: A. Percentage of female North Atlantic right whale four-year energy budgets allocated to pregnancy (black), lactation (grey) and female maintenance (white) costs, and mean additional costs associated with drag from entanglement in fishing gear (blue) over minimum (left) and maximum (right) entanglement durations for 8 females. Error bars show SD. B. Percentage of two-year energy budget allocated to pregnancy (black), lactation (grey) and female maintenance (white) for Southern minke (Lockyer, 1981a), grey (Villegas-Amtmann et al., 2015), Southern fin (Lockyer, 1981b, 1987) and blue (Lockyer, 1981b) whales. C. Cumulative Distribution Function (CDF) of the time to energetic equilibrium (to restore energy lost by a particular entanglement) for entangled female right whales $(n=8)$ based on their minimum (blue solid) and maximum (blue dashed) entanglement durations.

\subsection{Discussion}

Chronic entanglement in fishing gear affects marine animal populations worldwide (Fowler, 1987; Clapham and Mead, 1999; Read et al., 2006; Wegner and Cartamil, 2012). We used 
two approaches that yield comparable estimates of the energetics associated with increased drag from entanglement. These, in combination with individual history data have allowed us to show that:

- Propulsive requirements increase on average 1.58 fold, which over time affects individual survival (Figure 4).

- Entanglement results in the consumption of endogenous energy reserves on the order of magnitude as is consumed during lactation (Figure 3).

- Entanglement can lead to energetic demands similar in magnitude and duration to other life history events (Figure 2A, B).

- The onset and duration of these entanglements have significant impacts on the energy budget of individuals (Figure 2), especially reproductive females.

- Even short-term or low-drag entanglements can result in extended calving intervals in a k-strategist (Figure 5C).

\subsubsection{Entanglement cost and survival}

Emaciation is a common observation in marine animals chronically entangled in fishing gear (Fowler, 1987; Barco and Moore, 2010; Cassoff et al., 2011; Wegner and Cartamil, 2012; Barratclough et al., 2014), and is often attributed to increased energy consumption associated with added drag from the gear (Feldkamp, 1985; Feldkamp et al., 1988; van der Hoop et al., 2013b). We used two separate approaches to determine the costs of entanglement: from measured blubber thicknesses at death, and from measured drag forces to estimate energetic demands for swimming. These methods are in close agreement: differences in blubber thicknesses suggest that entangled right whales derive between $7.40 \times 10^{10} \mathrm{~J}$ and $1.20 \times 10^{11}$ $\mathrm{J}$ from lipid catabolism over the duration of a lethal chronic entanglement, while physical propulsion models and drag measurements suggest fifteen right whales expended $3.95 \times 10^{9}$ to $4.08 \times 10^{10} \mathrm{~J}$ more energy over the time course of their entanglements.

From propulsive power requirements, we determined that survival beyond disentanglement was related to the additional amount of work whales performed while entangled: the amount of drag imposed by the entangling gear (and the resulting power required to overcome it) was not a predictor of the fate of entangled individuals; what mattered is the amount of time over which the additional energetic costs are incurred (i.e., work). This is intuitive, as health impacts are a significant predictor of entanglement survival (Robbins et al., 2015), and deterioration in health of entangled whales requires time (Cassoff et al., 2011; Schick et al., 2013; Barratclough et al., 2014; Rolland et al., 2016). We therefore established a threshold of $8.57 \times 10^{9} \mathrm{~J}$ with which to assess other entanglement cases to estimate the time course to death or the point at which energetic reserves may become compromised. 


\subsubsection{Entanglement in the context of life history}

Large migratory animals undergo periods of fattening and fasting with remarkable adaptations for fat storage, mobilization and utilization that allow them to meet demands in natural energy-intensive periods. Our propulsive model suggests that drag from entanglement in fishing gear can incur energetic demands similar to costly life-history stages. Daily additional costs of swimming while entangled $\left(7.24 \times 10^{7}-7.52 \times 10^{8} \mathrm{~J} /\right.$ day $)$ are comparable to pregnancy $\left(1.84 \times 10^{8} \mathrm{~J} /\right.$ day; Fortune et al., 2013) and migration $\left(3.3 \times 10^{8} \mathrm{~J} /\right.$ day; van der Hoop et al., 2013b) and can be as high as costs of foraging $\left(5 \times 10^{8} \mathrm{~J} /\right.$ day; McGregor $2010)$ and reproduction $\left(7.9 \times 10^{8} \mathrm{~J} /\right.$ day; Klansjcek et al., 2007; Figure 2A). Integrated over time, entanglements can cost $4.56( \pm 5.30) \times 10^{9}$ to $4.73( \pm 7.95) \times 10^{10} \mathrm{~J}$ - as much as is energy expended to complete a migration $\left(7.3 \times 10^{9} \mathrm{~J}\right)$ and nearing what is required for an entire reproductive event $\left(5.8 \times 10^{11} \mathrm{~J}\right)$.

The timing of an additional energetic burden in the context of regular annual patterns, routines, and changes in energetic demands and variability cannot be ignored. We presented the relative and seasonally-varying costs of life history events of large baleen whales, as well as the time of onset, cost and duration of entanglements (Figure 2). The onset of these costs occurs in any month, when food may or may not be available, or when individuals may already be in negative energy balance, and last for many seasonal cycles (Figure 2C, D). Unlike migration or reproduction, where individuals undergo preparatory changes in body composition (e.g. Figure 3; Cherel et al., 1988; Miller et al., 2011; Christiansen et al., 2013) and organ function (Weber et al., 2009), entanglement is unanticipated and is not necessarily associated with any particular seasonal cue; whether an individual animal has laid down fat reserves to cope with the energetic costs of entanglement is a matter of chance. Entanglement onset in the context of seasonal variability in body condition (e.g. Figure 3B) and annual variability in prey availability and quality only add to the complexity of how entangled individuals may or may not be able to sustain the energetic demands of drag loading.

\subsubsection{Energetic costs and reproduction}

The connection between increased drag, altered behavior, additional energy demand, high stress and decreased breeding success has been established in studies addressing the effects of attaching scientific instruments (e.g., reviewed in Barron et al., 2010). Especially in birds and bats, added drag or weight from scientific instruments can lead to increases in energy expenditure, decreases in body condition, significant increases in corticosterones, and reduced nesting propensity and productivity (Barron et al., 2010; Elliott et al., 2012). Costs of transport significantly increase when instrumented (13.7\% - 100\%; Gessaman and

Nagy, 1988; Culik et al., 1994) even with devices designed to minimize increases in drag 
(17.4\%; Culik et al., 1994) or weight. Our results contribute to recent literature to suggest that entangled whales may follow the same model of response to prolonged increases in drag of $52 \%$ on average: decreases in body condition (Cassoff et al., 2011; Moore and Barco, 2013; van der Hoop et al., 2013b; Barratclough et al., 2014), elevated stress hormones (Hunt et al., 2006) and reduced reproductive success (Knowlton et al., 2012a).

For large whales, the most extreme variability in energetic demands and body condition are in females; body condition and blubber thicknesses increase significantly prior to pregnancy, and are depleted through to the end of lactation (e.g., Lockyer, 1986; Miller et al., 2012b; Williams et al., 2013). Models (Klansjcek et al., 2007; Villegas-Amtmann et al., 2015) and observations (Lockyer, 1978, 1986, 1987; Williams et al., 2013) suggest plasticity in calving intervals depending on a female's nutritional demands and environmental conditions; small changes in energy availability have large impacts on calving interval and age at first parturition. Klansjcek et al. (2007) show that reducing energy acquisition of North Atlantic right whales by $16 \%$ makes reproduction impossible, and (Villegas-Amtmann et al., 2015) estimate that annual energetic losses of as little as $4 \%$ can limit calf production or survival in gray whales. We show that, in close agreement with other large baleen whale species (Lockyer, 1981b,a, 1987; Villegas-Amtmann et al., 2015), female right whales allocate $2 \%$ of their four-year reproductive cycle on pregnancy, $23 \%$ on lactation, and $75 \%$ on maintenance and recovery of energetic stores (Figure 5A, B). Entanglement can add 0.3-7.8\% to the four-year energy budget, increasing the time to energetic equilibrium or female recovery in preparation for the next pregnancy by months to years. The calculations of extended calving intervals we present do not include the time over which females were entangled - on average 99-621 (range 11-2459) days. Though there have been cases of right whales becoming entangled while pregnant and while lactating, it is likely that the time while entangled may also contribute to time between reproductive events, especially in species with spatiotemporally restricted breeding. The timing of entanglement during the reproductive cycle is another important consideration, where other capital breeders seem to be especially sensitive to energetic disturbance during pregnancy rather than during lactation (Villegas-Amtmann et al., 2015).

Longer calving intervals (e.g. Figure 5A vs. B) suggest that reproduction is especially costly in balaenid whales (Nerini et al., 1984; Knowlton et al., 1994), making resource allocation essential. Knowlton et al. (2012a) showed that reproductive female right whales with severe entanglement wounds have significantly longer calving intervals than females with no or minor wounds. Additionally, females carrying gear or with severe entanglement histories are significantly less likely to calve again. The energetic drain from the integrated additional power requirements over the time course of an entanglement can therefore have immediate or long-term impacts on reproduction, critical to consider for both individualand population-level impacts. 
During reproduction, the blubber thickness of the mother is reduced by around $4.5 \mathrm{~cm}$ (Miller et al., 2011). Our results show that blubber layers of entangled juveniles and adults at necropsy were $5.5 \mathrm{~cm}$ and $2.1 \mathrm{~cm}$ thinner, respectively, compared to individuals in the same age classes who died from non-entanglement related causes (Figure 3A). Klansjcek et al. (2007) use the same ellipsoid method to estimate that a female right whale uses $5.8 \times 10^{11} \mathrm{~J}$ of energy in a reproductive event, from becoming pregnant to carrying a calf through weaning; we have shown that right whales catabolize a similar magnitude of lipids during an entanglement.

Both the blubber thickness and propulsive model methods illustrate how the cost of entanglement over time can affect energy availability. Adequate energy reserves are a major contributor to reproductive success in many mammals (Young, 1976; Frisch, 1984; Lockyer, 1986; Gittleman and Thompson, 1988) including right whales (Miller et al., 2011, 2012b). Baseline energy levels may be required to trigger ovulation: numerous studies have related insufficient energy availability with skipping reproduction (Wasser and Barash, 1983) or prolonged periods of anoestrous (Wright et al., 1992), and have identified threshold body conditions or blubber thicknesses beyond which the probability of pregnancy increases (Miller et al., 2011; Williams et al., 2013). On the 100-point scale used for right whale health assessments, there appears to be a threshold condition of 67 , below which reproduction does not occur; individuals in poor body condition are typically $<60$, including animals with entanglements (Rolland et al., 2016). The synergistic effects of metabolism, behavior, and chronic adrenal activation on stress-induced anovulation have also been clarified (Berga and Loucks, 2007). While the focus is often on females, male reproductive development, interest, performance and ability can also be affected by chronic or acute undernutrition and stress (Frisch, 1984). A significantly reduced androgen:estrogen ratio in a chronically entangled male right whale (Eg 1102, Figure 2), coincident with the highest observed fecal glucocorticoid levels, suggests that stress-related reproductive suppression can also occur in whales (Hunt et al., 2006).

\subsubsection{Comparing the bioenergetic methods}

The approaches taken here allow for independent estimates of energy expenditure related to a particular life history event. Changes in blubber thicknesses and estimated volumes only consider catabolism of lipid stored in the blubber layer, where the lipid content can vary with blubber depth and location along the body axis (Parry, 1949; Koopman et al., 2002). Entangled individuals may continue to forage or catabolize additional internal (e.g., muscular and visceral) lipid stores, so changes in blubber thicknesses or volumes likely provide a conservative estimate of total energy use. However, the blubber volume method integrates all costs of living by including basal metabolic, stress, thermoregulatory and health or repair costs that are currently not able to be measured. In addition, this method im- 
plicitly incorporates seasonal effects and energy requirements for swimming and overcoming entanglement-related drag. These methods highlight the difference between normal and entangled whales instead of attempting to estimate total costs for either condition. While whole-body bioenergetics models exist for cetaceans (Brodie, 1975; Kriete, 1995), including right whales (Fortune et al., 2013), it remains an unfortunate fact that the metabolic rates of large whales are unknown (e.g., see Gallivan, 1992); therefore using whole-body bioenergetic models to investigate the effects of entanglements is not possible.

Our method only incorporates measured differences in blubber thickness between entangled and non-entangled whales at death; it does not consider other changes in body girth in entangled whales, as there are few necropsy reports with entangled girth measurements and few instances of photogrammetry for entangled whales. Using normal body width minus a measured blubber thickness considers only changes in blubber volume, similar to Christiansen et al. (2013). The method excludes lipids metabolized from other body lipid depots (e.g., blood, viscera, muscle, bone). In most large whales, blubber contains majority of the total body adipose tissue (e.g., 96\% in fin whales; Pond and Mattacks, 1988); however, whales may be metabolizing additional lipid depots or during the most extreme fasting conditions, directly catabolizing protein as an energy source (Worthy and Lavigne, 1987; Cherel et al., 1992; Rea et al., 2007; McCue, 2010; Aguilar et al., 2014). Therefore, as it is based solely on lipid-based energy sources in the blubber layer, this method represents an underestimate of metabolized energy.

The blubber model does not consider changes in the lipid density of the blubber. Blubber is a heterogeneous tissue, where density and lipid content can vary with blubber depth, location along the body, body length, and nutritive and ontogenetic condition (Aguilar and Borrell, 1990; Koopman et al., 2002; Struntz et al., 2004; Dunkin et al., 2005, 2010). Significant changes in blubber lipid content can occur during seasonal fattening (Lockyer, 1981b; Aguilar and Borrell, 1990) or with reproductive status in balaenopterids (Lockyer, 1986, 1987). The rate at which blubber lipid content decreases with catabolism, and whether this decrease occurs in right whales as well, remains unknown, and therefore could not be included in this model. However, if blubber lipid content is reduced in emaciated right whales as it is in emaciated dolphins (Dunkin et al., 2005), our estimates will remain conservative.

The power model reflects the cost of entanglement on top of the costs of normal existence, i.e., for maintenance, thermoregulation and routine activity. It therefore provides a simplified estimate that ignores the status (e.g., nutritional, health, or life history) of the animal at the time of entanglement. Dates of the actual entangling event are extremely difficult to determine; while survey effort can help determine the dates last seen gear free or first seen entangled, the time frame between these dates can vary from 3 to 1037 days for cases presented in this study (Figure 2). Whales are almost always in healthy body condition at the time last seen prior to entanglement (Robbins et al., 2015). The rate at which body 
shape and composition deteriorate will be a function of the drag and energetic requirements, as well as changes in the associated hydro- and thermodynamics that will contribute back to energetic costs. The dynamics of this feedback remain unquantified and are too complex to include here.

While the power method includes a variable for swimming efficiency associated with entanglement (Williams, 1989; Cornick et al., 2006; Aoki et al., 2011; Nousek-McGregor et al., 2013; van der Hoop et al., 2013b), it assumes no change in swimming speed. Drag loading sometimes results in reduction in speed (Lang and Daybell, 1963; Boyd et al., 1997; Elliott et al., 2007; van der Hoop et al., 2014a); however, there are instances where speed is maintained and additional energy consumption occurs (Williams et al., 1993). Few data exist on how entangled animals alter their swimming behaviors: two right whales have been disentangled while wearing multi-sensor recording tags (DTAGs), from which ascent and descent speeds were resolved (Chapter 4; van der Hoop et al., 2013b). While one individual ( $\mathrm{Eg}$ 3911) shows significantly slower vertical descent and ascent speeds (46\% and $32 \%$ slower, respectively) when entangled compared to following disentanglement, the other individual ( $\mathrm{Eg}$ 4057) shows no difference in descent or ascent speeds between the two conditions (Chapter 4). Satellite-tag derived swimming speeds of entangled and nonentangled right whales (Baumgartner and Mate, 2005) do not indicate any difference in the average traveling speeds of these animals over the coarse resolution of ARGOS tags. Swimming speeds are often maintained in migratory species, where enough time must be left for essential activities such as feeding and reproduction (Weber et al., 2009). Our assumption that whales maintain speed likely provides an upper bound of power requirements.

\subsection{Conclusions}

Animals have adapted to prepare for, mitigate against, and recover from seasonal changes and natural fluctuations in energy demands and availability. We have shown that entanglement in fishing gear is an unpredictable event that can be extremely costly and last for

days to years. Wingfield et al. (1998) proposed the 'emergency life-history stage' where unexpected events lead to redirection of behavior from normal life history stages, brought about by a suite of physiological and behavioral responses that can be sustained only for so long without lasting effects; entanglement in fishing gear can be considered the same. Even over the wide range of fishing gears, entanglement durations, and fates of individuals in our study, our results suggest that drag from entanglement can impact blubber stores and require energy investment on the order of magnitude as a reproductive event or migration. Recovery from such physiological stress and disturbance may limit an individual's future reproductive success, making entanglement a potential contributor to fluctuations in population growth. Historically, whale conservation measures have focused on reducing mortality; 
a shift is required to also address morbidity and the sub-lethal impacts on individuals and their reproductive rates. 


\section{Chapter 6}

\section{Predicting lethal entanglements AS A CONSEQUENCE OF DRAG FROM FISHING GEAR}

This chapter has been submitted for publication: van der Hoop, J.M., Corkeron, P., Henry, A.G., Knowlton, A.R., and Moore, M.J.Predicting lethal entanglements as a consequence of drag from fishing gear Marine Pollution Bulletin Submission ID MPB-D-16-00969

JvdH developed concepts; ARK, AGH, PC and MJM guided analysis and application of results; ARK and AGH reviewed right whale case information; PC provided statistical advice; JvdH wrote the manuscript; PC, AGH, ARK and MJM contributed to the writing of the manuscript.

The supplemental materials for this chapter can be found in Appendix E. 


\subsection{Abstract}

Large whales are frequently entangled in fishing gear and sometimes swim while carrying gear for days to years. Entangled whales are subject to additional drag forces requiring increased thrust power and energy expenditure over time. To classify entanglement cases and aid potential disentanglement efforts, it is useful to know how long an entangled whale might survive, given the unique configurations of the gear they are towing. This study establishes an approach to predict drag forces on fishing gear that entangles whales, and applies this method to ten North Atlantic right whale cases to estimate the resulting increase in energy expenditure and the critical entanglement duration that could lead to death. Estimated gear drag ranged 11-275 N. Most entanglements were resolved before critical entanglement durations (mean \pm SD $216 \pm 260$ days) were reached. These estimates can assist real-time development of disentanglement action plans and U.S. Federal Serious Injury assessments required for protected species.

\subsection{Highlights}

- Large whales are often entangled in fishing gear for months to years.

- Drag and energy burden from entangling gear can be estimated at the time of a whale's detection.

- We develop tools for prognosis of specific entanglement cases to assist disentanglement action and inform stock assessment.

\subsection{Graphical abstract}
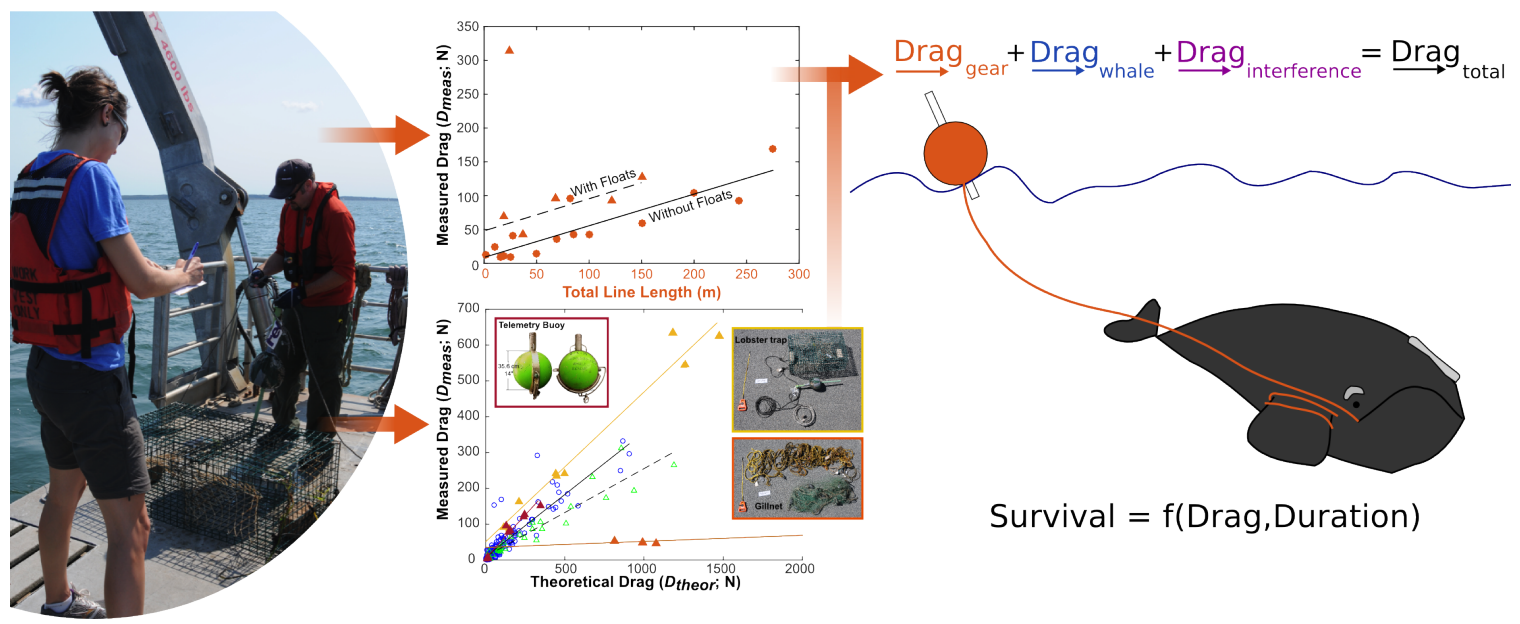


\subsection{Introduction}

Marine animals are frequently entangled in fixed fishing gear (Read et al., 2006; van der Hoop et al., 2013a), with larger whales often able to break free of anchor points. In doing so, some whales are able to continue to swim for days to years while carrying a portion of gear with them. For most large whales, the proximate source of entanglement is actively fished (vs. derelict) gear (Laist, 1997; Butterworth et al., 2012; Lyman, 2012), though the gear is dislodged, leaves the fishery site, and is carried by the animal. Efforts to disentangle large whales in particular have been developed in areas where incidence is especially high (e.g., by the Center for Coastal Studies in Provincetown, MA, U.S.A.) and information and experience gained by the teams involved in these efforts have been shared worldwide as the entanglement issue has been recognized as a global issue (IWC, 2010, 2011).

When entangled whales are reported, depending on the level of information provided at the initial report and the whale's proximity to a response effort, an evaluation is made as to whether the entanglement is likely life threatening. For life threatening cases, trained disentanglement teams develop action plans to determine whether the whale is a candidate for disentanglement, and if so what the response can or should involve (IWC, 2010). The plan considers the specific configuration of the gear on the animal and the animal's apparent health as described by observers or as documented in photographs or video. There is a sense of urgency to remove gear, and a clear set of protocols are implemented to properly assess the case and design a plan that prioritizes both animal and human safety. Depending on the species, environmental conditions, and gear, numerous disentanglement attempts may be required over days to months (Moore et al., 2010).

Entangled whales are subject to considerable drag forces (van der Hoop et al., 2013b, 2015), which demand increased thrust power and therefore energy expenditure over time. Whales can persist with chronic entanglements for years, yet most entangled North Atlantic right whales (hereafter right whales; Eubalaena glacialis) die within six months to a year after detection (Moore et al., 2006) if they are not successfully disentangled early on. Health impacts are the most predictive of subsequent survival of entangled right whales (Robbins et al., 2015). Longer entanglement durations are more likely to lead to severe injuries (Knowlton et al., 2015) and the total energy expenditure over the course of entanglement has been linked to individual fate (Chapter 5); the impact of entanglement drag over time is therefore a critical element to consider when developing response action plans or assessing whether an entanglement is life-threatening (NOAA, 2008; IWC, 2010).

How long can entangled right whales survive, given the unique configurations and dimensions of the gear they are towing? While it is possible to measure drag on some sets of gear (e.g., van der Hoop et al., 2013b, 2015), drag forces can also be estimated from wellestablished physical theory (Fridman, 1986; Faltinsen, 1993; Helmond, 2001; Keith et al., 
2004). To determine the relationship between measured and theoretical drag forces, both methods were applied to sets of fishing gear that had entangled or are similar to those entangling right whales. This relationship was then applied to entanglement cases for which drag forces had not been measured, to estimate (a) the drag experienced by these whales, (b) the resulting increase in energy expenditure, and the (c) potential longevity of each individual in its entangled condition.

\subsection{Methods}

Table 6-1: List of symbols and abbreviations.

\begin{tabular}{|l|l|l|}
\hline Symbol & Definition & Unit \\
\hline$\alpha$ & Incident flow angle & $\circ$ \\
\hline$A_{w}$ & Wetted surface area & $\mathrm{m}^{2}$ \\
\hline$C_{d}$ & Drag coefficient & \\
\hline$d$ & Line diameter & $\mathrm{m}$ \\
\hline$D$ & Drag force & $\mathrm{N}$ \\
\hline$D_{\text {corr }}$ & Corrected drag force & $\mathrm{N}$ \\
\hline$D_{f}$ & Drag forces on floats or traps & $\mathrm{N}$ \\
\hline$D_{I}$ & Interference drag force & $\mathrm{N}$ \\
\hline$D_{l}$ & Drag forces on line & $\mathrm{N}$ \\
\hline$D_{\text {meas }}$ & Measured drag force & $\mathrm{N}$ \\
\hline$D_{\text {theor }}$ & Theoretical drag force & $\mathrm{N}$ \\
\hline$D_{\text {tot }}$ & Entangled whale total drag force & $\mathrm{N}$ \\
\hline$D_{w}$ & Whale body drag force & $\mathrm{N}$ \\
\hline$\eta$ & Overall efficiency & \\
\hline$\eta_{m}$ & Metabolic efficiency & \\
\hline$\eta_{p}$ & Propulsive efficiency & \\
\hline$l$ & Total length & $\mathrm{m}$ \\
\hline$P_{T}$ & Thrust power & $\mathrm{W}$ \\
\hline$\rho$ & Density & $\mathrm{kg} / \mathrm{m}^{3}$ \\
\hline$q$ & Hydrodynamic stagnation pressure & $\mathrm{N}$ \\
\hline$t$ & Time & $\mathrm{s}$ \\
\hline$t_{\text {max }}$ & Maximum entanglement duration & $\mathrm{days}$ \\
\hline$t_{\text {min }}$ & Minimum entanglement duration & $\mathrm{days}$ \\
\hline$U$ & Speed & $\mathrm{m} / \mathrm{s}$ \\
\hline$W_{a}$ & Additional work & $\mathrm{J}$ \\
\hline$z$ & Tow point depth & $\mathrm{m}$ \\
\hline & & \\
\hline & & \\
\hline & & \\
\hline
\end{tabular}




\subsubsection{Measured gear sets}

Hydrodynamic drag forces on 21 sets of fishing gear removed from or similar to those entangling right whales were measured in a previous study via tensiometer $\left(D_{\text {meas }}\right.$; van der Hoop et al., 2015). Drag forces on these same gear sets are estimated here from theory (Fridman, 1986). Total length and line diameter were measured from dry gear. All symbols and abbreviations are listed in Table 1.

The drag force on fishing ropes can be estimated by

$$
D_{l}=C_{d} l d q
$$

where $C_{d}$ is the drag coefficient, $l$ the total length (not just trailing length; $\mathrm{m}$ ), and $d$ the diameter $(\mathrm{m})$ of the line, and $q$ is the hydrodynamic stagnation pressure $(\mathrm{N})$ :

$$
q=\frac{\rho U^{2}}{2}
$$

where $\rho$ is seawater density $\left(1025 \mathrm{~kg} / \mathrm{m}^{3}\right)$ and $U$ is the relative speed through water (i.e., including currents) at each tow point $(\sim 0.77,1.3$, and $2.1 \mathrm{~m} / \mathrm{s}) . C_{d}$ is estimated from Table 3.3 in Fridman (1986) based on the angle between the line and the flow direction $\alpha$ $\left(^{\circ}\right)$, calculated from the depth of each tow point $(z ; \sim 0,3$, and $6 \mathrm{~m})$ and the length of the line,

$$
\alpha=\sin \frac{z}{l} .
$$

Drag from floats, traps or buoys is estimated as

$$
D_{f}=C_{d} q A_{w}
$$

where $A_{w}$ is the wetted surface area $\left(\mathrm{m}^{2}\right)$ of each rigging component (see Appendix B1; van der Hoop et al., 2015) and corresponding $C_{d}$ values for typical rigging shapes from Table 3.5 in Fridman (1986). The total theoretical hydrodynamic drag $\left(D_{\text {theor }}\right)$ on a gear set is then the sum of the drag forces on the line $\left(D_{l}\right)$ and floats and/or traps $\left(D_{f}\right)$ if present:

$$
D_{\text {theor }}=D_{l}+D_{f}
$$

A linear model was fit to the theoretical $\left(D_{\text {theor }}\right)$ and measured drag $\left(D_{\text {meas }}\right)$ values, with float as a categorical covariate. This equation for corrected drag, $D_{c o r r}$, was then applied to ten other sets of entangling fishing gear that were not measured, but whose dimensions were sufficiently described to estimate drag forces from theory. 


\subsubsection{Non-Measured gear sets}

Ten sets of fishing gear entangling right whales were sufficiently described with dimensions to estimate drag forces from theory following equations 1 through 5. Body length and weight of the ten entangled whales were estimated from age at first entanglement from Moore et al. (2004), and maximum body width from length as in Fortune et al. (2012). These body dimensions were used to estimate drag forces on the whales' bodies, $D_{w}(\mathrm{~N})$, as in Eq. 8 in van der Hoop et al. (2015). Similar to the Measured gear sets above, gear dimensions were obtained from gear after it was collected. Total length refers to the length of all of the retrieved gear, rather than the length of trailing line; no effort was made to estimate dimensions of gear that was not removed or not retrieved. As such, all cases are underestimates of the total gear on the whales. Wetted area $\left(A_{w}\right)$ was estimated for all additional gear components (Appendix E1). Drag was estimated at $1.23 \mathrm{~m} / \mathrm{s}$, the upper 95\% CI of satellite-tag derived swimming speeds for right whales (Baumgartner and Mate, 2005; van der Hoop et al., 2012) and at a depth (z) of 0 m. These $D_{\text {theor }}$ were then corrected based on the linear relationship established above to yield a corrected drag value $D_{\text {corr }}$, so as to enable direct comparison with the measured drag forces $\left(D_{\text {meas }}\right)$ from van der Hoop et al. (2015).

Interference drag $\left(D_{I}, \mathrm{~N}\right)$ from each entangling gear set was estimated based on the location on the body, height, and frontal area at the attachment point (Jacobs, 1934; van der Hoop et al., 2015). The number of wraps on different body parts and the dimensions of the gear where it attaches greatly affect the magnitude of interference drag (van der Hoop et al., 2013a, 2015). The total drag on each entangled whale $\left(D_{t o t}\right)$ was then:

$$
D_{t o t}=D_{w}+D_{I}+D_{c o r r}
$$

Thrust power requirements to overcome drag for swimming when entangled $\left(P_{T, e} ; \mathrm{W}\right)$ and not entangled $\left(P_{T, n} ; \mathrm{W}\right)$ were calculated as:

$$
\begin{gathered}
P_{T, e}=\frac{D_{t o t} U}{\eta_{e}} \\
P_{T, n}=\frac{D_{w} U}{\eta_{n}},
\end{gathered}
$$

where $\eta$ is the maximum swimming efficiency (i.e., $\eta_{m} \times \eta_{p}$; muscular $\times$ propulsive) of a right whale when not entangled $\left(\eta_{n}=0.13\right)$ and entangled $\left(\eta_{e}=0.13\right)$ based on Chapter 4. Maximum propulsive efficiencies were applied instead of mean values for a more conservative estimate. As the simplest scenario, it was assumed that entanglement did not

affect an individual's swimming speed $\left(U_{e}=U_{n}\right)$, i.e., that animals do not slow down once entangled. 
The additional work $\left(W_{a}, \mathrm{~J}\right)$ required to swim when entangled was calculated as

$$
W_{a}=t\left(P_{T, e}-P_{T, n}\right)
$$

where $t$ is time (in seconds). van der Hoop et al. (Chapter 5) determined the amount of additional work performed by entangled right whales based on their minimum and maximum entanglement durations. Individuals who died performed significantly more work; the 0.75 quantile of minimum additional work performed by whales that did not survive their entanglements was $8.57 \times 10^{9} \mathrm{~J}$. This is therefore considered to be the critical level of additional energy expenditure, which if reached, may be fatal. $t$ was increased to determine the time required for these ten whales to reach this critical level of energy expenditure. The expected lethal entanglement durations were compared to the actual, observed minimum and maximum entanglement durations of each whale and were interpreted with disentanglement dates and the known fates of the individuals to determine whether or not energetic costs alone can predict mortality or serious injury in entangled whales.

Entanglement durations were calculated from sightings histories and disentanglement records of each whale (NARWC, 2015). Maximum entanglement durations $\left(t_{\max }\right)$ were calculated based on the last gear-free sighting before entanglement, and either first confirmed gear-free sighting following disentanglement, death (confirmed by carcass detection and identification), or presumed death (once an individual has not been sighted in 6 years; Knowlton et al., 1994). Minimum entanglement durations $\left(t_{m i n}\right)$ were calculated based on the first entangled sighting and either the date of disentanglement (including partial disentanglement), the date last seen entangled, or the date that a telemetry buoy, if it was attached during disentanglement efforts, ceased transmissions. To show the unique subset of entanglement case data used in this study, the sightings histories and minimum and maximum entanglement durations of the ten Non-Measured cases were compared with the 15 Measured cases and 47 others as described by (Knowlton et al., 2015).

\subsubsection{Disentanglement response}

Disentanglement response typically focuses on removing all or most of the gear from the whale, but in some cases shortening the length of trailing line to 1 body length is the best option available. To determine the drag reduction and increase in critical entanglement duration achieved in doing so, gear drag was estimated a second time with the length of gear equal to the body length of the entangled whale involved in each case (Table 2) and with floats and buoys removed if they were initially present. For one case (Eg 2151) where the gear was already $<1$ body length, the length of the gear was not changed. 


\subsubsection{Sensitivity to gear parameters}

To determine the sensitivity of the assessment tool, critical durations were estimated for all 10 cases by adding in levels of information. The first estimate included gear length only, then added the gear diameter and then the attachment point. If the entanglement included floats, four estimates were made: (1) the length of the gear with the presence of a float, then adding (2) gear diameter, (3) attachment point and finally (4) the specific dimensions of the float.

\subsection{Results}

Theoretical drag forces $\left(D_{\text {theor }}\right)$ vary consistently from measured drag values $\left(D_{\text {meas }}\right)$ at the same depths and speeds (Figures 1,2). The slopes between measured and theoretical drag are $0.335( \pm 0.161)$; however, the lobster trap and telemetry buoy have much greater slopes (0.418 and 0.483 respectively), and the gillnet gear has a much lower slope (0.017), than the

rest of the measured gear sets (Figure 2). Removing these three sets, measured drag can be predicted from drag estimated with theory, yielding a correction equation $\left(D_{c o r r}\right)$ of:

$$
D_{\text {corr }}=8.83+0.35 \times D_{\text {theor }}-0.10 \times D_{\text {theor }} \times \text { float },
$$

where float is a binary covariate $\left(\mathrm{RMSE}=27.2, \mathrm{Adj} . \mathrm{R}^{2}=0.846, p<0.0001\right)$. A separate relationship was fit to correct for an attached lobster trap $\left(D_{\text {corr }, \text { lobs }}\right)$ :

$D_{\text {corr }, \text { lobs }}=50.81+0.418 \times D_{\text {theor }},\left(\mathrm{RMSE}=41.8, \mathrm{Adj} . \mathrm{R}^{2}=0.965, p<0.0001\right)$

and the telemetry buoy $\left(D_{\text {corr }, \text { telem }}\right)$ :

$$
D_{\text {corr }, \text { telem }}=4.91+0.483 \times D_{\text {theor }},\left(\mathrm{RMSE}=15.4, \mathrm{Adj} . \mathrm{R}^{2}=0.932, p<0.0001\right)
$$

\subsubsection{Non-measured gear sets}

Corrected drag values for the ten sets of gear that were not towed ranged 11.5-281.0 N at $1.23 \mathrm{~m} / \mathrm{s}$. These corrected drag values $\left(D_{\text {corr }}\right)$ were combined with estimated whale drag $\left(D_{n}\right)$ and interference drag $\left(D_{I}\right)$ to yield the total drag estimated for each of the entangled whales swimming at $1.23 \mathrm{~m} / \mathrm{s}$ (Figure $3 \mathrm{~A})$. Total gear drag $\left(D_{\text {corr }}+D_{I}\right)$ contributed $86.0 \pm 94.6 \%$ to total body drag, but ranged from 7.6-260\% (Figure 3A). Generally, greatest gear drag contributions were seen in entanglements involving the youngest and therefore smallest animals ( $\operatorname{Eg} 3392=233 \%$, Eg $3281=260 \%)$, although some young animals also experienced low gear drag contributions $($ Eg $3120=38.9 \%$, Figure $3 \mathrm{~A})$. 


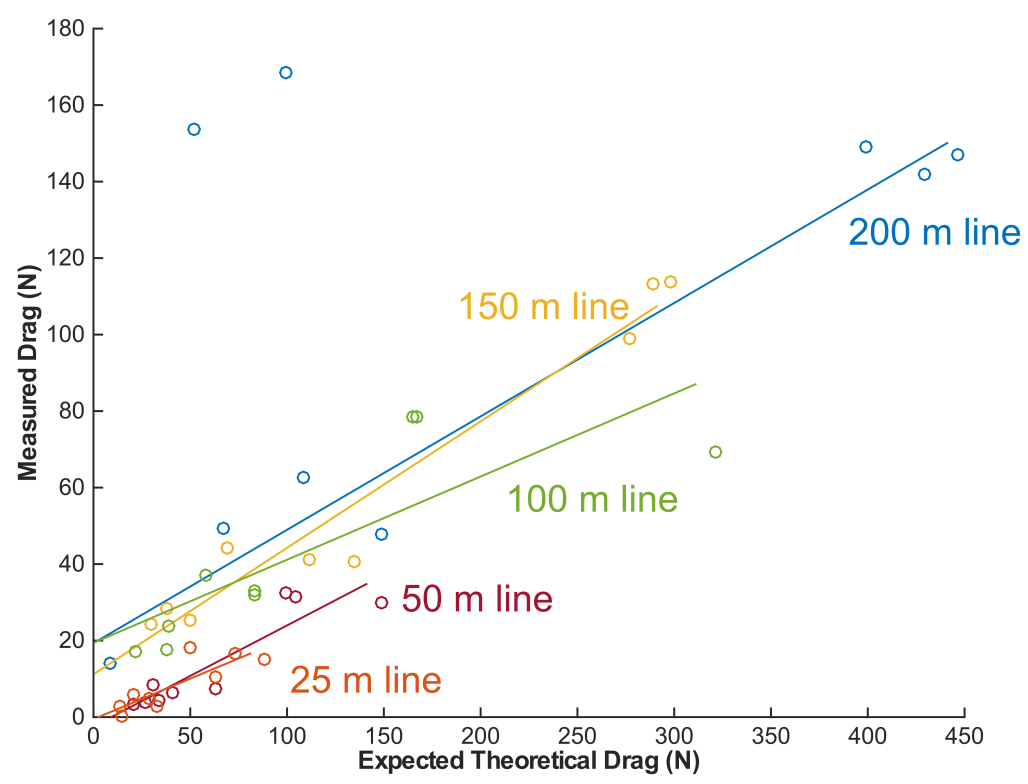

Figure 6-1: Expected theoretical forces vs. measured drag forces $(\mathrm{N})$ on five lengths of $8 \mathrm{~mm}$ diameter polypropylene rope. Colors represent different lengths of line (blue, $200 \mathrm{~m}$; yellow, $150 \mathrm{~m}$; green, $100 \mathrm{~m}$; red, $50 \mathrm{~m}$; orange, $25 \mathrm{~m}$. Each point represents a measurement and estimation at specific depths $(\sim 0,3$, and $6 \mathrm{~m})$ and speeds $(\sim 0.77,1.3$, and $2.1 \mathrm{~m} / \mathrm{s})$.

To overcome additional drag forces, these entangled whales expended significantly more power $\left(P_{T, e}=3030 \pm 1071 \mathrm{~W}\right)$ compared to when not entangled $\left(P_{T, n}=1870 \pm 923 \mathrm{~W}\right.$; paired $t$-test, $\left.t_{18}=2.5927, p=0.0184\right)$. Over the course of one day swimming at $1.2 \mathrm{~m} / \mathrm{s}$, these entangled whales would have to do $1.00 \times 10^{8} \mathrm{~J}$ more work $\left(W_{a}\right)$ than they would if not entangled (Figure 3B).

These 10 whales would have reached the critical additional energy requirement of $8.57 \times 10^{9}$ $\mathrm{J}$ in 216 \pm 260 days (range 38-914 days; Figure 3C, D), which can be compared to their observed entanglement durations (Figure 3D). One whale's minimum entanglement duration exceeded our critical additional work threshold (Eg 3120), whereas four whales' maximum duration exceeded the threshold (Figure 3D). Only two of the 10 whale cases died, 37 and 815 days before the critical entanglement duration was reached. 


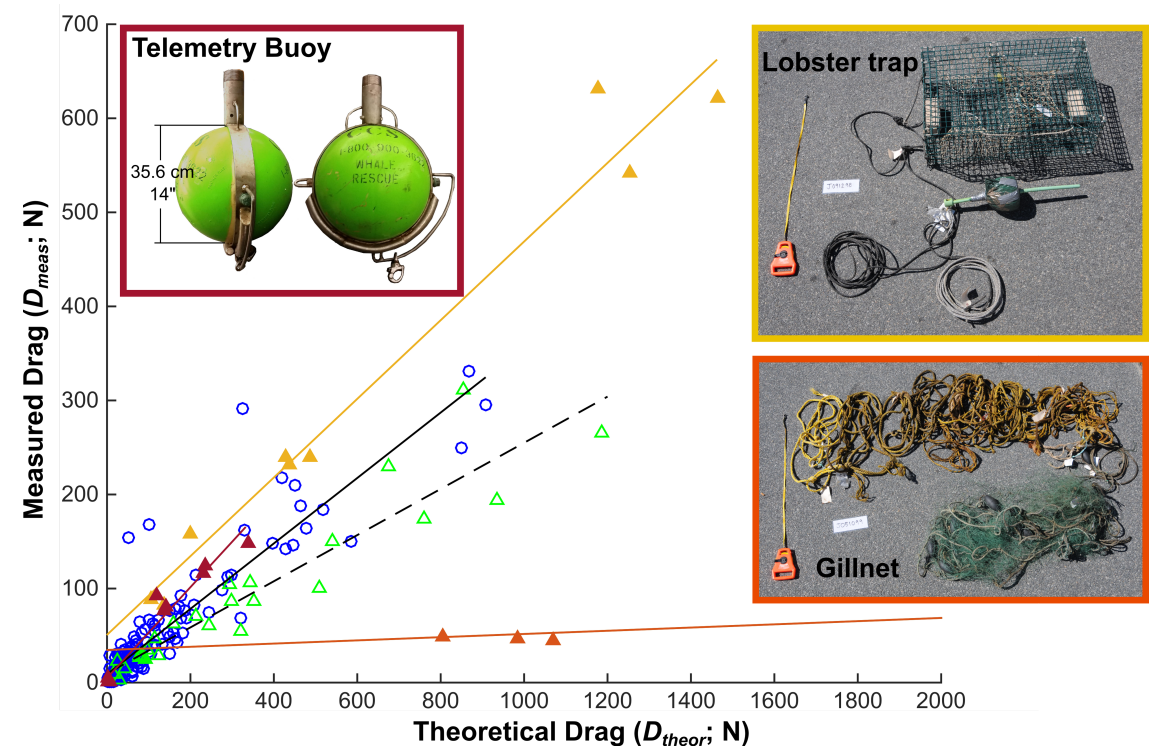

Figure 6-2: Expected theoretical vs. measured drag forces $(\mathrm{N})$ on 15 sets of fishing gear removed from entangled North Atlantic right whales, the satellite telemetry buoy, and 200, 150, 100, 50 and $25 \mathrm{~m}$ of $8 \mathrm{~mm}$ diameter polypropylene line. Each point represents a measurement and estimation at specific depths $(\sim 0,3$, and $6 \mathrm{~m})$ and speeds $(\sim 0.77,1.3$, and $2.1 \mathrm{~m} / \mathrm{s})$. Blue circles represent gear sets with only line; green triangles represent gear sets with additional floats or buoys; red, yellow, and orange triangles are the telemetry buoy, lobster pot and gill net gear, respectively, which are not used in the linear model fit between measured and theoretical drag for gear sets made up of only line (solid line), or with the presence of floats (dashed line) as a categorical covariate. See text for equations. 

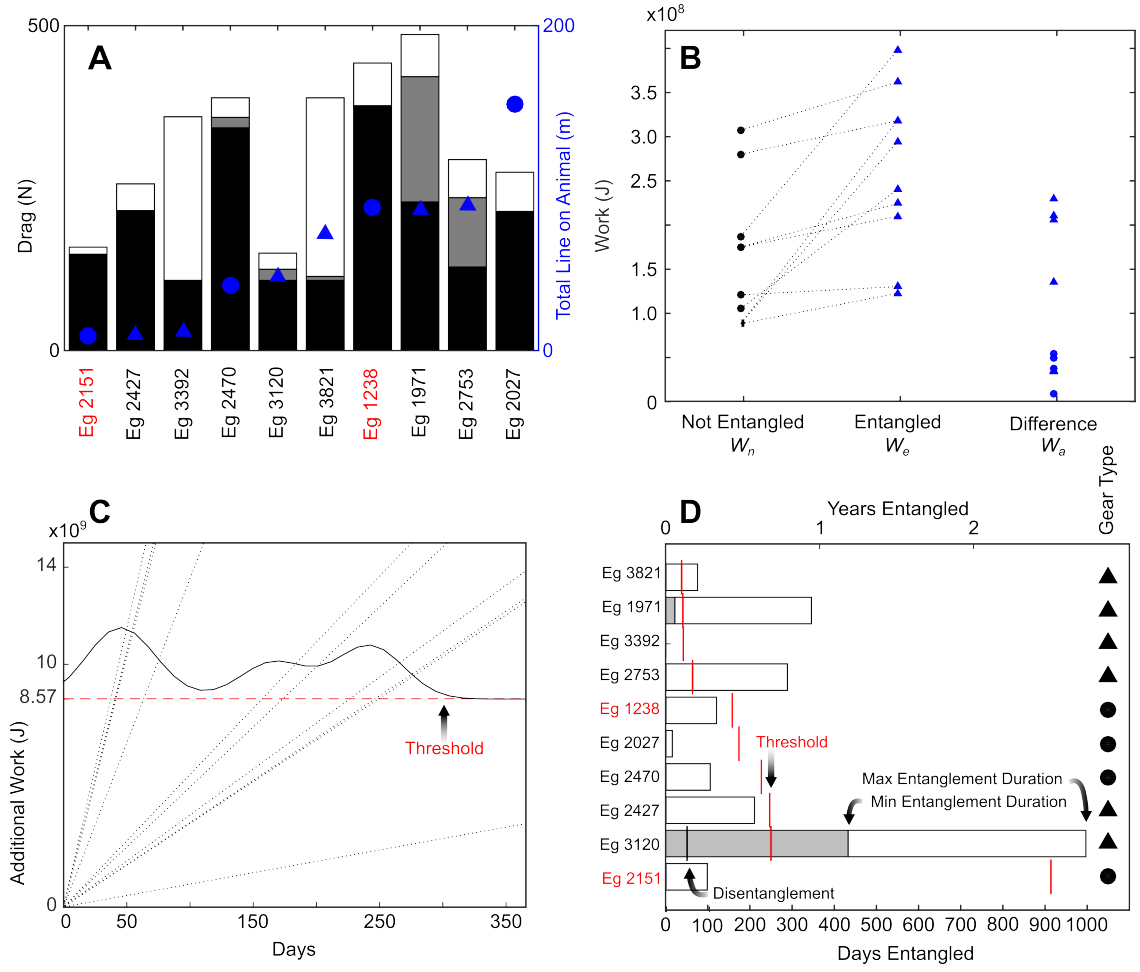

Figure 6-3: A: Drag forces $(\mathrm{N})$ on the bodies (black) of ten right whales (identified by Eg number), and interference (grey) and measured (white) drag from entangling gear. Red label text indicates whales that died. Blue markers represent the total length of line used in the drag estimate; circles represent gear made up of only line, triangles represent gear with floats or traps. See text for details. $\mathrm{B}$ : Total work $(\mathrm{J})$ required for ten right whales swimming for one day at $1.23 \mathrm{~m} / \mathrm{s}$ when not entangled ( $W_{n}$; black closed symbols) and entangled in fishing gear ( $W_{e}$; blue closed symbols), as well as the additional work required ( $W_{a}=W_{e} \neq W_{n}$; blue open symbols) when entangled. C: Additional work $\left(W_{a}, \mathrm{~J}\right)$ over increasing entanglement durations for each whale (black dotted lines) to determine the number of days until individuals expend minimum critical additional energy levels (red dashed line). The black line shows the distribution of days until minimum critical additional energy levels are reached. D: Minimum (grey) and maximum (white) observed entanglement durations of 10 right whales (Eg numbers) and time until minimum critical levels of additional energy expenditure (red lines) are reached. Red label text indicates whales that died. Black lines represent the day of disentanglement; if not visible, the whale was disentangled on the day of entanglement detection (day zero) except for Egs 1238 and 2151, neither of which were disentangled. 
Table 6-2: Details of fishing gear entanglements whose drag forces were estimated $(n=10)$. Sightings data provided the minimum and maximum entanglement periods (days), individual fate $(\mathrm{S}=$ survived; $\mathrm{D}=$ died) and age or minimum age at entanglement (years) for all whales, from which length and weight were estimated from Moore et al. (2004).

\begin{tabular}{|c|c|c|c|c|c|c|c|c|c|c|c|c|c|}
\hline & & & \multirow{3}{*}{$\begin{array}{l}\text { Estimated } \\
\text { Weight } \\
(\mathrm{kg})\end{array}$} & \multirow{2}{*}{\multicolumn{2}{|c|}{$\begin{array}{c}\text { Entanglement } \\
\text { Duration } \\
\text { (days) }\end{array}$}} & \multirow{3}{*}{$\begin{array}{l}\text { Fold Increase } \\
\text { in Drag }\end{array}$} & \multirow[t]{3}{*}{ Floats } & \multirow{3}{*}{$\begin{array}{c}\text { Non- } \\
\text { entangled } \\
\text { Power } \\
\left(P_{T, n}, \mathrm{~W}\right) \\
\end{array}$} & \multirow{3}{*}{$\begin{array}{c}\begin{array}{c}\text { Entangled } \\
\text { Power }\end{array} \\
\left(P_{T, e}, \mathrm{~W}\right) \\
\end{array}$} & \multirow{3}{*}{$\begin{array}{c}\begin{array}{c}\text { Additional } \\
\text { Power }\end{array} \\
\left(P_{T, a}, \mathrm{~W}\right) \\
\end{array}$} & \multirow{2}{*}{\multicolumn{2}{|c|}{ Additional Work }} & \multirow[t]{3}{*}{ Fate } \\
\hline & \multirow{2}{*}{$\begin{array}{c}\text { Entanglement } \\
\text { (years) }\end{array}$} & \multirow{2}{*}{$\begin{array}{l}\text { Length } \\
\text { (m) }\end{array}$} & & & & & & & & & & & \\
\hline & & & & Min & Max & & & & & & Min & Max & \\
\hline Eg 1238 & 20 & 14.28 & 38643 & 1 & 121 & 1.26 & 0 & 3558 & 4188 & 631 & $5.45 \times 10^{7}$ & $6.59 \times 10^{9}$ & $\mathrm{D}$ \\
\hline Eg 1971 & 8 & 13 & 17359 & 22 & 346 & 2.28 & 1 & 2165 & 4603 & 2438 & $4.63 \times 10^{9}$ & $7.29 \times 10^{10}$ & $\mathrm{~S}$ \\
\hline Eg 2027 & 7 & 12.82 & 15585 & 1 & 16 & 1.37 & 0 & 2026 & 2598 & 572 & $4.94 \times 10^{7}$ & $7.91 \times 10^{8}$ & $\mathrm{~S}$ \\
\hline Eg 2151 & 3 & 11.64 & 8490 & 1 & 99 & 1.15 & 0 & 1399 & 1508 & 109 & $9.38 \times 10^{6}$ & $9.29 \times 10^{8}$ & $\mathrm{D}$ \\
\hline Eg 2427 & 7 & 12.82 & 15585 & 1 & 211 & 1.28 & 1 & 2026 & 2428 & 403 & $3.48 \times 10^{7}$ & $7.34 \times 10^{9}$ & $\mathrm{~S}$ \\
\hline Eg 2470 & 17 & 14.05 & 33322 & 1 & 106 & 1.22 & 0 & 3244 & 3682 & 438 & $3.79 \times 10^{7}$ & $4.01 \times 10^{9}$ & $\mathrm{~S}$ \\
\hline Eg 2753 & 2 & 11.08 & 6717 & 1 & 289 & 2.45 & 1 & 1218 & 2782 & 1564 & $1.35 \times 10^{8}$ & $3.91 \times 10^{10}$ & $\mathrm{~S}$ \\
\hline Eg 3120 & 1 & 10.11 & 4943 & 433 & 997 & 1.49 & 1 & 1022 & 1420 & 398 & $1.49 \times 10^{10}$ & $3.43 \times 10^{10}$ & $\mathrm{~S}$ \\
\hline Eg 3392 & 1 & 10.11 & 4943 & 1 & Unk & 3.57 & 1 & 1022 & 3406 & 2383 & $2.06 \times 10^{8}$ & - & $\mathrm{S}$ \\
\hline Eg 3821 & 1 & 10.11 & 4943 & 1 & 76 & 3.86 & 1 & 1022 & 3681 & 2659 & $2.30 \times 10^{8}$ & $1.75 \times 10^{10}$ & $\mathrm{~S}$ \\
\hline
\end{tabular}




\subsubsection{Disentanglement response}

Partial disentanglements that cut trailing line to 1 body length and remove any floats from these 10 cases would reduce gear drag forces from on average $88.4( \pm 94.2) \mathrm{N}$ (range 11.1$274.9 \mathrm{~N}$ ) to $13.6( \pm 2.3) \mathrm{N}$ (range 11.1-18.1 N; Fig 4A). Total entangled whale drag would be reduced by $21( \pm 25) \%$ and up to $68 \%$ for cases where lobster traps were to be removed (Fig $4 \mathrm{~B})$. Additional work required for swimming would be significantly reduced $\left(t_{9}=4.3870, p=\right.$ 0.0018; Fig 4C) and critical duration would increase significantly by on average $304( \pm 295)$ days (range 0-824 days; $t_{9}=3.2567, p=0.0099$; Fig $4 \mathrm{D}$ ).
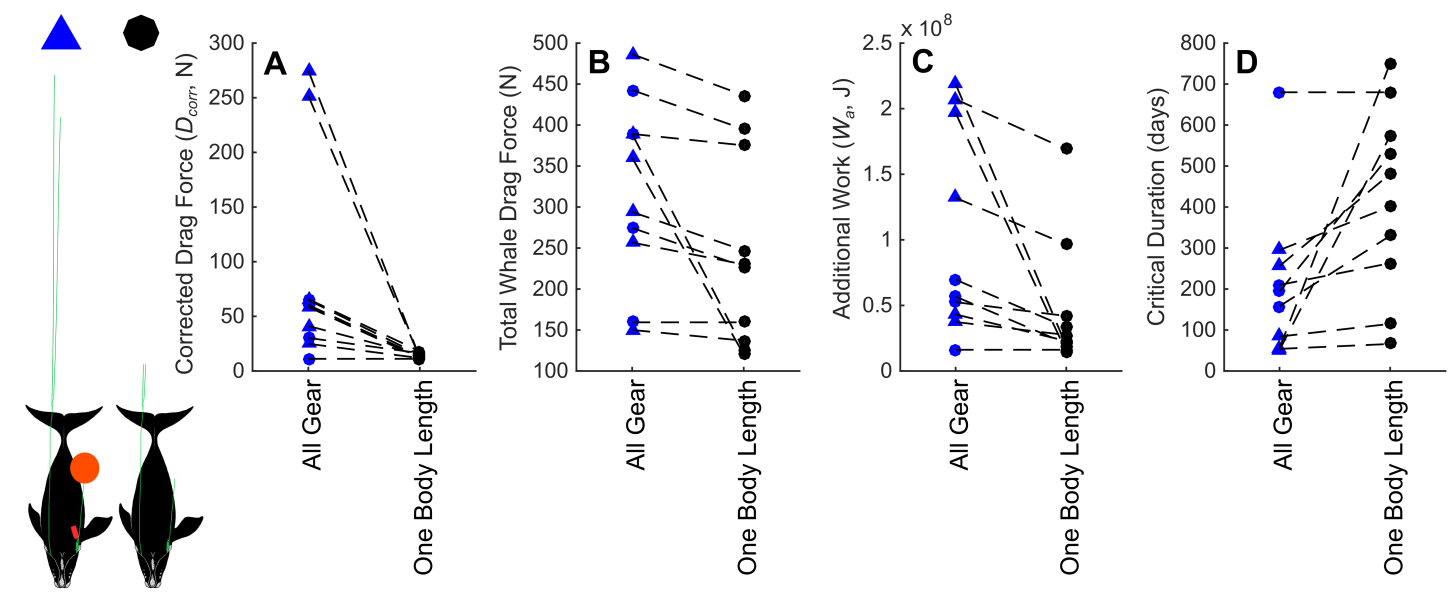

Figure 6-4: Partial disentanglement reduces drag and work, and increases critical entanglement duration. Corrected gear drag force (A), total whale drag force (B), additional work required to swim for 1 day (C) and critical entanglement duration (D) for ten North Atlantic right whales based on the fishing gear they were entangled in (blue), and that same gear when shortened to one body length, and with any floats or traps removed (black). See schematic to left. Circles represent gear sets with only line; triangles represent gear sets with additional floats or buoys.

\subsubsection{Sensitivity to gear parameters}

The critical duration estimate can include a range of gear specifics describing length, diameter, or attachment points and either the presence or absence or the dimensions of floats depending on the information available. The ten cases assessed in this study include the most refined estimates (all of the information). The sensitivity of the estimates of critical duration using different levels of information for each of the ten cases results in an average of $87( \pm 41)$ days difference (range $42-156$; Figure 5$)$. Including specific gear and float dimensions refines estimates but does not necessarily lead to more conservative or more liberal estimates. For example, including all gear and float dimensions can lead to a more conservative estimate if the float dimensions are smaller than the average floats that were measured in van der Hoop et al. (2015), resulting in a longer critical duration. Including dimensions can also lead to a less conservative estimate if gear is attached in a particularly 
disruptive configuration or if floats are larger than the average of the floats that were previously measured, resulting in a shorter critical estimate than would be estimated by simply gear length and float presence alone (Figure 5).

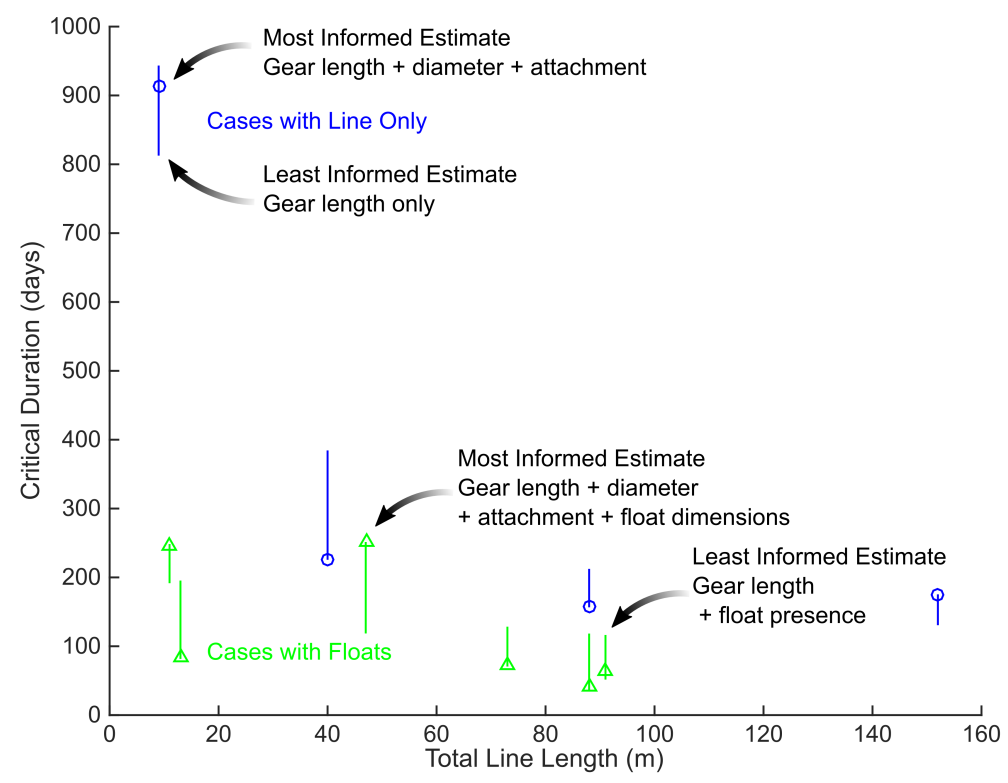

Figure 6-5: Uncertainty in gear configuration affects critical duration estimate by $\sim 3$ months. Estimates of critical duration (days) for 10 right whale entanglement cases made with different levels of gear information to show the effect of uncertainty in the gear configuration. Lines represent the range of critical duration when estimated with different levels of information; symbols denote the most informed estimate. Green is used for cases with floats, whereas blue represents cases with line only.

\subsection{Discussion}

Whales often become entangled in fishing gear, and can swim freely with a portion of gear attached to them. A combination of factors affect entanglement outcome, including gear strength and injury severity (Knowlton et al., 2015), individual condition (Pettis et al., 2004), and health impacts, entanglement duration and response efforts (Robbins et al., 2015). Post-entanglement survival has been linked to the additional energy consumption to overcome extra drag forces incurred over the duration of the entanglement (Chapter 5). This study compares measured and theoretical drag forces for configurations of fishing gear that have entangled or are similar to those entangling right whales, in order to estimate the drag on entangled whales at the time of their detection and to determine the potential time frame of their survival.

The consistent offset of measured to theoretical drag forces (e.g., Figures 1, 2) suggests that correcting values estimated from theory by a certain factor (Eqs. 10-12) can contex- 
tualize estimates of drag on unmeasured gear sets and enable comparisons with previously collected data. The consistent difference could be due to measurement (e.g., instrument sensitivity) or wave effects (e.g., propeller wash or hydrodynamic shielding from the vessel towing the gear) during the experiment that would have affected all measured gear sets in the same way. Correcting theoretical values to a standard, being the drag force measurements presented in van der Hoop et al. (2015), is essential to understand the relative risk of certain gear configurations in the context of previous studies as well as the impacts of drag loading on free-swimming animals.

\subsubsection{Assessing documented entanglements}

Ten right whale entanglement cases were documented with the necessary gear and individual information to estimate drag from theory and correct these values for comparison to previously measured drag forces (Appendix E2). These ten cases are expected to be lethal after $216 \pm 260$ days ( $\sim 7$ months; range 38-914 days, $\sim 1.25$ months to 2.5 years). This agrees with the observation in Moore et al. (2006) that most entangled right whales die within 6 months (182 days) to 1 year; however, entanglements can last much longer (Figure 6). The minimum entanglement durations exceeded the critical duration only once (Eg 3120, Figure $3 \mathrm{D})$.

Eg 3120 survived after being entangled for a minimum of 433 days, though the drag imposed by the known dimensions of the gear configuration was expected to be lethal within 250 days (Figure 3D). The entanglement was complex, crossing over the rostrum, with wraps and buoys at the peduncle; the drag from the gear contributed $38.9 \%$ to the total drag on the animal (Figure 3A). When observed 45-150 days into its entanglement, Eg 3120 appeared to be in good health, was in proximity of other whales and was feeding and defecating. 90 days later, the nuchal fat roll appeared diminished, suggesting poor body condition (Pettis et al., 2004). A partial disentanglement was successful in removing tail wraps on 24 August 2002, 243 days after its last gear-free sighting 23 Dec 2001 and 138 days after first entanglement detection 7 April 2002. The critical duration was not re-evaluated following partial disentanglement, as no data were available on the remaining gear but the partial disentanglement was conducted before the critical duration was reached. In a sighting 180 days after the partial disentanglement, the whale's overall condition appeared to have improved. Subsequent observations confirmed Eg 3120's increasing health status, and that the rest of the gear had been shed on its own. Depending on the health at the time of entanglement, a partial disentanglement occurring within the critical duration timeframe, and the ability for individuals to continue to feed (based on the time of year, location, the entanglement configuration and the effectiveness of disentanglement efforts; Chapter 5), individuals are able to persist with and recover from complex entanglements that initially impose substantial energetic costs. 
Two whales died before their critical entanglement duration was reached. Eg 2151 died after observed minimum and maximum entanglement durations of 1-99 days, much shorter than what was expected to be lethal given the amount of attached gear (i.e., the critical duration; 914 days; Figure 3D). The whale was last seen gear-free in August 1994, in good health near the end of the feeding season. At its first entangled sighting in November 1994, Eg 2151 had a tightly constricting wrap around its rostrum and upper jaw line, potentially embedded in the oral rete or impairing feeding. The whale appeared in poor condition with a heavy load of orange cyamids and thin appearance. Poor health, severe injuries, stress and the potential for feeding impairment, on top of increased energetic demand, may have led to a more rapid demise of this individual than expected, reflecting the other factors at play in survival.

In contrast, the cause of death of Eg 1238 was likely peracute underwater entrapment rather than chronic entanglement (Moore et al., 2013). Entanglement wounds did not have chronicity to them, with no signs of healing and no major cyamid proliferation. Had Eg 1238 not drowned, this method predicts a critical entanglement duration of 158 days. However, this case illustrates that gear is lethal in many different ways - long-term energy depletion, short-term severe injury or here, peracute entrapment.

The critical entanglement duration was therefore not entirely predictive of individual fate, as there is more to entanglement survival than just energetics. The configuration of the entanglement if it interferes with feeding, the timing of and body condition at the onset of entanglement (e.g. Eg 2151; Chapter 5) and the different types of entanglement injuries (e.g. Eg 1238; Cassoff et al., 2011; Moore et al., 2013) are important elements to consider. Additionally, drag is estimated from retrieved gear only: even in the event of full or partial disentanglement, not all gear is retrieved or measured. This study did not attempt to estimate drag from any remaining or unretrieved gear (e.g. the heavy monofilament line involved in Eg 3392; Appendix E1) because of a lack of scaled photographs with sufficient detail for all cases. Drag estimates for this case are therefore an underestimate and present a conservative estimate of the critical duration. Despite these limitations, critical entanglement duration is still useful in that it conservatively captures the energetic component of entanglement which can be combined with other elements to assessing entanglement cases in real time or retroactively under protected species evaluations (see below).

The ten cases presented here also represent a biased sample in that the whales for which gear is retrieved and analyzed are often disentangled or whose carcasses were discovered; eight of these ten cases were fully or partially disentangled very soon after their initial entanglement detection (minimum entanglement durations were mostly 1 day; Table 2) compared to other right whale cases (Figure 6A vs. B, C). Two cases had gear retrieved from carcasses (Table 2). Many chronically entangled whales are unable to be disentangled, and the likelihood of carcass detection or discovery is greatly reduced in these cases due to 
decreased body condition and therefore buoyancy; whales with limited blubber reserves likely sink at death. Specific data on the types and dimensions of gear involved in these chronic entanglement cases are therefore more difficult to obtain but for many cases, even those with incomplete gear information, this approach could provide a mechanism for obtaining a crude critical duration estimate to assist real-time decision making and stock assessment.

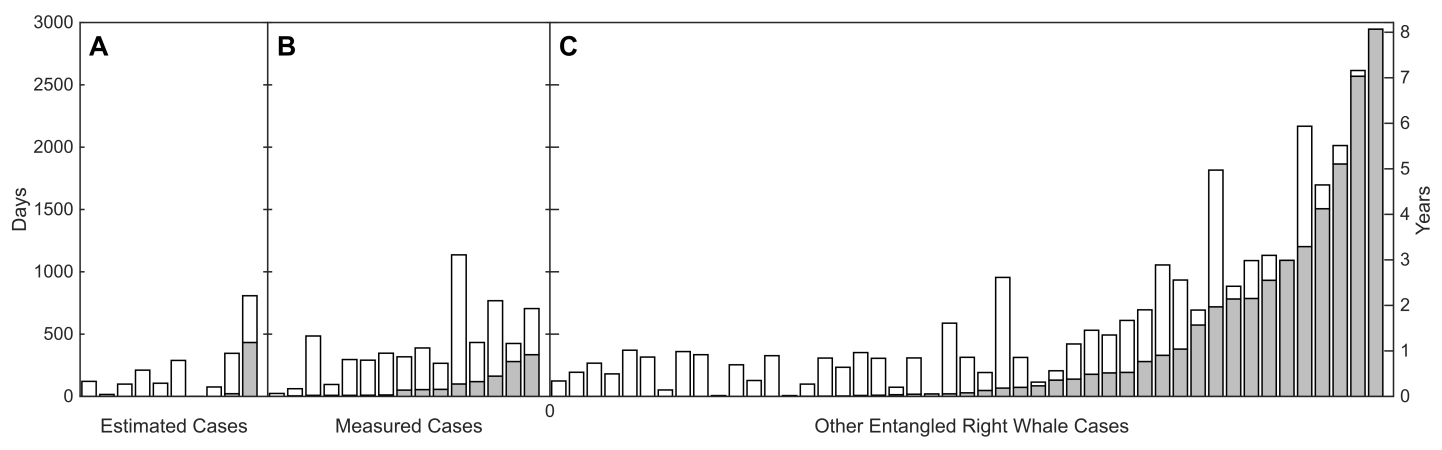

Figure 6-6: Minimum (grey) and maximum (white) entanglement durations (days) for right whale cases where drag forces on gear were Estimated (A; $n=10)$, Measured (B; $n=15)$, and for cases where insufficient data were available to estimate drag forces $(\mathrm{C} ; n=47)$.

\subsubsection{Application to entanglement assessment: Real-time}

When a whale is reported as entangled, a trained disentanglement team will develop a response action plan using information provided in the initial report. This plan confirms the entanglement and determines whether or not the whale is a candidate for disentanglement response based on the status of the population or stock, the gear characteristics, and the configuration risk of the entanglement on the individual (IWC, 2010). The entanglement configuration, based on verbal description or as it is captured in photographs, is assessed to determine the likelihood that the whale will shed the gear on its own, whether the whale is free-swimming or anchored, and the type and amount of gear involved. While considering human and animal safety as well as available resources, disentanglement operations are prioritized based on the health of the individual and the configuration of the entanglement. This study provides a method for real-time estimates of the drag imposed by the entanglement, and how long a whale entangled in a given gear configuration may survive, which can contribute to current assessment and response procedures.

Of the ten cases in this study, eight were fully or partially disentangled within 1 to 51 days of entanglement detection (Figure 3D). Robbins et al. (2015) show that most entanglement deaths occur within the first year of detection, and that human intervention increases survival probability, especially in the most high-configuration-risk cases. Even partial disentanglement can substantially reduce drag and therefore energetic impacts. Floats and buoys add $39 \mathrm{~N}$ of drag, so removing these additional elements can greatly reduce the total drag 
of entangling gear. van der Hoop et al. (2015) showed that cutting trailing line by $75 \%$ can decrease parasitic gear drag by $85 \%$; the current study demonstrates that reducing line to one body length and removing floats can extend the critical duration of these 10 entanglement cases by 304 days on average (Figure 4D). Early intervention is important to limit the deterioration of body condition and the compounding effects of low energy availability, injury and stress on individual health that could otherwise reach non-recoverable states.

\subsubsection{Application to entanglement assessment: Federal injury assessment}

The National Marine Fisheries Service (NMFS) is required to estimate annual levels of serious injury to marine mammals under the U.S. Marine Mammal Protection Act. Serious Injuries (SI) are those that may not be immediately lethal, but that are "more likely than not to result in mortality" (NMFS, 2012). The categories and criteria for large whales currently consider constricting wraps and deep lacerations as SI (Cole and Henry, 2013). While both of these situations are likely associated with the drag forces imposed by entangling gear and their duration, the evaluation criteria can and should apply the methods presented herein to incorporate energetic expenditure. Based on a limited sample set, van der Hoop et al. (Chapter 5) determined that the amount of drag (and the resulting power required to overcome it) from entangling gear was not itself a predictor of the fate of entangled individuals; however, the amount of time over which the additional energetic costs are incurred (i.e., work) is what affects individual health and survival; entanglement cases with higher drag will have shorter critical entanglement durations. Based on the results presented here, it is suggested that cases where the known or presumed duration of the entanglement exceeds the estimated critical duration should be considered SI. Appendix E2 includes a graphical approach and simplified formulae to be provided to those in charge of making SI determinations for NMFS.

As an example, these results and new formulae can be applied to three right whale cases: one that is not SI (Eg 4057), one where SI should be increased depending on future sightings (Eg 3111), and one where the whale is presumed dead and where critical duration gives additional certainty that the SI value should be increased to 1 (Eg 1019; Figure 7). Eg 4057 was observed entangled off Florida in February 2014 with extensive wounds at different stages of healing, though the entanglement appeared simple. With line woven through the baleen and trailing with no body wraps, the entanglement was defined as non-SI. Born in 2010, Eg 4057 was three years old at the time of entanglement detection. From age-lengthdrag curves (Figure A1), it is estimated that body drag for Eg 4057 swimming at $1.2 \mathrm{~m} / \mathrm{s}$ is $147 \mathrm{~N}$. The gear on Eg 4057 was described as $155 \mathrm{~m}$ of line, including a portion trailing $30 \mathrm{~m}$ aft of the flukes. No floats or buoys were involved in the original entangling gear. From van der Hoop et al. (2015), the entangling gear added $81.5 \mathrm{~N}$ of drag across speeds of $0.5-3.0 \mathrm{~m} / \mathrm{s}$. Assuming $1.6 \mathrm{~cm}\left(5 / 8^{\prime \prime}\right)$ diameter line, the corrected drag ( $D_{\text {corr }}$, Eq. 10) 
from the theoretical estimate $\left(D_{\text {theor }}\right.$, Eq. 1$)$ is $89 \mathrm{~N}$. The interference drag $\left(D_{I}\right)$ in this case is negligible, $<1 \mathrm{~N}$. Together, these estimates suggest a minimum critical entanglement duration of 118 days, which would be 14 June 2014 (Figure 7A).
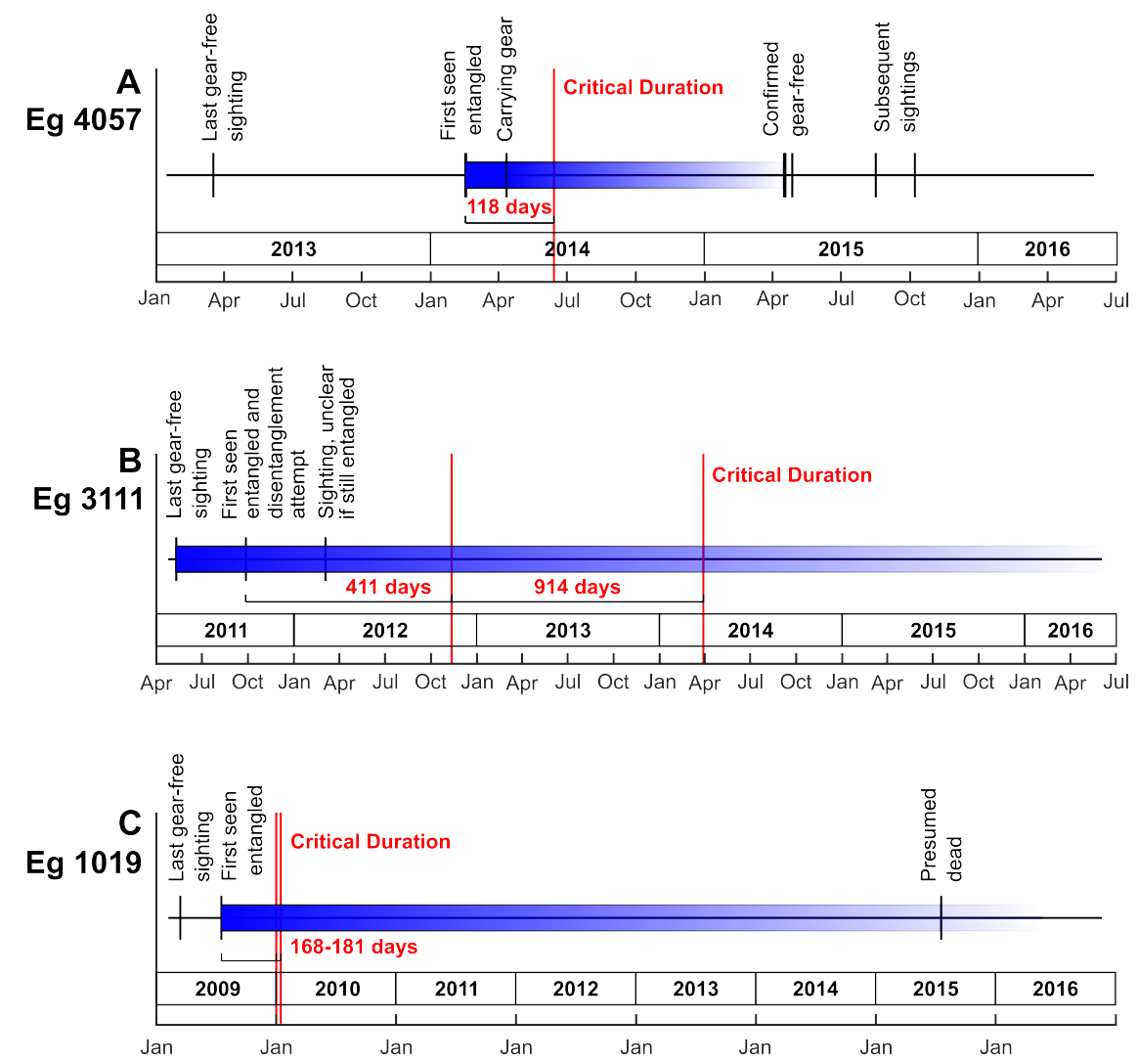

Figure 6-7: Instances of sighting (vertical black lines) during the entanglement timelines of right whales Eg 4057 (A), Eg 3111 (B) and Eg 1019 (C). Onset and duration are shown in blue, with the proposed critical entanglement duration for classification as Serious Injury (SI; red). The entanglement in panel A fades as the entanglement was shed between those confirmed sightings. Panels $\mathrm{B}$ and $\mathrm{C}$ fade through time as the animal may have shed gear during that time and the likelihood of present entanglement decreases with time, but note the method assumes no change in the gear during the entangled duration since last observation. Date ranges are due to uncertainty in gear dimensions on whales B and $\mathrm{C}$.

Eg 4057 was sighted still carrying gear on 12 April 2014, 55 days from the initial entanglement detection. A year later, multiple sightings in April 2015 confirmed that Eg 4057 was gear-free. Based on this timeline, Eg 4057's entanglement does not fit the proposed SI criterion in that it was not observed carrying gear beyond the minimum critical entanglement duration, i.e., the whale apparently shed the gear before the $147 \mathrm{~d}$ threshold. Entanglements that have lasted longer than the minimum critical duration should be considered serious injury, i.e., had Eg 4057 been sighted still carrying gear after 13 July 2014 or if retroactive analysis of catalogued photographs reveal evidence of entanglement at an earlier date (Figure 7A). It is common for whales to be already in compromised condition when first detected with gear. In this event it can be assumed that the whale had already 
been entangled for some period of weeks to months, but such a presumed duration cannot be added to the observed entanglement duration due to too many unknowns; future research to determine the rate of observable health declines or changes in body condition could enable these types of estimates.

Eg 4057 is a data-rich case, in contrast to Egs 3111 and 1019. Poorly documented cases often fall under more generalized SI criteria (e.g., L10) where they are prorated by 0.75 to account for uncertainty (NMFS, 2012; Cole and Henry, 2013). Eg 3111 was first sighted entangled in Sept 2011, and a disentanglement attempt occurred that same day. This attempt may have removed a portion of the gear, leaving 6-36 $\mathrm{m}$ remaining for a critical duration of 411-914 days. The whale was resighted 159 days later in March 2012 (Figure 7B) with improved skin condition and no gear was visible. However, sighting conditions prevented confirming if Eg 3111 had shed the remaining gear and the whale has yet to be seen since. If Eg 3111 is entangled at its next sighting or if it is not seen again by March 2018 and is therefore presumed dead, SI should be increased from 0.75 to 1. Eg 1019 was first seen entangled July 2009 with no further sightings, and was presumed dead in July 2015. A critical duration of 168-181 days was estimated based on 21.3-30.5 m of line with a 20 " ball buoy. The SI value for this case is 0.75 but should be increased to 1 : the critical duration gives additional certainty that the whale is more likely than not dead, i.e., seriously injured by definition (NMFS, 2012).

For this application, critical duration estimates should be adjusted following partial disentanglement (e.g., removal of floats, reducing training line) as small reductions in drag can significantly increase an individual's endurance (Figure 4D). However, if the disentanglement occurs after the critical duration is reached, the case should still be considered SI: energetic impacts may have already been sufficient to affect health and reduce survival probabilities even after disentanglement (e.g., Eg 3911, Moore et al., 2012; van der Hoop et al., 2013b).

\subsubsection{Methodological considerations}

Separating the lobster trap, telemetry buoy, and gillnet from the other gear sets is warranted based on the difference in the relationships between measured and theoretical drag, but also based on the gear characteristics themselves (Figure 2). The lobster trap and telemetry buoy are not only large and poorly streamlined, but are also heavy - the two-brick trap weighing $15.7 \mathrm{~kg}$ (34.6 lbs) and the telemetry buoy $18.2 \mathrm{~kg}$ (40.1 lbs) are the two heaviest sets of gear measured. In cases where there is evidence of weight attached to the trailing line, the equation for the lobster trap should be used. Although the amount of weight may not be known at the time of entanglement detection, the two-brick trap tested here (34.6 lbs) is lighter than most used in the Gulf of Maine lobster fishery (40-65 lbs; McCarron and Tetreault, 2012). Other pot gear may be lighter (e.g. blue crab $\sim 20$ lbs) or heavier (e.g. snow crab $\sim 40 \mathrm{lbs}$ ), or when whales tow more than one trap. Gillnet gear has a 
much lower measured drag than is expected from theory, likely due to the high surface area of the large mass of tangled gear (Figure 2). Water flow through or around the tangled mass is unknown, especially when it is wrapped around the whale's body. Drag is easier to estimate for simpler sets of gear (e.g., trailing line, Figure 1) or those with well-described accessories (e.g., floats or buoys, Figure 2); it is more difficult to estimate drag on gear that wraps the body. Computational fluid dynamics (CFD) may prove a more useful way to investigate the flow properties, drag conditions, and energetic cost associated with these types of entanglements.

There are now three ways to estimate drag from entangling fishing gear at the time of first entanglement: the methods presented herein, and the weight-drag and length-drag equations presented in van der Hoop et al. (2015). Whereas dry weight can be assessed long after gear is recovered from an animal that has been disentangled, this method is least likely to be beneficial when whales are first reported entangled. Gear length, however, is frequently described in entanglement reports. The length-drag equation in van der Hoop et al. (2015) requires only the length of the gear and the presence of floats to estimate the average added drag across all measured speeds $(1.27 \mathrm{~m} / \mathrm{s})$ with comparable fit $\left(\mathrm{R}^{2}=0.812\right.$, $\mathrm{RMSE}=21.2$ vs. $\left.\mathrm{R}^{2}=0.846, \mathrm{RMSE}=27.2\right)$. Estimating drag from theory incorporates not only the length of the line and the presence of floats, but also their dimensions (e.g., diameter, shape) and interference drag from the point of attachment. While interference drag can be insignificant in many cases (Figure 3A; example of Eg 4057 above), it can contribute up to $75 \%$ of total gear drag in others (e.g., Eg 1971, Figure 3A) where line wraps the body multiple times or where floats are located at the body's surface rather than trailing behind. Drag also can be estimated at whatever swimming speed is observed, depending on the behavior or health status of the animal; in this study, it is assumed that individuals maintain swimming speeds of $1.23 \mathrm{~m} / \mathrm{s}$, but speed is not always maintained in high drag conditions (Chapter 4; van der Hoop et al., 2014a) and right whale swimming speeds can range 0.4-4 m/s (Mate et al., 1997; Hain et al., 2013). Overall, drag should be estimated from theory and corrected to measured drag even with insufficient detail or a range of gear dimensions with an acknowledgement of potential inaccuracy and using the more conservative estimate e.g., Egs 3111 and 1019 above, where $30 \mathrm{~m}$ and $10 \mathrm{~m}$ differences in gear length estimates led to 503- and 13-day differences in critical durations (Figure 7B, C). Sensitivity analyses show that using different levels of information to describe the same gear configurations yields on average 87 day differences in critical duration estimates (Figure $5)$. The most informed estimate is not necessarily the most or least conservative, though it is the most accurate and most refined. The range in estimated critical duration is often within sightings gaps of entangled whales (e.g., Figure 7); it is therefore still useful to estimate this range with limited information for inclusion Serious Injury determinations at the federal level. 
The relationships established herein were applied to well-documented entanglement cases, where gear was removed, recovered, and measured. Efforts to recover gear from disentanglement operations or at death have allowed for a better understanding of the types of gear that frequently entangle whales, their common configurations on certain species (Johnson et al., 2005), their breaking strengths (Knowlton et al., 2015) and the drag forces they add to entangled animals (van der Hoop et al., 2013b, 2015). Even with disentanglement response, gear cannot be always be recovered; the majority of entanglements are only observed and described at sea. Estimates of range and size by humans are often inaccurate ( $10 \%$; Rohner et al., 2011) and variable (Øien and Schweder, 1992), though the magnitude, bias, and variability of estimation error decrease with experience (Baird and Burkhart, 2000). The methods presented in this study should be applied to cases where gear configurations are well documented (e.g., with photographs or video with a scale or reference object) or are described by experienced observers. This would ensure that gear dimensions, from which drag and survival estimates are derived, are as accurate as possible.

\subsubsection{Acknowledging variability}

The assessment of the critical duration is based on the additional energy required to overcome increased thrust production associated with entanglement drag (see Eq. 9). As such, the estimate is robust to uncertainty in whale age or length. It does not, however, take into account differences in energy stores available for different age or sex classes of whales.

This and previous studies (Johnson et al., 2005; van der Hoop et al., 2013b, 2015) show the variability in the types, dimensions, components, and configurations of gear that entangle right whales and other large whales. Variability in these elements affects the total drag; further, the points of attachment and the dimensions of the gear at the attachment points affect the relative contribution of interference drag vs. total drag (Figure 3A). Photographic and video documentation of the gear with spatial references or measurements are extremely helpful in estimating gear dimensions and placement on the whale. Efforts to document and draw entangling configurations have proven extremely useful for disentanglement response (IWC, 2010), for determination and definition of serious injuries to protected species (NOAA, 2008; Moore et al., 2013), and for assessment of the hydrodynamic effects of entanglement (herein; van der Hoop et al., 2013b, 2015).

There is also considerable variability in the dimensions of the whales that become entangled. Right whale calves and juveniles are more frequently found entangled than other life stages; though they make up only $29 \%$ of the population, over $50 \%$ of seriously entangled right whales are juveniles (Knowlton et al., 2012a). Similar trends hold in humpback whales (Knowlton et al., 2015) and other species (Fowler, 1987; Moore et al., 2009; McIntosh et al., 2016). The high incidence of entanglement in smaller animals means a larger relative size of the gear to the animal: gear with the same dimensions would contribute a greater amount to 
the animal's total entangled drag (Figure 3A). Swimming costs are therefore proportionally greater for smaller animals (Feldkamp, 1985).

Over the lifespan of a right whale (up to 70 years; Fortune et al., 2012), body length increases by $\sim 10 \mathrm{~m}$, and girths by $\sim 1.6 \mathrm{~m}$ (Fortune et al., 2012). Other natural life events can alter body shape significantly: pregnant right whales increase $4-25 \mathrm{~cm}$ in width in various positions along the body, lactating southern right whales (Eubalaena australis) can lose $21.8 \pm 6.1 \mathrm{~cm}$ in 3-4 months (Miller et al., 2012b) and migration can lead to significant reductions in body width (Perryman and Lynn, 2002) and weight (11-29\%; Rice and Wolman, 1971) in gray whales (Eschrichtius robustus). Unnatural, though extremely common in the right whale population (Knowlton et al., 2012a), chronic entanglement in fishing gear can reduce body diameter by $20 \%$ compared to mesomorphic right whales (van der Hoop et al., 2013a) and can reduce body weight by $28 \%$ (Barratclough et al., 2014). Almost all (49/50) photo identified entangled right whales are in good body condition at their last sighting prior to entanglement detection (Robbins et al., 2015).

Changes in body shape will affect whales' hydrodynamic efficiency and the relative contribution of gear drag, at variable and unknown rates (Appendix E3). If an adult whale (e.g., Eg 1223; 12 years old, 13.6 m, 32670 kg measured at necropsy; Barratclough et al., 2014) loses $28 \%$ of its body weight and $20 \%$ body diameter over the course of its entanglement, the whale's drag coefficient would decrease by $6.5 \%$ (0.0062 to 0.0058$)$ and its fineness ratio (body length/width) would increase by $25 \%$ (4.64 to 5.81), away from the optimal 4.5 (Ahlborn et al., 2009; Hoerner, 1965). These changes in body weight and girth would decrease drag by $24 \%$. In contrast, while maintaining body condition, the increase in length from juvenile (e.g., 2 years old, $11.1 \mathrm{~m}$ ) to adult (e.g., 28 years, $14.7 \mathrm{~m}$; a $33 \%$ increase) life stages leads to a $72.3 \%$ increase in drag force, and a $6 \%$ increase in drag coefficient, while the fineness ratio is essentially unchanged. There is therefore an interplay between increases in length with age, and decreases in body condition with entanglement duration, along with the nonlinear dynamics of both, that will be unique for every entangled whale based on age, configuration, health status, geographic location, and time of year. The estimates herein begin to combine these elements in their simplest form - considering individual length and girth at the onset of entanglement - but do not consider the intricate dynamics of these body shape changes and their effect on the drag regime.

\subsection{Conclusions}

It is possible to estimate drag from fishing gear at the time that an entangled whale is detected or reported. These estimates can be incorporated into the case assessment and development of disentanglement action plans (IWC, 2010). The observed and estimated critical entanglement durations should also be included in the decision-making process and 
can be valuable in determining whether entanglement cases are life-threatening or qualify as Serious Injuries for federally protected species (NOAA, 2008). This method could also be applied to other large whale species with significant entanglement-related mortality and injury rates (Cole and Henry, 2013; van der Hoop et al., 2013a). 


\section{Chapter 7}

\section{BOTTLENOSE DOLPHINS MODIFY BEHAVIOR TO REDUCE METABOLIC EFFECT OF TAG ATTACHMENT}

This chapter was originally published as van der Hoop, J. M., Fahlman, A., Hurst, T., Rocho-Levine, J., Shorter, K. A., Petrov, V. and Moore, M. J. (2014). Bottlenose dolphins modify behavior to reduce metabolic effect of tag attachment. Journal of Experimental Biology 217, 4229-4236.

JvdH, AF, KAS, VP, JR-L and MJM developed concepts; JR-L directed animal husbandry and training; JvdH, AF, KAS, VP, TH and JR-L performed experiments and simulations; JvdH and AF processed and analyzed data; JvdH, AF, KAS, VP, TH, JR-L and MJM wrote the manuscript. 


\subsection{Abstract}

Attaching bio-telemetry or -logging devices (tags) to marine animals for research and monitoring adds drag to streamlined bodies, thus affecting posture, swimming gaits and energy balance. These costs have never been measured in free-swimming cetaceans. To examine the effect of drag from a tag on metabolic rate, cost of transport and swimming behavior, four captive male dolphins (Tursiops truncatus) were trained to swim a set course, either non-tagged $(n=7)$ or fitted with a tag (DTAG2; $n=12)$, and surface exclusively in a flowthrough respirometer in which oxygen consumption $\left(\dot{V} \mathrm{O}_{2}\right)$ and carbon dioxide production $\left(\dot{V} \mathrm{CO}_{2} ; \mathrm{ml} / \mathrm{kg} / \mathrm{min}\right)$ rates were measured and respiratory exchange ratio $\left(\dot{V} \mathrm{O}_{2} / \dot{V} \mathrm{CO}_{2}\right)$ was calculated. Tags did not significantly affect individual mass-specific oxygen consumption, physical activity ratios (exercise $\dot{V} \mathrm{O}_{2} /$ resting $\dot{V} \mathrm{O}_{2}$ ), total or net cost of transport (COT; $\mathrm{J} / \mathrm{m} / \mathrm{kg}$ ) or locomotor costs during swimming or two-minute recovery phases. However, individuals swam significantly slower when tagged (by $11 \%$; mean \pm s.d., $3.31 \pm 0.35 \mathrm{~m} / \mathrm{s}$ ) than when non-tagged $(3.73 \pm 0.41 \mathrm{~m} / \mathrm{s})$. A combined theoretical and computational fluid dynamics model estimating drag forces and power exertion during swimming suggests that drag loading and energy consumption are reduced at lower swimming speeds. Bottlenose dolphins in the specific swimming task in this experiment slowed to the point where the tag yielded no increases in drag or power, while showing no difference in metabolic parameters when instrumented with a DTAG2. These results, and our observations, suggest that animals modify their behavior to maintain metabolic output and energy expenditure when faced with tag-induced drag.

\subsection{Introduction}

Bio-telemetry and bio-logging devices (tags) allow for direct measurements of movement and behavior in free-ranging animals. These technologies have been especially useful for the study of marine animals, which perform the majority of life functions out of view. Tag data have provided insights into the physiology, spatial ecology, acoustics and kinematics of marine animals, and have been used in combination with other measures (e.g. prey field sampling, genetics, oceanography) to interpret the role of a tagged animal in its environment. As technologies have improved, the cost and size of tags have been reduced, whereas sensing capabilities have increased. This has led to growth in the number and diversity of tags and study subjects (Crossin et al., 2014), as well as scientific efforts to deploy tags: the number of permits issued in the United States for tagging studies on marine turtles alone has tripled in the last decade (Jones et al., 2013).

However, the attachment of external devices is not benign (for reviews, see e.g. Wilson et al., 2006; McMahon et al., 2011). Whereas animal-specific rules intended to minimize tag 
impact have been established, e.g. that bird tags should not exceed 3 or $5 \%$ of the animal's body mass (Vandenabeele et al., 2011), there currently exist few guidelines for aquatic, terrestrial or flying mammals (American Society of Mammalogists, 1998). Of 559 published studies having deployed bio-logging tags on free-ranging marine mammals from 1965 until 2012 , only $2.5 \%$ addressed device influence, and only $1 \%$ focused on cetaceans (Costa and Gentry, 1986). The difficulty of establishing suitable controls by quantifying behavior and energetics from untagged animals at the same resolution as tagged animals probably limits the ability to perform such investigations (Shorter et al., 2013).

For marine mammals, hydrodynamic drag is of primary concern, where tag volume, shape, position and presence (and if so, size) of an antenna can significantly affect drag loading (Bannasch et al., 1994; Culik et al., 1994; Wilson et al., 2004; Jepsen et al., 2005; Vandenabeele et al., 2011). In an experimental study, Skrovan et al. (1999) showed that instrumented dolphins experience higher drag loading, especially when tags are quite large compared with the subject. To minimize the impact to the animal, design and analysis tools [e.g. computer-aided design (CAD) and computational fluid dynamics (CFD)] and physical models are used to estimate and predict drag coefficients and force balances of tag subjects with a given tag position or orientation (Bannasch et al., 1994; Pavlov et al., 2000; Yanov, 2001; Culik et al., 1994; Jones et al., 2013; Shorter et al., 2013).

Although these studies have provided useful estimates of the effect of drag from instruments, the models do not capture the full dynamics of a free-swimming animal. In situ measurements are required to determine how changes, such as body undulation, unsteady flow and tag movement (i.e. sliding) affect forces and moments on an animal, and to directly couple the effect of tag drag with changes in energy consumption. Previous studies on drag manipulation in marine mammals have shown changes in metabolic cost measured directly via respirometry (Feldkamp, 1987), or indirectly by metabolic heat production (Cornick et al., 2006). It is therefore reasonable to assume that increased drag from an instrument could translate into an increase in metabolic cost (Boyd et al., 1997; Jones et al., 2013). Using the doubly labeled water method, Boyd et al. (1997) found an average 19\% increase in oxygen consumption in female Northern fur seals at sea over a number of weeks, when wearing a tag estimated to increase drag by up to $70 \%$. To our knowledge, no investigations of this kind have been carried out with cetaceans.

As tag designs progress (Balmer et al., 2013; Shorter et al., 2013), it is crucial to quantify the impact of a tag on the subject and to determine whether amelioration is required. Here, the modeled increase in drag created by a tag is combined with an experimental study of the energetic cost of swimming with and without a tag. A conventional drag model is used to quantify the effect of different swimming speeds and forces on power output, and to provide insight into the experimental results. It is hypothesized that the added drag from the tag will result in increased energetic output during the swimming task. This hypothesis is tested 
using experiments with four trained bottlenose dolphins (Tursiops truncatus Montagu 1821) that perform a series of swimming tasks when wearing and not wearing a bio-logging tag (Digital Acoustic Recording Tag, DTAG2; Johnson and Tyack, 2003). Metabolic parameters and swimming speed of the animals were measured to determine the energetic and behavioral effects of instrumentation on a small cetacean. This work presents an experimental design for the direct measurement of behavioral modifications created by biologging tags on cetaceans for the first time.

\subsection{Results}

The conceptual model illustrates tradeoffs between swimming speed and drag forces when wearing and not wearing a tag (Fig. 1A). Individuals swam significantly slower (by $11 \%$; $F_{1,14}=7.24, P=0.0176$ ) when wearing a tag (mean \pm s.d. $3.31 \pm 0.35 \mathrm{~m} / \mathrm{s}$ ) than when non-instrumented $(3.73 \pm 0.41 \mathrm{~m} / \mathrm{s}$; Fig. $2 \mathrm{C})$. No individual variation in swimming speed was detected $\left(F_{3,14}=2.79, P=0.0794\right)$. Because of this observed decrease in swimming speed when wearing a tag, the model predicts an average change in drag of $-4.1 \mathrm{~N}$ (range from -13.3 to $0.4 \mathrm{~N}$ ) or $-7 \%$ (range from $-20.5 \%$ to $9.3 \%$ ) when instrumented (Fig. 1A, blue $=$ tag versus black $=$ no tag). Had individuals maintained the faster swimming speeds observed during the non-tagged trials, the modeled drag force would have increased by 10.1 N (8.2-11.6 N) or by 15\% (12.9-16.3\%; Fig. 1A, red). Estimates of power output between non-tagged and tagged trials at their observed speeds were not significantly different (Fig. 1B, blue, black; Student's t-test, $T_{17}=1.12, P=0.279$ ). However, maintaining a faster swimming speed with the increased drag loading created by the tag would require the animal to significantly increase power output during swimming, by 29-59\% (Fig. 1B, red; Student's t-test, $\left.T_{17}=-2.22, P=0.041\right)$. Slowing down to observed speeds reduced potential drag loading by $14.3 \mathrm{~N}(7.7-24.8 \mathrm{~N})$ and power expenditure by $530 \mathrm{~W}(270-920 \mathrm{~W})$ or $41.7 \%$ (26.8-61.5\%).

During the experiment, respiratory gases were measured for four male bottlenose dolphins (Table 1) from 11 to 15 November 2012. From this, metabolic rate was calculated before, during and after the animals completed a set swimming protocol, either noninstrumented $(n=7)$ or while wearing a DTAG2 (Fig. $3 ; n=12$ ). The number of trials per individual and the order in which they were performed are listed in Table 1. Across individuals, no significant linear trends in swimming $\dot{V} \mathrm{O}_{2}\left(P=0.130-0.581 ; R^{2}=0.08-0.96\right)$ or speed $\left(P=0.147-0.465 ; R^{2}=0.21-0.59\right)$ with trial number were apparent.

Individuals showed no difference in oxygen consumption rate $\left(\dot{V} \mathrm{O}_{2} ; \mathrm{ml} \mathrm{O}_{2} / \mathrm{kg} / \mathrm{min}\right)$ when wearing versus when not wearing a tag during rest, swim (Fig. 2A) or two-minute recovery phases (Table 2). Oxygen consumption rates were significantly different between individuals for all phases $\left(F_{3,14}=4.85,4.07,9.11 ; P=0.0162,0.0285,0.0013\right.$, respectively $)$. 

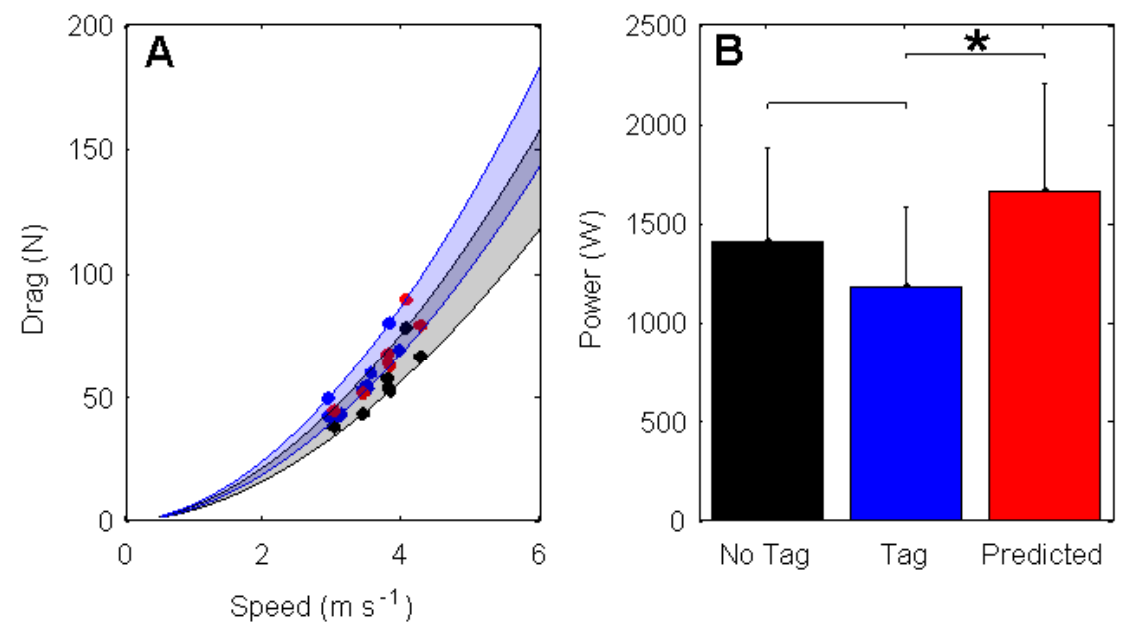

Figure 7-1: Bio-logging tags increase the drag forces experienced by bottlenose dolphins. (A) Envelope of the drag force $(\mathrm{N})$ estimated for the four bottlenose dolphins in this study when not wearing (black) and wearing (blue) bio-logging tags across a range of swimming speeds (lines; $\mathrm{m} / \mathrm{s}$ ) and for specific observed swimming speeds (dots; $\mathrm{m} / \mathrm{s}$ ). Red dots reflect the predicted drag loading in the tagged condition if individuals maintained their non-tagged swimming speed. (B) Mean \pm s.d. power output (W) estimated for when (blue) wearing a tag, swimming at observed speeds; and (black) not wearing a tag, swimming at observed speeds; and predicted for when (red) wearing a tag, if individuals had maintained their non-tagged swimming speed.

The slopes and intercepts of the respiratory exchange ratio $\left(\mathrm{RER}, \dot{V} \mathrm{O}_{2} / \dot{V} \mathrm{CO}_{2}\right.$.) throughout the recovery period did not significantly differ between tag and no-tag conditions (Table 2), and significant individual variability was evident in the slopes $\left(F_{3,14}=4.37, P=0.0228\right)$ but not the intercepts $\left(F_{3,14}=1.46, P=0.268\right)$ of the recovery RER. Measured RER values for resting $(1.00 \pm 0.02)$ were significantly greater than during swimming $(0.96 \pm 0.01$; $T_{36}=7.430, P<0.001$ ); there was no significant difference in RER between swimming and recovery $\left(0.97 \pm 0.01 ; T_{36}=-1.9405, P=0.060\right)$.

Table 7-1: Body sizes, resting metabolic rates and order of experimental trials. Measured body length, girth $(\mathrm{m})$ and weight $(\mathrm{W}, \mathrm{kg})$, calculated wetted surface area $\left(\mathrm{m}^{2}\right)$, mean \pm s.d. resting metabolic rate (RMR; $\mathrm{ml} \mathrm{O}_{2} / \mathrm{kg} / \mathrm{min}$ ) calculated over $N$ fed trials and the order of $N$ experimental trials $(\mathrm{C}=$ Control; $\mathrm{T}=$ Tag $)$ for four male bottlenose dolphins. Wetted surface area was calculated from mass as $A=0.08 W^{0.065}$ from (Fish, 1993), based on a number of odontocete species.

\begin{tabular}{|l|l|l|l|l|l|l|}
\hline Individual & $\begin{array}{l}\text { Length } \\
(\mathrm{m})\end{array}$ & $\begin{array}{l}\text { Girth } \\
(\mathrm{m})\end{array}$ & $\begin{array}{l}\text { Weight } \\
(\mathrm{kg})\end{array}$ & $\begin{array}{l}\text { Wetted sur- } \\
\text { face area } \\
\left(\mathrm{m}^{2}\right)\end{array}$ & $\begin{array}{l}\text { Mean+s.d. fed } \\
\text { RMR }(N \text { tri- } \\
\text { als })\end{array}$ & $\begin{array}{l}\text { Experimental } \\
\text { trial order }(N \\
\text { trials })\end{array}$ \\
\hline Kolohe & 2.61 & 0.44 & 186.9 & 2.3 & $6.33 \pm 1.54(7)$ & CTTCT $(5)$ \\
\hline Liko & 2.54 & 0.40 & 160.6 & 2.2 & $7.95 \pm 1.49(8)$ & CTTTCT $(6)$ \\
\hline Lono & 2.73 & 0.47 & 249.5 & 2.9 & $6.96 \pm 1.39(6)$ & TCT $(3)$ \\
\hline Nainoa & 2.46 & 0.41 & 165.6 & 2.2 & $5.22 \pm 1.43(7)$ & CTTTC $(5)$ \\
\hline
\end{tabular}

Whereas individuals had significantly different physical activity ratios (PAR; $F_{3,14}=$ 

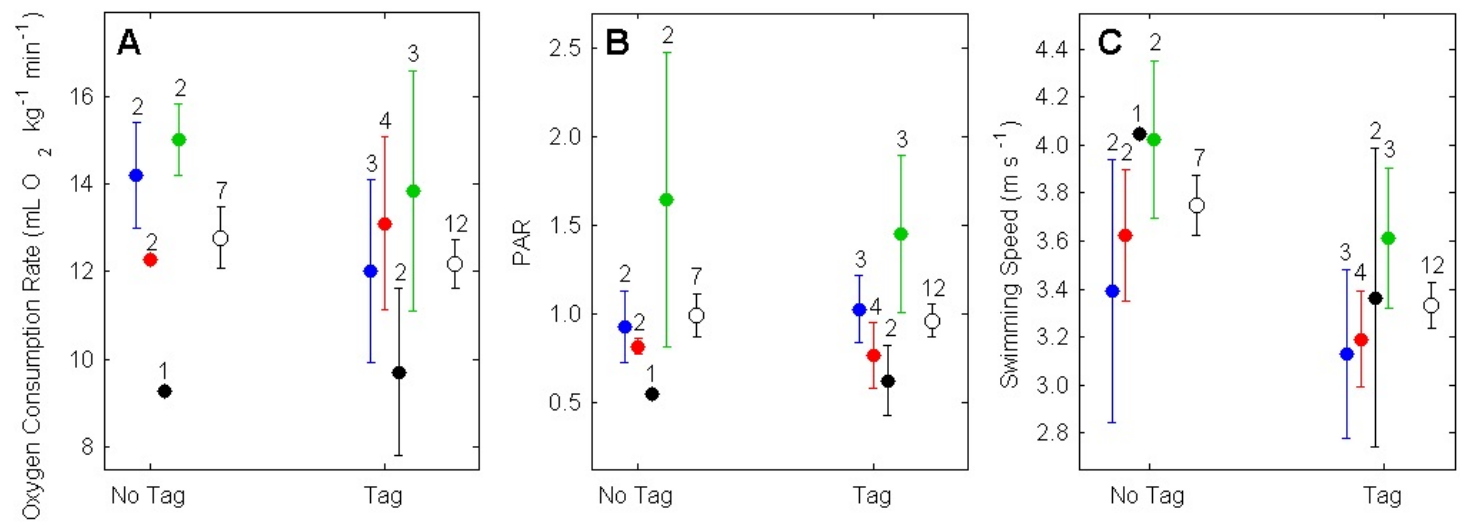

Figure 7-2: Bio-logging tags do not significantly affect the metabolic rate of bottlenose dolphins, but tagged dolphins swim at significantly lower speeds. (A) Individual mean \pm s.d. (Kolohe, blue; Liko, red; Lono, black; Nainoa, green) and marginal mean \pm s.e.m. (white) oxygen consumption rates $\left(\dot{V} \mathrm{O}_{2} ; \mathrm{ml} \mathrm{O}_{2} \mathrm{~kg}^{-1} \mathrm{~min}^{-1}\right)$. (B) Physical activity ratios (PAR, nondimensional) measured during swimming, and (C) swimming speeds $\left(\mathrm{m} \mathrm{s}^{-1}\right)$ of four bottlenose dolphins, when not wearing and when wearing a bio-logging tag.

$7.12, P=0.0039)$, being the energetic cost of a specific activity over the resting metabolic rate, there was no significant effect of wearing a tag (Fig. 2B, Table 2). This parameter indicates that on average the swimming task increased $\dot{V} \mathrm{O}_{2}$ by a factor of $2.01 \pm 0.89$ over resting values. Although it was expected, individual total and net cost of transport $\left(\mathrm{COT}_{\text {tot }}, \mathrm{COT}_{\text {net }} ; \mathrm{J} / \mathrm{m} / \mathrm{kg}\right)$ were not significantly greater when wearing $\left(\mathrm{COT}_{\text {tot }}=1.32 \pm 0.01\right.$, $\left.\mathrm{COT}_{\text {net }}=0.612 \pm 0.095\right)$ than when not wearing a tag $\left(\mathrm{COT}_{\text {tot }}=1.18 \pm 0.12, \mathrm{COT}_{\text {net }}=0.371\right.$ $\pm 0.385 ; T_{3}=1.49,1.41 ; P=0.116,0.125$, respectively; Table 2). Similarly, mean individual locomotor costs $(\mathrm{LC})$ were not significantly higher in tagged $(0.47 \pm 0.10)$ than in non-tagged trials $\left(0.32 \pm 0.32, T_{3}=0.993 ; P=0.197\right.$; Table 2$)$.

Pre-exercise resting metabolic rates were measured when individuals were fasted and when they had been fed up to $6.2 \mathrm{~kg}$ of a mix of herring, capelin and squid, depending on the time of day. Individuals had significantly higher resting oxygen consumption rates $\left(\dot{V} \mathrm{O}_{2}\right)$ when fed $(n=28$; mean \pm s.d. $6.65 \pm 1.73 \mathrm{ml} \mathrm{O} 2 / \mathrm{kg} / \mathrm{min})$ compared with fasted $(n=10$; $\left.4.34 \pm 0.53 \mathrm{ml} \mathrm{O} / \mathrm{kg} / \mathrm{min} ; F_{1,33}=21.44 ; P<0.001\right)$. There was no significant difference in $\operatorname{RER}\left(F_{1,33}=1.58 ; P=0.217\right)$ between fasted $(0.994 \pm 0.019)$ and fed $(1.00 \pm 0.0203)$ rest periods. Significant individual variability was observed in resting $\dot{V} \mathrm{O}_{2}\left(F_{3,33}=5.45 ; P=\right.$ $0.0037)$ and $\operatorname{RER}\left(F_{3,33}=3.38 ; P=0.0298\right)$. As such, individuals were fed during the experimental trials $(n=19)$.

\subsection{Discussion}

When faced with higher drag loading, either naturally (Williams, 1989), experimentally (Cornick et al., 2006), or inadvertently (van der Hoop et al., 2013b), marine mammals have 
Table 7-2: Oxygen consumption rates, physical activity ratios, respiratory exchange ratios and costs of transport. Mean \pm s.d. oxygen consumption rates $\left(\dot{V} \mathrm{O}_{2}\right)$ during rest, swim and recovery phases; physical activity ratio (PAR); slopes and intercepts fit to the respiratory exchange ratio (RER) during post-exercise recovery; total and net cost of transport (COT); and the contribution of locomotor costs to COT in four male bottlenose dolphins performing a swimming task while not wearing and while wearing a bio-logging tag. Test $(F$ and $t)$ and $P$ statistics are for the effect of tag in two-way ANOVA with no interaction and Student's $t$-tests, respectively.

\begin{tabular}{|l|l|l|l|l|}
\hline & No tag & Tag & $F_{\text {tag }(1,14)}$ & $P_{\text {tag }}$ \\
\hline Resting $\dot{V} \mathrm{O}_{2}(\mathrm{ml} \mathrm{O} / \mathrm{kg} / \mathrm{min})$ & $7.04 \pm 1.71$ & $7.01 \pm 2.10$ & 0.08 & 0.787 \\
\hline Swim $\dot{V} \mathrm{O}_{2}(\mathrm{ml} \mathrm{O} / \mathrm{kg} / \mathrm{min})$ & $13.2 \pm 2.2$ & $12.4 \pm 2.4$ & 0.46 & 0.508 \\
\hline Recovery $\dot{V} \mathrm{O}_{2}(\mathrm{ml} \mathrm{O} / \mathrm{kg} / \mathrm{min})$ & $13.4 \pm 2.4$ & $13.7 \pm 3.5$ & 0.01 & 0.922 \\
\hline PAR & $2.10 \pm 1.11$ & $1.96 \pm 0.79$ & 0.04 & 0.840 \\
\hline RER slope & $(7.60 \pm \pm$ & $(9.13 \pm \pm$ & 0.08 & 0.781 \\
& $7.33) \times 10^{-6}$ & $8.17) \times 10^{-6}$ & & \\
\hline RER intercept & $0.967 \pm 0.017$ & $0.961 \pm 0.013$ & 0.69 & 0.419 \\
\hline & & & $t_{3}$ & $P$ \\
\hline Total COT $(\mathrm{J} / \mathrm{m} / \mathrm{kg})$ & $1.18 \pm 0.12$ & $1.32 \pm 0.14$ & 1.493 & 0.116 \\
\hline Net COT $(\mathrm{J} / \mathrm{m} / \mathrm{kg})$ & $0.37 \pm 0.385$ & $0.612 \pm 0.095$ & -1.44 & 0.125 \\
\hline Locomotor cost contribution $(\%)$ & $0.32 \pm 0.32$ & $0.47 \pm 0.10$ & -0.993 & 0.197 \\
\hline
\end{tabular}

been shown to (1) increase swimming effort by increasing fluke stroke rate and/or amplitude (Williams, 1989; Cornick et al., 2006; Aoki et al., 2011), (2) reduce the use of stroke-and-glide gaits (Cornick et al., 2006) and (3) alter the speed and angle of dive ascents and descents (Boyd et al., 1997; van der Hoop et al., 2013b). Experiments have shown drag-attributed reductions in average swimming speed in Steller sea lions fitted with harnesses, increasing body drag by $23 \%$ (Cornick et al., 2006), and in maximum swimming speeds by attaching drag collars to bottlenose dolphins (Lang and Daybell, 1963) and wooden blocks to Antarctic fur seals (Boyd et al., 1997; Lang and Daybell, 1963).

In this work, it was expected that metabolic rate would significantly increase due to additional drag loading from wearing a tag. Instead, (1) an observed $11 \%$ reduction in swim speed when wearing the tag (Fig. 2C), (2) a lack of any significant effect on measured metabolic parameters (Fig. 2A,B) and (3) the reduced power output predicted by the model at slower swimming speeds (Fig. 1B) all suggest that tagged animals modulate their behavior to maintain energy expenditure when faced with greater drag forces. Individuals slowed to the point where the tag yielded no increases in drag or power (Fig. 1B). Similar reductions in speed have been associated with drag from tags or other instruments. Blomqvist and Amundin (2004) found significantly reduced activity levels in tagged bottlenose dolphins, in which fast-swimming behaviors significantly increased following tag removal. Similarly, bottlenose dolphins instrumented with a particularly large tag (14 kg, $\sim 22 \%$ of frontal area; Davis et al., 1999) swam on average 9-10\% slower than when noninstrumented (Skrovan 


\section{A}

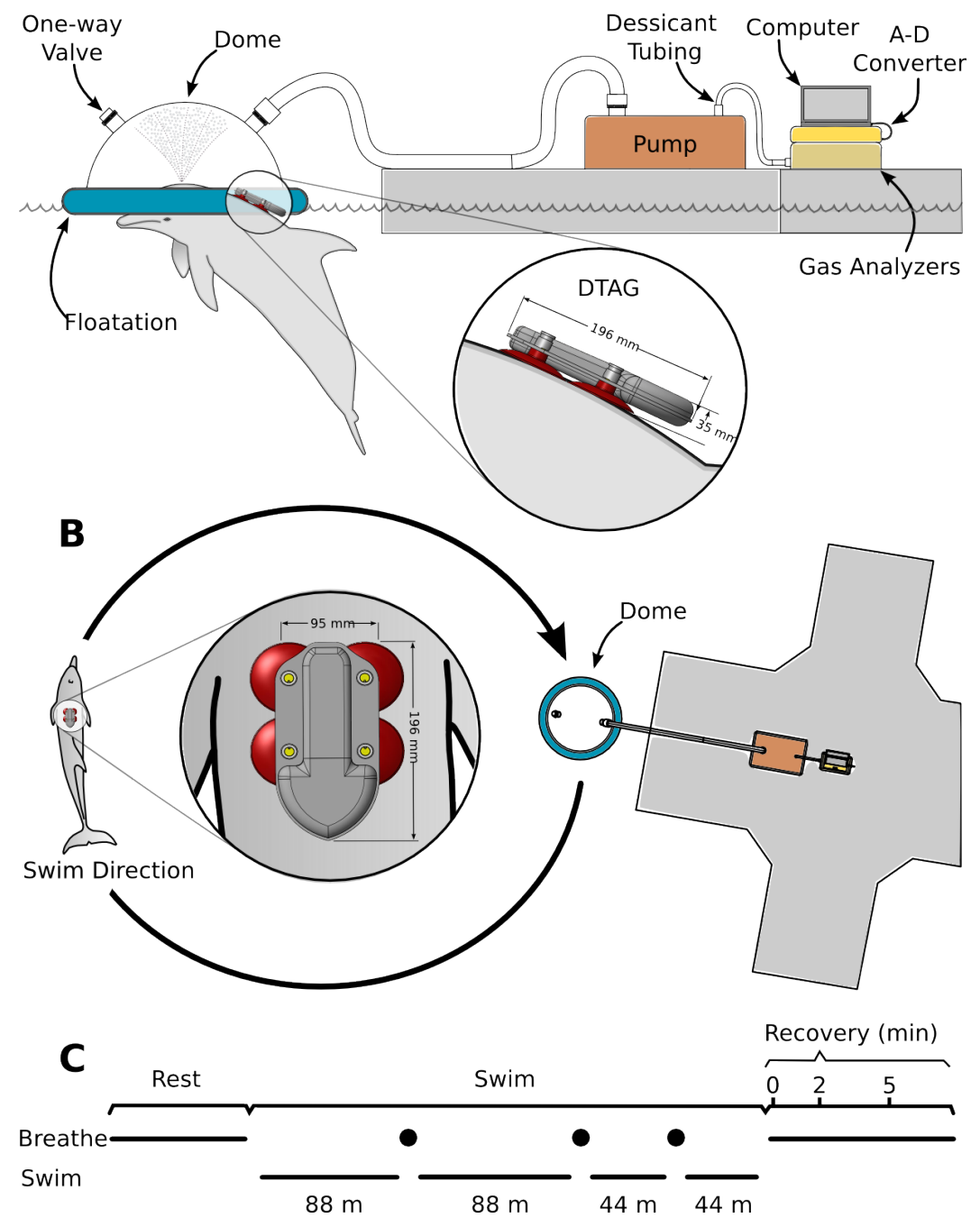

Figure 7-3: Experimental setup. (A) Dockside setup of a bottlenose dolphin wearing a DTAG2 in the respirometry system (see the Materials and methods for full description), (B) the $44 \mathrm{~m}$ swimming track departing from and returning to the respirometry dome, and $(\mathrm{C})$ the phases over which respirometry-based oxygen consumption rates were measured from four bottlenose dolphins Thick horizontal black lines represent time periods during which individuals were breathing in the respirometer (Breathe) or performing the swimming task of specific distances (Swim). Black dots represent pauses between specific laps in which individuals returned to the respirometer for two to three breaths.

et al., 1999), and drag collars of various diameters reduced maximum swimming speeds by $36 \%$ in bottlenose dolphins (Lang and Daybell, 1963).

Optimal swimming speed is a function of drag, but not of buoyancy or dive depth (Suzuki et al., 2014), and is proportional to (resting metabolic rate/drag) ${ }^{-3}$ (Alexander, 1999; Sato et al., 2010). Based on this relationship, the influence of tag-related drag should have decreased optimal swimming speeds in our experimental animals by $1.8 \%$ on average. Given that (1) the dolphins in this study were swimming at speeds much greater than optimal (ob- 
A

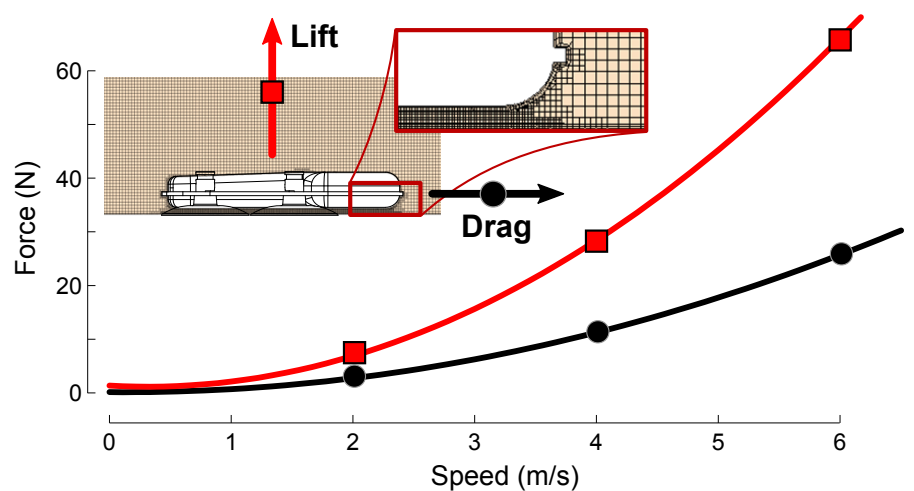

B

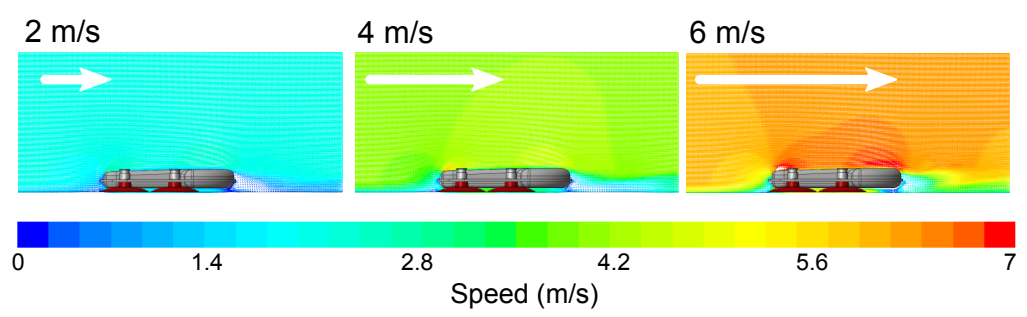

Figure 7-4: Lift and drag forces on a DTAG2 increase with speed. (A) Lift (red) and drag (black) forces $(\mathrm{N})$ on a DTAG2, and (B) flow visualization at uniform velocity profiles of 2,4 and $6 \mathrm{~m} / \mathrm{s}$ from computational fluid dynamics (CFD) simulations using STAR-CCM+ (version 9.04) over $9.6 \mathrm{~m}$ cells, with extra mesh refinement in the region located under the tag (A). See main text for further CFD details.

served 2.9-4.3 m/s versus estimated optimal 1.6-1.9 m/s), (2) metabolically optimal (within $10 \%$ of $\mathrm{COT}_{\mathrm{min}}$ ) speeds of bottlenose dolphins are 1.9-3.2 m/s (Yazdi et al., 1999) and (3) drag increases with the square of speed, it is not surprising that a greater reduction in speed was observed.

The swimming speeds of experimental trials were within routine swimming speeds of $T$. truncatus in aquaria $(1.2-6.0 \mathrm{~m} / \mathrm{s}$; Fish, 1993b) and while free-swimming (1.6-5.6 m/s; Rohr et al., 2002). Experimental studies have determined minimum COT ( COT $_{\min }$ ) to occur at 2.1 and $2.5 \mathrm{~m} / \mathrm{s}$; Williams et al., 1993; Yazdi et al., 1999). Although dolphins in this study swam above reported COT $_{\text {min }}$ speeds (at $2.9-4.3 \mathrm{~m} / \mathrm{s}$ ), they remained in the metabolically optimal range of swimming speeds for $33 \%$ of trials and showed a comparable average COT of $1.28 \mathrm{~J} / \mathrm{m} / \mathrm{kg}$ (compared with 1.29 and $1.16 \mathrm{~J} / \mathrm{m} / \mathrm{kg}$; Williams et al., 1993; Yazdi et al., 1999).

In this experiment the animals were required to swim completely submerged in order to capture all breaths in the respirometry dome (Fig. 3), thereby limiting the duration of the swimming phase. Previous studies (Taylor et al., 1987; Williams et al., 1993) conducted exercise tests on a number of mammal species over a minimum of 3 to 5 minutes, although $\dot{V} \mathrm{O}_{2}$ half times have yet to be established for marine mammals. As such, the swimming trials conducted in this experiment probably do not allow individuals to reach steady-state oxygen consumption. 
Whereas the tagged and untagged metabolic parameters measured during the experiment did not differ, they agree with previous studies on bottlenose dolphins. Mean fasted $\left(4.34 \pm 0.53 \mathrm{ml} \mathrm{O}_{2} / \mathrm{kg} / \mathrm{min}\right)$ and fed $(6.65 \pm 1.73 \mathrm{ml} \mathrm{O} 2 / \mathrm{kg} / \mathrm{min})$ resting metabolic rates (Table 1) fall within the range of those reported over the last 60 years $(4.0-7.6 \mathrm{ml} \mathrm{O} 2 / \mathrm{kg} / \mathrm{min}$; see Table 3 in Yazdi et al., 1999). It is not surprising that individuals showed different levels of physical fitness,as evidenced by significant individual variability in the effect of exercise on oxygen consumption rates (PAR) and recovery from exercise (slope of RER). It was expected that total and net COT would be greater for each individual when wearing a tag, given the decrease in speed and no difference in metabolic rate. High variability in both $\dot{V} \mathrm{O}_{2}$ and swimming speed probably affects the ability to detect statistically significant differences. However, mean $\mathrm{COT}_{\text {tot }}, \mathrm{COT}_{\text {net }}$ and locomotor cost contributions are increased when tagged (Table 2); net COT in particular is nearly double in instrumented individuals. COT was one of the few metabolic parameters that was not significantly different between individuals, which reinforces the frequent use of this measure for inter-individual and inter-species comparisons (Tucker, 1970; Schmidt-Nielsen, 1972; Williams, 1999). Tagged (6.52 1.42 $\mathrm{W} / \mathrm{kg})$, non-tagged $(7.83 \pm 1.66 \mathrm{~W} / \mathrm{kg})$ and predicted $(9.22 \pm 1.99 \mathrm{~W} / \mathrm{kg})$ power estimates fall well within the range $(0.3-23.7 \mathrm{~W} / \mathrm{kg})$ of mass-specific power estimates for $T$. truncatus using a variety of modeling methods across speeds 1-6 m/s (reviewed by Fish et al., 2014). Power may be separately estimated from $\mathrm{O}_{2}$ consumption rates, in which tagged $(5.72 \pm 0.27$ $\mathrm{W} / \mathrm{kg})$ and non-tagged power $(4.54 \pm 0.26 \mathrm{~W} / \mathrm{kg})$ are slightly greater than those calculated in Yazdi et al. (1999), but within the range of power measured at higher speeds $(2.9 \mathrm{~m} / \mathrm{s})$ in Williams et al. (1993). The disparity in power estimates from mechanical models and oxygen consumption measurements is an issue that remains unresolved in the field (Daniel, 1991).

Importantly, failure to demonstrate a statistically significant difference in metabolic parameters does not allow for the complete dismissal of instrument effects on metabolic rate, especially when considering limited sample sizes. It is possible that reduced swimming speeds observed in this study might be due to factors other than instrument drag and energy economy, potentially limiting the application of these results to wild populations. Interpretation of the swimming task by the dolphins might have been variable, as the discriminatory stimulus provided to animals was for a 'fast swim' although not at a specific pace. Individuals may have experienced additional wave drag from near-surface swimming (Hertel, 1969), required by the experimental protocol and limited by the depth of the study site.

The observed behavioral impacts of tag-associated drag remain applicable to wild animals. In certain scenarios, wild animals might be able to modulate their swimming behaviors without affecting fitness (prey capture, or competition with non-tagged conspecifics). However, animals might not be able to reduce their top velocities or acceleration; especially 
during high-speed pursuits chasing active prey (Aguilar Soto et al., 2008), the energetic cost due to extra drag would be considerable. In a social context, cohesion is often maintained between tagged and non-tagged members of a social group (Wursig, 1982), which would require increased power output and metabolic cost by tagged individuals to sustain pace (Fig. 1A,B). It is likely that tradeoffs between managing additional energy expenditure are balanced with the demands that foraging and social behaviors require: despite $13 \%$ and $10 \%$ slower ascent and descent rates during dives, respectively, Northern elephant seals with added drag experienced 65\% increases in field metabolic rate (Maresh et al., 2014). The short-term nature of the suction cup archival tags used here provides confidence that any metabolic or behavioral tag effects would occur over an extremely short proportion of a subject's life and that these effects would probably not carry over after the tag has fallen off. In addition to these concerns of animal welfare and scientific ethics, data reliability must be considered: it is crucial to ensure that tagged individuals exhibit normal behaviors for measurements to be meaningful and representative of the remainder of the population (e.g. Wilson et al., 2006).

Although the scope of this study limits the creation of hard design rules with respect to tag size and increased drag loading, the results presented here continue to support the argument for the creation of tags that minimize drag loading on the animal. The tag used in this study is an older generation model, the DTAG2. Modeled and measured drag forces on the current DTAG3 model have been described by Shorter et al. (2013), in comparison with two alternative model designs. The current DTAG3 is one-third smaller than the DTAG2 (frontal area $\left.24 \mathrm{~cm}^{2}\right)$, with smaller suction cups $(4.5 \times 1.5 \mathrm{~cm}$, diameter $\times$ height) holding the tag close to the attachment surface, thereby minimizing lift forces. A more streamlined urethane housing containing all of the tag elements (electronics, VHF and flotation) minimizes geometric disruptions in the flow around the housing, reducing drag forces. Similar to previous papers on tag design (Bannasch et al., 1994; Culik et al., 1994; Hazekamp et al., 2009; McMahon et al., 2011; Pavlov and Rashad, 2012), the study by Shorter et al. (2013) suggests that tag designs should: (1) minimize frontal cross-sectional areas and maintain a smooth exterior to reduce drag; (2) cover suction cups or other exposed features to reduce flow stagnation and wake generation; and (3) reduce lift by minimizing the attachment area and by adding flow channels or spoilers to reduce differences in flow speed above and below the housing, or redirect flow to counter lift.

In order to establish acceptable limits of drag associated with instrumentation (e.g. the $3 \%$ or $5 \%$ rules for birds), additional studies investigating the degree of impact of different amounts of drag loading are required and are underway. 


\subsection{Conclusions}

Wearing a tag during the prescribed swimming task presented in this work resulted in no detectable effect on the oxygen consumption rate of bottlenose dolphins. Behavioral changes in the form of reduced swimming speed appear to be a mechanism by which individuals avoid increased energy expenditure from tag-induced drag. Further studies to (1) measure differences in energy consumption when swimming at consistent, established speeds; (2) identify thresholds below which tag size does not affect metabolic cost; and (3) investigate individual response to increased drag via modulation of kinematics and swimming speed are currently underway and will better link the potential tradeoffs observed in this study.

\subsection{Materials and Methods}

\subsubsection{CFD and conceptual model}

A conceptual model was used to compare theoretical drag forces on instrumented and noninstrumented dolphins. Dolphin body drag $\left(D_{d} ; \mathrm{N}\right)$ was estimated based on the conventional model of a turbulent flat plate (Hoerner, 1965; Webb, 1975b; Fish and Rohr, 1999) with specific dimensions and estimated surface areas of the four dolphins used in the experiment (Table 1). The additional drag force imparted to the animal by the DTAG2 was estimated with computational fluid dynamics (CFD) simulations using STAR-CCM+ (version 9.04). This commercial code (STAR-CCM+, 2014) solves the transport equations for continuity and three-dimensional (3D) momentum on a very fine 3D mesh. The two-layer ReynoldsAveraged Navier-Stokes (RANS) approach for the solution of the k- $\epsilon$ transport equations was used to model turbulence (Rodi, 1991; STAR-CCM+, 2014). All simulations used trimmed cell mesh (9.6 m cells) with an extra mesh refinement in the region located under the tag and a prismatic cell layer at the wall (Fig. 4A). In order to achieve comparable simulation results to those presented by Shorter et al. (2013), the overall simulation domain consisted of a 1.7 -m-long duct with a $0.4 \mathrm{~m} \times 0.4 \mathrm{~m}$ square cross-section. During all of the simulations, the tag was located $1 \mathrm{~m}$ from the inlet with real wall (no slip) flow conditions on the lower wall, and ideal wall (free slip) conditions on the side and upper walls. Mesh sensitivity was performed using three different meshes (coarse, medium and fine) with 2 million, 9.6 million and 18 million cells, respectively. Variation in drag and lift forces from medium to fine mesh was $\sim 1 \%$. To estimate the inlet velocity profile effect, sensitivity analyses were performed on a shorter domain with the tag located $0.15 \mathrm{~m}$ from the inlet using two velocity profiles, fully developed and uniform, both with mean flow velocities of $4 \mathrm{~m} / \mathrm{s}$. The drag and lift forces from simulation with the fully developed flow were $15 \%$ and $10 \%$ lower than from uniform flow, respectively. Simulations using a uniform velocity profile were then conducted at mean flow velocity profiles of 2, 4, 6, 8 and $10 \mathrm{~m} / \mathrm{s}$. For all simulations, the side forces 
were considered as self-compensating, because of the tag symmetry. A polynomial function was used to interpolate forces at flow speeds between simulated points (Fig. 4B).

Table 7-3: List of symbols and abbreviations.

\begin{tabular}{|l|l|l|}
\hline Symbol & Definition & Unit \\
\hline$A$ & Wetted surface area & $\mathrm{m}^{2}$ \\
\hline$B P$ & Barometric pressure & $\mathrm{hPa}$ \\
\hline$C_{d}$ & Drag coefficient & \\
\hline COT & Cost of transport & $\mathrm{J} / \mathrm{m} / \mathrm{kg}$ \\
\hline COT $_{\text {min }}$ & Minimum cost of transport & $\mathrm{J} / \mathrm{m} / \mathrm{kg}$ \\
\hline COT $_{\text {net }}$ & Net cost of transport & $\mathrm{J} / \mathrm{m} / \mathrm{kg}$ \\
\hline $\mathrm{DTAG}$ & Digital acoustic recording tag & \\
\hline$D_{d}$ & Dolphin body drag & $\mathrm{N}$ \\
\hline$D_{t}$ & Tag drag & $\mathrm{N}$ \\
\hline$D_{T}$ & Total drag & $\mathrm{N}$ \\
\hline$F_{e}$ & Excurrent fraction of $\mathrm{O}_{2}$ & \\
\hline$F_{i}$ & Incurrent fraction of $\mathrm{O}_{2}$ & \\
\hline $\mathrm{LC}$ & locomotor costs & \\
\hline $\mathrm{PAR}$ & Physical activity ratio & \\
\hline$P_{L}$ & Locomotory power & $\mathrm{W}$ \\
\hline $\mathrm{RER}$ & Respiratory exchange ratio & \\
\hline$R h$ & Relative humidity & $\%$ \\
\hline$U$ & Speed & $\mathrm{m} / \mathrm{s}$ \\
\hline$\dot{V} \mathrm{CO}_{2}$ & Rate of carbon dioxide production & $\mathrm{ml} / \mathrm{kg} / \mathrm{min}$ \\
\hline$V_{e}$ & Excurrent flow rate & $\mathrm{L} / \mathrm{s}$ \\
\hline$\dot{V} \mathrm{O}_{2}$ & Rate of oxygen consumption & $\mathrm{ml} / \mathrm{kg} / \mathrm{min}$ \\
\hline$W V P$ & Water vapor pressure & $\mathrm{kPa}$ \\
\hline$\eta$ & Efficiency & \\
\hline$\rho$ & Fluid density & \\
\hline & & \\
\hline$W V$ & \\
\hline & & \\
\hline & & \\
\hline
\end{tabular}

A list of symbols and abbreviations are provided in Table 3. Total drag $\left(D_{T}\right)$ on an individual was the theoretical drag on each dolphin body $\left(D_{d}\right)$, plus the contribution of the tag $\left(D_{t}\right)$ when applicable:

$$
D_{T}=D_{d}+D_{t}
$$

$$
D_{T}=\frac{1}{2} \rho U^{2} A C_{d}+D_{t}
$$


where $\rho$ is fluid density (seawater; $\left.1025 \mathrm{~kg} / \mathrm{m}^{3}\right) ; U$ is swimming speed $(\mathrm{m} / \mathrm{s}) ; A$ is the wetted surface area of each dolphin calculated from weight $(W ; \mathrm{kg})$ as in Fish $(1993 \mathrm{~b})$ $\left(0.08 W^{0.065}\right.$; Table 1$)$; and $C_{d}$ is the profile drag coefficient (Blake, 1983; van der Hoop et al., 2013b). Locomotory power $\left(P_{L} ; \mathrm{W}\right)$ was estimated for each drag condition (tag and no tag) as:

$$
P_{L}=\frac{D_{T} U}{\eta}
$$

with an efficiency factor $\eta$ of 0.15 (Fish, 1993b; Fish and Rohr, 1999).

Drag augmentation factors (see e.g. Fish, 1993b; Fish and Rohr, 1999) were omitted as theoretical drag forces from the basic model alone agreed with those of post-parturition female (Noren et al., 2011) and noninstrumented bottlenose dolphins (Skrovan et al., 1999) estimated by glide deceleration. Locomotor power requirements were similar to those calculated by Fish (1993b) with a hydromechanical model [i.e. following Chopra and Kambe (1977)].

The model was populated with observed swimming speeds of individuals in tagged and non-tagged conditions. This conventional model illustrates our hypothesis and supports our experimental setup and approach by (1) estimating the drag forces and power requirements likely experienced during experimental swimming trials; and (2) assessing the potential energetic benefits achieved by reducing swimming speed when wearing a tag.

\subsubsection{Experiment}

To test the null hypothesis that wearing a tag does not affect metabolic rate, cost of transport, or swimming behaviors, four captive male T. truncatus (Table 1) were trained to perform a fully submerged swim around a set course and surface exclusively in a metabolic dome (Fig. 3), either noninstrumented or while wearing a bio-logging tag (DTAG2; Fig. 3). The individual determined the pace of the swimming task, i.e. swimming speed was not prescribed, and no speed target was provided. The order of tagged versus control (i.e. nontagged) trials was determined randomly, and was made more random by certain trials being unusable when an individual breathed outside of the dome. Metabolic rate was measured for the duration of each trial, consisting of pre-exercise rest, swim and recovery phases.

Animals were inactive under the respirometry dome during rest and recovery phases (Fig. 3C). The swimming course consisted of a $44 \mathrm{~m}$ circumference oval loop departing from and returning to the dome (Fig. 3B). Each trial consisted of six laps (Fig. 3C): two double laps, separated by two to three breaths in the respirometer, and two single laps, again separated by two to three breaths between excursions. The swimming phase was 1-1.5 min in duration, with average breath hold durations of $19 \mathrm{~s}$ (range 10-28 s). Animals were 
reinforced throughout the trial with positive encouragement and tactile stimulation, and with up to $1.8 \mathrm{~kg}$ of a mix of capelin, herring and squid 5 min into the recovery phase. Tags were attached by hand on the dorsal midline halfway between the blowhole and dorsal fin.

\subsubsection{Tags}

The DTAG2 is a bio-logging tag equipped with depth and temperature sensors, three-axis accelerometers and magnetometers sampling at $50 \mathrm{~Hz}$, and two hydrophones sampling at 192 $\mathrm{kHz}$ (Johnson and Tyack, 2003). A polyethylene casing houses the electronics, a syntactic foam float to provide positive buoyancy, a VHF radio beacon with a $44 \mathrm{~cm}$ antenna for tracking and four $6.3 \times 2 \mathrm{~cm}$ (diameter $\times$ height) suction cups for attachment $($ Fig. $3 \mathrm{~A}, \mathrm{~B})$. The fully assembled tag weighs $350 \mathrm{~g}$ in air and has a frontal area of $38 \mathrm{~cm}^{2}, \sim 3 \%$ of the frontal area of the smallest tagged dolphin based on girth.

\subsubsection{Respirometry}

A floating transparent acrylic dome (59 l internal volume; Stock no. 02- PD250CA-1687, California Quality Plastics Inc., Ontario, CA, USA) with circumferential buoyancy was used to collect respiratory gases and determine the rate of oxygen consumption $\left(\dot{V} \mathrm{O}_{2} ; \mathrm{ml}\right.$ $\left.\mathrm{O}_{2} / \mathrm{min}\right)$ and carbon dioxide production $\left(\dot{V} \mathrm{CO}_{2} ; \mathrm{ml} \mathrm{CO} / \mathrm{min}\right)$ by flow-through respirometry (Fig. 3A). A mass flow-meter (Flow Kit Model FK500, Sable Systems International, Las Vegas, NV, USA) pulled air into the dome through a tube ( $\sim 1$ volume $)$ connected to a low-resistance one-way valve at flow rates between 400-500 l/min. A subsample of this gas was passed via Nafion tubing to fast-response $\mathrm{O}_{2}$ and $\mathrm{CO}_{2}$ analyzers (ML206, Harvard Apparatus, Holliston, MA, USA), with data recorded at $20 \mathrm{~Hz}$ and saved to a laptop computer. The gas analyzers were calibrated before and after the experiment, using a commercial mixture of $5 \% \mathrm{O}_{2}, 5 \% \mathrm{CO}_{2}$ and balance $\mathrm{N}_{2}$; and before and after each experimental trial, using ambient air.

Whereas the mass flow meter automatically corrected to standard temperature and pressure (STP), post-processing was required to correct all volumes to standard temperature, pressure and dryness (STPD). Flow was corrected for humidity by:

$$
\text { Flow }=\frac{(B P-W V P)}{B P}
$$

where $B P$ was the average daily barometric pressure and $W V P$ is water vapor pressure estimated from the Antoine equation using the average daily air temperature (grand mean $25.4^{\circ} \mathrm{C}$, daily range $\left.21-29^{\circ} \mathrm{C}\right)$. Relative humidity $(R h)$ was assumed to be $100 \%$ in the dome due to regional air humidity measurements (grand mean $66.2 \%$, daily range 44-97\%) and the effect of exhalation. Assuming 90\% Rh instead of 100\% Rh resulted in a difference of $0.3 \%$ 
for flow rate and $0.5 \%$ for instantaneous $\dot{V} \mathrm{O}_{2}$, indicating little sensitivity to this parameter.

The accuracy of the respirometry system was determined by simultaneous $\mathrm{N}_{2^{-}}$and $\mathrm{CO}_{2}$ dilution tests (Fahlman et al., 2005), in which differences between the observed and expected values were within $2 \%$. Addition of $\mathrm{CO}_{2}$ confirmed minimal losses by dissolution in seawater (Fahlman et al., 2005). The effective volume of the system was 53 1, including the volume of the respirometer and the plastic hose to the analyzers (Bartholomew et al., 1981). With a flow rate of $450 \mathrm{l} / \mathrm{min}$, this resulted in a time constant of $0.11 \mathrm{~min}$. The time required to reach a $95 \%$ fractional transformation to a new steady state was 3.2 times this time constant, or $21 \mathrm{~s}$ (Fahlman et al., 2004).

From measured gas concentrations, $\dot{\mathrm{V}} \mathrm{O}_{2}\left(\mathrm{ml} \mathrm{O}_{2} / \mathrm{kg} / \mathrm{min}\right)$ was calculated as:

$$
\dot{V} O_{2}=\frac{\dot{V}_{e} \times\left(F_{i}-F_{e}\right)}{1-F_{i}\left(1_{R} Q\right)}
$$

where $\dot{V}_{e}$ is the excurrent flow rate; $F_{e}$ and $F_{i}$ the excurrent and incurrent fractions of $\mathrm{O}_{2}$, respectively; and RER the respiratory exchange ratio $\left(\dot{V} \mathrm{CO}_{2} / \dot{V} \mathrm{O}_{2}\right)$ (Koteja, 1996). Massspecific average $\dot{V} \mathrm{O}_{2}$ and $\dot{V} \mathrm{CO}_{2}$ were calculated for each phase by dividing the integrated instantaneous $\mathrm{O}_{2}$ consumption or $\mathrm{CO}_{2}$ production rates, respectively, over the duration (min) of the rest, swim (entire duration; i.e. time spent submerged and at the surface) and the first two minutes of the recovery (0-2 min after exercise) phase. Least-square linear regression analysis on the two-minute recovery phase RER was used to determine whether drag loading had an effect on the initial anaerobic metabolism (intercept) or the rate of return to resting values (slope).

The physical activity ratio (PAR; nondimensional) was calculated to detect the energetic cost of a specific activity over an individual's reference level (resting metabolic rate). In doing so, PAR controls for daily variability and for individual size and energy efficiency (Schutz et al., 2001). This method differs from the concept of metabolic equivalents (METs) only in that the resting energy expenditure is measured rather than estimated (Schutz et al., 2001; Byrne et al., 2005). PAR was calculated as the ratio of $\dot{V} \mathrm{O}_{2}$ during the swimming period and the pre-exercise rest period of a given trial.

Mass-specific cost of transport (COT; $\mathrm{J} / \mathrm{m} / \mathrm{kg}$ ) describes the energetic cost of covering a unit distance per unit mass (Schmidt-Nielsen, 1972) and was calculated as the average mass-specific metabolic rate during the swim and two-minute recovery phases combined (ml $\mathrm{O}_{2} \mathrm{~kg} / \mathrm{min}$; the exercise metabolic rate) divided by average swimming speeds $(\mathrm{m} / \mathrm{s})$. The average energy conversion for lipid, protein and carbohydrate sources of $20.1 \mathrm{~J} / \mathrm{ml} \mathrm{O} 2$ was used (Schmidt-Nielsen, 1997).

Both maintenance costs and locomotor costs (LC) contribute to cost of transport. The net cost of transport $\left(\mathrm{COT}_{\text {net }} ; \mathrm{J} / \mathrm{m} / \mathrm{kg}\right.$ ) can be calculated to provide a measure of locomotor 
cost normalized for both body mass and swimming speed (Williams, 1989; Rosen and Trites, 2002a):

$\mathrm{COT}_{\text {net }}=\frac{(\text { Exercise metabolic rate }- \text { Resting metabolic rate }) \times \text { Energy conversion factor }}{\text { Swimming speed }}(7.6)$

The contribution of LC to COT is then $\mathrm{COT}_{\text {net }}$ divided by COT. It is hypothesized that $\mathrm{COT}$ and $\mathrm{COT}_{\text {net }}$ would be greater and that $\mathrm{LC}$ would have larger contributions to COT in tagged trials.

\subsubsection{Statistical analysis}

To test whether individuals became conditioned to the respirometry apparatus or experimental protocol, linear models were fitted to swimming $\dot{V} \mathrm{O}_{2}$ and swimming speed versus trial number for each individual. Two-way ANOVA without interaction were used to test for the effect of individual and feeding condition (i.e. fasted or fed) on resting oxygen consumption rates $\left(\dot{V} \mathrm{O}_{2} ; \mathrm{ml} \mathrm{O} 2 / \mathrm{kg} / \mathrm{min}\right)$ and RER in rest periods. Two-way ANOVA without interaction were also used to test for the effect of wearing a tag on each individual's oxygen consumption rates $\left(\dot{V} \mathrm{O}_{2}\right)$ during the three trial phases (rest, swim, recovery) and PAR, and on least-square linear regression slopes and intercepts of RER over the recovery phase. Two-sample $t$-tests were used to compare RER between resting and swimming, and between swimming and recovery periods. One-sided paired $t$-tests were used to determine whether average COT, COTnet and LC for each individual were significantly greater when tagged versus not tagged. Swimming speed was estimated by dividing the distance of the swimming track $(44 \mathrm{~m})$ by the time required for an individual to complete each lap or set of laps. Two-way ANOVA without interaction were used to test whether swimming speeds of each individual were significantly different in tagged than in non-tagged trials. All data processing, statistical analyses and modeling were coded in MATLAB (R2011a; MathWorks, Inc., Natick, MA, USA). 


\section{Chapter 8}

\section{HYDRODYNAMIC DRAG OF BIO-LOGGING TAGS ON BOTTLENOSE DOLPHINS}

This chapter has been formatted to be submitted to Animal Biotelemetry, with authorship contributions from K. Alex Shorter, Victor Petrov, Julie Rocho-Levine and Michael Moore

JvdH, KAS, VP, JR-L and MJM developed concepts; JR-L directed animal husbandry and training; JvdH, KAS, MJM and JR-L completed experiments; VP and KAS completed simulations; JvdH analyzed data; JvdH, KAS, VP, JR-L and MJM wrote the manuscript.

The supplemental materials for this chapter can be found in Appendix F. 


\subsection{Abstract}

\section{Background}

External instrumentation affects the streamlined shape of animals, which contributes additional drag and lift to their natural force balance. To inform tag design, research permit evaluation and data validity, the contribution of tags of various sizes to the forces experienced by animals, as well as their behavioral responses, needs to be quantified. Many methods exist to assess passive drag forces on marine mammals; here we used a combination of computational fluid dynamics (CFD) and underwater video to estimate passive drag forces acting on bottlenose dolphins (Tursiops truncatus) during a controlled glide. To quantify the relative increase in drag coefficients created by a bio-logging tag over a range of loading conditions, we repeated the experiment with a tag (the DTAG3) and additional drag-inducing elements $(\operatorname{tag}+4, \operatorname{tag}+8)$ to determine the effects of increasing tag frontal area by up to eight times the tag alone.

\section{Results}

Measured drag coefficients of uninstrumented bottlenose dolphins ranged from 0.010 to 0.033. CFD simulations predicted an increase of $5.4 \%$ for the tag and $67.3 \%$ for the tag +4 . During the experiment, the tag alone increased drag coefficients by $7.9-26.2 \%$ and increasing the tag frontal area by factors of $4 \times$ and $8 \times$ increased drag coefficients by $75.6-126.4 \%$ and 185.8-192.5\% respectively. The ability of the animals to consistently perform the controlled gliding task declined beyond the tag +4 condition: variability in estimated drag coefficient increased, glide duration decreased and glide success was reduced.

\section{Conclusions}

Tags with large areas relative to the tag subject (e.g., the tag +8 ) have the potential to impede the animals' ability to perform even a simple gliding task. Both computational and experimental methods estimate drag forces with sufficient resolution to detect significant relative increases in drag due to bio-logging tags. Based on the results from both this work and the literature we recommend tags with hydrodynamic designs and $<3 \%$ wetted surface and $<10 \%$ frontal area of the study subject.

\subsection{Introduction}

Animal-borne sensors allow researchers to measure, visualize, and understand how and why animals move and interact with their environments (Wilson et al., 2008; Hussey et al., 2015; Kays et al., 2015). Over recent decades, device costs have decreased and sensor suites have been developed to address new scientific questions. Tag designs have diversified and have 
become more economical and available; as such, we have seen a tripling of permit applications to tag animals (Jones et al., 2013), as well as major increases in the number and diversity of subject species and the number of tags deployed on individual animals (McIntyre, 2014; Hussey et al., 2015).

Meanwhile, the scientific community has raised concern over the impacts of external tags (Wilson et al., 2006; McMahon et al., 2008). The terrestrial and flying animal research communities somewhat follow the ' $3 \%$ ' or ' $5 \%$ rules' whereby tag weight should not exceed $3 \%$ or $5 \%$ of the weight of the subject. The basis and validity of these rules have been questioned (Barron et al., 2010), and the relevance for marine taxa is especially limited: hydrodynamic handicapping from altered drag, lift and buoyant forces is a greater issue than added weight (Vandenabeele et al., 2011). Few true recommendations exist for tag development or deployment on specific species; however, recent efforts to describe acceptable increases in tag area relative to the tag subject or the percentage increase in drag caused by tags (Jones et al., 2013) are important advances.

Force balance and the thrust output required to overcome drag are central to estimating energetic expenditure; quantifying force balance is therefore essential to determine the potential for and magnitude of effects of tags on energy consumption or swimming behavior (e.g., van der Hoop et al., 2014a). The direct measurement of propulsive forces during swimming are difficult to measure, but accurate estimates of drag loading and direct measurement of the animal's specific acceleration can be used to calculate estimates of the propulsive force generate during swimming (Martin Lopez et al., 2016; Ware et al., 2016).

Many methods are available to estimate passive drag forces, when an animal is not swimming (vs. active drag during propulsion). Glide deceleration (Lang and Daybell, 1963; Bilo and Nachtigall, 1980) remains one of best methods to determine passive drag coefficients of live, free-swimming animals (see Fish et al., 2014, for a review). The method employs the concept that when an animal passively glides horizontally through the water the forces acting on the body can be simplified to only the drag forces opposing the movement of the body and therefore slowing it down. The rate of deceleration can then be used to calculate the drag force, being equal to mass times acceleration (Lang and Daybell, 1963; Bilo and Nachtigall, 1980). Methods using glide data are only useful to describe or quantify gliding behaviors, but still provide a baseline for relative comparison of different drag conditions (e.g., with instrumentation (Skrovan et al., 1999) or during and after pregnancy (Noren et al., 2011)), using true surfaces and in-water conditions.

In addition to glide deceleration, wind tunnels with physical models (Hanson, 2001; Jones et al., 2013), tow experiments with live animals in a rigid body position (Feldkamp, 1987), and particle image velocimetry (Fish et al., 2014) have been used to determine drag forces and coefficients for static and swimming animals. These techniques facilitate the measurement of drag loading in a controlled experimental environment, but provide less 
realistic measurement environments or situations. To improve environmental context, data from the tags themselves (Miller et al., 2004; Aoki et al., 2011; Ware et al., 2016), along with estimated body parameters, have been used to estimate drag forces on free-swimming animals. In contrast with the various experimental methods described above, computational fluid dynamics (CFD) models are becoming an increasingly popular method to simulate the expected effects of instrumentation and to refine tag design (Hazekamp et al., 2009; Pavlov and Rashad, 2012; Balmer et al., 2013; Shorter et al., 2013; van der Hoop et al., 2014a). Working in a simulation environment enables a rapid evaluation of a range of tag designs in multiple fluid flow conditions but can be difficult to compare with experimental results.

In this work we investigate two questions: (A) How much does a specific bio-logging tag design (DTAG3) change the drag forces as compared to uninstrumented animals? (B) Can we use our current tag design and experimental set-up to evaluate the effects of larger or multiple tags deployed on a single individual? To investigate these questions we used computational fluid dynamics and two methods of analysis of position data from underwater video of gliding behavior in bottlenose dolphins (Tursiops truncatus) to (1) assess and compare drag coefficients under increasing drag-loading conditions created with a bio logging tag (DTAG3) with attachable elements to determine (2) the relative load of the tags and (3) their impact on individual behavior. We hypothesized that the tag alone would yield

minimal but measurable increases in drag coefficient, and that increased frontal area from additional drag-loading elements would further increase drag coefficients of instrumented bottlenose dolphins.

\subsection{Methods}

\subsubsection{Computational Fluid Dynamics (CFD)}

Building on previous work (Shorter et al., 2013), we developed CAD (computer-aided design) models of the tag and additional elements on representative animal geometry in simulation to determine the relative additional forces imparted by the tag and on additional draginducing elements $(\operatorname{tag}+2, \operatorname{tag}+4)$ designed to increase the tag's frontal area by up to eight times the tag alone (Figure 1). Due to time constraints we were unable to complete CFD simulations for the tag +8 . We used Autodesk Inventor 2013 (Autodesk Inc., San Rafael, CA, U.S.A.) to create the 3D models of the tag geometry, and scaled the dolphin geometry to match the average length of the animals in the experimental trials $(2.48 \mathrm{~m})$ at Dolphin Quest Oahu (Honolulu, HI, U.S.A.) using STAR-CCM+ software (version 9.04; CD-adapco, Melville, NY, USA/London, England).

We performed CFD simulations of the combined dolphin-tag model in STAR-CCM + . This commercial code (STAR-CCM+, 2014) solves the transport equations for continuity and 3-D momentum on a very fine 3-D mesh. We modeled turbulence with the two-layer 


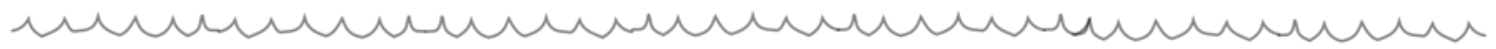

(A)
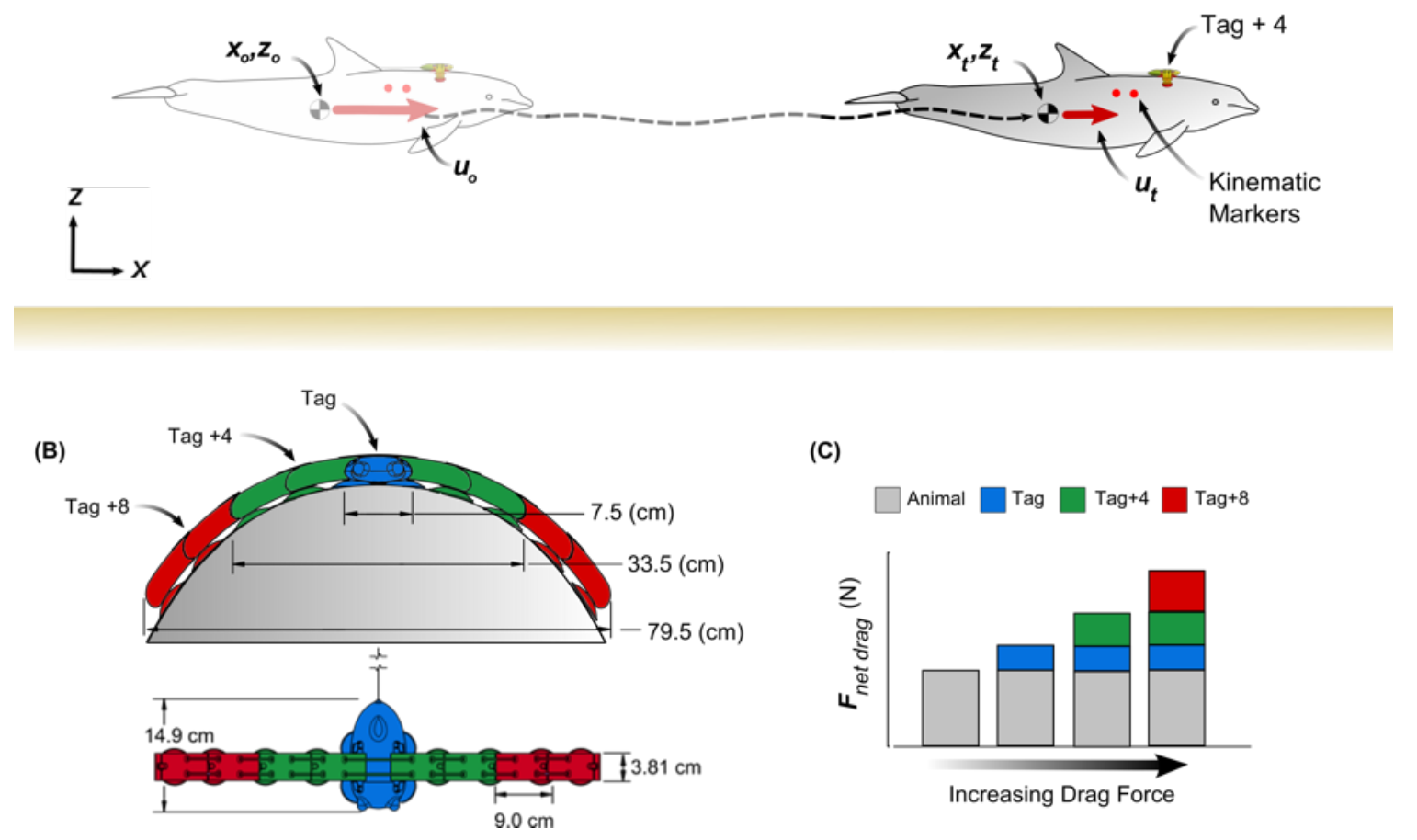

Figure 8-1: Illustration of the experimental setup used to collect center of mass position and velocity for different drag loading conditions: Animal, Tag, Tag +4 and Tag +8 . Planar video of the animals during a glide was collected using underwater cameras. Dots of zinc oxide on the dolphin's skin were used to create kinematic markers visible in the video. The position $(\mathrm{x}, \mathrm{z})$ of each zinc oxide dot was tracked through each glide video frame and used to calculate the velocity of the animal during the trial.

Reynolds-Averaged Navier-Stokes (RANS) approach for the solution of the $k$-transport equations (Rodi, 1991; STAR-CCM+, 2014). We first performed mesh sensitivity tests in 2D with a dolphin middle cross-section profile to estimate required cell size. We tested three different mesh sizes $($ coarse $=0.05 \mathrm{~m}$, medium $=0.025 \mathrm{~m}$, and fine $=0.01 \mathrm{~m}$ ) with 0.365 million, 0.998 million and 6.50 million cells, respectively. Variation in drag and lift forces from medium to fine mesh was $1.85 \%$. The $3 \mathrm{D}$ simulations were performed with trimmed cell mesh ( $0.25 \mathrm{~m}$ cell base size) with 50-57 million cells; the number of cells depends on the tag configuration tested - more drag elements lead to more cells due to small gaps. The 3D domain consisted of a cylinder $10.5 \mathrm{~m}$ long with $6 \mathrm{~m}$ diameter (Figure F1). During all of the simulations of the combined dolphin-tag model, the tag was located $0.74 \mathrm{~m}$ from the rostrum midway between the blow hole and dorsal fin. We then conducted simulations with a uniform velocity profile at mean flow velocity profiles of 1-6 m/s. For all simulations, the side force acting on the tag was negligible. We used a polynomial function to interpolate forces at flow speeds between simulated points. We calculated drag coefficients $\left(C_{d}\right)$ from 
simulated drag forces $(D ; \mathrm{N})$ as

$$
C_{d}=\frac{2 D}{\rho A_{w} U^{2}}
$$

\subsubsection{Experimental setup}

To investigate the effect of tags of increasing size on the total rigid body drag of bottlenose dolphins, two male bottlenose dolphins at Dolphin Quest Oahu were trained to glide with no locomotory movement on cue between two trainers standing or floating in water 8-12 $\mathrm{m}$ apart (Figure 1). The experimental lagoon was 1.5-2.4 $\mathrm{m}$ deep. One trainer released the dolphins, which held a rigid body position as they were propelled and released to glide towards the other trainer. Dots of zinc oxide on the dolphin's skin were used to create kinematic markers visible in the video to calculate speed, described below. We filmed glides perpendicular to the path of the animal at $30 \mathrm{fps}$ with a Canon PowerShot G12 digital camera in a waterproof housing (Canon WP-DC34). We mounted the camera and housing on a monopod, which was rested on the bottom of the lagoon to reduce the camera's motion from water movement.

The dolphins were asked to perform glides without the tag (control), when wearing a DTAG3 (tag) and when wearing the tag with either four $(\operatorname{tag}+4)$ or eight $(\operatorname{tag}+8)$ urethane drag-adding elements attached on each side of the tag (Figure 1).

\subsubsection{Video analysis}

We analyzed underwater footage of dolphins gliding in Tracker (v. 4.87, Brown, 2014; Figure 1). We used the following criteria to select glides for analysis: (i) no visible body movement, (ii) no visible changes in the horizontal plane (i.e., the dolphin did not noticeably move closer to or away from the camera), (iii) minimal changes in depth, (iv) $1 \mathrm{~s}$ minimum glide duration, and $(\mathrm{v})$ the change in acceleration was $\leq 0$ (i.e., there was a detectable decrease in speed; Stelle et al., 2000; Noren et al., 2011). Glide duration was calculated from the time the kinematic markers entered the frame to when the dolphins showed detectable body movement. We calculated success rates based on the number of glides the dolphins were asked to perform and the number of glides that were successful, having met the above criteria, for each individual and each tag condition.

We digitized two clearly visible points close to the dolphins' center of mass (zinc oxide marks for 41 trials, or the front and back of the DTAG3, for 4 trials) at 30 fps for each glide video segment. These points provided kinematic markers to track through the camera frame; the distance between the points were measured when applied to the animal and provided a length reference in the video frame. We digitized this two-dimensional position data ( $\mathrm{x}, \mathrm{z}$; 
Figure 1) for every frame, from which we computed velocity $(U ; \mathrm{m} / \mathrm{s})$ as:

$$
U=\sqrt{\left(\frac{d x}{d t}\right)^{2}+\left(\frac{d z}{d t}\right)^{2}} .
$$

The speed at which glides began was the speed at which the tracking points (zinc oxide) became visible in the video footage. We estimated drag coefficients by two methods from these derived velocity data (Figure 2). We measured the distance between the two kinematic markers throughout the glide to determine the potential for parallax distortion. We provide a list of symbols and abbreviations in Table 2 .

\subsubsection{Video $C_{d}$ : Time-varying}

For both reference points in each glide, we filtered measurements of velocity through time with a $1 \mathrm{~s}$ moving average (Figure 2). We then estimated acceleration $\left(a ; \mathrm{m} / \mathrm{s}^{2}\right)$ at each time point from the averaged velocity. To achieve an estimate of the drag coefficient $\left(C_{d}\right)$ at each time point, we computed:

$$
C_{d}=\frac{2 \times 1.06 W a}{\rho A_{w} U^{2}}
$$

where $W$ is the dolphin's body weight (kg; Table 1), 1.06 represents the added mass from water entrainment, is seawater density $\left(1021 \mathrm{~kg} / \mathrm{m}^{3} ; 24^{\circ} \mathrm{C}\right.$, salinity 31$)$, and $A_{w}$ is the wetted surface area $\left(\mathrm{m}^{2}\right)$ calculated from Fish (1993a). We calculated frontal area from girth, which was measured approximately two fingers in front of the dorsal fin after animals exhaled (Table 1). We used the mean of both time-varying $C_{d}$ estimates for each of the two kinematic markers in statistical analyses.

Table 8-1: Weights $(\mathrm{kg})$ and lengths $(\mathrm{m})$ of the two bottlenose dolphins in the study, their estimated wetted surface area calculated from Fish (1993a) and the number of glides they each successfully performed in different tag conditions. Girth was measured approximately two fingers in front of the dorsal fin after animals exhaled.

\begin{tabular}{|l|l|l|l|l|l|l|l|l|l|}
\hline $\begin{array}{l}\text { Dolphin } \\
\text { ID }\end{array}$ & $\begin{array}{l}\text { Weight } \\
(W ; \mathrm{kg})\end{array}$ & $\begin{array}{l}\text { Length } \\
(l ; \mathrm{m})\end{array}$ & $\begin{array}{l}\text { Girth } \\
(\mathrm{m})\end{array}$ & $\begin{array}{l}\text { Frontal area } \\
\left(A_{f} ; \mathrm{m}^{2}\right)\end{array}$ & $\begin{array}{l}\text { Wetted sur- } \\
\text { face area } \\
\left(A_{w} ; \mathrm{m}^{2}\right)\end{array}$ & \multicolumn{5}{|c|}{ Number of glides } \\
\hline & & & & & & $\begin{array}{l}\text { No } \\
\text { Tag }\end{array}$ & Tag & $\begin{array}{l}\text { Tag } \\
+4\end{array}$ & $\begin{array}{l}\text { Tag } \\
+8\end{array}$ \\
\hline $63 \mathrm{H} 4$ & 176 & 2.52 & 1.23 & 0.1204 & 2.39 & 7 & 6 & 11 & 0 \\
\hline $01 \mathrm{~L} 5$ & 154 & 2.37 & 1.21 & 0.1165 & 2.19 & 4 & 5 & 4 & 8 \\
\hline
\end{tabular}




\section{$C_{d}:$ Time-Varying}

$U$

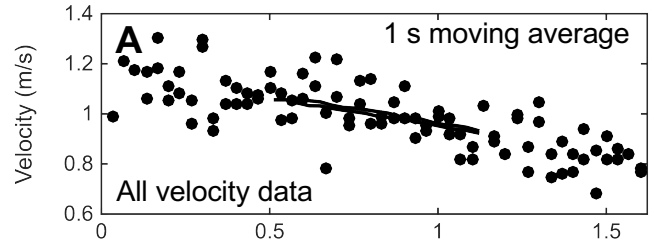

$a=\frac{d U}{d t}$

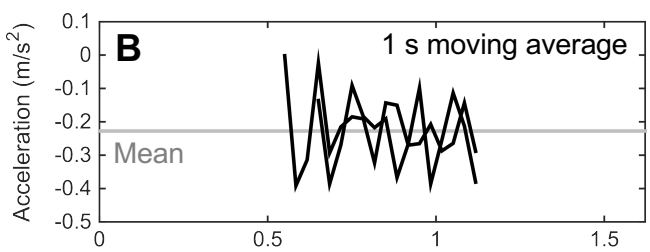

$$
C_{d}=\frac{2 \times 1.06 M_{b} a}{\rho A_{w} U^{2}}
$$

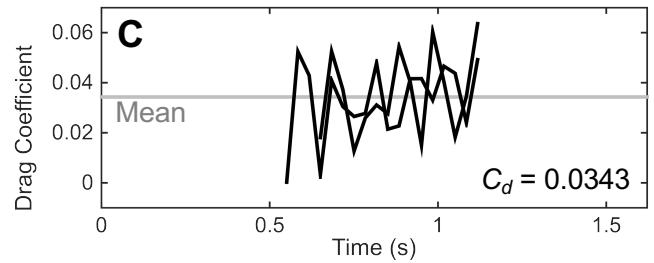

$C_{d}$ : Velocity Fit

$$
\begin{aligned}
& \left.f=\frac{C_{d} \rho A_{w} U^{2}}{C_{d}^{2} \rho A_{w}}\right) \underbrace{2}_{c} U^{2} \\
& a=\underbrace{\frac{d U}{d t}}_{c}=a=c U^{2} \\
& \frac{-1}{c} \cdot \frac{1}{U(t)}+\frac{1}{c} \cdot \frac{1}{U_{o}}=t-f_{o}^{0} \\
& \frac{d U}{c U^{2}}=d t \\
& \frac{1}{c} \int_{U_{o}}^{U_{f}} \frac{1}{U^{2}} d U=\int_{t_{o}}^{t_{f}} d t \\
& \left.\frac{1}{c} \cdot \frac{1}{U^{2}}\right|_{U_{f}} ^{U_{o}}=t_{f}-t_{o}
\end{aligned}
$$

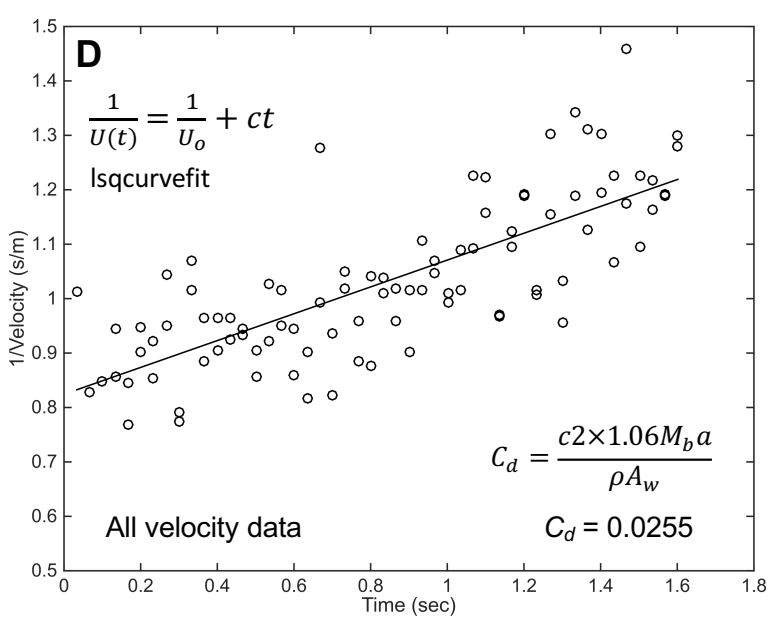

Figure 8-2: Methods to estimate drag coefficients from underwater video data. The time-varying method for estimating drag coefficients $\left(C_{d}\right)$ uses the $1 \mathrm{~s}$ moving average of velocity $(U, \mathrm{~m} / \mathrm{s} ; \mathrm{A})$ and acceleration $\left(a, \mathrm{~m} / \mathrm{s}^{2} ; \mathrm{B}\right)$ to calculate $C_{d}(\mathrm{C})$ at each time point. Data are shown for both kinematic markers on one individual during one glide. The velocity fit method fits the inverse of velocity through time (D) with least-squares. Data displayed for both methods are from the same glide.

\subsubsection{Video $C_{d}$ : Velocity fit}

Following the derivation in Figure 2, we fit the inverse of the linear velocity vs. time relationship by least squares to estimate drag coefficients (Bilo and Nachtigall, 1980). Both 
Table 8-2: List of symbols and abbreviations.

\begin{tabular}{|l|l|l|}
\hline Symbol & Definition & Unit \\
\hline$a$ & Acceleration & $\mathrm{m} / \mathrm{s}^{2}$ \\
\hline$A_{f}$ & Frontal area & $\mathrm{m}^{2}$ \\
\hline$A_{w}$ & Wetted surface area & $\mathrm{m}^{2}$ \\
\hline$C_{d}$ & Drag coefficient & \\
\hline CFD & Computational Fluid Dynamics & \\
\hline$D$ & Drag force & $\mathrm{N}$ \\
\hline$M_{b}$ & Body mass & $\mathrm{kg}$ \\
\hline$l$ & Length & $\mathrm{m}$ \\
\hline$\rho$ & Density & $\mathrm{kg} / \mathrm{m}^{3}$ \\
\hline$R e$ & Reynolds number & \\
\hline$t$ & Time & $\mathrm{s}$ \\
\hline$U$ & Velocity & $\mathrm{m} / \mathrm{s}$ \\
\hline$v$ & Kinematic viscosity & $\mathrm{m}^{2} / \mathrm{s}$ \\
\hline$W$ & Weight & $\mathrm{kg}$ \\
\hline$x$ & x-position in video frame & \\
\hline$z$ & z-position in video frame (depth) & \\
\hline
\end{tabular}

methods incorporated the wetted surface areas (from CFD) and weights (measured dry weight) of the tags and the additional urethane elements (Table 3) into the surface area and mass terms in the equations in Figure 2.

\subsubsection{Drag comparison}

We calculated the percent increase in drag coefficient between the tagged conditions and the non-tagged animal, estimated by each of the three methods as:

$$
\frac{C_{d, \text { tagged }}-C_{d, \text { animal }}}{C_{d, \text { animal }}} \times 100
$$

This provides a reference of the increase in a non-dimensional quantity for comparison of the effects of tags of different dimensions (Jones et al., 2013). We also calculated the Reynolds number $(R e)$ to compare drag coefficients within this study and to other values in the literature:

$$
R e=\frac{l U}{v},
$$

where $l$ is the body length ( $\mathrm{m}$; Table 1$), U$ the speed at the start of the glide $(\mathrm{m} / \mathrm{s})$ and $v$ the kinematic viscosity of seawater $\left(1.05 \times 10^{-6}\right)$. 


\subsubsection{Statistical analysis}

We used two-way ANOVA with post-hoc Tukey's HSD to determine whether drag coefficients from CFD simulations were significantly different between tag conditions (no tag, tag, $\operatorname{tag}+2$, tag +4 ), while considering the effect of velocity. We used Bartlett's test to test for homogeneity of variances in the video-derived drag coefficients and glide duration with drag loading. We used a non-parametric Kruskal-Wallis to test for the effect of drag loading on drag coefficient and glide duration due to unequal variances among tag treatments. When a significant difference was detected, we used Dunn's post-hoc test for multiple comparisons. We could not apply two-factor models because of non-parametric data and an unbalanced design due to variable success rates among individuals and treatments.

\subsection{Results}

\subsubsection{Drag Coefficients - Computational Fluid Dynamics}

Qualitatively, the CFD simulations show that the disturbance of the flow in the different loading conditions generally occurs in a local region around the tag (Figure 3). In this region the velocity of the fluid is increased over the top of the body and an area of low pressure is created behind the tag. These disturbances to the flow result in increased lift and drag forces on the dolphin body. The drag coefficient of the representative dolphin geometry in CFD simulations ranged 0.009-0.013. As speed increased from 1-6 m/s, drag forces on the animal body (control) increased from $15 \mathrm{~N}$ to $387 \mathrm{~N}$ (Table 3). Drag coefficients were significantly different between tag conditions (two-way ANOVA $F_{3,15}=2345, p<0.0001$ ). The tag added 1-25 $\mathrm{N}$ across these speeds, increasing the drag coefficient by only $5.4 \%$ to $0.0106( \pm 0.001$; post-hoc Tukey's HSD $p=0.0002)$. The tag +2 increased the drag coefficient to $0.0136( \pm 0.001)$, by $34.7 \%$ over the non-tagged dolphin $(p<0.0001)$; drag forces increased by $4-158 \mathrm{~N}$ to $19-545 \mathrm{~N}$ total. The tag +4 package increased drag forces to $22-686 \mathrm{~N}$, adding 8-299 N, while the drag coefficient was $0.017( \pm 0.001), 67.3 \%$ greater than the non-tagged control condition (Figure 4A, B; $p<0.0001$ ).

\subsubsection{Drag coefficients - Video - Time-varying}

A total of 45 glides fit the necessary criteria to estimate drag coefficients from underwater video by two methods (Table 1). The average variance of the distance between kinematic markers during each of the glides was only $0.0007 \mathrm{~m}$, indicating little effect of distortion by the camera lens or its housing. The mean (SD) drag coefficient of non-tagged bottlenose dolphins $(n=11)$ was $0.033( \pm 0.006)$. The variance in drag coefficient was significantly different between tag treatments (Bartlett's $\mathrm{K}^{2}=53.28, \mathrm{df}=3, p<0.0001$ ), increasing especially between the tag +4 and $\operatorname{tag}+8$ configurations (Figure $4 \mathrm{~A}$ ). There was no detectable 

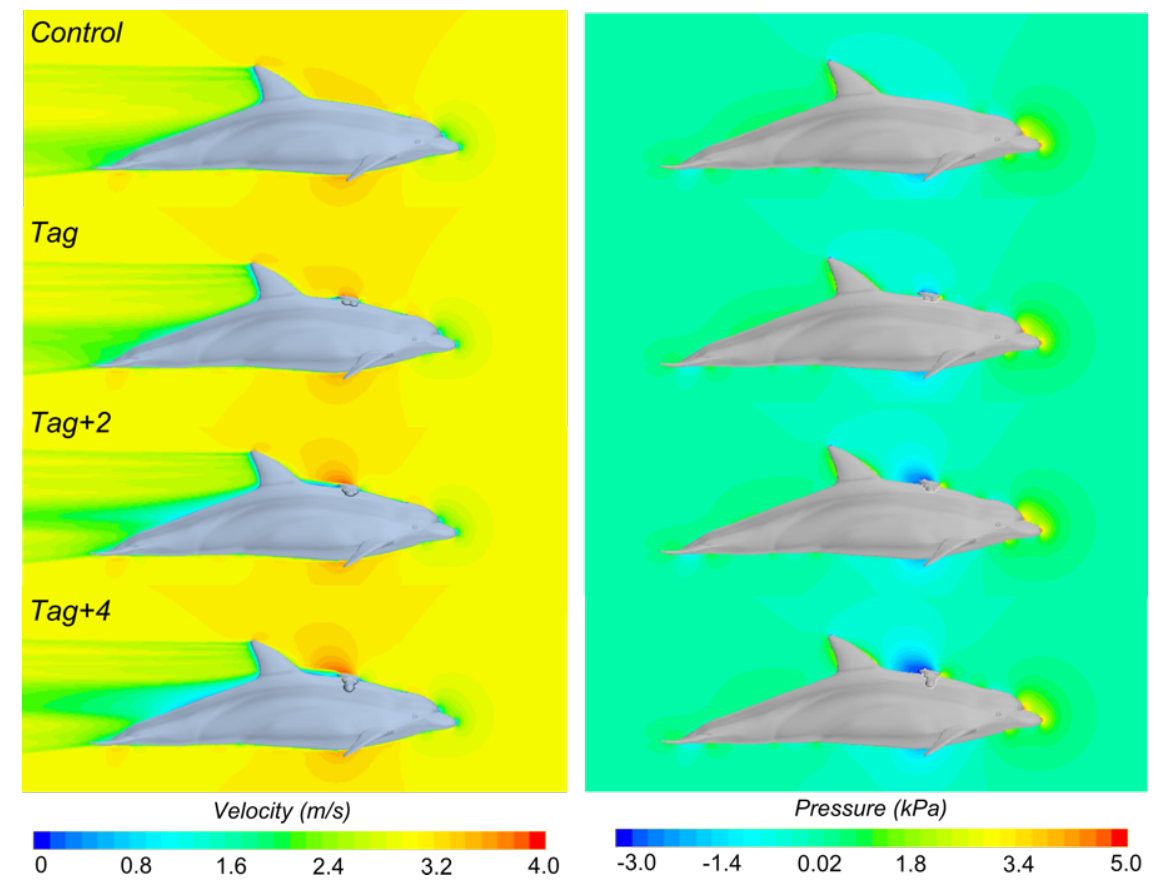

Figure 8-3: Computational Fluid Dynamics simulation results from the dolphin and combined dolphin-tag models that illustrate how the tag and added drag elements affect the velocity of the flow (left) and the resulting pressure differentials (right) around the model of the animal.

significant increase in drag coefficients with added drag when estimated from the timevarying method (Kruskal-Wallis $X^{2}=4.2102, \mathrm{df}=3, p=0.2396$; Figure 4A).

\subsubsection{Drag coefficients - Video - Velocity fit}

The drag coefficient of non-tagged bottlenose dolphins $(n=11)$ was $0.025( \pm 0.013)$ and again, the variance in drag coefficient is significantly different between tag treatments (Bartlett's $\mathrm{K}^{2}=32.261$, df $=3, p<0.0001$ ). Drag coefficients estimated with the velocity fit method significantly increased with added drag (Kruskal-Wallis $X^{2}=11.666$, df $=3, p=0.0086$; Figure $4 \mathrm{~B})$. There was no detectable increase between no tag and tag conditions $(p=0.7454)$. The tag +4 significantly increased $C_{d}$, by $126.4 \%(p=0.0314)$ and the tag +8 by $192.5 \%(p=0.0200)$ compared to when the dolphins were not instrumented. The tag +4 and $\operatorname{tag}+8$ also led to significantly greater $C_{d}$ compared to the tag-only $(p=$ 0.0124 and 0.0087 , respectively).

\subsubsection{Comparison of methods}

We used three methods to estimate drag coefficients of bottlenose dolphins when not instrumented and when wearing tags of increasing frontal area: one method applied computational fluid dynamics, and the other two measured passive drag using the same underwater video footage. The CFD estimates were lower than video-based estimates, but were still within 
Table 8-3: Dimensions and drag coefficients of the tag and tag + configurations and mean $( \pm \mathrm{SD})$ drag coefficients estimated from simulation (computational fluid dynamics, CFD), and with two experimental methods from video recordings: time-varying and velocity model. Percent increase is relative to the No Tag (Control) condition. Note CFD are not available for the $\operatorname{tag}+8$ and experimental methods were not completed for the tag +2 .

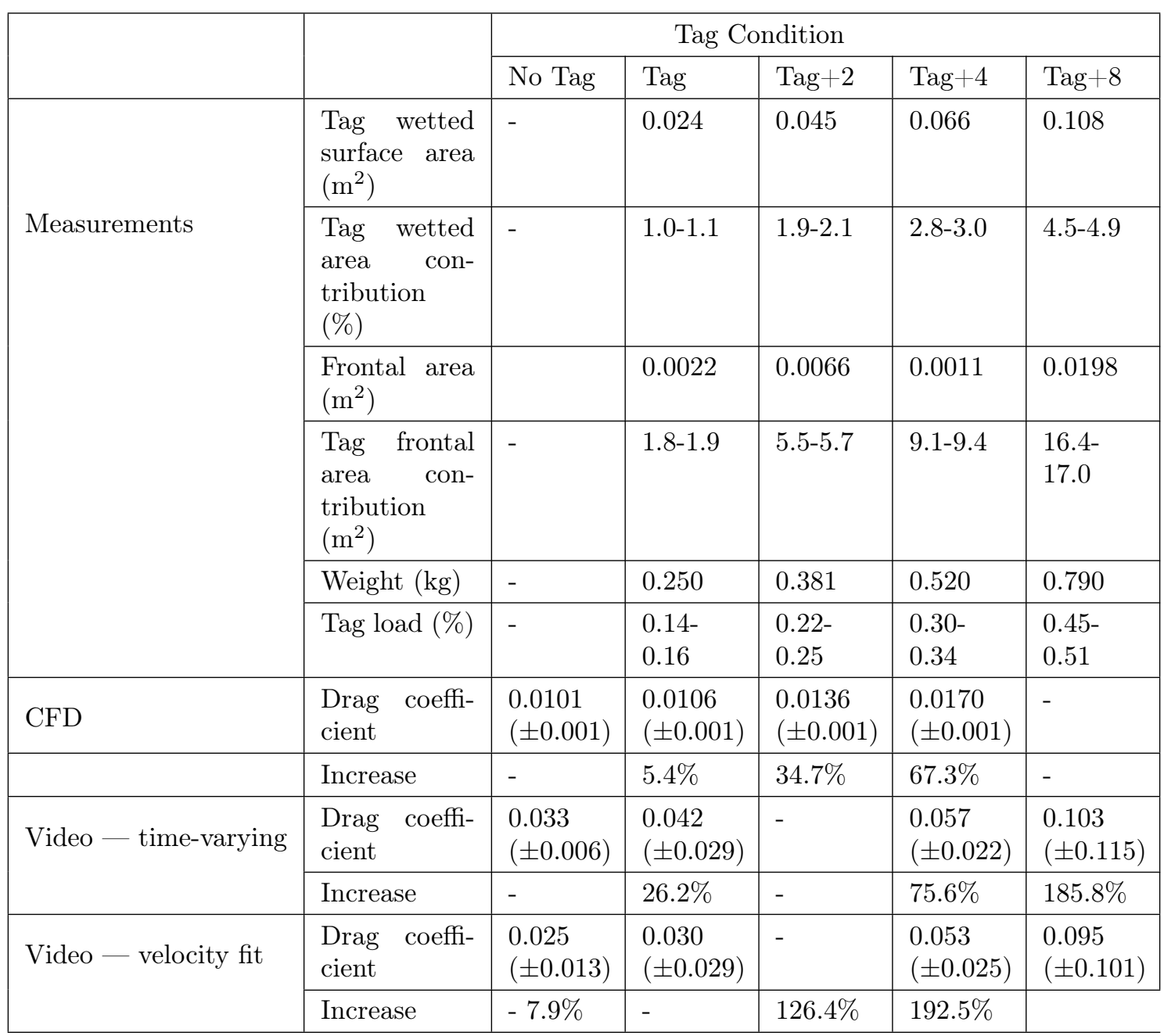

the error of some of the velocity-based estimates (Figure 3A, B). Values obtained from the video and CFD are within the envelope of drag coefficients estimated from experimental and modeling studies, especially for drag-added dolphins (Figure 5; e.g., Lang and Daybell, 1963; Noren et al., 2011). All estimates were higher than the theoretical flat plate estimates for laminar and turbulent boundary layers (black solid and dashed lines; Figure 5), as are most estimates from the near-recent literature.

The two video methods used the same video data for each glide, but computed $C_{d}$ in different ways. Estimates were in close agreement, fitting the $1: 1$ line with $\mathrm{R}^{2}=0.9029$ (Figure 6B). Drag coefficients obtained with the time-varying method were especially higher than the velocity fit method at the lowest drag values $\left(C_{d}=0-0.05\right.$; Figure $\left.6 \mathrm{~B}\right)$.

Based on these differences in drag coefficients, estimated drag forces at $1 \mathrm{~m} / \mathrm{s}$ are variable 

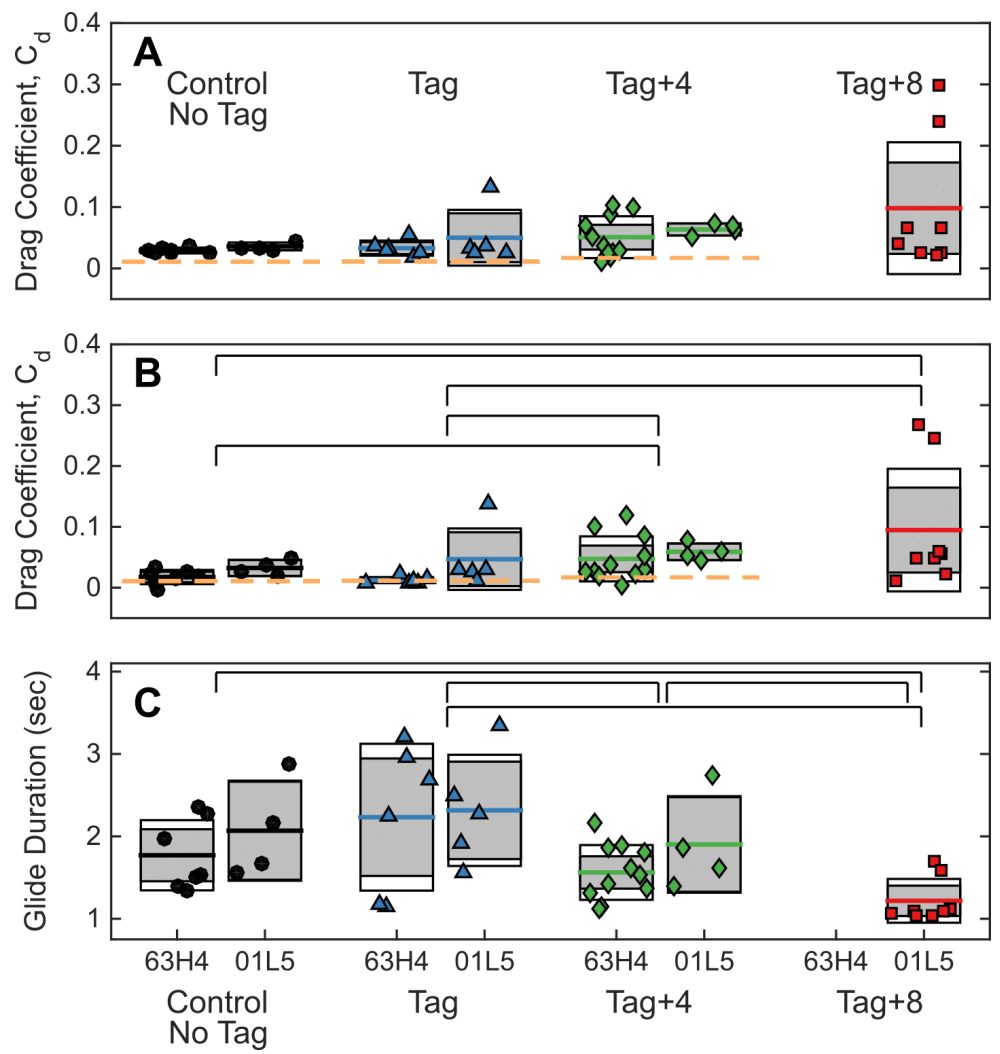

Figure 8-4: Drag coefficients as estimated from underwater video with the (A) time-varying and (B) velocity fit methods, compared with CFD (orange horizontal lines), and (C) the durations of the glides used for the method for two bottlenose dolphins (IDs 63H4 and 01L5) in control (black circles; no tag), tag (blue triangles), tag +4 (green diamonds) and tag +8 (red squares) drag loading configurations. Boxplots show the mean (thick line), standard deviation (grey) and standard error (white). Brackets in B and C denote groups that are significantly different by Kruskall Wallace with Dunn's Multiple Comparison tests. Note CFD were not completed for the tag +8 .

based on the method applied. CFD estimates are consistently lower than the two video-based estimates. At such low speeds, the impact of the tag is barely evident in CFD, increasing drag forces by $1 \%,<1 \mathrm{~N}$. All methods showed an increase in the estimated drag forces with increasing frontal area of the tag package.

\subsubsection{Tag effect}

Variance in glide duration was not equal among tag conditions (Bartlett's $\mathrm{K}^{2}=8.8455$, $\mathrm{df}=$ $3, p=0.03142$ ). Tag condition had a significant effect on glide duration (Kruskal-Wallis $X^{2}$ $=15.731, \mathrm{df}=3, p=0.0013)$ : while there was no difference in glide duration between control and tag $(p=0.3142)$ or $\operatorname{tag}+4(p=0.3497)$ conditions, drag loading with the tag +8 led to significantly shorter glides $(p=0.0036$, Figure $4 \mathrm{C})$. Additionally, success rates declined as drag was added (Figure 7). While both individuals were asked to perform glides in all conditions, dolphin $63 \mathrm{H} 4$ was never able to successfully complete a glide with the tag +8 . 

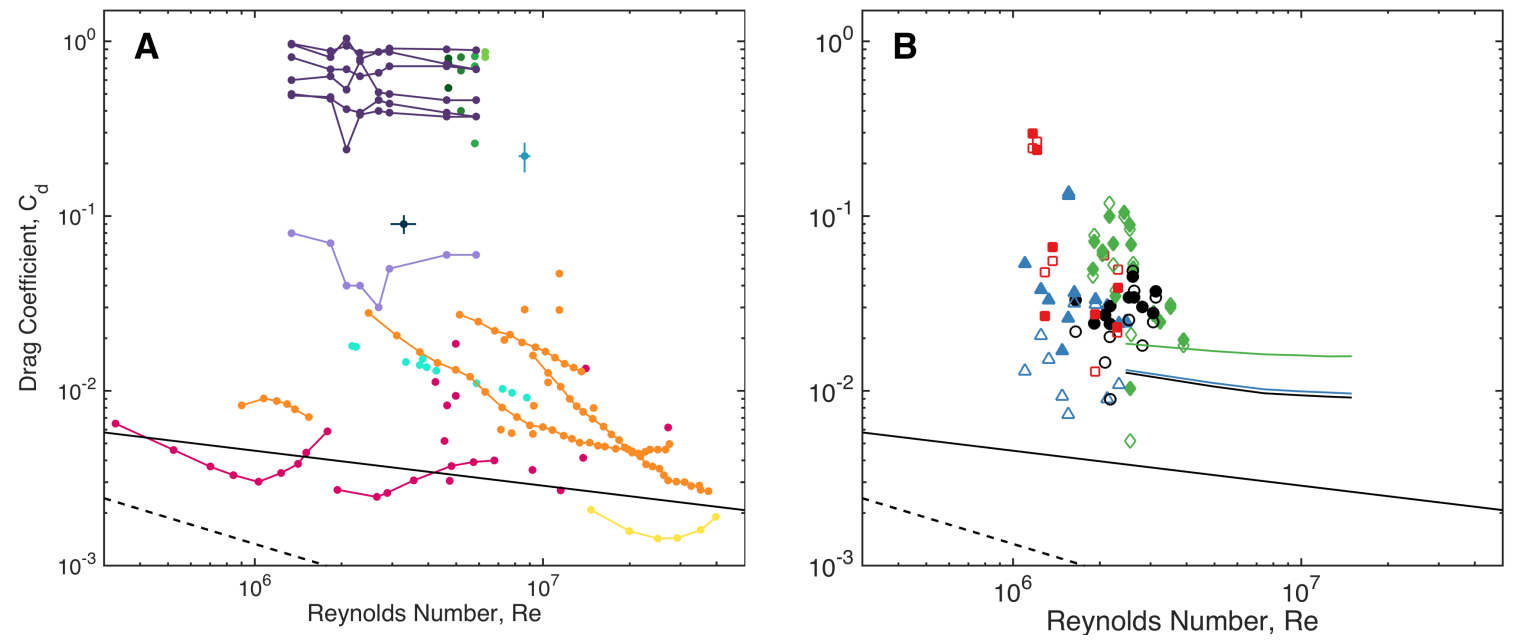

Figure 8-5: Drag coefficients of dolphins and porpoises estimated in historical data (A) compared to this study (B). Data from the literature (A) are presented as points, joined when from the same experimental series and are colored by experimental approach or conditions: light purple $=$ porpoise model, dark purple $=$ porpoise model with various satellite tag designs (Hanson, 2001); green = dolphin wearing collars of increasing thickness (Lang and Daybell, 1963); light blue = pregnant dolphins, dark blue $=$ same dolphins 18 months post-parturition (Noren2011); teal $=$ Digital Particle Imag e Velocimetry (Fish et al., 2014); pink = hydrodynamic models for dolphins; orange = gliding dolphins, rigid models, and towed bodies; yellow = dolphin NACA 66 model. Data from Lang and Norris (1966); Purves et al. (1975); Webb (1975b); Chopra and Kambe (1977) are as presented in Fish et al. (2014). Data from this study (B) follow the same convention as other figures (black circles $=$ no tag; blue triangles $=\operatorname{tag}$; green diamonds $=\operatorname{tag}+4$; red squares $=\operatorname{tag}+8$ ). Colored lines reflect results from Computational Fluid Dynamics.

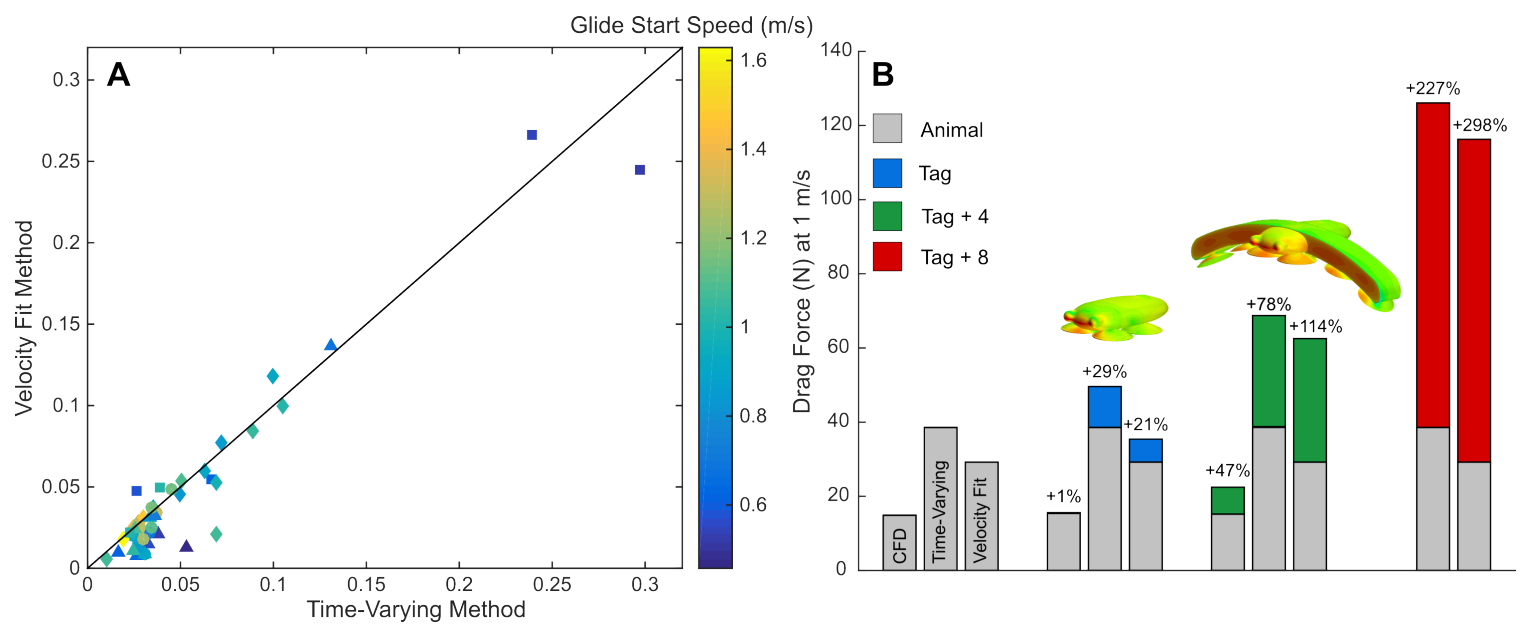

Figure 8-6: Drag coefficients and forces on tagged dolphins with three methods. A: A comparison of the drag coefficients derived by these two methods, colored by the speed at the start of the glide. The black line represents 1:1. B: Contribution of drag forces $(\mathrm{N})$ at $1 \mathrm{~m} / \mathrm{s}$ from the animal's body (grey) and the tag, $\operatorname{tag}+4$ and $\operatorname{tag}+8$ configurations for the three methods used to determine drag coefficients. Note there are no CFD simulations available for tag +8 .

\subsection{Discussion}

This study used computational and experimental methods to estimate passive drag coefficients of instrumented dolphins and assessed the influence of drag loading. Computational 


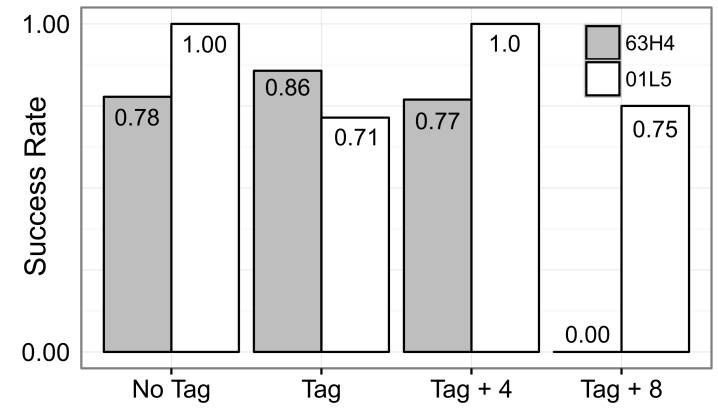

Figure 8-7: Success rates of glide performance of two male bottlenose dolphins (ID 63H4, grey; ID 01L5, white) when not wearing tags, and when instrumented with a bio-logging tag (Tag), and the tag plus additional drag-adding elements attached on each side (Tag +4 and $\mathrm{Tag}+8)$.

and experimental data collected from live animals can both be used to quantify and illustrate the effect of drag loading from tags; these complementary approaches are useful in that they include different sources of error and assumptions. In particular, the experimental results presented in this work can be used to evaluate, inform and improve the models used to generate the simulation results.

\subsubsection{Comparison of methods}

This study applied computational and experimental approaches to estimate drag coefficients in swimming animals to determine the relative effects of instrumentation. Both approaches allowed for comparison between drag loading conditions and to determine the effect of the tag over the control or no-tag scenario. Though the differences in coefficients seem minimal (Table 3), the biological effect is large (Figures 4C, 6B, 7). Simulation results from CFD avoid behavioral or postural effects that may affect experimental results, though they ignore the biology of the system. Simulations reflect only steady-flow conditions across a realistic range of speeds $(1-6 \mathrm{~m} / \mathrm{s})$ that could not be achieved in the experimental trials. CFD provides flow visualization which can illustrate the reasons behind such marked increases in drag (Figure 3; Shorter et al., 2013): the bluff elements used to increase the frontal area of the tag create large areas of fluid damming (high pressure in front of the tag) and stagnate flow (low pressure) behind the tag. This pressure differential acting over the large crosssectional area results in the intended increase in drag forces that are detected in not only the CFD but also the experimental approaches.

Digitizing underwater video footage of gliding animals to obtain position data through time uses live animals with true (vs. computer-generated) body surface characteristics. Glide measurements still represent passive drag and likely simplified estimates of loading, as animals are typically swimming, turning, and moving. Limb movements and changes in body posture can occur, and not all of them may be noticeable (Harris, 1937; Webb, 1975a). 
Body positioning, fluid-surface interactions, camera effects, and the lack of an antenna in CFD simulations all help to clarify why drag coefficients from experimental glide methods were greater than CFD and some of the estimates in the literature (Figure 4). Energy is dissipated as waves are formed at the air-water interface (Vogel, 1994). Wave drag can be significant, especially at these depths of $<1.5 \mathrm{~m}$, but would have been consistent within trials, and between animals and days. Similarly, the substratum could confer edge effects, though animals performed glides in the middle of the available water column $(1.5-2.4 \mathrm{~m}$ deep). Similar to wave effects, bottom effects should at least be consistent between trials. Parallax, user error in digitizing and static positioning of the video recording system with respect to the animals can introduce variability in the glide data, though little distortion was evident based on consistent measurements between the two kinematic markers in each frame throughout glide recordings. The detailed tag geometries included in CFD simulations did not include the $26.5 \mathrm{~cm}$-long, $1 \mathrm{~mm}$ diameter flexible antenna for VHF radio tracking. Antennae can contribute significant drag, though the effect of flexible antenna in the line of flow is less of a concern (Wilson et al., 2004).

This study applied two methods to derive velocity, acceleration, and then the drag coefficient from experimental position data: one takes into account the time-varying nature of each parameter (time-varying method), and the other smooths much of the variation in velocity by fitting with linear regression (velocity-fit method). The velocity-fit method has been applied to e.g., humans (Kjendlie and Stallman, 2008; Barbosa et al., 2015), seals (Williams and Kooyman, 1985), sea lions (Feldkamp, 1987; Stelle et al., 2000), penguins (Clark and Bemis, 1979; Bilo and Nachtigall, 1980) and dolphins (Videler and Kammermans, 1985; Skrovan et al., 1999; Noren et al., 2011), providing for comparison to data in the literature (Figure 4). This method assumes that drag coefficients are constant over the range of velocities encountered during the glide (Bilo and Nachtigall, 1980). The timevarying method is sensitive to changes in measured velocity and acceleration, where the drag coefficient increases throughout the glide (Figure 2). Depending on the duration of the glide or the magnitude of change in velocity, the assumptions of Bilo and Nachtigall (1980) may be less valid (Williams, 1987); in that instance, the time-varying approach may be more appropriate. However, this method incorporates higher variability into estimates, likely leading to a lack of detection of significant differences in drag coefficients between the conditions that was detected with the CFD and velocity-fit methods.

\subsubsection{Use of tag data to estimate drag}

Velocity estimates can be derived from data collected from tags with impellers (Aoki et al., 2011; Suzuki et al., 2014), by applying measured correlations between flow noise and swimming speed (Goldbogen et al., 2008) and when dive pitch is sufficiently high (Miller et al., 2004). These studies have used initial and final velocities during glides to estimate drag 
coefficients of tagged animals. Though the tags in this study recorded sound, estimates of speed from flow noise require per-deployment calibration from high pitch measurements, which were not available here. Additionally, flow noise calibrations are likely too noisy to derive the small changes in velocity (mean $0.27 \mathrm{~m} / \mathrm{s}$; range $0.02-0.58 \mathrm{~m} / \mathrm{s}$ ) at the very low speeds in this study $(0.4-1.6 \mathrm{~m} / \mathrm{s})$.

Tag accelerometers measure animal movement and gravitational acceleration which have been separated (Martin Lopez et al., 2015, 2016; Ware et al., 2016) to derive speed estimates from accelerometers over short (1-3 s) periods. Thus, drag coefficients can be estimated from accelerometer tag data, with the potential to separate form drag from other (e.g. wave, tag) components (Ware et al., 2016), to determine drag forces acting on animals or from their tag packages without measured controls.

\subsubsection{Simulated, measured and natural speeds}

The swimming speeds used in simulation $(1-6 \mathrm{~m} / \mathrm{s} ; \sim 0.4-2.5 \mathrm{l} / \mathrm{s})$ reflect the natural range of swimming speeds in dolphin species. Although dolphins are known to have a high capacity for maximum swimming speeds when trained (6.5-8.2 m/s; 2.5-3.8 l/s; Rohr et al., 2002) or when performing burst behaviors $(15 \mathrm{~m} / \mathrm{s} ; 5.9 \mathrm{l} / \mathrm{s}$; Lockyer, 1987), routine speeds are much lower (1.6-5.6 m/s; Wursig and Wursig, 1979; Irvine et al., 1982; Shane, 1990; Mate et al., 1995; Ridoux et al., 1997). Minimum transport costs $\left(C O T_{\min }\right)$ occur at swimming speeds of 2.1-2.5 m/s ( $1 \mathrm{l} / \mathrm{s}$; Williams et al., 1993; Yazdi et al., 1999).

Glide speeds in the experimental trials were much slower, 0.4-1.6 m/s. Most studies that employ glide speed decay or deceleration methods rely on study animals to swim at speed and cease swimming. Here, the dolphins performed modified stroke and glide swimming behavior by being propelled and released to glide passively through the field of view. The speed at which these glides began was the speed at which the tracking points (zinc oxide) became visible in the video footage.

The fluid environment during glides is complex. Glide speeds translate to Reynolds numbers between $1-4 \times 10^{6}$, while CFD simulations range $2 \times 10^{6}-1 \times 10^{7}$. Transition from laminar to turbulent flow occurs somewhere between $5 \times 10^{5}$ to $1 \times 10^{7}$, depending on the shape and smoothness of the body (Vogel, 1994). During a glide, velocity increases at the rostrum and along the front of the body in areas of high pressure, maintaining a thin boundary layer with low viscous forces (Figure 2; Ungerechts et al., 1998). Velocity and pressure decrease as flow passes the widest point of the body, $0.34-0.45 \times l$ (Fish, 1993); the transition to turbulent flow is most likely to occur at this point (Blake, 1983). Pressure drag increases due to the imbalance of pressure loads in front of and behind this widest point (Vogel, 1994; Ungerechts et al., 1998). Tag dimensions and placement can create greater imbalance in these pressure fields (Figure 2) or initiate earlier boundary layer separation which increases drag (Culik et al., 1994). For non-instrumented animals at low speeds 
during glides, observations suggest that the boundary layer remains attached up to the base of the flukes (Fish and Hui, 1991; Rohr et al., 1998). Larger tags and at higher speeds may lead to short-term separation (Figure 2), though reattachment is likely, especially with active movement (Anderson et al., 2001). Boundary layer thickness and flow conditions differ between passively gliding and actively swimming animals. Because propulsive movements may increase drag by $2-4 \times$ depending on the swimming style (Lighthill, 1969; Webb, 1975b), passive drag estimates do not necessarily reflect the active force balance. Still, they provide relative changes in drag forces associated with instrumentation that are still valid during natural behaviors, as has been done here.

We carried out these experiments and simulations with the DTAG placed in an optimal location for small cetaceans: between the blowhole and dorsal fin, in the forward-facing orientation. Tags deployed in capture-release programs (Moore et al., 2016) are typically placed in this preferred position; however, pole-based deployments on cetaceans of any size lead to attachments in any orientation or location. Tags placed on different regions of the body may have considerably different hydrodynamic regimes and will therefore contribute different drag and lift forces and pitching moments: when placed ahead of the point of maximal girth tags can lead to early flow separation and large increases in frontal area (Bannasch et al., 1994), but those placed too far caudally can result in body destabilization (Culik and Wilson, 1991; Healy et al., 2004; Vandenabeele et al., 2015). Suction cup tags such as the DTAG can slide into a new position or orientation as drag forces over the tag exceed suction forces in the cup holding it to the animal (Moore et al., 2016). When oriented sideways or in any direction off-axis, the DTAG will have a greater frontal area, and will expose the antenna to transverse flow; experimental and simulated drag forces on the DTAG3 vary by $\pm 10 \mathrm{~N}$ depending on orientation (Shorter et al., 2013). Different attachment locations increased one tag's drag by $0-10 \%$ on turtle casts in a wind tunnel (Jones et al., 2011); a similar effect of a DTAG attached in an alternate orientation or that has moved throughout a deployment could be expected.

\subsubsection{Tag effect}

Bottlenose dolphins remain relatively hydrodynamically efficient when instrumented with the DTAG3 alone, with a measured 5-26\% increase in drag coefficient, no decrease in glide duration, and no effect on glide success rate. Jones et al. (2013) suggest a qualitative framework to establish acceptable levels of instrument drag; the DTAG3 roughly falls within their 'green' range of 0-20\% drag increase based on two of three measurement methods in this study. While the DTAG3 still increases the drag loading on the animal these results indicate that, as currently designed, this design may be appropriate for use on cetaceans as small as a bottlenose dolphin. Hydrodynamic tag designs that are $<1 \%$ of the wetted area and $<2 \%$ of the frontal area of the wearer are recommended. 
Increasing loading beyond the DTAG3's dimensions leads to further increases in estimated drag coefficients. The tag $+2, \operatorname{tag}+4$ and $\operatorname{tag}+8$ conditions have bluff bodies with no hydrodynamic fairing. While these designs represent $\sim 2, \sim 3$ and $\sim 5 \%$ of the wetted area of the dolphins, they are $\sim 6, \sim 9$ and $\sim 17 \%$ of their frontal area. These treatments increased drag coefficients by $67-126 \%$ and 186-193\% (Table 3). Further, animal performance of the gliding task seems to deteriorate beyond the tag +4 condition. Variability in estimated drag coefficient increased, glide duration decreased, and glide success was reduced (Figures 3,6). These results agree with the concerns from previous work showing behavioral changes with instrumentation (van der Hoop et al., 2014a; Broell et al., 2016), and especially with large tags (Wilson et al., 1986; Littnan et al., 2004; McMahon et al., 2008; Berga et al., 2014; Maresh et al., 2014).

\subsubsection{Tag size recommendations}

Attaching tags to animals increases the wearer's mass and surface area, contributing to changes in the overall drag coefficient (Equations 1 and 3). Mass is of less concern in a buoyant medium, but frontal or wetted area and the shape of the device can have a large impact on the added drag loading created by the tag. When designing a study, it is important to know the amount of drag a particular tag or set of tags may add to the research subject, and whether or not this drag is sufficient to affect the animal's behavior or physiology, or the data that are collected. However, every researcher intending on using tags should not be charged with the task of completing CFD simulations or experimental assessments of these parameters. Percent increases in drag coefficient scale with the percent increase in wetted and frontal areas of the tag setups used in this study (Figure F2). Based on these relationships and the animal responses presented herein, a recommendation can be made on the appropriate tag dimensions for a given body size or surface area of the tag subject. Tags that increase wetted area by $\leq 1 \%$ and frontal area by $\leq 2 \%$ (e.g., the DTAG3 deployed here) increase $C_{d}$ by $5-26 \%$ (Table 3), or increase drag forces experienced by the animal at $1 \mathrm{~m} / \mathrm{s}$ by $1-29 \%$ (Figure 5). Following Jones et al. (2013), tags of these dimensions are suitable for deployment, especially over the short-term. Tags between 1-3\% of a cetacean's wetted surface area or between $2-10 \%$ of their frontal area, such as the tag +4 tested here, increase drag coefficient by $67-126 \%$ (Table 3) and increase drag forces by $47-114 \%$ when swimming at $1 \mathrm{~m} / \mathrm{s}$ (Figure 6). With this level of drag loading, behavior (e.g. Figure 7) and energetics (Chapters 9, 10) can be affected.

Based on these results, we used body lengths and wetted and frontal surface areas from Bose et al. (1990) and Fish (1998) to calculate contours of tag sizes that would fall into the acceptable size ranges for deployment on specific odontocete species (Figure 8). This graphic can be used to determine tag areas that do not exceed our recommendations based on the $3 \%$ wetted area or $10 \%$ frontal area of a study species based on their body size, or to 
identify tag designs that should be attached with caution or for particularly short durations. The DTAG3 and tag +4 configurations fall along these contours, as they form the basis for the diagram. A tag with the wetted or frontal areas of the tag +8 is too large for deployment on bottlenose dolphins, but may be acceptable for larger species (body length $>4 \mathrm{~m}$, e.g. beaked or killer whales).
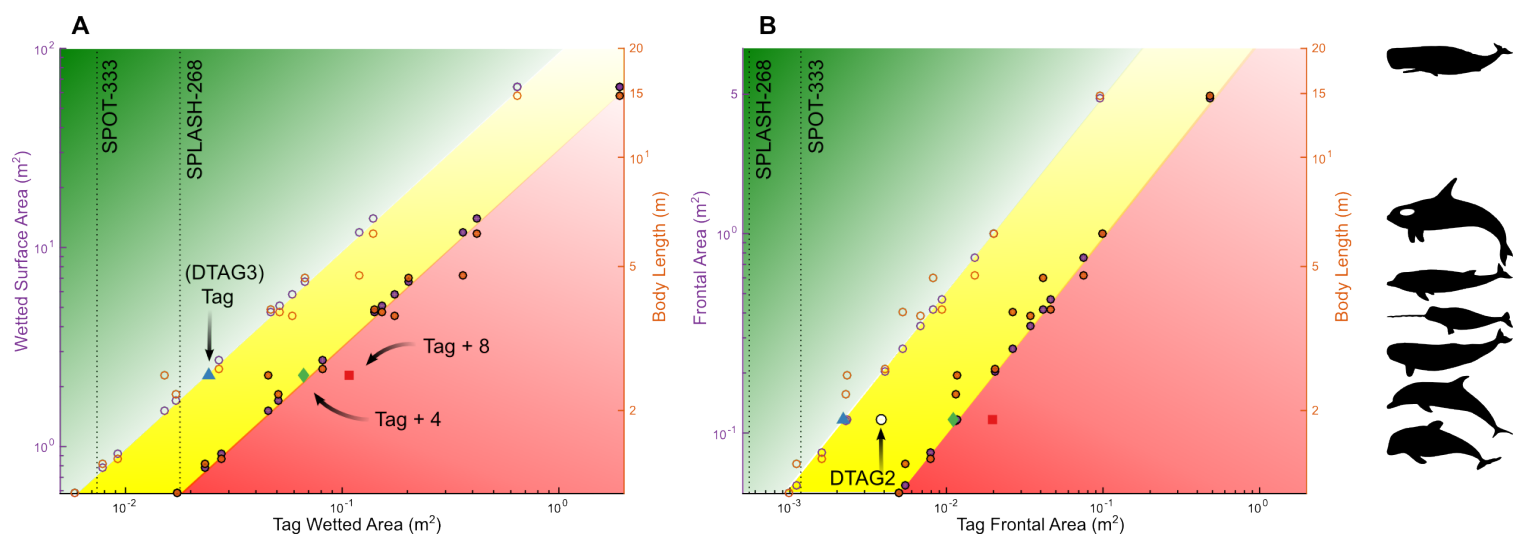

Figure 8-8: Recommended tag dimensions (wetted and frontal areas; $\mathrm{m}^{2}$ ) for cetacean species based on their body length (m; orange) and wetted (A) and frontal (B) areas $\left(\mathrm{m}^{2}\right.$; purple). Open circles represent acceptable tag dimensions that would increase the subject's drag by $5-26 \%$ as measured in this study; filled circles represent tag dimensions that increase drag by $67-126 \%$. Tags whose wetted area fall within the green and yellow areas represent those that can be considered 'good' and 'satisfactory' for deployment on the species in question. Deployments of tags on species that fall in the red area should be avoided, especially on the long-term. The tags used in this study are shown as blue, green, and red markers. Two Wildlife Computers tags, the SPOT-333 (LIMPET) and SPLASH-268 tags are also included for reference. Body length and animal surface area values are from Bose et al. (1990); Fish (1998).

How do other tags on the market compare? For reference, we have included dimensions of the SPOT-333 (LIMPET) and SPLASH-268 tags from Wildlife Computers (Redmond, WA, U.S.A.). Based on wetted areas, our results suggest that both tags are suitable for deployment on small cetaceans, but that the larger SPLASH-268 tag could be considered best for animals $>2 \mathrm{~m}$ long (Figure 8A). However, the SPLASH-268 tag is designed to attach with a single pin to sit behind the dorsal fin, adding negligible frontal area to the tag subject, making it suitable for deployment on even the smallest odontocetes (Figure 8B). This shows the balance between frontal and wetted areas that is struck when designing a tag, depending on the intended study species and attachment mechanism. The shape of the tag housing and its placement is critical to consider when assessing a tag's suitability based on these figures. Shape optimization of a tag with the same frontal area reduced the drag burden on tagged penguins by 37-46\% at routine swimming speeds (Bannasch et al., 1994).

Similarly, attaching multiple tags can lead to interactive effects that cannot be predicted from combined areas alone (Jones et al., 2011). Thirty percent of marine mammal biologging studies reviewed in (McIntyre, 2014) reference data from more than one device per 
animal (McIntyre Pers. Comm. ${ }^{1}$; this is likely a conservative estimate as many studies report only the number of devices per animal that generated the data for the paper, rather than the total number of devices attached. The tag size recommendations herein are not intended to evaluate the suitability of tags that will be deployed coincident with others on a single animal.

Both researchers and tag companies have taken active roles to work together in reducing device size (Bannasch et al., 1994; Hazekamp et al., 2009; Pavlov and Rashad, 2012; Balmer et al., 2013), refining the tools on the market. For example, the transition from the DTAG2 to current DTAG3 model reduced the frontal area by $\sim 1 / 3$, making it more suitable for deployments on smaller species (Figure 8B; Shorter et al., 2013). Many early-generation tags fall well within the 'red' zone presented here (e.g., side-mount tags, (Hanson, 2001; Geertsen et al., 2004; Berga et al., 2014); or large camera tags, (Skrovan et al., 1999; Littnan et al., 2004)), which did elicit significant responses from tag wearers. Luckily, recent advances and refinements have provided better tag design alternatives that should be favored by researchers and permitting agencies. We recommend the use of tags that have small wetted and frontal surface areas and that incorporate hydrodynamic design elements to reduce the potential effects of drag loading on the animals; other tagging effects from e.g., handling stress and attachment mechanisms still hold.

Finally, the size, shape and resulting amount of drag added to tag subjects should be considered relative to the intended deployment duration. The short-term (12-48 h) deployments of external suction-cup tags such as the DTAG contribute little additional work and energy consumption in the context of a monthly or annual energy budget (Jones et al., 2013, see e.g.,). Tags deployed for weeks or months (e.g. external tags glued to the animal's surface) should be especially small and hydrodynamically shaped. While this consideration would minimize the energetic impact of tagging, it does not address the issue of recording unnatural behaviors due to tag effects for a tag deployment of any duration (Broell et al., 2016).

\subsection{Conclusions}

Transmitting and archival tags used for animal science have been miniaturized due to technological advances and to help reduce impacts. Fortunately, more and more studies are assessing the impacts of tags across taxa (Culik et al., 1994; Barron et al., 2010; Jones et al., 2013; van der Hoop et al., 2014a; Broell et al., 2016). These results suggest that computational and experimental methods can be used to determine the increase in drag associated with tags and whether this impact is appropriate over the intended duration of tag attach-

\footnotetext{
${ }^{1}$ Trevor McIntyre, PhD. Mammal Research Institute, Department of Zoology and Entomology, University of Pretoria, South Africa
} 
ment. This work follows on Jones et al. (2013) to provide a suggested framework with which to evaluate appropriate tag sizes across a range of body sizes of cetacean tag subjects, in the event that CFD or experimental approaches cannot be conducted for a particular project. 
Chapter 9

\section{ENERGY ECONOMY IN BOTTLENOSE DOLPHINS UNDER DIFFERENT LEVELS OF INSTRUMENTATION DRAG \\ LOADING}

This chapter has been formatted to be submitted to the Journal of Experimental Biology, with authorship contributions from Andreas Fahlman, K. Alex Shorter, Victor Petrov, Julie Rocho-Levine, and Michael Moore

JvdH, AF, KAS, JR-L and MJM developed concepts; JvdH, AF, KAS, JR-L and MJM performed fieldwork; JR-L directed animal husbandry and training; JvdH and AF processed and analyzed data; KAS and VP conducted and analyzed simulations; JvdH, AF, KAS, VP, JR-L and MJM wrote the manuscript.

The supplemental materials for this chapter can be found in Appendix G. 


\subsection{Abstract}

Instrumenting animals with tags contributes additional resistive forces (weight, buoyancy, lift and drag) that may result in additional energy costs. Alternatively, this additional metabolic expense can be avoided if behavior is adjusted to reduce movement costs in an effort to maintain power output. We sought to increase the cost of a swimming task by increasing drag on bottlenose dolphins by up to 2.4 times, to investigate the metabolic effect of instrumentation. We refined a previous research protocol to determine whether (1) metabolic rate increases systematically with greater levels of drag loading or (2) whether individuals employ strategies to compensate for the increased loading, such as (a) reducing speed, (b) reducing swimming distance or (c) avoiding surface wave drag. We detected no significant difference in oxygen consumption rates when four male dolphins performed a repeated swimming task, but they reduced their swimming speeds by up to $45 \%$ in the largest loading condition: dolphins changed their behavior to optimize energy economy. In repeated swimming trials where we added and removed drag incrementally $(\sim 15 \mathrm{~N}$ at $3 \mathrm{~m} / \mathrm{s})$, dolphins adjusted their swimming paths to cut corners as drag increased, and reduced their speeds in line with predictions from Computational Fluid Dynamics simulations. Dolphins did not avoid additional surface wave drag by swimming at greater depths. These results indicate how animals employ smart strategies to economize movement within the constraints of a task and their environment, and that tagged animal behavior cannot always be considered representative of the untagged population.

\subsection{Introduction}

Tagging studies strive to collect novel data in an environment where observations are difficult - where tags are needed to measure animal movement as well as environmental and physiological variables to help interpret animal behavior or performance (Johnson et al., 2009; Crossin et al., 2014; Hussey et al., 2015). Tags do, however, contribute additional weight and bulk, and more relevant in the marine realm, buoyancy, lift and drag. It is important to know the impact of these devices not only on the tag subject's vital rates (e.g., Barron et al., 2010; Best et al., 2014; van der Hoop et al., 2014a), but also on their behavior which we often assume to be representative of the untagged population (Ropert-Coudert and Wilson, 2004; Vandenabeele et al., 2011; Broell et al., 2016).

The physiological and behavioral changes associated with handling, attachment, healing, or sedation that may be involved in the tagging procedure can be difficult to separate, especially over short-term studies (Elliott et al., 2012; Jepsen et al., 2015). A more physically-grounded principle that should be consistent among tag subjects is that resistive forces (e.g., drag, weight or buoyancy) contributed by the tag should require more work to 
overcome. Additional thrust must be produced to counter drag, or more lift is required to counter weight, though weight is of less concern in water due to buoyancy. Drag forces, specifically, increase with speed-squared and therefore present a steep trade-off (Jones et al., 2013). Animals can maintain speed under drag-loading but will need to consume more energy to produce the additional power required. Alternatively, individuals can compensate for the added loading created by the tag by reducing their speed to maintain a 'normal' power output. Diving vertebrates may have several compensatory mechanisms; for example, there may be changes in dive behavior (Webb et al., 1998) that do not result in detectable changes in energy requirements (Fahlman, 2008).

In a previous study (van der Hoop et al., 2014a), bottlenose dolphins (Tursiops truncatus) changed their swimming behavior in response to drag loading from a bio-logging tag (DTAG2; Johnson and Tyack, 2003). Dolphins performing a repeated swimming task slowed down to the point where the tag yielded no increases in drag or power; in doing so, individuals' energy consumption rates were no different. These results suggested that dolphins modify their behavior to maintain metabolic output and energy expenditure when faced with tag-induced drag. Other strategies exist to reduce the metabolic cost of these conditioned swimming tasks. Changing their movement path between markers would enable individuals could reduce their total distance over time (Alexander, 2003; Ohashi et al., 2007). Additional drag forces from surface wave effects could be avoided by swimming deeper in the water column (Hoerner, 1965; Hertel, 1969; Blake, 2009); or, by swimming deeper, individuals could take advantage of bottom effects that confer considerable propulsive benefits (Rayner, 1991; Webb, 1993). Building on our previous work, this research extends the experimental protocol monitoring swimming speeds and movement patterns to investigate the impact of multiple levels of drag loading to determine whether (1) metabolic rate increases systematically with greater levels of drag loading or (2) whether individuals employ strategies to reduce metabolic cost, such as (a) reducing speed, (b) reducing swimming distance or (c) avoiding surface wave drag or benefitting from substratum effects.

\subsection{Methods}

\subsubsection{Overview}

We trained four male bottlenose dolphins (Table 1) with operant conditioning to complete two types of swimming tasks to determine the effect of drag loading conditions on individuals' metabolic rates, swimming speeds, patterns and kinematics (fine-scale movement patterns). The first swimming task (Metabolic Trials, below) involved measuring individuals' metabolic rates before and after a continuous 10 min lap swim under three drag conditions (no tag, tag, tag +8 ; Drag Conditions, below). In the second swimming task (Incremental Loading Trials, below), animals swam one lap at a time, as drag was adjusted piece-by-piece through 
all drag conditions ( 6 total: no tag, $\operatorname{tag}, \operatorname{tag}+2, \operatorname{tag}+4, \operatorname{tag}+6, \operatorname{tag}+8)$. All experimental trials were conducted in a man-made lagoon up to $3.5 \mathrm{~m}$ deep at Dolphin Quest, Oahu in Honolulu, HI, U.S.A. from 23 Sept - 15 Oct 2013. All experiments were by voluntary participation without restraints and the dolphin could refuse to participate or withdraw at any point during an experimental trial. Prior to initiating the study, we desensitized animals to the equipment and trained them for novel research-associated behaviors. We provide a list of symbols and abbreviations in Table 2 .

Table 9-1: Measurements and number and order of experimental metabolic trials. Metabolic trials were performed under control ( $\mathrm{C}$, no tag), tag ( $\mathrm{T}$, DTAG3), and tag $+8(\mathrm{~T}+8)$ conditions. Girth was measured approximately two fingers in front of the dorsal fin after animals exhaled.

\begin{tabular}{|l|l|l|l|l|l|l|l|}
\hline $\begin{array}{l}\text { Dolphin } \\
\text { ID }\end{array}$ & $\begin{array}{l}\text { Length } \\
(\mathrm{m})\end{array}$ & $\begin{array}{l}\text { Girth } \\
(\mathrm{m})\end{array}$ & $\begin{array}{l}\text { Body } \\
\text { Diameter } \\
(\mathrm{m})\end{array}$ & $\begin{array}{l}\text { Weight } \\
(\mathrm{kg})\end{array}$ & $\begin{array}{l}\text { N trials } \\
\text { com- } \\
\text { pleted }\end{array}$ & $\begin{array}{l}\text { Days be- } \\
\text { tween trials }\end{array}$ & $\begin{array}{l}\text { Metabolic } \\
\text { trial order }\end{array}$ \\
\hline 6JK5 & 2.61 & 1.4 & 0.45 & 198 & 9 & $\begin{array}{l}2,1,2,1,4, \\
2,4,6\end{array}$ & $\begin{array}{l}\mathrm{C}, \mathrm{T}+8, \mathrm{C}, \\
\mathrm{T}, \mathrm{T}+8, \mathrm{~T}, \\
\mathrm{C}, \mathrm{T}, \mathrm{T}+8\end{array}$ \\
\hline 99L7 & 2.54 & 1.21 & 0.39 & 167 & 4 & $2,5,6$ & $\mathrm{C}, \mathrm{T}, \mathrm{C}, \mathrm{T}$ \\
\hline 9FL3 & 2.73 & 1.49 & 0.47 & 240 & 9 & $\begin{array}{l}2,4,2,1,2, \\
2,1,2\end{array}$ & $\begin{array}{l}\mathrm{C}, \mathrm{T}, \mathrm{T}+8, \\
\mathrm{C}+8, \mathrm{~T}, \\
\mathrm{C}, \mathrm{T}+8, \mathrm{~T}\end{array}$ \\
\hline 9ON6 & 2.46 & 1.28 & 0.41 & 171 & 4 & $1,5,4$ & $\mathrm{~T}+8, \mathrm{C}, \mathrm{T}, \mathrm{C}$ \\
\hline
\end{tabular}

\subsubsection{Drag conditions}

We designed multiple drag conditions based on the dimensions of the DTAG (version 3; Johnson and Tyack, 2003; Shorter et al., 2013), a suction-cup attached bio-logging tag approximately $15 \mathrm{~cm}$ in length (Figure 1). Along with control (no tag) and tag (DTAG) conditions, we created additional urethane elements to attach to either side of the DTAG with zip ties. These drag-adding elements each had approximately the same cross-sectional area as the tag itself, but adding two elements disproportionately increases the tag setup's total drag from $1.6 \mathrm{~N}$ to 34.6 at $3 \mathrm{~m} / \mathrm{s}$ due to their shape (Chapter 8 ). We refer to the resulting increased drag-loading conditions by the number of elements added, e.g., the tag +4 condition includes the tag and four elements total (Figure 1A, B) and the tag +8 condition includes the tag and eight elements total (Figure 1D). For all conditions, we attached the tag to the back of the animal, between the blowhole and dorsal fin to replicate the location of tags placed on wild animals. Metabolic trials were conducted without the tag (control), and with the tag and tag +8 configurations; incremental loading trials included all drag loading conditions. 


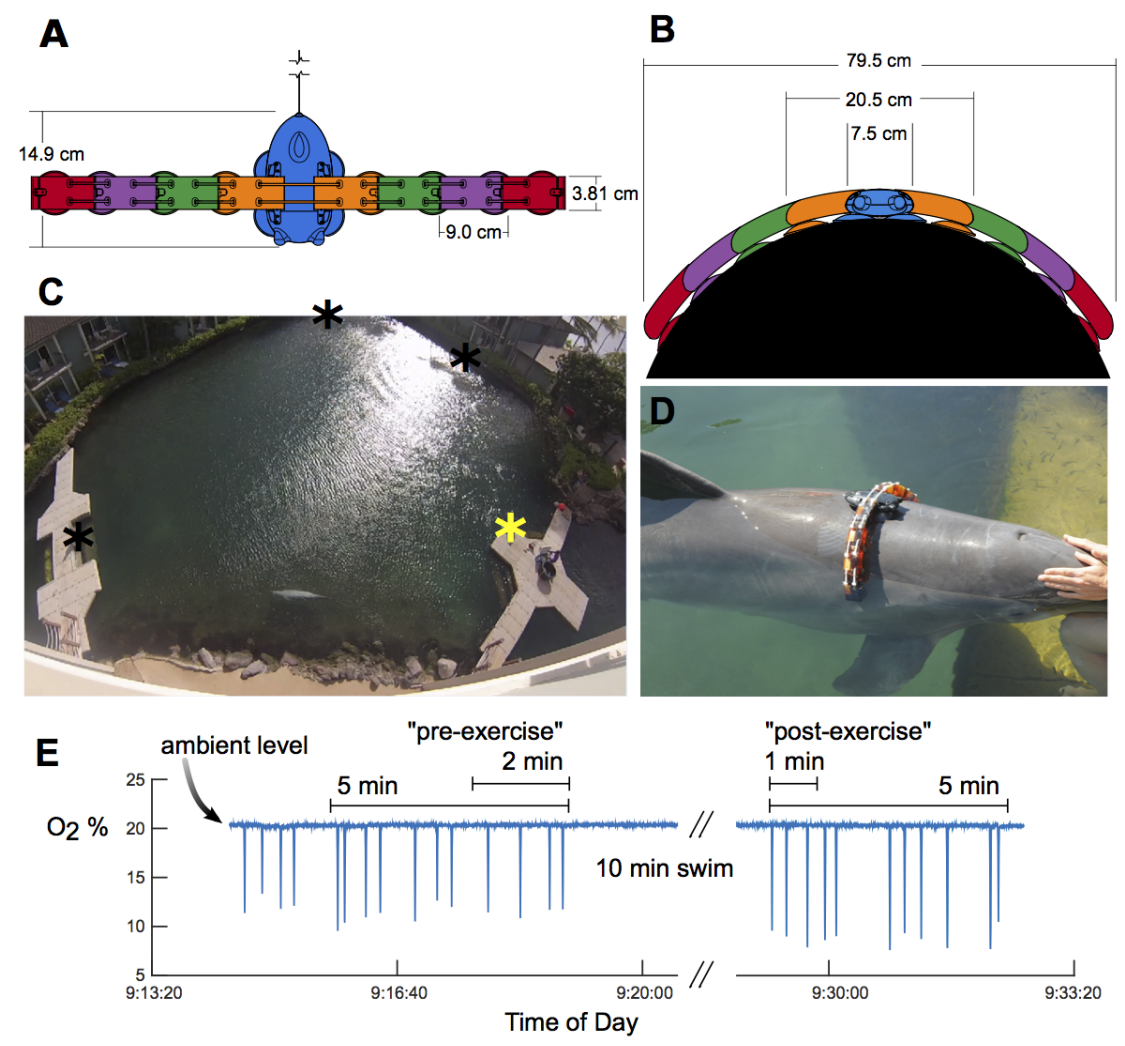

Figure 9-1: The experimental setup. Top (A) and frontal (B) views of the DTAG (blue) and additional elements that were attached to increase drag loading. Each element has approximately the same cross-sectional area as the tag alone. (C) Dolphins swam between waypoints in the lagoon (stars), and respirometry was measured at the main dock station (yellow star). (D) A bottlenose dolphin equipped with the tag +8 configuration: four elements attached on each side of a DTAG (black). (E) Example of oxygen concentration $\left(\mathrm{O}_{2} \%\right)$ measured with the respirometer over the duration of a metabolic trial, with 2-minute 'pre-exercise' and 1-minute 'post-exercise' and 10-minute swim periods highlighted. Sudden drops in $\mathrm{O}_{2} \%$ reflect inhalations by the dolphin.

\subsubsection{Swimming task 1: Metabolic trials}

To determine the metabolic impacts of increasing drag, we trained four dolphins to swim clockwise between four waypoints in the lagoon where trainers were stationed (one lap; Figure 1C) for a minimum of ten minutes (maximum = 10:33 min:sec). We did not control swimming speed. We measured dolphins' metabolic rate before and after this ten-minute swimming task (see Respirometry, below). Animals breathed freely while swimming and none of the breaths during exercise were captured using the respirometer. We chose the drag condition for each trial at random for each individual; however, Dolphin 99L7 did not perform any trials with tag +8 due to earlier unsuccessful attempts to complete the task with high drag loading. A trial was terminated if the dolphin was not completing the task as directed, or if the animal chose to stop. Each dolphin performed only one swimming trial per day. All metabolic trials were completed between 8:37 and 11:18 am, when animals were 
post-prandial.

\subsubsection{Swimming task 2: Incremental loading trials}

To determine the effects of small ( $\sim 15 \mathrm{~N}$ at $3 \mathrm{~m} / \mathrm{s})$, incremental changes in drag loading and unloading on swimming behaviors, two bottlenose dolphins swam one lap between the same waypoints as above (Figure 1), but in the counter-clockwise direction. The first lap began with the tag +8 loading condition. After each lap, we symmetrically removed drag elements: $\operatorname{tag}+8, \operatorname{tag}+6, \operatorname{tag}+4, \operatorname{tag}+2, \operatorname{tag}$, control. We then re-built the tag set up, adding drag elements after each lap: control, $\operatorname{tag}, \operatorname{tag}+2, \operatorname{tag}+4, \operatorname{tag}+6, \operatorname{tag}+8$. We measured the time it took to complete each lap with a stopwatch, and recorded the swimming paths of the dolphins with an camera mounted above the lagoon (see Aerial Video, below). We did not measure metabolic rate during this second swimming task. We undertook trials between 12:00 and 15:05 on 14 Oct (ID 6JK5) and 15 Oct (ID 9FL3) 2013. The time between laps was on average 2:19 and 2:45 minutes (IDs 6JK5 and 9FL3, respectively) with a range of 0:30 - 8:38 min. Individuals had been fed before the trial, and were reinforced between laps. Approximately two hours elapsed between unloading and loading portions of the trial for both individuals.

\subsubsection{Respirometry}

To measure dolphins' respiratory flow rates and expired oxygen $\left(\mathrm{O}_{2}\right)$ and carbon dioxide $\left(\mathrm{CO}_{2}\right)$, we placed a custom-made Fleisch type pneumotachometer (Micah Brodsky, V.M.D. Consulting, Miami, FL) with a low-resistance laminar flow matrix (Item No. Z9A887-2, Merriam Process Technologies, Cleveland, OH) over the blowhole (Fig. 1 in Fahlman et al., 2015). We connected a differential pressure transducer (MPX-2.5 mbar type 339/2, Harvard Apparatus, Holliston, MA) to the pneumotachometer with two, $310 \mathrm{~cm}$ lengths of $2.0 \mathrm{~mm}$ inner diameter (I.D.), firm walled, flexible tubing. We calibrated the measured flow rates by integrating the signal from the pressure transducer with a $7.0 \mathrm{l}$ calibration syringe (Series 4900, Hans-Rudolph Inc, Shawnee, KS) immediately before and after each metabolic trial. We used a series of pump cycles at various flow speeds to calibrate the total volume and flow rates of expiratory and inspiratory phases (Fahlman et al., 2015).

A subsample of the gas immediately outside the blow-hole was drawn at a flow rate of $200 \mathrm{ml} \mathrm{min}-1$ through a $310 \mathrm{~cm}$ length of $2 \mathrm{~mm}$ I.D., firm walled, flexible tubing and a $30 \mathrm{~cm}$ length of $1.5 \mathrm{~mm}$ I.D. Nafion tubing, to a fast-response $\mathrm{O}_{2}$ and $\mathrm{CO}_{2}$ analyzer (ML206, Harvard Apparatus, Holliston, MA, USA). The response times for a $90 \%$ change to equilibrium for $\mathrm{O}_{2}$ and $\mathrm{CO}_{2}$ were $67 \mathrm{msec}$ and $94 \mathrm{msec}$, respectively (Fahlman et al., 2015). The gas analyzers sampled at $200 \mathrm{~Hz}$; we phase-corrected resulting respiratory gas signals to match the respiratory flow rates to account for the time lag for gas to flow through the

tubing. We estimated the $\mathrm{O}_{2}$ consumption rate $\left(\dot{V} \mathrm{O}_{2}, 1 \mathrm{O}_{2} / \mathrm{min}\right)$ as previously described 
Table 9-2: List of symbols and abbreviations.

\begin{tabular}{|l|l|l|}
\hline Symbol & Definition & Unit \\
\hline$a$ & Derived coefficient & \\
\hline$b$ & Derived coefficient & \\
\hline $\mathrm{CFD}$ & Computational Fluid Dynamics & \\
\hline $\mathrm{CO}_{2}$ & Carbon dioxide & \\
\hline$D$ & Drag force & $\mathrm{N}$ \\
\hline $\mathrm{O}_{2}$ & Oxygen & \\
\hline $\mathrm{PAR}$ & Physical Activity Ratio & \\
\hline$U$ & Speed & $\mathrm{m} / \mathrm{s}$ \\
\hline$U_{r e d}$ & Reduced speed & $\mathrm{m} / \mathrm{s}$ \\
\hline$\dot{V} \mathrm{O}_{2}$ & Oxygen consumption rate & $1 \mathrm{O}_{2} / \mathrm{min}$ and $\mathrm{ml} \mathrm{O}_{2} / \mathrm{kg} / \mathrm{min}$ \\
\hline
\end{tabular}

in Fahlman et al. (2015), calculating the instantaneous oxygen consumption by multiplying the expiratory flow rate and $\mathrm{O}_{2}$ content. We then integrated the instantaneous $\dot{V} \mathrm{O}_{2}$ to yield the total volume of $\mathrm{O}_{2}$ exchanged during each breath. We summed these $\mathrm{O}_{2}$ volumes for each breath during the trial period and divided by the duration of sub-portions of the trial (before or after exercise, see below) to provide an average $\dot{V} \mathrm{O}_{2}$ for those time periods. We report $\dot{V} \mathrm{O}_{2}$ as measured $\left(\mathrm{l} \mathrm{O}_{2} / \mathrm{min}\right)$ and as mass-specific $\left(\mathrm{ml} \mathrm{O}_{2} / \mathrm{kg} / \mathrm{min}\right)$ by dividing by the measured weight of the individuals during the month of the study period (Table 1 ).

We calibrated the gas analyzers before and after the experiment using a commercial mixture of $5 \% \mathrm{O}_{2}, 5 \% \mathrm{CO}_{2}$, and $90 \% \mathrm{~N}_{2}$ (blend accuracy 0.10\%; Praxair, Inc; Danbury, CT, USA). We used ambient air to check the calibration before and after each experimental trial. We obtained hourly mean air temperature and humidity measurements from the National Weather Service database for the times of the trials (National Weather Service, 2015). All gas volumes were converted to standard temperature pressure dry (STPD). Exhaled air was assumed to be saturated at $37^{\circ} \mathrm{C}$, and inhaled air volume was corrected for ambient temperature and relative humidity.

We designed a specific experimental design to assess the metabolic changes associated with variation in drag, as follows. The dolphin was asked to remain neutrally buoyant at the water surface next to the trainer for at least $5 \mathrm{~min}$ immediately prior to exercise while the pneumotachometer was placed over the blow hole allowing the respiratory flow, gas content and metabolic rate to be determined (Figure 1C, E). The 'pre-exercise' metabolic rate was determined to be the average $\dot{V} \mathrm{O}_{2}$ over the last $2 \mathrm{~min}$ of this period. By this time the variability in the instantaneous $\dot{V} \mathrm{O}_{2}$ had decreased. Following the swimming task, the dolphin returned immediately to the measurement station, where we continuously measured the respiratory flow and gas composition for a minimum of $5 \mathrm{~min}$. We defined the first 1 min of this period as the 'post-exercise' period over which we averaged $\dot{V} \mathrm{O}_{2}$ to estimate the 
metabolic rate.

We calculated the Physical Activity Ratio (PAR) to reflect the energetic cost of the swimming task over an individual's resting metabolic rate, similar to the concept of Metabolic Equivalents (METs; Schutz et al., 2001; Hills et al., 2014):

$$
P A R=\frac{\dot{V} O_{2} 1 \text { min post-exercise recovery }}{\dot{V} O_{2} 2 \text { min pre-exercise recovery }}
$$

PAR therefore controls for variability between days (e.g., due to temperature effects) and individual size and energy efficiency.

\subsubsection{Lap timing}

The time required for individuals to complete a lap of either swimming task was recorded with a hand-held timer. Lap times began or restarted when the animal left or returned to the measurement station (Figure 1C). Lap times were available for all metabolic swimming trials but one (ID 6JK5, control condition, 23 Sept 2013).

\subsubsection{Aerial video}

We installed a wall-mounted GoPro camera (Hero3 5.0 MegaPixel) to record aerial video footage of the lagoon (Figure 1) during the incremental loading trials. The fixed camera mount allowed for videos to be recorded with the same field of view and remained in place for a single trial; the camera angle changed between trials and the results can therefore not be compared across individuals. We used consistent waypoints in all frames to align videos for visual comparison within a trial (e.g. Figure 1C). We digitized the point of the dolphin's rostrum in Tracker (version 4.87; Brown, 2014) video tracking software. The camera recorded at $24 \mathrm{fps}$, and we digitized every 10 frames for an analyzed frame rate of $2.4 \mathrm{fps}$. We used the measured width of the dock (Figure 1C) as a scale marker in the video frame. The video footage was not corrected for the oblique camera angle, so only relative changes in the swimming paths were compared.

\subsubsection{Expected drag and speeds}

In a previous study (Chapter 8), we ran computational fluid dynamics (CFD) simulations to estimate the drag forces on a bottlenose dolphin without a tag (control) and when tag packages of increasing size were added ( $\operatorname{tag}, \operatorname{tag}+2$, $\operatorname{tag}+4$; Figure 2 ). We fit power functions to the relationship between speed $(U ; \mathrm{m} / \mathrm{s})$ and $\operatorname{drag}(D ; \mathrm{N})$ from CFD simulations in simulated drag conditions (Chapter 8),

$$
D=a U^{b}
$$


where $a$ and $b$ are polynomial coefficients for the power function derived by least squares (polyfit.m; MATLAB 2014b). CFD simulations were not able to be completed for tag +6 and tag +8 conditions; however, we calculated the difference in drag forces between the available simulations ( $\operatorname{tag}$ vs. $\operatorname{tag}+2, \operatorname{tag}+2$ vs. $\operatorname{tag}+4$ ) to determine the additional loading from a pair of drag elements, which allowed us to predict drag forces on tag +6 and tag +8 configurations (Figure 2).

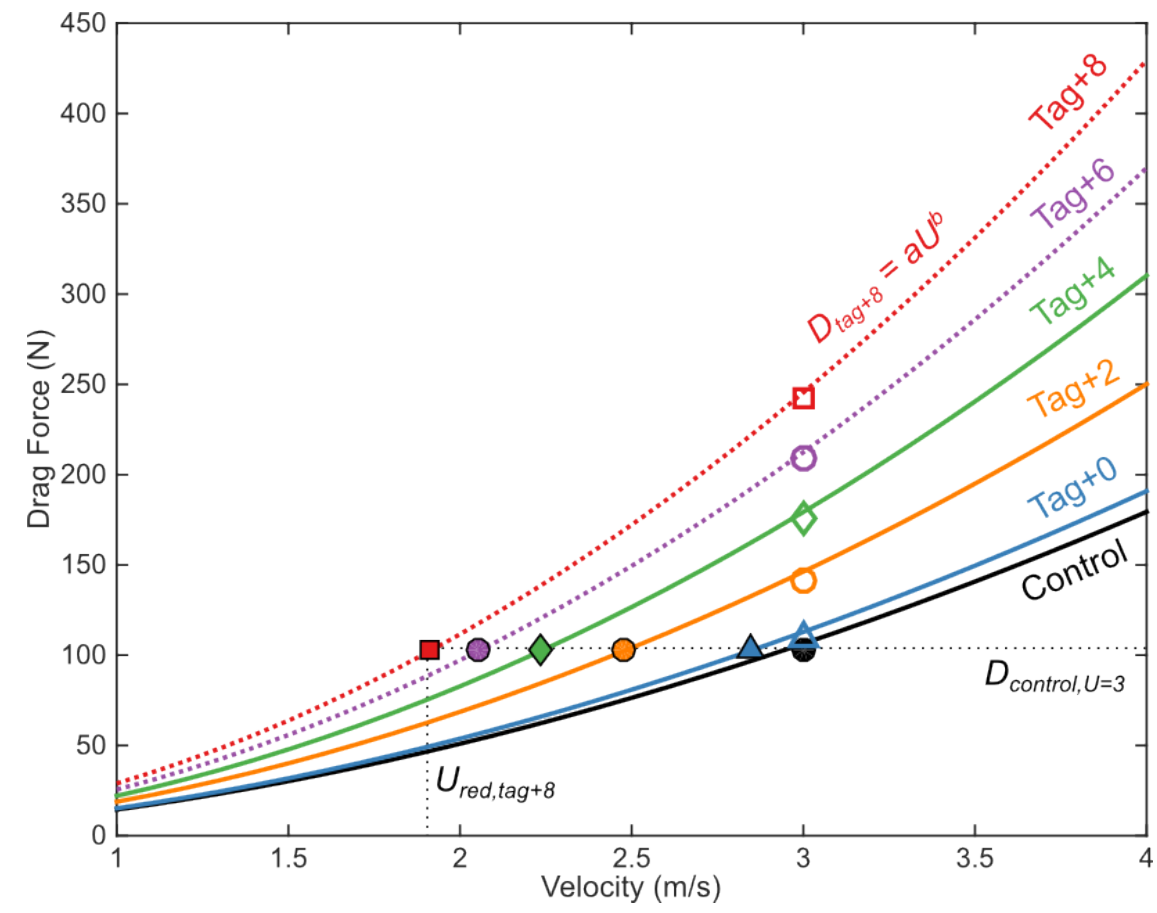

Figure 9-2: Drag forces on a bottlenose dolphin (black) and a dolphin instrumented with a tag (blue) and tags with additional drag-adding elements as simulated with Computational Fluid Dynamics (solid lines) and as predicted from CFD simulations (dotted lines). Coefficients for the equations for each condition (e.g. $D_{t a g+8}=a U^{b}$ ) are listed in Table 3. With added drag, dolphins can maintain speed (e.g., $3 \mathrm{~m} / \mathrm{s}$ ) but experience higher drag forces (open symbols) or can reduce speed (e.g., $\left.U_{r e d, t a g+8}\right)$ to maintain the drag force they experience when not wearing a tag (e.g., $\left.D_{\text {control }, U=3}\right)$ and must overcome (solid symbols).

In a concurrent experiment, we measured swimming speeds with a GPS-equipped remotecontrolled boat (Chapter 10; Shorter et al. In Prep), allowing us to determine that dolphins in the present study's control condition swam $\sim 3 \mathrm{~m} / \mathrm{s}$ (Figure G1). We calculated the expected increase in drag forces if dolphins maintained speeds at $3 \mathrm{~m} / \mathrm{s}$ under all drag loading conditions as:

$$
\frac{D_{\text {condition }, U=3}}{D_{\text {control }, U=3}}
$$

where $D_{\text {control }, U=3}$ is the drag force experienced at $3 \mathrm{~m} / \mathrm{s}$ in the control condition $(102 \mathrm{~N}$, Chapter 8 ) and $D_{\text {condition }, U=3}$ is the drag force experienced at $3 \mathrm{~m} / \mathrm{s}$ in other tag conditions (condition $=\operatorname{tag}, \operatorname{tag}+2, \operatorname{tag}+4, \operatorname{tag}+6, \operatorname{tag}+8$; Figure 2, open circles). Alternatively, we 
calculated the reduction in speed necessary to maintain drag forces as those in the control condition at $3 \mathrm{~m} / \mathrm{s}$ in the control condition $\left(D_{\text {control }, U=3}\right.$; Figure 2, closed circles). We calculated reduced speeds $\left(U_{\text {red }} ; \mathrm{m} / \mathrm{s}\right)$ - the speed at which drag forces would be maintained at $102 \mathrm{~N}$ - for each tag condition as

$$
U_{\text {red,condition }}={\frac{D_{\text {control }, U=3}}{a}}^{1 / b}
$$

\subsubsection{Tag analysis}

The DTAG contains a pressure sensor (Keller Series PA L3, range 200 bar) that sampled at $250 \mathrm{~Hz}$, down-sampled to $20 \mathrm{~Hz}$ for analysis, used to estimate the animals' depth in the lagoon. We calibrated pressure sensors on the tag with custom algorithms, including concurrent temperature measurements to compensate for temperature effects on the pressure data (Johnson and Tyack, 2003; Johnson, 2015). We assumed the top 1\% of the depth record during each trial reflected the water's surface. To determine whether tagged animals with higher drag $(\operatorname{tag}+8)$ were occupying greater depth to avoid surface wave drag or to benefit from substratum effects, we calculated the amount of time spent in depth bins of 0-0.5 $\times, 0.5$ $1.5 \times$ and $>1.5 \times$ body diameter. We estimated body diameter from dolphin morphometrics

as body girth $/ \pi$ (Table 1 ); girth was measured approximately two fingers in front of the dorsal fin after animals exhaled.

\subsubsection{Statistical analysis}

We used two-way ANOVA to determine whether PAR or post-exercise metabolic rate $\left(\dot{V} \mathrm{O}_{2}\right)$ were significantly different between individuals or drag conditions, with the expectation that PAR and would increase with drag loading. We used two-way ANOVA with post-hoc Tukey's test to determine whether lap durations differed significantly between drag loading conditions, while controlling for individual behavior.

\subsection{Results}

\subsubsection{Metabolic trials}

Four dolphins performed 26 metabolic trials under different drag loading conditions (control, tag, tag +8$)$, the number and order of which we list in Table 1. Mean air temperature and humidity were $27.3 \pm 1.0^{\circ} \mathrm{C}$ (range $25.0-29.4^{\circ} \mathrm{C}$ ) and $62 \pm 5 \%(48-69 \%$ ) during the times of the trials. The average water temperature in the lagoon was $24.6 \pm 0.5^{\circ} \mathrm{C}$.

The PAR indicates that the swimming task increased $\dot{V} \mathrm{O}_{2}$ by a factor of $3.32 \pm 2.74$ over resting values, with no detectable difference between individuals (two-way ANOVA; $\left.F_{3,20}=1.51 ; p=0.2423\right)$ or drag conditions $\left(F_{2,20}=0.79 ; p=0.4697\right.$; Figure 3$)$. Individuals 
had significantly different post-exercise $\left(F_{3,20}=7.63 ; p=0.0014\right)$ and mass-specific $\dot{V} \mathrm{O}_{2}$ $\left(F_{3,20}=4.69 ; p=0.0122\right)$, though we did not detect a significant effect of drag on either $\left(F_{2,20}=0.06\right.$ and $0.08 ; p=0.9460$ and 0.9199 , respectively; Figure 4). Further, drag condition significantly increased lap durations (two-way ANOVA; $F_{2,19}=29.28 ; p<0.0001$ ); average lap durations were no different between control and tag-only conditions (Tukey HSD; $p=0.4545)$ but, when instrumented with the tag +8 , laps were significantly longer than control and tag conditions (by $45 \%$ and 33\% respectively; Tukey HSD; $p<0.0001$ for both; Figure 5).
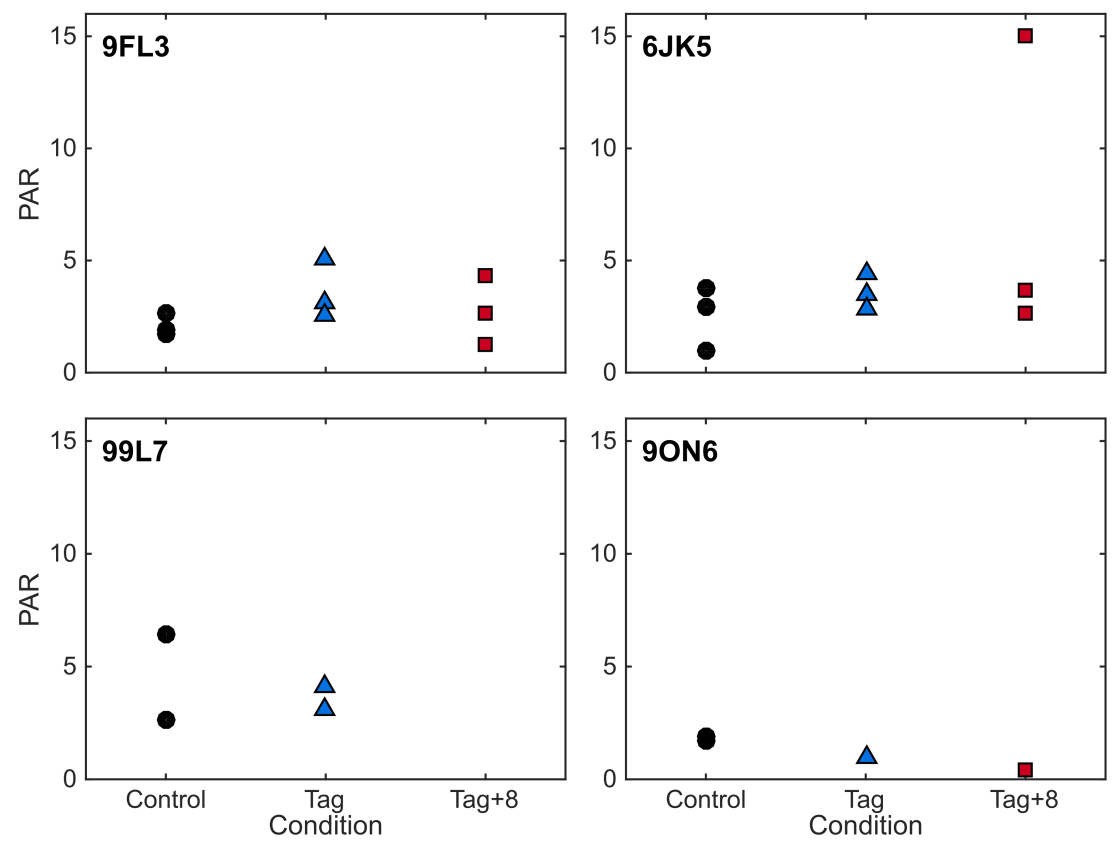

Figure 9-3: Physical Activity Ratio (PAR - the energetic cost of the swimming task over an individual's resting metabolic rate) calculated for each swimming trial performed by four bottlenose dolphins (IDs 9FL3, 6JK5, 99L7 and 9ON6) under control (no tag), tag and tag +8 conditions. Data points for represent separate trials.

\subsubsection{Incremental loading trials}

In the second swimming task, two dolphins completed the counter-clockwise laps as drag was removed and then added (Figure 6). Dolphins gradually increased the diameter of their swimming path as drag loading decreased, increasing the distance traveled (Figure 6A, B); as drag loading was increased again, the swimming path became progressively constricted and shortened (Figure 6C, D). Swimming speed changed progressively with unloading and loading: both individuals completed the swimming lap faster as drag was reduced, and lap durations gradually increased again as drag was incrementally added (Figure 7). Percent increases in lap duration over the control condition were similar to what was expected from 

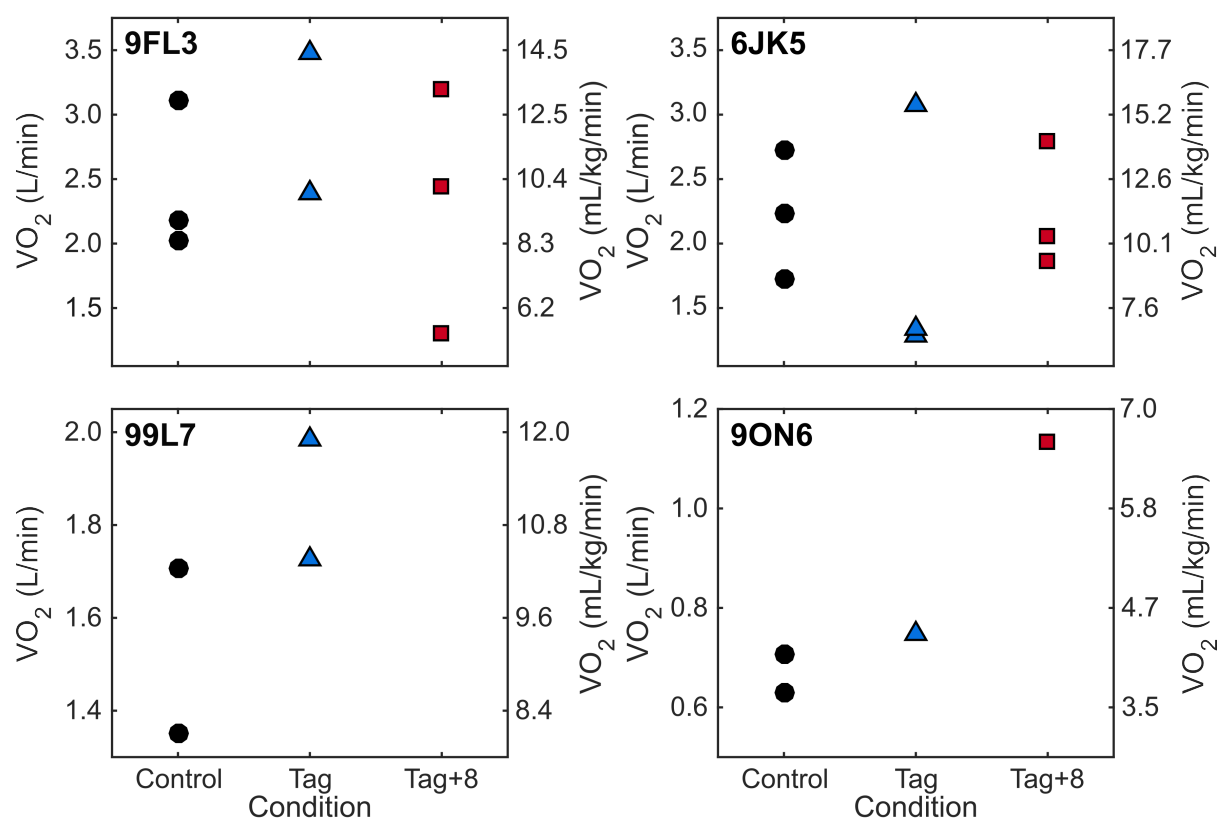

Figure 9-4: Standard and mass-specific oxygen consumption rates $\left(\dot{V} \mathrm{O}_{2} ; \mathrm{L} / \mathrm{min}\right.$ and $\left.\mathrm{mL} / \mathrm{kg} / \mathrm{min}\right)$ of four bottlenose dolphins (IDs 9FL3, 6JK5, 99L7 and 9ON6) measured after swimming trials under control (no tag), tag and tag +8 conditions. Data points represent separate trials.

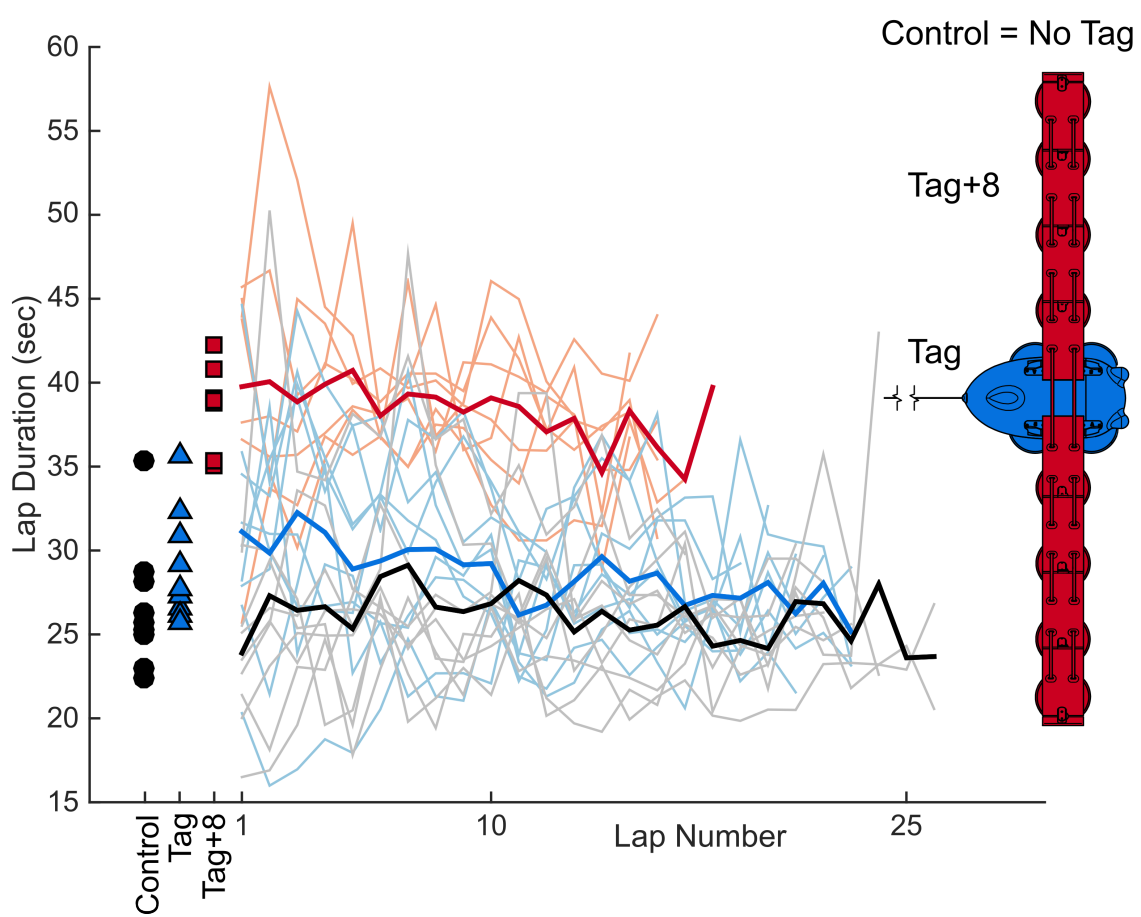

Figure 9-5: Mean lap duration increases with drag loading. Mean lap duration across all trials (symbols) and the timed duration of each lap (light lines) with per-condition averages (thick lines). 
CFD simulations if individuals were slowing down to maintain drag forces (Figure 7). The two dolphins slowed on average $13 \pm 6 \%$ when wearing just the tag, and slowed $23 \pm 6 \%$, $56 \pm 47 \%, 39 \pm 17 \%$ and $45 \pm 12 \%$ with the tag $+2,+4,+6$ and +8 configurations, respectively (Figure 7). CFD simulations predicted similar speed reductions ranging from $5 \%$ from the tag only, to $36 \%$ for the tag +8 (Table 3, Figure 7).

Table 9-3: Equations for drag with speed from computational fluid dynamics (CFD) simulations of a dolphin without a tag and with tags of increasing size, the absolute $(\mathrm{N})$ and percent increase in drag force at $3 \mathrm{~m} / \mathrm{s}$, and the absolute $(\mathrm{m} / \mathrm{s})$ and percent reduction in speed required to maintain drag forces when not wearing a tag at $3 \mathrm{~m} / \mathrm{s}$.

\begin{tabular}{|l|l|l|l|l|l|}
\hline $\begin{array}{l}\text { Drag Con- } \\
\text { dition }\end{array}$ & Equation & $\begin{array}{l}\text { Drag Force } \\
(\mathrm{N})\end{array}$ & $\begin{array}{l}\text { Percent } \\
\text { Increase }\end{array}$ & $\begin{array}{l}\text { Speed } \\
(\mathrm{m} / \mathrm{s})\end{array}$ & $\begin{array}{l}\text { Percent } \\
\text { Reduction }\end{array}$ \\
\hline No tag & $D=14.5 U^{1.8}$ & 102 & 0 & 3 & 0 \\
\hline Tag & $D=15.2 U^{1.8}$ & 109 & 6 & 2.8 & 5 \\
\hline Tag +2 & $D=18.8 U^{1.9}$ & 142 & 38 & 2.5 & 17 \\
\hline Tag +4 & $D=22.1 U^{1.9}$ & 176 & 72 & 2.2 & 25 \\
\hline Tag +6 & $D=25.5 U^{1.9}$ & 209 & 104 & 2.1 & 32 \\
\hline Tag +8 & $D=29.0 U^{1.9}$ & 243 & 137 & 1.9 & 36 \\
\hline
\end{tabular}

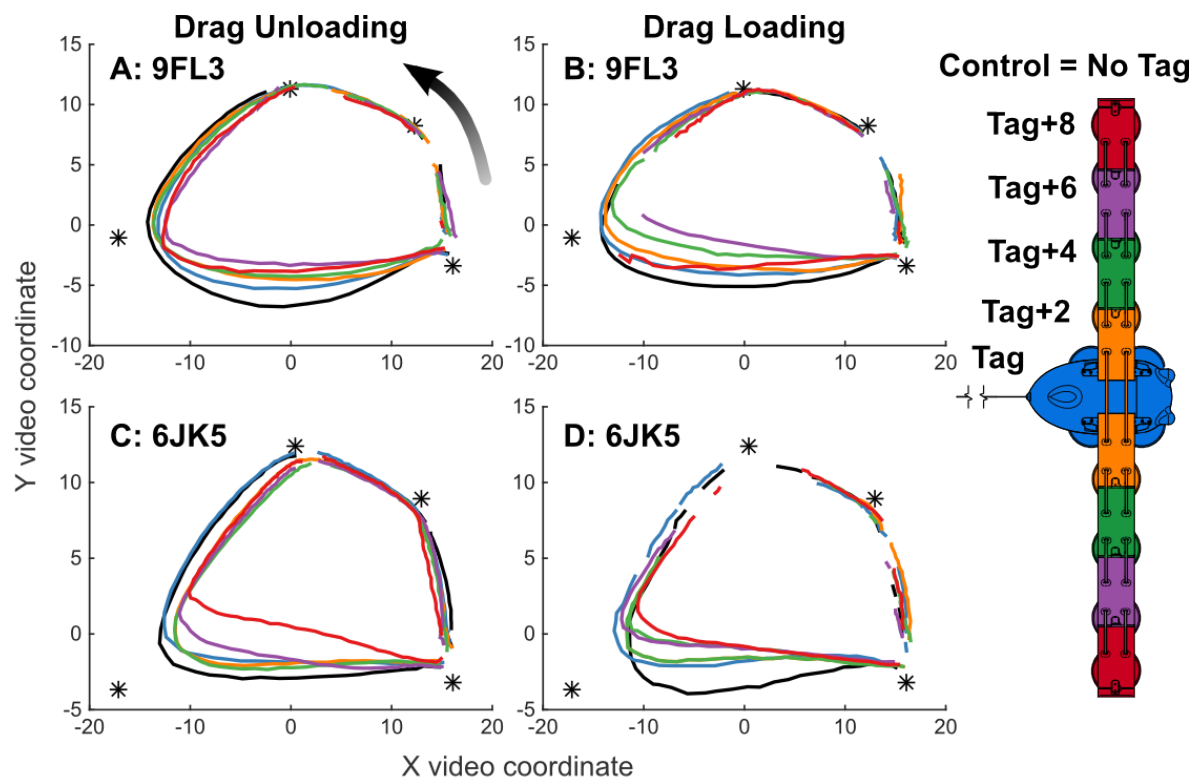

Figure 9-6: Dolphins progressively changed swimming paths with drag unloading and loading. Paths of swimming dolphins 9FL3 (A, B) and 6JK5 $(\mathrm{C}, \mathrm{D})$ tracked from aerial video footage as they swam counter-clockwise between waypoints (stars). Drag loading was progressively decreased (A, $\mathrm{C})$ and then increased $(\mathrm{B}, \mathrm{D})$ through drag loading conditions without the tag and up to the tag +8 (right). Gaps in paths reflect time periods when the dolphins were not visible for $>2$ time steps, $\sim 1$ second. 


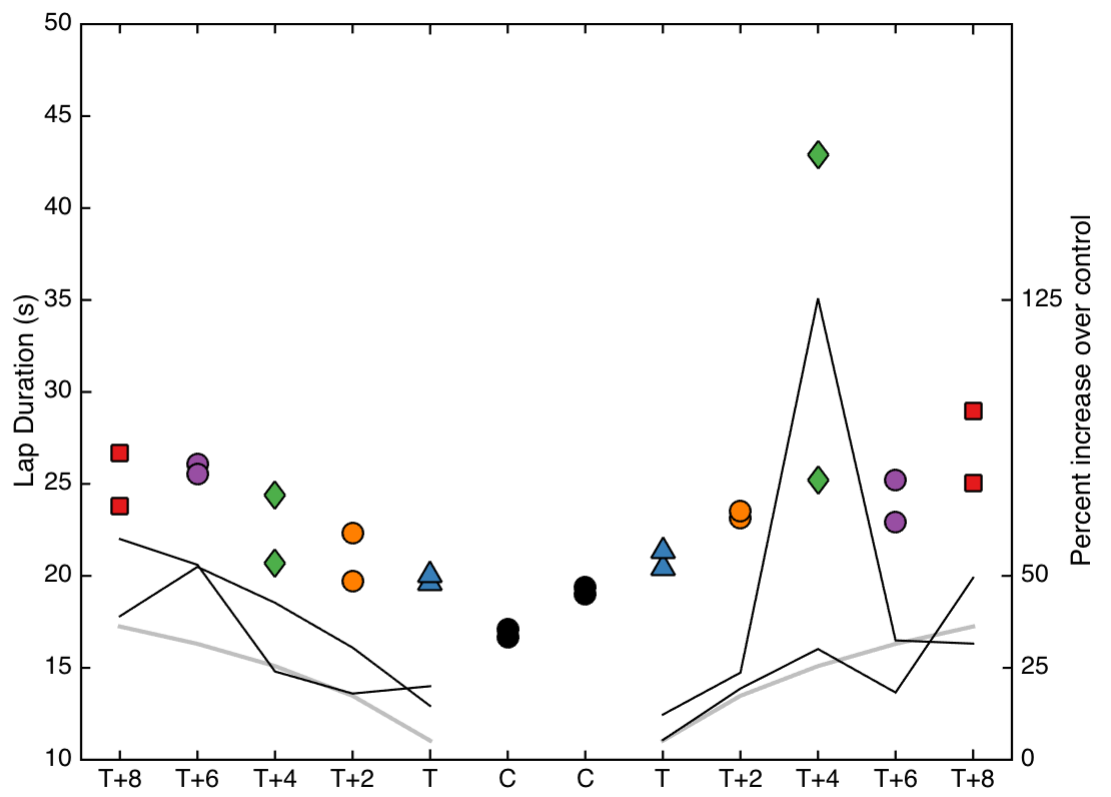

Figure 9-7: Dolphins changed speed with incremental changes in drag loading, in line with what is expected from simulation models. Time required to complete a swimming lap (seconds) in each experimental condition as drag elements and a bio-logging tag $(\mathrm{T})$ are removed and then added to manipulate force loading on two bottlenose dolphins (symbols). Lines represent the percent increase in lap duration over the duration observed in the control condition $(\mathrm{C})$.

\subsubsection{Tag analysis}

Mean root mean square error for the depth calibration of pressure sensors was $0.034 \mathrm{~m}$ (range 0.017-0.056 m). Mean maximum depths measured by the tags were $0.90( \pm 0.11) \mathrm{m}$ and $0.90( \pm 0.09) \mathrm{m}$ for the tag and tag +8 conditions, respectively. When tagged with $\operatorname{tag}+8$, individuals did not appear to spend more time at greater depth to avoid surface wave drag (Figure 8 ). The greatest proportion of time was spent at $0.5-1.5 \times$ body diameter $(0.20-0.71$ $\mathrm{m}): 74.4 \pm 0.9 \%$ in the tagged condition, and $73.0 \pm 0.09 \%$ in the tag +8 condition (Figure 8 ).

\subsection{Discussion}

Animals employ smart strategies such as gliding or soaring to economize movement; similarly, tagged animals may compensate for drag from bio-logging tags by modifying swimming biomechanics or behavior to reduce energy consumption. To investigate this, we refined a protocol from a previous study (van der Hoop et al., 2014a) to determine if animals expended more energy to swim to counter the hydrodynamic changes associated with tags of increasing size, or if and how they employed strategies to reduce metabolic cost in these highdrag conditions. Tags for scientific studies significantly increase drag forces on the animal 

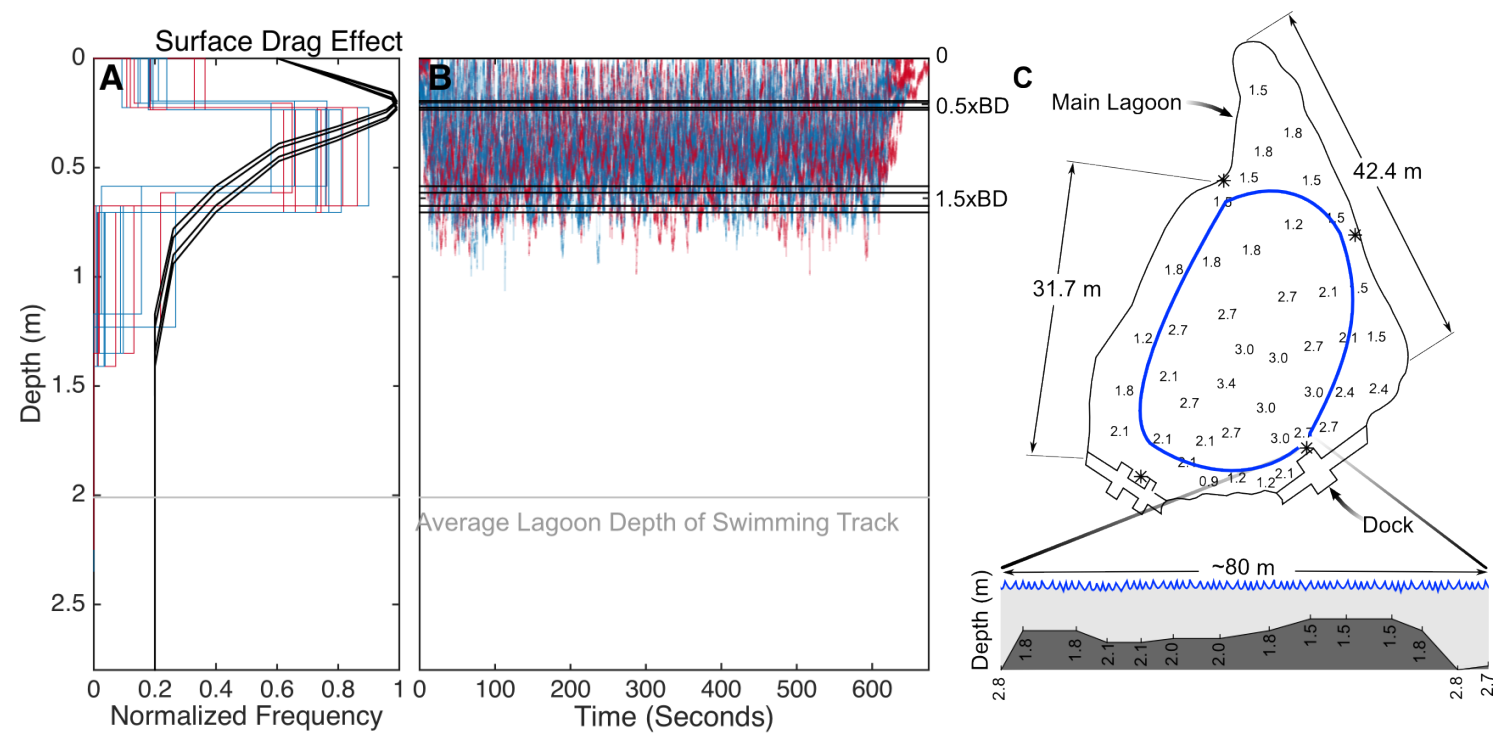

Figure 9-8: Dolphins swam in surface waters in both drag conditions. Proportion of time spent (colors) and effect of surface drag (black; Hertel 1966) at depth $(\mathrm{m})$ in bins of $<0.5 \times, 0.5-1.5 \times$ and $>$ $1.5 \times$ body diameter (BD; A) and all depth profiles (B) of dolphins instrumented with a bio-logging tag (blue) and the tag +8 drag elements (red) during metabolic swimming tasks. Four surface drag effects and body diameters are calculated for each animal (black lines). The lagoon is up to $3.5 \mathrm{~m}$ deep $(\mathrm{C})$ though the average depth of the lagoon during the swimming track (blue; C) is $2 \mathrm{~m}$.

subjects. CFD simulations show areas of fluid damming in front of the tag, and stagnate flow behind it (Chapter 8; Shorter et al., 2013). Observations during our experiments confirm how these flows were predicted to be altered, forming bubbles and turbulence in the wake of the drag elements (Figure 9).
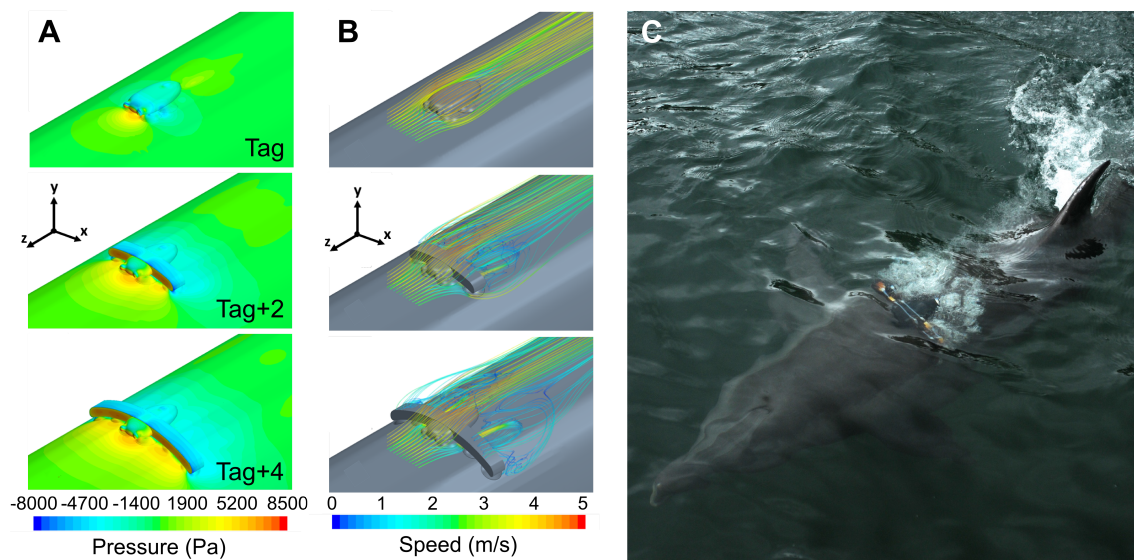

Figure 9-9: Observed water flow patterns over the tag were similar to those simulated with CFD. Simulated water pressure (A) and speed (B) as they flow over the DTAG3 (tag, top) and additional drag elements used to increase the frontal area by factors of two (tag +2 , middle) and four $(\operatorname{tag}+4$, bottom) in $4 \mathrm{~m} / \mathrm{s}$ inline flow. Pressure is mapped to the surface geometry and is shown on the left, and streamlines with the corresponding fluid speed are shown on the right. Observations (C) of similar flow patterns on one of the instrumented dolphins $(\operatorname{tag}+4)$. 
We found no detectable difference in oxygen consumption rates or overall energetic cost (e.g., PAR) of increased drag with a tag or the tag +8 configuration (Figures 3,4 ). We did not expect a significant effect of the tag-only configuration, given lower drag loading (1.06 times, $7 \mathrm{~N}$ at $3 \mathrm{~m} / \mathrm{s}$ ) compared to the larger DTAG2 used in van der Hoop et al. (2014a, 1.17 times; $20 \mathrm{~N}$ at $3 \mathrm{~m} / \mathrm{s}$ ) which also yielded no detectable metabolic effect. Despite improvements to the previous study with a novel, high-resolution, breath-by-breath respirometry technique (Fahlman et al., 2015); a longer exercise period to ensure steady-state metabolism was reached; and a greater sample size, we still did not detect a measurable metabolic effect of the tag. Further, we added a drag condition that was designed to increase tag drag by a factor of 8. Extrapolating from CFD simulations of smaller tag conditions $(\operatorname{tag}+2, \operatorname{tag}+4$; Figure 2), the $\operatorname{tag}+8$ configuration should have increased total drag forces on the dolphins by 2.4 times $(242 \mathrm{~N}$ at $3 \mathrm{~m} / \mathrm{s})$, but we detected no consistent or significant metabolic effect (Figures 3,4).

The animals did adjust both their swimming path and speed in response to the drag loading. When drag was reduced by adjusting the number of additional drag elements attached to the tag, dolphins progressively increased their swimming speed, responding to small ( $\sim 15 \mathrm{~N}$ at $3 \mathrm{~m} / \mathrm{s}$ ) changes in overall body drag. During subsequently re-loading, individuals progressively slowed down (Figure 7). These individuals are therefore able to perceive small changes in their total body force balance (i.e., their hydrodynamics) and adapt their behavior in response; dolphins slowed on average by $13 \%$ when wearing just the tag, and reduced their speeds in line with predictions from Computational Fluid Dynamics simulations to maintain drag forces, therefore thrust production and energy consumption (Figure 7). During the metabolic trials, dolphins increased the time it took to complete a lap (i.e., decreased their swimming speed) as drag loading was increased. Individuals slowed by $45 \%$ over the control condition, while not significantly increasing their energy expenditure despite 2.4 times as much drag (tag +8 configuration; Figure 5 ). The effect of the tag without added drag elements was less dramatic, with no detectable difference in lap duration between the control and tag conditions (Figure 5).

Our previous study (van der Hoop et al., 2014a) suggested that dolphins reduced their swimming speed to maintain overall drag forces to maintain energy expenditure under increased drag loading. CFD and theoretical models confirmed that tagged dolphins were reducing speed to the point at which energy costs were not higher than they would be if they were not wearing the tags. Others have observed significantly reduced activity levels in tagged bottlenose dolphins (Blomqvist and Amundin, 2004) and porpoises (Geertsen et al., 2004), and significantly lower speeds in instrumented (Lang and Daybell, 1963; Skrovan et al., 1999) or pregnant dolphins (Noren et al., 2011). Other swimmers show similar behavioral accommodation in response to drag from external tags (reviewed in e.g., van der Hoop et al., 2014a; Jepsen et al., 2015). 
From an evolutionary standpoint, animals should seek to minimize energetic costs, especially those involved in everyday actions (e.g., routine swimming; Sparrow and Newell, 1998). Movements can be adapted to minimize metabolic energy expenditure with respect to constraints imposed by a task (swim from A to B), the environment (low drag, high drag), and the organism itself (body shape, size). By perceiving a difference in conditions between tasks and adapting behavior as such in order to reduce expenditure, animals are economical (Sparrow and Newell, 1998; Halsey, 2016). We have also shown how incremental changes underlie this adaptive response to maintain energy economy, and the sensitivity of these dolphins to changes in their hydrodynamic regime. The stepwise control of velocity during incremental loading trials allowed dolphins to achieve the principle of least effort (reviewed in Sparrow and Newell, 1998), maintaining the economy of the task.

Speed is not the only variable that can be adapted to optimize energy economy in response to changing conditions. During the incremental loading trials, two dolphins changed their speeds and also the shape of their swimming paths reducing their total distance but still accomplishing the goal of reaching all of the waypoints in the experiment (Figure 6). Another possibility for dolphins to have improved their energy economy would be to alter the depth of their swimming. At the surface, wave drag can increase drag by up to 5 times; this effect is strongest at depths of $0.5 \times$ body diameter and becomes negligible beyond $3 \times$ body diameter (Hoerner, 1965; Hertel, 1969; Blake, 2009). At the bottom, swimming against the substratum can confer energetic benefits, especially for lift-based swimmers such as cetaceans (Webb, 1993). Interaction with a surface reduces drag by limiting energy dissipation of the vortices shed by the tail and can reduce the cost of transport up to $15 \%$ (Rayner, 1991; Webb, 1993). We therefore investigated the potential for animals to avoid increased metabolic costs by minimizing surface wave drag or preferentially interacting with the bottom. We expected animals to swim at greater depths when wearing the $\operatorname{tag}+8$, to reduce overall drag forces they would experience. However, animals swam at the same depths when instrumented with the tag alone and with the tag +8 (Figure 8 ). Swimming at $3 \mathrm{~m} / \mathrm{s}$, the tag +8 would have increased drag by $2.4 \times$ (Figure 2 , Table 3 ); swimming even $0.5 \mathrm{~m}$ deeper would halve the surface effect (Figure 8). Individuals were therefore not avoiding or minimizing drag forces by swimming deeper; however, the shallow depth $(<3.5 \mathrm{~m}, 6.4-7.7 \times$ body diameter; $\sim 2 \mathrm{~m}$ for the route of the swimming task, $4.3-5.1 \times$ body diameter) of the experimental lagoon may have influenced animals' ability to avoid surface effects. Whether swimming animals change their surfacing and diving behaviors to reduce time in high-drag surface waters or to preferentially interact with a solid substrate in compensation for drag loading remains unknown in deeper ocean environments.

Animals are not always able to prioritize energy economy; the dolphins in this study were able to reduce their speeds as there was no constraint to maintain it. Tradeoffs exist between behaviors that maximize economy (e.g., swimming slowly, gliding) and those that 
maximize fitness (Halsey, 2016) such as predator evasion, prey pursuit (Goldbogen et al., 2007; Aguilar Soto et al., 2008) and maintaining social cohesiveness (Wursig, 1982). Fish and eels with external tags show significantly increased metabolic rates when maintaining swimming speeds, though critical speeds (the time and velocity at which fish fatigue) is often reduced (reviewed in Jepsen et al., 2015). Dolphins are capable of high-powered efforts that significantly increase energy expenditure (Williams et al., 1993; Yazdi et al., 1999). Previous studies detected increases in oxygen consumption when dolphins generated swimming forces up to $85 \mathrm{Kg}(=834 \mathrm{~N})$ for $5-7 \mathrm{~min}$ which would be comparable to the tag +6 at $6 \mathrm{~m} / \mathrm{s}$ (Williams et al., 1993). A follow-up study increasing the animals' motivation to maintain speed at $3 \mathrm{~m} / \mathrm{s}$ for 5 minutes did yield an increase in metabolic rate when wearing the tag (Chapter 10; Shorter et al. Unpublished Data). The dolphins in this study likely had the ability to perform the task at greater speeds with the highest drag configuration, but chose not to.

It is important to consider how these results contribute a greater understanding of movement and energy in free-swimming cetaceans (Engel et al., 2010). Free-swimming dolphins are active throughout the day with bouts of activity; most populations spend $\sim 50-60 \%$ of time traveling, 15-20\% feeding, and 15-20\% milling about (Hanson and Defran, 1993; Powell and Wells, 2011; Wells et al., 2013). Resting behaviors are low in certain populations (e.g., Hanson and Defran, 1993) but comprise 7-18\% of time in others (Wells et al., 2013). Activity budgets suggest that animals at Dolphin Quest Oahu spend $\sim 72 \%$ of their time swimming, actively fluking and gliding $42 \%$ of the time (Shorter et al. Submitted). Average dive times for coastal Tursiops spp. range 20-40 s (reviewed in Shane, 1990; Mate et al., 1995); dives at Dolphin Quest are on average $22 \mathrm{~s}$ (Shorter et al. Submitted). More detailed comparisons of activity and behavior in wild and managed dolphin populations are not currently available (Wells et al., 2013); however, based on activity budgets and dive comparisons, the responses of managed dolphins to swimming tasks of intermediate duration (5-10 min) and intensity are expected to apply to free-swimming populations.

Observed speed reductions were greater than expected from simulations for both the metabolic trials and the incremental loading trials (Table 3). CFD simulations assume static and uniform flow conditions across a rigid-body dolphin model (Chapter 8; Hazekamp et al., 2009; Pavlov and Rashad, 2012; Shorter et al., 2013) and therefore model passive drag forces only. Swimming dolphins interact with non-uniform and variable flow, interact with the water's surface, and generate additional (i.e., active) drag forces as they propel their bodies through the water (Lighthill, 1969; Webb, 1975b). These real-world factors that are not incorporated into simulations may explain the discrepancy between observed and expected speed reductions. For tag development and design purposes, it should be noted that CFD simulations present only the passive component of total body drag and therefore lead to an underestimate of the overall increase in drag and potential impact on speed reduction. 


\subsection{Conclusions}

We sought to quantify the metabolic cost of bio-logging tags on bottlenose dolphins during a controlled swimming task. When performing the tasks at their freely-chosen rate, dolphins methodically adjusted their swimming speed and no change in metabolic cost was detected. Studies with the goal of determining the metabolic effects of instrumentation require protocols that collect a diversity of data (e.g., position, fine-scale movement) to detect alternative or adaptive strategies subjects can use when faced with drag loading. Further studies to constrain the task to determine metabolic impact when high-speed behaviors are maintained are required. 
Chapter 10

\section{CONSEQUENCES OF TAG-RELATED DRAG LOADING IN DOLPHINS WHEN CIRCUMSTANCES PRECLUDE SLOWING DOWN}

This chapter includes authorship contributions from Andreas Fahlman, K. Alex Shorter, Julie RochoLevine, Tom Hurst and Michael Moore

JvdH, AF, KAS, JRL, MM developed protocols; JRL coordinated husbandry and training of animals; JvdH, AF, KAS, JRL, TH, MM performed field work; JvdH and AF processed the data; JvdH analyzed the data; JvdH wrote the manuscript; AF, KAS, JRL, TH, MM contributed to the writing of the manuscript.

The supplemental materials for this chapter can be found in Appendix H. 


\subsection{Abstract}

Two strategies tend to be adopted by animals when drag is added e.g., by carrying external bio-logging tags: (1) speed is decreased to minimize costs of transport; or (2) speeds are maintained, incurring additional energy demands due to drag from the tags. Previous studies on the effects of drag from bio-logging tags detected no difference in the measured metabolic rate or simulated energy output of bottlenose dolphins (Tursiops truncatus) with drag loading, but that individuals significantly slowed down, likely to avoid increased energetic costs. In this study, we wanted to examine energy expenditure of a swimming task under different levels of drag loading when speed was unchanged by conditioning the animals to swim at the steady speed of a remote-controlled ( $\mathrm{RC}$ ) boat. The energetic cost of the swimming task increased when animals were wearing the tag compared to when they swam without an instrument, but the metabolic effect did not continue to increase with further increases in drag $(\operatorname{tag}+4)$. However, dolphins increased their relative distance from the RC boat in these high-drag trials. These results demonstrate that there may be an energetic cost associated with the added drag from even a reasonably small hydrodynamic tag, with implications for design and interpretation of metabolic and field tag experiments.

\subsection{Introduction}

Animal movement has evolved in part by energetic constraints, where fitness is maximized by minimizing energy consumption. The economical approach is to perform a task while expending the least amount of energy as possible (Alexander, 1989; Sparrow and Newell, 1998). This is often achieved by moving at speeds where transport costs are minimized (Williams, 1999; Wickler et al., 2000; Rosen and Trites, 2002b; Weber et al., 2009; Halsey, 2016), or by taking advantage of environmental factors such as winds (Hedenstrom, 1993; Weimerskirch et al., 2000), tides (Weihs, 1978; Metcalfe et al., 1990; Forward and Tankersley, 2001), or buoyancy (Webb et al., 1998; Fahlman, 2008; Aoki et al., 2011; Miller et al., 2012b; Adachi et al., 2014). Economy includes but is not the same as efficiency. Efficiency is the ratio of work done relative to the metabolic energy required at multiple levels in the system (e.g. muscular, hydraulic, propulsive; Zamparo et al., 2002; Blake, 2005), Together with elements such as frequency and speed, efficiency contributes to economy, the energetic cost of a task.

Different pressures or constraints on a task will result in alternative strategies. For example, two strategies have been observed in animals when drag is added e.g., by carrying external bio-logging tags: (1) speed is decreased to minimize costs of transport (Wilson et al., 1986; Skrovan et al., 1999; van der Hoop et al., 2014a) and overall energy expenditure is conserved by shifting behavioral budgets (Feldkamp et al., 1988; Blomqvist and Amundin, 
2004; Elliott et al., 2014), or (2) speeds are maintained, incurring additional energy demands due to drag from the tags (Costa and Gentry, 1986; Culik et al., 1993; Methling et al., 2011; Tudorache et al., 2014; Jepsen et al., 2015; Maresh et al., 2015). Individuals can also adopt a strategy somewhere between these two end points (Culik and Wilson, 1991; Boyd et al., 1997; Cornick et al., 2006) depending on social, environmental, or time constraints. At the upper end of performance, critical speeds (the speed at which individuals fatigue, Brett, 1964) are often reduced, limiting the maximum swimming capacity of tagged animals compared to controls (reviewed in e.g., Tudorache et al., 2014; Jepsen et al., 2015).

Previous studies on the effects of drag from bio-logging tags have shown that bottlenose dolphins (Tursiops truncatus) under human care with few competing constraints do not increase energy output under drag loading, and will slow down to avoid increased energetic costs (Chapter 9; van der Hoop et al., 2014a). However, these animals do not have pressures often present in free-ranging individuals to maintain speed to pursue prey, evade predators, or maintain cohesion within a social group. Are there measurable metabolic effects of drag loading from instrumentation when animals swim at consistent, high speeds? In this study, we used a remote-controlled (RC) boat to constrain the swimming speed of bottlenose dolphins as they performed a swimming task under different levels of drag loading.

\subsection{Methods}

\subsubsection{Overview}

We trained two bottlenose dolphins (Table 1) with operant conditioning to swim following the pace of a remote-controlled boat to determine whether the swimming metabolic rate would increase if the animals maintained swimming speeds and therefore experienced higher drag forces under loading conditions. Individuals performed the swimming task in three drag conditions (control [no tag], tag, tag +4 ; Drag Conditions, below). All trials were by voluntary participation without restraints; dolphins could refuse to participate or withdraw at any point during a trial. We desensitized animals to the equipment and trained them for these research-associated behaviors prior to initiating the study. We provide a list of symbols and abbreviations in Table 2.

Table 10-1: Measurements and number and order of experimental trials performed under control (C, no tag), tag ( $\mathrm{T}, \mathrm{DTAG} 3)$, and tag $+4(\mathrm{~T}+4)$ drag-loading conditions.

\begin{tabular}{|l|l|l|l|l|}
\hline Dolphin ID & Length $(\mathrm{m})$ & Weight $(\mathrm{kg})$ & Trial Order & Days between trials \\
\hline 9FL3 & 2.73 & 240 & $\mathrm{~T}, \mathrm{~T}+4, \mathrm{C}$ & 2,1 \\
\hline 9ON6 & 2.46 & 171 & $\mathrm{~T}, \mathrm{C}, \mathrm{T}+4$ & 1,2 \\
\hline
\end{tabular}


Table 10-2: List of symbols and abbreviations.

\begin{tabular}{|l|l|l|}
\hline Symbol & Definition & Unit \\
\hline $\mathrm{CFD}$ & Computational Fluid Dynamics & \\
\hline $\mathrm{CO}_{2}$ & Carbon dioxide & \\
\hline $\mathrm{COT}$ & Cost of Transport & $\mathrm{J} / \mathrm{kg} / \mathrm{m}$ \\
\hline$D$ & Drag force & $\mathrm{N}$ \\
\hline $\mathrm{fps}$ & Frames per second & $/ \mathrm{s}$ \\
\hline $\mathrm{O}_{2}$ & Oxygen & \\
\hline $\mathrm{PAR}$ & Physical Activity Ratio & \\
\hline $\mathrm{RC}$ & Remote-controlled & \\
\hline$U_{\text {red }}$ & Reduced speed & $\mathrm{m} / \mathrm{s}$ \\
\hline$\dot{V} \mathrm{CO}_{2}$ & Carbon dioxide production rate & $\mathrm{l} \mathrm{CO}_{2} / \mathrm{min}$ \\
\hline$\dot{V} \mathrm{O}_{2}$ & Oxygen consumption rate & $\mathrm{l} \mathrm{O}_{2} / \mathrm{min}$ \\
\hline
\end{tabular}

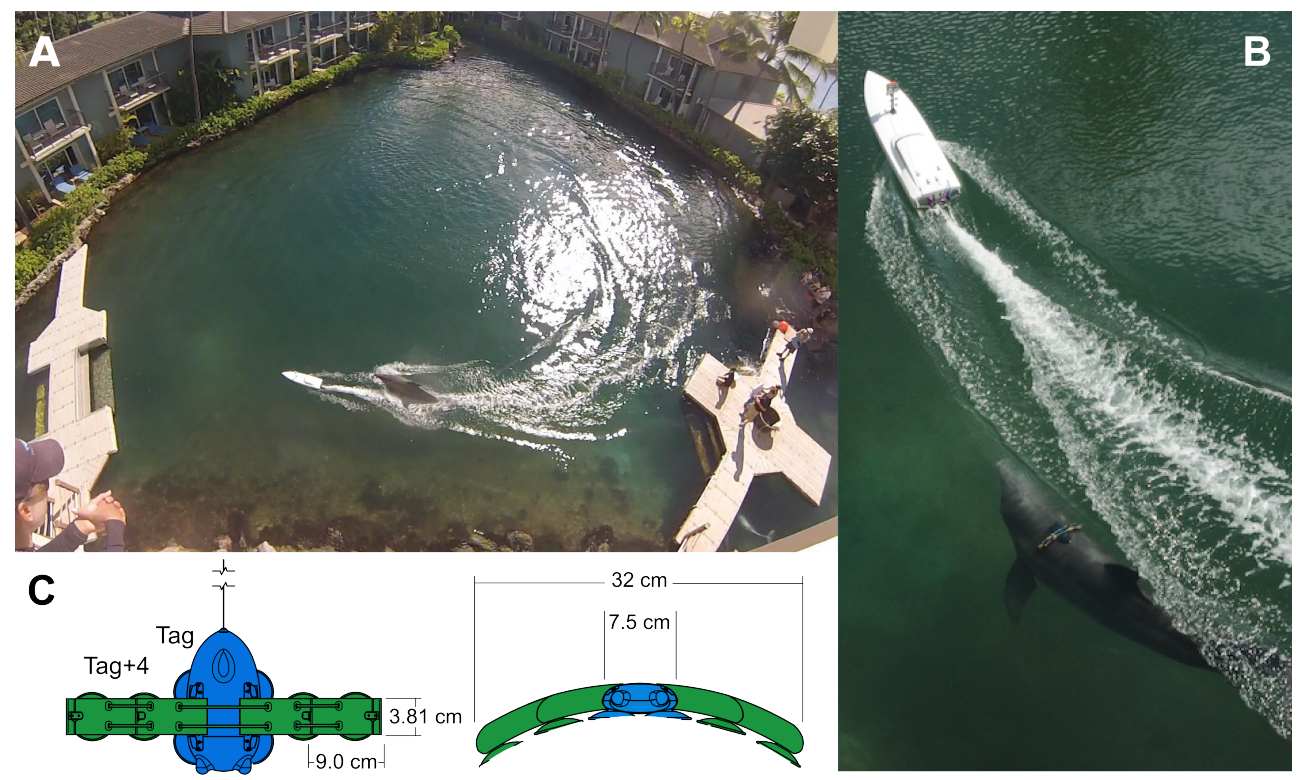

Figure 10-1: The experimental setup. Bottlenose dolphins swam around the experimental lagoon (A) following a remote controlled boat (B) when not wearing a tag, or when wearing the tag $(\mathrm{C}$; blue) or tag +4 ( $\mathrm{C}$; green). Metabolic rate was measured at the dock (A; lower right) before and after each swimming trial.

\subsubsection{Drag conditions}

We designed three drag conditions as described in Chapter 9, based on the dimensions of the DTAG3 (Figure 1; Johnson and Tyack, 2003; Shorter et al., 2013). We conducted repeated swimming trials with control (no tag), tag (DTAG) and tag +4 conditions, which were expected to increase drag loading over the non-tagged condition by 1.06 $\mathrm{E}$ and 1.64CE, 
respectively, at $3 \mathrm{~m} / \mathrm{s}$ based on Computational Fluid Dynamics simulations (CFD; Chapters $8,9)$. We attached the tag or tag +4 setup on the back of the animal between the blowhole and dorsal fin to replicate the location of tags placed on wild animals.

\subsubsection{Swimming task}

We operated a GPS-equipped remote-controlled boat at $3 \mathrm{~m} / \mathrm{s}$ to set pace for the dolphins as they swam for 5 minutes around an experimental lagoon at Dolphin Quest Oahu (Honolulu, HI, U.S.A.; Figure 1). The custom-built RC boat had a fiberglass hull (1.24 $\mathrm{m}$ long, 0.33 m beam; Aeromarine Laminates, Seaford, DE, U.S.A.) which housed an electric motor powered by $18.5 \mathrm{~V} 8000 \mathrm{mAmp}$ lithium batteries. The boat's propeller had a tubular shield. We controlled the $\mathrm{RC}$ boat with a $2.4 \mathrm{gHz}$ radio receiver and electronic speed controller (TR624 Tactic; Hobbico, Inc; Champaign, IL, U.S.A.) and recorded and monitored speed $(0.1 \mathrm{~m} / \mathrm{s}$ accuracy) at $10 \mathrm{~Hz}$ via GPS (GPS-v4 and Boat Data Recorder; Eagle Tree Systems, Bellevue WA U.S.A.). We measured dolphins' metabolic rates immediately before and after each swimming trial using a respirometer; none of the breaths during exercise were captured using the respirometer.

\subsubsection{Respirometry}

We measured breath-by-breath respiratory flow rates, expired oxygen $\left(\mathrm{O}_{2}\right)$ and carbon dioxide $\left(\mathrm{CO}_{2}\right)$ content with a custom-made pneumotachometer (Fahlman et al., 2015) before and after the swimming tasks, as described in Chapter 9. Briefly, we collected data with the pneumotachometer for at least 5 minutes before and 5 minutes after the swimming task. From the recorded values, we calculated pre-exercise metabolic rate (oxygen consumption rate; $\dot{V} \mathrm{O}_{2} ; \mathrm{l} \mathrm{O}_{2} / \mathrm{min}$ ) during the last 2 minutes before the swimming task began, and the post-exercise metabolic rate during the first minute after the swimming task ended (Fahlman et al., 2015). We calculated the Physical Activity Ratio (PAR) as:

$$
P A R=\frac{\dot{V} \mathrm{O}_{2} 1 \mathrm{~min} \text { post-exercise recovery }}{\dot{V} \mathrm{O}_{2} 2 \mathrm{~min} \text { post-exercise recovery }}
$$

which reflects the energetic cost of the swimming task over an individual's resting metabolic rate to control for variability between days and between individual size and energy efficiency (Black et al., 1996; Schutz et al., 2001; Hills et al., 2014). Animals breathed freely during the swimming task. We undertook trials when animals were post-prandial, between 9:01-10:15 am, 11-15 October 2013. During the trials, mean air temperature was $27.6 \pm 0.6^{\circ} \mathrm{C}$, relative humidity $63.2 \pm 3.90 \%$, and ambient pressure $1015.4 \pm 0.6 \mathrm{hPa}$. The water temperature in the lagoon was $24.8 \pm 0.8^{\circ} \mathrm{C}$. 


\subsubsection{Aerial video}

We recorded video of the metabolic measurements and swimming task with a stationary camera (GoPro HD Hero 3 Black, San Mateo, CA; 24 fps) installed above the lagoon (Figure 1). As described in Chapter 9, we aligned videos from different trials with consistent waypoints in the video frame. We digitized the path of the dolphin and boat with Tracker video tracking software (version 4.87; Brown, 2014) every 10 frames for a frame rate of $2.4 \mathrm{fps}$ for analysis. We calculated the distance in coordinates between the position of the dolphin and the boat at the left-most point in the lap (i.e., as the difference between the minima of the $\mathrm{x}$-coordinates of both dolphin and boat position time series). To determine whether individuals maintained speed when following the boat, we compared the average lap durations among trials with different drag conditions with two-way ANOVA and post-hoc Tukey's HSD test in R ( $\mathrm{R}$ Core Team, 2015). We also compared lap durations from boat trials with those from previous work swimming the same course when individuals swam at their preferred speeds (Chapter 9).

\subsection{Results}

Two dolphins each performed the swimming task once under all three drag conditions: without the tag, with the tag, and with the tag +4 (Table 1). For both individuals, the effect of exercise on oxygen consumption increased between the control and tag conditions (Figure 2). Oxygen consumption rates after exercise with the tag were 94\% (1.00 l O $2 / \mathrm{min})$ and $14 \%\left(0.16 \mathrm{l} \mathrm{O}_{2} / \mathrm{min}\right)$ higher than the control for the two animals, respectively. Exercise with the tag increased energy consumption over resting values (PAR) by 3.85 and 1.70 times, being 4.67 and 1.53 times greater than the effect of exercise with no tag (PAR; Figure 2).

With further increases in drag (tag +4$)$, oxygen consumption rates increased beyond the control condition, but were lower than the tag condition. The effect of exercise with the $\operatorname{tag}+4$ was intermediate $(\mathrm{PAR}=2.58$ and 1.36 ; Figure 2$)$ between the two conditions with much lower (tag) and no drag loading (control).

We detected no significant difference in lap duration when following the boat under different drag conditions (Tukey's HSD; $p \geq 0.9869$ for three comparisons). Lap durations were no different during the $\mathrm{RC}$ boat trials compared to the preferred, self-selected swimming speeds of dolphins in the control condition in a previous study (Chapter 9; $p \geq 0.8700$ for three comparisons; Figure 3). With the tag +4 , individuals increased their relative distance from the RC boat (Figure 4). Both individuals swam further to the inside of the boat's path, reducing the total distance they swam (Figure 4). While they continued to perform the task of swimming alongside the boat, their strategy for doing so changed in the highest loading condition. 

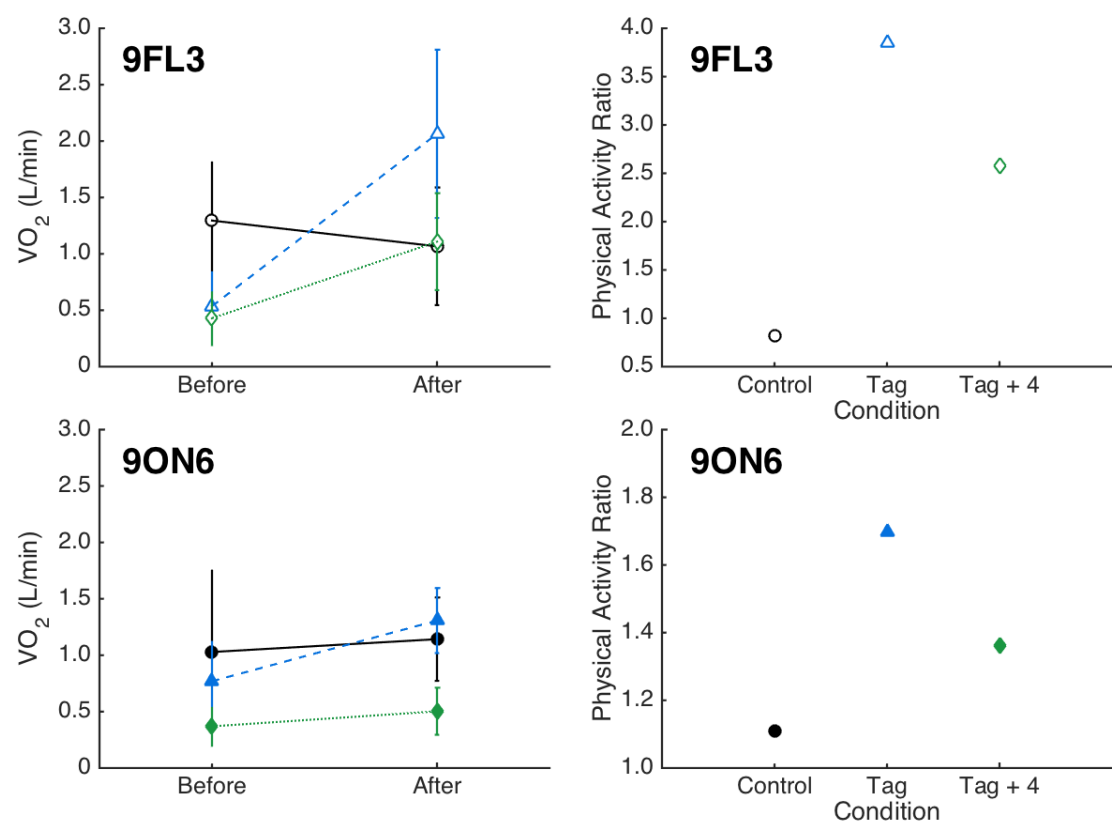

Figure 10-2: Energy expenditure of bottlenose dolphins during a 5-min swimming task increased under drag loading. Mean (error bar $=\mathrm{SD}$ ) oxygen consumption rate $\left(\dot{V} \mathrm{O}_{2}\right)$ before and after (left) and Physical Activity Ratio (right) of two bottlenose dolphins (IDs 9FL3 and 9ON6) after a 5 min metabolic trial swimming next to a remote-controlled boat driven at $\sim 3 \mathrm{~m} / \mathrm{s}$. Dolphins performed the task with no tag (control, black circles), and instrumented with the tag (blue triangles) and tag +4 (green diamonds).

\subsection{Discussion}

Under increased drag loading one economical approach is to slow down to avoid increased energetic costs as our previous studies in bottlenose dolphins have shown (Chapter 9; van der Hoop et al., 2014a). However, there are situations where increased speed is required to evade predators, capture prey, or maintain social cohesion. In this study, we wanted to examine dolphins' energy expenditure if the swimming speed of the animal was constrained.

With the speed constraint, energy expenditure in two dolphins did increase when wearing even a small bio-logging tag. In simulations with the tag and representative dolphin geometry at $3 \mathrm{~m} / \mathrm{s}$, the tag increased drag on the model by $7 \mathrm{~N}(6 \%$; Chapters 8,9$)$. Individuals maintained the speed constraint (Figure 3) and followed the path of the boat fairly consistently (Figure 4). With further increases in drag $(\operatorname{tag}+4)$, metabolic rates were higher than when individuals did not wear the tag, but were lower than when they wore the tag alone (Figure 2). However, individuals increased their relative distance from the RC boat (Figure 4). Although lap durations were maintained (Figure 3), the dolphins appear to have reduced the relative distance traveled by swimming to the inside of the boat. We can infer that by doing so, the animals may have therefore reduced their swimming speed during the tag +4 loading condition. 


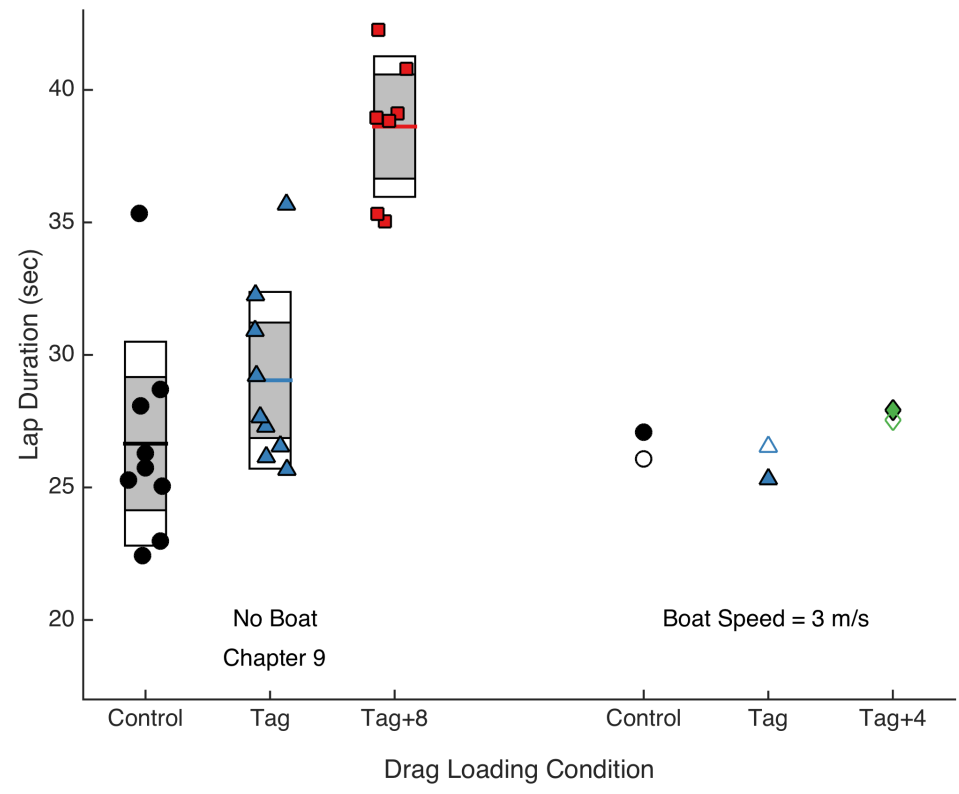

Figure 10-3: Dolphins maintained their lap duration when following a remote-controlled boat. Average time for two bottlenose dolphins (ID 9FL3 open symbols; 9ON6 closed symbols) to complete a swimming lap at their preferred speeds (no boat, Chapter 9) and when following a remote controlled boat at $3 \mathrm{~m} / \mathrm{s}$ under control (no tag; black circles), tag (blue triangles), tag +4 (green diamonds) and tag +8 (red squares) drag loading conditions.

Previous studies that have used boats to set a swimming pace for cetaceans describe similar observations. Beluga whales trailed further behind their pace boat at speeds $>2.5$ $\mathrm{m} / \mathrm{s}$, above their expected $\mathrm{COT}_{\min }$ speeds of 1.6-2.0 m/s (Shaffer et al., 1997). At speeds beyond $3.1 \mathrm{~m} / \mathrm{s}$, one whale shifted further back to swim in the boat's wake, while the other refused to perform the task (Shaffer et al., 1997). Individuals are therefore willing to increase energy expenditure to a point, and otherwise look for alternatives to reduce the energy expenditure of a task as it is perceived.

The boat speed used to set pace for the dolphins was $\sim 3 \mathrm{~m} / \mathrm{s}$, above speeds of minimum transport costs $\left(\mathrm{COT}_{\min } ; 2.1-2.5 \mathrm{~m} / \mathrm{s}\right.$; Williams et al., 1993; Yazdi et al., 1999) but in line with the preferred swimming speeds of individuals in control trials when swimming pace was not set (Figure 3; Chapter 9). The faster swimming speed, with an expected $72 \%$ increase in drag at that speed, and a lower oxygen consumption rate compared to trials with the tag alone raises the possibility that the dolphins were exercising above their lactate threshold and relying on anaerobic metabolism.

Lactate threshold and the maximum aerobic capacity $(\max )$ has been previously tested in bottlenose dolphins. Williams et al. (1993) found that a $145 \mathrm{~kg}$ male reached max of $29.4 \mathrm{ml} \mathrm{O} 2 / \mathrm{kg} / \mathrm{min}$ under a $192 \mathrm{~kg}$ workload. The dolphin could only swim under a 202 $\mathrm{kg}$ workload for 4 minutes. We cannot compare oxygen consumption rates measured during 

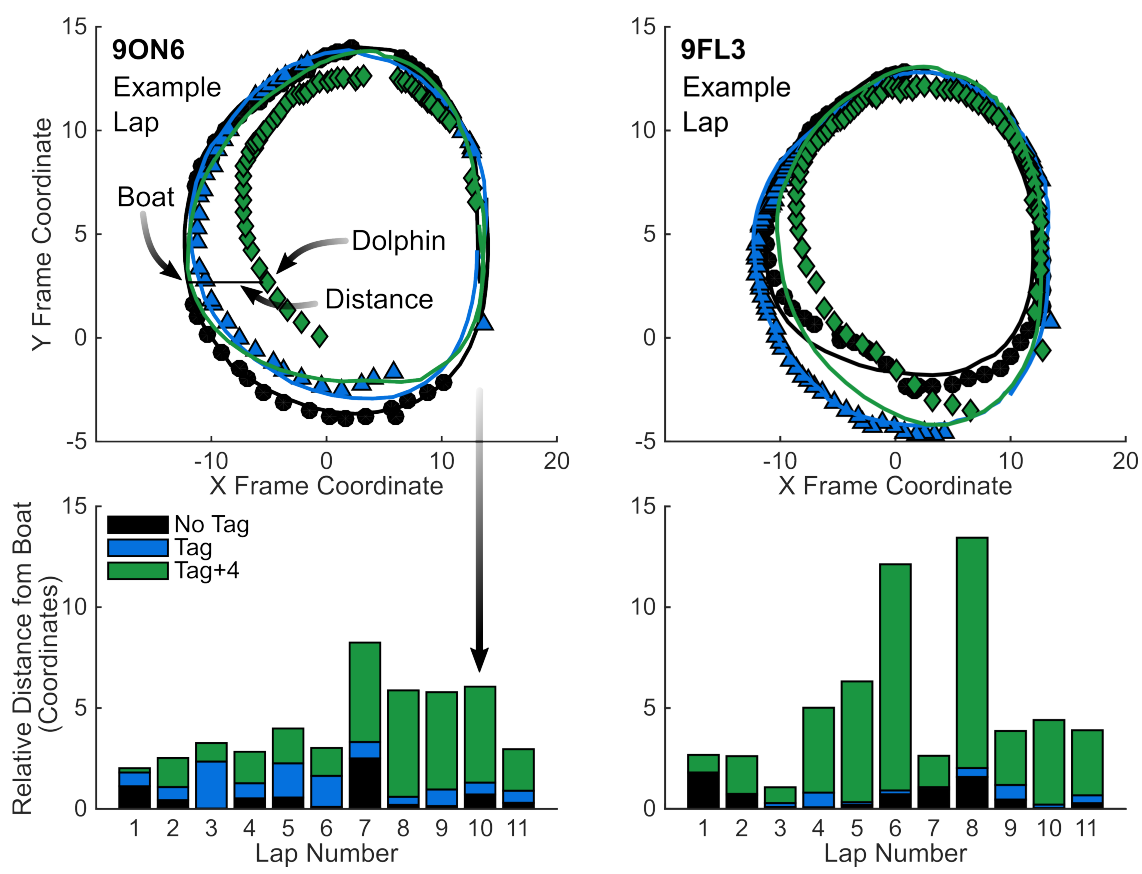

Figure 10-4: Dolphins deviated from the path of the boat as drag loading increased. Top: Representative paths of two bottlenose dolphins (IDs 9ON6 left, 9FL3 right; symbols) during the second-last lap of metabolic trials as they swam following a remote-controlled boat (lines) at $3 \mathrm{~m} / \mathrm{s}$. Dolphins performed the task with no tag (control, black and circles), and instrumented with the tag (blue and triangles) and tag +4 (green and diamonds). Bottom: Relative distance in the video frame between the position of the dolphin and the position of the boat throughout the 5-minute swimming task.

exercise (e.g., Goforth, 1990; Williams et al., 1993; Yazdi et al., 1999) with those measured only during recovery from exercise (herein). Anaerobic metabolism may be indicated by high end-expiratory $\mathrm{CO}_{2}$ concentrations, or an unequal relationship between $\mathrm{CO}_{2}$ production $\left(\dot{V} \mathrm{O}_{2}\right)$ and $\mathrm{O}_{2}$ consumption $\left(\dot{V} \mathrm{CO}_{2}\right)$; neither pattern is consistently observed for the few trials for these individuals in any drag condition (Figure H1). Future studies should include measurements of blood lactate levels to confirm aerobic vs. anaerobic metabolism in these types of exercise swimming tasks.

Thrust force production for swimming bottlenose dolphins has been measured at 50-700 $\mathrm{N}$ during steady, routine swimming at 0.6-3.7 m/s (Fish et al., 2014). Even at $3 \mathrm{~m} / \mathrm{s}$, thrust production was $500 \mathrm{~N}$ (Fish et al., 2014); the addition of $7 \mathrm{~N}$ of drag from a bio-logging tag, or $\sim 70 \mathrm{~N}$ from the tag +4 (Chapter 8 ) may seem little relative to the capacity of bottlenose dolphins. These results demonstrate that there may be an energetic cost associated with the added drag from even a reasonably small hydrodynamic tag, with considerable effects on dolphins' swimming speeds and behavior (herein; Chapter 9) and metabolic rate depending on which strategy they choose under drag loading conditions.

It is important to consider how this and other studies on exercise physiology in managed dolphins apply to managed populations. Routine speeds in free-swimming dolphins range $<1$ to $5 \mathrm{~m} / \mathrm{s}$ (Wursig and Wursig, 1979; Irvine et al., 1982; Shane, 1990; Mate et al., 1995; 
Ridoux et al., 1997), where activity is cycled between high- and low-energy behaviors (Wells et al., 2013, e.g. reviewed in). Based on similar dive durations and activity budgets between coastal bottlenose dolphins (Shane, 1990; Mate et al., 1995; Wells et al., 2013) and managed animals at Dolphin Quest (Shorter et al. Submitted), we expect the physiological responses measured here are applicable across these populations. 
Chapter 11

Conclusion AND OUTLOOK 


\subsection{Synthesis}

This thesis has quantified the magnitude and effects of drag loading in two scenarios: entanglement in fishing gear in large whales, and external tag attachment in small cetaceans. I found that entanglement in fishing gear increases drag by up to 3 fold (Chapters 2, 3), whereas current bio-logging tag designs (DTAG2 and DTAG3) increase drag by $10-28 \%$ and $\sim 6 \%$, respectively, on bottlenose dolphins (Chapters 7,8 ). I examined changes in swimming and diving behaviors associated with this added drag in both systems (Chapters 2, 4, 7, 9, 10) and modeled (Chapter 5) and measured (Chapters 7, 9, 10) the metabolic costs of swimming with increased drag.

These two systems are complementary, and provide an opportunity for the comparison of how animals in different environments and with different constraints respond to drag loading. There are similarities between the systems, and also important differences; for small cetaceans, the issue of entanglement is often not one of drag, and drag is of little concern when tagging large whales. Below, I provide some synthesis among chapters along with important distinctions, and describe future applications and areas of research inspired by this work.

\subsubsection{Energy economy}

A central theme in this thesis is energy economy - how animals minimize energetic costs. Drag loading sometimes results in reduction in speed (Lang and Daybell, 1963; Boyd et al., 1997; Elliott et al., 2007; van der Hoop et al., 2014a); however, there are instances where speed is maintained and additional energy consumption occurs (Williams et al., 1993; Methling et al., 2011; Tudorache et al., 2014; Jepsen et al., 2015). In this thesis, experimental trials with bottlenose dolphins showed that individuals reduced their swimming speed to maintain overall drag forces to maintain energy expenditure under increased drag loading (Chapters 7, 9). Our experimental approach required conditioning animals to keep pace with a remote-controlled boat in order for them to maintain $\sim 3 \mathrm{~m} / \mathrm{s}$ swimming speeds with even a $6 \%$ increase in drag (DTAG3; Chapter 10) as opposed to slowing down to reduce additional energy costs.

Few data exist on large whale swimming speeds, including those relevant to changes in force balance. I resolved dive descent and ascent speeds from DTAGs deployed on two right whales; one whale (Eg 3911) showed significantly slower descent (46\%) and ascent (32\%) rates with an $80 \%$ increase in drag (Chapters 2, 4; Table 1). However, the other individual (Eg 4057) showed little difference in descent and ascent rates, despite a $51 \%$ increase in drag (Chapter 4). A comparison of speeds from satellite telemetry data of entangled (D. Sandilands, pers. comm. ${ }^{1}$ ) and non-entangled whales (Baumgartner and Mate, 2005) did

\footnotetext{
${ }^{1}$ Center for Coastal Studies, 115 Bradford Street, Provincetown, MA 02657
} 
not indicate any differences in swimming speed between these groups, though the resolution of GPS data limit such an analysis (see Next Steps, below).

Table 11-1: A comparison of changes in drag and speed as reported in this thesis.

\begin{tabular}{|l|l|l|}
\hline & Change in drag & Change in speed \\
\hline Chapter $2-\operatorname{Eg} 3911$ & $80.0 \%$ & $-33 \%$ \\
\hline Chapter $4-\operatorname{Eg} 4057$ & $51.2 \%$ & $+24 \%$ \\
\hline Chapter 7 - DTAG2 & $9.7 \%$ & $-11 \%$ \\
\hline Chapters 8, 9- DTAG3 & $6.0 \%$ & $-13 \%$ \\
\hline Chapters 8, 9- tag +8 & $137.0 \%$ & $-45 \%$ \\
\hline
\end{tabular}

\subsubsection{Swimming efficiency}

Swimming efficiency is one of the many factors that contributes to energy economy. In Chapter 4 I was able to estimate a right whale's propulsive efficiency (i.e., the ratio of thrust produced relative to the power supplied to the system), and how or whether this was affected by changes in kinematics and/or speed in response to drag loading from entanglement in fishing gear. The method used data from bio-logging tags, which are also available from the experimental work in dolphins (Chapters 9, 10). Applying this same method to swimming data from the dolphins (e.g., comparing the tag and tag + conditions) would provide an analogous situation with more controlled parameters to investigate how or whether swimming efficiency contributes to energy economy in these systems.

\subsubsection{Positioning of added drag elements}

The position of additional drag is important in determining its impact. Chapters 3 and 6 show the magnitude and variability of interference drag in different entanglement configurations: the relative contributions of interference drag to total drag depends on the point of attachment, the element's shape, and the boundary layer flow (Jacobs 1934). Chapters 8 and 9 show the same effect with instrumentation. Although it was not visible at low speeds during our dolphin glide experiment (Chapter 8), the tag + configurations caused major flow disturbance as was observed in CFD simulations; the resulting bubble formation posterior to the tag + elements especially at high speeds led to much higher drag (Figure 9 in Chapter 9). The location of tag placement is often limited by animal morphology (e.g., desire to attach to the dorsal fin), attachment mechanism (e.g., glued to a seal's head vs. attached via suction-cup behind a dolphin's blowhole), and tag needs (e.g., for VHF or satellite antennae to maximize time out of water). While tags can be deployed with ideal or near-ideal placements, tags with flexible attachment mechanisms (i.e., suction cup tags) often move during a deployment. Within those constraints, it is important to iterate on tag designs to 
develop tags that minimize frontal and wetted areas (Chapter 8) with hydrodynamic designs (Shorter et al., 2013) that sit in areas where flow has already separated or where flow disturbance is minimized (Bannasch et al., 1994; Jones et al., 2013). No matter the source of added drag, its placement on a body is critical to evaluate as it will enhance or reduce the total effect of loading.

Interference drag is also relevant when researchers add more than one tag to a subject in order to collect a diversity of data types. Jones et al. (2011) showed the effect of placing multiple tags in different configurations on turtles; the combined increase in drag is not equal to the sum of the drag of each of the elements. Attaching tags in series vs. in parallel, and interactive effects between the spacing of the tags, are also elements for researchers to consider when planning bio-logging tag studies. Thirty percent of marine mammal biologging studies reviewed in McIntyre (2014) reference data from more than one device per animal (McIntyre Pers. Comm. ${ }^{2}$; this is likely a conservative estimate as many studies report only the number of devices per animal that generated the data for the paper, rather than the total number of devices attached. The tag size recommendations herein are not intended to evaluate the suitability of tags that will be deployed coincident with others on a single animal.

\subsubsection{Duration of impact}

Chapters 3,5 and 6 emphasize the importance of the amount of time for which animals are subject to increased drag. Entangled whales free-swimming with high-drag configurations can experience considerable loading, but these events are often not lethal over short (daylong) timescales (Chapter 5). Alternatively, relatively low-drag configurations can lead to death when carried for months to years (Chapter 5, Moore et al., 2006). The amount of additional work (power $\mathrm{x}$ time) required to overcome additional drag is equally important when evaluating tag impacts. External suction-cup tags such as the DTAG provide shortterm $(12-48 \mathrm{~h})$ attachments; they therefore contribute little additional work and energy consumption in the context of a monthly or annual energy budget (see e.g. Jones et al., 2013). The size, shape and resulting amount of drag added to tag subjects should be considered relative to the intended deployment duration: tags deployed for weeks or months (e.g. external tags glued to the animal's surface) should be especially small and hydrodynamically shaped. While this consideration would minimize the energetic impact of tagging, it does not address the issue of recording unnatural behaviors due to tag effects for a tag deployment of any duration (e.g. Broell et al., 2016).

\footnotetext{
${ }^{2}$ Trevor McIntyre, PhD. Mammal Research Institute, Department of Zoology and Entomology, University of Pretoria, South Africa
} 


\subsubsection{Relative impact of tags vs. entanglement in dolphins vs. whales}

Entanglement in fishing gear is an issue for small cetaceans as well as large whales. Entrapment and injury in gear are most common for porpoise and dolphin species (Kuiken et al., 1994; Moore et al., 2013); even with subsequent release, these types of interactions can result in sub-lethal impacts (Wilson et al., 2014a). Individuals are found with gear still attached, where gear has mostly been identified as monofilament and crab float lines (Wells et al., 1998; NOAA, 2008; Wells et al., 2008; Barco and Moore, 2010; Moore et al., 2013). Drag on the gear is sufficient to lead to sharp laceration and cause gear to migrate through appendages over weeks to months, sometimes resulting in amputation (NOAA, 2008; Barco and Moore, 2010; Moore et al., 2013). In this context, drag on entangling fishing gear carried by small cetaceans may not have significant metabolic effects as would be evidenced by emaciation in these cases, but causes direct injury with the potential to significantly alter swimming ability (Webb, 1973).

Bio-logging and -tracking tags have been deployed on large whales for decades (Schevill and Watkins, 1966; Watkins and Schevill, 1977). As technology has advanced to miniaturize sensors, tag designs have been iterated upon and have become smaller and more hydrodynamic. Drag from instrumentation is therefore of little concern when tagging large whales; most short-term suction-cup tags (e.g., the DTAG2, DTAG3, Acousonde) represent $~ 0.1 \%$ of the frontal areas of right whales and $\leq 0.01 \%$ of their wetted areas - far within the recommended tag size range (Chapter 8 ). There are a few unique cases where large camera tags can still be considered cumbersome for even the blue whale, the largest animal on the planet (Calambokidis et al., 2007). Camera tags can and have been refined to smaller sizes (Goldbogen et al., 2016); we encourage further efforts to do so.

\section{$11.2 \quad$ Next steps}

\subsubsection{Tag drag}

The work presented in this thesis with colleagues at Dolphin Quest Oahu represents an iterative study design in response to observed behaviors after our initial experiment in 2012 (Chapter 7). The major application of this work was in suggesting design criteria for tags to minimize any behavioral and metabolic effects of instrumentation (as presented in Chapters $7,9,10)$. Future work should continue to refine the experimental protocol to condition animals to maintain speed and perform the desired task (Chapter 10). Kinematic studies similar to those completed for entangled right whales (Chapter 4) should be undertaken to see if and how small cetaceans adjust their power output and whether drag loading results in detectable changes in their propulsive efficiency.

Additionally, it is important to better understand swimming speeds of tagged cetaceans 
and their behaviors in social groups (e.g., Macfarlane, 2015) in order to know what environmental and/or social constraints exist on tagged dolphins in other research environments where tags are deployed.

\subsubsection{Entanglement drag}

\section{Application to other species}

This thesis has demonstrated the degree to which entanglement in fishing gear affects North Atlantic right whale behavior, movement and physiology with major implications on individual life history and reproductive capacity and therefore population trajectory. Entanglement is an issue for species other than right whales; it is the leading cause of death to large whale species in the North Atlantic (van der Hoop et al., 2013a, 2014b) and is a serious conservation concern to populations worldwide (Robbins et al., 2007; Neilson et al., 2009; IWC, 2011). Global disentanglement efforts have been able to retrieve gear from whales (Chapters $2,3,4)$, and analysis of this gear reflects the distribution patterns of these highly migratory species. For example, right whales have carried gear for years, between habitats (Chapters $3,5,6)$; entangled humpback whales have traveled over 2450 nautical miles (4500 km) from Alaska to Hawaii (Lyman, 2014). North Pacific humpback whales are a species that are often chronically entangled in similar gear configurations, and show signs of emaciation. These populations are data-rich and would be an excellent next model for investigation or application of this research.

The relationships between drag force and gear dimensions in Chapter 3 are applicable across species as they detail the entangling gear only. Applying these relationships and putting these estimated drag forces in the context of other species (Chapter 6) would require age:length relationships and length:width specifications for the species in question, likely available in the literature (Kraus, 1985; Perryman and Lynn, 2002; Geraci and Lounsbury, 2005; Durban et al., 2015). It could be assumed, as a first approximation, that humpback, fin (Balaenoptera physalus), sei (B. borealis), minke (B. acutorostrata) and blue (B. musculus) whales have the same survival capacity as right whales, which would offer a conservative approach. However, balaenopterids are likely less energetically robust to the constant increase in drag associated with entanglement as they have lower total body lipid reserves and are not as well-adapted to continuous high-drag swimming that balaenids require for ram feeding (Pivorunas, 1979; Woodward, 2006; Simon et al., 2009). These overestimates of survival for other species would therefore favor the fishery.

\section{Demographic modeling}

This research has essentially defined chronic entanglement as an additional, anthropogenic life history stage (Chapter 5). I have estimated individual and often sub-lethal energetic costs 
associated with entanglement in fishing gear that can be integrated into demographic models. Doing so improves previous estimates of how entanglement affects population growth in large whales, which have typically counted detected mortalities (e.g., Read et al., 2006; van der Hoop et al., 2013a, 2014b; McIntosh et al., 2016) or compared natural and entanglementrelated mortality to birth rates (Fowler et al., 1990; Volgenau et al., 1995). Individual Based Models (IBMs) incorporate changes in individual characteristics through time, and how these characteristics contribute to the population as a whole; these models have been used to investigate population-level effects of individual condition in right whales (Klansjcek et al., 2007; Schick et al., 2013). The Schick et al. (2013) model includes entanglement status and severity classes, but do not necessarily align with the energetic demand associated with specific gear configurations.

Entanglement could also be included as a life history stage in matrix models (e.g., Caswell et al., 1999; Fujiwara and Caswell, 2001). Transition probabilities to this life history stage would be governed by sightings data; individuals would transition from the stage back to their previous demographic state (e.g. juvenile male) or to death based on entanglement severity (Knowlton et al., 2012b, 2015), magnitude of the drag from the gear (Chapter 3; van der Hoop et al., 2015), critical duration (Chapter 6), and individual sightings data. Other right whale health assessment methods and models (Pettis et al., 2004; Schick et al., 2013; Rolland et al., 2016) are under way. The importance of drag (Chapters 2, 3, 4; van der Hoop et al., 2013b, 2015) and the resulting energetic cost (Chapters 5, 6) of entanglements has been demonstrated by the work in this thesis; entanglement duration, not just wound severity, should be considered in these heath assessment and individual-based models.

\section{Effects of consuming lipid stores}

Adipose tissue has multiple functions in marine mammals: it provides (1) almost limitless energy storage; (2) a source of buoyancy; (3) streamlining; (4) insulation from water which has a high heat capacity; and (5) immune and endocrine functions (Parry, 1949; Pond, 1978; Pond and Mattacks, 1988). Lipid depots are non-uniform. For example, blubber thickness is variable across the body surface and the blubber layer shows vertical stratification with different levels of energy density and metabolic activity (Pond, 1978; Koopman et al., 2002; Struntz et al., 2004). Despite differentiation of lipid store metabolism, consuming large volumes of blubber lipids to meet nutritional demands likely affects the ability of blubber to function in its other roles.

The consumption or contribution to body lipid stores as animals fast or feed affects their overall body density. This relationship has been extremely useful in detecting changes in body condition in diving animals (e.g., Biuw et al., 2003). Right whales with thicker blubber layers are more positively buoyant, and use this to their advantage when ascending from dives (Nousek-McGregor et al., 2013). One entangled right whale in good body condition (Eg 
4057) did not show large changes in dive behavior with $157 \mathrm{~N}$ of added buoyancy (Chapter 4). The other whale (Eg 3911) had significantly reduced dive depth and duration (Chapters 2, 4). The magnitude of the added buoyancy $(1058 \mathrm{~N})$ combined with the extreme emaciation of that case likely influenced the magnitude of the response. How diving behavior changes in an individual through the process of emaciation, regardless of its mechanism or any gear attached, would be interesting to know.

Blubber plays a structural role, affecting e.g. body streamlining (Pabst, 1990). With starvation, blubber loss is greatest in mid-body regions with little change at either end of the body; this pattern is consistent across marine mammal species including elephant seals (Slip et al., 1992), porpoises (Koopman et al., 2002), right whales (Miller et al., 2012b), and other large mysticetes (Lockyer et al., 1985). Compartmentalization of the body's total lipid store with preferential metabolism from certain body regions and layers (Koopman et al., 2002) likely maintains the function of blubber as a structural tissue, streamlining the body in the rostral and caudal regions, and acting as a biological spring for oscillatory propulsion (Pabst, 1990, 2000). Still, these changes in body shape influence fineness ratios and the location of maximum girth of the body, which can decrease total body drag by $24 \%$ in a severely emaciated right whale (Appendix III, Chapter 6).

Blubber provides insulation, limiting heat loss to water. The effects of blubber metabolism on the thermoregulatory capacity has received little focus. Emaciated animals have the lowest insulation (Dunkin et al., 2005), which results from not only a change in blubber thickness (i.e., quantity) but also its thermal conductivity (i.e., quality). Overheating may be an issue for large whale species with thick blubber layers and low surface area-to-volume ratios; structures in the oral cavity to dump heat while foraging have been identified in many mysticetes (e.g. reviewed in Heyning, 2001). The positive feedback whereby emaciated animals must increase their metabolic rate to compensate for higher rates of heat loss compared to non-emaciated animals, in turn likely consuming more blubber and therefore further reducing insulation likely does not pose a great threat for large whales. However, this may remain a risk for smaller species subject to chronic entanglement with resulting emaciation (Feldkamp et al., 1988; DeLong et al., 1990).

Energy expenditure is not constant during fasting, but is a function of starvation time, body size, activity patterns, and fat content (Oritsland et al., 1985; Cherel et al., 1988, 1992; Caloin, 2004). There is varying evidence for whether metabolic rate is increased or suppressed in response to emaciation. Metabolic effects will likely be different depending on whether the animal experiences a predictable (e.g., seasonal) fast where costs are 'known' and energy is stored in preparation vs. imposed fasts (e.g., Rosen et al., 2014) or periods of unexpected, prolonged energetic deficit (e.g., chronic entanglement; Wang et al., 2006).

Lipid-based metabolism generates more water per gram ( $1.07 \mathrm{~g} \mathrm{H}_{2} \mathrm{O} / \mathrm{g}$ fat) compared to carbohydrate $(0.56 \mathrm{~g} / \mathrm{g})$ or protein $(0.39 \mathrm{~g} / \mathrm{g})$ substrates (Costa, 2009). Fasting or re- 
liance on endogenous energy stores will increase metabolic body water, which reduces renal function demands and results in the production of dilute urine (Bentley, 1963; Ortiz, 2001; Aguilar et al., 2014). Fasting in terrestrial or other marine environments often requires osmoregulatory adaptations to cope with water limitations (Ortiz, 2001; Costa, 2009); fasting baleen whales do not face these same water limitations (Hui, 1981; Aguilar et al., 2014).

Consumption of endogenous energy reserves in the form of blubber can result in mobilization of contaminant burden. Many toxins present in the marine environment are lipophilic and therefore can be sequestered and concentrated in the lipid depots of the body, e.g., the blubber layer (Klansjcek et al., 2007; Montie et al., 2010). Previous studies have detected environmental contaminants in right whales (Woodley et al., 1991; Montie et al., 2010) and their prey (Weisbrod et al., 2000), as well as evidence for biological response to these toxicants (Angell et al., 2004). While toxicants may have little effect while bound and sequestered, it is uncertain to what extent these contaminants can be released to circulate as individuals consume large volumes of their blubber reserve during entanglement through the process of emaciation (Debier et al., 2006). Models suggest increased toxicant concentrations in right whales by way of blubber catabolism related to the timing of seasonal variability or energy restriction relative to the reproductive season (Klansjcek et al., 2007). Data from pinnipeds give evidence for the relationship between contaminants and lipid dynamics; concentrations and circulation increase with decreasing lipid reserves (Debier et al., 2006) and dilute with mass gain (Hall et al., 2008). These toxicants may then reduce fertility or lead to mortality (e.g., Martineau et al., 2002), though direct evidence of toxicant-related mortality in baleen whales is still lacking (O'Shea and Brownell, 1994). Whether chronically entangled, emaciated right whales do in fact show higher circulating levels of contaminants, and how these contaminants may work in concert with increased stress, low energy and infection are unknown but important questions in understanding the factors and process of health deterioration due to entanglement.

Total body lipid plays an important role in immune function. Immune responses are often reduced in humans and animals with nutrient, especially fat, limitations (reviewed in Pond, 1996) and facing energetic trade-offs associated with cold stress or reproduction (Peck

et al., 2016, and references therein). Reduced capacity to mount an immune response to injury or trauma associated with entanglement could be impacted by low lipid reserves or also the chronic elevation of adrenal hormones.

\section{Stress}

Although stress is beneficial on the short-term to respond to and recover from a stimulus or perceived threat, remaining in a stressed state in the long-term is maladaptive. Elevation of adrenal hormones, catecholamines and neurotransmitters involved in regulating the metabolic, immune and cardiovascular responses to stress results cannot be indefinitely 
maintained; maintaining this unbalanced 'allostatic state' can impair future stress response and fecundity and result in immune and other physiological dysfunction (McEwen and Wingfield, 2003, 2010; Houser et al., 2016).

Fecal glutocorticoids are considerably elevated in chronically entangled whales (Hunt et al., 2006, 2015). The connection between increased drag, altered behavior, additional energy demand, high stress and decreased breeding success has been established in studies addressing the effects of attaching scientific instruments (e.g., reviewed in Barron et al., 2010). Especially in birds and bats, added drag or weight from scientific instruments can lead to increases in energy expenditure, decreases in body condition, significant increases in corticosteroids, and reduced nesting propensity and productivity (Barron et al., 2010; Elliott et al., 2012). Our results contribute to recent literature to suggest that entangled whales may follow the same model of response to prolonged increases in drag of $52 \%$ on average: decreases in body condition (Cassoff et al., 2011; Moore and Barco, 2013; van der Hoop et al., 2013a; Barratclough et al., 2014), elevated stress hormones (Hunt et al., 2006) and reduced reproductive success (Knowlton et al., 2012b). Future research along this cascade including clarifying the mechanisms behind the interactions between stress, energetic demand, nutrition, and metabolism are required, but especially difficult in large whale species (Hunt et al., 2015).

\section{Movement patterns}

Experimental approaches (Chapters 7, 9, 10) suggest that bottlenose dolphins change their swimming paths and reduce swimming speeds in response to drag loading (see Section 11.1.1). To determine whether drag from entanglement elicits similar changes, I completed a preliminary comparison of satellite tag-derived swimming speeds of entangled and nonentangled right whales; however, high error and low fix rates likely limited the analysis. Dive descent and ascent rates from two DTAGs revealed opposing responses, and may have been confounded by differences in individual buoyancy. The question remains: Do entangled whales swim more slowly? Advances in telemetry resolution may provide insight on how entangled individuals move. Further, given the tradeoffs between speed and drag and the relative costs of entanglement and migration (Chapter 5), do entangled whales maintain migratory timing? Sightings data may determine whether individuals that are entangled or in otherwise poor health or body condition continue to appear in breeding and feeding areas with their conspecifics, or whether (see e.g., Shaw and Levin, 2011; Jachowski et al., 2013)?

\section{The entangled life history stage}

The time course, energetic expenditures, and physiological changes associated with entanglement in fishing gear (Chapters 2-6) all lend towards treating entanglement as its own life history stage (Chapter 5). Wingfield et al. (1998) suggested that in response to certain 
perturbations, animals reduce specific behaviors (i.e., 'low-resilience behaviors' such as those that are social in nature; Nicol, 2015) in favor of emergency behaviors, and mobilize energy stores. This life history stage, like others, is brought about by a suite of physiological and behavioral responses that can be sustained only for so long without lasting effects. The concept ties together the effects of injury and trauma, prolonged elevation of stress hormones, and nutritional stress associated with entanglement. Life-cycle events involve large-scale changes in morphology, physiology and behavior regulated by hormonal responses. In the same way and in the more extreme scenarios, drag (as shown in both systems investigated in this thesis) can induce large-scale changes in an individual's force balance (Chapters 2, 3, 8), leading to increased energy consumption (Chapter 10) and behavioral adaptation (Chapters $4,7,8,9,10)$. Animal energy and time budgets are often altered under drag loading: entangled animals spend more time resting (Feldkamp, 1985; Fowler, 1987; Feldkamp et al., 1988), and tagged animals can be much less energetic (Blomqvist and Amundin, 2004; Geertsen et al., 2004; Barron et al., 2010). Applying the physiological and behavioral results of this thesis to the way we approach animal ecology (e.g., Ricklefs and Wikelski, 2002; Cooke et al., 2013; Tomlinson et al., 2014; Jachowski and Singh, 2015) and incorporating these effects in demographic models would truly address the consequences of entanglement which occur long before death. 
Appendix A

ChAPTER 2 SUPPlEMENTAL INFORMATION 


\section{A.1 Supplemental Material}

We used four methods to estimate body weight from length. (1) Age-weight and lengthweight functions (Moore et al. 2004) approximated the weight of a 2-yr-old or $950 \mathrm{~cm}$ right whale to 6,717 and $6,396 \mathrm{~kg}$, respectively, though the paucity of the data at these age values suggests a more plausible range of 8,000-10,000 kg. (2) An additional age-dependent lengthweight function (Fortune 2012) estimated $10,551 \mathrm{~kg}$. (3) To address the degree of emaciation of the individual and its effect on the above weight estimates, we estimated width-to-total body length ratios at intervals of $10 \%$ of the body length from the tip of the rostrum and compared to width-to-length ratios measured using vertical aerial photogrammetry of 10 adult female right whales (Miller et al. 2012) (Table S1). This comparison suggests Eg 3911 was on average $20 \%$ thinner than other adult female right whales, allowing for a weight estimation of between $6,400 \mathrm{~kg}$ and $8,440 \mathrm{~kg}$. (4) We reduced other scaling factors for gray whales (Sumich 1986) and generic cetaceans (Geraci and Lounsbury 2005) by $20 \%$ to account for emaciation to obtain estimates of $7,048 \mathrm{~kg}$ and $7,200 \mathrm{~kg}$, respectively.

Table A-1: Timeline of events on 15 January 2011 in Sedation/Entangled, Disentangled, and Recovery phases of Eg 3911.

\begin{tabular}{|l|l|l|l|l|l|l|l|l|}
\hline & \multicolumn{7}{|c|}{ Width-to-total body length ratio } \\
\hline & 0.1 & 0.2 & 0.3 & 0.4 & 0.5 & 0.6 & 0.7 & 0.8 \\
\hline Mesomorphic right whales $(\mathrm{n}=10)$ & 0.149 & 0.191 & 0.226 & 0.22 & 0.207 & 0.176 & 0.126 & 0.063 \\
\hline Eg3911 & 0.132 & 0.175 & 0.199 & 0.195 & 0.156 & 0.121 & 0.078 & 0.051 \\
\hline Eg3911:Mesomorphic ratio & 0.887 & 0.92 & 0.88 & 0.887 & 0.751 & 0.684 & 0.617 & 0.798 \\
\hline Mean ratio & & & & & & & & 0.803 \\
\hline
\end{tabular}


Appendix B

Chapter 3 Supplemental Information 


\section{B.1 Estimating wetted surface area and Froude number of gear configurations.}

\section{B.1.1 Wetted surface area estimates}

Wetted surface area was estimated based on the area of a cylinder with a specified length, $l$, and radius, $r$ :

$$
A_{w}=2 \pi r l
$$

For most gear sets, this calculation was straightforward and based on the single line diameter and dimensions; however, for the following six gear sets made up of multiple line types, floats, or buoys, total wetted surface area was estimated as the sum of surface areas of the components of each gear set. For all attached floats and buoys, half the wetted surface area is calculated, assuming that half of each item is in contact with the water.

\section{J070602}

Gear details:

Imperial: $121 \mathrm{ft}$ of $3 / 8^{\prime \prime}$ diameter line, with two $32^{\prime \prime} \times 7 / 8^{\prime \prime}$ buoy sticks and an $8^{\prime \prime}$ trawl can buoy

Metric: $37 \mathrm{~m}$ of $9.5 \mathrm{~mm}$ diameter line, with two $813 \mathrm{~mm} \times 22 \mathrm{~mm}$ buoy sticks and a $203 \mathrm{~mm}$ trawl can buoy

Line $A_{w}=2 \pi(0.0048 \mathrm{~m})(37 \mathrm{~m})=1.12 \mathrm{~m}^{2}$

Buoy $A_{w}=\frac{\pi(0.203 \mathrm{~m})^{2}}{2}=0.0647 \mathrm{~m}^{2}$

Buoy Sticks $A_{w}=2\left(\frac{2 \pi(0.0111 \mathrm{~m})(0.0813 \mathrm{~m})}{2}\right)=0.113 \mathrm{~m}^{2}$

Total $A_{w}=1.30 \mathrm{~m}^{2}$

\section{$\mathrm{J} 120305$}

Gear details:

Imperial: $41 \mathrm{ft}$ of 5/16" diameter line, $360 \mathrm{ft}$ of $3 / 8^{\prime \prime}$ diameter line, $3 \times 10^{\text {" diameter }}$ trawl can buoys

Metric: $12 \mathrm{~m}$ of $8 \mathrm{~mm}$ diameter line, $110 \mathrm{~m}$ of $9.5 \mathrm{~mm}$ diameter line, $3 \times 250 \mathrm{~mm}$ diameter trawl can buoys

Line $A_{w}=2 \pi(0.0040 \mathrm{~m})(12 \mathrm{~m})+2 \pi(0.0048 \mathrm{~m})(110 \mathrm{~m})=3.62 \mathrm{~m}^{2}$

Buoys $A_{w}=\frac{\pi(0.254 \mathrm{~m})^{2}}{2} \times 3=0.30 \mathrm{~m}^{2}$

Total $A_{w}=3.92 \mathrm{~m}^{2}$

$\mathrm{J} 120604$ 
Gear details:

Imperial: $24 \mathrm{ft}$ of sinking 3/8" diameter line, $468 \mathrm{ft}$ floating 3/8" line, $18^{\prime \prime}$ diameter balloon, $7^{\prime \prime} \times 14$ " bullet float Metric: $7 \mathrm{~m}$ of sinking $9.5 \mathrm{~mm}$ diameter line, $143 \mathrm{~m}$ of 9.5 $\mathrm{mm}$ diameter line, $45 \mathrm{~cm}$ diameter balloon, $17.8 \times 35.6 \mathrm{~cm}$ bullet float

Line $A_{w}=2 \pi(0.0048 \mathrm{~m})(7 \mathrm{~m})+2 \pi(0.0048 \mathrm{~m})(143 \mathrm{~m})=4.52 \mathrm{~m}^{2}$

Buoys $A_{w}=\frac{\pi(0.457 \mathrm{~m})^{2}}{2}=0.328 \mathrm{~m}^{2}$

Float $A_{w}=\frac{\pi(0.0889 \mathrm{~m})^{2}+2 \pi(0.0889 \mathrm{~m})(0.356 \mathrm{~m}-0.0889 \mathrm{~m})+2 \pi(0.0889 \mathrm{~m})^{2}}{2}=0.112 \mathrm{~m}^{2}$

Total $A_{w}=4.96 \mathrm{~m}^{2}$

\section{$\mathrm{J} 120808$}

Gear details:

Imperial: $129 \mathrm{ft}$ of 3/8" diameter line, $600 \mathrm{ft}$ of $5 / 16^{\prime \prime}$ diameter line

Metric: $43 \mathrm{~m}$ of $9.5 \mathrm{~mm}$ diameter line, $200 \mathrm{~m}$ of $8 \mathrm{~mm}$ diameter line

Line $A_{w}=2 \pi(0.0048 \mathrm{~m})(43 \mathrm{~m})+2 \pi(0.0040 \mathrm{~m})(200 \mathrm{~m})=6.32 \mathrm{~m}^{2}$

Total $A_{w}=6.32 \mathrm{~m}^{2}$

\section{J091298}

Gear details:

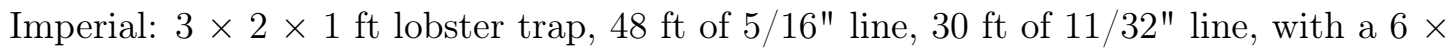
8" acorn float on a 30" Plante spindle.

Metric: $0.91 \times 0.61 \times 0.30 \mathrm{~m}$ lobster trap, $15 \mathrm{~m}$ of $8 \mathrm{~mm}$ diameter line, $9 \mathrm{~m}$ of $9 \mathrm{~mm}$ diameter line, with a $15 \times 20 \mathrm{~cm}$ acorn float on a $76 \mathrm{~cm}$ Plante spindle.

Line $A_{w}=2 \pi(0.004 \mathrm{~m})(15 \mathrm{~m})+2 \pi(0.0044 \mathrm{~m})(9 \mathrm{~m})=0.63 \mathrm{~m}^{2}$

Buoy $A_{w}$, assuming the buoy is a half ellipsoid with $a=0.101 \mathrm{~m} ; b=c=0.076 \mathrm{~m}$ using Knud Thomsen's formula, where $p=1.6075,=$

$$
S A \simeq \frac{4 \pi\left(\frac{a^{p} b^{p}+a^{p} c^{p}+b^{p} c^{p}}{3}\right)^{1 / p}}{2}=0.0446 \mathrm{~m}^{2}
$$

Spindle $A_{w}=2 \pi(0.0127 \mathrm{~m})(0.5588 \mathrm{~m})=0.0448 \mathrm{~m}^{2}$

Total float $A_{w}=0.0447 \mathrm{~m}^{2}$ Trap $A_{w}$, assuming $0.038 \mathrm{~m}(1.5 ")$ mesh size and $0.0025 \mathrm{~m}$ $\left(1 / 10^{\prime \prime}\right)$ wire diameter: The trap consists of six panels, two each of (a) $0.91 \times 0.61 \mathrm{~m}$, (b) $0.61 \times 0.30 \mathrm{~m}$, and $(\mathrm{c}) 0.91 \times 0.30 \mathrm{~m}$. The mesh area $\left(A_{M}\right)$ of each panel $(\mathrm{a}, \mathrm{b}, \mathrm{c})$ was calculated as:

$$
A_{M}=N \times K \times 2 M \times W,
$$

where $N$ is the number of wire columns, $K$ is number of wire rows, $M$ is the mesh size and $W$ the wire diameter. The number of columns and rows is determined by the size of the panel divided by the mesh size (Fridman and Dvernik 1973, Reid 1977).

Trap $A_{w} \simeq 2((24 \times 16)+(16 \times 8)+(24 \times 8)) \times 2 \times 0.038 \mathrm{~m} \times 0.0025 \mathrm{~m}=0.268 \mathrm{~m}^{2}$

Total $A_{w}=0.6258+0.0447+0.268=0.9385 \mathrm{~m}^{2}$ 


\section{J051099}

Gear details:

Imperial: $216 \mathrm{ft}$ of $1 / 2^{\prime \prime}$ diameter line and $5 \mathrm{ft}$ of $5 / 8^{\prime \prime}$ diameter line, with a gillnet towed in an approximately $3 \times 1.5 \times 1.5 \mathrm{ft}$ shape.

Metric: $66 \mathrm{~m}$ of $12.7 \mathrm{~mm}$, and $2 \mathrm{~m}$ of $16 \mathrm{~mm}$ diameter line, with a gillnet towed in an approximately $1 \times 0.5 \times 0.5 \mathrm{~m}$ shape.

Line $A_{w}=2 \pi(0.00635 \mathrm{~m})(66 \mathrm{~m})+2 \pi(0.0079 \mathrm{~m})(2 \mathrm{~m})=2.73 \mathrm{~m}^{2}$

Gillnet $A_{w}=2.5 \mathrm{~m}^{2}$

Total $A_{w}=2.73+2.5=5.23 \mathrm{~m}^{2}$

\section{Telemetry Buoy}

Gear details:

Imperial: 14" diameter buoy towed on $62 \mathrm{ft}$ of $5 / 16^{\prime \prime}$ diameter line

Metric: $35.6 \mathrm{~cm}$ diameter buoy towed on $19 \mathrm{~m}$ of $8 \mathrm{~mm}$ diameter line

Line $A_{w}=2 \pi(0.0040 \mathrm{~m})(19 \mathrm{~m})=0.478 \mathrm{~m}^{2}$

Buoy $A_{w}=\frac{\pi(0.356 \mathrm{~m})^{2}}{2}=0.199 \mathrm{~m}^{2}$

Total $A_{w}=0.677 \mathrm{~m}^{2}$

\section{B.1.2 Froude number estimates}

The Froude number ( $F n$; dimensionless) was calculated for floats on certain gear sets across the range of measured tow speeds $(U ; \mathrm{m} / \mathrm{s})$ as

$$
F n=\frac{U}{\sqrt{g l}},
$$

where $g$ is the acceleration due to gravity $\left(9.8 \mathrm{~m} / \mathrm{s}^{2}\right)$ and $\mathrm{l}$ is the length of the float $(\mathrm{m})$ at the water line level, assuming that each float is half submerged.

J070602: two $0.8 \mathrm{~m}$ buoy sticks and an $0.2 \mathrm{~m}$ diameter trawl can, total $l=1.0 \mathrm{~m}$.

J120305: $3 \times 0.25 \mathrm{~m}$ diameter trawl can buoys, total $l=0.75 \mathrm{~m}$.

J120604: $0.178 \mathrm{~m} \times 0.356 \mathrm{~m}$ bullet float that we assume floats at an angle and therefore has $l=0.25 \mathrm{~m}$ and a $0.45 \mathrm{~m}$ diameter balloon float, total $l=0.7 \mathrm{~m}$.

Telemetry Buoy: $0.356 \mathrm{~m}$ diameter buoy, $l=0.356 \mathrm{~m}$. 
B.2 Sets of fishing gear removed from entangled North Atlantic right whales used in this experiment 


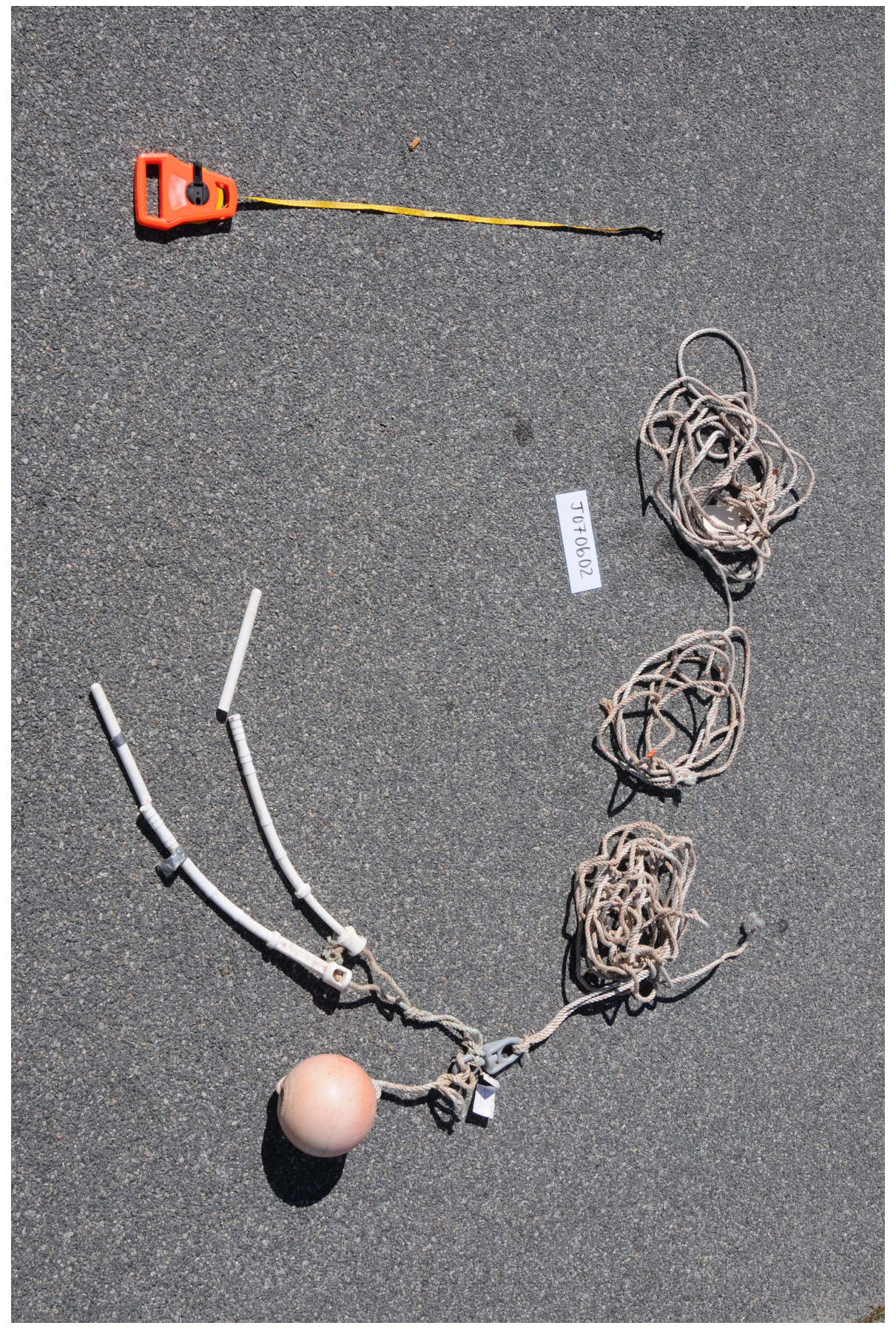




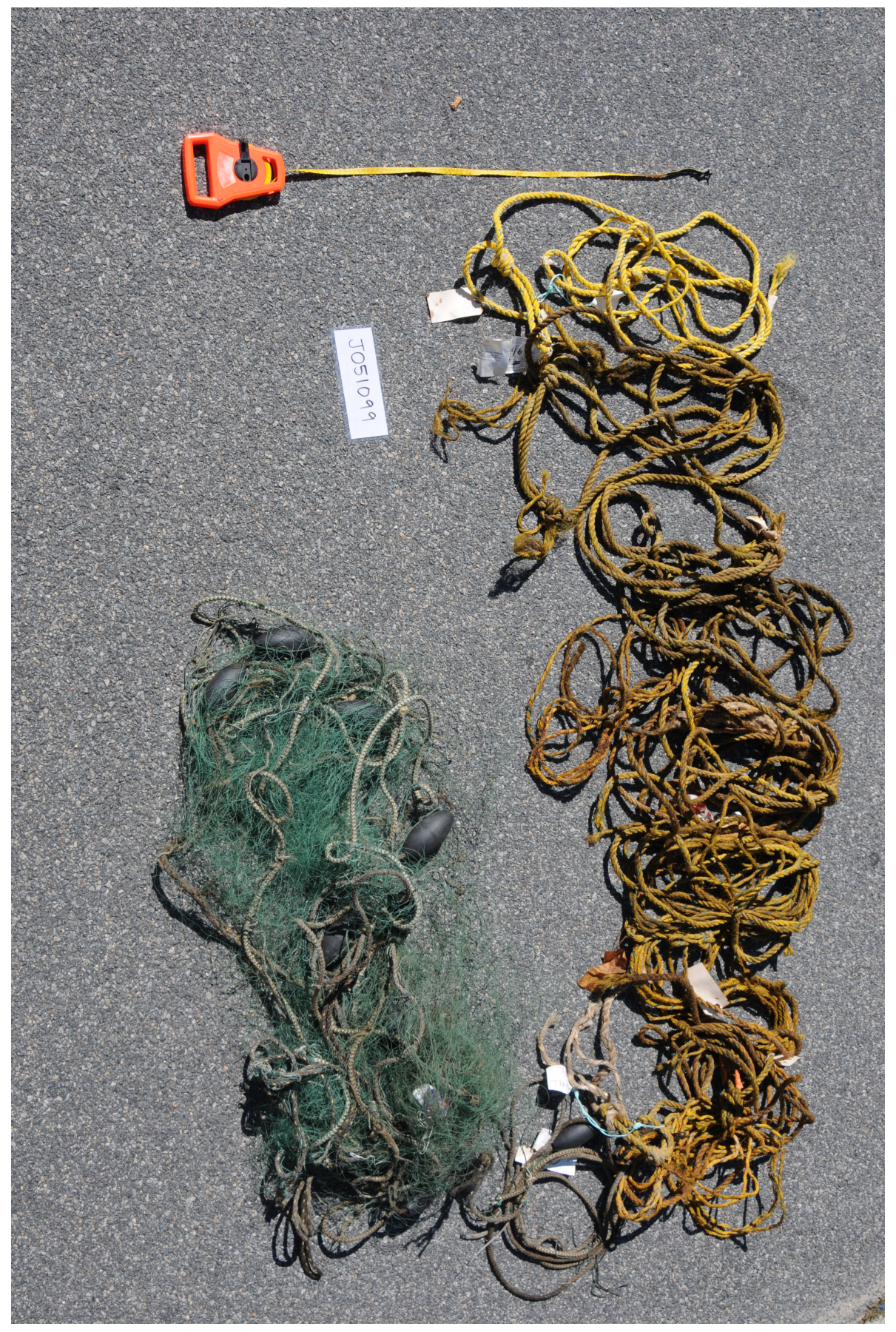




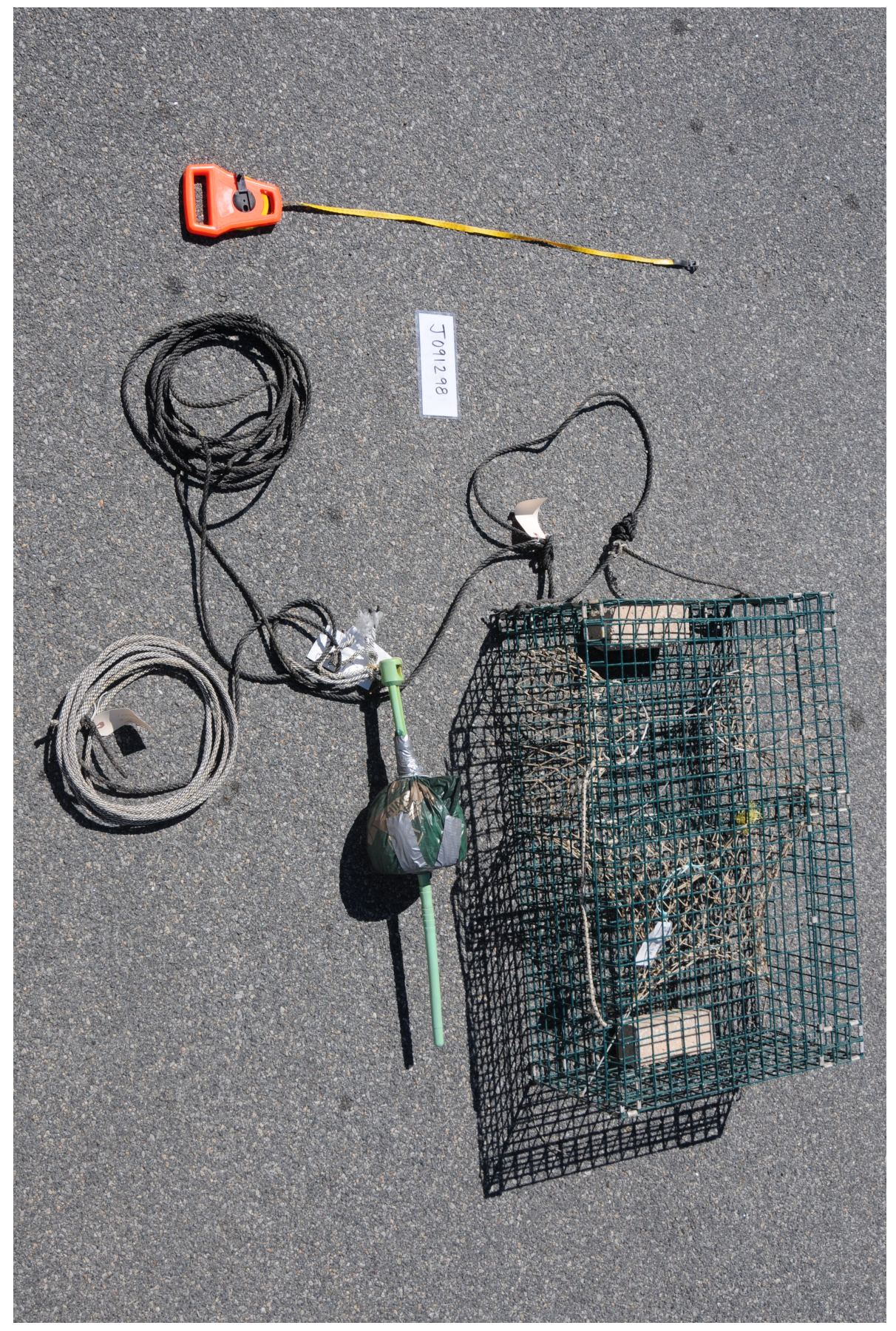




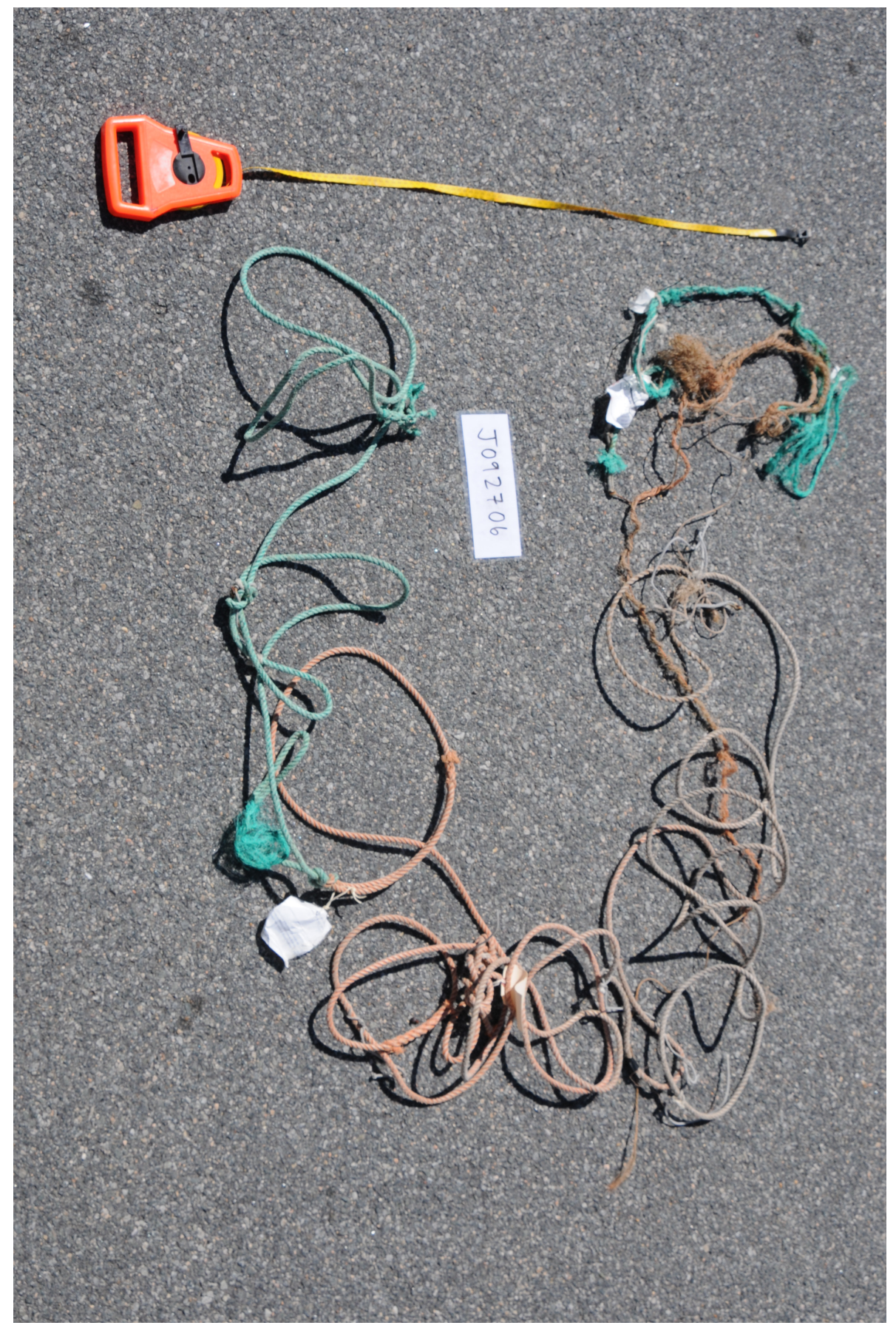




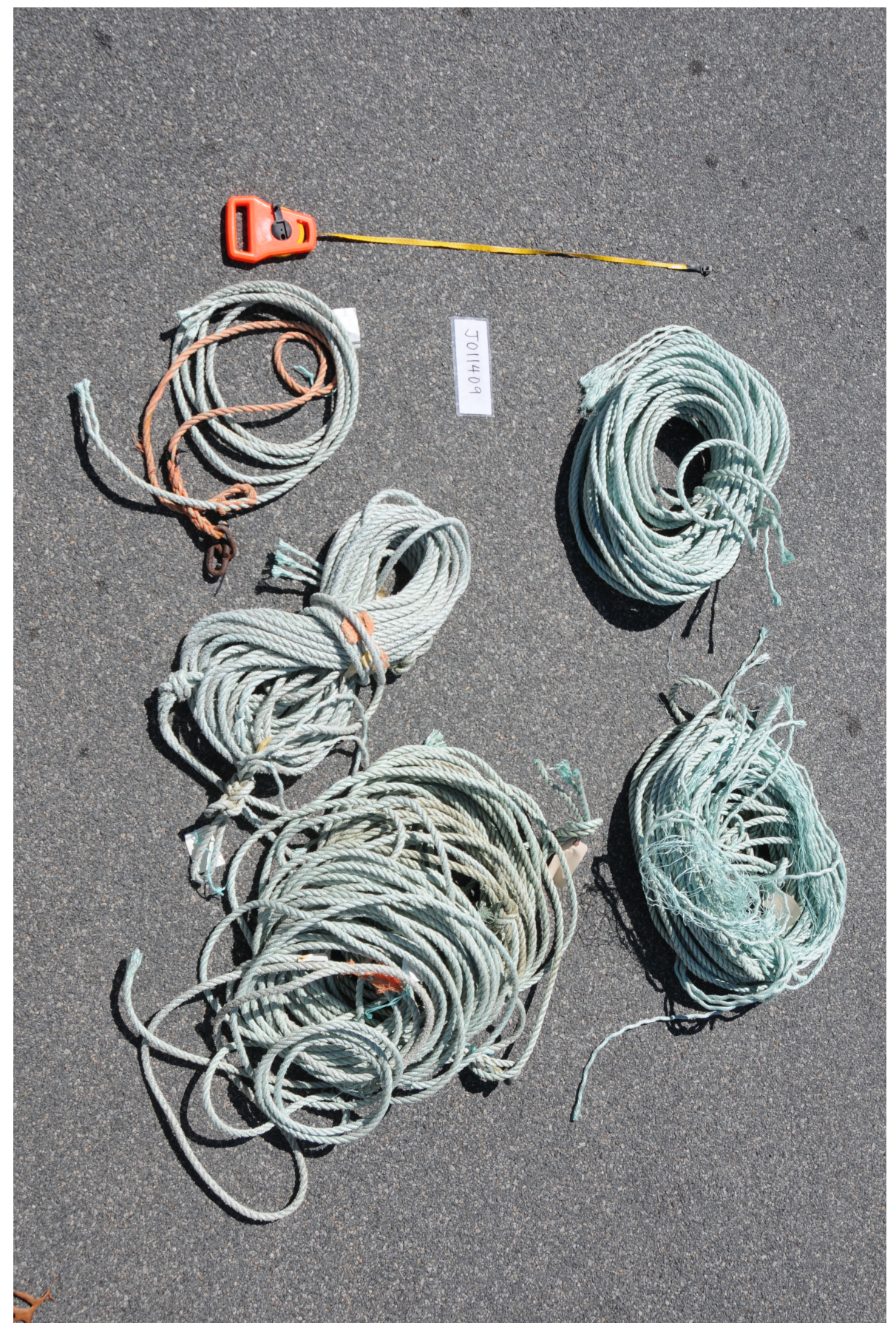




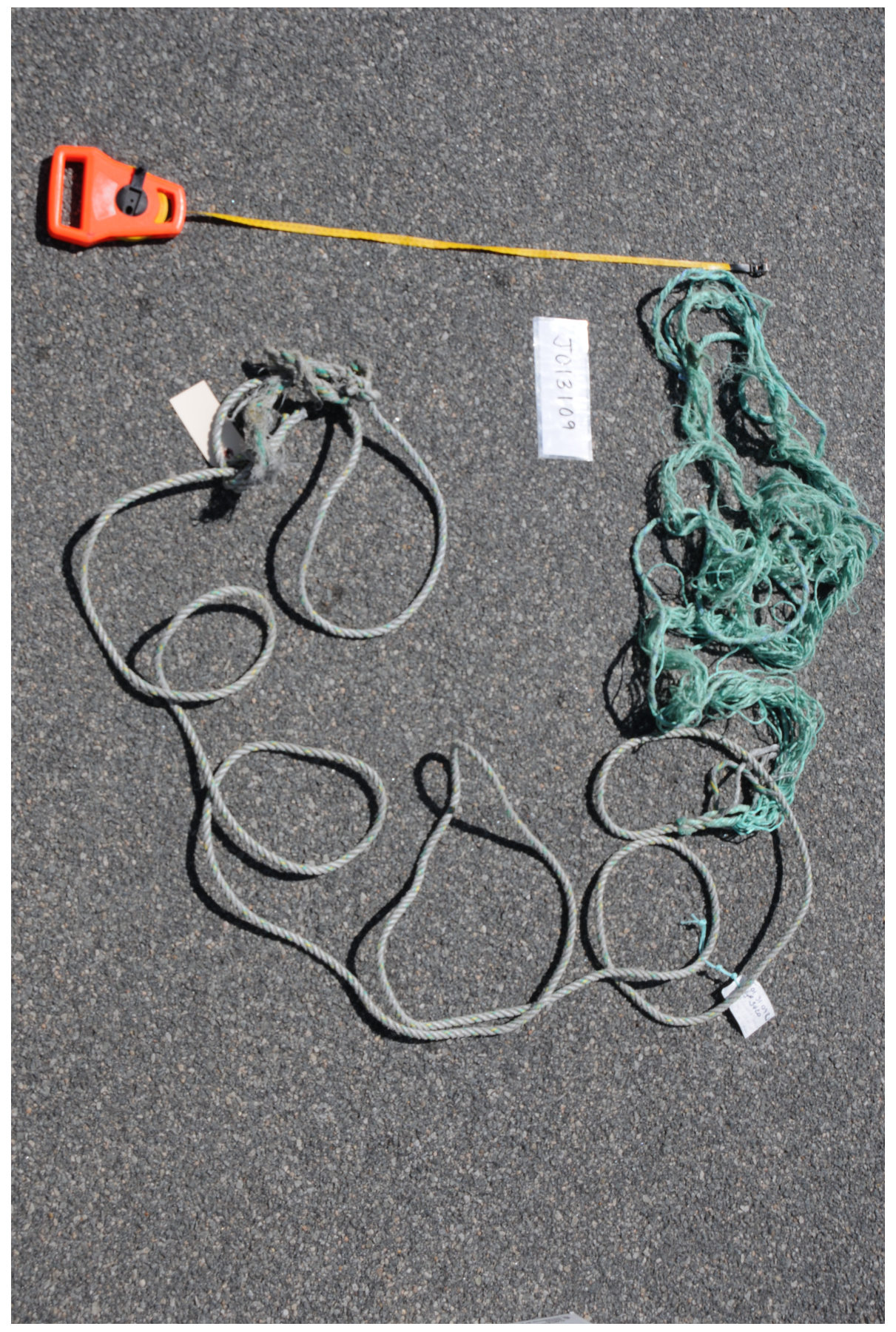




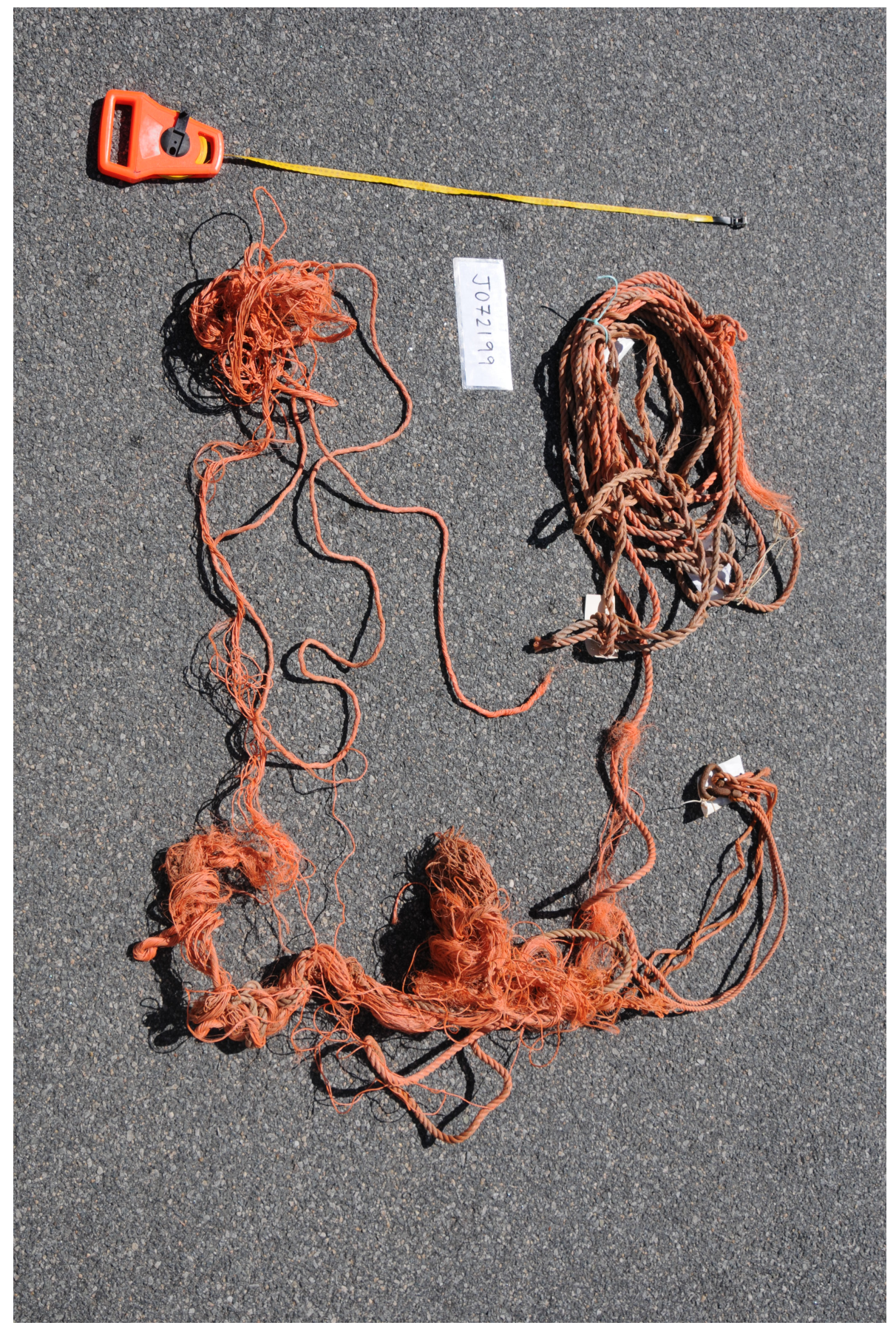




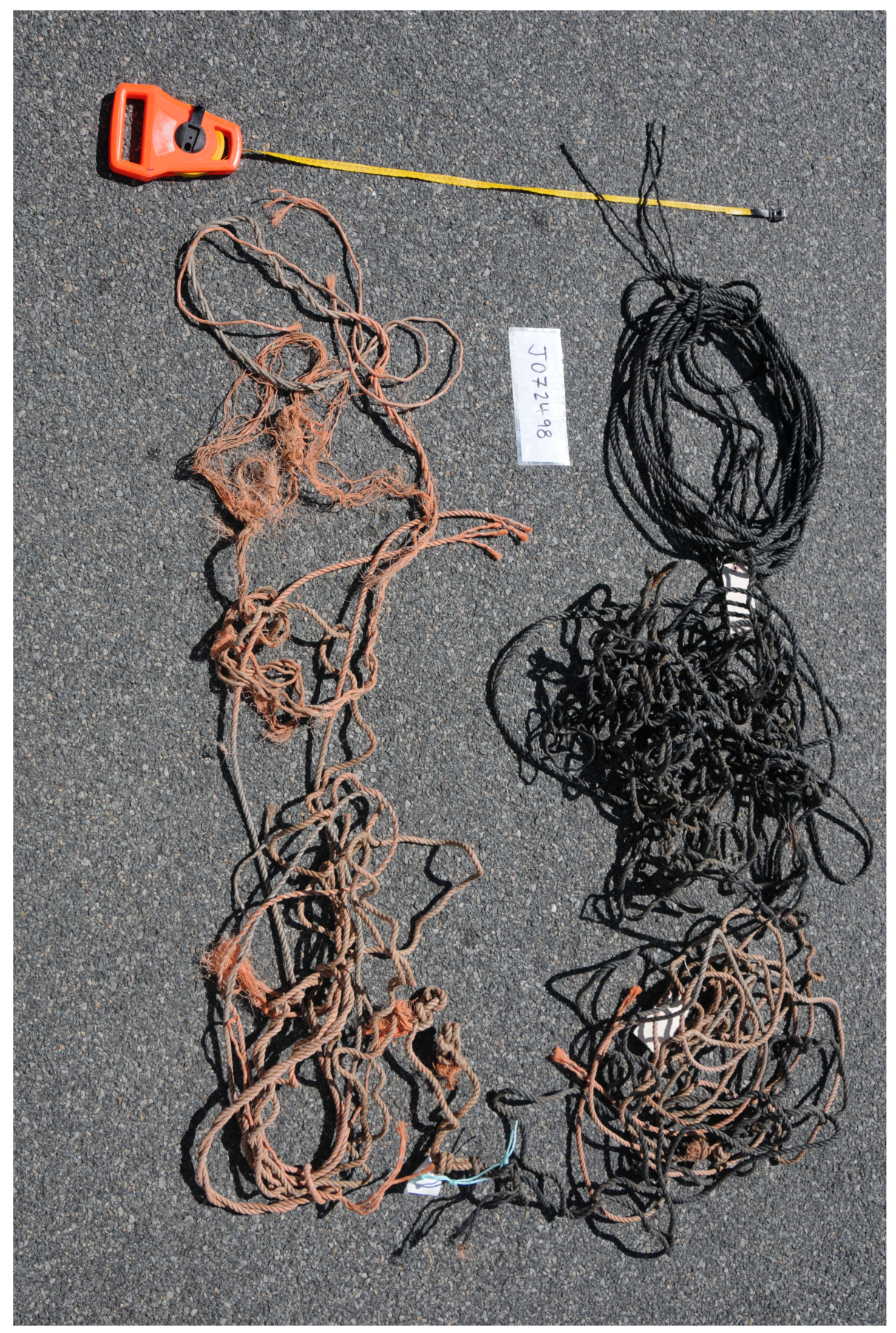




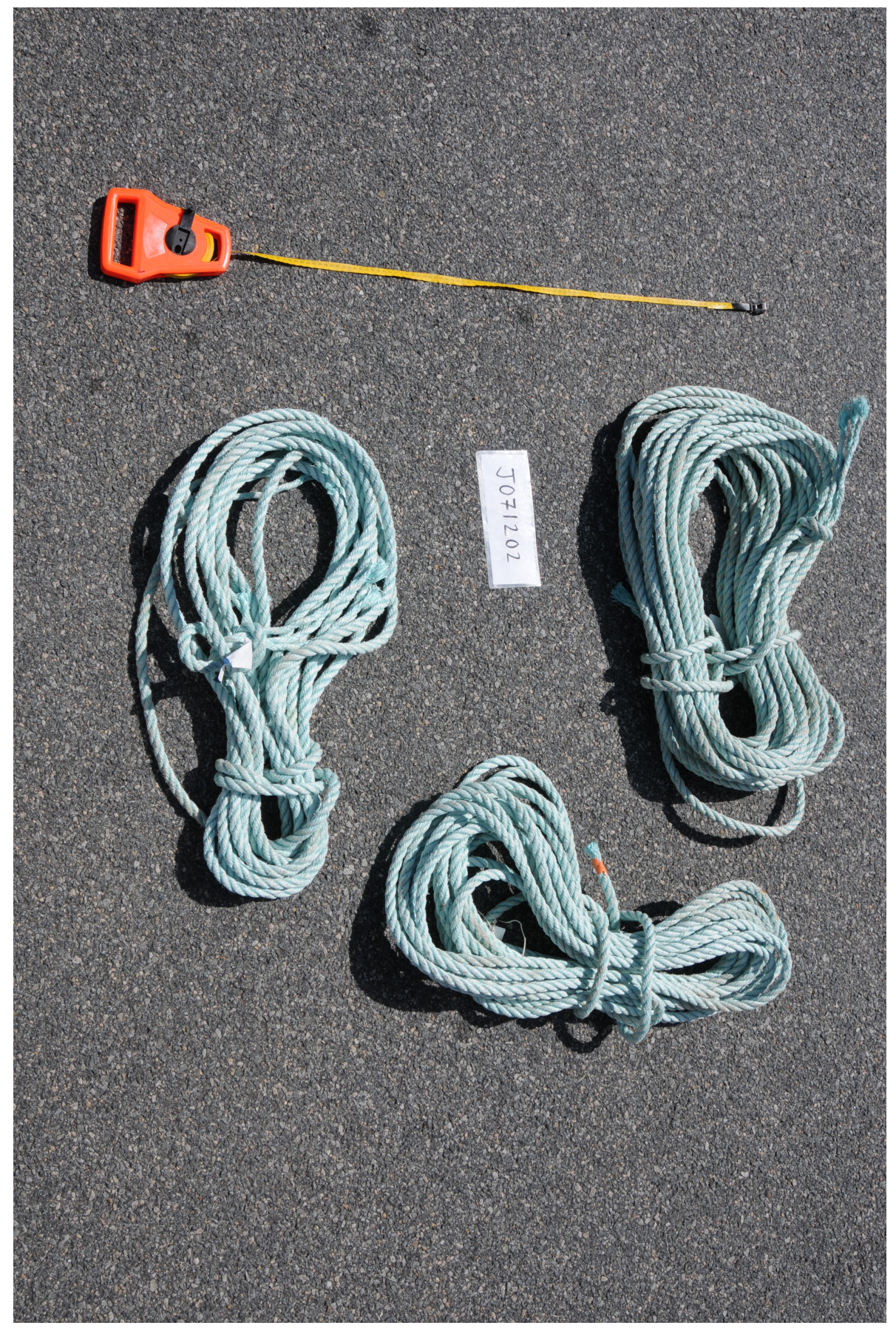




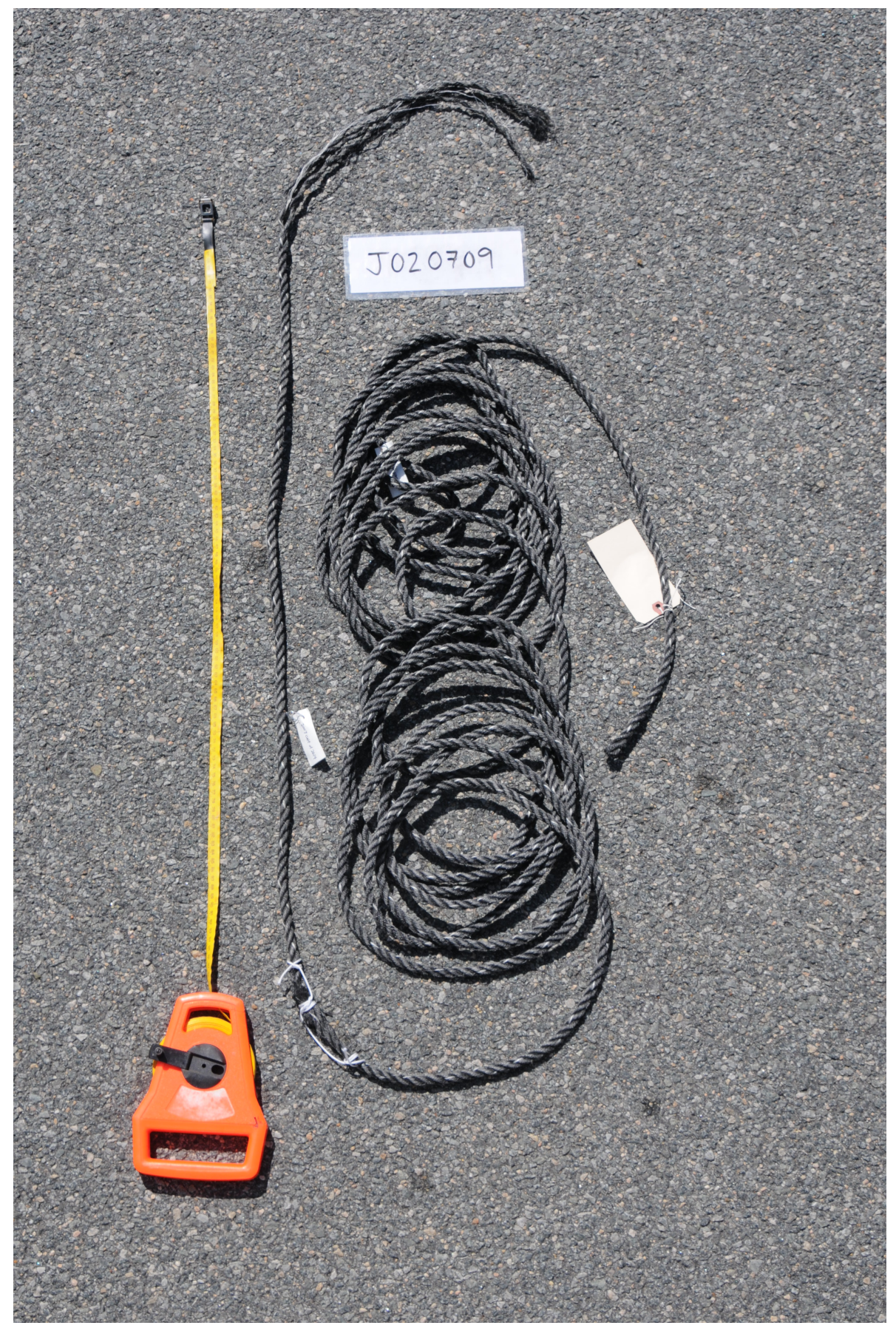




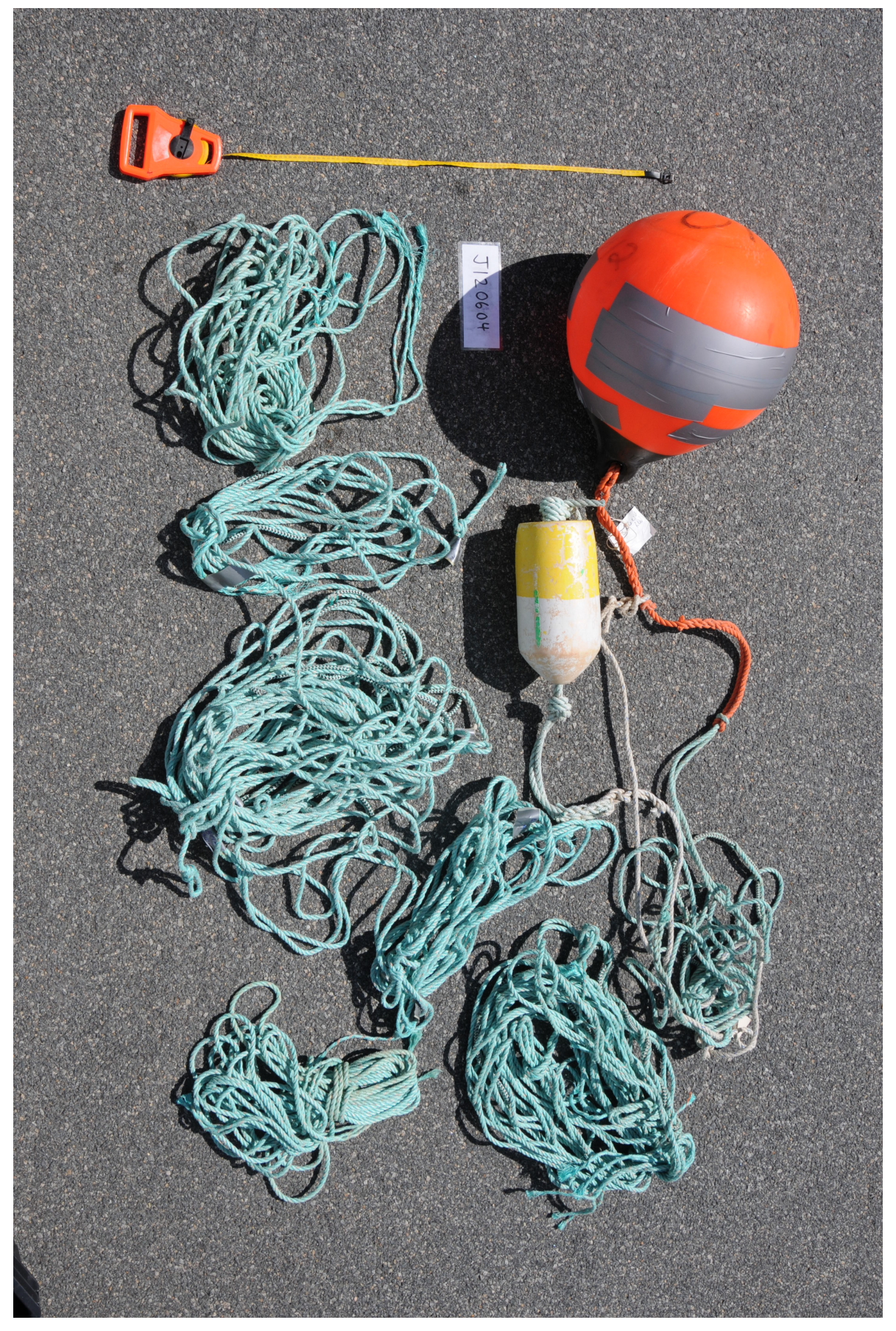




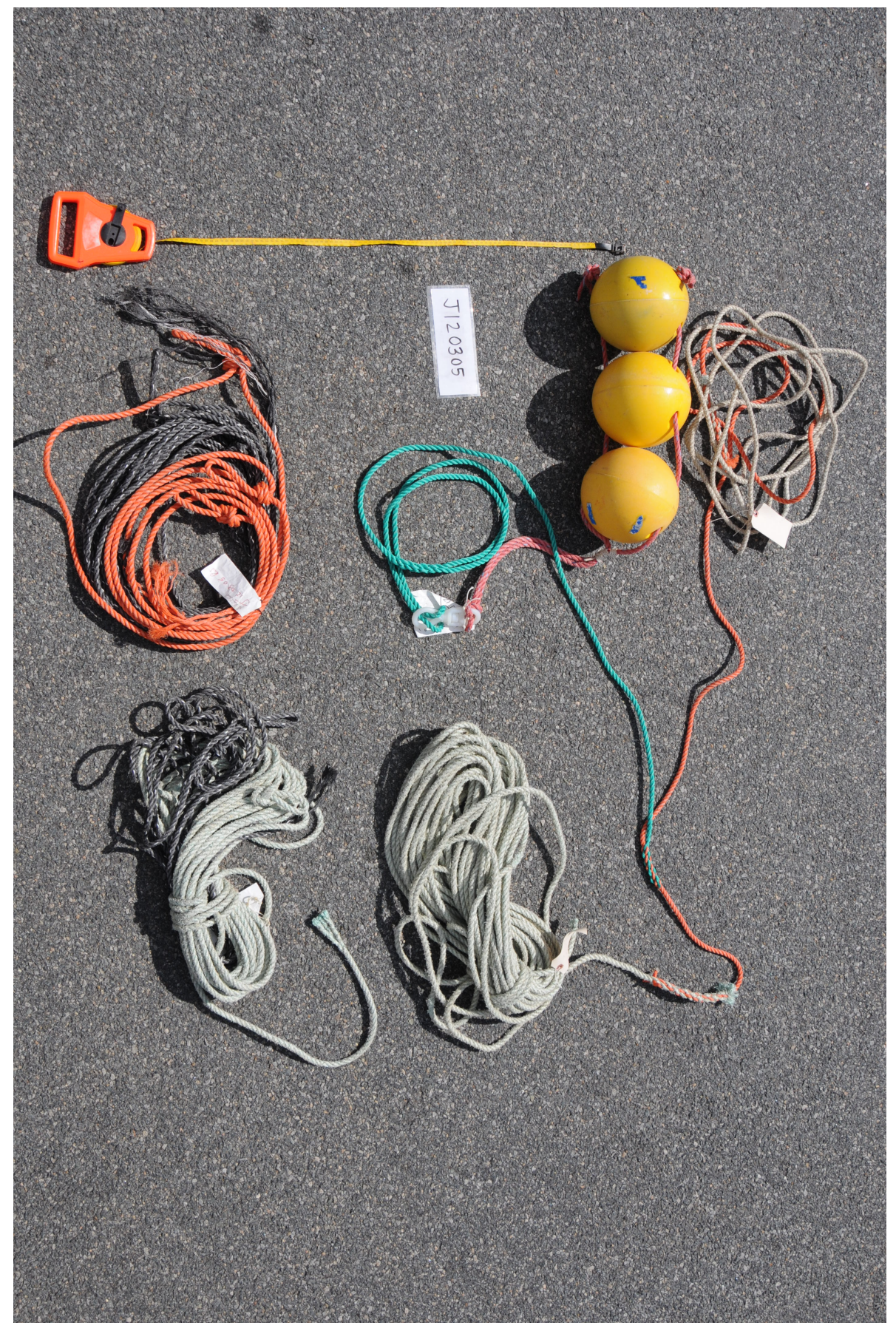




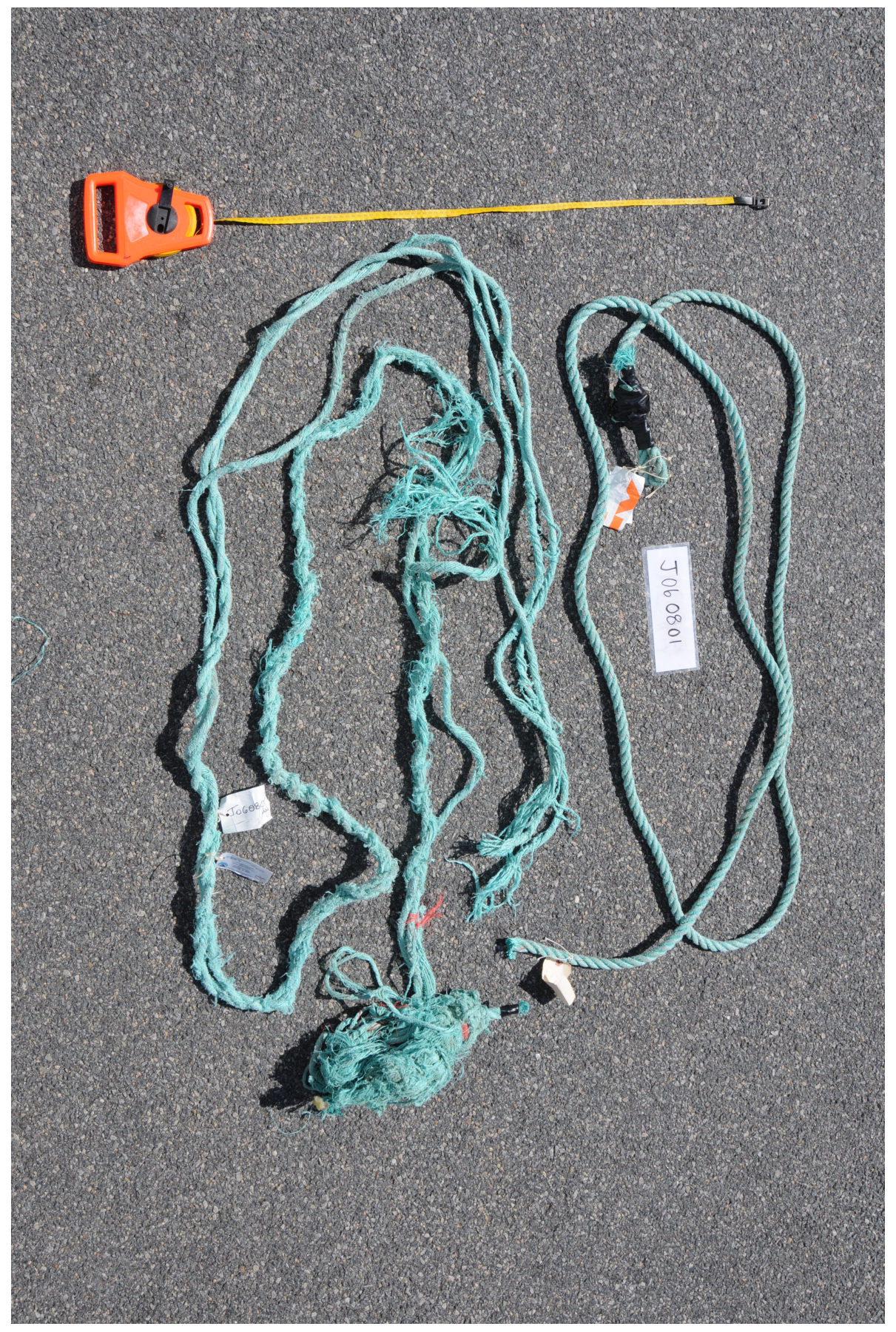




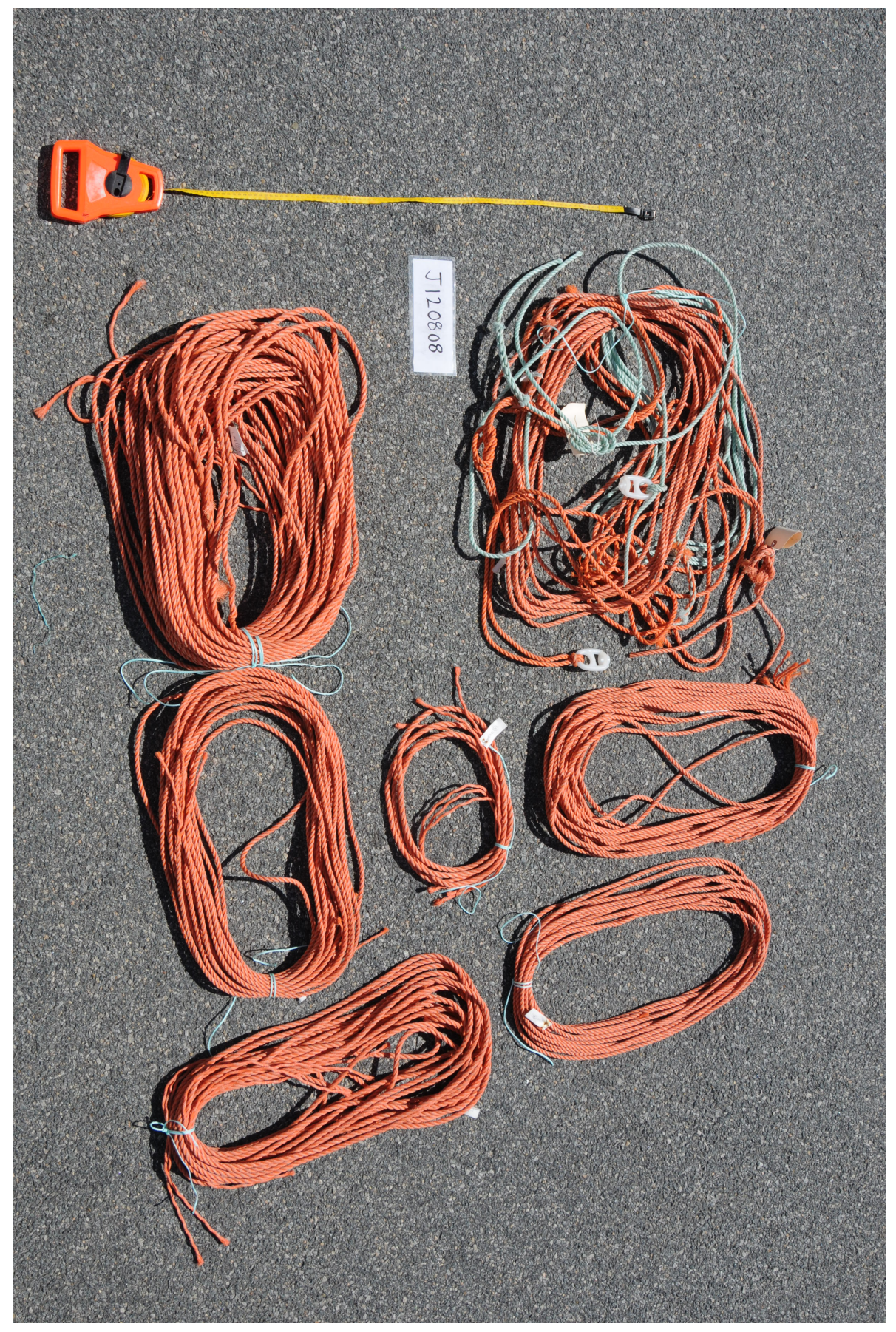




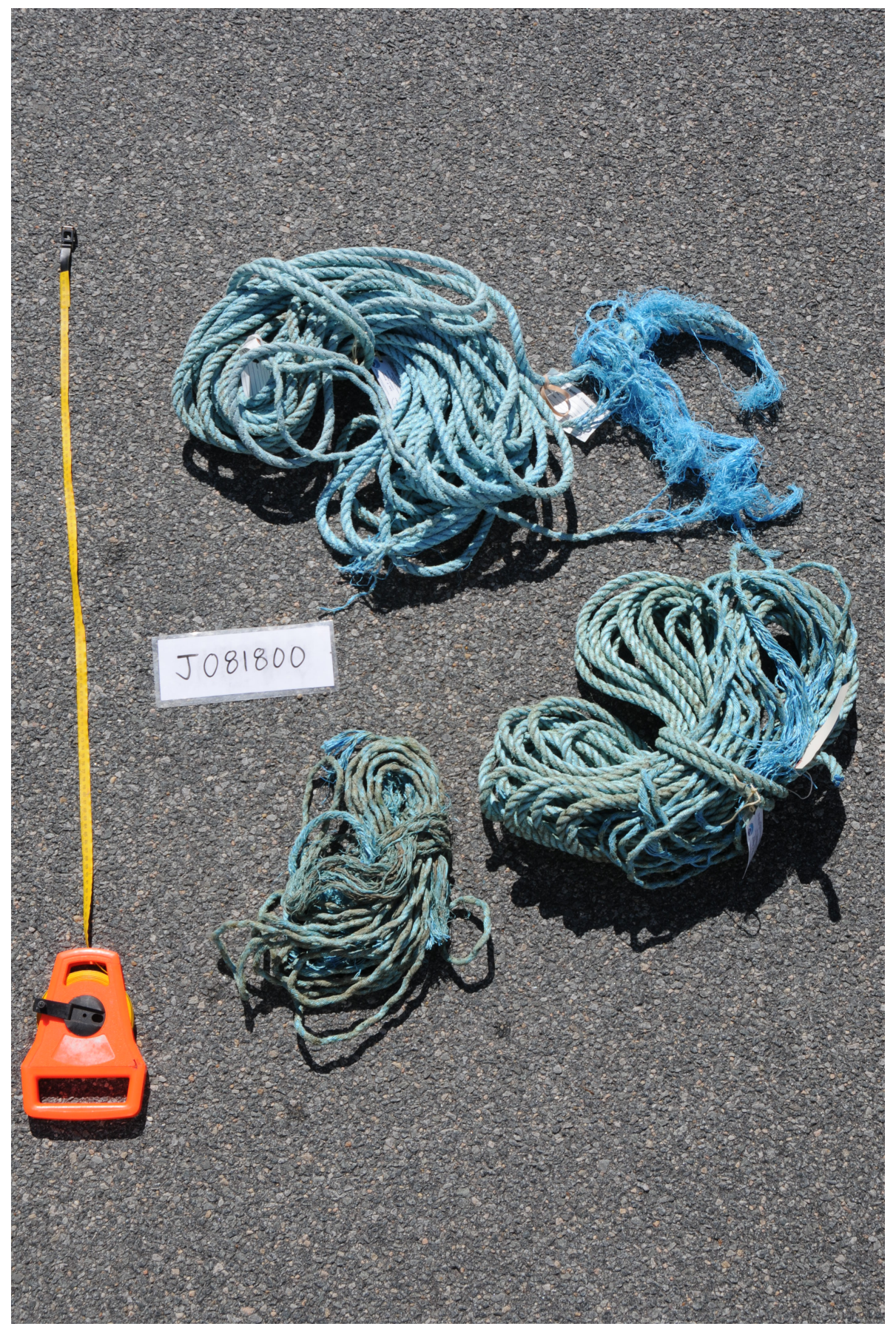




\section{B.3 Supplemental Figures}
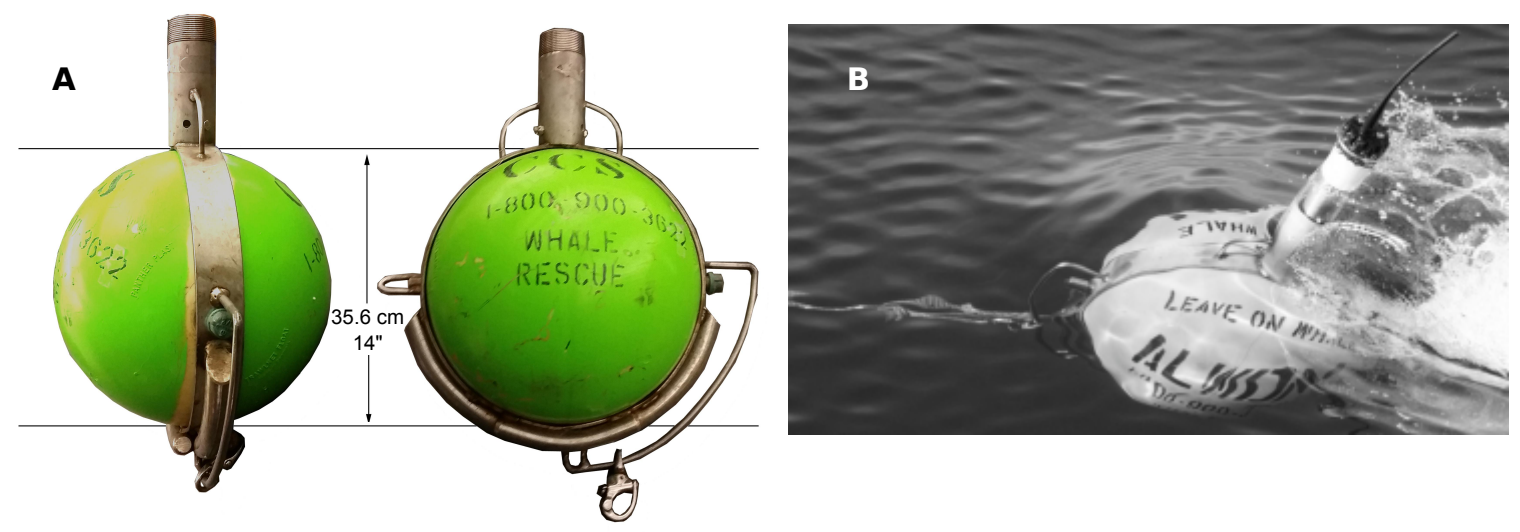

Figure B.1: Dimensions (A) and in situ photograph (B) of the satellite telemetry buoy used for tracking entangled whales for later disentanglement attempts.
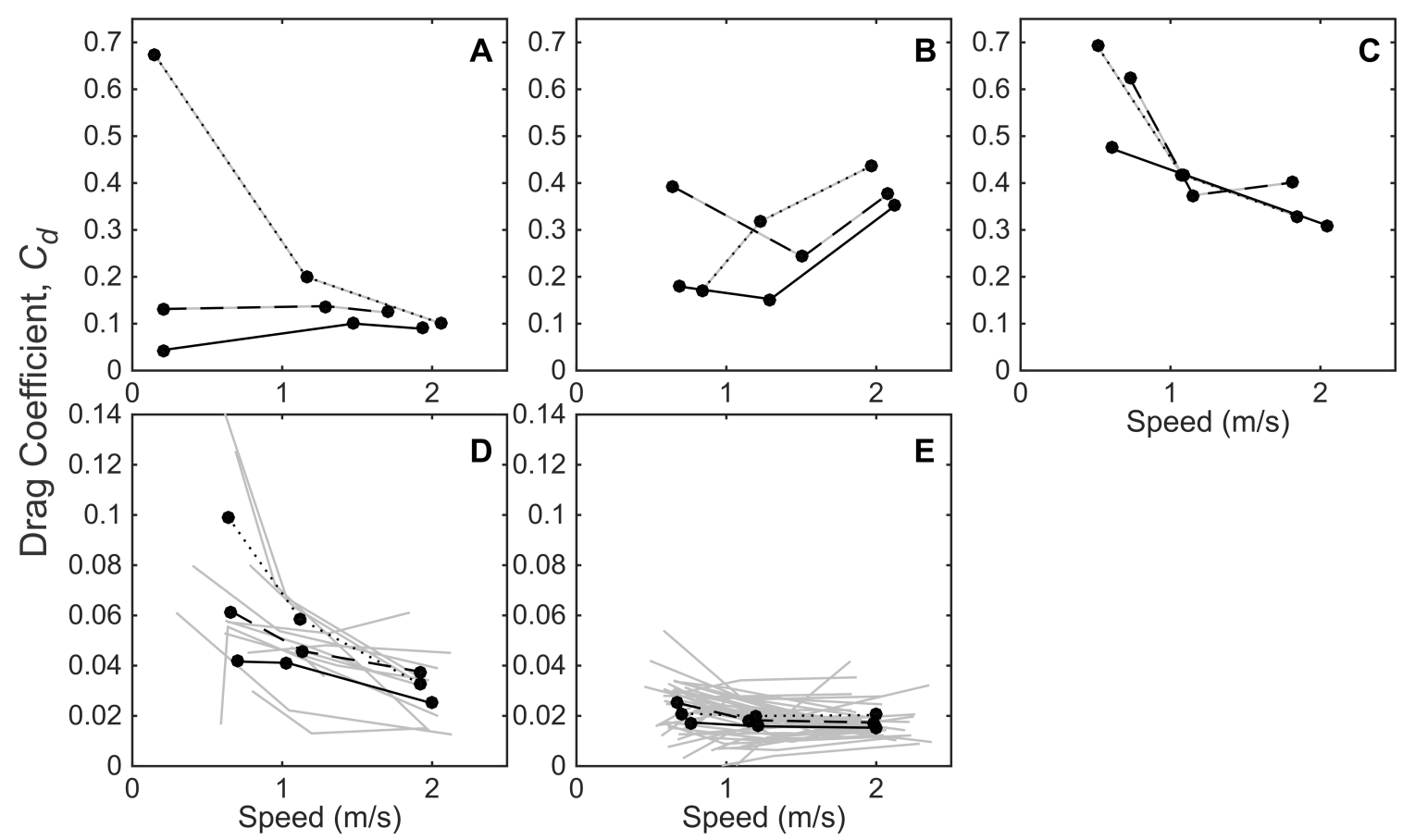

Figure B.2: Groups of gear have similar drag coefficients and responses to depth and speed. Average drag coefficients (Cd) of five clusters (A through E; by hierarchical clustering) of 21 sets of fishing gear removed from or similar to those entangling North Atlantic right whales across speeds, at depths of $0 \mathrm{~m}$ (solid black), $3 \mathrm{~m}$ (dashed black), and $6 \mathrm{~m}$ (dotted black). Grey lines illustrate estimated drag coefficients for all gear within that cluster, from which averages are calculated. Note the difference in the $\mathrm{y}$-axis limits for panels $\mathrm{A}, \mathrm{B}, \mathrm{C}$ vs. D, E. 


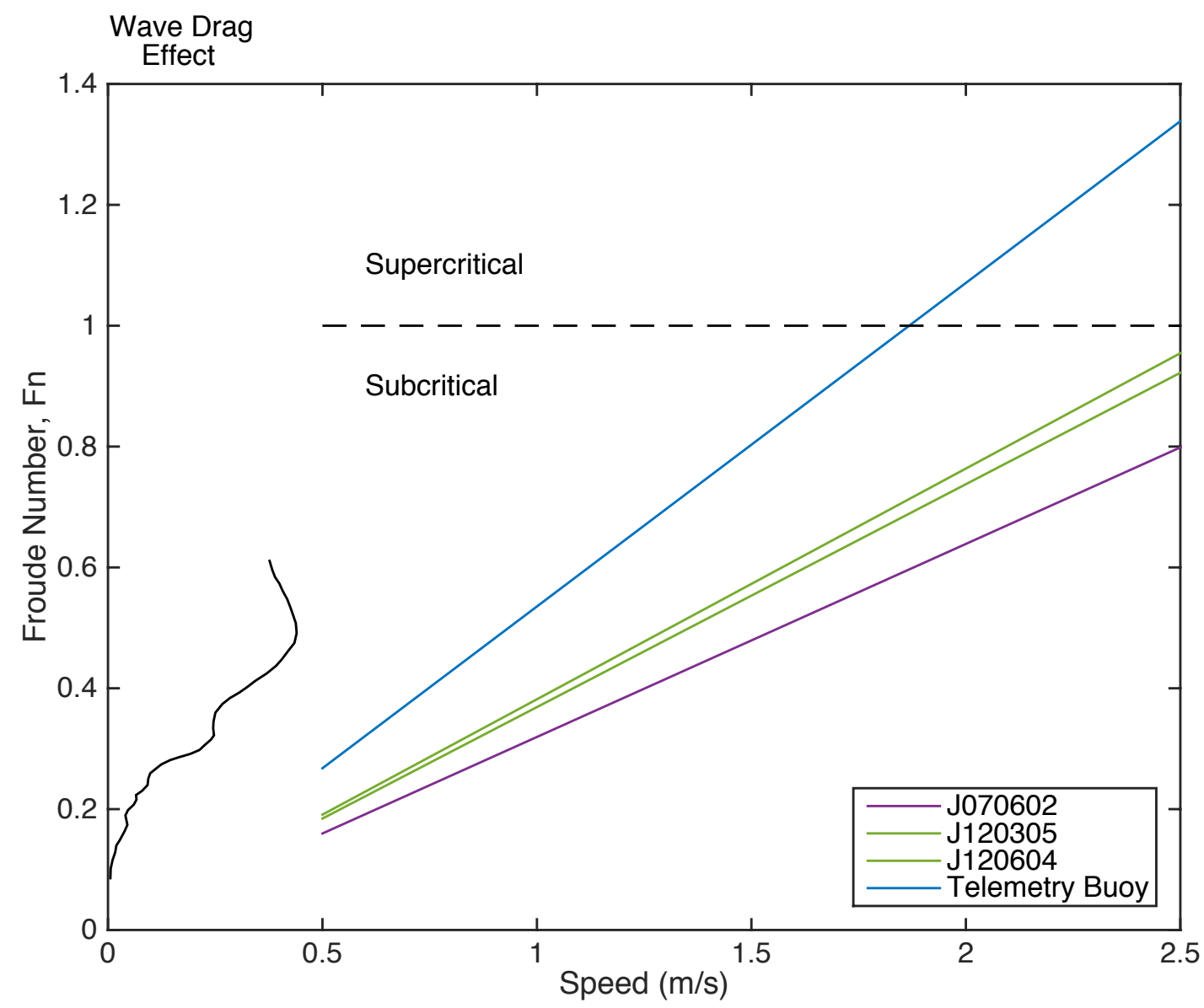

Figure B.3: Froude number $(\mathrm{Fn})$ with speed $(\mathrm{m} / \mathrm{s})$ for three sets of fishing gear removed from entangled right whales (J070602, J120305 and J120604) and the satellite telemetry buoy. Colors represent different gear clusters (see text for details). The magnitude of the wave drag effect is shown for a range of Fn (relative; see Lighthill 1978).
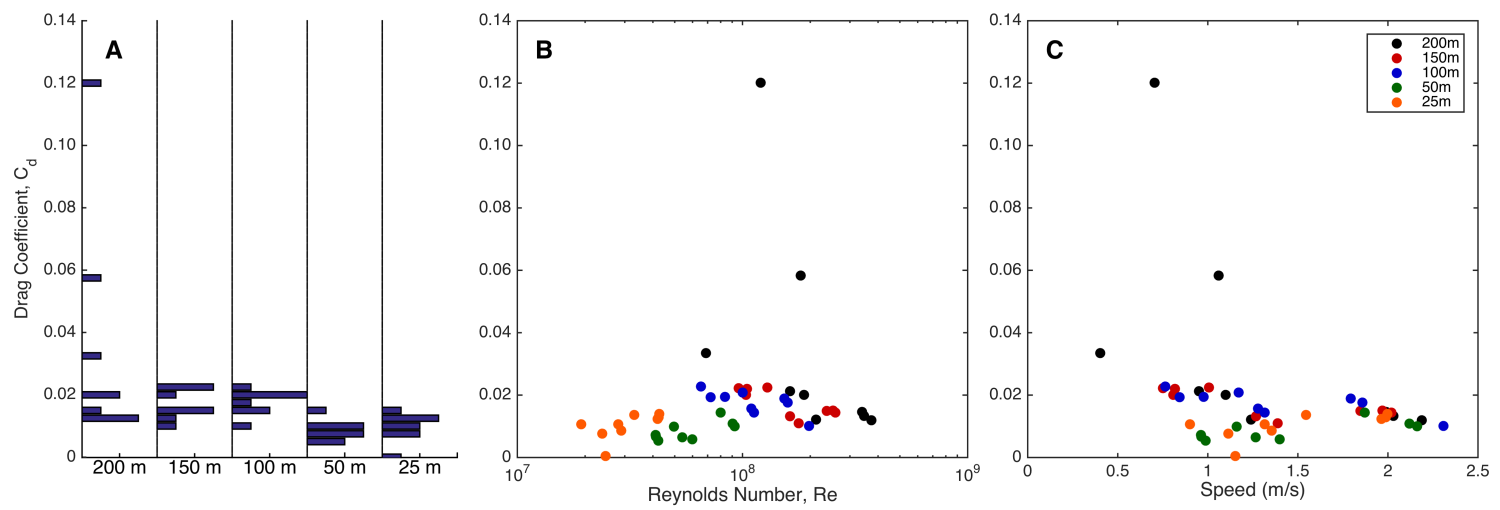

Figure B.4: Changes in drag coefficient with towed line length (A), Reynolds number (Re; B) and speed $(\mathrm{C})$. 
Appendix C

Chapter 4 Supplemental Information 


\section{C.1 Supplemental Figures}
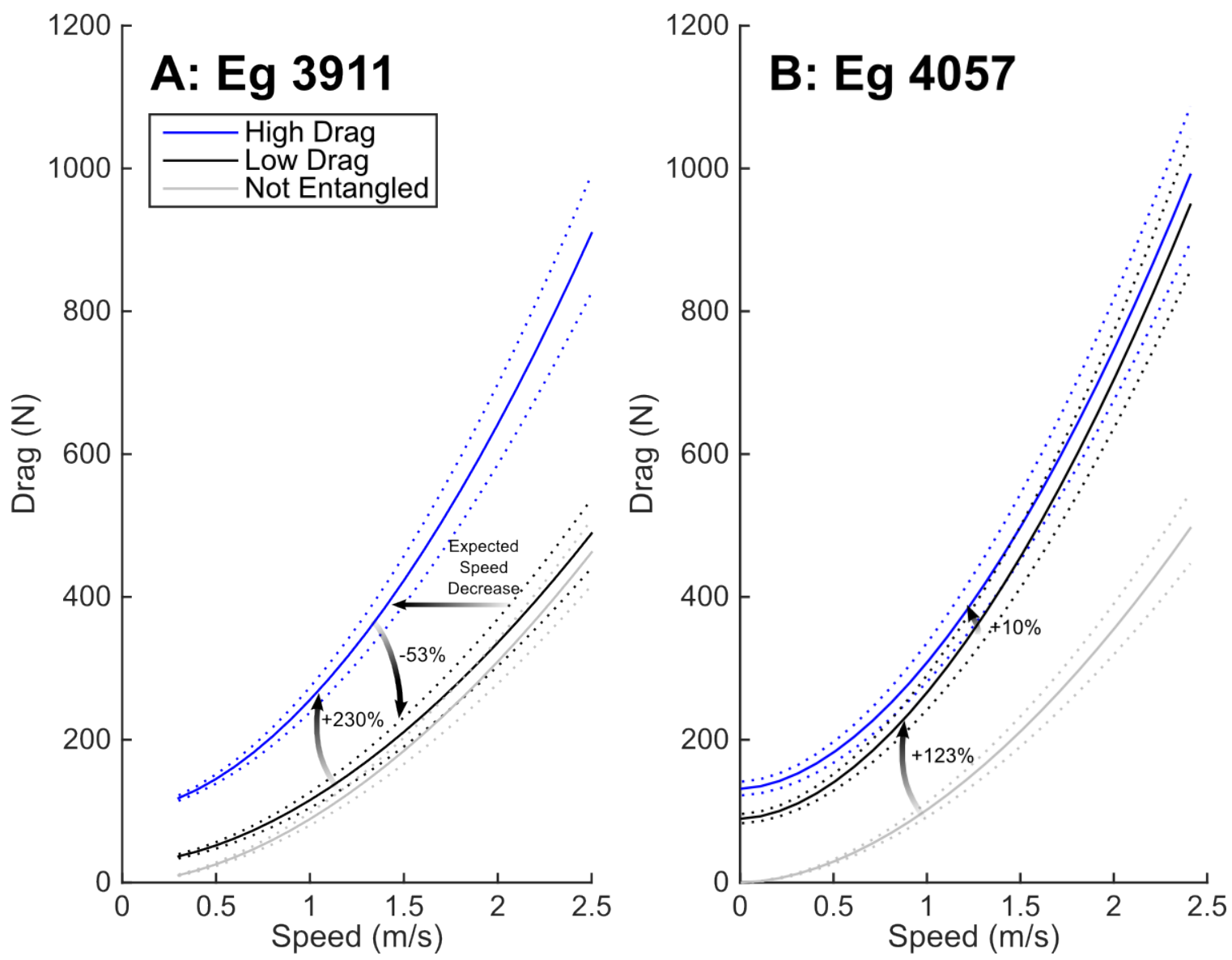

Figure C.1: Drag forces on right whales Eg 3911 (A) and 4057 (B) when not entangled (grey), entangled in minimal fishing gear (low drag; black) and entangled in the high drag condition (blue). Dotted lines represent low and high estimates based on $\pm 10 \%$ uncertainty for oscillation and gear drag estimates. Arrows and numbers indicate the amount of drag increase over non-entangled conditions, or the change in drag during the disentanglement procedure. See text for details. 

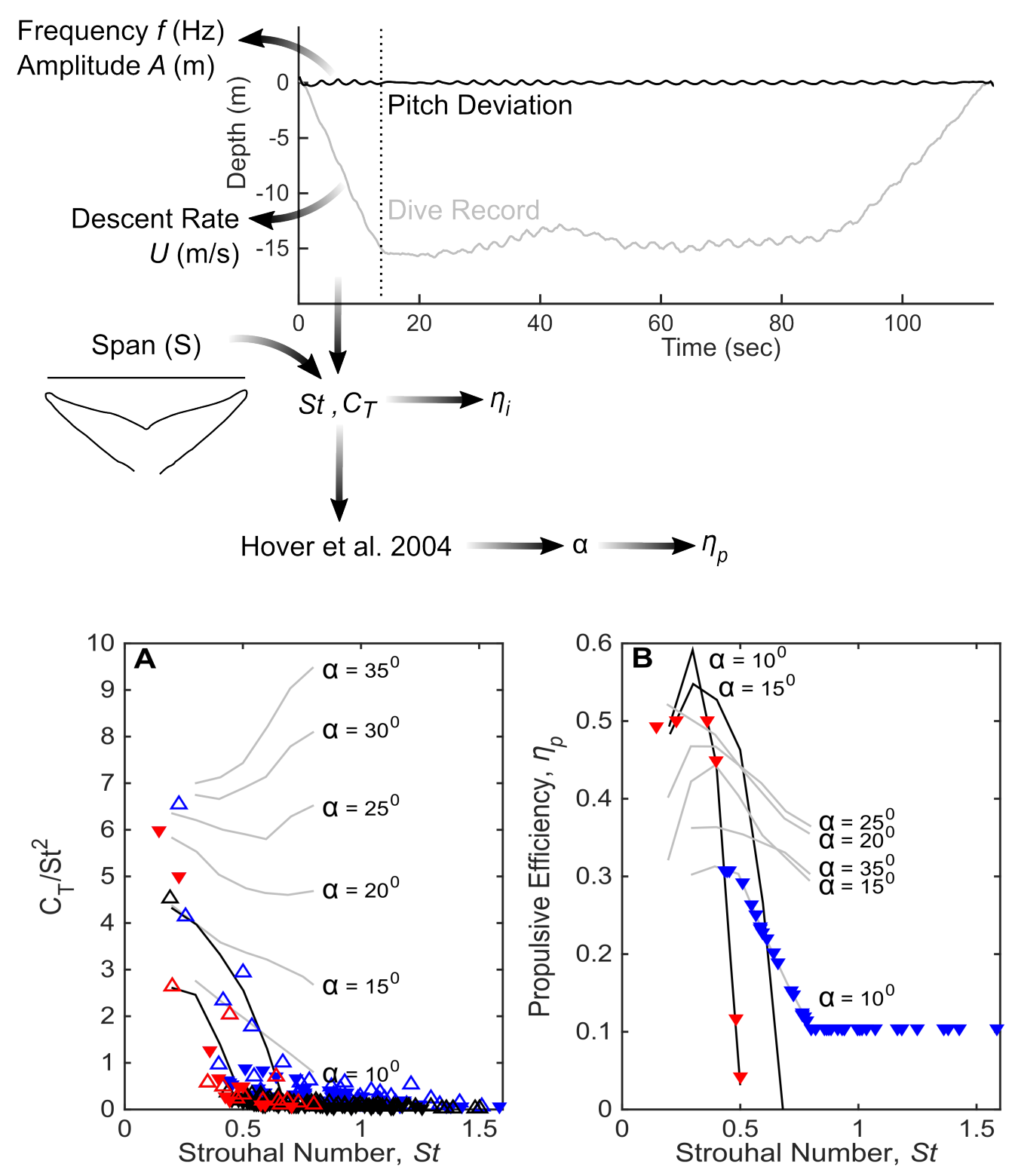

Figure C.2: Description of the estimation of propulsive efficiency from tag data and studies on flexible foils. Top schematic shows the variables obtained during the descent of a dive and whale tail fluke measurement for estimation of the thrust coefficient $\left(C_{T}\right)$ and Strouhal number $(S t)$. Ideal efficiency $\left(\eta_{i}\right)$ is estimated from $C_{T}$ (see text Eq. 4). Data from Hover et al., 2004 are used to estimate angle of attack $(\alpha)$ from contours $C_{T} / S t^{2}$ vs. $S t(\mathrm{~A})$, and propulsive efficiency $\left(\eta_{p}\right)$ is estimated for values of $S t$ along contours of $\alpha$ (B). Estimates are made for descents (inverted filled triangles) and ascents (open triangles) of entangled (blue) and disentangled (black) dives. Red triangles represent dives where $S t<0.4$, where estimates are from harmonic profiles (black). Grey contours represent sawtooth wave profiles. 

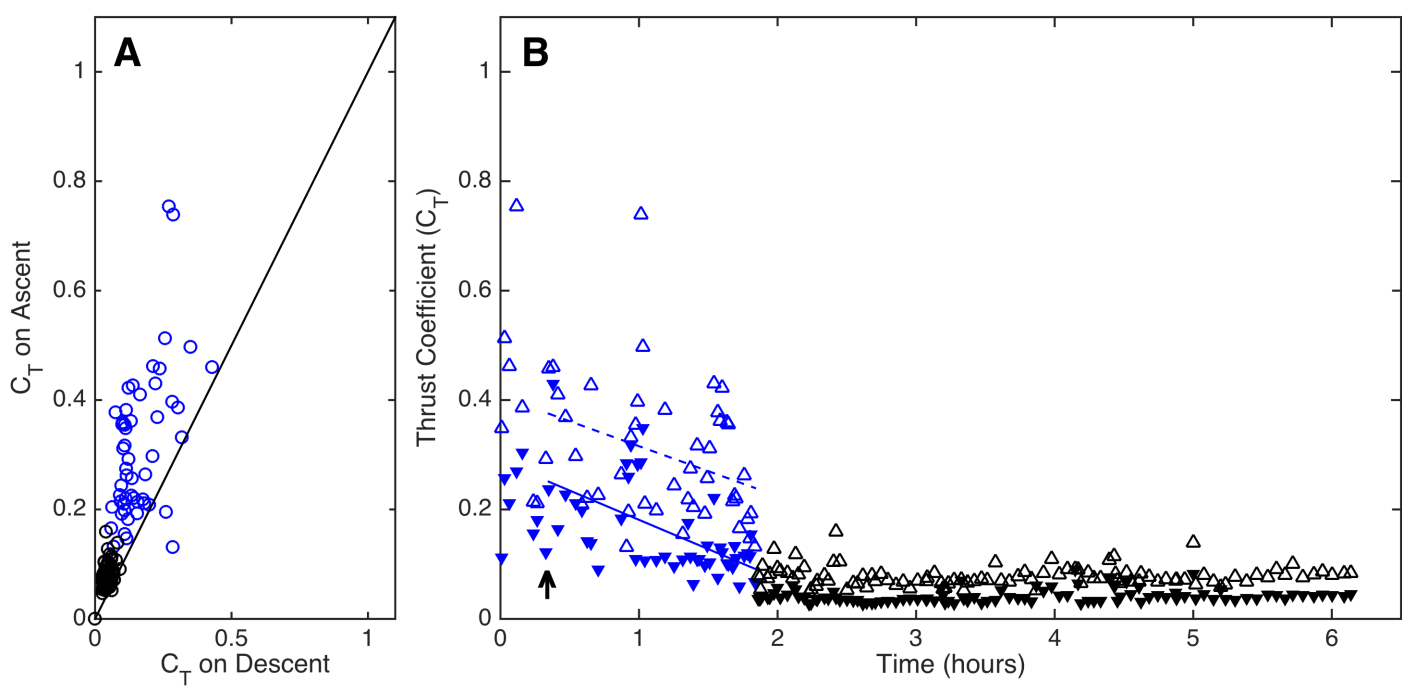

Figure C.3: A. Thrust coefficient $\left(C_{T}\right)$ on the descent vs. ascent of each dive of right whale Eg 3911 in low (black) and high (blue) drag conditions. B. Thrust coefficient on dive descents (filled, inverted triangles) and ascents (open triangles) through time during the tag deployment. Blue lines represent linear model fit for descent (solid) and ascent (dashed) following drug injection (time denoted by arrow). 
Appendix D

Chapter 5 Supplemental Information 


\section{D.1 Supplemental Figures}
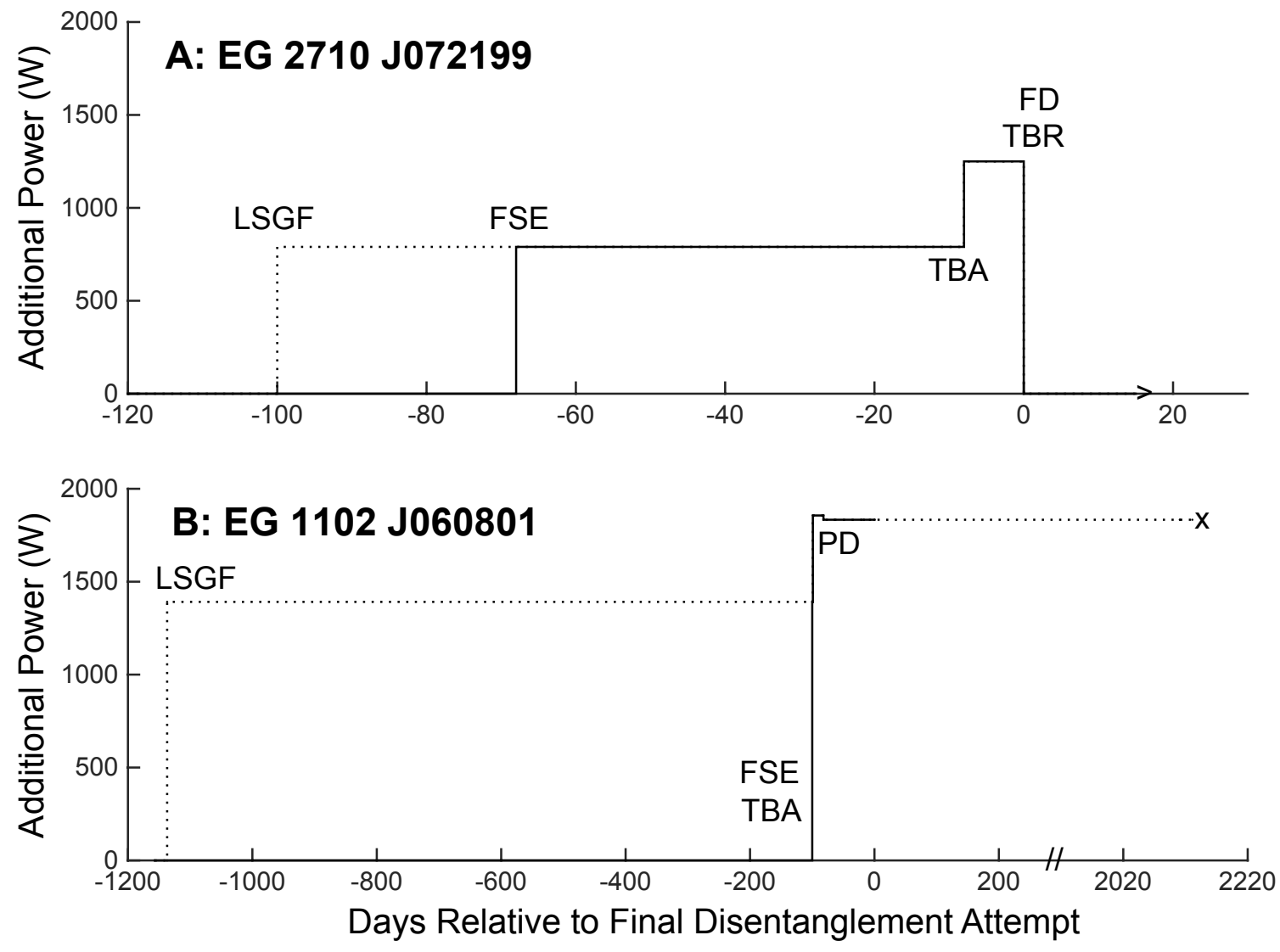

Figure D.1: Time course of disentanglement for cases EG 2710/J072199 (A) and EG 1102/J060801 (B), where zero represents the day of the final disentanglement attempt. LSGF = Last Seen GearFree, FSE = First Seen Entangled, TBA = telemetry buoy added; TBR = telemetry buoy removed; $\mathrm{PD}=$ partial disentanglement $\mathrm{FD}=$ full disentanglement; $\mathrm{x}=$ death . 
Appendix E

Chapter 6 SupPlemental Information 


\section{E.1 Wetted areas of floats or traps on unmeasured gear sets.}

Whale Eg 1427, Gear J071212: $46 \mathrm{~cm}$ diameter buoy

Buoy $A_{w}=\frac{\pi(0.46 \mathrm{~m})^{2}}{2}=0.33 \mathrm{~m}^{2}$

Whale Eg 1971, Gear J062497: 43 cm diameter buoy (A3 Polyform)

Buoy $A_{w}=\frac{\pi(0.43 \mathrm{~m})^{2}}{2}=0.29 \mathrm{~m}^{2}$

Whale Eg 2427, Gear J072001: $43 \mathrm{~cm}$ diameter buoy (A3 Polyform)

Buoy $A_{w}=\frac{\pi(0.43 \mathrm{~m})^{2}}{2}=0.29 \mathrm{~m}^{2}$

Whale Eg 2753, Gear J060599: LD-3, $34.3 \mathrm{~cm} \times 73.7 \mathrm{~cm}$ Scan float

Buoy $A_{w}$, assuming the buoy is a half ellipsoid with $a=0.3685 \mathrm{~m} ; b=c=0.1715 \mathrm{~m}$ using Knud Thomsen's formula, where $p=1.6075,=$

$$
S A \simeq \frac{4 \pi\left(\frac{a^{p} b^{p}+a^{p} c^{p}+b^{p} c^{p}}{3}\right)^{1 / p}}{2}=0.336 \mathrm{~m}^{2}
$$

Whale Eg 3120, Gear J040702: $13 \times 28 \mathrm{~cm}$ lobster buoy

Buoy $A_{w}$, assuming the buoy is a half ellipsoid with $a=0.14 \mathrm{~m} ; b=c=0.064 \mathrm{~m}$, as above $=0.0475 \mathrm{~m}^{2}$

Whale Eg 3392, Gear J070903: $0.91 \times 0.61 \times 0.30 \mathrm{~m}$ lobster trap and $15 \times 33 \mathrm{~cm}$ lobster buoy

Buoy $A_{w}$, assuming the buoy is a half ellipsoid with $a=0.17 \mathrm{~m} ; b=c=0.075 \mathrm{~m}$, as above $=$ $0.0656 \mathrm{~m}^{2}$ Trap $A_{w}$, assuming $0.038 \mathrm{~m}$ (1.5") mesh size and $0.0025 \mathrm{~m}\left(1 / 10^{\prime \prime}\right)$ wire diameter: The trap consists of six panels, two each of (a) $0.91 \times 0.61 \mathrm{~m}$, (b) $0.61 \times 0.30 \mathrm{~m}$, and (c) $0.91 \times 0.30 \mathrm{~m}$. The mesh area $\left(A_{M}\right)$ of each panel $(\mathrm{a}, \mathrm{b}, \mathrm{c})$ was calculated as:

$$
A_{M}=N \times K \times 2 M \times W,
$$

where $N$ is the number of wire columns, $K$ is number of wire rows, $M$ is the mesh size and $W$ the wire diameter. The number of columns and rows is determined by the size of the panel divided by the mesh size (Fridman and Dvernik 1973, Reid 1977).

Trap $A_{w} \simeq 2((24 \times 16)+(16 \times 8)+(24 \times 8)) \times 2 \times 0.038 \mathrm{~m} \times 0.0025 \mathrm{~m}=0.268 \mathrm{~m}^{2}$

Total $A_{w}=0.0656+0.268=0.336 \mathrm{~m}^{2}$.

This whale also had heavy gauge $\left(\sim 5 / 16^{\prime \prime}\right.$ dia) monofilament line wrapped around the peduncle, likely as a result of a separate and previous interaction. Drag on this gear was not estimated as no measurements were available. Drag estimates for this case are therefore an underestimate. 
Whale Eg 3821, Gear J092609: $1.06 \times 0.61 \times 0.30 \mathrm{~m}$ lobster trap

Trap $A_{w}$, assuming $0.038 \mathrm{~m}\left(1.5^{\prime \prime}\right)$ mesh size and $0.0025 \mathrm{~m} \mathrm{(1/10")}$ wire diameter: The trap consists of six panels, two each of (a) $1.06 \times 0.61 \mathrm{~m}$, (b) $0.61 \times 0.30 \mathrm{~m}$, and (c) $1.06 \times 0.30$ m. The mesh area $\left(A_{M}\right)$ of each panel $(\mathrm{a}, \mathrm{b}, \mathrm{c})$ was calculated as:

$$
A_{M}=N \times K \times 2 M \times W,
$$

where $N$ is the number of wire columns, $K$ is number of wire rows, $M$ is the mesh size and $W$ the wire diameter. The number of columns and rows is determined by the size of the panel divided by the mesh size (Fridman and Dvernik 1973, Reid 1977).

Trap $A_{w} \simeq 2((28 \times 16)+(16 \times 8)+(28 \times 8)) \times 2 \times 0.038 \mathrm{~m} \times 0.0025 \mathrm{~m}=0.304 \mathrm{~m}^{2}$

\section{E.2 Guide for calculating minimum entanglement duration for Serious Injury (SI) determinations}

\section{E.2.1 Motivation}

The known or presumed duration of an entanglement should be considered as a criterion in Serious Injury (SI) determinations. We suggest that this duration be compared to the critical entanglement duration, the time it takes for additional energy expenditure $\left(W_{a}, \mathrm{~J}\right)$ to reach the threshold lethal energy expenditure level $\left(8.57 \times 10^{9} \mathrm{~J}\right)$ in van der Hoop et al. (Chapter 5). A MATLAB function (CriticalDuration.m) calls upon multiple functions and scripts developed for and from the equations in this paper and provides a simplified method to assist SI determinations.

\section{E.2.2 Procedure}

A graphical representation of the MATLAB function CriticalDuration.m is provided below. The minimum inputs to the function are the whale's length (whaleLength; $\mathrm{m}$ ) or age (whaleAge; years) and the length of the entangling gear (gearLength; m) and presence or absence of floats (float; binary 0 or 1 ). Additional information on the dimensions of the floats, the diameter of the line, and the attachment points of gear on the whale will help refine the estimate of theoretical drag $\left(D_{\text {theor }} ; \mathrm{N}\right)$ which is then corrected to units comparable to those measured for this and previous studies (van der Hoop et al., 2016). The whale and gear drag are combined to estimate the additional work $\left(W_{a} ; \mathrm{J}\right)$ required for the entanglement, from which the critical duration is determined. We suggest that a criterion for SI be if the critical duration exceeds the known entanglement duration. 
[critDur] = CriticalDuration(whaleLength, whaleAge, gearLength, float,gearDiam, attachment)

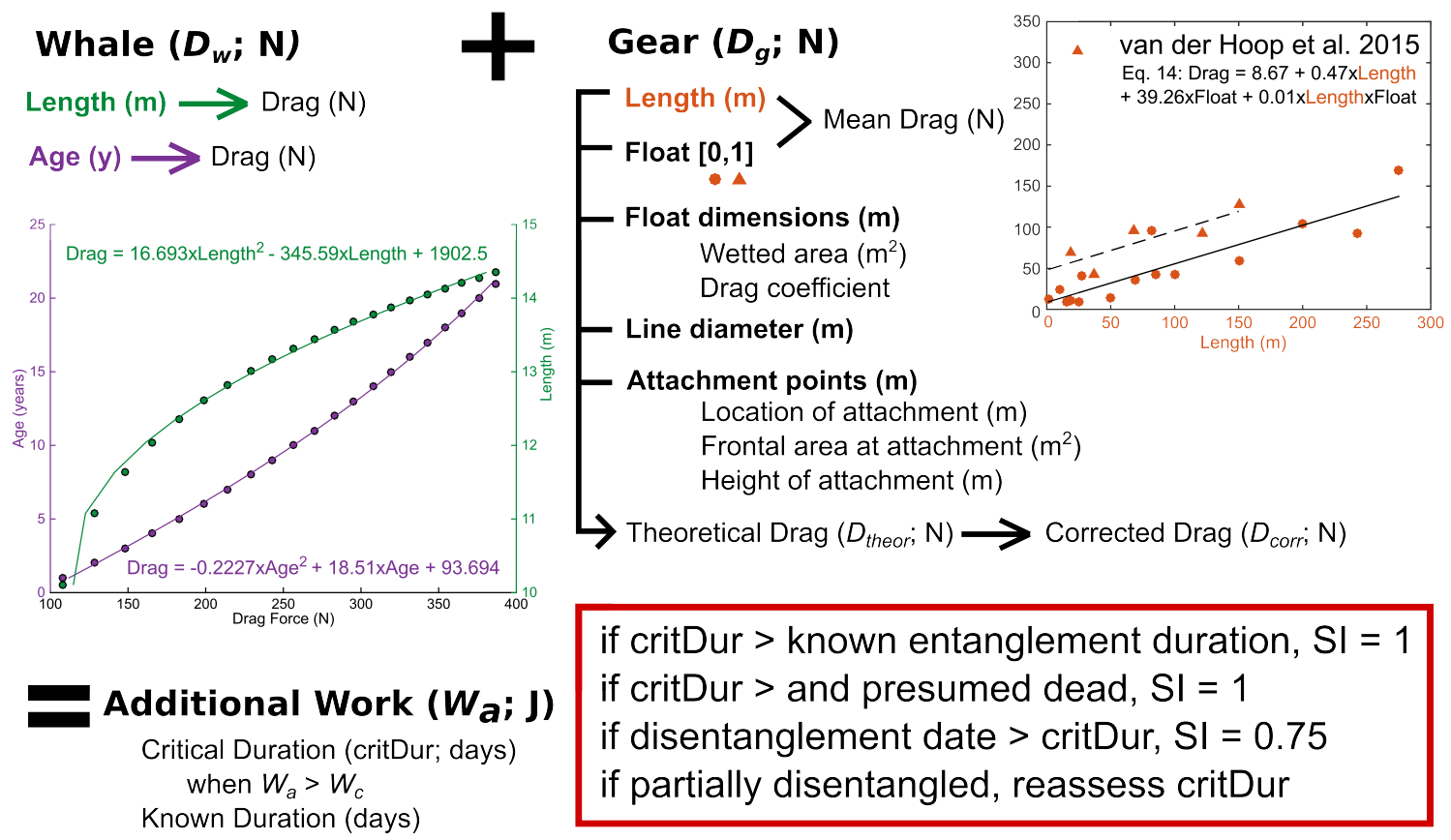

Figure E.1: Critical Duration and Serious Injury evaluation flow chart

\section{E.3 Changes in whale drag with body dimensions}

\section{E.3.1 Motivation}

Morphology and morphometry differ among marine mammal families, likely related to specialization for foraging modes and ecological niche. Even closely related species (e.g., balaenopterids) show surprising morphometric differences that can affect their swimming performance and hydrodynamics (Woodward et al., 2006).

Within a species, considerable morphometric variation exists. Over the lifespan of a right whale (up to 70 years; Fortune et al., 2012), body length increases by $10 \mathrm{~m}$, and girths by $1.6 \mathrm{~m}$ (Fortune et al., 2012). Other natural life events can alter body shape significantly: pregnant right whales increase $4-25 \mathrm{~cm}$ in width in various positions along the body, lactating southern right whales (Eubalaena australis) can lose $21.8 \pm 6.1 \mathrm{~cm}$ in 3-4 months (Miller et al., 2012) and migration can lead to significant reductions in body width (Perryman and Lynn, 2002) and weight (11-29\%; Rice and Wolman, 1971) in gray whales (Eschrichtius robustus). Unnatural, though extremely common in the right whale population (Knowlton et al., 2012), chronic entanglement in fishing gear can reduce body diameter by $20 \%$ compared to mesomorphic right whales (van der Hoop et al., 2013b) and can reduce body weight by $28 \%$ (Barratclough et al., 2014).

How do these changes affect hydrodynamics and drag forces on a whale's body? 


\section{E.3.2 Methods}

The theoretical rigid-body drag force $\left(F_{D} ; \mathrm{N}\right)$ was calculated based on a turbulent spindle model (Webb, 1975),

$$
F=\frac{1}{2} \rho U^{2} A_{w} C_{d}
$$

where $\rho$ is seawater density $\left(1025 \mathrm{~kg} / \mathrm{m}^{3}\right), U$ is swimming speed $(\mathrm{m} / \mathrm{s})$, and $A_{w}$ is the total wetted surface area $\left(\mathrm{m}^{2}\right)$ calculated from body weight $W(\mathrm{~kg})$ as $A_{w}=0.08 W^{0.65}$ (Fish, 1993). $C_{d}$ is the drag coefficient, calculated as

$$
C_{d}=C_{f}\left[1+1.5\left(\frac{d_{\max }}{l}\right)^{3 / 2}+7\left(\frac{d_{\max }}{l}\right)^{3}\right]
$$

where $C_{f}$ is the frictional drag component computed from the Reynolds number $(R e)$,

$$
C_{f}=0.072\left(R e^{-1 / 5}\right)
$$

and $d_{\max }$ and $l$ are the maximum body diameter $(\mathrm{m})$ and total body length $(\mathrm{m})$ of a right whale.

To determine how total body drag, drag coefficient, and fineness ratio $\left(F R ; l / d_{\max }\right)$ change with body dimensions, $d_{\max }, l$, and $W$ were varied to reflect the morphometrics of (1) four right whales of varying ages, lengths, and body conditions (Table A1), (2) a mesomorphic right whale from age 2 to 28 with body length- and width-at-age from Moore et al. 2004 and Fortune et al. 2012, and (3) right whale Eg 1223 (Table A1) with constant body length but with reductions of $28 \%$ in body weight (Barratclough et al., 2014) and 20\% in maximum body diameter (van der Hoop et al., 2013b; Figure 2C).

The percent change in drag coefficient, total body drag force, and fineness ratio were calculated between the minimum and maximum values $(1)$ of the four whales or $(2,3)$ of the range obtained from varying different body dimensions.

\section{E.3.3 Results}

Drag increases with body size (Figure A1A), where at $1.2 \mathrm{~m} / \mathrm{s}$ (95\% CI swimming speed; Baumgartner and Mate, 2005) drag ranges from $34-327$ N, a $162 \%$ difference. Drag coeffi-

cients range $2.9 \times 10^{-3}$ to $3.6 \times 10^{-3}$, differing no more than $20 \%$, while fineness ratios vary by $24 \%$, from 4.55 to 5.73 .

During an individual's growth from age 2 to 28 , the total drag forces increase by $72 \%$, 
Table E-1: Individual ID, age, measured length $(\mathrm{m})$, weight $(\mathrm{Kg})$, diameter $(\mathrm{m})$, and notes on the body condition or cause of death of four North Atlantic right whales. $\mathrm{E}=$ estimated, $\mathrm{W}=$ weighed, $\mathrm{S}=$ sum of weight of parts.

\begin{tabular}{|l|l|l|l|l|l|}
\hline Whale ID & Age & Length $(\mathrm{m})$ & Diameter $(\mathrm{m})$ & Weight $(\mathrm{Kg})$ & Notes \\
\hline Eg 3911 & 2 & 10 & 2.20 & $7000(\mathrm{E})$ & Entangled, emaciated \\
\hline MH89-424-Eg & $<1$ & 4.12 & 0.719 & $1227(\mathrm{~W})$ & Perinatal \\
\hline Eg 1014 & 28 & 13.7 & 2.96 & $52640(\mathrm{~S})$ & Vessel Collision \\
\hline Eg 1223 & 12 & 13.6 & 2.93 & $32670(\mathrm{~W})$ & Vessel Collison \\
\hline
\end{tabular}
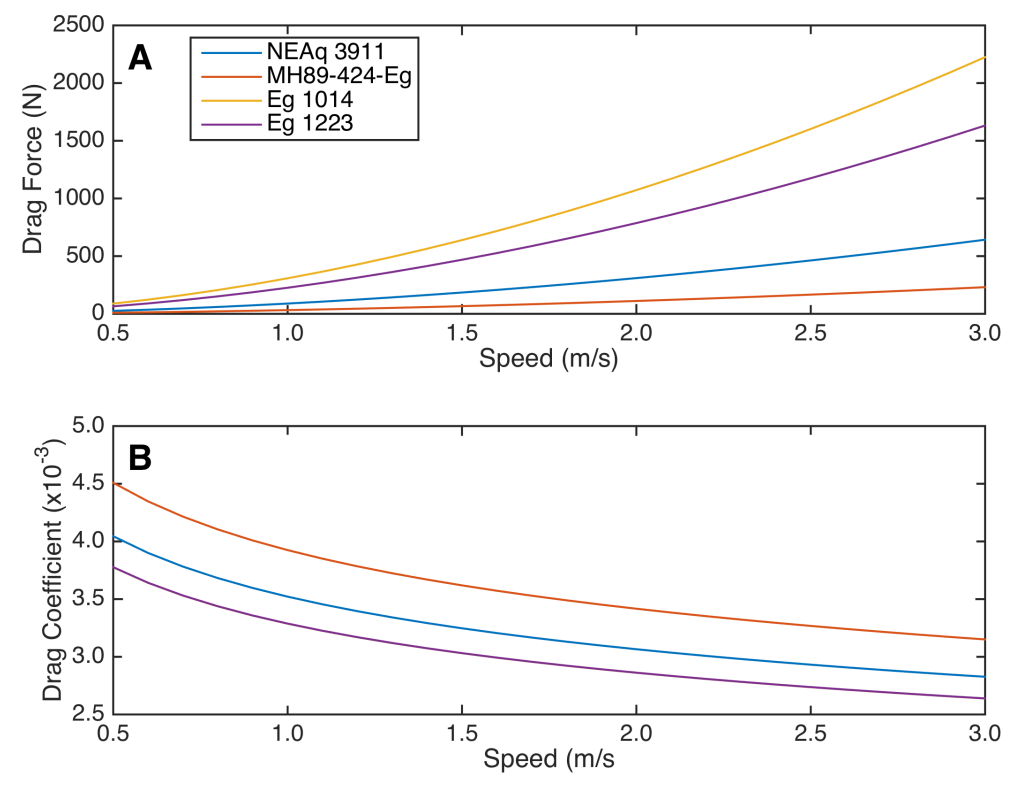

Figure E.2: Drag forces (A) and coefficients (B) of four North Atlantic right whales of different body dimensions across modeled speeds.

from 123 to $437 \mathrm{~N}$ at $1.2 \mathrm{~m} / \mathrm{s}$ (Figure A2A). The drag coefficient decreases $7.1 \%$ (Figure $\mathrm{A} 2 \mathrm{~B}$ ). With normal growth, the fineness ratio remains almost unchanged at 4.19 (range 4.08-4.23; a 3.7\% change; Figure A2C).

As body width and weight decrease through the course of an entanglement, an individual's total body drag force may decrease by $21.5 \%$ at $1.2 \mathrm{~m} / \mathrm{s}$, with a $6.5 \%$ reduction in drag coefficient and a $21.7 \%$ increase in $F R$. 

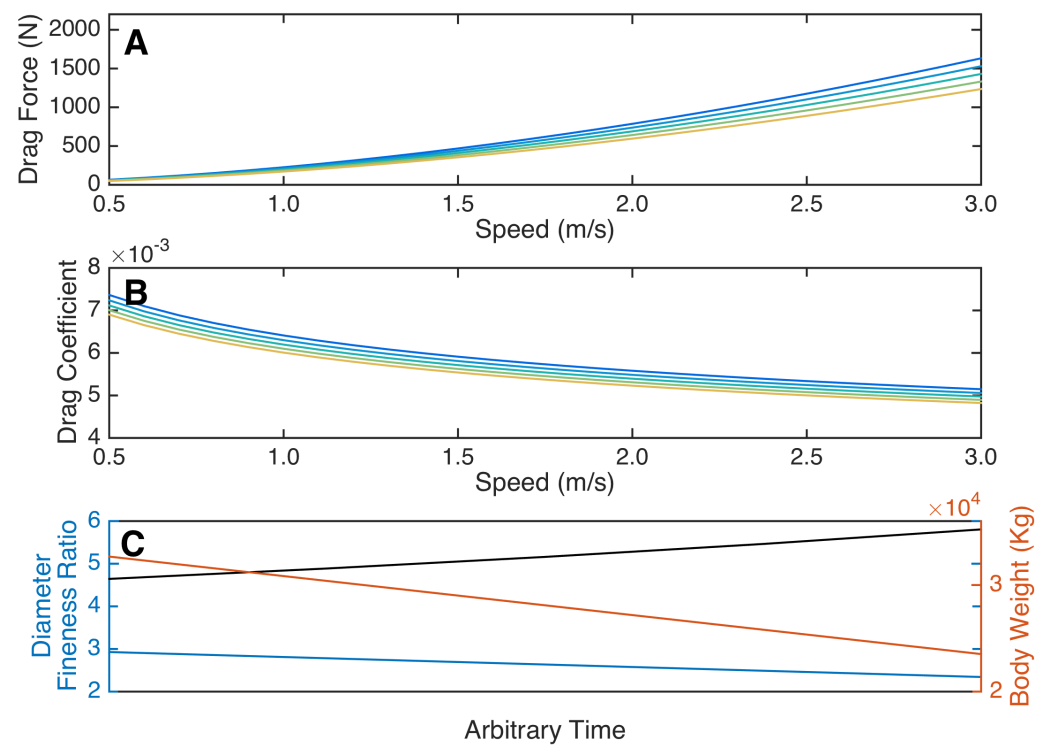

Figure E.3: Total body drag force (N; A) and drag coefficient (B) with speed modeled for a North Atlantic right whale from age 2 (darkest blue) to 28 (yellow), and modeled body diameter (blue), fineness ratio (black) and body weight (orange) with age.
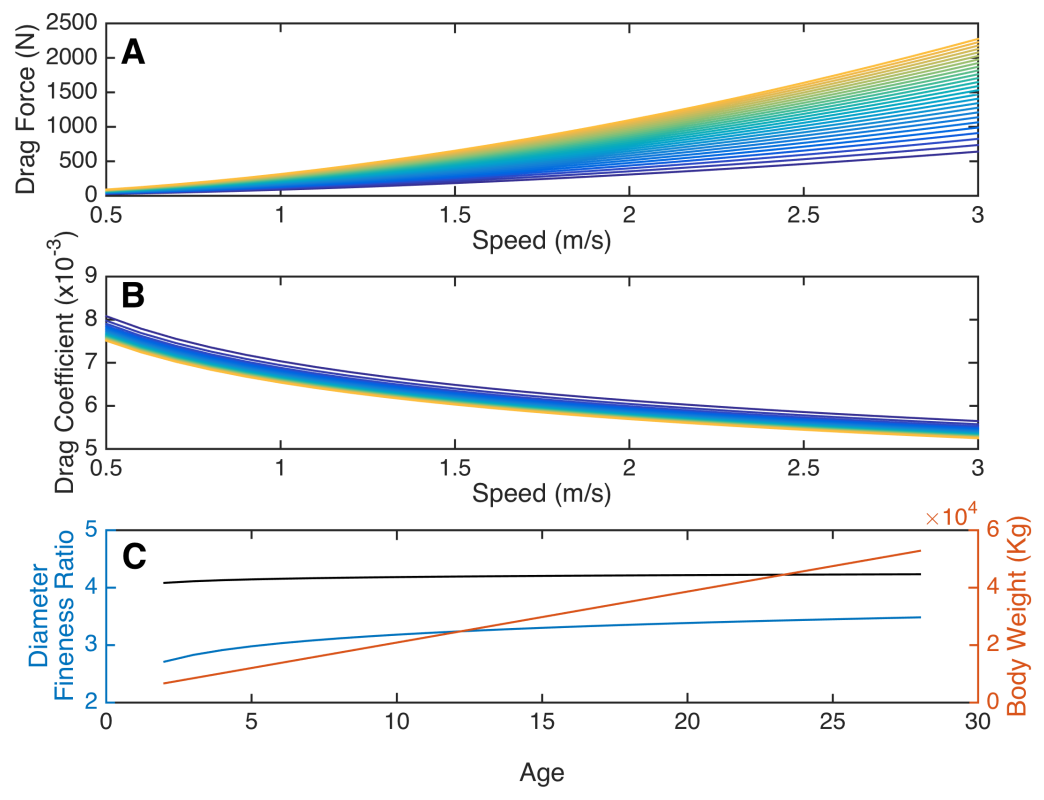

Figure E.4: Total body drag force (N; A) and drag coefficient (B) with speed modeled for a North Atlantic right whale Eg 1223 with healthy body dimensions (blue) and as it loses $20 \%$ of body width and $28 \%$ of body weight (yellow). Body diameter (C; blue) and weight (orange) are reduced through time and affect the fineness ratio (black). 
Appendix $\mathrm{F}$

ChAPTER 8 SUPPLEMENTAL INFORMATION 


\section{F.1 Supplemental Figures}

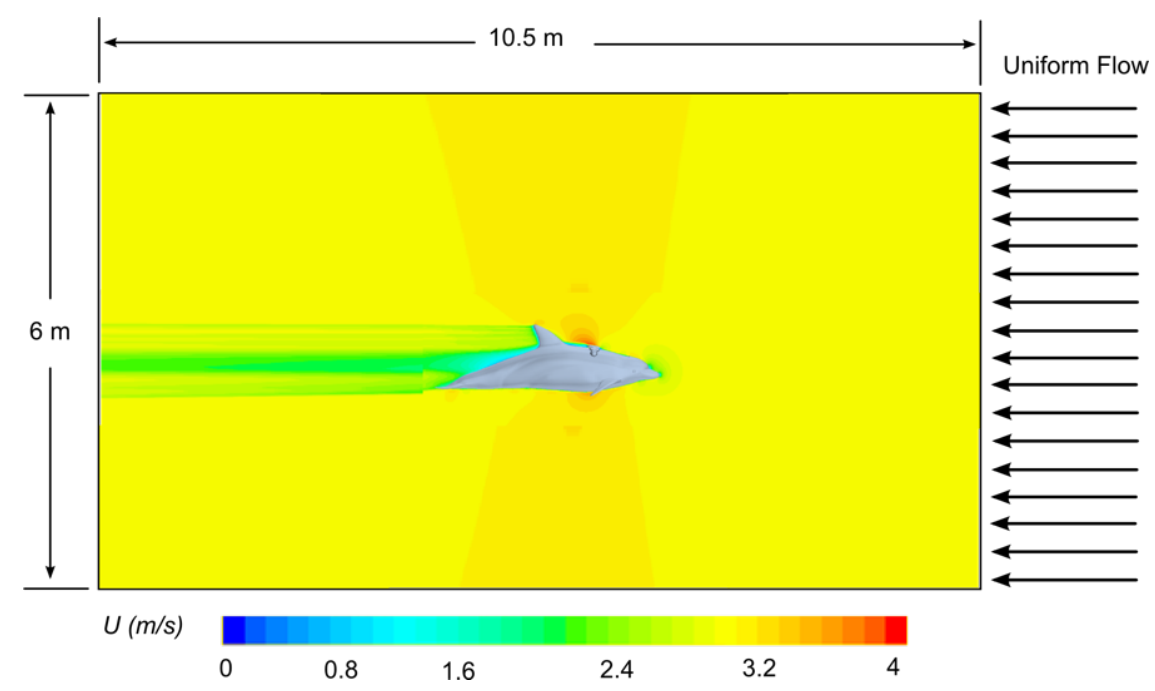

Figure F.1: Illustration of the dolphin-tag model in the computational domain used for the simulations of all tag conditions. Velocity results from a representative simulation in $3 \mathrm{~m} / \mathrm{s}$ steady state flow are included in the figure.
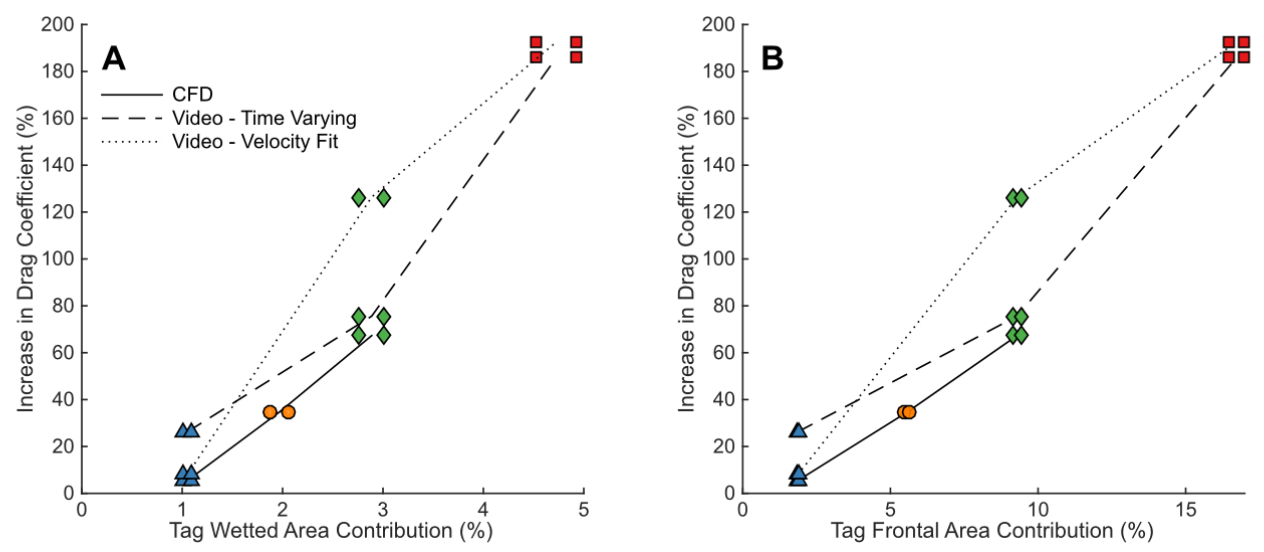

Figure F.2: Percentage increase in drag coefficient with increasing tag wetted area $(\mathrm{A})$ and frontal area (B) contribution to the tag wearer. Symbols represent the tag (blue triangles), $\operatorname{tag}+2$ (orange circles), $\operatorname{tag}+4$ (green diamonds) and tag +8 (red squares); solid lines represent data from Computational Fluid Dynamics (CFD). Dashed and dotted lines are from underwater video time-varying and velocity-fit methods, respectively. 
Appendix G

Chapter 9 Supplemental Information 


\section{G.1 Supplemental Figures}

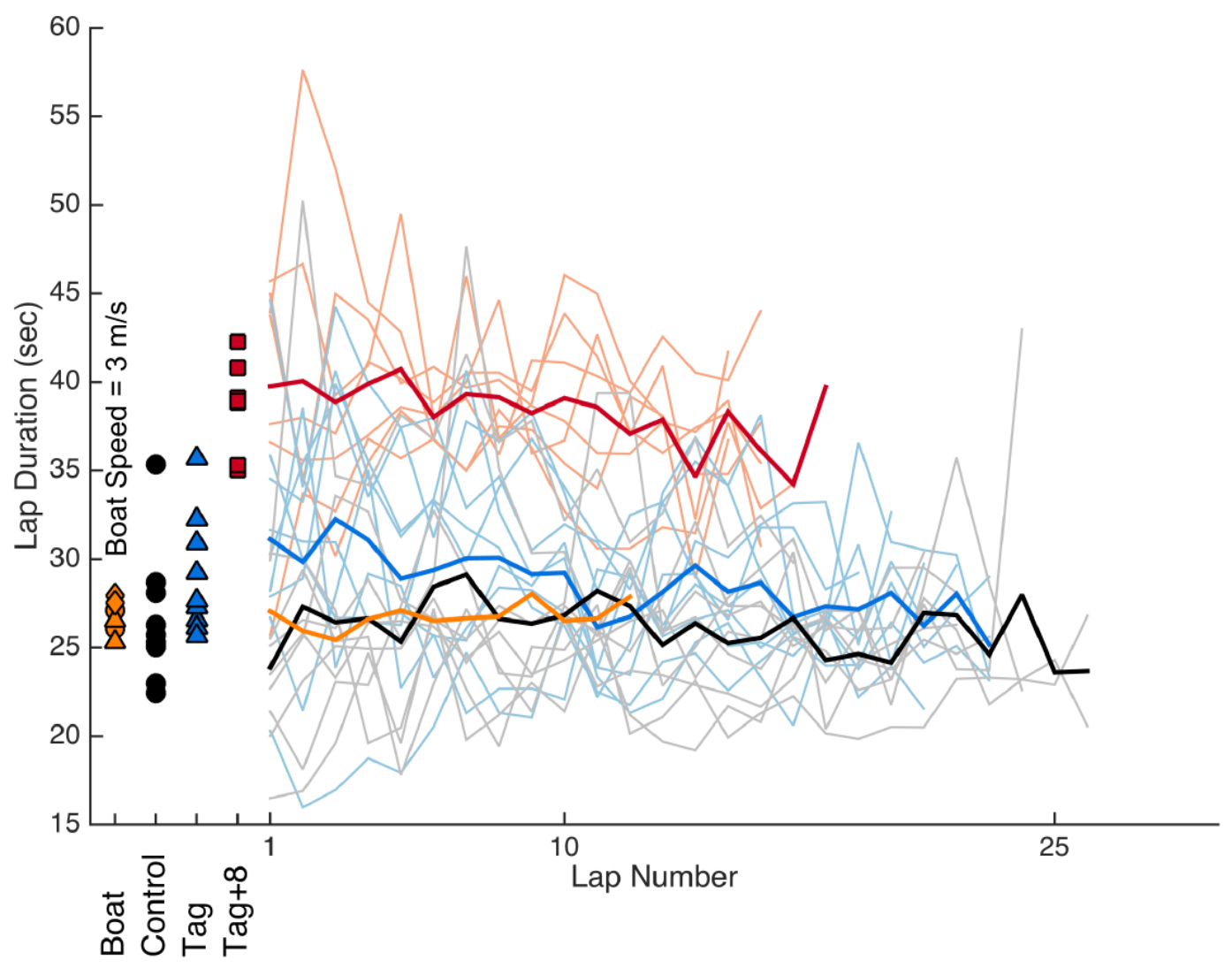

Figure G.1: Mean lap duration for all trials paced with a remote controlled boat at $3 \mathrm{~m} / \mathrm{s}$ (orange), and in control (black), tag (blue), and tag +8 (red) conditions with no prescribed swimming pace (left symbols) as well as the duration of each lap (thin lines) and per-lap averages (thick lines) for all trials. 
Appendix $\mathbf{H}$

\section{Chapter 10 Supplemental Information}




\section{H.1 Supplemental Figures}
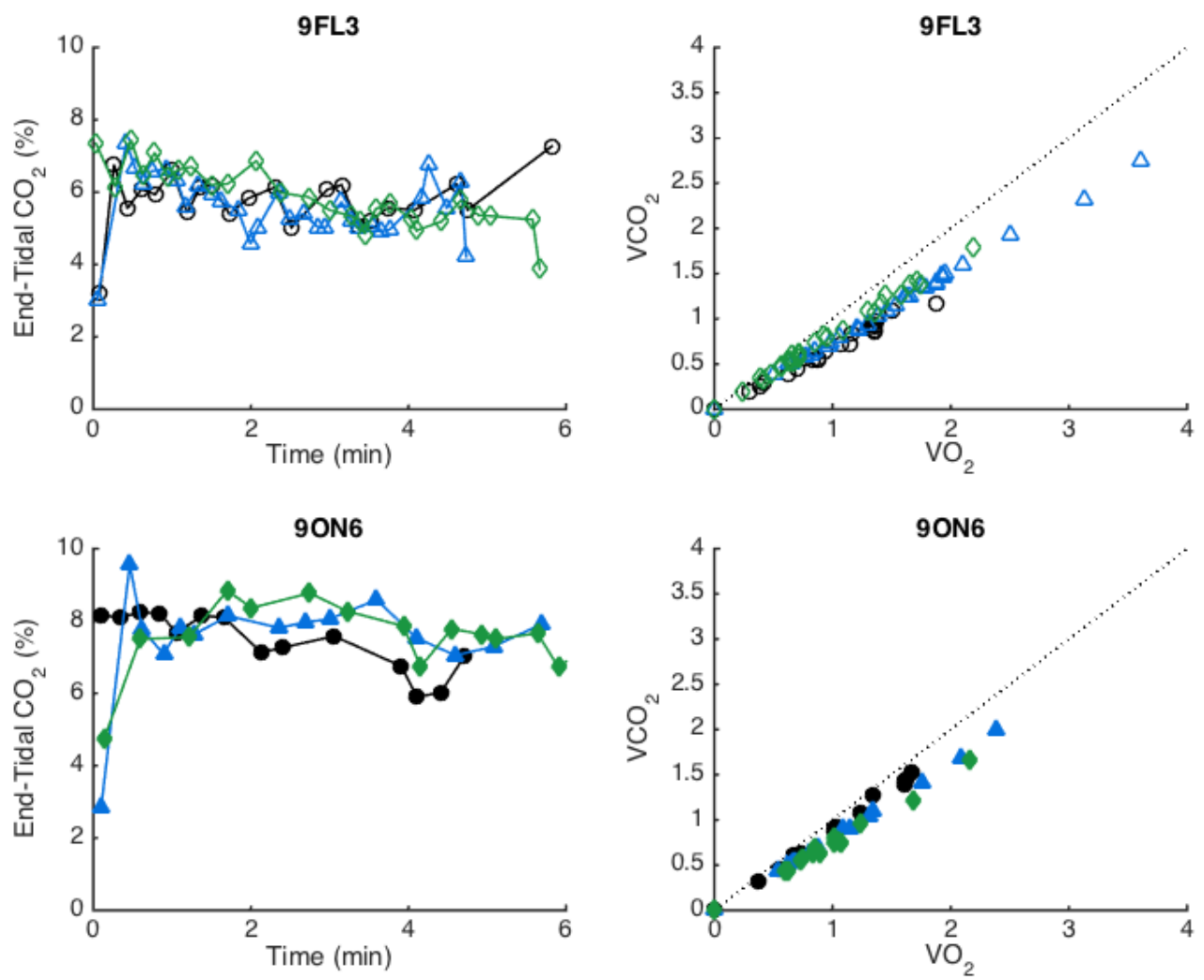

Figure H.1: End-expiratory carbon dioxide $\left(\mathrm{CO}_{2}\right)$ concentrations (left) and the relationship between instantaneous oxygen consumption $\left(\dot{V} \mathrm{O}_{2}\right)$ and carbon dioxide production $\left(\dot{V} \mathrm{CO}_{2}\right)$ for two bottlenose dolphins (ID 9FL3 top, open symbols; 9ON6 bottom, closed symbols) through the recovery period after a 5-min exercise task when not wearing a tag (black circles) and when wearing a tag (blue triangles) and the tag +4 (green diamonds). The black dotted line (right panels) is 1:1 for reference. 


\section{BIBLIOGRAPHY}

Adachi, T., Maresh, J. L., Robinson, P. W., Peterson, S. H., Costa, D. P., Naito, Y., Watanabe, Y. Y., and Takahashi, A. The foraging benefits of being fat in a highly migratory marine mammal. Proceedings of the Royal Society B-Biological Sciences, 281 (1797):2014-2020, 2014. doi: 10.1098/rspb.2014.2120.

Aguilar, A. and Borrell, A. Patterns of lipid content and stratification in the blubber of fin whales (Balaenoptera physalus). Journal of Mammalogy, 71(4):544-554, 1990. doi: $10.2307 / 1381793$.

Aguilar, A., Giménez, J., Gómez-Campos, E., Cardona, L., and Borrell, A. $\delta 15 n$ value does not reflect fasting in mysticetes. PLoS ONE, 9(3):e92288, 2014. doi: 10.1371/journal. pone.0092288.

Aguilar Soto, N., Johnson, M. P., Madsen, P. T., Diaz, F., Dominguez, I., Brito, A., and Tyack, P. Cheetahs of the deep sea: deep foraging sprints in short-finned pilot whales off Tenerife (Canary Islands). Journal of Animal Ecology, 77(5):936-947, 2008. doi: 10.1111/j.1365-2656.2008.01393.x.

Ahlborn, B. K., Blake, R. W., and Chan, K. H. S. Optimal fineness ratio for minimum drag in large whales. Canadian Journal of Zoology, 87(2):124-131, 2009. doi: 10.1139/z08-144.

Alexander, D. E. Drag coefficients of swimming animals: effects of using different reference areas. Biological Bulletin, 179(2):186-190, 1990.

Alexander, R. M. Optimization and gaits in the locomotion of vertebrates. Physiological Reviews, 69(4):1199-1227, 1989.

Alexander, R. M. Bioenergetics: One price to run, swim, or fly? Nature, 397(6721):651-653, 1999. doi: $10.1038 / 17687$.

Alexander, R. Principles of Animal Locomotion. Princeton University Press, 2003. ISBN 9780691086781.

American Society of Mammalogists. Guidelines for the capture, handling, and care of mammals as approved by the American Society of Mammalogists. Journal of Mammalogy, 79 (4):1416-1431, 1998.

Anderson, E. J., McGillis, W. R., and Grosenbaugh, M. A. The boundary layer of swimming fish. Journal of Experimental Biology, 204:81-102, 2001.

Anderson, J. M., Streitlien, K., Barrett, D. S., and Triantafyllou, M. S. Oscillating foils of high propulsive efficiency. Journal of Fluid Mechanics, 360:41-72, 1998.

Andrews, R., Pitman, R., and Ballance, L. Satellite tracking reveals distinct movement patterns for Type B and Type C killer whales in the southern Ross Sea, Antarctica. Polar Biology, 31(12):1461-1468, 2008. doi: 10.1007/s00300-008-0487-z. 
Angell, C. M., Wilson, J. Y., Moore, M. J., and Stegeman, J. J. Cytochrome P450 1A1 expression in cetacean integument: implications for detecting contaminant exposure and effects. Marine Mammal Science, 20(3):554-566, 2004.

Aoki, K., Watanabe, Y. Y., Crocker, D. E., Robinson, P. W., Biuw, M., Costa, D. P., Miyazaki, N., Fedak, M. A., and Miller, P. J. Northern elephant seals adjust gliding and stroking patterns with changes in buoyancy: validation of at-sea metrics of body density. Journal of Experimental Biology, 214(17):2973-2987, 2011. doi: 10.1242/jeb.055137.

Archie, E. A. Wound healing in the wild: stress, sociality, and energetic costs affect wound healing in natural populations. Parasite Immunology, 35:374-385, 2013. doi: 10.1111/ pim.12048.

Arthur, L. H., McLellan, W. A., Piscitelli, M. A., Rommel, S. A., Woodward, B. L., Winn, J. P., Potter, C. W., and Ann Pabst, D. Estimating maximal force output of cetaceans using axial locomotor muscle morphology. Marine Mammal Science, 31:1401-1426, 2015. doi: $10.1111 / \mathrm{mms} .12230$.

Baird, R. W. and Burkhart, S. M. Bias and variability in distance estimation on the water: implications for the management of whale watching. Proceedings of the International Whaling Commission (IWC) Meeting, Document S:10 pp., 2000.

Baldwin, K. and Pickett, T. The time tension line cutter evaluation: Fishermen pilot study and controlled experiments. Technical Report Final Report for NOAA Contract NA08NMF4270419, Durham, NH, 2009.

Balmer, B. C., Wells, R. S., Howle, L. E., Barleycorn, A. A., McLellan, W. A., Ann Pabst, D., Rowles, T. K., Schwacke, L. H., Townsend, F. I., Westgate, A. J., and Zolman, E. S. Advances in cetacean telemetry: A review of single-pin transmitter attachment techniques on small cetaceans and development of a new satellite-linked transmitter design. Marine Mammal Science, 30:656-673, 2013. doi: 10.1111/mms.12072.

Bannasch, R., Wilson, R. P., and Culik, B. Hydrodynamic aspects of design and attachment of a back-mounted device in penguins. Journal of Experimental Biology, 194:83-96, 1994.

Barbosa, T. M., Morais, J. E., Forte, P., Neiva, H., Garrido, N. D., and Marinho, D. A. A comparison of experimental and analytical procedures to measure passive drag in human swimming. PLoS One, 10(7):e0130868, 2015. doi: 10.1371/journal.pone.0130868.

Barco, S. G. and Moore, K. M. Handbook for recognizing, evaluating and documenting human interaction in stranded cetaceans and pinnipeds. Technical report, 2010. URL https://repository.library.noaa.gov/view/noaa/4429.

Barratclough, A., Jepson, P. D., Hamilton, P. K., Miller, C. A., Wilson, K., and Moore, M. J. How much does a swimming, underweight, entangled right whale (Eubalaena glacialis) weigh? Calculating the weight at sea, to facilitate accurate dosing of sedatives to enable disentanglement. Marine Mammal Science, 30(4):1589-1599, 2014. doi: 10.1111/mms. 12132 .

Barron, D. G., Brawn, J. D., and Weatherhead, P. J. Meta-analysis of transmitter effects on avian behaviour and ecology. Methods in Ecology and Evolution, 1(2):180-187, 2010. doi: 10.1111/j.2041-210X.2010.00013.x. 
Bartholomew, G. A., Vleck, D., and Vleck, C. M. Instantaneous measurements of oxygen consumption during pre-flight warm-up and post-flight cooling in Sphingid and Saturnid moths. Journal of Experimental Biology, 90:17-32, 1981.

Batchelor, G. K. An Introduction to Fluid Dynamics. Cambridge University Press, 2000. ISBN 9781139643467.

Baumgartner, M. F. and Mate, B. R. Summertime foraging ecology of North Atlantic right whales. Marine Ecology Progress Series, 264:123-135, 2003. doi: 10.3354/meps264123.

Baumgartner, M. F. and Mate, B. R. Summer and fall habitat of North Atlantic right whales (Eubalaena glacialis) inferred from satellite telemetry. Canadian Journal of Fisheries and Aquatic Sciences, 62(3):527-543, 2005. doi: 10.1139/f04-238.

Baumgartner, M. F., Cole, T. V. N., Clapham, P. J., and Mate, B. R. North Atlantic right whale habitat in the lower Bay of Fundy and on the SW Scotian Shelf during 1999-2001. Marine Ecology Progress Series, 264:137-154, 2003.

Beck, C. A., Bowen, W. D., and Iverson, S. J. Seasonal changes in buoyancy and diving behaviour of adult grey seals. Journal of Experimental Biology, 203(15):2323-2330, 2000.

Bentley, P. J. Composition of the urine of the fasting humpback whale (Megaptera nodosa). Comparative Biochemistry and Physiology, 10:257-259, 1963.

Berga, A. S., Wright, A. J., Galatius, A., Svergaard, S., and Tieilmann, J. Do larger tag packages alter diving behavior in harbor porpoises? Marine Mammal Science, 2014.

Berga, S. L. and Loucks, T. L. Stress induced anovulation. In Fink, G., editor, Encyclopedia of Stress, volume 3. Oxford Academic Press, Oxford, GB, 2007.

Bertram, J. E. Constrained optimization in human walking: cost minimization and gait plasticity. Journal of Experimental Biology, 208(6):979-991, 2005. doi: 10.1242/jeb.01498.

Bertram, J. E. Locomotion: Why we walk the way we walk. Current Biology, 25(18):R795-7, 2015. doi: 10.1016/j.cub.2015.08.035.

Best, P. B., Mate, B., and Lagerquist, B. Tag retention, wound healing, and subsequent reproductive history of southern right whales following satellite-tagging. Marine Mammal Science, 2014. doi: 10.1111/mms.12168.

Bill, R. G. and Herrnkind, W. F. Drag reduction by formation movement in spiny lobsters. Science, 193(4258):1146-1148, 1976. doi: 10.1126/science.193.4258.1146.

Bilo, D. and Nachtigall, W. A simple method to determine drag coefficients in aquatic animals. Journal of Experimental Biology, 87:259-357, 1980.

Biuw, M., McConnell, B., Bradshaw, C. J. A., Burton, H., and Fedak, M. A. Blubber and buoyancy: monitoring the body condition of free-ranging seals using simple dive characteristics. Journal of Experimental Biology, 206(19):3405-3423, 2003. doi: 10.1242/ jeb.00583.

Black, A., Coward, W., Cole, T., and Prentice, A. Human energy expenditure in affluent societies: an analysis of 574 doubly-labelled water measurements. European Journal of Clinical Nutrition, 50(2):72-92, 1996. ISSN 0954-3007. 
Blake, R. W. Fish locomotion. Cambridge University Press, Cambridge, UK, 1983.

Blake, R. W. Efficiency and Economy in Animal Physiology. Cambridge University Press, 2005. ISBN 9780521019064.

Blake, R. W. Biological implications of the hydrodynamics of swimming at or near the surface and in shallow water. Bioinspiration ES Biomimetics, 4(1):15004, 2009.

Blomqvist, C. and Amundin, M. An acoustic tag for recording directional pulsed ultrasounds aimed at free-swimming bottlenose dolphins (Tursiops truncatus) by conspecifics. Aquatic Mammals, 30(3):345-356, 2004. doi: 10.1578/am.30.3.2004.345.

Bonnard, M. and Pailhous, J. Intentional compensation for selective loading affecting human gait phases. Journal of Motor Behavior, 23(1):4-12, 1991.

Bose, N. and Lien, J. Propulsion of a Fin Whale (Balaenoptera physalus): Why the Fin Whale is a Fast Swimmer. Proceedings of the Royal Society B-Biological Sciences, 237 (1287):175-200, 1989.

Bose, N. and Lien, J. Energy absorption from ocean waves: a free ride for cetaceans. Proceedings of the Royal Society of London B, 240:591-605, 1990.

Bose, N., Lien, J., and Ahia, J. Measurements of the bodies and flukes of several cetacean species. Proceedings of the Royal Society B-Biological Sciences, 242:163-173, 1990.

Boyd, I. L., McCafferty, D. J., and Walker, T. R. L. B. B. Variation in foraging effort by lactating Antarctic fur seals: response to simulated increased foraging costs. Behavioural Ecology and Sociobiology, 40:135-144, 1997.

Brett, J. R. The respiratory metabolism and swimming performance of young sockeye salmon. Journal of the Fisheries Research Board of Canada, 21(5):1183-1226, aug 1964. doi: $10.1139 /$ f64-103.

Brillant, S. W., Vanderlaan, A. S. M., Rangeley, R. W., and Taggart, C. T. Quantitative estimates of the movement and distribution of North Atlantic right whales along the northeast coast of North America. Endangered Species Research, 27(2):141-154, 2015. doi: $10.3354 /$ esr00651.

Brodie, P. F. Cetacean energetics, an overview of intraspecific size variation. Ecology, 56 (1):152-161, 1975.

Broell, F., Burnell, C., and Taggart, C. T. Measuring abnormal movements in free-swimming fish with accelerometers: implications for quantifying tag and parasite load. Journal of Experimental Biology, 219(5):695-705, 2016. doi: 10.1242/jeb.133033.

Brown, D. Tracker Video Analysis and Modeling Tool, 2014.

Budiman, J., Fuwa, S., and Ebata, K. Fundamental studies on the hydrodynamic resistance of small pot traps. Fisheries Science, 70:952-959, 2004.

Butterworth, A., Clegg, I., and Bass, C. Untangled - Marine Debris: a global picture of the impact on animal welfare and of animal-focused solutions. Technical Report of the World Society for the Protection of Animals, London, 2012. 
Byrne, N. M., Hills, A. P., Hunter, G. R., Weinsier, R. L., and Schutz, Y. Metabolic equivalent: one size does not fit all. Journal of Applied Physiology, 99(3):1112-1119, 2005. doi: 10.1152/japplphysiol.00023.2004.

Calambokidis, J., Schorr, G. S., Steiger, G. H., Francis, J., Bakhtiari, M., Marshall, G., Oleson, E. M., Gendron, D., and Robertson, K. Insights into the underwater diving, feeding, and calling behavior of blue whales from a suction-cup- attached video-imaging tag (crittercam). Marine Technology Society Journal, 41(4):19-29, 2007.

Caloin, M. Modeling of lipid and protein depletion during total starvation. American Journal of Physiology: Endocrinology and Metabolism, 287(4):E790-8, 2004. doi: 10. 1152/ajpendo.00414.2003.

Carrillo, J. M., Overstreet, R. M., Raga, J. A., and Aznar, F. J. Living on the Edge: Settlement Patterns by the Symbiotic Barnacle Xenobalanus globicipitis on Small Cetaceans. PLoS One, 10(6):e0127367, 2015. doi: 10.1371/journal.pone.0127367.

Cassoff, R. M., Moore, K. M., McLellan, W. A., Barco, S. G., Rotstein, D. S., and Moore, M. J. Lethal entanglement in baleen whales. Diseases of Aquatic Organisms, 96(3): 175-185, 2011. doi: 10.3354/dao02385.

Caswell, H., Fujiwara, M., and Brault, S. Declining survival probability threatens the North Atlantic right whale. Proceedings of the National Academy of Science, 96:3308-3313, 1999.

Cavatorta, D., Starczak, V., Prada, K. E., and Moore, M. J. A note on the friction of different ropes in right whale (Eubalaena glacialis) baleen: an entanglement model. Journal of Cetacean Research and Management, 7(1):39-42, 2005.

Cherel, Y., Robin, J.-P., and Le Maho, Y. Physiology and biochemistry of long-term fasting in birds. Canadian Journal of Zoology, 66:159-166, 1988.

Cherel, Y., Robin, J.-P., Heitz, A., Calgari, C., and Le Maho, Y. Relationships between lipid availability and protein utilization during prolonged fasting. Journal of Comparative Physiology B, 162:305-313, 1992.

Chopra, M. G. and Kambe, T. Hydromechanics of lunate-tail swimming propulsion. Part 2. Journal of Fluid Mechanics, 79(1):49-69, 1977. doi: doi:10.1017/S0022112077000032.

Christiansen, F., Vikingsson, G. A., Rasmussen, M. H., and Lusseau, D. Minke whales maximise energy storage on their feeding grounds. Journal of Experimental Biology, 216 (3):427-436, 2013. doi: 10.1242/jeb.074518.

Clapham, P. J. and Mead, J. G. Megaptera novaeangliae. Mammalian Species, 604:1-9, 1999.

Clark, B. D. and Bemis, W. Kinematics of swimming of penguins at the detroit zoo. Journal of Zoology, 188(3):411-428, 1979. ISSN 1469-7998.

Cole, T. V. N. and Henry, A. G. Serious injury determinations for baleen whale stocks along the Gulf of Mexico, United States East Coast and Atlantic Canadian Provinces, 2007-2011. Technical Report 13-24, 2013. 
Cooke, S. J., Sack, L., Franklin, C. E., Farrell, A. P., Beardall, J., Wikelski, M., and Chown, S. L. What is conservation physiology? Perspectives on an increasingly integrated and essential science. Conservation Physiology, 1(1):cot001, 2013. doi: 10.1093/conphys/ $\cot 001$.

Cornick, L. A., Inglis, S. D., Willis, K., and Horning, M. Effects of increased swimming costs on foraging behavior and efficiency of captive Steller sea lions: Evidence for behavioral plasticity in the recovery phase of dives. Journal of Experimental Marine Biology and Ecology, 333:306-314, 2006. doi: 10.1016/j.jembe.2006.01.010.

Costa, D. Osmoregulation, pages 801-806. Academic Press, San Diego, CA, 2009.

Costa, D. and Gentry, R. L. Free-ranging energetics of northern fur seals. In Gentry, R. L. and Kooyman, G. L., editors, Fur Seals: Maternal strategies on land and at sea. Princeton University Press, Princeton, NJ, 1986.

Crossin, G. T., Cooke, S. J., Goldbogen, J. A., and Phillips, R. A. Tracking fitness in marine vertebrates: current knowledge and opportunities for future research. Marine Ecology Progress Series, 496:1-17, 2014. doi: 10.3354/meps10691LB-Crossin2014.

Culik, B. M., Wilson, R. P., and Bannasch, R. Flipper-bands on penguins: what is the cost of a life-long commitment? Marine Ecology Progress Series, 98:209-214, 1993.

Culik, B. M., Bannasch, R., and Wilson, R. P. External devices on penguins: how important is shape? Marine Biology, 118:353-357, 1994.

Culik, B. and Wilson, R. P. Swimming energetics and performance of instrumented Adelie penguins (Pygoscelis Adeliae). Journal of Experimental Biology, 158(1):355-368, 1991.

Daniel, T. L. Efficiency in aquatic locomotion: limitations from single cells to animals. Efficiency and economy in animal physiology. Cambridge University Press, Cambridge, pages 83-95, 1991.

Davis, R. W., Collier, S. O., Hagey, W., Williams, T. M., and Le Boeuf, B. J. A video system and three dimensional dive recorder for marine mammals: Using video and virtual reality to study diving behavior. In Fifth European Conference on Biotelemetry, Strasbourg, France, 1999.

Debier, C., Chalon, C., Le Boeuf, B. J., de Tillesse, T., Larondelle, Y., and Thome, J. P. Mobilization of PCBs from blubber to blood in northern elephant seals (Mirounga angustirostris) during the post-weaning fast. Aquatic Toxicology, 80(2):149-157, 2006. doi: 10.1016/j.aquatox.2006.08.002.

DeLong, R. L., Gearin, P. J., Bengtson, J. L., Dawson, P., and Feldkamp, S. D. Studies on the effects of entanglement on individual Northern fur seals. In Shomura, R. S. and Godfrey, M. L., editors, Proceedings of the Second International Conference on Marine Debris, 2-7 April 1989, volume US Departm, pages 492-493, Honolulu, Hawaii, 1990.

Devore, J. Probability and statistics for engineering and the sciences. Thomson Higher Education, Belmont, California, seventh edition, 2008. 
Dunkin, R. C., McLellan, W. A., Blum, J. E., and Pabst, D. A. The ontogenetic changes in the thermal properties of blubber from Atlantic bottlenose dolphin Tursiops truncatus. Journal of Experimental Biology, 208(8):1469-1480, 2005. doi: 10.1242/jeb.01559.

Dunkin, R. C., McLellan, W. A., Blum, J. E., and Pabst, D. A. The buoyancy of the integument of Atlantic bottlenose dolphins (Tursiops truncatus): Effects of growth, reproduction, and nutritional state. Marine Mammal Science, 26(3):573-587, 2010. doi: 10.1111/j.1748-7692.2009.00353.x.

Durban, J. W., Fearnbach, H., Barrett-Lennard, L. G., Perryman, W. L., and Leroi, D. J. Photogrammetry of killer whales using a small hexacopter launched at sea1. Journal of Unmanned Vehicle Systems, 3(3):131-135, 2015. doi: 10.1139/juvs-2015-0020.

Duysens, J., Clarac, F., and Cruse, H. Load-regulating mechanisms in gait and posture: comparative aspects. Physiological Reviews, 80(1):84-133, 2000.

Ebbeling, C. J., Hamill, J., and Crussemeyer, J. A. Lower extremity mechanics and energy cost of walking in high-heeled shoes. Journal of Orthopaedic and Sports Physical Therapy, 19(4):190-196, 1994.

Elliott, K. H., Davoren, G. K., and Gaston, A. J. The influence of buoyancy and drag on the dive behaviour of an Arctic seabird, the Thick-billed Murre. Canadian Journal of Zoology, 85:352-361, 2007. doi: 10.1139/Z07-012.

Elliott, K. H., McFarlane-Tranquilla, L., Burke, C. M., Hedd, A., Montevecchi, W. A., and Anderson, W. G. Year-long deployments of small geolocators increase corticosterone levels in murres. Marine Ecology Progress Series, 466:1-7, 2012. doi: 10.3354/meps09975.

Elliott, K. H., Le Vaillant, M., Kato, A., Gaston, A. J., Ropert-Coudert, Y., Hare, J. F., Speakman, J. R., and Croll, D. Age-related variation in energy expenditure in a long-lived bird within the envelope of an energy ceiling. Journal of Animal Ecology, 83(1):136-146, 2014. doi: $10.1111 / 1365-2656.12126$.

Eloy, C. Optimal Strouhal number for swimming animals. ArXiv, page eprint arXiv:1102.0223, 2011.

Fahlman, A. The pressure to understand the mechanism of lung compression and its effect on lung function. Journal of Applied Physiology, 104(4):907-908, 2008. doi: 10.1152/ japplphysiol.00081.2008.

Fahlman, A., Handrich, Y., Woakes, A. J., Bost, C. A., Holder, R., Duchamp, C., and Butler, P. J. Effect of fasting on the $\mathrm{VO}_{2}-f h$ relationship in king penguins Aptenodytes patagonicus. American Journal of Physiology Regulatory Integrative and Comparative Physiology, 287:R870-R877, 2004.

Fahlman, A., Schmidt, A., Handrich, Y., Woakes, A. J., and Butler, P. J. Metabolism and thermoregulation during fasting in king penguins, Aptenodytes patagonicus, in air and water. American Journal of Physiology Regulatory Integrative and Comparative Physiology, 289:R670-R679, 2005. doi: 10.1152/ajpregu.00130.2005.-We.

Fahlman, A., Loring, S. H., Levine, G., Rocho-Levine, J., Austin, T., and Brodsky, M. Lung mechanics and pulmonary function testing in cetaceans. The Journal of Experimental Biology, 218(13):2030-2038, 2015. doi: 10.1242/jeb.119149. 
Faltinsen, O. Sea loads on ships and offshore structures. Cambridge University Press, 1993. ISBN 9780521458702.

Fedak, M. A., Lovell, P., and Grant, S. M. Two approaches to compressing and interpreting time-depth information as collected by time-depth recorders and satellite-linked data recorders. Marine Mammal Science, 17(1):94-100, 2001.

Feldkamp, S. D. The effects of net entanglement on the drag and power output of a California sea lion, Zalophus californianus. Fishery Bulletin, 83(4):692-695, 1985.

Feldkamp, S. D. Swimming in the California sea lion: morphometrics, drag and energetics. Journal of Experimental Biology, 131:117-135, 1987.

Feldkamp, S. D., Costa, D. P., and DeKrey, G. K. Energetic and behavioral effects of net entanglement on juvenile northern fur seals, Callorhinus ursinus. Fishery Bulletin, 87: 85-94, 1988.

Fertl, D. and Newman, W. A. Barnacles A2 - Perrin, William F. In Würsig, B. and Thewissen, J. G. M., editors, Encyclopedia of Marine Mammals (Second Edition), pages 89-91. Academic Press, London, 2009. ISBN 978-0-12-373553-9. doi: http://dx.doi.org/ 10.1016/B978-0-12-373553-9.00025-0.

Fish, F. E. Influence of hydrodynamic design and propulsive mode on mammalian swimming energetics. Australian Journal of Zoology, 42:79-101, 1993a.

Fish, F. E. Power output and propulsive efficiency of swimming bottlenose dolphins (Tursiops truncatus). Journal of Experimental Biology, 185(1):179-193, 1993b.

Fish, F. E. Kinematics of ducklings swimming in formation: consequences of position. Journal of Experimental Zoology, 273:1-11, 1995.

Fish, F. E. Comparative kinematics and hydrodynamics of odontocete cetaceans: morphological and ecological correlates with swimming performance. Journal of Experimental Biology, 201(20):2867-2877, 1998.

Fish, F. E. Performance constraints on the maneuverability of flexible and rigid biological systems. In Eleventh International Symposium on Unmanned Untethered Submersible Technology, pages 394-406, Durham, NH, 1999. Autonomous Undersea Systems Institute.

Fish, F. E. Maneuverability by the sea lion Zalophus californianus: turning performance of an unstable body design. Journal of Experimental Biology, 206(4):667-674, 2003. doi: $10.1242 /$ jeb.00144.

Fish, F. E. and Hui, C. A. Dolphin swimming - a review. Mammal Review, 21(4):181-195, 1991.

Fish, F. E. and Lauder, G. V. Passive and active flow control by swimming fishes and mammals. Annual Review of Fluid Mechanics, 38:193-224, 2006. doi: 10.1146/annurev. fluid.38.050304.092201.

Fish, F. E. and Rohr, J. J. Review of dolphin hydrodynamics and swimming performance. Technical Report 18, US Navy SPAWAR Systems Center, San Diego, CA, 1999. 
Fish, F. E., Howle, L. E., and Murray, M. M. Hydrodynamic flow control in marine mammals. Integrative and Comparative Biology, 48(6):788-800, 2008. doi: 10.1093/icb/icn029.

Fish, F. E., Legac, P., Williams, T. M., and Wei, T. Measurement of hydrodynamic force generation by swimming dolphins using bubble DPIV. Journal of Experimental Biology, 217(2):252-260, 2014. doi: 10.1242/jeb.087924.

Fortune, S. M. E. and Trites, A. W. North Atlantic right whale growth and energetics. PhD thesis, University of British Columbia, Vancouver, B.C., 2012.

Fortune, S. M. E., Trites, A. W., Perryman, W. L., Moore, M. J., Pettis, H. M., and Lynn, M. S. Growth and rapid early development of North Atlantic right whales (Eubalaena glacialis). Journal of Mammalogy, 93(5):1342-1354, 2012. doi: 10.1644/n-MAMM-A-297. 1.

Fortune, S. M. E., Trites, A. W., Mayo, C. A., Rosen, D. A. S., and Hamilton, P. K. Energetic requirements of North Atlantic right whales and the implications for species recovery. Marine Ecology Progress Series, 478:253-272, 2013. doi: 10.3354/meps10000.

Forward, R. B. and Tankersley, R. A. Selective tidal-stream transport of marine animals. Oceanography and Marine Biology: an Annual Review, 39:305-353, 2001.

Fowler, C. W. Marine debris and northern fur seals: a case study. Marine Pollution Bulletin, 18(6B):326-335, 1987.

Fowler, C. W., Merrick, R., and Baker, J. D. Studies of the population level effects of entanglement on Northern fur seals. In Shomura, R. S. and Godfrey, M. L., editors, Proceedings of the Second International Conference on Marine Debris, 2-7 April 1989, pages 453-474, Honolulu, Hawaii, 1990.

Fridman, A. L. Calculations for fishing gear designs. FAO Fishing Manuals. Fishing News Books, Farhnam, Surrey. 1986.

Fridman, A. L. and Dvernik, A. V. Development of a method for the calculation of the resistance of a trawl net. Fischerei Forschung, 11(2):7-13, 1973.

Frisch, R. E. Body fat, puberty and fertility. Biological Reviews, 59(2):161-188, 1984.

Fujiwara, M. and Caswell, H. Demography of the endangered North Atlantic right whale. Nature, 414:537-541, 2001.

Gailey, R., ALlen, K., Castles, J., Kucharik, J., and Roeder, M. Review of secondary physical conditions associated with lower-limb amputation and long-term prosthesis use. Journal of Rehabilitation Research and Development, 45(1):15-30, 2008.

Gallivan, G. A. What are the metabolic rates of cetaceans? Physiological Zoology, 65(6): 1285-1297, 1992.

Geertsen, B. M., Teilmann, J., Kastelein, R. A., Vlemmix, H. N. J., and Miller, L. A. Behaviour and physiological effects of transmitter attachments on a captive harbour porpoise (Phocoena phocoena). Journal of Cetacean Research and Management, 6(2):139-146, 2004. 
Geraci, J. R. and Lounsbury, V. J. Marine mammals ashore: a field guide for strandings. National Aquarium in Baltimore, Baltimore, Maryland, 2nd edition, 2005.

Gessaman, J. Ecological energetics of homeotherms: a view compatible with ecological modeling. Utah State University Press, Logan, Utah, USA, 1973.

Gessaman, J. and Nagy, K. A. Transmitter loads affect the flight speed and metabolism of homing pigeons. The Condor, 90(3):662-668, 1988.

Gittleman, J. L. and Thompson, S. D. Energy allocation in mammalian reproduction. American Zoologist, 28:863-875, 1988.

Goforth, H. J. Ergometry (exercise testing) of the bottlenose dolphin, book section 32, pages 559-574. Academic Press, San Diego, CA, 1990.

Goldbogen, J. A., Pyenson, N. D., and Shadwick, R. E. Big gulps require high drag for fin whale lunge feeding. Marine Ecology Progress Series, 349:289-301, 2007. doi: 10.3354/ meps07066.

Goldbogen, J. A., Calambokidis, J., Croll, D. A., Harvey, J. T., Newton, K. M., Oleson, E. M., Schorr, G., and Shadwick, R. E. Foraging behavior of humpback whales: kinematic and respiratory patterns suggest a high cost for a lunge. Journal of Experimental Biology, 211(23):3712-3719, 2008. doi: 10.1242/jeb.023366.

Goldbogen, J. A., Calambokidis, J., Oleson, E., Potvin, J., Pyenson, N. D., Schorr, G., and Shadwick, R. E. Mechanics, hydrodynamics and energetics of blue whale lunge feeding: efficiency dependence on krill density. Journal of Experimental Biology, 214(1):131-146, 2011. doi: $10.1242 /$ jeb.048157.

Goldbogen, J. A., Stimpert, A. K., Deruiter, S. L., Calambokidis, J., Friedlaender, A. S., Schorr, G. S., Moretti, D. J., Tyack, P. L., and Southall, B. L. Using accelerometers to determine the calling behavior of tagged baleen whales. The Journal of Experimental Biology, 2014. doi: 10.1242/jeb.103259.

Goldbogen, J., Cade, D., Calambokidis, J., Stimpert, A., and Friedlaender, A. Insights into the Underwater Behavior, Species Interactions, and Biomechanics of Baleen Whales using Suction-Cup Attached Video and Inertial Sensors. The FASEB Journal, 30(1 Supplement): 90.3, 2016.

Hain, J. H. W., Hampp, J. D., McKenney, S. A., Albert, J. A., and Kenney, R. D. Swim speed, behavior, and movement of North Atlantic right whales (Eubalaena glacialis) in coastal waters of Northeastern Florida, USA. PloS one, 8(1):e54340, 2013. doi: 10.1371/ journal.pone.0054340.g002.

Hall, A. J., Gulland, F. M. D., Ylitalo, G. M., Greig, D. J., and Lowenstine, L. Changes in blubber contaminant concentrations in California sea lions (Zalophus californianus) associated with weight loss and gain during rehabilitation. Environmental Science and Technology, 42(11):4181-4187, 2008.

Halsey, L. G. Terrestrial movement energetics: current knowledge and its application to the optimising animal. Journal of Experimental Biology, 219(10):1424-1431, 2016. doi: $10.1242 /$ jeb.133256. 
Hamilton, P. K., Knowlton, A. R., and Marx, M. K. Right whales tell their own stories: the photo-identification catalog. In Kraus, S. D. and Rolland, R. M., editors, The urban whale: North Atlantic right whales at the crossroads, pages 75-104. Harvard University Press, Cambridge, MA, 2007.

Hamilton, P. K. and Cooper, L. A. Changes in north atlantic right whale (Eubalaena glacialis) cow-calf association times and use of the calving ground: 1993-2005. Marine Mammal Science, 26(4):896-916, 2010. doi: 10.1111/j.1748-7692.2010.00378.x.

Hamner, W. A., Stone, G. S., and Obst, B. S. Behavior of southern right whales, Eubalaena australis, feeding on the Antarctic krill, Euphausia superba. Fishery Bulletin, 86(1):143$150,1988$.

Hanson, M. B. An evaluation of the relationship between small cetacean tag design and attachment durations: a bioengineering approach. Phd thesis, University of Washington, Seattle, WA, 2001.

Hanson, M. T. and Defran, R. H. The behaviour and feeding ecology of the pacific coast bottlenose dolphin, tursiops truncatus. Aquatic Mammals, 19(3):127-142, 1993.

Harris, J. E. The mechanical significance of the position and movements of the paired fins in the teleostei. Tortugas Laboratory Papers, 31:173-189, 1937.

Hazekamp, A. A. H., Mayer, R., and Osinga, N. Flow simulation along a seal: the impact of an external device. European Journal of Wildlife Research, 56(2):131-140, 2009. doi: 10.1007/s10344-009-0293-0.

Healy, M., Chiaradia, A., Kirkwood, R., and Dann, P. Balance: a neglected factor when attaching external devices to penguins. Memoirs of National Institute of Polar Research, 58:179-182, 2004.

Hedenstrom, A. Migration by soaring or flapping flight in birds: the relative importance of energy cost and speed. Philosophical transactions of the Royal Society of London. Series B, Biological sciences, 342:353-361, 1993.

Heglund, N. C., Willems, P. A., Penta, M., and Cavagna, G. A. Energy-saving gait mechanics with head-supported loads. Nature, 375:52-54, 1995.

Helmond, I. Towed Vehicles. In Steele, J. H., editor, Encyclopedia of Ocean Sciences, pages 2994-3003. Academic Press, Oxford, 2001. ISBN 978-0-12-227430-5. doi: 10.1006/rwos. 2001.0309 .

Henry, A. G., Cole, T. V. N., Garron, M., Ledwell, W., and Reid, A. Mortality and serious injury determinations for baleen whale stocks along the Gulf of Mexico, United States East Coast and Atlantic Canadian provinces, 2006-2010. US Department of Commerce, Northeast Fisheries Science Center Reference Document 12-11; 24 p. Available from: National Marine Fisheries Service, 166 Water Street, Woods Hole, MA 02543-1026, or online at http://nefsc.noaa.gov/publications/, 2012.

Hertel, H. Hydrodynamics of swimming and wave-riding dolphins. In Andersen, H. T., editor, The Biology of Marine Mammals, pages 31-63. Academic Press, New York, 1969. 
Hertel, H. and Technica, S. Structure, form, movement. Reinhold Publishing, New York, NY, 1966.

Heyning, J. E. Thermoregulation in feeding baleen whales: Morphological and physiological evidence. Aquatic Mammals, 27(3):284-288, 2001.

Hills, A. P., Mokhtar, N., and Byrne, N. M. Assessment of physical activity and energy expenditure: an overview of objective measures. Frontiers in Nutrition, 1:5, 2014. doi: $10.3389 /$ fnut.2014.00005.

Hind, A. T. and Gurney, W. S. C. The metabolic cost of swimming in marine homeotherms. Journal of Experimental Biology, 200:531-542, 1997.

Hoerner, S. F. Fluid dynamic drag. Published by Author, Midland Park, New Jersey, 1965.

Hofmeyr, G. J., Bester, M. N., Kirkman, S. P., Lydersen, C., and Kovacs, K. M. Entanglement of Antarctic fur seals at Bouvetoya, Southern Ocean. Marine Pollution Bulletin, 52 (9):1077-1080, 2006. doi: 10.1016/j.marpolbul.2006.05.003.

Houser, D. S., Champagne, C. D., Crocker, D. E., Kellar, N. M., Cockrem, J., Romano, T., Booth, R. K., and Wasser, S. K. Natural Variation in Stress Hormones, Comparisons Across Matrices, and Impacts Resulting from Induced Stress in the Bottlenose Dolphin. In Popper, A. N. and Hawkins, A., editors, The Effects of Noise on Aquatic Life II, volume 875, chapter 56, pages 467-471. Springer New York, 2016. ISBN 978-1-4939-2980-1.

Hover, F. S., Haugsdal, Ø., and Triantafyllou, M. S. Effect of angle of attack profiles in flapping foil propulsion. Journal of Fluids and Structures, 19(1):37-47, 2004 . doi: 10.1016/j.jfluidstructs.2003.10.003.

Hui, C. A. Seawater consumption and water flux in the common dolphin Delphinus delphis. Physiological Zoology, 54(4):430-440, 1981.

Hunt, K. E., Rolland, R. M., and Kraus, S. D. Conservation physiology of an uncatchable animal: the North Atlantic right whale (Eubalaena glacialis). Integrative and Comparative Biology, 2015. doi: 10.1093/icb/icv071.

Hunt, K. E., Rolland, R. M., Kraus, S. D., and Wasser, S. K. Analysis of fecal glucocorticoids in the North Atlantic right whale (Eubalaena glacialis). General and Comparative Endocrinology, 148(2):260-272, 2006. doi: 10.1016/j.ygcen.2006.03.012.

Hussey, N. E., Kessel, S. T., Aarestrup, K., Cooke, S. J., Cowley, P. D., Fisk, A. T., Harcourt, R. G., Holland, K. N., Iverson, S. J., Kocik, J. F., Mills Flemming, J. E., and Whoriskey, F. G. Aquatic animal telemetry: A panoramic window into the underwater world. Science, 348(6240), 2015. doi: 10.1126/science.1255642.

Irvine, A. B., Wells, R. S., and Scott, M. D. An evaluation of techniques for tagging small odontocete cetaceans. Fishery Bulletin, 80(1):135-143, 1982.

IWC. Report of the Workshop on Welfare Issues Associated with the Entanglement of Large Whales. Technical Report IWC/62/15, Maui, HI, 2010.

IWC. Report of the Second IWC Workshop on Welfare Issues Associated with the Entanglement of Large Whales with a Focus on Entanglement Response. Technical Report IWC/64/WKM\&AWI REP1, Provincetown, MA, 2011. 
Jachowski, D. S. and Singh, N. J. Toward a mechanistic understanding of animal migration: incorporating physiological measurements in the study of animal movement. Conservation Physiology, 3(1):cov035, 2015. doi: 10.1093/conphys/cov035.

Jachowski, D. S., Montgomery, R. A., Slotow, R., Millspaugh, J. J., and Franklin, C. Unravelling complex associations between physiological state and movement of African elephants. Functional Ecology, 27(5):1166-1175, 2013. doi: 10.1111/1365-2435.12118.

Jacobs, E. N. Airfoil section characteristics as affected by protuberances. National Advisory Committee for Aeronautics (NACA). Technical Report 446, 1934.

Jepsen, N., Schreck, C., Clements, S., and Thorstad, E. B. A brief discussion on the 2\% tag/bodymass rule of thumb. In Spedicato, M. T., Lembo, G., and Marmulla, G., editors, Aquatic telemetry: advances and applications. Proceedings of the Fifth Conference on Fish Telemetry held in Europe, Ustica, Italy, 9-13 June 2003. FAO/COISPA, Rome, 2005.

Jepsen, N., Thorstad, E. B., Havn, T., and Lucas, M. C. The use of external electronic tags on fish: an evaluation of tag retention and tagging effects. Animal Biotelemetry, 3:49, 2015. doi: 10.1186/s40317-015-0086-z.

Johnson, A., Salvador, G., Kenney, J., Robbins, J., Kraus, S., Landry, S., and Clapham, P. Fishing gear involved in entanglements of right and humpback whales. Marine Mammal Science, 21(4):635-645, 2005. doi: 10.1111/j.1748-7692.2005.tb01256.x.

Johnson, M. and Tyack, P. A digital acoustic recording tag for measuring the response of wild marine mammals to sound. IEEE Journal of Oceanic Engineering, 28(1):3-12, 2003. doi: 10.1109/JOE.2002.808212.

Johnson, M., Aguilar de Soto, N., and Madsen, P. T. Studying the behaviour and sensory ecology of marine mammals using acoustic recording tags: a review. Marine Ecology Progress Series, 395:55-73, 2009. doi: 10.3354/meps08255.

Johnson, M. DTAG Toolbox for MATLAB, 2015.

Jones, T. T., Bostrom, B., Carey, M., Imlach, B., Mikkelsen, J., Ostafichuk, P., Eckert, S., Opay, P., Swimmer, Y., Seminoff, J., and Jones, D. R. Determining transmitter drag and best-practice attachment procedures for sea turtle biotelemetry. NOAA Technical Memorandum NMFS, SWFSC-480:1-58, 2011.

Jones, T. T., Van Houtan, K. S., Bostrom, B. L., Ostafichuk, P., Mikkelsen, J., Tezcan, E., Carey, M., Imlach, B., Seminoff, J. A., and Rands, S. Calculating the ecological impacts of animal-borne instruments on aquatic organisms. Methods in Ecology and Evolution, 4: 1178-1186, 2013. doi: 10.1111/2041-210x.12109.

Kays, R., Crofoot, M. C., Jetz, W., and Wikelski, M. Terrestrial animal tracking as an eye on life and planet. Science, 348(6240), 2015. doi: 10.1126/science.aaa2478.

Keith, W. L., Cipolla, K. M., Hart, D. R., and Furey, D. A. Drag measurements on long, thin cylinders at small angles and high reynolds numbers. NUWC-NPT. Technical Report 11,555, Newport, RI, 2004.

Kjendlie, P.-L. and Stallman, R. K. Drag characteristics of competitive swimming children and adults. Journal of Applied Biomechanics, 24:35-42, 2008. 
Klansjcek, T., Nisbet, R. M., Caswell, H., and Neubert, M. G. A model for energetics and bioaccumulation in marine mammals with applications to the right whale. Ecological Applications, 17(8):2233-2250, 2007.

Knowlton, A. R., Kraus, S. D., and Kenney, R. D. Reproduction in North Atlantic right whales (Eubalaena glacialis). Canadian Journal of Zoology, 72(7):1297-1305, 1994.

Knowlton, A. R., Hamilton, P. K., Marx, M. K., Pettis, H. M., and Kraus, S. D. Monitoring North Atlantic right whale Eubalaena glacialis entanglement rates: a 30 yr retrospective. Marine Ecology Progress Series, 466:293-302, 2012a. doi: 10.3354/meps09923.

Knowlton, A. R., Hamilton, P. K., and Pettis, H. M. Status of reproductive females in the North Atlantic right whale population and impacts of human activities on their reproductive success. Report for NOAA Cooperative Agreement Number NA09OAR4320129. Technical report, $2012 \mathrm{~b}$.

Knowlton, A. R., Robbins, J., Landry, S., McKenna, H. A., Kraus, S. D., and Werner, T. Implications of fishing rope strength on the severity of large whale entanglements. Conservation Biology, 30(2):318-328, 2015. doi: 10.1111/cobi.12590.

Kojeszewski, T. and Fish, F. E. Swimming kinematics of the Florida manatee (Trichechus manatus latirostris): hydrodynamic analysis of an undulatory mammalian swimmer. Journal of Experimental Biology, 210(14):2411-2418, 2007. doi: 10.1242/jeb.02790.

Koopman, H. N., Pabst, D. A., McLellan, W. A., Dillaman, R. M., and Read, A. J. Changes in blubber distribution and morphology associated with starvation in the harbor porpoise (Phocoena phocoena): Evidence for regional differences in blubber structure and function. Physiological and Biochemical Zoology, 75(5):498-512, 2002.

Kooyman, G. L. and Ponganis, P. J. Emperor penguin oxygen consumption, heart rate and plasma lactate levels during graded swimming exercise. Journal of Experimental Biology, 195:199-209, 1994.

Koteja, P. Measuring energy metabolism with open-flow respirometric systems: which design to choose? Functional Ecology, 10(5):675-677, 1996.

Krahn, M. M., Herman, D. P., Ylitalo, G. M., CSloan, C. A., Burrows, D. G., Hobbs, R. C., Mahoney, B. A., Yanigada, G. K., Calambokidis, J., and Moore, S. E. Stratification of lipids, fatty acids and organochlorine contaminants in blubber of white whales and killer whales. Journal of Cetacean Research and Management, 6(2):175-189, 2004.

Kraus, S. D. A review of the status of right whales (Eubalaena glacialis) in the western North Atlantic with a summary of research and management needs. Technical Report to the U.S. Marine Mammal Commission, Bethesda, Maryland, 1985.

Kraus, S. D., Prescott, J. H., Knowlton, A. R., and Stone, G. E. Migration and calving of right whales (Eubalaena glacialis) in the western North Atlantic. Report of the International Whaling Commission, 10(Special Issue):139-144, 1986.

Kraus, S. D., Hamilton, P. K., Kenney, R. D., Knowlton, A. R., and Slay, C. K. Reproductive parameters of the North Atlantic right whale. Journal of Cetacean Research and Management, 2(Special Issue):231-236, 2001. 
Kriete, B. Bioenergetics in the killer whale, Orcinus orca. PhD thesis, University of British Columbia, Vancouver, B.C., 1995.

Kuiken, T., Simpson, V. R., Allchin, C. R., and Benne, P. M. Mass mortality of common dolphins (Delphinus delphis) to incidental capture in fishing gear. Veterinary Record, 134: 81-89, 1994.

Lagier, R. Spondylosis in the whale - an anatomicoradiological study of an osteophyte. Scandanavian Journal of Rheumatology, 6:245-249, 1977.

Laist, D. W. Impacts of marine debris: entanglement of marine life in marine debris including a comprehensive list of species with entanglement and ingestion records. In Coe, J. M. and Rogers, D. B., editors, Marine Debris, chapter 10, pages 99-139. Springer, New York, 1997.

Lang, T. G. and Daybell, D. A. Porpoise performance tests in a sea-water tank. NOTS Technical Publication 3063. NAVWEPS Report 8060. 61 pp., page 32, 1963.

Lang, T. G. and Norris, K. S. Swimming speed of a pacific bottlenose porpoise. Science, 151:588-590, 1966.

Laursen, B., Ekner, D., Simonsen, E. B., Voigt, M., and Sjøgaard, G. Kinetics and energetics during uphill and downhill carrying of different weights. Applied ergonomics, 31(2):159$166,2000$.

Ledwell, W., Curren, K., Huntington, H., and Hood, C. The whale man of Newfoundland and Labrador: Jon Lien 1939-2010. Canadian Field-Naturalist, 124(4):384-398, 2010.

Lighthill, M. J. Hydromechanics of aquatic animal propulsion. Annual Review of Fluid Mechanics, 1(1):413-446, 1969. doi: doi:10.1146/annurev.fl.01.010169.002213.

Lighthill, M. J. Large-amplitude elongated-body theory of fish locomotion. Proceedings of the Royal Society of London. Series B, Biological Sciences, 179(1055):125-138, 1971.

Lighthill, M. J. Waves in fluid. Cambridge University Press, New York, 1978.

Littnan, C. L., Baker, J. D., Parrish, F. A., and Marshall, G. J. Effects of video camera attachment on the foraging behavior of immature Hawaiian monk seals. Marine Mammal Science, 20(2):345-352, 2004.

Lockyer, C. A theoretical approach to the balance between growth and food consumption in fin and sei whales, with special reference to the female reproductive cycle. Report of the International Whaling Commission, 28:243-249, 1978.

Lockyer, C. Estimation of the energy costs of growth, maintenance and reproduction in the female Minke whale, (Balaenoptera acutorostrata), from the southern hemisphere. Report of the International Whaling Commission, 31:337-343, 1981a.

Lockyer, C. Growth and energy budgets of large baleen whales from the southern hemisphere. In Research, F., editor, Mammals in the seas, volume 3, pages 379-484. Food and Agriculture Organization, 1981b. ISBN 9789251005132. 
Lockyer, C. Body fat condition in Northeast Atlantic fin whales, Balaenoptera physalus, and its relationship with reproduction and food resource. Canadian Journal of Fisheries and Aquatic Sciences, 43:142-147, 1986.

Lockyer, C. Evaluation of the role of fat reserves in relation to the ecology of North Atlantic fin and sei whales. In Cetacean Bioenergetics. 1987.

Lockyer, C. H., McConnell, L. C., and Waters, T. D. Body condition in terms of anatomical and biochemical assessment of body fat in North Atlantic fin and sei whales. Canadian Journal of Zoology, 63:2328-2338, 1985.

Lovvorn, J. R., Watanuki, Y., Kato, A., Naito, Y., and Liggins, G. A. Stroke patterns and regulation of swim speed and energy cost in free-ranging brunnich's guillemots. Journal of Experimental Biology, 207(26):4679-95, 2004. doi: 10.1242/jeb.01331.

Lyman, E. G. 2011-ĂŞ2012 season summary on large whale entanglement threat and reports received around the Main Hawaiian Islands. Technical Report of the Hawaiian Islands Humpback Whale National Marine Sanctuary, 2012.

Lyman, E. G. 2013-2014 Hawai'i large whale entanglements and response efforts around the Main Hawaiian Islands. Technical Report of the Hawaiian Islands Humpback Whale National Marine Sanctuary, 2014.

Macfarlane, N. B. W. The choreography of belonging: Toothed whale spatial cohesion and acoustic communication. Phd thesis, Massachusetts Institute of Technology and Woods Hole Oceanographic institution, Cambridge, MA, 2015.

Maresh, J. L., Simmons, S. E., Crocker, D. E., McDonald, B. I., Williams, T. M., and Costa, D. P. Free-swimming northern elephant seals have low field metabolic rates that are sensitive to an increased cost of transport. Journal of Experimental Biology, 217(9): 1485-1495, 2014. doi: 10.1242/jeb.094201.

Maresh, J. L., Adachi, T., Takahashi, A., Naito, Y., Crocker, D. E., Horning, M., Williams, T. M., and Costa, D. P. Summing the strokes: energy economy in northern elephant seals during large-scale foraging migrations. Movement Ecology, 3(1):22, 2015. doi: 10.1186/ s40462-015-0049-2.

Martin Lopez, L. M., Miller, P. J., Aguilar de Soto, N., and Johnson, M. Gait switches in deep-diving beaked whales: biomechanical strategies for long-duration dives. Journal of Experimental Biology, 218(9):1325-38, 2015. doi: 10.1242/jeb.106013.

Martin Lopez, L. M., de Soto, N. A., Miller, P., and Johnson, M. Tracking the kinematics of caudal-oscillatory swimming: a comparison of two on-animal sensing methods. Journal of Experimental Biology, 2016. doi: 10.1242/jeb.136242.

Martineau, D., Lemberger, K., Dallaire, A., Labelle, P., Lipscomb, T. P., Michel, P., and Mikaelian, I. Cancer in wildlife, a case study: beluga from the St. Lawrence Estuary, Quebec, Canada. Environmental Health Perspectives, 110(3):285-292, 2002.

Mate, B., Nieukirk, S. L., and Kraus, S. Satellite-monitored movements of the Northern right whale. Journal of Wildlife Management, 61(4):1393-1405, 1997. 
Mate, B. R., Rossbach, K. A., Neukirk, S. L., Wells, R. S., Irvine, A. B., Scott, M. D., and Read, A. J. Satellite-monitored movements and dive behavior of a bottlenose dolphin (Tursiops truncatus) in tampa bay, florida. Marine Mammal Science, 11(4):452-463, 1995.

Mayo, C. A. and Marx, M. K. Surface foraging behaviour of the North Atlantic right whale, Eubalaena glacialis, and associated zooplankton characteristics. Canadian Journal of Zoology, 68(10):2214-2220, 1990. doi: 10.1139/z90-308.

McCarron, P. and Tetreault, H. Lobster pot gear configurations in the Gulf of Maine. Technical Report of the Maine Lobstermens Association, 2012.

McCue, M. D. Starvation physiology: reviewing the different strategies animals use to survive a common challenge. Comparative Biochemistry and Physiology A: Molecular and Integrative Physiology, 156(1):1-18, 2010. doi: 10.1016/j.cbpa.2010.01.002.

McEwen, B. S. and Wingfield, J. C. What is in a name? Integrating homeostasis, allostasis and stress. Hormones and Behavior, 57(2):105-111, 2010. doi: 10.1016/j.yhbeh.2009.09. 011 .

McEwen, B. S. and Wingfield, J. C. The concept of allostasis in biology and biomedicine. Hormones and Behavior, 43(1):2-15, 2003. doi: 10.1016/s0018-506x(02)00024-7.

McGregor, A. E. N. The cost of locomotion in North Atlantic right whales Eubalaena glacialis. PhD thesis, Duke University, Durham, NC, 2010.

McIntosh, R. R., Kirkwood, R., Sutherland, D. R., and Dann, P. Drivers and annual estimates of marine wildlife entanglement rates: A long-term case study with Australian fur seals. Marine Pollution Bulletin, 101:716-725, 2016. doi: http://dx.doi.org/10.1016/ j.marpolbul.2015.10.007.

McIntyre, T. Trends in tagging of marine mammals: a review of marine mammal biologging studies. African Journal of Marine Science, pages 1-14, 2014. doi: 10.2989/1814232x. 2014.976655 .

McMahon, C. R., Collier, N., Northfield, J. K., and Glen, F. L. B. M. Taking the time to assess the effects of remote sensing and tracking devices on animals. Animal Welfare, 20: 515-521, 2011.

McMahon, C. R., Field, I. C., Bradshaw, C. J. A., White, G. C., and Hindell, M. A. Tracking and data-ĂŞlogging devices attached to elephant seals do not affect individual mass gain or survival. Journal of Experimental Marine Biology and Ecology, 360(2):71-77, 2008. doi: 10.1016/j.jembe.2008.03.012.

McNamara, J. M. and Houston, A. I. Optimal annual routines: behaviour in the context of physiology and ecology. Philosophical Transactions of the Royal Society of London B Biological Sciences, 363(1490):301-19, 2008. doi: 10.1098/rstb.2007.2141.

Mendenhall, W. and Sincich, T. A Second Course in Statistics: Regression Analysis. Prentice Hall, 2011. ISBN 9780321691699.

Metcalfe, J. D., Arnold, G. P., and Webb, P. W. The energetics of migration by selective tidal stream transport: an analysis for plaice tracked in the southern North Sea. Journal 
of the Marine Biological Association of the United Kingdom, 70(01):149-162, 1990. doi: doi:10.1017/S0025315400034275.

Methling, C., Tudorache, C., Skov, P. V., and Steffensen, J. F. Pop Up Satellite Tags Impair Swimming Performance and Energetics of the European Eel (Anguilla anguilla). PLoS ONE, 6(6):e20797, 2011. doi: 10.1371/journal.pone.0020797.

Millar, J. S. and Hickling, G. J. Fasting endurance and the evolution of mammalian body size. Functional Ecology, 4(1):5-12, 1990.

Miller, C. A., Reeb, D., Best, P. B., Knowlton, A. R., Brown, M. W., and Moore, M. J. Blubber thickness in right whales Eubalaena glacialis and Eubalaena australis related with reproduction, life history status and prey abundance. Marine Ecology Progress Series, 438: 267-283, 2011. doi: 10.3354/meps09174.

Miller, C. A., Best, P. B., Perryman, W. L., Baumgartner, M. F., and Moore, M. J. Body shape changes associated with reproductive status, nutritive condition and growth in right whales Eubalaena glacialis and E. australis. Marine Ecology Progress Series, 459:135-156, 2012a. doi: 10.3354/meps09675.

Miller, P. J., Biuw, M., Watanabe, Y. Y., Thompson, D., and Fedak, M. A. Sink fast and swim harder! Round-trip cost-of-transport for buoyant divers. Journal of Experimental Biology, 215(20):3622-3630, 2012b. doi: 10.1242/jeb.070128.

Miller, P. J. O., Johnson, M., Tyack, P., and Terray, E. A. Swimming gaits, passive drag and buoyancy of diving sperm whales Physeter macrocephalus. Journal of Experimental Biology, 207(11):1953-1967, 2004. doi: 10.1242/jeb.00993.

Montie, E. W., Letcher, R. J., Reddy, C. M., Moore, M. J., Rubinstein, B., and Hahn, M. E. Brominated flame retardants and organochlorine contaminants in winter flounder, harp and hooded seals, and North Atlantic right whales from the Northwest Atlantic Ocean. Marine Pollution Bulletin, 60(8):1160-1169, 2010. doi: http://dx.doi.org/10. 1016/j.marpolbul.2010.04.002.

Moore, E., Lyday, S., Roletto, J., Litle, K., Parrish, J. K., Nevins, H., Harvey, J., Mortenson, J., Greig, D., Piazza, M., Hermance, A., Lee, D., Adams, D., Allen, S., and Kell, S. Entanglements of marine mammals and seabirds in central California and the north-west coast of the United States 2001-2005. Marine Pollution Bulletin, 58(7):1045-1051, 2009. doi: 10.1016/j.marpolbul.2009.02.006.

Moore, K. T. and Barco, S. G. Handbook for recognizing, evaluating, and documenting human interaction in stranded cetaceans and pinnipeds. U.S. Department of Commerce, NOAA Technical Memorandum, NOAA-TM-NMFS-SWFSC-510. Technical report, 2013.

Moore, M. J., Bogomolni, A., Bowman, R., Hamilton, P. K., Harry, C. T., Knowlton, A. R., Landry, S., Rotstein, D. S., and Touhey, K. Fatally entangled right whales can die extremely slowly. In OCEANS 2006, pages 1-3, 2006.

Moore, M. J., Walsh, M., Bailey, J., Brunson, D., Gulland, F. M. D., Landry, S., Mattila, D., Mayo, C. A., Slay, C. K., Smith, J. S., and Rowles, T. Sedation at sea of entangled North Atlantic right whales (Eubalaena glacialis) to enhance disentanglement. PloS one, 5(3):e9597, 2010. doi: 10.1371/journal.pone.0009597.g001. 
Moore, M. J., van der Hoop, J. M., Barco, S. G., Costidis, A. M., Gulland, F. M., Jepson, P. D., Moore, K. T., Raverty, S., and McLellan, W. A. Criteria and case definitions for serious injury and death of pinnipeds and cetaceans caused by anthropogenic trauma. Diseases of Aquatic Organisms, 103(3):229-264, 2013. doi: 10.3354/dao02566.

Moore, M. J., Shorter, K. A., Hurst, T., van der Hoop, J., Bocconcelli, A., Johnson, M., Tyack, P., Rittschof, D., Nowacek, D. P., and Howle, L. E. Improving attachments of non-invasive (type iii) electronic data loggers to cetaceans - final report. Report, Office of Naval Research, Arlington, VA, 2016. URL http: //www .onr.navy.mil/en/Science-Technology/Departments/Code-32/All-Programs/ Atmosphere-Research-322/Marine-Mammals-Biology/Annual-Report-FY15.aspx.

Moore, M., Andrews, R., Austin, T., Bailey, J., Costidis, A., George, C., Jackson, K., Pitchford, T., Landry, S., Ligon, A., McLellan, W., Morin, D., Smith, J., Rotstein, D., Rowles, T., Slay, C., and Walsh, M. Rope trauma, sedation, disentanglement, and monitoring-tag associated lesions in a terminally entangled North Atlantic right whale (Eubalaena glacialis). Marine Mammal Science, 29:E98-E113, 2012. doi: 10.1111/j.1748-7692.2012.00591.x.

Moore, M. J. and van der Hoop, J. M. The painful side of trap and fixed net fisheries: chronic entanglement of large whales. Journal of Marine Biology, 2012:1-4, 2012. doi: $10.1155 / 2012 / 230653$.

Moore, M. J., Knowlton, A. R., Kraus, S. D., McLellan, W. A., and Bonde, R. K. Morphometry, gross morphology and available histopathology in North Atlantic right whale (Eubalaena glacialis) mortalities (1970-2002). Journal of Cetacean Research and Management, 6:199-214, 2004.

Morin, D. and Kenney, J. 2010 Large Whale Entanglement Report. Technical report, National Marine Fisheries Service, Gloucester, MA, 2011.

Muijres, F. T., Spedding, G. R., Winter, Y., and Hedenström, A. Actuator disk model and span efficiency of flapping flight in bats based on time-resolved PIV measurements. Experiments in Fluids, 51(2):511-525, 2011. doi: 10.1007/s00348-011-1067-5.

NARWC. North Atlantic Right Whale Consortium Database. New England Aquarium, Boston, MA, U.S.A., 2011.

NARWC. North Atlantic Right Whale Consortium Database, 18 Feb 2015. New England Aquarium, Boston, MA, U.S.A., 2015.

National Weather Service. Weather observations for the past three days - honolulu, honolulu international airport, 2015. URL http://w1. weather.gov/data/obhistory/PHNL.html.

Neilson, J. L., Straley, J. M., Gabriele, C. M., and Hills, S. Non-lethal entanglement of humpback whales (Megaptera novaeangliae) in fishing gear in northern Southeast Alaska. Journal of Biogeography, 36(3):452-464, 2009. doi: 10.1111/j.1365-2699.2007.01820.x.

Nerini, M. K., Braham, H. W., Marquette, W. M., and Rugh, D. J. Life history of the bowhead whale, Balaena mysticetus (mammalia: Cetacea). Journal of Zoology, 204(4): 443-468, 1984. ISSN 1469-7998. doi: 10.1111/j.1469-7998.1984.tb02381.x. URL http: //dx.doi.org/10.1111/j.1469-7998.1984.tb02381.x. 
NFWF. Status of humpback whales and human impacts. Report, National Fish and Wildlife Foundation. Submitted by Cascadia Research Collective, Olympia, WA 98501, 2007.

Nicol, C. J. The Behavioural Biology of Chickens. CABI, 2015. ISBN 9781780642499.

NMFS. Report of the workshop on large whale disentanglement: Learning from the past and moving towards the future. page 68, Boston, MA, 2003. National Marine Fisheries Service Northeast Regional Office.

NMFS. Process for distinguishing serious from non-serious injury of marine mammals. National Marine Fisheries Service Policy Directive PD 02-038. Department of Commerce, National Oceanic and Atmospheric Adminstration, National Marine Fisheries Service., page 4 pp. Available at: http://www.nmfs.noaa.gov/direc, 2012.

NOAA. Endangered Fish and Wildlife; Final Rule to Implement Speed Restrictions to Reduce the Threat of Ship Collisions with North Atlantic Right Whales. Federal Register, 73(198):60173-60191, 2008.

Noren, S. R., Redfern, J. V., and Edwards, E. F. Pregnancy is a drag: hydrodynamics, kinematics and performance in pre- and post-parturition bottlenose dolphins (Tursiops truncatus). Journal of Experimental Biology, 214(24 LB - Noren2011):4151-4159, 2011. doi: $10.1242 /$ jeb.059121.

Nousek-McGregor, A. E., Miller, C. A., Moore, M. J., and Nowacek, D. P. Effects of body condition on buoyancy in endangered North Atlantic right whales. Physiological and Biochemical Zoology, 87(1):160-171, 2013. doi: 10.1086/671811.

Nowacek, D. P., Johnson, M. P., Tyack, P. L., Shorter, K. A., McLellan, W. A., and Pabst, D. A. Buoyant balaenids: the ups and downs of buoyancy in right whales. Proceedings of the Royal Society B-Biological Sciences, 268(1478):1811-1816, 2001. doi: 10.1098/rspb. 2001.1730 .

Nudds, R. L., John, E. L., Keen, A. N., and Shiels, H. A. Rainbow trout provide the first experimental evidence for adherence to a distinct Strouhal number during animal oscillatory propulsion. Journal of Experimental Biology, 217(13):2244-2249, 2014. doi: $10.1242 /$ jeb.102236.

Ohashi, K., Thomson, J. D., and D'Souza, D. Trapline foraging by bumble bees: Iv. optimization of route geometry in the absence of competition. Behavioral Ecology, 18(1): 1-11, 2007. doi: 10.1093/beheco/arl053.

Øien, N. and Schweder, T. Estimates of bias and variability in visual distance measurements made by observers during shipboard surveys of northeastern Atlantic minke whales. Report of the International Whaling Commission, 42:407-412, 1992.

Opila-Correia, K. A. Kinematics of high-heeled gait with consideration for age and experience of wearers. Archives of Physical Medicine and Rehabilitation, 71(11):905-909, 1990.

Oritsland, N. A., Pasche, A. J., Markussen, N. H., and Ronald, K. Weight loss and catabolic adaptations to starvation in grey seal pups. Comparative Biochemistry and Physiology, 82A(4):931-933, 1985. 
Ortiz, R. M. Osmoregulation in marine mammals. Journal of Experimental Biology, 204: 1831-1844, 2001.

O'Shea, T. J. and Brownell, R. J. Organochlorine and metal contaminants in baleen whales: a review and evaluation of conservation implications. Science of The Total Environment, 154:179-200, 1994.

Pabst, D. A. Axial muscles and connective tissues of the bottlenose dolphin. In Leatherwood, J. S. and Reeves, R. R., editors, The Bottlenose Dolphin, pages 51-67. Academic Press, New York, 1990.

Pabst, D. A. To bend a dolphin: Convergence of force transmission designs in cetaceans and scombrid fishes. American Zoologist, 40:146-155, 2000.

Parks, S. E., Warren, J. D., Stamieszkin, K., Mayo, C. A., and Wiley, D. Dangerous dining: surface foraging of North Atlantic right whales increases risk of vessel collisions. Biology letters, 8(1):57-60, 2012. doi: 10.1098/rsbl.2011.0578.

Parry, D. A. The structure of whale blubber, and a discussion of its thermal properties. Quarterly Journal of Microscopical Science, 90(1):13-25, 1949.

Pavlov, D. S., Lupandin, A. I., and Skorobogatov, M. A. The effects of flow turbulence on the behavior and distribution of fish. Journal of Ichthyology, 40(2):S232-S261, 2000.

Pavlov, V. V. and Rashad, A. M. A non-invasive dolphin telemetry tag: computer design and numerical flow simulation. Marine Mammal Science, 28(1):E16-E27, 2012. doi: 10.1111/j.1748-7692.2011.00476.x.

Peck, H. E., Costa, D. P., Crocker, D. E., and Goldbogen, J. Body reserves influence allocation to immune responses in capital breeding female northern elephant seals. Functional Ecology, 30(3):389-397, 2016. doi: 10.1111/1365-2435.12504.

Perryman, W. L. and Lynn, M. S. Evaluation of nutritive condition and reproductive status of migrating gray whales (Echrichtius robustus) based on analysis of photogrammetric data. Journal of Cetacean Research and Management, 4(2):155-164, 2002.

Pettis, H. M., Rolland, R. M., Hamilton, P. K., Brault, S., Knowlton, A. R., and Kraus, S. D. Visual health assessment of North Atlantic right whales (Eubalaena glacialis) using photographs. Canadian Journal of Zoology, 82(1):8-19, 2004. doi: 10.1139/z03-207.

Pike, E. Tidal influence on diel movement of North Atlantic right whales (Eubalaena glacialis) in the Bay of Fundy. Thesis, 2008. URL http://dukespace.lib.duke.edu/ dspace/handle/10161/498.

Pircio, A. W., Gylys, J. A., Cavanagh, R. L., Buyniski, J. P., and Bierwagen, M. E. Pharmacology of butorphanol, a 3,14-dihydroxymorphinan narcotic-antagonist analgesic. Archives Internationales de Pharmacodynamie et de Therapie, 220:231-257, 1976.

Pivorunas, A. The Feeding Mechanisms of Baleen Whales. American Scientist, 67(4):432440, 1979.

Pond, C. M. Morphological aspects and the ecological and mechanical consequences of fat deposition in wild vertebrates. Annual Review of Ecology and Systematics, 9:519-570, 1978. 
Pond, C. M. Interactions between adipose tissue and the immune system. Proceedings of the Nutrition Society, 55:111-126, 1996.

Pond, C. M. and Mattacks, C. A. The distribution, cellular structure, and metabolism of adipose tissue in the fin whale, Balaenoptera physalus. Canadian Journal of Zoology, 66 (2):534-537, 1988. doi: 10.1139/z88-077.

Ponganis, P. J., Kooyman, G. L., Zornow, M. H., Castellini, M. A., and Croll, D. A. Cardiac output and stroke volume in swimming harbor seals. Journal of Comparative Physiology B, 160:473-482, 1990.

Potvin, J., Goldbogen, J. A., and Shadwick, R. E. Metabolic expenditures of lunge feeding rorquals across scale: implications for the evolution of filter feeding and the limits to maximum body size. PloS one, 7(9):e44854, 2012. doi: 10.1371/journal.pone.0044854.

Powell, J. R. and Wells, R. S. Recreational fishing depredation and associated behaviors involving common bottlenose dolphins (tursiops truncatus) in sarasota bay, florida. Marine Mammal Science, 27(1):111-129, 2011. ISSN 08240469. doi: 10.1111/j.1748-7692.2010. 00401.x.

Prandtl, L. Essentials of fluid dynamics: With applications to hydraulics aeronautics, meteorology, and other subjects. 1952 (various printings). Hafner Pub. Co., 1952.

Pullar, J. D. and Webster, A. J. F. The energy cost of fat and protein deposition in the rat. British Journal of Nutrition, 37:355-363, 1977.

Purves, P. E., Dudok van Heel, W. H., and Jonk, A. Locomotion in dolphins Part I: Hydrodynamic experiments on a model of the bottle-nosed dolphin, Tursiops truncatus, (Mont.). Aquatic Mammals, 3:5-31, 1975.

Rayleigh, L. On the resistance of fluids. Philosophical Magazine, 5(2):430-441, 1876.

Rayner, J. A vortex theory of animal flight. part 1. the vortex wake of a hovering animal. Journal of Fluid Mechanics, 91(4):697-730, 1979.

Rayner, J. On the aerodynamics of animal flight in ground effect. Philosophical transactions of the Royal Society of London. Series B, Biological sciences, 334:119-128, 1991.

Rea, L. D., Rosen, D. A. S., and Trites, A. W. Utilization of stored energy reserves during fasting varies by age and season in Steller sea lions. Canadian Journal of Zoology, 85(2): 190-200, 2007. doi: 10.1139/z06-204.

Read, A. J., Drinker, P., and Northridge, S. Bycatch of marine mammals in U.S. and global fisheries. Conservation Biology, 20(1):163-169, 2006. doi: 10.1111/j.1523-1739. 2006.00338.x.

Rechsteiner, E. Seasonal resting metabolic rate and food intake of captive Pacific whitesided dolphins (Lagenorhynchus obliquidens). Aquatic Mammals, 39(3):241-252, 2013. doi: 10.1578/am.39.3.2013.241.

Reves, J. G., Fragen, R. J., Vinik, H. R., and Greenblatt, D. J. Midazolam: pharmacology and uses. Anesthesiology, 62(3):310-324, 1985. 
Rice, D. W. and Wolman, A. A. The life history and ecology of the gray whale (Eschrichtius robustus). American Society of Mammalogy Special Publication, 3:1-142, 1971.

Ricklefs, R. E. and Wikelski, M. The physiology/life-history nexus. Trends in Ecology and Evolution, 17:462-468, 2002.

Ridoux, V., Guinet, C., Liret, C., Creton, P., Steenstrup, R., and Baeauplet, G. A video sonar as a new tool to study marine mammals in the wild: measurements of dolphin swimming speed. Marine Mammal Science, 13(2):196-206, 1997.

Robbins, J. Scar-based inference into Gulf of Maine humpback whale entanglement: 2010. Technical Report EA133F09CN0253, National Marine Fisheries Service, Northeast Fisheries Science Center, Woods Hole, MA, 2012.

Robbins, J. and Knowlton, A. R. Apparent survival of North Atlantic right whales after entanglement in fishing gear. Technical Report NA09OAR4320129, National Marine Fisheries Service, Northeast Fisheries Science Center, Woods Hole, MA, 2012.

Robbins, J., Barlow, J., Burdin, A. M., Calambokidis, J., Gabriele, C., Clapham, P. J., Ford, J., LeDuc, R., Mattila, D. K., Quinn, T., Rojas-Bracho, L., Straley, J., Urban, J., Wade, P. R., Weller, D. W., Witteveen, B. H., Wynne, K., and Yamaguchi, M. Comparison of humpback whale entanglement across the North Pacific Ocean based on scar evidence. Technical Report SC/59/BC15, to the Scientific Committee of the International Whaling Commission, 2007.

Robbins, J., Knowlton, A. R., and Landry, S. Apparent survival of North Atlantic right whales after entanglement in fishing gear. Biological Conservation, 191:421-427, 2015. doi: $10.1016 /$ j.biocon.2015.07.023.

Roberts, S. B. and Young, V. R. Energy costs of fat and protein deposition in the human infant. American Journal of Clinical Nutrition, 48:951-955, 1988.

Rodi, W. Experience with two-layer models combining the k-e model with a one-equation model near the wall. In 29th Aerospace Sciences Meeting AIAA 91-0216, Reno, NA, 1991.

Rohner, C. A., Richardson, A. J., Marshall, A. D., Weeks, S. J., and Pierce, S. J. How large is the world's largest fish? Measuring whale sharks Rhincodon typus with laser photogrammetry. Journal of Fish Biology, 78(1):378-385, 2011. doi: 10.1111/j.1095-8649. 2010.02861.x.

Rohr, J. J., Latz, M. I., Fallon, S., Nauen, J. C., and Hendricks, E. Experimental approaches towards interpreting dolphin-stimulated bioluminescence. Journal of Experimental Biology, 201:1447-1460, 1998.

Rohr, J. J., Fish, F. E., and Jr., G. J. W. Maximum swim speeds of captive and free-ranging delphinids: critical analysis of extraordinary performance. Marine Mammal Science, 18 (1):1-19, 2002.

Rolland, R. M., Schick, R. S., Pettis, H. M., Knowlton, A. R., Hamilton, P. K., Clark, J. S., and Kraus, S. D. Health of North Atlantic right whales (Eubalaena glacialis) over three decades: from individual health to demographic and population trends. Marine Ecology Progress Series, 542:265-282, 2016. doi: 10.3354/meps11547MARINE. 
Ropert-Coudert, Y. and Wilson, R. P. Subjectivity in bio-logging science: do logged data mislead? Memoirs of National Institute of Polar Research, 58:23-33, 2004.

Rosen, D. A. and Trites, A. W. Changes in metabolism in response to fasting and food restriction in the Steller sea lion (Eumetopias jubatus). Comparative Biochemistry and Physiology B, 132:389-399, 2002a.

Rosen, D. A. and Trites, A. W. Cost of transport in steller sea lions, Eumetopias jubatus. Marine Mammal Science, 18(2):513-524, 2002b.

Rosen, D. A. S., Volpov, B. L., and Trites, A. W. Short-term episodes of imposed fasting have a greater effect on young northern fur seals (Callorhinus ursinus) in summer than in winter. Conservation Physiology, 2(1):cou021, 2014. doi: 10.1093/conphys/cou021.

Rutz, C. and Hays, G. C. New frontiers in biologging science. Biology letters, 5(3):289-292, 2009. doi: $10.1098 / \mathrm{rsbl} .2009 .0089$.

Salvador, G. and Kenney, J. Large Whale Gear Research Summary. Technical report, 2002.

Sarwahi, V., Boachie-Adjei, O., Backus, S. I., and Taira, G. Characterization of gait function in patients with postsurgical sagittal (flatback) deformity: a prospective study of 21 patients. Spine (Phila Pa 1976), 27(21):2328-2337, 2002. doi: 10.1097/01.BRS.0000030304. 83145.01.

Sato, K., Shiomi, K., Watanabe, Y., Watanuki, Y., Takahashi, A., and Ponganis, P. J. Scaling of swim speed and stroke frequency in geometrically similar penguins: they swim optimally to minimize cost of transport. Proceedings of the Royal Society of London. Series B, Biological Sciences, 277(1682):707-714, 2010. doi: 10.1098/rspb.2009.1515.

Schevill, W. E. and Watkins, W. A. Radio-tagging of whales. Technical report, 1966.

Schick, R. S., Kraus, S. D., Rolland, R. M., Knowlton, A. R., Hamilton, P. K., Pettis, H. M., Kenney, R. D., and Clark, J. S. Using hierarchical Bayes to understand movement, health, and survival in the endangered North Atlantic right whale. PLoS ONE, 8(6):e64166, 2013. doi: 10.1371/journal.pone.0064166.g001.

Schmidt-Nielsen, K. Locomotion: Energy cost of swimming, flying and running. Science, $177: 222-228,1972$

Schmidt-Nielsen, K. Animal Physiology: Adaptation and Environment. Cambridge University Press, 1997. ISBN 9780521570985.

Schutz, Y., Weinsier, R. L., and Hunter, G. R. Assessment of free-living physical activity in humans: an overview of currently available and proposed new measures. Obesity Research, 9(6):368-379, 2001.

Selinger, J. C., O'Connor, S. M., Wong, J. D., and Donelan, J. M. Humans can continuously optimize energetic cost during walking. Current Biology, 25(18):2452-2456, 2015. doi: 10.1016/j.cub.2015.08.016.

Semmlow, J. Signals and systems for bioengineers: a MATLAB-based introduction. Academic Press, Waltham, MA, 2nd editio edition, 2012. 
Setsaas, T. H. and Bester, M. N. Goose barnacle (Lepas australis) infestation of the Subantarctic fur seal (Arctocephalus tropicalis). African Zoology, 41(2):305-307, 2006. doi: 10.3377/1562-7020(2006)41[305:gblaio]2.0.co;2.

Shaffer, S. A., Costa, D. P., Williams, T. M., and Ridgway, S. H. Diving and swimming performance of white whales, Delphinapterus leucas: an assessment of plasma lactate and blood gas levels and respiratory rates. Journal of Experimental Biology, 200:3091-3099, 1997.

Shane, S. H. Behavior and ecology of the bottlenose dolphin at Sanibel Island, Florida. In Leatherwood, J. S. and Reeves, R. R., editors, The Bottlenose Dolphin, pages 245-265. Academic Press, New York, London, 1990.

Shaw, A. K. and Levin, S. A. To breed or not to breed: a model of partial migration. Oikos, 120(12):1871-1879, 2011. ISSN 00301299. doi: 10.1111/j.1600-0706.2011.19443.x.

Shimojo, H., Sengoku, Y., Miyoshi, T., Tsubakimoto, S., and Takagi, H. Effect of imposing changes in kick frequency on kinematics during undulatory underwater swimming at maximal effort in male swimmers. Human Movement Science, 38C:94-105, 2014. doi: 10.1016/j.humov.2014.09.001.

Shorter, K. A., Murray, M. M., Johnson, M., Moore, M. J., and Howle, L. E. Drag of suction cup tags on swimming animals: Modeling and measurement. Marine Mammal Science, 30:726-746, 2013. doi: 10.1111/mms.12083LB-Shorter2013.

Simon, M., Johnson, M., Tyack, P., and Madsen, P. T. Behaviour and kinematics of continuous ram filtration in bowhead whales (Balaena mysticetus). Proceedings of the Royal Society of London. Series B, Biological Sciences, 276(1674):3819-3828, 2009. doi: 10.1098/rspb.2009.1135.

Simon, M., Johnson, M., and Madsen, P. T. Keeping momentum with a mouthful of water: behavior and kinematics of humpback whale lunge feeding. Journal of Experimental Biology, 215(21):3786-3798, 2012. doi: 10.1242/jeb.071092.

Skrovan, R. C., Williams, T. M., Berry, P. S., Moore, P. W., and Davis, R. W. The diving physiology of bottlenose dolphins (Tursiops truncatus). II. Biomechanics and changes in buoyancy at depth. Journal of Experimental Biology, 202(20 LB - Skrovan1999):27492761, 1999.

Slip, D. J., Gales, N. J., and Burton, H. R. Body mass loss, utilization of blubber and fat, and energetic requirements of male southern elephant seals, Mirounga leonina, during the moulting fast. Australian Journal of Zoology, 40:235-243, 1992.

Sparrow, W. A. and Newell, K. M. Metabolic energy expenditure and the regulation of movement economy. Psychonomic Bulletin 85 Review, 5(2 LB - Sparrow1998):173-196, 1998. doi: 10.3758/bf03212943.

Speakman, J. R. and Krol, E. Limits to sustained energy intake. xiii. recent progress and future perspectives. Journal of Experimental Biology, 214(2):230-241, 2010. ISSN 00220949 1477-9145. doi: 10.1242/jeb.048603.

STAR-CCM+. User Guide. CD-adapco, 2014. 
Stelle, L. L., Blake, R. W., and Trites, A. W. Hydrodynamic drag in Steller sea lions (Eumetopias jubatus). Journal of Experimental Biology, 203:1915-1923, 2000.

Struntz, D. J., McLellan, W. A., Dillaman, R. M., Blum, J. E., Kucklick, J. R., and Pabst, D. A. Blubber development in bottlenose dolphins (Tursiops truncatus). Journal of Morphology, 259(1):7-20, 2004. doi: 10.1002/jmor.10154.

Suzuki, I., Sato, K., Fahlman, A., Naito, Y., Miyazaki, N., and Trites, A. W. Drag, but not buoyancy, affects swim speed in captive Steller sea lions. Biology Open, 0:1-8, 2014. doi: 10.1242 /bio. 20146130 .

Szabo, A. and Duffus, D. Mother-offspring association in the humpback whale, Megaptera novaeangliae: following behaviour in an aquatic mammal. Animal Behaviour, 75(3):10851092, 2008. ISSN 00033472. doi: 10.1016/j.anbehav.2007.08.019.

Taber, S. M. and Thomas, P. O. Calf development and mother-calf spatial relationships in southern right whales. Animal Behaviour, 30:1072-1083, 1982.

Taylor, C. R., Karas, R. H., Weibel, E. R., and Hoppeler, H. Adaptive variation in the mammalian respiratory system in relation to energetic demand: II. Reaching the limits to oxygen flow. Respiration Physiology, 69:7-26, 1987.

Thomas, P. O. and Taber, S. M. Mother-infant interaction and behavioral development in southern right whales, Eubalaena australis. Behaviour, 88(1):42-60, 1984.

Tomlinson, S., Arnall, S. G., Munn, A., Bradshaw, S. D., Maloney, S. K., Dixon, K. W., and Didham, R. K. Applications and implications of ecological energetics. Trends in Ecology and Evolution, 29(5):280-290, 2014. doi: 10.1016/j.tree.2014.03.003.

Triantafyllou, G. S., Triantafyllou, M. S., and Grosenbaugh, M. A. Optimal thrust development in oscillating foils with application to fish propulsion. Journal of Fluids and Structures, 7(2):205-224, 1993. doi: http://dx.doi.org/10.1006/jfls.1993.1012.

Triantafyllou, M. S., Triantafyllou, G. S., and Yue, D. K. P. Hydrodynamics of Fishlike Swimming. Annual Review of Fluid Mechanics, 32(1):33-53, 2000. doi: doi:10.1146/ annurev.fluid.32.1.33.

Tucker, V. A. Energetic cost of locomotion in animals. Comparative Biochemistry and Physiology, 34(4):841-846, 1970.

Tudorache, C., Burgerhout, E., Brittijn, S., and van den Thillart, G. The effect of drag and attachment site of external tags on swimming eels: experimental quantification and evaluation tool. PLoS One, 9(11):e112280, 2014. doi: 10.1371/journal.pone.0112280.

Ungerechts, B. E., Daly, D., and Zhu, J. P. What dolphins tell us about hydrodynamics. Journal of Swimming Research, 13:1-7, 1998.

Urban, J., Flores de Sahagun, V., Jones, M. L., Swartz, S. L., Mate, B., Gomez-Gallardo, A., and Guerrero-Ruiz, M. Gray whales with loss of flukes adapt and survive. Marine Mammal Science, 20(2):335-338, 2004. 
van der Hoop, J. M., Fahlman, A., Hurst, T., Rocho-Levine, J., Shorter, K. A., Petrov, V., and Moore, M. J. Bottlenose dolphins modify behavior to reduce metabolic effect of tag attachment. Journal of Experimental Biology, 217:4229-4236, 2014a. doi: 10.1242/jeb. 108225 .

van der Hoop, J. M., Vanderlaan, A. S. M., and Taggart, C. T. Absolute probability estimates of lethal vessel strikes to North Atlantic right whales in Roseway Basin, Scotian Shelf. Ecological Applications, 22(7):2021-2033, 2012. doi: 10.1890/11-1841.1.

van der Hoop, J. M., Moore, M. J., Barco, S. G., Cole, T. V., Daoust, P. Y., Henry, A. G., McAlpine, D. F., McLellan, W. A., Wimmer, T., and Solow, A. R. Assessment of management to mitigate anthropogenic effects on large whales. Conservation Biology, 27 (1):121-133, 2013a. doi: 10.1111/j.1523-1739.2012.01934.x.

van der Hoop, J. M., Moore, M. J., Fahlman, A., Bocconcelli, A., George, C., Jackson, K., Miller, C., Morin, D., Pitchford, T., Rowles, T., Smith, J., and Zoodsma, B. Behavioral impacts of disentanglement of a right whale under sedation and the energetic cost of entanglement. Marine Mammal Science, 30:282-307, 2013b. doi: 10.1111/mms.12042.

van der Hoop, J. M., Vanderlaan, A. S. M., Cole, T. V. N., Henry, A. G., Hall, L., MaseGuthrie, B., Wimmer, T., and Moore, M. J. Vessel strikes to large whales before and after the 2008 Ship Strike Rule. Conservation Letters, 8:24-32, 2014b. doi: 10.1111/conl.12105.

van der Hoop, J. M., Corkeron, P., Kenney, J., Landry, S., Morin, D., Smith, J., and Moore, M. J. Drag from fishing gear entangling North Atlantic right whales. Marine Mammal Science, 32(2):619-642, 2015. doi: 10.1111/mms.12292.

van Es, A. J. H. The energetics of fat deposition during growth. Nutrition and Metabolism, 21:88-104, 1977.

Vandenabeele, S. P., Shepard, E. L., Grogan, A., and Wilson, R. P. When three per cent may not be three per cent; device-equipped seabirds experience variable flight constraints. Marine Biology, 159(1):1-14, 2011. doi: 10.1007/s00227-011-1784-6.

Vandenabeele, S. P., Shepard, E. L. C., Gremillet, D., Butler, P. J., Martin, G. R., and Wilson, R. P. Are bio-telemetric devices a drag? Effects of external tags on the diving behaviour of great cormorants. Marine Ecology Progress Series, 519:239-249, 2015. doi: 10.3354/meps11058.

Vanderlaan, A. S. M. and Taggart, C. T. Estimating risk to the North Atlantic right whale (Eubalaena glacialis) from ocean-going vessels and fishing gear. PhD thesis, Dalhousie University, Halifax, Nova Scotia, 2010.

Videler, J. J. and Kammermans, P. Differences between upstroke and downstroke in swimming dolphins. Journal of Experimental Biology, 119:265-274, 1985.

Videler, J. J. and Weihs, D. Energetic advantages of burst-and-coast swimming of fish at high speeds. Journal of Experimental Biology, 97:169-178, 1982.

Villegas-Amtmann, S., Schwarz, L. K., Sumich, J. L., and Costa, D. P. A bioenergetics model to evaluate demographic consequences of disturbance in marine mammals applied to gray whales. Ecosphere, 6(10):art183, 2015. doi: 10.1890/es15-00146.1. 
Vogel, S. Life in Moving Fluids: the physical biology of flow. Princeton University Press, Princeton, New Jersey, 2nd edition, 1994.

Volgenau, L., Kraus, S. D., and Lien, J. The impact of entanglements on two substocks of the western North Atlantic humpback whale, Megaptera novaeangliae. Canadian Journal of Zoology, 73:1679-1698, 1995.

Wahl, M. Marine epibiosis. I. Fouling and antifouling: some basic aspects. Marine Ecology Progress Series, 58:175-189, 1989.

Wahl, M. Fouled snails in flow: potential of epibionts on Littorina littorea to increase drag and reduce snail growth rates. Marine Ecology Progress Series, 138:157-168, 1996.

Wang, T., Hung, C. C., and Randall, D. J. The comparative physiology of food deprivation: from feast to famine. Annu Rev Physiol, 68:223-251, 2006. doi: 10.1146/annurev.physiol. 68.040104.105739.

Ware, C., Trites, A. W., Rosen, D. A., and Potvin, J. Averaged Propulsive Body Acceleration (APBA) can be calculated from biologging tags that incorporate gyroscopes and accelerometers to estimate swimming speed, hydrodynamic drag and energy expenditure for steller sea lions. PLoS One, 11(6):e0157326, 2016. doi: 10.1371/journal.pone.0157326.

Warnes, G. R., Bolker, B., Bonebakker, L., Gentleman, R., Huber, W., Liaw, A., Lumley, T., Maechler, M., Magnusson, A., Moeller, S., and Schwartz, M. gplots: Various $\mathrm{R}$ programming tools for plotting data. $R$ package version 2.14.2. http://CRAN.Rproject.org $/$ package $=$ gplots, 2014.

Wasser, S. K. and Barash, D. P. Reproductive suppression among female mammals: Implications for biomedicine and sexual selection theory. Quarterly Review of Biology, 58(4): 513-538, 1983.

Watkins, W. A. and Schevill, W. E. The development and testing of a radio whale tag. Technical Report DTIC Document ADA044807, Office of Naval Research, Arlington, VA, 1977.

Watson, K. P. and Granger, R. A. Hydrodynamic effect of a satellite transmitter on a juvenile green turtle (Chelonia mydas). Journal of Experimental Biology, 201:2497-2505, 1998.

Webb, P. M. Efficiency of pectoral-fin propulsion of Cymatogaster aggregata. In Wu, T. Y., Brokaw, C. J., and Brennen, C., editors, Swimming and Flying in Nature, volume 2, pages 573-584. Plenum Press, New York, 1975a.

Webb, P. M., Crocker, D. E., Blackwell, S. B., Costa, D. P., and Le Boeuf, B. J. Effects of buoyancy on the diving behavior of northern elephant seals. Journal of Experimental Biology, 201:2349-23585, 1998.

Webb, P. W. Kinematics of Pectoral Fin Propulsion in Cymatogaster Aggregata. Journal of Experimental Biology, 59(3):697-710, 1973.

Webb, P. W. Hydrodynamics and energetics of fish propulsion. Bulletin of the Fisheries Research Board of Canada, 190:1-158, 1975b. 
Webb, P. W. The effect of solid and porous channel walls on steady swimming of steelhead trout, oncorhynchus mykiss. Journal of Experimental Biology, 178:97-108, 1993.

Weber, P. W., Howle, L. E., Murray, M. M., and Fish, F. E. Lift and drag performance of odontocete cetacean flippers. Journal of Experimental Biology, 212(14):2149-2158, 2009. doi: $10.1242 /$ jeb.029868.

Wegner, N. C. and Cartamil, D. P. Effects of prolonged entanglement in discarded fishing gear with substantive biofouling on the health and behavior of an adult shortfin mako shark, Isurus oxyrinchus. Marine Pollution Bulletin, 64(2):391-394, 2012. doi: 10.1016/ j.marpolbul.2011.11.017.

Weihs, D. Tidal stream transport as an efficient method for migration. Journal du Conseil / Conseil Permanent International pour l'Exploration de la Mer, 38(1):92-99, 1978.

Weimerskirch, H., Guionnet, T., Martin, J., Shaffer, S. A., and Costa, D. P. Fast and fuel efficient? Optimal use of wind by flying albatrosses. Proceedings of the Royal Society B Biological Sciences, 267(1455):1869-1874, 2000. doi: 10.1098/rspb.2000.1223.

Weiner, J. Physiological limits to sustainable energy budgets in birds and mammals: ecological implications. Trends in Ecology and Evolution, 7(11):384-388, 1992.

Weisbrod, A., Shea, D., Leblanc, G., Moore, M., and Stegeman, J. J. Organochlorine bioaccumulation and risk for whales in a northwest Atlantic food web. Marine Environmental Research, 50(1):440-441, 2000. doi: http://dx.doi.org/10.1016/S0141-1136(00)00216-6.

Wells, R. S., McHugh, K. A., Douglas, D. C., Shippee, S., McCabe, E. B., Barros, N. B., and Phillips, G. T. Evaluation of potential protective factors against metabolic syndrome in bottlenose dolphins: feeding and activity patterns of dolphins in sarasota bay, florida. Frontiers in Endocrinology, 4:139, 2013. doi: 10.3389/fendo.2013.00139.

Wells, R. S., Hoffmann, S., and Moors, T. L. Entanglement and mortality of bottlenose dolphins, Tursiops truncatus, in recreational fishing gear in Florida. Fishery Bulletin, 96: 647-650, 1998.

Wells, R. S., Allen, J. B., Hofmann, S., Bassos-Hull, K., Fauquier, D. A., Barros, N. B., DeLynn, R. E., Sutton, G., Socha, V., and Scott, M. D. Consequences of injuries on survival and reproduction of common bottlenose dolphins (Tursiops truncatus) along the west coast of Florida. Marine Mammal Science, 24:774-794, 2008. doi: 10.1111/j.1748-7692.2008.00212.x.

Wells, R. S., Allen, J. B., Lovewell, G., Gorzelany, J., Delynn, R. E., Fauquier, D. A., and Barros, N. B. Carcass-recovery rates for resident bottlenose dolphins in Sarasota Bay, Florida. Marine Mammal Science, 31:355-368, 2014. doi: 10.1111/mms.12142.

West, G. C. Seasonal variation in the energy balance of the tree sparrow in relation to migration. The Auk, 77(3):306-329, 1960.

Wickler, S. J., Hoyt, D., Cogger, E. A., and Hirschbein, M. H. Preferred speed and cost of transport: the effect of incline. The Journal of Experimental Biology, 203:2185-2200, 2000 . 
Williams, R., Vikingsson, G. A., Gislason, A., Lockyer, C., New, L., Thomas, L., and Hammond, P. S. Evidence for density-dependent changes in body condition and pregnancy rate of North Atlantic fin whales over four decades of varying environmental conditions. ICES Journal of Marine Science, 70(6):1273-1280, 2013. doi: 10.1093/icesjms/fst059.

Williams, R., Gero, S., Bejder, L., Calambokidis, J., Kraus, S. D., Lusseau, D., Read, A. J., and Robbins, J. Underestimating the damage: interpreting cetacean carcass recoveries in the context of the Deepwater Horizon/BP incident. Conservation Letters, 4(3):228-233, 2011. doi: 10.1111/j.1755-263X.2011.00168.x.

Williams, T. M. Approaches for the study of exercise physiology and hydrodynamics in marine mammals. In Huntley, A. C., Costa, D. P., Worthy, G. A. J., and Castellini, M. A., editors, Approaches to Marine Mammal Energetics, chapter 9, pages 127-145. Allen Press, Lawrence, KS, 1987.

Williams, T. M. Swimming by sea otters: adaptations for low energetic cost locomotion. Journal of Comparative Physiology A, 164:815-824, 1989.

Williams, T. M. The evolution of cost efficient swimming in marine mammals: limits to energetic optimization. Philosophical Transactions of the Royal Society of London. Series B: Biological Sciences, 354:193-201, 1999. doi: 10.1098/rstb.1999.0371.

Williams, T. M. Intermittent swimming by mammals: a strategy for increasing energetic efficiency during diving. American Zoologist, 41:166-176, 2001.

Williams, T. M. and Kooyman, G. L. Swimming performance and hydrodynamic characteristics of harbor seals Phoca vitulina. 58(5):576-589, 1985.

Williams, T. M., Friedl, W. A., and Haun, J. E. L. B. W. The physiology of bottlenose dolphins (Tursiops truncatus): heart rate, metabolic rate and plasma lactate concentration during exercise. Journal of Experimental Biology, 179:31-46, 1993.

Willis, K., Horning, M., Rosen, D. A. S., and Trites, A. W. Spatial variation of heat flux in Steller sea lions: evidence for consistent avenues of heat exchange along the body trunk. Journal of Experimental Marine Biology and Ecology, 315(2):163-175, 2005. doi: 10.1016/j.jembe.2004.09.018.

Wilson, R. P. and McMahon, C. R. Measuring devices on wild animals: what constitutes acceptable practice? Frontiers in Ecology and the Environment, 4(3):147-154, 2006.

Wilson, R. P., Kreye, J. M., Lucke, K., and Urquhart, H. Antennae on transmitters on penguins: balancing energy budgets on the high wire. Journal of Experimental Biology, 207(15):2649-2662, 2004. doi: 10.1242/jeb.01067.

Wilson, R. P., White, C. R., Quintana, F., Halsey, L. G., Liebsch, N., Martin, G. R., and Butler, P. J. Moving towards acceleration for estimates of activity-specific metabolic rate in free-living animals: the case of the cormorant. Journal of Animal Ecology, 75(5): 1081-1090, 2006. doi: 10.1111/j.1365-2656.2006.01127.x.

Wilson, R. P., Shepard, E. L. C., and Liebsch, N. Prying into the intimate details of animal lives: use of a daily diary on animals. Endangered Species Research, 4:123-137, 2008. doi: 10.3354/esr00064. 
Wilson, R. S., Niehaus, A. C., Gwendolyn, D., Hunter, A., and Smith, M. Does individual quality mask the detection of performance trade-offs? A test using analyses of human physical performance. Journal of Experimental Biology, 217:545-551, 2014a. doi: 10. 1242/jeb.092056.

Wilson, R. P., Grant, W. S., and Duffy, D. C. Recording Devices on Free-Ranging Marine Animals: Does Measurement Affect Foraging Performance? Ecology, 67(4):1091-1093, 1986. doi: $10.2307 / 1939832$.

Wilson, R. P., Grundy, E., Massy, R., Soltis, J., Tysse, B., Holton, M., Cai, Y., Parrott, A., Downey, L. A., Qasem, L., and Butt, T. Wild state secrets: ultra-sensitive measurement of micro-movement can reveal internal processes in animals. Frontiers in Ecology and the Environment, 12(10):582-587, 2014b. doi: 10.1890/140068.

Wingfield, J. C., Maney, D. L., Breuner, C. W., Jacobs, J. D., Lynn, S., Ramenofsky, M., and Richardson, R. D. Ecological bases of hormone-behavior interactions: the "emergency life history stage". American Zoologist, 38:191-206, 1998.

Winn, J. P., Woodward, B. L., Moore, M. J., Peterson, M. L., and Riley, J. G. Modeling whale entanglement injuries: An experimental study of tissue compliance, line tension, and draw-length. Marine Mammal Science, 24(2):326-340, 2008. doi: 10.1111/j.1748-7692. 2008.00184.x.

Woodley, T. H., Brown, M. W., Kraus, S., and Gaskin, D. E. Organochlorine levels in North Atlantic right whale (Eubalaena glacialis) blubber. Archives of Environmental Contamination and Toxicology, 21:141-145, 1991.

Woodward, B. L. Locomotory strategies, dive dynamics, and functional morphology of the mysticetes: Using morphometrics, osteology, and Dtag data to compare swim performance in four species of baleen whales. PhD thesis, University of Maine, Orono, ME, 2006.

Woodward, B. L., Winn, J. P., and Fish, F. E. Morphological specializations of baleen whales associated with hydrodynamic performance and ecological niche. Journal of Morphology, 267(11):1284-1294, 2006a. doi: 10.1002/jmor.10474.

Woodward, B. L., Winn, J. P., Moore, M. J., and Peterson, M. L. Development of a towed telemetry tag for tracking the long term movements of right whales at sea. Technical Report NA04NMF4720400, Woods Hole, MA, 2006b.

Worthy, G. A. J. and Lavigne, D. M. Mass loss, metabolic rate, and energy utilization by harp and gray seal pups during the postweaning fast. Physiological Zoology, 60(3): 352-364, 1987.

Wright, I. A., Rhind, S. M., Whyte, T. K., and Smith, A. J. Effects of body condition at calving and feeding level after calving on LH profiles and the duration of the post-partum anoestrous period in beef cows. Animal Science, 55(01):41-46, 1992.

Wursig, B. Radio tracking dusky porpoises in the South Atlantic. Mammals in the seas, 4: 145-160, 1982.

Wursig, B. and Wursig, M. Behavior and ecology of the bottlenose dolphin, Tursiops truncatus, in the south atlantic. Fishery Bulletin, 77(2):399-412, 1979. 
Yanov, V. G. Effect of additional loading on kinematics and hydrodynamics of dolphins. Biophysics, 46(3):563-572, 2001.

Yazdi, P., Kilian, A., and Culik, B. M. Energy expenditure of swimming bottlenose dolphins (Tursiops truncatus). Marine Biology, 134:601-607, 1999.

Young, R. A. Fat, Energy and Mammalian Survival. American Zoologist, 16:699-710, 1976.

Zamparo, P., Pendergast, D. R., Termin, B., and Minetti, A. E. How fins affect the economy and efficiency of human swimming. Journal of Experimental Biology, 205:2665-2676, 2002.

Zollikofer, C. P. E. Stepping patterns in ants III. Influence of load. Journal of Experimental Biology, 192:119-127, 1994. 